\title{
A Search for Weakly Interacting Particles with the Cryogenic Dark Matter Search Experiment
}

\author{
Dissertation \\ zur \\ Erlangung der naturwissenschaftlichen Doktorwürde \\ (Dr. sc. nat.) \\ vorgelegt der \\ Mathematisch-naturwissenschaftlichen Fakultät \\ der \\ Universität Zürich \\ Tobias Bruch
aus
Deutschland \\ Promotionskomitee \\ Prof. Dr. Laura Baudis (Vorsitz) \\ Prof. Dr. Claude Amsler \\ Dr. Eirini Tziaferi
}

Zürich 2010 



\section{Zusammenfassung}

In den letzten Jahrzenten haben kosmologische Beobachtungen zu einem stimmigen Modell des Universums geführt in dem 85\% der Materie nicht baryonisch, nicht leuchtend und nicht relativistisch zur Zeit der Strukturbildung war. Theorien jenseits des Standardmodells der Teilchenphysik liefern eine Fülle an Kandidaten für diese ungesehene "Dunkle Materie". Schwachwechselwirkende massive Teilchen (weakly interacting massive particles; WIMPs) sind eine Klasse von Kandidaten, die durch die thermische Produktion im frühen Universum motiviert werden. WIMPs (oder jeglicher andere Kandidat für Dunkle Materie), die in einem die Galaxie einschliessenden spherischen und isothermen Halo (das Standard Halo Model; SHM) verteilt sind, können in erdgebundenen Detektoren nachgewiesen werden.

Im Standardmodell der Entstehung von Scheibengalaxien werden massive Satteliten bevorzugt in die Galaxieebene gezogen, in der sie sich auflösen und dadurch eine Dunkle Materie Scheibe (Dark Disc) ensteht. Die in Bezug auf die Erde geringe Geschwindigkeit, von Dunkle Materie Teilchen in dieser Scheibe verstärkt die Detektionsraten bei niedrigen Rückstossenergien in direkten Detektionsexperimenten. Für WIMP Massen $\gtrsim 50 \mathrm{GeV} / \mathrm{c}^{2}$ und einen Energiebereich von $5-20 \mathrm{keV}$ sind die Detektionsraten bis zu einem Faktor 3 größer. Der Vergleich dieser Raten mit den Raten bei höheren Energien ist vermutlich sensitiv zur WIMP Masse. Dies erlaubt eine bessere Bestimmung der Masse insbesondere für Massen $\gtrsim 100 \mathrm{GeV} / \mathrm{c}^{2}$. Zusätzlich wird das jährliche Modulationssignal signifikant verstärkt und die Phase der Modulation ist relativ zum Halo um $\sim 3$ Wochen versetzt. Die Variation der gemessen Phase mit der Rückstossenergie ermöglicht die Bestimmung der Masses des Teilchens, wenn die Parameter der Dark Disc durch zukünftige astronomische Beobachtungen bestimmt wurden.

Dunkle Materie Teilchen können nicht nur direkt in terrestrischen Laboratorien detektiert werden, sondern auch indirekt durch die Detektion der aus der Annihilation stammenden Teilchen. Frühere Vorhersagen des Neutrino Flusses aus WIMP Annihilationen in der Erde und der Sonne basierten auf der Annhame, dass die Dunkle Materie entsprechend dem SHM verteilt ist. Obwohl die lokale Dichte der Dark Disc vergleichbar zur Dichte des Halo ist, führt die höhere Phasenraumdichte bei niedrigen Geschwindigkeiten zu einer enormen Steigerung der Einfangraten in der Sonne und Erde. Für typische Parameter der Dark Disc sind die aus der Erde stammenden Muonflüsse um drei Größenordungen höher als die vom Halo vorhergesagten Flüsse. Für die Sonne sind die resultierneden Flüsse um eine Größenordnung höher. Diese erhöhten Flüsse verbessern die Aussichten für Neutrino Teleskope WIMP Parameter zu messen oder einzuschränken signifikant. Der aus der Erde stammende Fluss ist stark abhängig von den gewählten Parametern der Dark Disk, während die Vorhersagen für die Sonne sehr robust sind.

Das Cryogenic Dark Matter Search (CDMS) Experiment sucht nach den seltenen Wechselwirkungen der im Halo verteilten WIMPs mit erdgebundenen Atomkernen. Das CDMS 
Experiment befinded sich im Soudan Underground Laboratory im nördlichen Minnesota und benutzt Halbleiterkristalle die auf Millikelvin Temperaturen gekühlt werden. Die Detektoren sind zur Auslese des Ionisations und des athermischen Phononsignals einer Wechselwirkung in den Kristallen instrumentiert. Die gleichzeitige Messung beider Signale ermöglichte eine hervorragende Diskriminierung von Wechselwirkungen die hauptsächlich von Teilchen aus radioaktiven Zerfällen stammen. Diese Ereignisse sind Untergrundereignisse in der Suche nach Kernrückstössen die von WIMP Wechselwirkungen verursacht werden. Eine detaillierte Studie des Untergrundes führte zu einem Modell, das den gemessenen elektromagnetischen Untergrung erklären kann, und eine verlässliche Vorhersage des radiogenen Neutronen Untergrundes ermöglicht.

Die Analyse zur Suche nach Kernrückstossen von WIMP Wechselwirkungen resultierte in der Detektion von zwei Kandidaten in der Signalregion. Basierend auf dem erwarteten Untergrund für diese Analyse beträgt die Wahrscheinlichkeit zwei oder mehr Ereigniskandidaten zu detektieren 23\%. Aufgrund dieser hohen Wahrscheinlichkeit kann das Resultat dieser Analyse nicht als statistisch signifikante Detektion von WIMP Wechselwirkungen gedeutet werden. Die Daten setzen eine obere Grenze bei einem 90\% Konfidenzlevel auf den WIMP-Nucleon Spin-unabhängigen Wirkungsquerschnitt von $7.0 \times 10^{-44} \mathrm{~cm}^{2}$ für eine WIMP Masse von $70 \mathrm{GeV} / \mathrm{c}^{2}$. Die Kombination dieser Ergebnisse mit allen vorherigen Datensätzen des CDMS-II Experimentes resultiert in einer oberen Grenze auf den WIMPNucleon Spin-unabhängigen Wirkungsquerschnitt von $3.8 \times 10^{-44} \mathrm{~cm}^{2}$ für eine WIMP Masse von $70 \mathrm{GeV} / \mathrm{c}^{2}$. Dieses kombinierte Resultat setzt die weltweit führenden oberen Grenzen auf den WIMP-Nucleon Spin-unabhängigen Wirkungsquerschnitt für WIMP Massen oberhalb von $\sim 44 \mathrm{GeV} / \mathrm{c}^{2}$. Zusätzlich schliesst dieses Resultat Parameterraum des Inelastischen Dunkle Materie Modelles aus, welches die Diskrepanz zwischen der Detektion des jährlichen Modulationssignals im DAMA Experiment und den Nullresultaten anderer direkter Detektionsexperimente erklären könnte.

Das Axion, welches postuliert wurde um das starke-CP Problem der Quantenchromodynamik zu lösen, könnte in der Sonne produziert werden. Diese solaren Axionen könnten in Kristalldetektoren durch die Primakov-Konversion zu Photonen detektiert werden. Die Suche nach solaren Axion, die in dieser Arbeit vorgestellt wird, wurde zum ersten Mal mit dem CDMS Experiment durchgeführt. Das Erreichen einer Energieschwelle von $2 \mathrm{keV}$ für Elektronrückstösse ermöglichte die Suche nach der Konversion von solaren Axionen in Photonen in den Germaniumkristalldetektoren. In dieser Analyse konnten keine Konversionen von solaren Axionen nachgewiesen werden. Somit konnte einen obere Grenze auf die Primakov-Kopplung $g_{a \gamma \gamma}$ von $2.4 \times 10^{-9} \mathrm{GeV}^{-1}$ mit einem Konfidenzlevel von $95 \%$ für Axionmassen unterhalb von $0.1 \mathrm{keV} / \mathrm{c}^{2}$ gesetzt werden. Diese obere Grenze ist die erste basierend auf einer präzisen Kenntnis der Orientierung der Kristallachsen in Kristallbasierten Experimenten die nach solaren Axionen suchen.

Die Analyse des niederenergetischen Elektronrückstoss Spektrums des CDMS Experimtes in einem Energiebereich von $2-8.5 \mathrm{keV}$ bestimmte die detektierte Ereignissrate und lieferte Details über mögliche Untergrundquellen. In der Analyse konnte kein signifikanter Hinweis eines in der Energie lokalisierten Beitrages zur detektierten Rate über dem angenommen Untergrundmodell nachgewiesen werden. Die oberen Grenzen auf einen 
zusätzlichen Beitrag zur beobachteten Ereignissrate ermöglichen eine Einschränkung der Kopplung von pseudoskalaren Dunkle Materie Teilchen, wie dem Axion, die im Halo verteilt sind. Die Analyse setzt eine weltweit führende, experimentelle obere Grenze auf die axioelektrische Kopplung $g_{a \bar{e} e}$ von $1.4 \times 10^{-12}$ mit einem $90 \%$ Konfidenzelevel für eine Axionmasse von $2.5 \mathrm{keV} / \mathrm{c}^{2}$. Dieses Resultat schliesst die Interpretation des von DAMA detektierten jährlichen Modulationssignals aufgrund der Wechselwirkungen von Axionen für Axionmassen grösser als $1.4 \mathrm{keV} / \mathrm{c}^{2}$ aus. In einem allgemeinen Modell der Konversion eines Dunkle Materie Teilchens in elektromagnetische Energie ist die, bei einem Konfidenzlevel von $90 \%$ gesetzte, obere Grenze von 0.246 events $/ \mathrm{kg} /$ day bei $3.15 \mathrm{keV}$ geringer als die totale von DAMA über dem Untergrund detektierte Ereignissate. Da kein entsprechendes Teilchenmodell existiert, welches die Skalierung des Wirkungsquerschnittes in NaI und Ge vorgeben würde, wird eine $Z^{2}$ Skalierung angenommen. Mit dieser Annahme bleibt die von DAMA detektierte Ereignissrate höher als die von CDMS gesetzte obere Grenze. Unter der konservativen Annahme, dass die Modulationsamplitude 6\% der totalen Rate beträgt, können obere Grenzen auf die Modulationsamplitude gesetzt werden, die einen Faktor $\sim 2$ geringer sind als die von DAMA beobachtete Modulationsamplitude. Dies ermöglicht die Einschränkung von möglichen Erklärungen dieser Modulation. Eine direkte Suche nach einem modulierten Beitrag zur Ereignissrate im niederenergie Spektrum des CDMS Experimentes setzt eine obere Grenze auf die Modulationsamplitude die noch keine ausreichende Sensitivität hat um die von DAMA aufgestellte Behauptung zu überprüfen. 



\section{Abstract}

Cosmological observations in the last decades have led to a concordance model of the universe, where $\sim 85 \%$ of matter is non-baryonic, non-luminous and non-relativistic at the time of structure formation. Theories of physics beyond the Standard Model of particle physics propose a wide array of candidates for the nature of this unseen "dark matter". Weakly interacting massive particles (WIMPs) are a class of candidates which is well motivated by thermal production models for dark matter in the early universe. WIMPs (or any other dark matter candidate), distributed in a spherical isothermal halo surrounding our galaxy (the standard halo model; SHM), could be detected in terrestrial detectors.

In the standard model of disc galaxy formation, a dark matter disc forms as massive satellites are preferentially dragged into the disc-plane and dissolve. The low velocity of the dark matter particles in the dark disc with respect to the Earth enhances detection rates at low recoil energy in direct detection experiments. For WIMP masses $\gtrsim 50 \mathrm{GeV} / \mathrm{c}^{2}$, the detection rates increase by up to a factor of 3 in the $5-20 \mathrm{keV}$ recoil energy range. Comparing this with rates at higher energies may be sensitive to the WIMP mass, providing stronger mass constraints particularly for masses $\gtrsim 100 \mathrm{GeV} / \mathrm{c}^{2}$. The annual modulation signal is significantly boosted and the modulation phase is shifted by $\sim 3$ weeks relative to the dark halo. The variation of the observed phase with recoil energy determines the particle's mass, once the dark disc properties are fixed by future astronomical surveys.

Dark matter particles cannot only be detected directly in laboratories, but also indirectly by their annihilation products. Previous predictions of the neutrino flux from WIMP annihilation in the Earth and the Sun have assumed that galactic dark matter is distributed according to the SHM. Although the dark disc has a local density comparable to the dark halo, its higher phase space density at low velocities greatly enhances capture rates in the Sun and Earth. For typical dark disc properties, the resulting muon flux from the Earth is increased by three orders of magnitude over the SHM, while for the Sun the increase is one order of magnitude. This significantly increases the prospects of neutrino telescopes to fix or constrain parameters in WIMP models. The flux from the Earth is extremely sensitive to the detailed properties of the dark disc, while the flux from the Sun is more robust.

The Cryogenic Dark Matter Search (CDMS) experiment seeks to directly detect the rare interactions of WIMPs distributed in the local halo with terrestrial nuclei. The CDMS experiment operates an array of semiconductor crystals at millikelvin temperatures in the Soudan Underground Laboratory in northern Minnesota. The detectors are instrumented to measure the ionization and athermal phonon signals of each particle interaction. The combined measurement provides a powerful discrimination against interactions of particles generated primarily by radioactive decays. Such events are considered as background events in the search for WIMP induced nuclear recoils. A detailed study of the background resulted in a model which can fully explain the observed electromagnetic background and allows to reliably predict the expected radiogenic neutron background. 
An analysis searching for nuclear recoils from WIMP interactions resulted in the observation of two events in the signal region. Based on the background estimate for this analysis, the probability of observing two or more background events is $23 \%$. Hence, the result of this analysis cannot be interpreted as a statistically significant detection of WIMP interactions in the detectors. This data sets an upper limit on the WIMP-nucleon elasticscattering spin-independent cross section of $7.0 \times 10^{-44} \mathrm{~cm}^{2}$ for a WIMP mass of $70 \mathrm{GeV} / \mathrm{c}^{2}$ at the $90 \%$ confidence level. Combining this result with all previous CDMS-II data gives an upper limit on the WIMP-nucleon spin-independent cross section of $3.8 \times 10^{-44} \mathrm{~cm}^{2}$ for a WIMP mass of $70 \mathrm{GeV} / \mathrm{c}^{2}$. The combined result places world-leading upper limits on the WIMP-nucleon spin-independent cross section for WIMP masses above $\sim 44 \mathrm{GeV} / \mathrm{c}^{2}$. In addition, this result excludes new parameter space of inelastic dark matter models, which can resolve the discrepancy between the annual modulation signature observed by DAMA and the null observation of other direct detection experiments.

Axions, which have been postulated to solve the strong-CP problem of quantum chromodynamics, may be produced in the interior of the Sun. These solar axions could then be detected in crystal detectors by their Primakov conversion to photons. The search for solar axions described in this thesis has been performed for the first time with the CDMS experiment. The energy threshold of $2 \mathrm{keV}$ for electron recoil events achieved in this analysis allows a search for possible solar axion conversions into photons in the germanium crystal detectors. The solar axion search analysis resulted in a null observation of solar axion conversions and sets an upper limit on the Primakov coupling $g_{a \gamma \gamma}$ of $2.4 \times 10^{-9} \mathrm{GeV}^{-1}$ at the $95 \%$ confidence level for axion masses less than $0.1 \mathrm{keV} / \mathrm{c}^{2}$. This limit benefits from the first precise measurement of the absolute crystal plane orientations in crystal based experiments searching for solar axions.

An analysis of the low-energy electron-recoil spectrum of the CDMS experiment provides details on the observed counting rate and possible background sources in the energy range from $2-8.5 \mathrm{keV}$. No significant excess of a peaked contribution to the total counting rate above the background model is observed. The upper limits on the counting rate are used to constrain the couplings of pseudoscalar dark matter particles. This analysis sets a world-leading experimental upper limit on the axio-electric coupling $g_{a \bar{e} e}$ of $1.4 \times 10^{-12}$ at the $90 \%$ confidence level for an axion mass of $2.5 \mathrm{keV} / \mathrm{c}^{2}$. This analysis also excludes an interpretation of the DAMA annual modulation result in terms of axion interactions for axion masses above $1.4 \mathrm{keV} / \mathrm{c}^{2}$. In a more general framework of a conversion of a dark matter particle into electromagnetic energy, the $90 \%$ confidence upper limit of 0.246 events $/ \mathrm{kg} /$ day at $3.15 \mathrm{keV}$ is lower than the total rate above background observed by DAMA. In the absence of any specific particle model to provide the scaling in cross section between $\mathrm{NaI}$ and $\mathrm{Ge}$, a $\mathrm{Z}^{2}$ scaling is assumed. With this assumption the observed rate in DAMA remains higher than the upper limit from CDMS. Under the conservative assumption that the modulation amplitude is $6 \%$ of the total rate, upper limits on the modulation amplitude, which are a factor of $\sim 2$ less than observed by DAMA, are obtained, constraining some possible interpretations of this modulation. A direct search for a modulated counting rate contribution in the CDMS low-energy spectrum sets upper limits on the modulation amplitude which are not yet sensitive enough to probe the DAMA claim. 


\section{Contents}

List of Figures vi vi vis

List of Tables $\quad \mathrm{x}$

Introduction $\quad x i$

Preface

1 Evidence for Dark Matter in the Universe 1

1.1 Cosmology Framework . . . . . . . . . . . . . . . . . . . . . . . . . 1

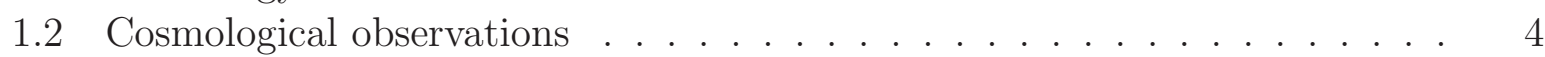

1.2.1 Nucleosvnthesis in the early universe . . . . . . . . . . . . . . . . . . 4

1.2.2 Cosmic Microwave Background . . . . . . . . . . . . . . 6

$1.2 .3 \quad$ Large-Scale Structure Formation . . . . . . . . . . . . . . . . . . . 6

1.3 Astronomv . . . . . . . . . . . . . . . . . . . . . . . . . . . . 8

1.3.1 Galaxy Clusters . . . . . . . . . . . . . . . . . . . . . 8

1.3 .2 Galaxy dynamics . . . . . . . . . . . . . . . . . . . . . . 10

2 Candidates for Dark Matter 13

2.1 Weakly interacting massive particles . . . . . . . . . . . . . . . . . 14

2.1 .1 Supersymmetry . . . . . . . . . . . . . . . . . . . . . . . . . . . . . . . . . . .

2.2 Axions . . . . . . . . . . . . . . . . . . . . . . . . . . . . . . . 19

2.2 .1 The $\theta$-vacua . . . . . . . . . . . . . . . . . . . . . . . . . . 19

2.2 .2 Electric dipole moment of the neutron . . . . . . . . . . . . . . 20

2.2 .3 The axion solution . . . . . . . . . . . . . . . . 20

3 Direct detection of Dark Matter 23

3.1 WIMP - nucleus scattering . . . . . . . . . . . . . . . . . . . . . . . . . . . . . . . . .

3.1 .1 Energv spectra . . . . . . . . . . . . . . . . . . 24

3.1 .2 Scalar WIMP - nucleus scattering . . . . . . . . . . . . . . 27

3.1.3 Axial WIMP - nucleus scattering . . . . . . . . . . . . . . . . . 28

3.2 Dark matter halos and local phase-space density . . . . . . . . . . . . . . . 30

3.2 .1 The Milky Way's Dark Disd . . . . . . . . . . . . . . . . . . . . 30

3.3 Experimental tasks $\ldots \ldots \ldots \ldots \ldots \ldots$ 


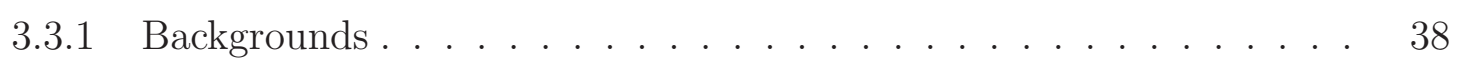

3.3 .2 Event-bv-event discrimination $\ldots \ldots \ldots \ldots$

3.3 .3 Direct detection technologies . . . . . . . . . . . . . . . . . . . . 39

4 Indirect Detection Signals in Presence of the Dark Disc 44

4.1 Indirect detection . . . . . . . . . . . . . . . . . . . 44

4.2 Capture from the dark disc . . . . . . . . . . . . . . . . . . . . . 45

4.3 The dark matter candidate . . . . . . . . . . . . . . . . . . . . . . . . . 47

4.4 Neutrino Flux from the Sun and Earth . . . . . . . . . . . . . . . . . . 48

4.4 .1 The $\operatorname{Sun} \ldots \ldots \ldots \ldots \ldots$

4.4 .2 The Earth . . . . . . . . . . . . . . . . . . . . . . . . . . . . . 48

4.4 .3 Sensitivity to the population bound to the Solar System . . . . . . 49

4.5 Discussion . . . . . . . . . . . . . . . . . . . . . . . . 51

5 Crvogenic Dark Matter Search 55

5.1 CDMS-II at the Soudan Underground Laboratory . . . . . . . . . . . . . 55

5.1 .1 Crvogenics . . . . . . . . . . . . . . . . . . . . 56

5.1 .2 Shielding of the experiment . . . . . . . . . . . . . 57

5.2 Cold hardware . . . . . . . . . . . . . . . . . . . . . . . . 60

5.2 .1 Detector housing . . . . . . . . . . . . . . . . . . . . 60

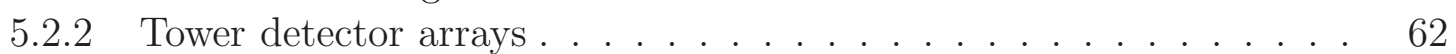

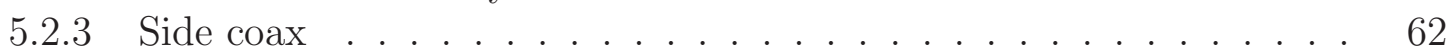

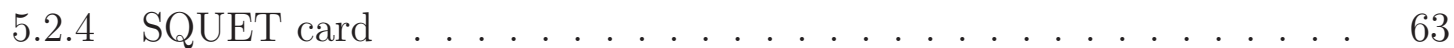

5.2 .5 Stripline . . . . . . . . . . . . . . . . . . . . . . . 63

5.3 Warm electronics . . . . . . . . . . . . . . . . . . . . . . . 64

5.3 .1 Front-end boards . . . . . . . . . . . . . . . . . . . . 64

5.3 .2 Receiver trigger-filter boards . . . . . . . . . . . . . . . . . . 64

5.3 .3 Data acquisition hardware $\ldots \ldots \ldots \ldots \ldots . \ldots \ldots$

5.4 Data acquisition software and data handling . . . . . . . . . . . 66

$\begin{array}{lll}6 & \text { CDMS detectors } & 67\end{array}$

6.1 Crvstal substrate characteristics $\ldots \ldots \ldots \ldots \ldots$

6.2 The ionization signal $\ldots \ldots \ldots \ldots \ldots \ldots . \ldots \ldots$

6.2 .1 Charge transport and collection . . . . . . . . . . . . . . . . 72

6.2 .2 The ionization readout . . . . . . . . . . . . . . . . . . . . 73

6.2 .3 Ionization pulse reconstruction . . . . . . . . . . . . . . . . 75

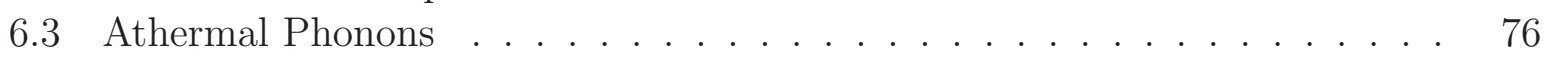

6.3 .1 Phonon propagation . . . . . . . . . . . . . . 77

6.4 Phonon collection . . . . . . . . . . . . . . . . . . . . 78

6.4 .1 Transition-edge sensors . . . . . . . . . . . . . . . . . . . . . . . . $\quad 79$

6.4 .2 SQUID based TES readout $\ldots . \ldots \ldots \ldots$. . . . . . . . . . . 81

6.4 .3 Phonon pulse reconstruction . . . . . . . . . . . . . . . . . . . . 82

6.5 Background discrimination techniques . . . . . . . . . . . . . . . 85 
6.5 .1 The dead-laver $\ldots \ldots \ldots \ldots$. . . . . . . . . . . . . . . . . 87

6.5 .2 Face dependency of surface events . . . . . . . . . . . . . . . 88

7 Calibration of the CDMS detectors $\quad 90$

7.1 Ionization signal . . . . . . . . . . . . . . . . . . . . . . . . 90

7.1 .1 Position dependence $\ldots \ldots \ldots$. . . . . . . . . . . . . . 91

7.1 .2 Energy calibration . . . . . . . . . . . . . . . . . 9 92

7.1 .3 Silicon detectors . . . . . . . . . . . . . . . . . . . . . . . . . . 93

7.2 Phonon signal . . . . . . . . . . . . . . . . . . . . . . . . . . . 93

7.3 Energy and Position correction . . . . . . . . . . . . . . . 96

7.3 .1 Energy dependence . . . . . . . . . . . . . . . . . . . . 99 96

7.3 .2 Position dependence $\ldots \ldots \ldots \ldots$. . . . . . . . . . . . . 96

7.3.3 Phonon correction table . . . . . . . . . . . . . . . . . . . . . 98

7.3 .4 Correction of the data . . . . . . . . . . . . . . . . . . 100

7.4 Ionization vield . . . . . . . . . . . . . . . . . . . . . . . . . . . . 101

7.4 .1 Electron-recoil band $\ldots \ldots \ldots$. . . . . . . . . . . . . . . . 101

7.4 .2 Nuclear-recoil band . . . . . . . . . . . . . . . . . . . . . . . 101

7.4 .3 Comparison with Lindhard prediction . . . . . . . . . . . . . . . . 103

8 Backgrounds 105

8.1 Gamma background . . . . . . . . . . . . . . . . . . . . . . 105

8.1 .1 Gamma background simulations . . . . . . . . . . . . . . . . 108

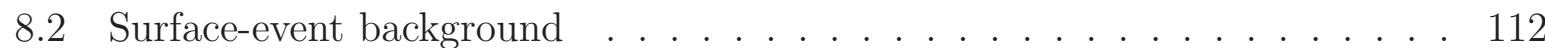

8.2 .1 Ambient gamma contribution . . . . . . . . . . . . . . . . 112

$8.2 .2 \quad{ }^{210} \mathrm{~Pb}$ surface contamination . . . . . . . . . . . . . . . . 113

8.3 Neutron background . . . . . . . . . . . . . . . . . . . . . . . . 117

8.3 .1 Radiogenic neutrons . . . . . . . . . . . . . . . . . . . . 117

8.3 .2 Cosmogenically produced neutrons . . . . . . . . . . . . . . . . 119

9 Analysis of WIMP - Search data 121

9.1 Blinding of the data . . . . . . . . . . . . . . . . . . . . . . . 121

9.2 Data quality . . . . . . . . . . . . . . . . . . . . . . . . . . . 122

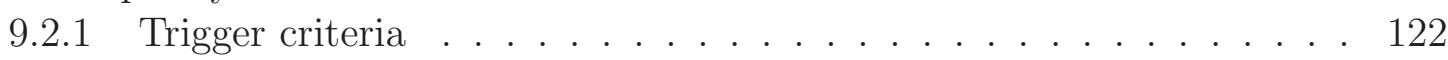

9.2 .2 Detectors considered in the analysis . . . . . . . . . . . . . . . . . 123

9.2 .3 Data selection . . . . . . . . . . . . . . . . . . . . . . . . . . 124

9.2 .4 Helium films on the detectors . . . . . . . . . . . . . . . . 125

9.2 .5 Activation after neutron calibrations . . . . . . . . . . . 126

9.2 .6 Neutralization state of the detectors . . . . . . . . . . . . . . . . 128

9.2 .7 Periods of elevated charge noise . . . . . . . . . . . . . . . . . . . 129

9.2 .8 Malfunctioning detector regions . . . . . . . . . . . . . . . . 130

9.2 .9 Electronic glitches and trigger stability . . . . . . . . . . . 131

9.2 .10 MINOS neutrino beam . . . . . . . . . . . . . . . . . . . . 131

9.3 Reconstruction quality cuts . . . . . . . . . . . . . . . . . . . . 132 
$9.3 .1 \quad$ Ionization pulse reconstruction . . . . . . . . . . . . . . . . . . . . 132

9.3 .2 Crvocooler cycle . . . . . . . . . . . . . . . . . . . . . . 133

9.3 .3 Phonon pre-pulse baseline . . . . . . . . . . . . . . . . . . . . 135

9.3.4 Phonon pulse start time . . . . . . . . . . . . . . . . . . . . 135

9.3.5 Position correction manifold quality . . . . . . . . . . . . . . . . 136

9.4 Event selection cuts . . . . . . . . . . . . . . . . . . . . . 136

9.4 .1 Veto-anticoincident events . . . . . . . . . . . . . . . 136

$9.4 .2 \quad$ Single-scatters . . . . . . . . . . . . . . . . . . . . . . . . . . . 138

9.4 .3 Fiducial volume . . . . . . . . . . . . . . . . . . . . . . . . . . . 140

9.4 .4 Surface-event rejection . . . . . . . . . . . . . . . . . . . . . 142

9.4 .5 Ionization vield selection . . . . . . . . . . . . . . . . . . . . . 147

9.4 .6 Expected gamma leakage . . . . . . . . . . . . . . . . . . . . . . . . 148

9.4 .7 Analysis thresholds . . . . . . . . . . . . . . . . . . . 152

9.5 Total exposure f. . . . . . . . . . . . . . . . . . . . . . . 154

9.6 Analysis efficiency . . . . . . . . . . . . . . . . . . . . . . . . . 156

$9.7 \quad$ Spectrum averaged exposure $\ldots \ldots \ldots$. . . . . . . . . . . . . . . . . 157

$\begin{array}{ll}10 \text { WIMP - Search results } & 158\end{array}$

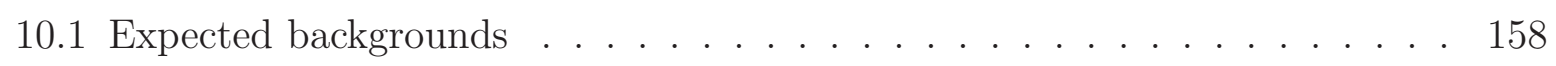

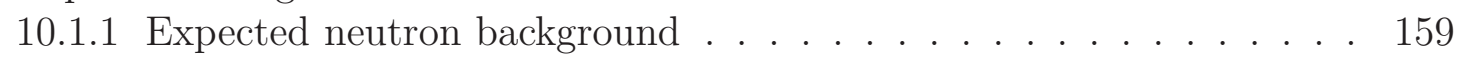

10.1 .2 Surface-event leakage . . . . . . . . . . . . . . . . . . . . . 161

10.2 Unblinding the signal region . . . . . . . . . . . . . . . . . . . . . . . 164

10.3 Post unblinding checks of the candidate events . . . . . . . . . . . . . 168

10.3 .1 Ionization pulse reconstruction pathology . . . . . . . . . . . . . . . 170

10.4 Limits on the WIMP-nucleon elastic scattering cross-section . . . . . . . . 172

10.4 .1 Spin-independent coupling . . . . . . . . . . . . . . . 173

10.4 .2 Spin-dependent coupling . . . . . . . . . . . . . . . . . . 175

10.5 DAMA result in light of the inelastic dark matter model . . . . . . . . . . 177

10.6 Discussion and future prospects for CDMS . . . . . . . . . . . . . . 181

11 Constraining the WIMP scattering cross section and mass 182

11.1 Determination of confidence regions . . . . . . . . . . . . . . . . . . 182

11.1 .1 Classical construction of confidence regions . . . . . . . . . . . . . 184

11.1.2 Confidence intervals from the Feldman-Cousins ordering principle .187

11.2 Application of the Feldman Cousins method . . . . . . . . . . . . . . 190

11.2 .1 Treatment of backgrounds . . . . . . . . . . . . . . . . . 191

11.2 .2 Uncertainties on astronomical parameters . . . . . . . . . . . . 194

11.2.3 Applying Markov Chains to sample over nuisance parameters . . . . 195

12 Search for Solar Axions with the CDMS Experiment 204

12.1 Solar Axions . . . . . . . . . . . . . . . . . . . . . 204

12.2 Expected conversion rate . . . . . . . . . . . . . . . . 205

12.3 Solar axion search analysis . . . . . . . . . . . . . . . . . . . . . 211 
12.3 .1 Data selection . . . . . . . . . . . . . . . . . . . . . . . . 211

12.3.2 Low-energv electron-recoil spectrum . . . . . . . . . . . . . . . . . 219

12.3 .3 Likelihood analysis . . . . . . . . . . . . . . . . . . . 220

12.4 Discussion . . . . . . . . . . . . . . . . . . . . 226

$\begin{array}{ll}13 \text { Low-energy electron-recoil spectrum analysis } & 227\end{array}$

13.1 CDMS low-energy electron-recoil spectrum . . . . . . . . . . . . . . . 228

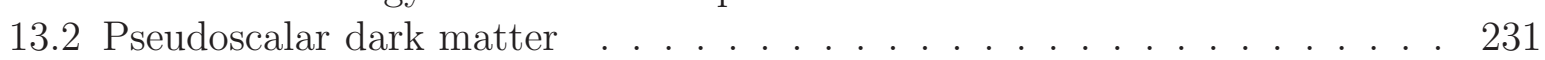

13.3 Electromagnetic conversion dark matter . . . . . . . . . . . . . . . . 234

13.4 Annual modulation . . . . . . . . . . . . . . . . . . . . . 237

$\begin{array}{ll}14 \text { Conclusions } & 240\end{array}$

Acknowledgment I

Literature II 


\section{List of Figures}

1.1 Matter content of the universe . . . . . . . . . . . . . 4

1.2 Light element abundances measurements . . . . . . . . . . . . . . 5

1.3 Power spectrum of the CMB temperature anisotropies . . . . . . . . . . . 6

1.4 Gravitational lensing of Abell $2218 \ldots \ldots \ldots \ldots$

1.5 Images of the Bullet cluster $\ldots \ldots \ldots \ldots$

1.6 Spiral galaxies rotation curves . . . . . . . . . . . . . . . . . . . . 11

1.7 Rotation curve of the Milky Way . . . . . . . . . . . . . . . . . 12

2.1 Comoving number density $\ldots \ldots \ldots \ldots$

2.2 Gauge coupling unification . . . . . . . . . . . . . . . . 16

2.3 Feynman diagram contributing to the neutron's electric dipole moment. . . 20

3.1 Helm form factors of selected materials . . . . . . . . . . . . . . . 28

3.2 Differential and integrated WIMP scattering rates . . . . . . . . . . . 29

3.3 Merging satellite simulation results . . . . . . . . . . . . . . 31

3.4 Rotational velocities at the solar neighborhood . . . . . . . . . . . . . . 32

3.5 Differential recoil rates for the dark disc and the SHM . . . . . . . . . . 34

3.6 Threshold recoil energy below which the dark disc component dominates . $\quad 35$

3.7 Total differential rate for a Ge target . . . . . . . . . . . . . . . . 35

3.8 Annual modulation enhancement by the dark disc component . . . . . . . 36

3.9 Phase shift of the annual modulation . . . . . . . . . . . . . 37

3.10 Photographs of interactions in the COUPP detector . . . . . . . . . . 43

4.1 Muon flux at the Earth from neutrinos originating in the Sun . . . . . . . 49

4.2 Phase space density at low velocities for the SHM and the dark disd . . . . 50

4.3 Muon flux at the Earth from neutrinos originating in the Earth . . . . . 52

4.4 Total muon flux at the Earth's surface . . . . . . . . . . . . . . 53

5.1 Lavout of the Soudan Underground Laboratory . . . . . . . . . . . . 56

5.2 Schematic of the experimental setup . . . . . . . . . . . . . . 58

5.3 Schematic of the cold hardware in the icebox . . . . . . . . . . . . . . . 61

5.4 Sketch of a CDMS-II Tower . . . . . . . . . . . . . . . . . . . 62

5.5 Photograph and layout of the CDMS SQUET cards . . . . . . . . . . 63

5.6 Final 5 Tower configuration of the Soudan icebox . . . . . . . . . . . 64 
6.1 Physical lavout of a ZIP detector substrate as seen from the top . . . . . . 68

6.2 Schematic sensor configuration of a ZIP detector . . . . . . . . . . . . . . . 69

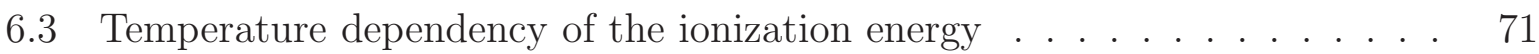

6.4 Lavout of the ZIPs' ionization electrodes . . . . . . . . . . . . . . . . . . . 73

6.5 Ionization signal amplifier circuit $\ldots \ldots \ldots$. . . . . . . . . . . 74

6.6 Ionization channel noise spectrum . . . . . . . . . . . . . . . . . . . 75

6.7 Schematic of the phonon sensors' layout . . . . . . . . . . . . . . . . 79

6.8 Quasiparticle assisted phonon measurement . . . . . . . . . . . . . . . . 80

6.9 Schematic of the phonon channel bias and readout electronics . . . . . . . 81

6.10 Position reconstruction based on phonon pulse characteristics . . . . . . . . 84

6.11 Position reconstruction based on an empirical model . . . . . . . . . . . . . 85

6.12 Ionization vield discrimination of ZIP detectors . . . . . . . . . . . . . . . 86

6.13 Face selected surface-events . . . . . . . . . . . . . . . . . . 89

7.1 Position dependence of the optimal filter amplitudes . . . . . . . . . . . . . . 91

7.2 Position corrected ionization signal . . . . . . . . . . . . . . . . . . . . . . . . . . . . . . .

7.3 Calibrated ionization signal energy spectrum . . . . . . . . . . . . . . . . . 93

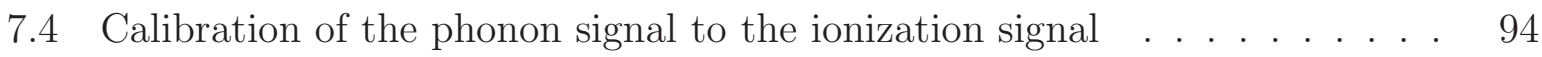

7.5 Position reconstruction based on phonon energy partition . . . . . . . . . . 95

7.6 Calibrated phonon signal energy spectrum . . . . . . . . . . . . . . . . . . 95

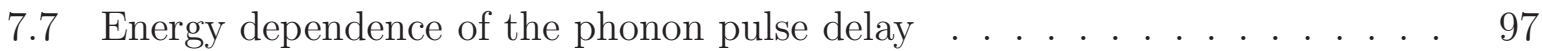

7.8 Energy dependence of the phonon pulse rise-time $\ldots \ldots \ldots$. . . . . . . 97

7.9 Slice of the 3 -dimensional position manifold . . . . . . . . . . . . . . . . 98

7.10 Position dependence of the phonon pulse delav . . . . . . . . . . . . . . . . . 99

7.11 Position dependence of the phonon pulse rise-time . . . . . . . . . . . . . . . 99

7.12 Electron-recoil selection by the ionization vield parameter . . . . . . . . . . 102

7.13 Nuclear-recoil selection by the ionization vield parameter . . . . . . . . . 103

7.14 Comparison of the nuclear-recoil band centroids with the Lindhard theory 104

8.1 Low-background spectrum of all germanium detectors . . . . . . . . . . . . 107

8.2 Comparison of the observed and simulated spectrum for one Tower . . . . 109

8.3 Comparison of the total observed and simulated spectrum . . . . . . . . . 111

$8.4{ }^{210} \mathrm{~Pb}$ decay scheme . . . . . . . . . . . . . . . . . . . . . . . . 113

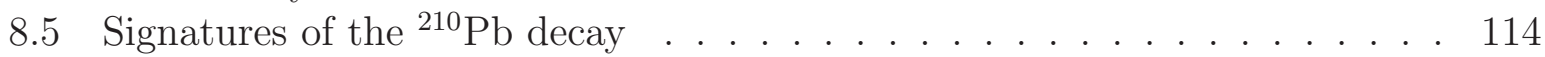

8.6 Correlation of nearest neighbor events . . . . . . . . . . . . . . . . . . . . . 115

9.1 Electron-recoil rate after neutron calibrations . . . . . . . . . . . . . . . . 127

9.2 Low energy calibration and resolution . . . . . . . . . . . . . . . . . . 127

9.3 Malfunction detector regions . . . . . . . . . . . . . . . . . . . . . 130

9.4 Definition and efficiency of the charge reconstruction quality cut . . . . . . 133

9.5 Noise power spectrum of T1Z4's inner ionization channel . . . . . . . . . . . 134

9.6 Histogram of WIMP search veto events for a representative veto panel . . . 137

9.7 Variation of the mean and resolution of the noise distribution with time . . 139 
9.8 Efficiency of the singles cut throughout the data taking period . . . . . . . 140

9.9 Efficiency of the ionization signal based fiducial volume cut . . . . . . . . . 141

9.10 Ionization vield distribution of face selected surface-events . . . . . . . . . 143

9.11 Simple timing discriminator . . . . . . . . . . . . . . . . . . . . . . . . . . . . . . . . 144

9.12 Energy distribution of surface-events . . . . . . . . . . . . . . . . . . . . . . . . . . . . . . . . 14

9.13 Surface-event rejection cut . . . . . . . . . . . . . . . . . . . . . 146

9.14 Surface-event rejection cut efficiency . . . . . . . . . . . . . . . . . . . . 147

9.15 Efficiency of the ionization vield based nuclear-recoil selection . . . . . . . 148

9.16 Yield distributions of simulated energies for a representative detector . . . 149

9.17 Scaling of the gamma simulations to the recorded low background spectrum 150

9.18 Cumulative expected gamma leakage in the nuclear-recoil band . . . . . . . 151

9.19 Variation of the ionization threshold with time . . . . . . . . . . . . 153

9.20 Combined ionization threshold and electron recoil rejection cut efficiency . 154

9.21 Nuclear-recoil acceptance as a function of recoil energy . . . . . . . . . . . 156

10.1 Normalized vield and timing of the combined unblinded data . . . . . . . . 166

10.2 Normalized vield and timing for individual detectors . . . . . . . . . . . . . 167

10.3 Ionization vield, energy and timing of the two candidate events . . . . . . . 169

10.4 Inner-electrode ionization signal fit of candidate event detected in T3Z4 . 171

10.5 Start time bin determination by the maximal amplitude and $\chi^{2}$ algorithm . 171

10.6 Limits on the SI WIMP-nucleon elastic scattering cross section . . . . . . . 174

10.7 Limits on the SD WIMP-nucleon elastic scattering cross section . . . . . . 176

10.8 Modulation signature observed by the DAMA collaboration . . . . . . . . . 177

10.9 Comparison of the differential rates for elastic and inelastic scattering . . . 179

10.10Constraints on the DAMA allowed inelastic dark matter parameter space . 180

11.1 Likelihood ratio distribution with varving number of events . . . . . . . . . 185

11.2 Classical confidence belts for a Poisson process . . . . . . . . . . . . . . 187

11.3 Confidence belt based on the Feldman - Cousins ordering principle . . . . . . 188

11.4 Comparison of the likelihood function approach with Feldman-Cousins . . 189

11.5 Confidence regions for eight signal and zero background events . . . . . . . . 191

11.6 Energy distribution of single-scatters in the nuclear-recoil signal region . 192

11.7 Confidence regions for eight events while varving the expected background 193

11.8 Confidence levels with true astrophysical parameters . . . . . . . . . . . . 194

11.9 Confidence levels biased by wrong astrophysical parameters . . . . . . . . 195

11.10MCMC sampling of a correlated 2-dimensional Gaussian . . . . . . . . . . 196

11.11Frequency confidence intervals for the example of a detection of 30 events . 197

11.12 Confidence regions from the analytical approach in the MCMC . . . . . . . 198

11.13Comparison of the MCMC sampled and the likelihood function contours . 198

11.14Upper and lower limits on the mass and cross section . . . . . . . . . . . 199

11.15Acceptance regions from a MCMC sampling of the astrophysical parameters 200

11.16 Sampling of the astrophvsical parameters distribution . . . . . . . . . . 200

11.17Acceptance regions and sampling of the background distribution . . . . . . 202 
12.1 Spectral flux of solar axions at the Earth . . . . . . . . . . . . . . 205

12.2 Solar axion conversion rate . . . . . . . . . . . . . . . . . . . . 208

12.3 Detector crvstal plane alignment . . . . . . . . . . . . . . . . . . . . . 208

12.4 Detector and seasonal dependency of solar axion conversions . . . . . . . 210

12.5 Electron-recoil distribution . . . . . . . . . . . . . . . . . . . . . . 212

12.6 Singles cut threshold for the detectors used in this analvsis . . . . . . . . . 213

12.7 Ionization threshold for the detectors used in this analvsis . . . . . . . . . . 214

12.8 Efficiency of the charge threshold and nuclear-recoil rejection cut . . . . . . 215

12.9 Efficiency of the charge $\chi^{2}$ and fiducial volume cut . . . . . . . . . . . 216

12.10Efficiency of the phonon trigger and combined detection efficiency . . . . . 216

12.11Longterm gamma rates after neutron calibrations . . . . . . . . . . . . 218

12.12Low-energy electron-recoil spectrum . . . . . . . . . . . . . . . . . . . . 219

12.13Efficiency corrected. co-added low-energy spectra . . . . . . . . . . . . . 220

12.14Profile likelihood function for the scaling factor . . . . . . . . . . . . . . . 222

12.15Upper limit on the photon-axion coupling constant . . . . . . . . . . 224

12.16Systematic uncertainty on the upper limit on the coupling constant $g_{a \alpha \text { a }}$. . 225

13.1 Fit to the low-energy spectrum . . . . . . . . . . . . . . . . . . . 228

13.2 Upper limits on an excess rate above background . . . . . . . . . . . . . . 231

13.3 Comparison of the upper limits with the low-energy spectrum . . . . . . . 232

13.4 Constraints on the axio-electric coupling constant . . . . . . . . . . . . . . 233

13.5 Fit to the low-energy spectrum of the DAMA/LIBRA experiment . . . . 234

13.6 Comparison of the upper limits in CDMS with the DAMA/LIBRA results 236

13.7 Count rate as a function of date in the $2-4 \mathrm{keV}$ range . . . . . . . . . . . . 238

13.8 Constraints on the phase and amplitude of a modulated counting rate . . . 239 


\section{List of Tables}

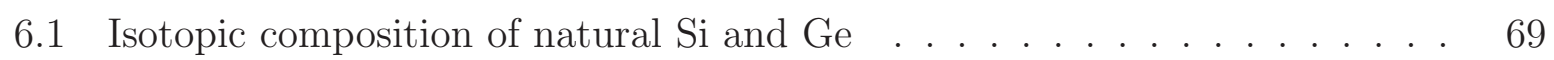

6.2 Temperature dependent band gap parameters . . . . . . . . . . . . 70

8.1 Contamination levels of materials within the inner lead shield . . . . . . 110

8.2 Various fitted rates from the ${ }^{210} \mathrm{~Pb}$ model . . . . . . . . . . . . . . . . 116

8.3 Contamination levels of materials within the outer polvethylene shield . . . 118

8.4 Expected neutron rates from radioactive contamination . . . . . . . . . 118

8.5 Number of unvetoed and vetoed nuclear recoil single-scatters . . . . . . . 120

9.1 Crvstal substrates in the CDMS-II setup . . . . . . . . . . . . . 123

9.2 Mass of the crvstals in the CDMS-II setup . . . . . . . . . . . . . 155

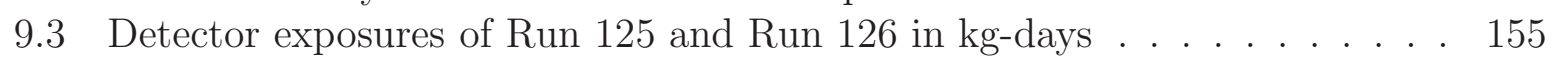

9.4 Detector exposures of Run 127 and Run 128 in kg-days . . . . . . . . . . 155

10.1 Expected rates and number of single-scatter nuclear-recoils . . . . . . . . 159

10.2 Number of single-scatter events in the $2 \sigma$ nuclear-recoil band . . . . . . . 165

11.1 Values of the quantile $Q_{\alpha}$ for different values of the confidence level . . . 184

11.2 Quantities used in an example confidence interval construction . . . . . . . 188

11.3 Limits on the particle properties with fixed astrophvsical parameters . . . . 199

11.4 Limits on the particle properties with sampled astrophvsical parameters . . 201

11.5 Mass uncertaintv bv sampling the astrophvsical parameters . . . . . . . . . 201

11.6 Limits on the particle properties with sampled expected background . . . . 202

12.1 Reciprocal lattice vectors of germanium . . . . . . . . . . . . . . . . 207

12.2 Detector alignment . . . . . . . . . . . . . . . . . . . . 209

13.1 Rate $[$ events $/ \mathrm{kg} / \mathrm{dav} / \mathrm{keV}]$ in the $2-8.5 \mathrm{keV}$ energy range. . . . . . . . . . 229 


\section{Introduction}

The last few decades have resulted in an understanding of the universe, which is not dominated by the usual baryonic matter, but by some unseen "dark components". The baryons only make up a tiny fraction $(\sim 5 \%)$ of the energy content of the universe, while the dark components split into "dark matter" $(\sim 25 \%)$ and "dark energy" $(\sim 70 \%)$. The modern picture of the universe along with the evidences for dark energy and especially dark matter are summarized in Chapter 1. Both dark components have a strong influence on the evolution of the universe and the structure of the universe at the present day. Especially the dark matter component has a great impact on the evolution of large-scale structures and formation of galaxies. Discovering the nature of these two components is thus the most pressing question of modern cosmology.

To date only theoretical models of both components exist, since their physical nature has not yet been revealed by experiments. It is believed that dark matter is composed of new particles which point to physics beyond the Standard Model. Chapter 2 gives a brief introduction to possible dark matter candidates arising in supersymmetric extensions of the Standard Model or in attempts to explain experimental observations which are incompatible with the Standard Model. The detection of such dark matter particles would give a first hint to a central question of science: What is the universe made of?

This dissertation describes the search for dark matter particles, which should be distributed in a halo around the Milky Way. The general detection principle of weakly interacting massive particles is described in Chapter 3. To predict interaction rates in direct detection experiments, assumptions on the local phase-space density of the dark matter particles have to be made. Recent N-body simulations of galaxy formation including baryons suggest that a macroscopic dark matter structure apart from an isothermal halo forms during the galaxy's evolution. The effect of this new dark matter disc on direct detection experiments is also described in this Chapter.

Dark matter particles cannot only be detected directly in laboratories, but also indirectly by their annihilation products. A predicted signature of dark matter particle annihilation are neutrino fluxes emerging from the interior of the Sun and Earth. These neutrino fluxes are generated by annihilation of dark matter particles which became gravitationally captured in the Sun and Earth. Since the capture rate and thus the resulting annihilation rate depends on the initial velocity of the dark matter particles, these rates are significantly enhanced by the lower velocity population in the dark matter disc. Chapter 4 studies the impact of the dark matter disc on the detectable fluxes from dark matter annihilation in current and future neutrino telescopes. 
The main part of this dissertation focuses on the search for weakly interacting particles (WIMPs) with the Cryogenic Dark Matter Search (CDMS) experiment. Semiconductor crystals operated at milikelvin temperatures are used in the CDMS experiment to search for a signal from dark matter particle interactions. In a search for rare particle interactions it is crucial to reduce external backgrounds as much as possible. An active muon scintillator veto and passive shielding consisting of layers of polyethylene and lead are used in the experimental setup to reduce such backgrounds. The experimental setup, as well as the cryogenic infrastructure and the readout electronics of the detectors are presented in Chapter 5. Chapter 6 focuses on the detector technology, discussing details of the readout of the ionization and phonon signal and the additional discrimination power of the detectors between electron and nuclear recoils, based on the ratio of these two signals. The calibration of the detectors, using electron and nuclear recoils from calibration sources is explained in detail in Chapter 7 . For any direct detection experiment it is not only crucial to reduce background interactions as much as possible, but also to identify the sources of these backgrounds. Chapter 8 gives a summary of the backgrounds induced by ambient gammas and surface contamination of the crystals. From the results presented in this Chapter all observed backgrounds in the CDMS experiment can be explained, and a reliable prediction on the expected neutron background rate can be made. The CDMS experiment has been designed to search for nuclear recoils induced by weakly interacting massive particles scattering off nuclei in the detectors. Chapter 9 is dedicated to details of the analysis in the search for nuclear recoils, listing all selection criteria imposed on the data and evaluating the signal efficiency. A great advantage of the CDMS experiment is the capability of operating in a nearly background free regime, with an expected background of less than one event for the current analysis. The expected background and results from this analysis are summarized in Chapter 10.

Upon the detection of dark matter interactions, there are two parameters of interest which can be determined from direct detection experiments. These are the particle's mass and the WIMP-nucleon scattering cross section. Given the expected low statistics of the first detections it is questionable if the standard procedures for determining confidence regions in the parameter space can be used. In the limiting case of low statistics a Feldman and Cousins based procedure would be adequate for determining the confidence regions. In addition the astrophysical input parameters used in the analysis of direct detection experiments may introduce a systematical bias in the confidence regions. To take these systematics into account in the determination of the confidence regions a Markov Chain Monte Carlo algorithm approach is studied. This approach samples over the nuisance parameters and returns the confidence regions which appropriately take into account the systematic uncertainties introduced by the nuisance parameters. Both of these studies are summarized in Chapter 11.

Although being designed for the search for nuclear recoils, the CDMS experiment can also search for different signatures of weakly interacting particles. A candidate particle would be the axion, which has been postulated to solve the strong CP-problem arising in quantum chromodynamics. If this particle exists it would be produced in the interior of stars such as the Sun. Chapter 12 describes the search for solar axions with the CDMS 
experiment which has been performed for the first time. Axions would materialize in the detectors via the Primakov effect, for which, similar to Bragg scattering, the crystal lattice can interact in a coherent way giving a strong enhancement of the conversion rate. Thus, the expected conversion rate depends on the position of the Sun with respect to the detectors, providing a unique signature of solar axion conversions in the detectors.

In a more general model, interactions from dark matter particles could be detected as electron recoils rather than nuclear recoils. Any dark matter particle which would produce an electron recoil in the detectors would be rejected in the search for nuclear recoils. It is thus interesting to investigate electron recoils which are usually regarded as background events. A candidate for such interactions would be pseudoscalar dark matter, but also a very model independent analysis is used to search for an excess in detected rate above background. Chapter 13 is dedicated to an analysis of the low-energy electron-recoil spectrum of the CDMS experiment. Such analyzes are of special interest in the light of the claim of the Dark Matter (DAMA) collaboration, who observe and annual modulation of the counting rate in their detectors. The results from this analysis are compared to the DAMA claim, constraining possible interpretations of the observed annual modulation signature. 



\section{Preface}

The CDMS-II experiment is not the work of any one person, but a collaborative effort by more than fifty scientists and engineers. Thus, not all of the work described in this dissertation is the author's own. For clarification, this preface summarizes my own involvement with the CDMS collaboration and the contributions to the experiment's scientific results. In addition, studies described in this dissertation which I have done apart from my contributions to the CDMS-II experiment are mentioned.

In the text I have tried to properly attribute figures and results generated by others, but avoid the citation of internal notes which are not publicly available. I apologize in advance to the reader for any errors or oversights.

\section{The Milky Way's Dark Disc}

Using results from N-Body simulations, which predict a macroscopic dark matter disc structure in the Milky Way, I evaluated the impacts of this dark matter structure on direct detection experiments (Section 3.2.1). The results from this study have been published [1], for which I was the corresponding author.

Due to the lower velocities of particles in the dark disc, this component has a significant impact on indirect detection of WIMP dark matter with neutrino telescopes. I calculated the resulting capture rates in the Sun and Earth and obtained significantly increased muonfluxes detectable in current and future neutrino telescopes (Chapter 4). The results from this study have been published [2], for which I was the corresponding author.

\section{Calibration of the CDMS-II detectors}

For the calibration of the detectors, I checked the correct calibration of the ionization and phonon channel with a Monte Carlo simulation (see Section 7.1.2 and 7.2) and verified that the calibrations are valid down to recoil energies of $10 \mathrm{keV}$ (Section 9.2.5).

I also defined the electron-recoil bands (see Section 7.4.1) and analyzed the compatibility of the nuclear-recoil band centroids with the Lindhard theory (Section 7.4.3).

\section{Backgrounds of the CDMS-II experiment}

I performed the ambient gamma Monte Carlo simulations described in Section 8.1.1 which explain the observed low-background spectrum and which were used to deduce the con- 
tamination levels of the materials close to the detectors. These contamination levels were partially used to estimate the expected neutron background for the WIMP search analysis.

I also evaluated the ambient gamma induced surface-event rate described in Section 8.2.1, which in combination with the rate expected from ${ }^{210} \mathrm{~Pb}$ surface contamination of the detectors, can explain the total observed surface-event rate in the CDMS-II experiment (see Section 8.2). The result from this study will be published in a forthcoming publication for which I will be a leading author [3].

\section{WIMP search analysis}

During my involvement with the CDMS collaboration I took part in the analysis of Run 123/4 and Run 125-128. The analysis of the data sets is described in Chapter 9, focusing on the Run 125-128 analysis. I took part in the organization and analysis of these two runs with particular emphasis on the following parts:

- Overall organization of the data quality cuts and definition of the WIMP search signal region.

- Definition of an inner-electrode selection cut at high energies for background studies (Section 9.4.3).

- Definition of the series dependent multiples-rejection cut (Section 9.4.2).

- Initial development of the optimization scheme for the surface-event rejection cut, as well as the cuts selecting the face separated surface-event sample used in the definition of the surface-event rejection cut (Section 9.4.4).

- Analysis of the electron-recoil leakage into the nuclear-recoil band and definition of the associated rejection cut (Section 9.4.6).

- Evaluation (in collaboration with Sebastian Arrenberg) of the imposed cut selection efficiencies and the final signal efficiency (Section 9.6).

The results from the analysis of the Run 123/4 data have been published [4]. The main WIMP search results presented in this dissertation (Run 125-128) have been published in Science [5].

\section{Constraining the WIMP-nucleon scattering cross sec- tion and mass}

Upon the detection of dark matter interactions, there are two parameters of interest which can be determined from direct detection experiments. These are the particle's mass and the WIMP-nucleon scattering cross section. Given the expected low statistics of the first detections, it is questionable if the standard procedures for determining confidence regions 
in the parameter space can be used. I studied the applicability of the likelihood function approach in the limiting case of low statistics, and developed a Feldman and Cousins based procedure which can be used for determining confidence regions in the low statistics limit. In addition, the astrophysical input parameters used in the analysis of direct detection experiments may introduce a systematical bias in the confidence regions. To take these systematics into account in the determination of the confidence regions, I developed a Markov Chain Monte Carlo algorithm. This algorithm samples over the nuisance parameters and returns the confidence regions which appropriately take into account the systematic uncertainties introduced by the nuisance parameters. Both of these studies are summarized in Chapter 11.

\section{Solar axion search analysis}

I played a leadership role in the first solar axion search analysis described in Chapter 12 . My contributions to this analysis have been:

- Development of the code calculating the expected conversion rate in the detectors (Section 12.2).

- Defining the data selection for this analysis (Section 12.3.1).

- Evaluation (in collaboration with Sebastian Arrenberg) of the imposed cut selection efficiencies and the final signal efficiency (Section 12.3.1 and 12.3.2 ).

- Evaluation of the detectors' energy resolution functions at low energies (Section 12.3.2).

- Development of the likelihood analysis to search for a signal of solar axion conversions in the detectors (Section 12.3.3).

- Calculation of the upper limit on the axion-photon coupling constant and its systematic uncertainty (Section 12.3.3).

The results from this analysis, which has been performed for the first time with the CDMS-II experiment, have been published [6], for which I was a lead author.

\section{Low-energy electron-recoil analysis}

I performed the more general analysis of the low-energy electron-recoil analysis described in chapter 13. In this analysis I

- Developed the likelihood analysis to search for a signal above background in the detectors (Section 13.1).

- Calculated the upper limit on the axio-electric coupling constant (Section 13.2). 
- Analyzed the compatibility of the upper limits set in this analysis with the DAMA claim in a very general model of an electromagnetic conversion of dark matter particles (Section 13.3).

- Developed a Feldman Cousins analysis to search for a hint of an annual modulation in the data (Section 13.4).

The results of this analysis have been published in two publications. The results on the axio-electric coupling have been part of [6], while the general analysis of the low-energy electron recoil spectrum and the comparison to the DAMA claim were a second publication [7], for which I was the corresponding author. 


\section{Chapter 1}

\section{Evidence for Dark Matter in the Universe}

The first hint for a "missing mass problem" was discovered in 1933 by Zwicky. Since then, many observations have lead to the conclusion that $\sim 25 \%$ of the universe's energy composition is made of some unknown non-baryonic matter. Due to the non-observation in the electromagnetic spectrum this form of matter is called "dark matter". The current cosmological model of the universe's evolution as well as the understanding of small scale structures such as galaxies is in need of this dark matter. Although there are alternative approaches by modifying the gravitational laws [8, 9] the concept of dark matter convincingly solves the discrepancies between the observations and expectations from a pure baryonic-matter content of the universe on all scales. Although the existence of dark matter is solely inferred from its gravitational effects it has become an accepted part of modern astrophysics.

After introducing the modern concordance model of cosmology, the main evidences for the existence of dark matter, ranging from the very early universe to the properties of galaxies at the present day are briefly discussed.

\subsection{Cosmology Framework}

A full discussion of the cosmology framework is beyond the scope of this work, so this section merely focuses on the terminology and an overview of the framework in which the problem of dark matter will be introduced. More details on the cosmological theory and supporting evidences can be found in modern textbooks (see for example [10, 11, 12]).

Modern cosmology is based on general relativity, and the assumption that the universe is homogeneous and isotropic. Although there is a large deviation from isotropy at the scales of galaxies, when averaged over gigaparsec scales, one part of the universe looks much like another. Though the universe does not change much as we move through space it shows a great variation with time. The first evidence for the universe's evolution is its expansion discovered in 1929 by Edwin Hubble [13. Applying the conditions of homogeneity and 
isotropy to the space time metric of general relativity, gives the Friedmann-RobertsonWalker metric:

$$
d s^{2}=c^{2} d t^{2}-R^{2}(t)\left(\frac{d r^{2}}{1-k r^{2}}+r^{2}\left(d \theta^{2}+\sin ^{2} \theta d \phi^{2}\right)\right) .
$$

In this expression, $t$ is time, $(r, \theta, \phi)$ are dimensionless comoving spherical coordinates, $k$ sets the curvature of the universe $(k=-1,0,1$ for negatively curved, flat, or positively curved), and $R(t)$ is a scale factor evolving with time. The time evolution of this metric is determined by the Einstein field equations. Thus, the time evolution of the metric depends on the universe's contents. The contents of the universe are usually separated in three categories differentiated by their relative values of energy density $\rho$ and relativistic pressure $p$ :

- Matter: Non-relativistic material $(p=0)$. The energy density of matter evolves as $\sim 1 / R(t)^{3}$ as the universe expands.

- Radiation: Relativistic particles (such as photons, neutrinos) with positive relativistic pressure $\left(p=+\rho c^{2} / 3\right)$. Taking into account relativistic effects of the radiation's momentum, the energy density of radiation dilutes faster than that of matter $\sim 1 / R(t)^{4}$.

- Vacuum energy: Energy density associated with space-time itself, which has negative relativistic pressure $\left(p=-\rho c^{2}\right)$. The energy density does not change as the universe expands.

Defining a dimensionless scale factor $a \equiv R / R_{0}$, where $R_{0}=R\left(t_{0}\right)$ is the scale factor at the present day, and setting $c=1$ the Einstein equations become the Friedmann-Lemaitre equations:

$$
\begin{aligned}
\left(\frac{\dot{a}}{a}\right)^{2} \equiv H^{2}(t) & =\frac{8 \pi G}{3}\left(\rho_{M}+\rho_{R}+\rho_{\Lambda}\right)-\frac{k}{a^{2}} \\
-\frac{\ddot{a}}{a} & =\frac{4 \pi G}{3}\left[\left(\rho_{M}+\rho_{R}+\rho_{\Lambda}\right)+3\left(p-\rho_{\Lambda}\right)\right]
\end{aligned}
$$

where the indices $M, R$ and $\Lambda$ stand for matter, radiation and vacuum energy. In addition the Hubbel parameter $H(t)$ has been defined. From equation (1.2) follows that the universe has zero curvature if the total energy density $\rho=\rho_{M}+\rho_{R}+\rho_{\Lambda}$ equals a critical density $\rho_{c} \equiv \frac{3 H^{2}(t)}{8 \pi G}$. Expressing terms in fraction of this critical density: $\Omega_{x}=\rho_{x} / \rho_{c}$ the FriedmanLemaitre equations can be written in the following useful form:

$$
\begin{aligned}
\Omega-1 & =\frac{k}{a^{2} H^{2}(t)} \\
\dot{H}(t) & =-H^{2}(t)\left(1+\frac{1}{2} \Omega_{M}+\Omega_{R}-\Omega_{\Lambda}\right)
\end{aligned}
$$


where $\Omega=\Omega_{M}+\Omega_{R}+\Omega_{\Lambda}$. Given the scaling laws listed above (e.g $\left.\Omega_{M} \propto 1 / a^{3}\right)$ these equations may be solved for the evolution of the scaling factor $a(t)$. Boundary conditions implied in the solution of the equations at the present day are usually indicated by " 0 " subscripts: $a_{0}=1, H_{0}, \Omega_{0, M}, \Omega_{0, R}, \Omega_{0, \Lambda}, \Omega_{0}$. These parameters are the basic parameters of the modern cosmological model. The three components of $\Omega$ depend differently upon $a$, so the balance between them varies with time. Radiation dominates the universe's evolution at very early times, while vacuum energy dominates at very late times. If $\Omega_{0}=1$, then $k=0$ and $\Omega=1$ throughout the universe's history. If not, $\Omega \rightarrow 1$ as time advances.

Cosmological time is usually measured in terms of the scale factor $a(t)$. The scale factor is most easily observed through its effect on electromagnetic radiation: light emitted with a wavelength $\lambda_{1}$ at a time when the scale factor was $a\left(t_{1}\right)$ will be observed at a wavelength $\lambda_{2}=\lambda_{1} \frac{a\left(t_{2}\right)}{a\left(t_{1}\right)}$ when the scale factor reaches $a\left(t_{2}\right)$. This relation is often expressed as the cosmological redshift $z=a(t)-1$, ranging from zero at the present day back to $z \rightarrow \infty$ at the Big Bang. Cosmological redshift can be determined directly from the observed frequencies of features in the spectra of cosmological objects. Measuring cosmological time in terms of the scaling factor is useful because many attributes of the universe - for example its temperature and density - depend directly on the scale factor, while varying the contents of the cosmological model will change the corresponding $a(t)$.

The modern concordance model of cosmology is known as " $\Lambda$ cold dark matter" $(\Lambda C D M)$ cosmology. Here $\Lambda$ symbolizes the cosmological constant, the simplest form of vacuum energy or "dark energy". In this model $\Omega \approx 1$ and at present day is dominated by the contributions $\Omega_{0, M}$ and $\Omega_{0, \Lambda}$. The term "cold" indicates that most of the universe's matter content was non-relativistic during the formation of large-scale structure. This model is supported by a wide variety of measurements. The agreement between three very different classes of measurements is illustrated in figure 1.1. Studies of the cosmic microwave background (CMB), along with observations of baryon acoustic oscillations in the galaxy cluster distribution and observation of distant Type Ia supernova describe a consistent set of cosmological parameters: $\Omega_{0, B}=0.0456 \pm 0.0015$ (baryonic matter), $\Omega_{0, D M}=0.228 \pm 0.013$ (non-baryonic dark matter) and $\Omega_{0, \Lambda}=0.726 \pm 0.015$ (dark energy) [15] . 


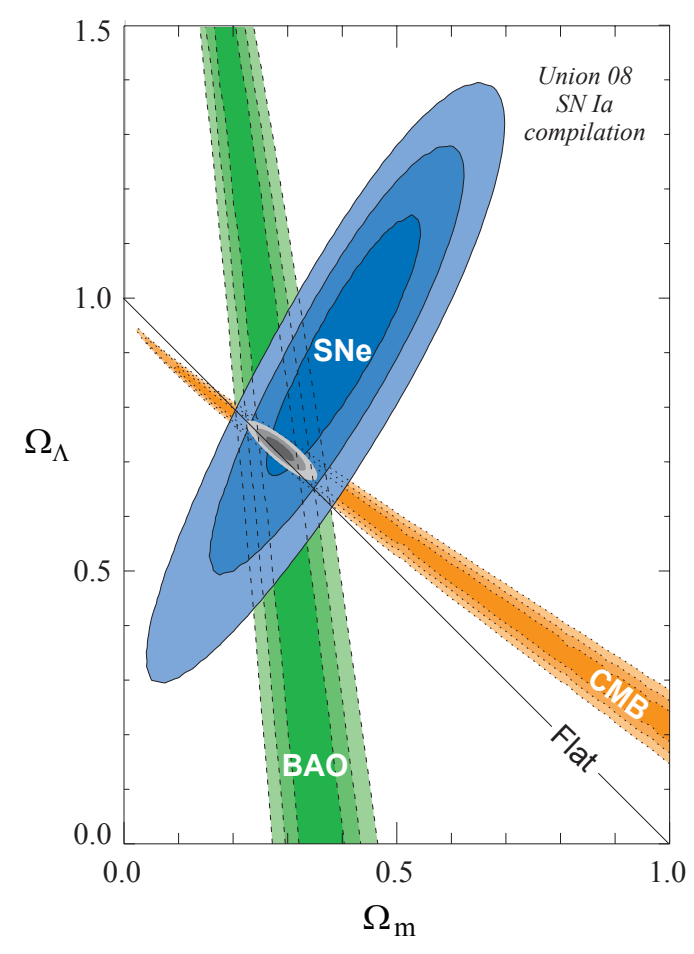

Figure 1.1: Constraints on the current matter density ( $\Omega_{0, M}$ denoted as $\Omega_{m}$ in the figure) and dark energy density ( $\Omega_{0, \Lambda}$ denoted as $\Omega_{\Lambda}$ in the figure). Colored regions represent $1 \sigma$, $2 \sigma$ and $3 \sigma$ confidence regions from current measurements of the cosmic microwave background (CMB), baryon acoustic oscillations (BAO) and measurements of Type Ia supernovae (SNe). Figure taken from [14.

\subsection{Cosmological observations}

Cosmological observations and measurements reveal the existence and necessity of dark matter in the universe's evolution. The current state of knowledge is that, from the first few minutes after the Big Bang on, dark matter has determined the evolution of the universe. The three main cosmological arguments for the existence of dark matter are briefly described below.

\subsubsection{Nucleosynthesis in the early universe}

During the first few minutes after the Big Bang light elements are synthesized from free protons and neutrons. The study of the abundances of light elements, notably ${ }_{1}^{2} \mathrm{H},{ }_{2}^{3} \mathrm{He}$, ${ }_{2}^{4} \mathrm{He},{ }_{3}^{3} \mathrm{Li}$, in the framework of the Big Bang Nucleosynthesis (BBN) model, yields strong constraints on the baryonic matter in the Universe, $\Omega_{b}$. The primordial abundance of light elements depends crucially on the conditions during the period in which such fusion was possible. In particular the baryon-to-photon ratio $\eta=n_{b} / n_{\gamma} \times 10^{-10}$ determines the production of light elements in the BBN (see for example [16]). The photon density sets the 


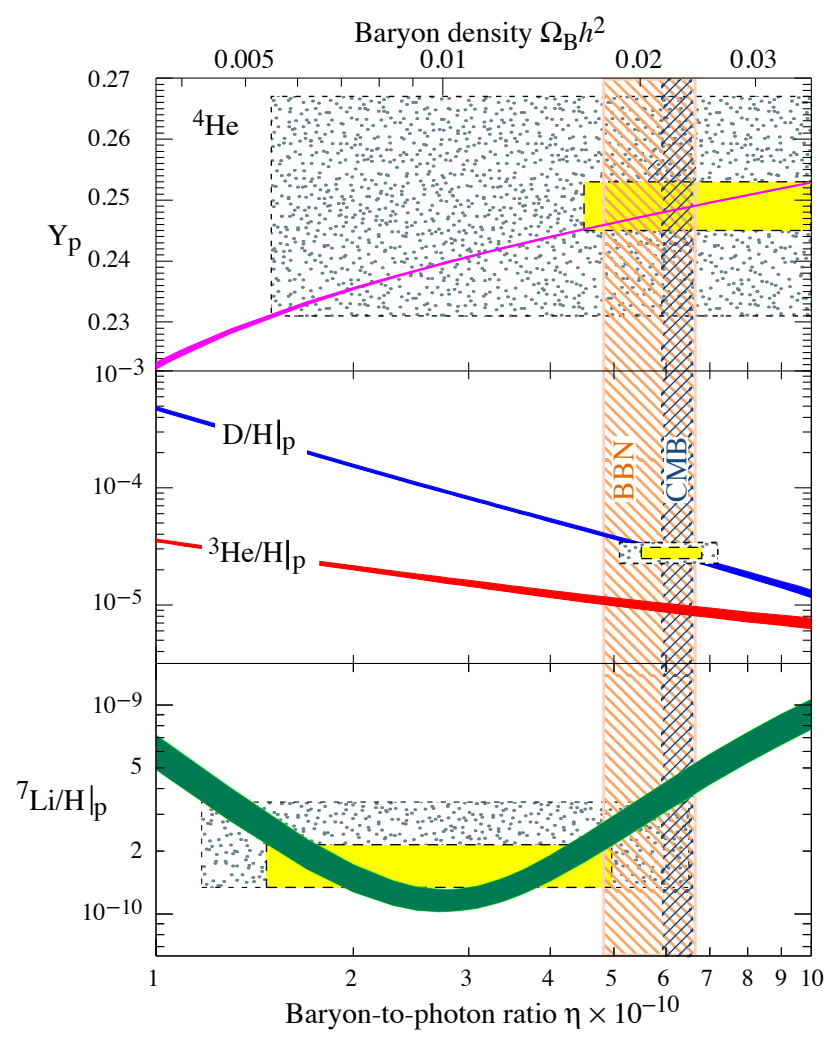

Figure 1.2: Primordial light-element abundances from measurements and predictions in the BBN model. Bands show $95 \%$ confidence level predictions from the standard cosmological BBN model of light element abundances as a function of the baryon to photon ratio during nucleosynthesis. Abundances shown are number ratios to $\mathrm{H}$ expect ${ }_{2}^{4} \mathrm{He}$, which is a ratio by mass $\left(\mathrm{Y}_{p}\right)$. Small boxes indicate the statistical $2 \sigma$ regions of the measurements, while large boxes incorporate systematic errors. The vertical lines indicate the $95 \%$ confidence level ranges from CMB measurements and the combined nucleosynthesis result. Figure taken from [18].

length of the nucleosynthesis, through its control of the universe's expansion rate. Given equal photon densities a greater baryon density leads to a faster fusion to ${ }_{2}^{4} \mathrm{He}$ and fewer nucleons left over in ${ }_{1}^{2} \mathrm{H}$ and ${ }_{2}^{3} \mathrm{He}$. Since the photon density is well-known from the cosmic microwave background's (CMB) temperature, light element ratios provide an excellent "baryometer" with which to measure the abundance of ordinary matter.

Deuterium is the most powerful of these baryometers, since it is no longer created in stars. Since the ${ }_{1}^{2} \mathrm{H}$ abundance is sensitive to $\eta$ and decreases with time, any measurement of the ${ }_{1}^{2} \mathrm{H}$ abundance at high redshift yields a direct upper limit on $\eta$. Current measurements from quasar absorption lines [17] indicate $\eta \approx 5.5$ [18]. These measurements imply a baryon density of $\Omega \approx 0.04$ (see figure 1.2). Ordinary, baryonic matter thus only constitutes a small fraction of the universe's total matter density. The dominant dark matter contribution must therefore be non-baryonic. 


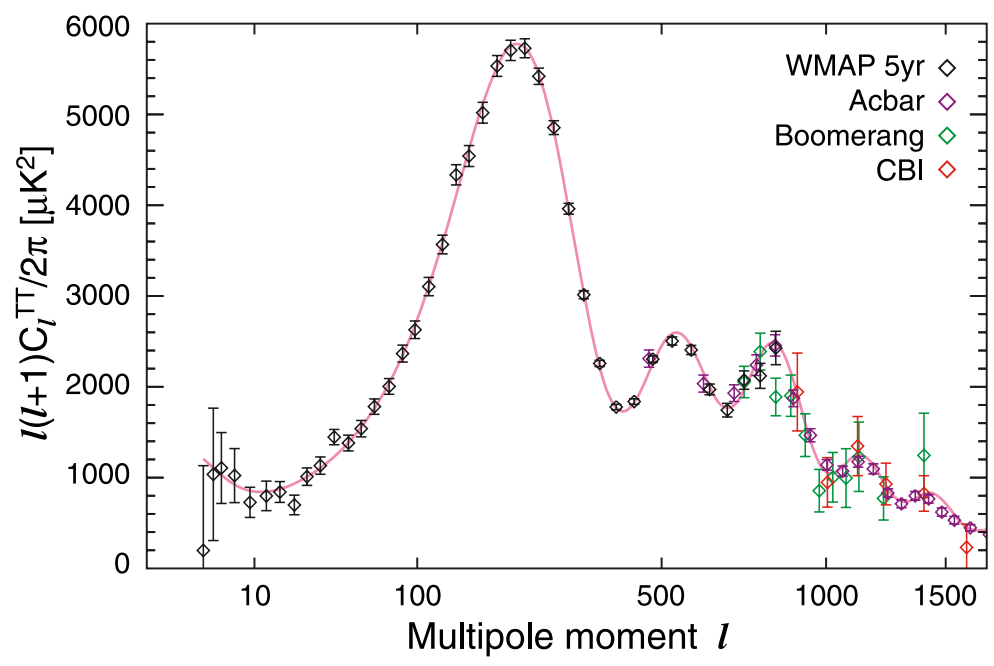

Figure 1.3: Power spectrum of primary CMB temperature anisotropies derived from the WMAP 5 -year data and observations at small angular scales. The red curve in the figure indicates the prediction of the best fit $\Lambda C D M$ cosmology. Figure taken from [19].

\subsubsection{Cosmic Microwave Background}

The CMB is the universe's primordial black body radiation, last scattered when the universe was $\sim 400,000$ years old. Superimposed on the black body spectrum at $2.73 \mathrm{~K}$ the $\mathrm{CMB}$ has anisotropies at the $10 \mu \mathrm{K}$ level. The CMB temperature fluctuations map inhomogeneities in the photon-baryon plasma at the era of decoupling. The inhomogeneities can be interpreted as incoherent acoustic waves in the plasma of the last-scattering surface. The densities of baryonic and non-baryonic matter have a strong impact on these oscillations: the inertia of the oscillating plasma is increased by baryons, while dark matter reduces the driving effect of those oscillations upon the gravitational potential. The angular power spectrum of the CMB anisotropies is thus a sensitive probe of cosmological parameters, encoded in the height and position of spectral peaks. Figure 1.3 shows the power spectrum of CMB fluctuations in temperature, as measured by the Wilkinson Microwave Anisotropy Probe (WMAP) and other recent experiments [15, 19]. The best fit parameters of a $\Lambda C D M$ model to the $\mathrm{CMB}$ anisotropies match those observed in other cosmological measurements.

\subsubsection{Large-Scale Structure Formation}

The basic problem in the formation and growth of the universes' large-scale structure is to understand the evolution from the small perturbations in the very homogeneous early universe, to galaxies, clusters etc., observed in the universe today. The cosmic microwave background shows anisotropies at the $10^{-5}$ level (see section 1.2.2). Since baryons and photons were tightly coupled at this era these fluctuations are a snapshot of the level of 
baryon inhomogeneity of the early universe. Hence, the structures we see in the universe today would originate in this initial baryonic inhomogeneities. Regions with slightly higher density than their surroundings collapsed under their own gravity, forming the seed of galaxies and clusters. In the standard theory of structure formation these over- and underdensities are characterized by the density contrast field:

$$
\delta(\vec{x}, t) \equiv \frac{\rho(\vec{x}, t)-\bar{\rho}(t)}{\bar{\rho}(t)} .
$$

where $\bar{\rho}(t)$ is the universe's mean energy density at time $t$. For small density contrasts $(\delta \ll 1)$ the evolution of the power spectrum of $\delta$ can be solved by linear perturbation theory. Solutions for $\delta>1$ require numerical simulations. For a discussion on the full relativistic theory of gravitational collapse in the expanding universe I refer the reader to [10, 11, 20]. The main results in terms of the existence of dark matter are summarized below:

- In the very early universe radiation pressure prevents perturbations in the photonbaryon plasma from growing significantly after their entry in the causal horizon. The plasma undergoes acoustic oscillations during this period, lasting until the baryons decouple from the photons at the epoch of last scattering.

- The density contrasts in pressurless matter, e.g. dark matter, grow only logarithmically during radiation domination. These density contrasts begin to grow as $\delta \sim a(t)$ once the universe becomes matter-dominated.

- After decoupling of the baryons the photon-baryon plasma can grow in proportion to $a(t)$. After decoupling, the baryons fall into the existing potential wells of dark matter perturbations within a few expansion times.

The last point is the crucial part of the argument for dark matter. The observation of structure at the present day demands $\delta \gtrsim 10^{-3}$ at the era of last scattering, far greater than observed in the CMB small scale anisotropies $(l \sim 1000)$. The anisotropies observed in the CMB have not had time to grow to the structures observed in the universe today. Non-baryonic dark matter solves this problem by decoupling the CMB from the total matter density of the universe. The nearly smooth baryon distribution seen in the CMB small-scale anisotropies rapidly falls into the gravitational potentials of dark matter overdensities, which had more time to grow. 


\subsection{Astronomy}

Astronomical observations reveal the properties of structures in the universe such as galaxies and galaxy clusters. Explaining the observed phenomena, a cosmological independent argument for the existence of dark matter can be made. The main arguments, inferred solely from the gravitational impact of dark matter on visible matter, are discussed below.

\subsubsection{Galaxy Clusters}

Galaxy clusters are the largest gravitational bound systems in the universe. They not only contain visible galaxies but also a much larger fraction of a hot intracluster medium (ICM), and are excellent systems in which to observe the effects of unseen matter. Three methods give strong evidences for a large amount of dark matter in galaxy clusters. These are the cluster dynamics, X-ray emission from the ICM and gravitational lensing which will be discussed in detail below.

\section{Cluster dynamics}

In 1933 Fritz Zwicky measured the line-of-sight velocities of galaxies in the Coma cluster [21, 22]. From the galaxies' velocity dispersion and with Hubble's estimate of the mass of a typical galaxy, Zwicky obtained the total kinetic energy of all galaxies in the cluster. The distance of the cluster had been determined from the mean redshift of the galaxies and with the cluster's angular diameter the physical dimensions of the cluster could be estimated. Using the viral theorem:

$$
2\langle T\rangle=-\langle V\rangle \approx \frac{G M_{\text {cluster }}^{2}}{2<r\rangle}
$$

where $\langle T\rangle$ is the kinetic energy and $\langle V\rangle$ is the potential energy, the cluster's mass could be derived. The result was, that the gravitational mass was much higher than expected based on the luminosity of the galaxies. Such measurements are usually expressed in the massto-light ratio, M/L, normalized to one for the mass and luminosity of the sun. The mass M is inferred from gravitational dynamics using the viral theorem and L from the visible light emitted from the galaxies of the cluster. Zwicky inferred a mass-to-light ratio of several hundred for the Coma cluster, leading to the postulation of unseen matter. Zwicky's result was inflated by an overestimation of the Hubble constant $H_{0}$, but modern estimates of $\mathrm{M} / \mathrm{L}$ are similarly high, supporting the same basic conclusion: galaxy clusters are dominated by unseen matter.

\section{X-ray emission}

The hot intracluster gas can be used to map the gravitational potential of galaxy clusters. Most of the baryonic matter in clusters is actually not gathered into stars but remains in the ICM captured in the cluster's gravitational well. In accordance with the viral theorem 
this gas is heated to $>10^{6} \mathrm{~K}$ as it falls into the center of the gravitational well and emits X-rays by thermal Bremsstrahlung. The emission spectrum depends on the depth of the gravitational well. Hence the observation of X-ray emission of the ICM not only reveals the mass of the ICM, but can also be used to map the dark matter mass distribution. Maps of such X-ray emission by instruments like the Chandra [23] and XMM-Newton [24] space telescopes indicate cluster masses consistent with those from cluster dynamics and gravitational lensing.

\section{Gravitational lensing}

The mass of galaxy clusters can also be inferred from their effect upon light rays from distance sources [25, 26]. In the weak-field low velocity limit of general relativity the gravitational potential well of a galaxy cluster or other massive objects acts as a complex lens, distorting the images of more distant galaxies or quasars in the vicinity of the lens. The major advantage of this approach is that it provides a measurement of the total mass independent of the assumption of viral equilibrium. Mass estimates from gravitational lensing cannot be biased by incomplete gravitational collapse, unlike estimates from cluster dynamics or X-ray emissions.

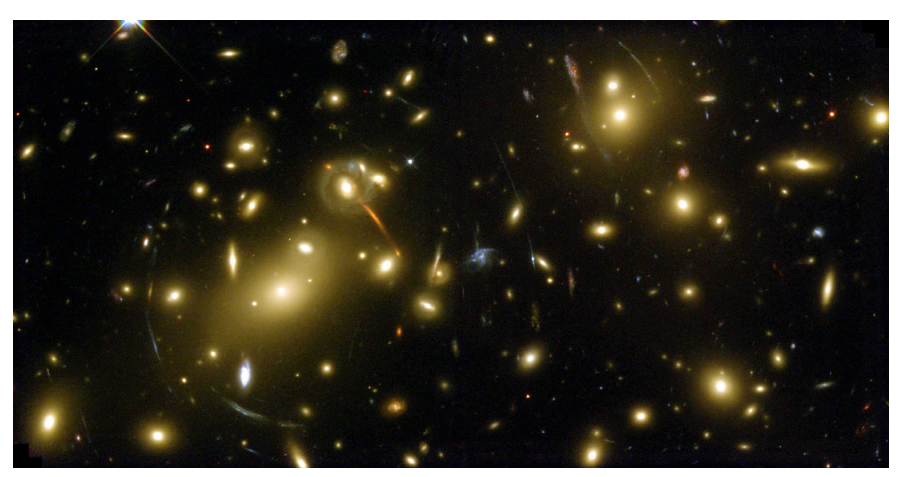

Figure 1.4: Image of the Abell 2218 cluster from the Hubbel space telescope, showing prominent arcs from gravitational lensing. Image from the Space Telescope Science Institute.

The projected mass density along the line of sight may distort the images from distant sources into obvious arcs or rings, or results in a multiply-imaged source. Figure 1.4 shows Abell 2218, a rich galaxy cluster surrounded by prominent arcs. From the actual distortion of the source image the total mass of the gravitational lens can be inferred (see for example [27, 28]).

\section{The "Bullet cluster"}

A dramatic confirmation of the dark matter content of galaxy clusters has been recently observed in the "Bullet cluster" 1E057-558, a pair of colliding clusters visible shortly after their first intersection. The mass distributions of the hot gas and the total mass of this system were reconstructed through a multi-wavelength program [29]. Figures 1.5](a) and 


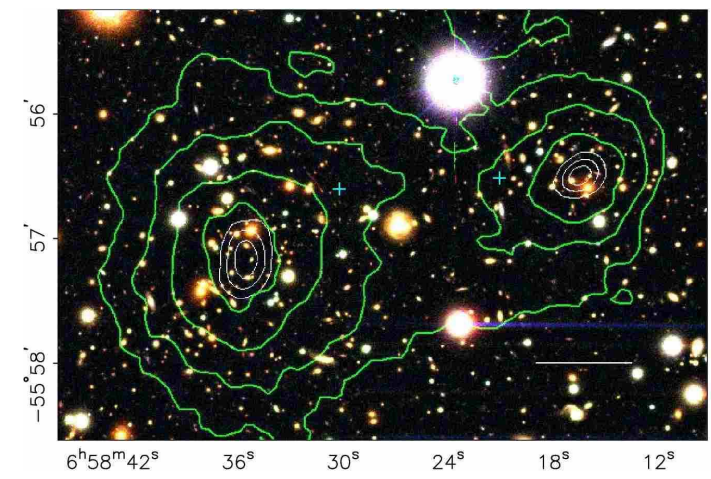

(a)

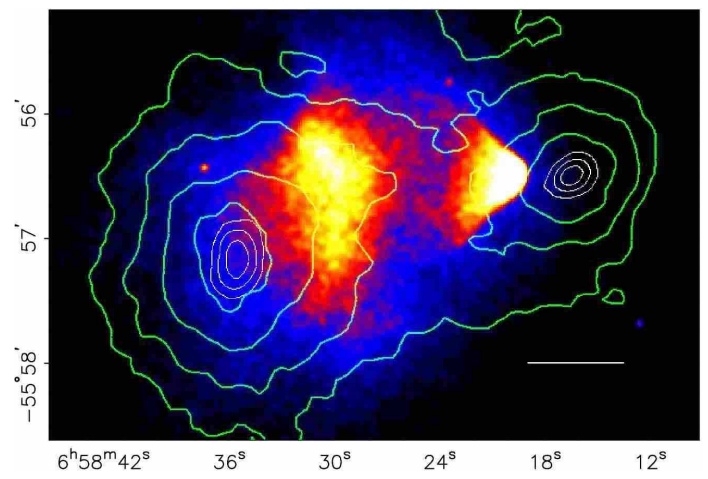

(b)

Figure 1.5: Images of the Bullet cluster (1E0657-558) overlaid with weak gravitational lensing mass contours. (a) Optical image from the Hubble Space telescope. (b) X-ray image from the Chandra X-Ray Observatory. Images taken from 29.

1.5 (b) show the lensing mass contours overlaid on the optical (a) and X-ray (b) images of the structure. The prominent segregation between the total matter (traced by gravitational lensing) and the smaller portion of baryonic matter (traced by X-rays) is a very strong evidence for the dark matter model. The collisionless dark matter halos (as well as the galaxies themselves) have passed through each other essentially unchanged, while the two populations of ICM have been shocked by interactions during the collision and remain in the center of the two colliding structures. This segregation provides the confirmation that the bulk of a cluster's mass is not merely baryonic gas, but is in some essentially unseen non-collisional form. A second system (MACS J0025.4-1222) has been characterized [30], confirming this interpretation.

\subsubsection{Galaxy dynamics}

The total mass enclosed in a region of space can be inferred from the observation of the motion of nearby visible objects. Hence the motion of stars carries information about the surrounding mass distribution. One of the clearest evidences for the existence of dark matter comes from the rotational dynamics of spiral galaxies. Spiral galaxies consist of a central bulge surrounded by a rotating disc of younger stars. The measurement of the galactic rotation speed as a function of radius from the galactic bulge have been performed for a large number of spiral galaxies. The measurements are based on observed Doppler shifts in various spectral features [31. For different radii different spectral features are used; in the central region the rotational transition of $\mathrm{CO}_{2}$, in the disc the visible hydrogen lines and in the outer disc and halo the $21 \mathrm{~cm}$ HI line. Especially the $21 \mathrm{~cm} \mathrm{HI} \mathrm{line} \mathrm{is}$ particularly useful, as it is detectable at radii well outside the visible galactic disc.

If light traces the matter distribution, it is expected from Newtonian mechanics, that the orbital velocity of galactic material at radii outside the visible disc decreases as $\sim r^{-1 / 2}$. 


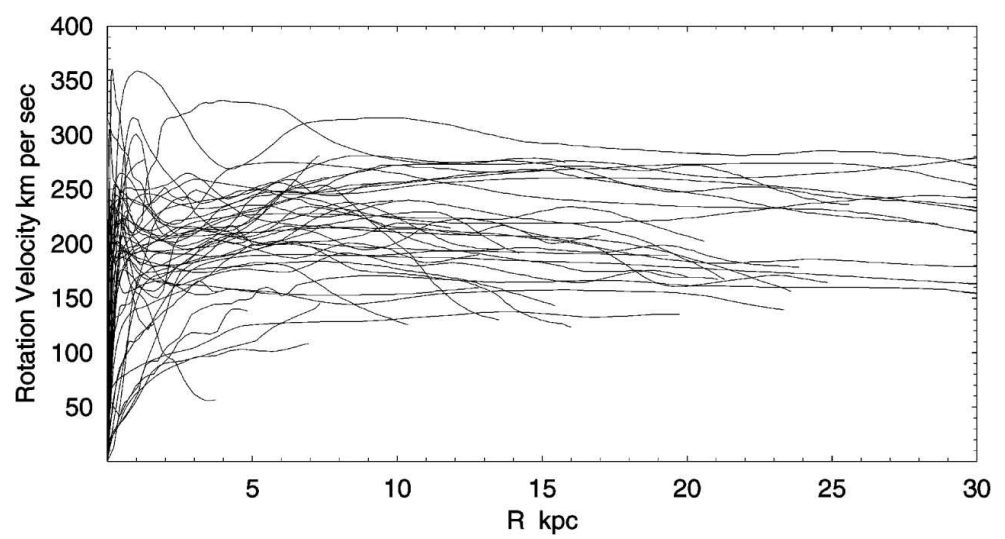

Figure 1.6: Superposition of rotation curves from various spiral galaxies. Velocities are measured with a variety of techniques at different radii (see text). Figure taken from [31.

However, the observed rotation curves shown in figure 1.6 are remarkably flat at high radii. This observation is consistent with Newtonian mechanics for a roughly spherical mass distribution with total enclosed mass proportional to the radius $(\mathrm{M}(\mathrm{r}) \propto \mathrm{r})$. These measurements suggest that the visible matter is embedded in a substantially larger "dark matter halo", which extends well beyond the visible disc. Without the gravitational mass of this halo, the disc at large radii would fly apart.

Using models for the mass distributions in the bulge and disc of the Milky Way, the expected behavior of the orbital velocity as a function of radius can be derived. Figure 1.7 shows measured velocities as a function of the radius from the galactic center and the expectation from the baryonic mass distributions of the Milky Way. It can be clearly seen that the prediction does not match the observations for the Milky Way. However, by adding a dark halo the measurements are very well described by the expected orbital velocities. The choice of the mass distribution in the dark halo is a priori arbitrary and a large range of distributions, although sometimes unphysical, can describe the data [33]. In $\Lambda C D M$ cosmology the formation of halos can be modeled and predictions about the parameters of the dark matter mass distribution can be made. These results are used in figure 1.7 for the prediction of the dark matter component in the expected orbital velocities.

If the full dark matter mass distribution would be known, the local dark matter density at the position of the Sun could be calculated. However, plenty models fit the measurements quite well, all giving different values for the local dark matter density. The range of allowed densities at the Sun's vicinity is $\rho_{D M} \approx 0.1-0.8 \mathrm{GeV} / \mathrm{cm}^{3}$. 


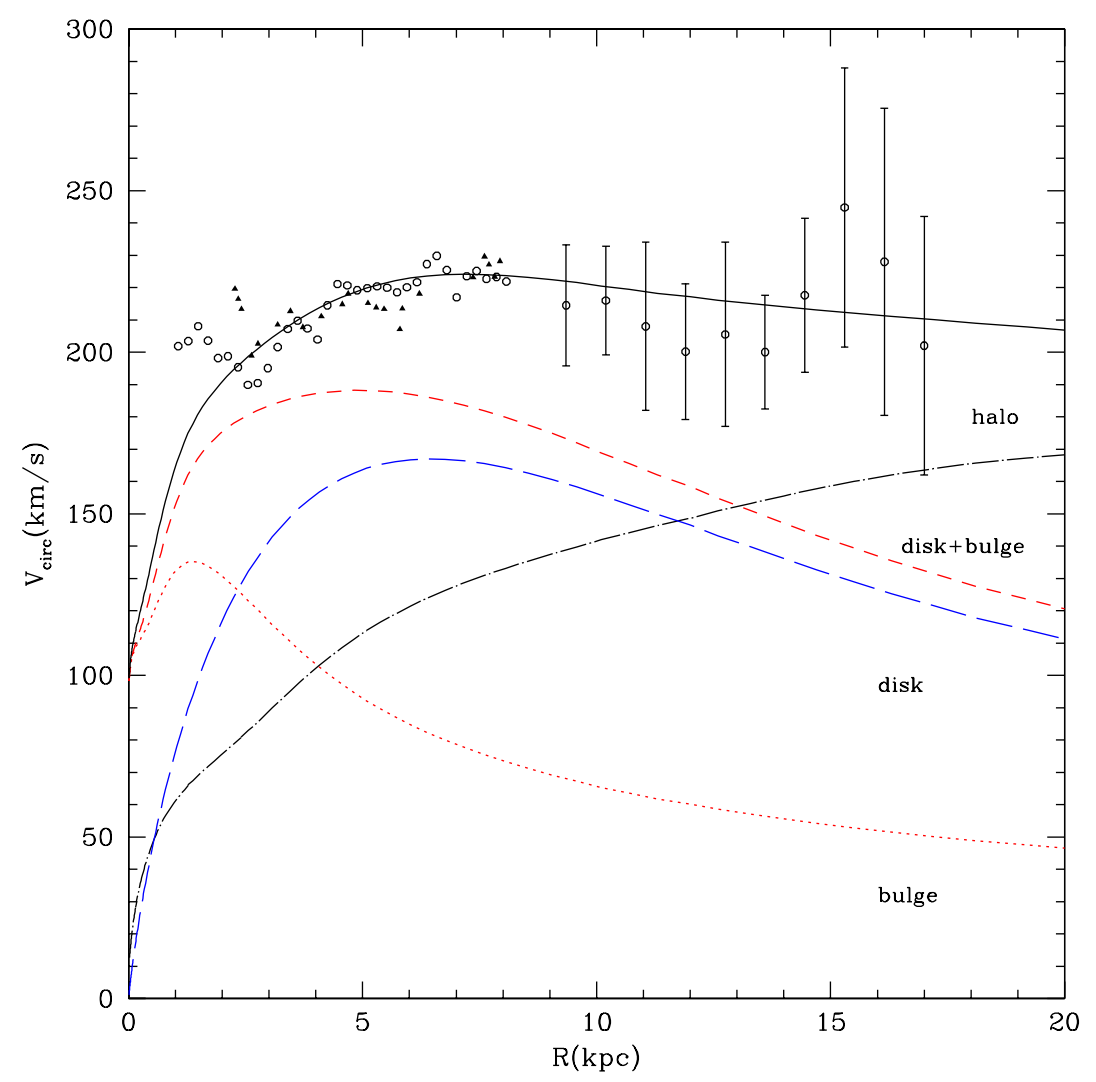

Figure 1.7: Measurements of the rotational velocity at different radii of the Milky Way. The data can only be described if in addition to the expectations from the baryonic disc and bulge a contribution from a dark matter halo is taken into account. Figure taken from 32 . 


\section{Chapter 2}

\section{Candidates for Dark Matter}

The current model of dark matter encompasses that dark matter is predominantly nonbaryonic. This is supported by evidence from nucleosynthesis and the cosmic microwave background (see Section 1.2.1 and 1.2.2). Non-baryonic matter should also be weakly interacting, supported by upper-limits on the self-interaction from astrophysical observations (see Section 1.3.1). In order to be present in the universe today, dark matter needs to be stable, or at least have a lifetime which is long compared to the age of the universe. Finally, dark matter has to be "cold" - that is slow moving. A cold dark matter candidate must be non-relativistic throughout the formation of large-scale structures. The large random velocities of relativistic dark matter particles ("hot dark matter") would disrupt the hierarchical formation of large-scale structures (see Section 1.2.3). Beyond these conditions, very little about dark matter is known. In particular the identity of the particles which constitute the dark matter remain a mystery.

Numerous candidates for dark matter have been proposed. A major candidate for dark matter are weakly interacting massive particles which can arise in extensions of the Standard Model of particle physics such as Supersymmetry [34, 35, 36, 37] or extra spatial dimensions which could either be flat [38] or warped [39]. Other massive particles are "SuperWIMPs" 40], "SIMPZillas" 41] or "Super-heavy relics" 42, 43. There are also candidates which are very light. The most natural candidate would be the Standard Model neutrinos since there is experimental evidence that these particles have a small mass [44, 45, 46, 47]. Another candidate would be the "axion" which has been postulated to solve the strong-CP problem of quantum chromodynamics.

The mentioned candidates are far from listing all possible candidates which have been proposed as dark matter candidates. A full discussion of each possible candidate would be beyond the scope of this work. Thus, only two classes of candidates, WIMPs and Axions, and their theoretical motivation which are the most relevant for the work presented in this dissertation are introduced. 


\subsection{Weakly interacting massive particles}

Weakly interacting massive particles (WIMPs) are a generic class of dark matter candidates, widely regarded as the most promising. These hypothetical particles have masses $1 \mathrm{GeV} \lesssim M c^{2} \lesssim 10 \mathrm{TeV}$ and couplings on the order of the weak scale. The strength of the WIMP hypothesis comes from the fact, that very general considerations of cosmology and particle physics point independently to the existence of such particles.

A massive particle $\chi$ which is in thermal equilibrium in the early universe, will remain in thermal equilibrium until the universe's expansion rate renders the annihilation rate inefficient $\left(\Gamma_{\chi \chi} \lesssim H\right)$. If the particle is non-relativistic at this point, the equilibrium number density is suppressed exponentially at low temperatures:

$$
n_{\chi}(T) \simeq g\left(\frac{M_{\chi} k T}{2 \pi \hbar}\right)^{3 / 2} e^{-\frac{M_{\chi} c^{2}}{k T}} .
$$

An accurate calculation of the relict density of such particles (number density after freezeout) requires numerical solutions of the Boltzmann equation in an expanding universe:

$$
\frac{d n_{\chi}(t)}{d t}+3 H n_{\chi}(t)=-\left\langle\sigma_{\chi \chi} v\right\rangle\left[n_{\chi}^{2}(t)-\left(n_{\chi}^{(e q)}\right)^{2},\right]
$$

where $\left\langle\sigma_{\chi \chi} v\right\rangle$ is the velocity average annihilation cross section. An approximate solution for the relict density is [48]:

$$
\Omega_{\chi} h^{2} \simeq \frac{0.1 p b \cdot c}{\left\langle\sigma_{\chi \chi} v\right\rangle}
$$

The relict density thus depends on the annihilation cross section of the particles. A particle with a higher annihilation cross section can remain longer in thermal equilibrium, leading to further Boltzmann suppression and thus a lower relic density. Figure 2.1 illustrates the general character of the Boltzmann equation solutions and the dependency of the relic density on the velocity averaged annihilation cross section.

The approximate relic density given in equation (2.3) suggests that such particles will have a relic abundance comparable to the observed one for non-baryonic dark matter $\left(\Omega_{D M} h^{2} \simeq 0.1\right.$, see section 1.1), if the annihilation cross section is in the typical order of magnitude of weak cross sections ( $\sim$ pb scale). Hence any stable, massive particle interacting through the weak nuclear force would naturally be a dark matter candidate. Such a particle would also satisfy the constraints from cosmological observations.

For completely different reasons, the Standard Model of particle physics [49, 50] also argues for the existence of new particles at the weak scale $(\sim 100 \mathrm{GeV})$. In the Standard Model interactions among the fermionic fields are mediated by spin-1 gauge fields. Weak interactions are mediated by massive bosons: the $W^{ \pm}\left(\mathrm{M}_{W}=83 \mathrm{GeV} / \mathrm{c}^{2}\right)$ and $\mathrm{Z}^{0}\left(\mathrm{M}_{Z}=\right.$ $\left.91 \mathrm{GeV} / \mathrm{c}^{2}\right)$. These particles acquire mass by the spontaneous breaking of an $\mathrm{SU}(2) \times \mathrm{U}(1)$ gauge symmetry to the simpler $\mathrm{U}(1)$ of electromagnetism. This symmetry is broken by the formation of a spinless SU(2)-doublet field, the Higgs Boson with a mass comparable to that of the $W^{ \pm}$and $\mathrm{Z}^{0}$. With the addition of the Higgs Boson (which has thus far 


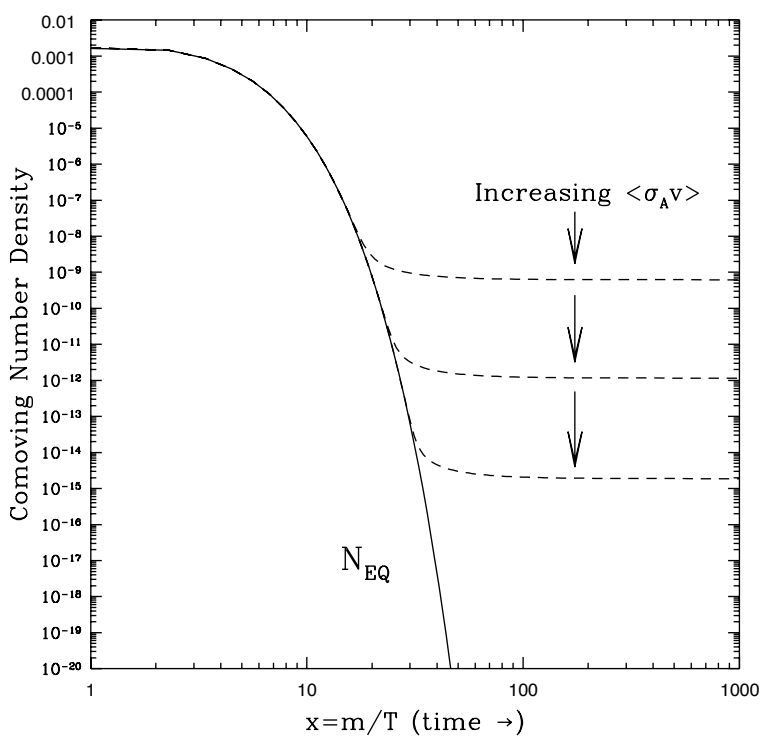

Figure 2.1: Comoving number density of WIMPs as a function of temperature (measured in units of the particle's mass) in the universe. The solid curve shows the equilibrium abundance, while dashed lines show the dependency of the abundance with increasing velocity averaged annihilation cross section. Figure taken from [10]

remained undiscovered in experiments) and of neutrino mass terms, the Standard Model is in excellent agreement with experimental data to date.

Despite the successes of the Standard Model, it has several problems which suggest that it is not the fundamental theory of nature. The most interesting hint for new particles is the hierarchy problem: physics beyond the Standard Model are needed to stabilize the large hierarchy between the weak $\left(10^{3} \mathrm{GeV}\right)$ and Planck $\left(10^{19} \mathrm{Gev}\right)$ scales without fine-tuning. The masses of Standard Model particles are subject to radiative corrections due to loop diagrams. The contributions of these diagrams are formally divergent, but become finite if it is assumed that new physics appear at some high energy scale $\Lambda$ (e.g. the Planck scale) to regulate these loops. Such corrections are of no concern as long as their magnitude slightly depends upon $\Lambda$ (e.g. logarithmically), but if they are large (e.g. proportional to a positive power of $\Lambda$ ) it is expected that they drive the relevant masses towards $\Lambda$. The Standard Model must thus be fine-tuned to prevent the Higgs mass (and thus the $\mathrm{W}^{ \pm}$and $\mathrm{Z}^{0}$ masses) from sitting near the Planck scale. New particles at the weak-scale needed to solve the hierarchy problem are natural WIMP candidates.

\subsubsection{Supersymmetry}

Supersymmetric extensions of the Standard Model often provide natural candidates for WIMP dark matter. Below the basics of supersymmetry will be reviewed. For further details on supersymmetric theories and their phenomenology I refer the reader to [34, 35, 36, 37. A full discussion on supersymmetric dark matter can be found in [48]. 

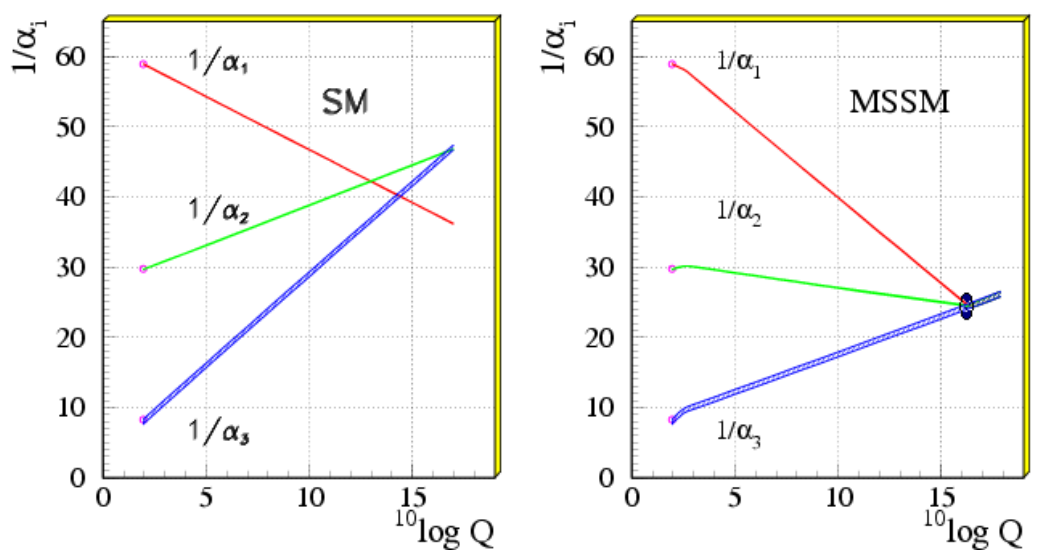

Figure 2.2: Gauge coupling unification in grand unified theories (GUTs) without (left panel) and with (right panel) $\mathrm{TeV}$-scale supersymmetric particles. In both panels the inverse of the gauge group's coupling constant is shown as a function of the renormalization energy scale. Figure taken from [52].

Supersymmetric models propose a symmetry between fermionic and bosonic fields and are based on the observation that radiative corrections due to fermion and boson loops have opposite signs. If each fermionic degree of freedom in the Standard Model is matched to a corresponding "sfermion" of the same mass and gauge charges, their radiative corrections cancel exactly. Similar cancellations occur if each gauge boson degree of freedom is matched to a fermion "gaugino" of the same mass and charges. Supersymmetry must be clearly broken since such particles have not been observed. The "superpartners" cancel out the worst of the radiative corrections, even if the masses do not exactly match, leaving only a logarithmically dependence on the mass difference between the partners. If these new particles have masses at the $\mathrm{TeV}$ scale, radiative corrections are limited to this scale and the weak scale is naturally stabilized.

Supersymmetry (SUSY) can also resolve one of the long standing problems of grand unified theories (GUTs), which unify the Standard Model gauge interactions at high energies as part of a larger gauge group [51]. For this to be true, radiative corrections have to drive the various couplings to a common value at some high energy scale. These corrections depend upon the mass and couplings of the involved particles, and for the Standard Model particles the couplings almost unify (see left panel in figure 2.2). The addition of supersymmetric partners at $\mathrm{TeV}$ masses alter the radiative corrections, allowing the coupling constants to unify at some high energy scale (see right panel in figure 2.2). The higher unification scale also raises the proton lifetime sufficiently in some SUSY GUT models to evade experimental constraints [53, 54, 55] 


\section{The minimal supersymmetric standard model}

The simplest extension of the Standard Model is the Minimal Supersymmetric Standard Model (MSSM) [56, 57. The MSSM adds the minimal number of particles necessary to incorporate the Standard Model in a supersymmetric theory:

1. A Majorana fermion for each Standard Model gauge boson before electroweak symmetry breaking: $\tilde{b}^{0}, \tilde{w}^{0}, \tilde{w}^{ \pm}, \tilde{g}_{i}^{0}$.

2. Two scalars for each Dirac fermion, representing left and right chiralities: $\tilde{e}_{L, R}, \tilde{u}_{L, R}$, etc.

3. A complex Higgs doublet is required to give masses to both up-type and down-type quarks.

4. Four Majorana fermion higgsinos, two charged and two neutral: $\tilde{h}^{ \pm}, \tilde{h}_{1,2}^{0}$.

In the MSSM mixing of electroweak Majorana fermions with identical quantum numbers produces new physical mass eigenstates:

- Four Majorana neutralinos $\chi_{i}^{0}(i=1,2,3,4)$, each a mixture of $\tilde{b}^{0}, \tilde{w}^{0}$ and $\tilde{h}_{1,2}^{0}$.

- Two pairs of Majorana charginos $\chi_{j}^{ \pm}(j=1,2)$, each a mixture of $\tilde{w}^{ \pm}$and $\tilde{h}^{ \pm}$.

With the most general supersymmetric Lagrangian with this particle content, which is invariant under the Standard Model gauge groups, the usual gauge and Yukawa interactions of the Standard Model fields are obtained, as well as a new mass parameter $\mu$ giving mass to the Higgs bosons. In addition, there are several terms which mediate interactions that violate baryon or lepton conservation. For example these new interactions would permit tree-level proton decay by processes which are only suppressed by the $\mathrm{TeV}$-scale mass of the superpartners. This suppression is not enough to be consistent with current experimental limits on the proton life time. To satisfy experimental constraints, a realistic theory of lowenergy supersymmetry must thus forbid (or at least strongly suppress) such new processes.

This can be achieved by introducing a new discrete symmetry called $R$-parity. An MSSM particle's $R$-parity can be expressed as:

$$
R=(-1)^{2 j+3 B+L}
$$

where $j$ is the particle's spin, $B$ is its baryon number and $L$ is its lepton number. All Standard Model particles and Higgs bosons have an $R$-parity of +1 , while their superpartners have an $R$-parity of -1 . If $R$-parity is conserved all lepton and baryon violating couplings are eliminated. Furthermore, this renders the lightest supersymmetric partner (LSP) absolutely stable, since the lightest $R=-1$ cannot decay without violating $R$-conservation. In any supersymmetric model with $R$-conservation the LSP is thus a natural dark matter candidate. 
In the MSSM it is not attempted to explain SUSY-breaking from first principles, but merely to describe the low-energy effective field theory after it is broken. The MSSM thus introduces in the supersymmetric Lagrangian the most general set of interactions which break supersymmetry without re-introducing the $\Lambda^{n}$ divergence. These "soft "SUSYbreaking terms encompass gaugino masses, scalar masses and trilinear couplings among the various fields. By doing so, the full MSSM introduces more than 100 new free parameters - masses, trilinear couplings, mixing angles, and CP-violating phases - beyond those of the Standard Model.

\section{Constrained supersymmetric models}

Due to the vast number of free parameters in the MSSM, phenomenological studies of supersymmetry are generally carried out in restricted subspaces of the MSSM parameter space. The most popular of these is the constrained MSSM (CMSSM) [58. This model imposes a series of strong constraints inspired by GUTs and theories of gravity-mediated SUSY-breaking:

- All scalar masses unify to a common value $M_{0}$ at the GUT scale.

- All gaugino masses unify to a common value $M_{1 / 2}$ at the GUT scale.

- All trilinear couplings take a common value $A_{0}$ at the GUT scale.

- Electroweak symmetry is broken by radiative corrections to the Higgs potential, following the Coleman-Weinberg mechanism [59].

After imposing these conditions the entire SUSY spectrum can be characterized using only five free parameters:

1. The unified scalar mass $M_{0}$

2. The unified gaugino mass $M_{1 / 2}$

3. The unified trilinear coupling $A_{0}$

4. The ratio of the two Higgs vacuum expectation values, specified as $\tan \beta \equiv \frac{v_{2}}{v_{1}}$

5. The sign of the Higgs mass parameter $\mu$

This simplified model is almost certainly incorrect as the true representation of nature, but hopefully encompasses most of the major mechanisms at work in the correct theory. 


\subsection{Axions}

The Lagrangian of quantum chromodynamics includes a CP-violating term which would, if present, endow the neutron with an electric dipole moment. This is in contradiction with current experimental upper limits on the neutron's electric dipole moment. A solution to this problem is the postulation of a new field resulting in a new particle: the axion. Depending on the mass of the axion it is a valid and cosmological relevant dark matter candidate. The following sections introduce the strong CP-problem and the axion solution to the problem.

\subsubsection{The $\theta$-vacua}

The Lagrangian of quantum chromodynamics (QCD) is:

$$
\mathscr{L}=-\frac{1}{2 g^{2}} \operatorname{Tr} F_{\mu \nu} F^{\mu \nu}+\frac{1}{2} \partial_{\mu} a \partial^{\mu} a+\bar{q}(i \not D-M)
$$

where $M$ is the diagonal quark mass matrix obtained by symmetry breaking of the electroweak gauge group. The solution to the field equation for a pure $\mathrm{SU}(2)$ Yang-Mills theory derived from (2.5) are called instantons (see for example [60]). The instanton describes a solution of the gauge field equations in which a vacuum evolves into another vacuum. These vacua can be classified by the Pontryagin index $q=-\infty, \ldots,-1,0,+1, \ldots,+\infty$. The Yang-Mills vacuum is therefore infinitely degenerate, consisting of an infinite number of non-equivalent vacua. The question now is, what is the amplitude for a transition from one vacua to another? Classically it would be zero since there is an energy hump between two vacua. However quantum mechanics allows tunneling between different vacua given by a barrier penetration amplitude. Thus in a Yang-Mills quantum theory the vacuum is infinitely degenerate, but with non-zero transition amplitudes between gauge rotated vacua. Thus, it follows that the ground state of the Hilbert space may be written as [61]

$$
\left|\theta>=\sum_{n=-\infty}^{\infty} e^{i n \theta}\right| n>
$$

Since QCD contains $\mathrm{SU}(2)$ as a subgroup it admits the instanton solutions.

In the $\theta$ vacuum $\mathrm{CP}$ violating interactions must be considered. These can be parametrized as $\bar{\theta}=\theta_{0}+\theta_{\text {weak }}$, giving an interaction term in the Lagrangian of :

$$
\mathscr{L} \equiv \frac{\bar{\theta}}{64 \pi^{2}} \epsilon^{\mu \nu \rho \sigma} F_{\mu \nu}^{a} F_{\rho \sigma}^{a}
$$

where $\theta_{0}$ is the angle given above the electroweak scale and $\theta_{\text {weak }}$ is the value introduced by the electroweak CP violation. The observable nonzero vacuum angle $\bar{\theta}$ has led to the so called "strong CP problem". The interaction term (2.7) gives rise to huge CP violating effects in QCD unless the parameter $\bar{\theta}$ is very small. The effects of this interaction can be searched for in CP violating static properties of (almost) stable hadrons. One of these measurements is the upper bound on the neutron's electric dipole moment which will be discussed below. 


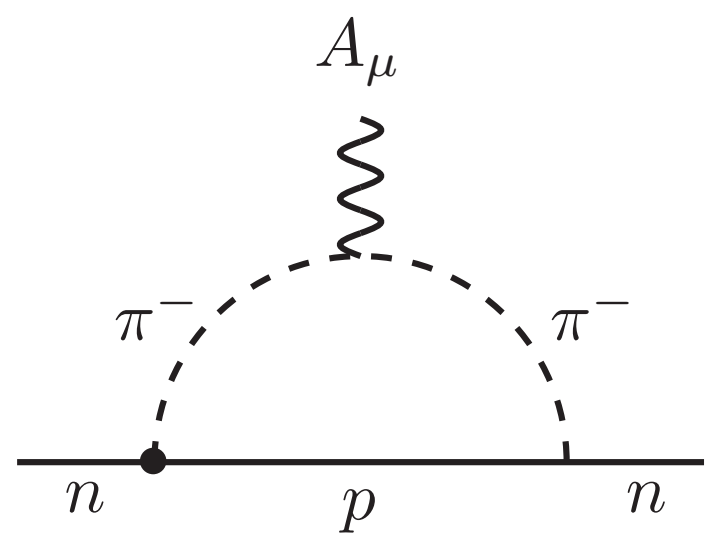

Figure 2.3: Feynman diagram contributing to the neutron's electric dipole moment. The bullet point represents the $\mathrm{CP}$ violating effect. Figure taken from 62

\subsubsection{Electric dipole moment of the neutron}

An electric dipole moment of the neutron can arise through diagrams shown in figure 2.3. The CP violation is present by a mismatch between the $\mathrm{CP}$ conserving right hand side vertex and the $\mathrm{CP}$ violating left hand side vertex. The neutron's electric dipole moment can be estimated as:

$$
\left|d_{n}\right|=\frac{g_{\pi n n} \cdot \overline{g_{\pi n n}}}{4 \pi^{2} m_{n}} \ln \left(\frac{m_{n}}{m_{\pi}}\right) e c m
$$

where the $\mathrm{CP}$ violating scalar coupling $\overline{g_{\pi n n}}$ (bullet point in figure 2.3 ) is estimated as [62]:

$$
\overline{g_{\pi n n}}=\hat{\theta} \frac{Z}{(Z+1)}
$$

where $Z=m_{u} / m_{d} \approx 0.5$ is the up/down quark mass ratio.

With these formulas the electric dipole moment of the neutron can be calculated. Taking $g_{\pi n n} \simeq 13$ [62] one obtains $\left|d_{n}\right|=4.5 \times 10^{-15} \bar{\theta}$ ecm. The upper bound on the neutron's electric dipole moment $\left|d_{n}\right|<2.9 \times 10^{-26}$ ecm [63] thus requires:

$$
|\hat{\theta}|<0.7 \times 10^{-11}
$$

This extremely small upper bound on $\hat{\theta}$ is the so called "strong CP problem" . The value of $|\hat{\theta}|<10^{-11}$ is perfectly allowed but it is not explained why it is so small. The CP problem is thus a kind of naturalness problem.

\subsubsection{The axion solution}

The most attractive solution for the strong CP problem is based on the idea by Peccei and Quinn of making $\bar{\theta}$ a dynamical field [64, 65]. Without a Peccei-Quinn U(1) symmetry $\bar{\theta}$ is a coupling of the theory, different values of $\bar{\theta}$ describe different theories. The Peccei-Quinn 
(PQ) mechanism, e.g the dynamical interpretation of $\bar{\theta}$, interprets different values of $\bar{\theta}$ as different vacuum states in a given theory. If it can be proven that $\bar{\theta}=0$ is the chosen true vacuum, then $\bar{\theta}$ will roll down to 0 regardless of the initial value of $\bar{\theta}$. The interpretation of the dynamical $\bar{\theta}$ as a field $a$ by $\bar{\theta}=a / f_{a}$ in the QCD Lagrangian fixes $\bar{\theta}=0$. The QCD Lagrangian with gluon fields, massive quarks and this field can be written as:

$$
\mathscr{L}=-\frac{1}{4 g^{2}} F_{\mu \nu}^{a} F^{\mu \nu a}+\frac{1}{2} \partial_{\mu} a \partial^{\mu} a+\sum_{i} \bar{q}_{i}\left(i \not D-m_{i}\right) q_{i}+\frac{a}{32 \pi^{2} F_{a}} F_{\mu \nu}^{a} \tilde{F}^{\mu \nu a}+\frac{\theta^{\prime}}{32 \pi^{2}} F_{\mu \nu}^{a} \tilde{F}^{\mu \nu a}
$$

where $\theta^{\prime}$ is the vacuum angle given by the initial choice of $\theta_{Q C D}$ and by the effect of the electroweak symmetry breaking. An effective vacuum angle will be $\left\langle a>/ f_{a}+\theta^{\prime}\right.$. Shifting the field $a \rightarrow a-f_{a} \theta^{\prime}$ we can neglect $\theta^{\prime}$. By considering the potential energy of the vacuum it can be shown (see for example [61]), that a minima of the potential energy is at $a=0$ or

$$
\bar{\theta}=<a>/ f_{a}=0
$$

Thus the dynamical $\bar{\theta}$ field solves the strong CP problem.

It has to be noted that in the derivation of this result weak interactions have been neglected. If weak $\mathrm{CP}$ violation is taken into account new diagrams contribute to the potential. These result in a minimum of the potential of $|\bar{\theta}| \approx 10^{-17}[66$. Therefore, the PQ mechanism does not give $\bar{\theta}=0$ but a tiny value due to weak CP violation.

Weinberg [67] and Wilczek [68] realized that an inevitably consequence of the PQ mechanism is a new pseudoscalar boson, the axion, which is the Nambu-Goldstone boson of the PQ symmetry. Although the original Peccei-Quinn-Weinberg-Wilczek axion model has been ruled out, "invisible" axion models allow a wide range of axion masses and axionmatter couplings. A detailed discussion of these models would be beyond the scope of this work, so I refer the reader to 61] for a in-depth discussion.

Here it should only be noted that the PQ symmetry is explicitly broken at low energies and axions acquire a small mass of

$$
m_{a}=\frac{\sqrt{Z}}{(Z+1)} \frac{f_{\pi} m_{\pi}}{f_{a}}
$$

where $f_{p i}=92 \mathrm{MeV}$ is the pion decay constant and $f_{a}$ is the PQ scale or axion decay constant.

Another important property of axions is their two photon interaction,

$$
\mathscr{L}_{a \gamma \gamma}=\frac{g_{a \gamma \gamma}}{4} F_{\mu \nu} F^{\mu \nu} a=-g_{a \gamma \gamma} \vec{E} \cdot \vec{B} a
$$

where $F$ is the electromagnetic field-strength tensor, and $\vec{E}$ and $\vec{B}$ are the electric and magnetic fields, respectively. The coupling constant is

$$
g_{a \gamma \gamma}=\frac{a}{2 \pi f_{a}}\left(\frac{E}{N}-\frac{2}{3} \frac{4+Z}{1+Z}\right)=\frac{a}{2 \pi}\left(\frac{E}{N}-\frac{2}{3} \frac{4+Z}{1+Z}\right) \frac{1+Z}{\sqrt{Z}} \frac{m_{a}}{m_{\pi} f_{\pi}}
$$


Here $\mathrm{E}$ and $\mathrm{N}$ are the model dependent electromagnetic and color anomaly of the axial current associated with the axion field. Frequently cited generic examples of axion models are the Kim-Shifman-Vainshtein-Zakharov (KSVZ) model [69, 70] where $E / N=0$ and the Dine-Fischler-Srednicki-Zhitnitskii (DFSZ) model [71, 72] where $E / N=8 / 3$, but a much broader range of possibilities exists [73]. 


\section{Chapter 3}

\section{Direct detection of Dark Matter}

Direct detection experiments aim to detect dark matter particles via their elastic scattering off atomic nuclei. The rate of these interactions depends on astrophysical assumptions about the phase-space of dark matter particles in the vicinity of the Earth. A quite general ansatz for the expected recoil rate can be derived without any assumption on the actual WIMP nucleus coupling. Depending on the coupling, the properties of the target nuclei have to be taken into account in the evaluation of the expected scattering rate.

Recent developments in the N-body simulations of dark halo formation, which include the effect of the baryons showed that apart from the standard halo a macroscopic dark matter disc can form during the galaxy's evolution. Dark matter particles in this disc populate a different phase-space than particles in the halo and this alters the expected rates in direct detection experiments.

Each direct detection experiment faces the challenge of identifying candidate events among a far larger background rate. The techniques for reducing and discriminating background events from WIMP candidates used in modern direct detection experiments are introduced, and the different technologies used by several experiments will be explained.

\subsection{WIMP - nucleus scattering}

Since the Earth moves through the dark matter halo as the Solar system moves around the galactic center, dark matter interactions may occur in earthbound detectors. If a dark matter particle elastically scatters off a nucleus in a detector a sufficient sensitive detector may be able to observe this interaction [74]. Based on an argument of "crossing symmetry" between the annihilation and scattering process it is plausible that WIMP interaction cross sections are of similar magnitude as the WIMP self-annihilation cross section needed to set the WIMP relic density to the observed value (see Section 2.1). This sets the characteristic WIMP-nucleus scattering cross section in the regime of weak scale interactions. It has to be noted that this is just a qualitative argument, since many model dependent considerations can alter the relationship between scattering and annihilation-cross section. Due to the weak scale nature of WIMP interactions, the rate of interactions is expected to be much 
less than one per day of exposure and kilogram of target mass.

Even without assuming a specific WIMP model, the expected differential recoil rates in a direct detection experiment can be calculated based on assumptions on the distribution of WIMPs in the Milky Way's halo. The derivation of the differential recoil rate is discussed in the next section. The model dependence is taken into account in the cross section entering the differential recoil rate formula. A priori the model dependent WIMPnucleon scattering amplitudes which determine the cross section of the interaction can take a variety of different forms. However it has been shown quite generally [75], that only scalar and axial-vector terms survive in the extreme non-relativistic limit of the interaction Lagrangian. In this limit the general interaction Lagrangian becomes:

$$
\mathcal{L}_{\chi N} \approx 4 \chi^{\dagger} \chi\left(f_{p} \eta_{p}^{\dagger} \eta_{p}+f_{n} \eta_{n}^{\dagger} \eta_{n}\right)+16 \sqrt{2} G_{F} \chi^{\dagger} \frac{\vec{\sigma}}{2} \chi \cdot\left(a_{p} \eta_{p}^{\dagger} \frac{\vec{\sigma}}{2} \eta_{p}+a_{n} \eta_{n}^{\dagger} \frac{\vec{\sigma}}{2} \eta_{n}\right)
$$

where $\chi$ is the WIMP wave function, $\eta_{p(n)}$ is the proton (neutron) Weyl spinor and $\vec{\sigma}$ is the spin operator (Pauli spin matrices). The WIMP-nucleon interaction is thus characterized by four couplings: the "spin-independent" (SI) couplings $f_{p}$ and $f_{n}$ and the "spindependent" (SD) couplings $a_{p}$ and $a_{n}$. The cross section for spin-independent scattering is described in section 3.1 .2 and for spin-dependent scattering in section 3.1.3.

\subsubsection{Energy spectra}

In this section the energy spectra of WIMP - nuclear recoils is derived, following the calculations of [76]. Let us consider a target nuclei with atomic mass $M_{T(u)}$ measured in AMU 1. The event rate per unit mass is then given by

$$
d R=\frac{N_{0}}{M_{T(u)}} \cdot \sigma \cdot v \cdot d n
$$

where $N_{0}$ denotes the Avogardo number, $\sigma$ the WIMP - nucleus cross section, $v$ the velocity of the WIMP impacting on the nucleus and $d n$ is the differential particle density given by

$$
d n=\frac{n_{0}}{k} \cdot f\left(\mathbf{v}, \mathbf{v}_{E}\right) \cdot d^{3} \mathbf{v}
$$

Here $n_{0}$ is the mean dark matter particle density:

$$
n_{0}=\frac{\rho_{D M}}{M_{D M}}
$$

for a WIMP with mass $M_{D M}$ and local density $\rho_{D M}$. In equation $3.3 k$ denotes a normalization constant such that

$$
\int_{0}^{v_{e s c}} d n \equiv n_{0}
$$

\footnotetext{
${ }^{1}$ Atomic Mass Unit
} 
In this equation the galactic escape velocity $v_{e s c}$ of the dark matter particles has been introduced. Comparing equations (3.5) and (3.3) shows, that $k$ is given by the integral over the velocity distribution $f\left(\mathbf{v}, \mathbf{v}_{E}\right)$. This integral can be written as:

$$
k=\int_{0}^{2 \pi} d \phi \int_{-1}^{+1} d(\cos \theta) \int_{0}^{v_{e s c}} f\left(\mathbf{v}, \mathbf{v}_{E}\right) v^{2} d v
$$

Assuming a Maxwellian velocity distribution:

$$
f\left(\mathbf{v}, \mathbf{v}_{E}\right)=e^{-\left(\mathbf{v}+\mathbf{v}_{E}\right)^{2} / v_{0}^{2}}
$$

where $\mathbf{v}$ is the velocity with respect to the target, $\mathbf{v}_{E}$ is the Earth velocity with respect to the dark matter halo, and $v_{0}$ is a characteristic velocity, usually set to the galactic rotation velocity. Then, in the simplest case with $v_{\text {esc }}=\infty$

$$
k=k_{0}=\left(\pi v_{0}^{2}\right)^{3 / 2},
$$

whereas for a distribution which is truncated at $\left|\mathbf{v}+\mathbf{v}_{E}\right|=v_{e s c}, k$ would be given by

$$
k=k_{1}=k_{0} \cdot\left[\operatorname{erf}\left(\frac{v_{e s c}}{v_{0}}\right)-\frac{2}{\pi^{1 / 2}} \cdot \frac{v_{e s c}}{v_{0}} \cdot e^{-v_{e s c}^{2} / v_{0}^{2}}\right] .
$$

Defining $R_{0}$ as the event rate per unit mass for $v_{E}=0, v_{e s c}=\infty$ and with the zero momentum transfer cross section $\sigma_{0}$,

$$
R_{0}=\frac{2}{\pi^{1 / 2}} \cdot \frac{N_{0}}{M_{T(u)}} \cdot \frac{\rho_{D M}}{M_{D M}} \cdot \sigma_{0} \cdot v_{0}
$$

equation (3.2) can be rewritten as:

$$
d R=R_{0} \cdot \frac{k_{0}}{k} \cdot \frac{1}{2 \pi v_{0}^{4}} \cdot v f\left(\mathbf{v}, \mathbf{v}_{E}\right) d^{3} v
$$

The recoil energy of a nucleus struck by a dark matter particle with a kinetic energy,

$$
E=\frac{1}{2} \cdot M_{D M} v^{2}
$$

scattered at an angle $\theta$ (in the center of mass frame) is given by:

$$
E_{R}=\frac{1}{2} E \cdot r \cdot(1-\cos \theta)
$$

In this formula $r$ denotes a kinematic factor including the reduced mass of the WIMP nucleus scattering:

$$
r=\frac{4 M_{D M} M_{T}}{\left(M_{D M}+M_{T}\right)^{2}}
$$


Here $M_{T}$ is the actual mass of the nucleus in $\mathrm{kg} 2$. Assuming isotropic scattering, so that the recoils are uniformly distributed in $E_{R}$, over the range $0 \leq E_{R} \leq E \cdot r$ we can derive the differential energy-recoil spectrum by averaging over the incident energy spectrum:

$$
\frac{d R}{d E_{R}}=\int_{E_{\min }}^{E_{\max }} \frac{1}{E r} d R(E)=\frac{1}{E_{0} r} \int_{v_{\min }}^{v_{\max }} \frac{v_{0}^{2}}{v^{2}} d R(v)
$$

$E_{\text {min }}=E_{R} / r$ is the smallest particle energy which can give a recoil energy of $E_{R}$.

Using equation (3.11) the differential energy - recoil spectrum is given by:

$$
\frac{d R}{d E_{R}}=\frac{R_{0}}{E_{0} r} \cdot \frac{k_{0}}{k} \cdot \frac{1}{2 \pi v_{0}^{2}} \int_{v_{\min }}^{v_{\max }} \frac{1}{v} \cdot f\left(\mathbf{v}, \mathbf{v}_{E}\right) \cdot d^{3} \mathbf{v}
$$

Evaluating the integral over the velocity distribution, the differential recoil rate can be written as [77]:

$$
\frac{d R}{d E_{R}}=\left\{\begin{array}{cc}
\frac{R_{0}}{E_{0} r} \frac{k_{0}}{k_{1}}\left\{\frac{\sqrt{\pi} v_{0}}{4 v_{E}}\left[\operatorname{erf}\left(\frac{v_{\min }+v_{E}}{v_{0}}\right)-\operatorname{erf}\left(\frac{v_{\min }-v_{E}}{v_{0}}\right)\right]-e^{\left.-\frac{v_{e s c}^{2}}{v_{0}^{2}}\right\}}\right. & \text { I } \\
\frac{R_{0}}{E_{0} r} \frac{k_{0}}{k_{1}}\left\{\frac{\sqrt{\pi} v_{0}}{4 v_{E}}\left[\operatorname{erf}\left(\frac{v_{e s c}}{v_{0}}\right)-\operatorname{erf}\left(\frac{v_{\min }-v_{E}}{v_{0}}\right)\right]-\frac{v_{e s c}+v_{E}-v_{\min }}{2 v_{E}} e^{-\frac{v_{e s c}^{2}}{v_{0}^{2}}}\right\} & \text { II } \\
0 & \text { III }
\end{array}\right.
$$

where the three cases correspond to the following three velocity intervals:

$$
\begin{aligned}
\text { I } & 0<v_{\text {min }}<v_{e s c}-v_{E} \\
\text { II } & v_{e s c}-v_{E}<v_{\text {min }}<v_{e s c}+v_{E} \\
\text { III } & v_{e s c}+v_{E}<v_{\text {min }}
\end{aligned}
$$

\section{Annual modulation}

The Earth moves through the dark matter halo at a relative velocity $v_{E}$, the vector sum of the Earth's orbital velocity around the Sun and the Sun's orbital velocity around the galactic center. The Earth's net velocity through the halo thus varies approximately sinusoidally during the course of its orbit, with an approximate velocity of:

$$
v_{E} \approx 244+15 \cos (2 \pi t) \mathrm{km} / \mathrm{s},
$$

where $t$ is the time measured in years since the maximum velocity near June 2nd. For a more detailed evaluation of the Earth's velocity I refer the reader to [76]. This movement of the Earth around the Sun introduces an annual modulation of the expected counting rate in direct detection experiments. The effect of the modulation in the incident velocity distribution results in a modulation of the detected counting rate of the order of a few percent, depending on the assumed halo model. However, the detection of such an annual modulation would be a clear statistical signature of WIMP interactions.

\footnotetext{
${ }^{2}$ In practice it is very useful to convert masses in $\mathrm{GeV}$ and express velocities in units of $\mathrm{c}$.
} 


\subsubsection{Scalar WIMP - nucleus scattering}

Scalar interactions are spin-independent WIMP-nucleon interactions corresponding to a coupling to the nucleon density operators. The coupling to protons and neutrons is characterized by the coupling constants $f_{p}$ and $f_{n}$ of equation (3.1). In the limit of vanishing momentum transfer, the scattering amplitudes of single nucleons add coherently giving a total scattering amplitude of

$$
M_{S I} \propto\left(Z f_{p}+(A-Z) f_{n}\right)
$$

for a nucleus with atomic number $\mathrm{Z}$ and mass number $\mathrm{A}$. Thus, the spin-independent WIMP-nucleon cross section becomes

$$
\sigma_{0}^{S I}=\frac{4}{\pi} \mu_{D M-T}^{2}\left[Z f_{p}+(A-Z) f_{n}\right]^{2},
$$

where $\mu_{D M-T}$ is the reduced mass of the WIMP nucleus system. In general, supersymmetric and similar models predict $f_{p} \approx f_{n}$ [4], giving a cross section $\sigma_{0}^{S I} \propto A^{2}$. Thus, the coherent enhancement of the cross section makes WIMP targets built of heavy nuclei vastly more sensitive to WIMP interactions than targets composed of light nuclei.

\section{Nuclear form factors}

In real collisions with non-zero momentum transfer, the various WIMP-nucleon scattering amplitudes will not interfere perfectly constructively. If the momentum transfer, defined as:

$$
q=\sqrt{\left(2 M_{T} E_{R}\right)}
$$

increases, such that the wavelength $\bar{h} / q$ is no longer large compared to the nuclear radius, the cross section begins to fall with increasing $q$. This effect can be taken into account by a form factor, $F\left(q r_{n}\right)$, where $r_{n}$ is an effective nuclear radius. The effective nuclear radius $r_{n}$ can be approximately found by fitting muon scattering data to a Fermi distribution. From this data an analytical function for $r_{n}$ can be derived:

$$
r_{n}^{2}=c^{2}+\frac{7}{3} \pi^{2} a^{2}-5 s^{2}
$$

The values of the parameters $c, a$ and $s$ are determined empirical with best estimates:

$$
c \simeq\left(1.23 A^{1 / 3}-0.60\right) f m \quad a \simeq 0.52 \mathrm{fm} \quad s \simeq 0.9 \mathrm{fm}
$$

The analytical form of the nuclear form factor proposed by Helm [76] is conventionally used for calculating the energy - recoil spectra.

$$
F\left(q r_{n}\right)=3 \frac{\left(\sin \left(q r_{n}\right)-q r_{n} \cos \left(q r_{n}\right)\right)}{\left(q r_{n}\right)^{3}} \cdot e^{(q s)^{2} / 2}
$$




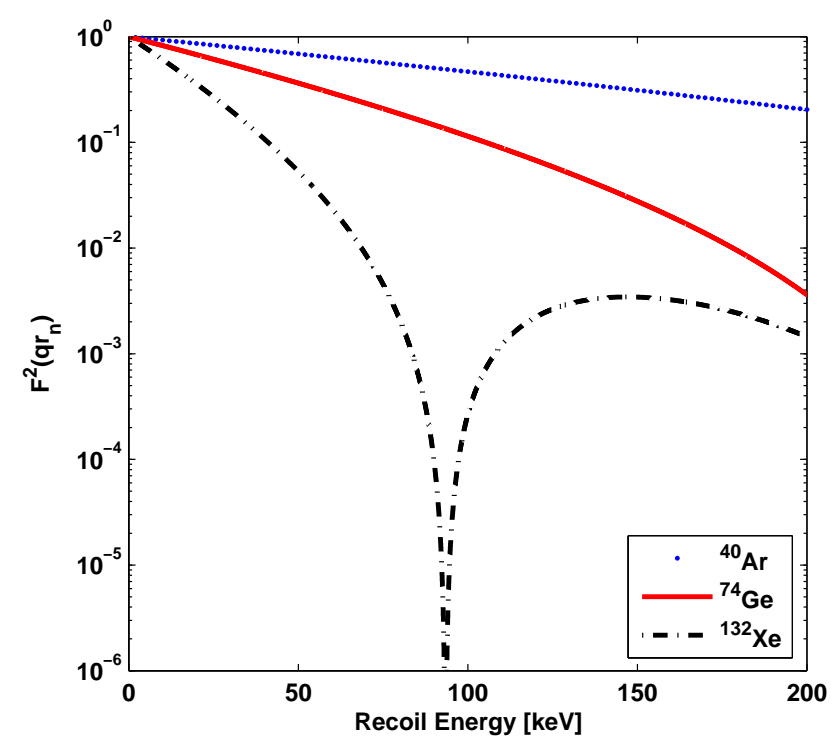

Figure 3.1: Helm form factor of selected materials used in modern direct dark matter experiments. For each material the isotope with the highest natural abundance is shown.

Hence the cross section used for the calculation of the energy - recoil spectra behaves as:

$$
\sigma^{S I}\left(q r_{n}\right)=\sigma_{0}^{S I} \cdot F^{2}\left(q r_{n}\right)
$$

In figure 3.1 the form factors for several isotopes, used in modern direct detection experiments, calculated according to formula (3.24) are shown.

The form factor suppression of the cross section is greater for heavier targets which counteracts the $A^{2}$ sensitivity enhancement over light nuclides. The two panels in figure 3.2 show the differential recoil rate as a function of energy (left) and the integrated rate above threshold (right). In general the differential recoil spectra are featureless and approximately exponentially falling with recoil energy. The effect of the form factor suppression is best seen in the right panel of figure 3.2. For example a target made of heavy nuclei, (e.g. Xe) must run at a low threshold to see the same event rate as a comparable mass of Ge.

\subsubsection{Axial WIMP - nucleus scattering}

For spin-dependent WIMP-nucleon interactions, axial-vector couplings give amplitudes proportional to the spin of the WIMP and nucleus. The coupling to the spin of protons and neutrons is characterized by the coefficients $a_{p}$ and $a_{n}$ in formula (3.1). Since the interaction amplitude switches the sign if the spin of the scattering target is flipped, the spin-dependent WIMP-nucleon interaction amplitudes with two nucleons of opposite spin will interfere destructively in the zero-momentum transfer limit. Since nucleons pair by opposite spin in the nucleus, the interaction rate is dominated by unpaired nucleons. In the limit of zero momentum transfer, the WIMP-nucleon spin-dependent interaction cross 

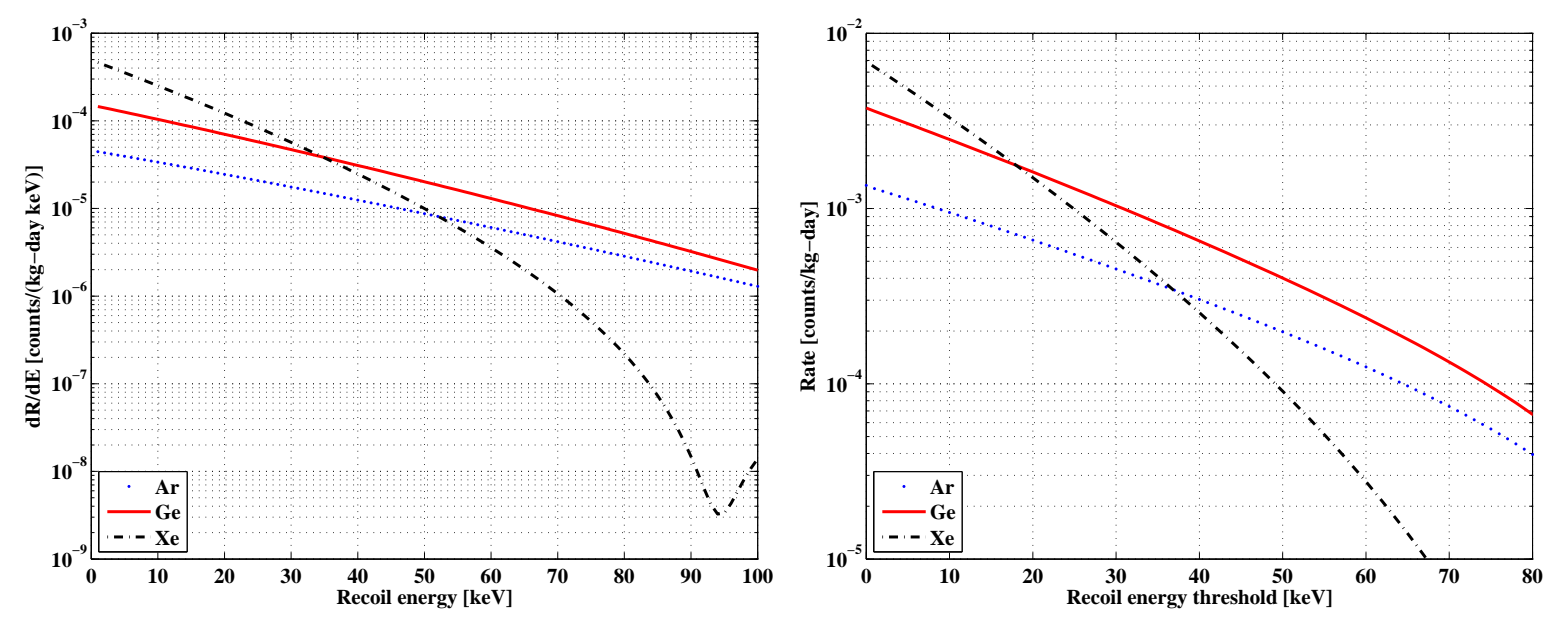

Figure 3.2: Left panel: Differential recoil spectra for several target materials. The recoil spectra are featureless and approximately exponentially falling with recoil energy. Right panel: Integrated rate above recoil energy threshold for chosen target materials (the differential rates have been integrated from threshold to $100 \mathrm{keV}$ recoil energy). In both figures the mass of the dark matter particle is assumed to be $100 \mathrm{GeV} / \mathrm{c}^{2}$, having a spin-independent cross section of $\sigma_{S I}=10^{-44} \mathrm{~cm}^{2}$.

section is:

$$
\sigma_{0}^{S D}=\frac{32(J+1)}{\pi J} G_{F}^{2} \mu_{D M-T}^{2}\left[a_{p}\left\langle S_{p}\right\rangle+a_{n}\left\langle S_{n}\right\rangle\right]^{2},
$$

where $J$ is the nuclear spin and $\left\langle S_{p(n)}\right\rangle$ is the expectation value for the nucleus proton (neutron) spin. The spin expectation values must be obtained from detailed nuclear structure calculations [78, 79]. These calculations show that an odd proton (neutron) nucleus may have a small but non-zero $\left\langle S_{n}\right\rangle\left(\left\langle S_{p}\right\rangle\right)$ due to polarization effects within the nucleus.

In the case of spin-dependent scattering it is not convenient to take $a_{p} \approx a_{n}$, since their ratio may vary in sign and magnitude depending on the chosen dark matter particle model. This means that in the finite-momentum-transfer case the form factor cannot be factored out of the cross section in a model-independent way. The preferred way to deal with this is to follow [80] by writing the WIMP-nucleus differential cross section in the form

$$
\frac{d \sigma_{S D}}{d q^{2}}=\frac{8 G_{F}^{2}}{(2 J+1) v^{2}} S(q)
$$

where $v$ is the WIMP velocity and

$$
S(q) \equiv a_{0}^{2} S_{00}(q)+a_{0} a_{1} S_{01}(q)+a_{1}^{2} S_{11}(q)
$$

with $a_{0} \equiv a_{p}+a_{n}$ and $a_{1} \equiv a_{p}-a_{n} . S(q)$ describes the effects of finite momentum transfer, as well as the values for the neutron and proton spin expectations, which must be computed for each nuclei separately using nuclear structure models [78, 79]. 


\subsection{Dark matter halos and local phase-space density}

In case of direct detection, it is necessary to know the dark matter's phase-space structure to predict rates. In early calculations, the Standard Halo Model (SHM) assumed no rotation and the density distribution was taken to be a spherical isothermal sphere with a core radius of several kpc. More recent modeling includes the cuspier central profiles from $\Lambda \mathrm{CDM}$ simulations [81, 82], producing changes of $\mathcal{O}(10 \%)$ with respect to the SHM [83]. Larger boosts have been claimed if dark matter is highly clumped [84, but it is more likely that we live outside a clump, leading to a modest reduction in the local density [85].

\subsubsection{The Milky Way's Dark Disc}

Dark matter only simulations have extremely high resolution, but they may not be addressing the "next to leading order" of the model because they do not include the effect of the baryons. Read et al. (2008) recently demonstrated that massive satellites are preferentially dragged into the baryonic disc-plane by dynamical friction where they dissolve leaving a thick dark matter disc [86, 87, 88]. The precise properties of the dark disc depend on the stochastic merger history and cosmology. However, given the expected merger history for a typical Milky Way in $\Lambda \mathrm{CDM}$, they found a dark disc with density in the range $\rho_{\mathrm{d}} / \rho_{\mathrm{h}} \sim 0.2-2$ at the solar neighborhood (where $\rho_{\mathrm{h}}$ is the density of the SHM).

In a first approach [87] dark matter only simulations have been used to estimate the expected merger history of a Milky Way mass galaxy, and then a stellar disc has been added to the simulations to measure its effect. The results of a simulation are shown in Figure 3.3. The left panel shows the accreted stars (red) and dark matter (blue) at the end of a simulation, where a Large Magellanic Cloud (LMC) mass satellite merged at $\theta=10^{\circ}$ to the stellar disc. The stars and the dark matter have settled into accreted discs. The right panel shows the corresponding velocity distribution in $\mathrm{v}_{\phi}$ (rotation velocity) at the solar neighborhood (a cylinder $8<\mathrm{R}<9 \mathrm{kpc},|\mathrm{z}|<0.35 \mathrm{kpc}$ ). The underlying dark matter halo is shown in green and is not rotating; the accreted stars and dark matter (red and blue) have kinematics similar to that of the underlying stellar disc (black). It is important to stress that all satellites regardless of their initial inclination contribute some accreted material that is focused into the disc plane. Thus, it is expected that the accreted dark and stellar discs will compromise several accreted satellites; the most massive low-inclination mergers being the most important contributors.

In a second, fully self-consistent, approach cosmological hydrodynamic simulations of Milky Way mass galaxies have been used [86]. Figure 3.4 shows the distribution of rotational velocities at the solar neighborhood for a particular simulation. The left panel shows the dark matter (black), stars (red), the matter accreted from the four most massive disrupted satellites (green, blue, magenta and cyan), and the sum of all matter accreted from these satellites (black dotted). A double Gaussian fit to the dark matter distribution is shown in blue with single Gaussian fits to the dark disc and halo shown as blue/dotted lines. The clear peak of the rotation velocity of the dark matter near the rotation velocity of the stars (red) indicates the formation of the co-rotating dark disc. The best fit double 


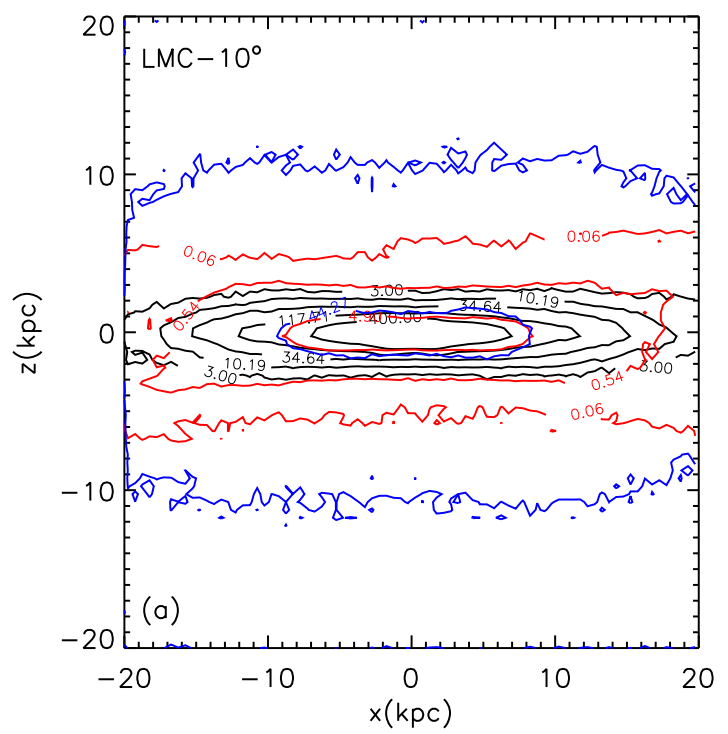

(a)

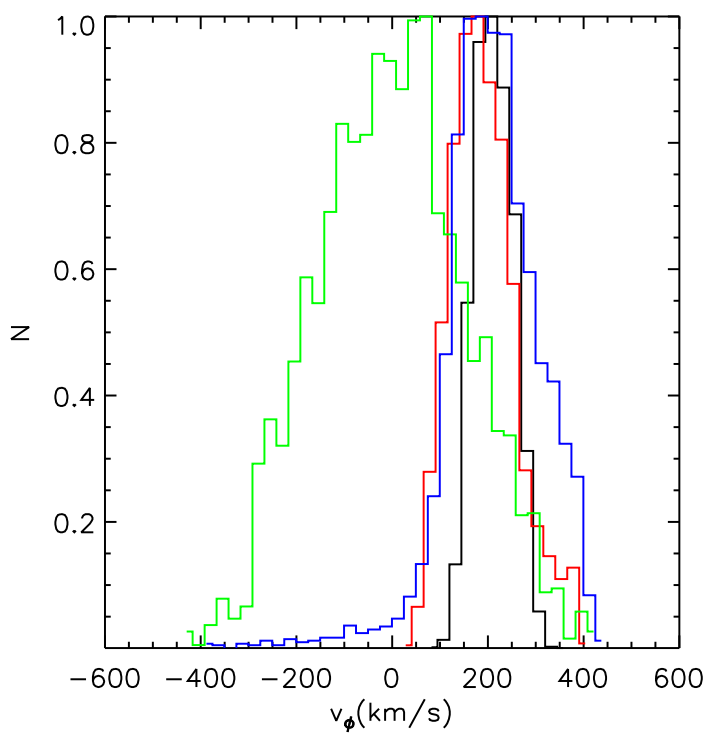

(b)

Figure 3.3: (a): The accreted stars (red) and dark matter (blue) at the end of a simulation where a LMC satellite merged at $\theta=10^{\circ}$ to the Milky Way's stellar disc; the black contours show the underlying Milky Way's stellar distribution. Figure taken from [87. (b): The corresponding velocity distribution in $\mathrm{v}_{\phi}$ (rotation velocity) at the solar neighborhood; the underlying Milky Way's dark matter halo is shown in green. The rotation velocity of the accreted dark matter (blue) and stars (red) clearly follows the velocity distribution of the stars at the solar neighborhood (black) and differs significantly from the dark halo (green). Figure taken from 89]

Gaussian parameters are marked in the top left of the figure showing that the dark disc lags the velocity of the stars by $64.07 \mathrm{~km} / \mathrm{s}$. The right panel shows the velocity distribution of the dark matter from the same simulation when the effect of the baryonic component (the stars) is not taken into account. This clearly shows that including the effect of the baryons leads to the formation of a dark matter disc. The accreted stellar disc shares similar kinematics to the dark disc. Depending on assumptions about the mass to light ratio of accreted satellites, these stars can make up $10-50 \%$ of the Milky Way stellar thick disc [87. The lower bound $\rho_{\mathrm{d}} / \rho_{\mathrm{h}}=0.2$ is particularly conservative since it is produced by just one merger of LMC mass within $20^{\circ}$ of the disc plane. In $\Lambda$ CDM two such low inclination mergers per Milky Way (and seven in total at all inclinations) are expected.

An upper bound on $\rho_{\mathrm{d}} / \rho_{\mathrm{h}}$ may be obtained from the kinematics of stars at the solar neighborhood [90, 91, 92]. The latest measurements from Hipparchos give a conservative upper bound of $\rho_{\mathrm{d}} / \rho_{\mathrm{h}}<3$, including systematic errors [93, 94]; with $\rho_{\mathrm{d}} / \rho_{\mathrm{h}}<2$ being likely. If more than half of the thick disc owes to accretion, the likely dark disc density would be near the upper limit. As such values of $\rho_{\mathrm{d}} / \rho_{\mathrm{h}}=[0.5,1,2]$ are considered here. The disc density $\rho_{\mathrm{d}}$ is an excess over $\rho_{\mathrm{h}}$, locally increasing the dark matter density. 


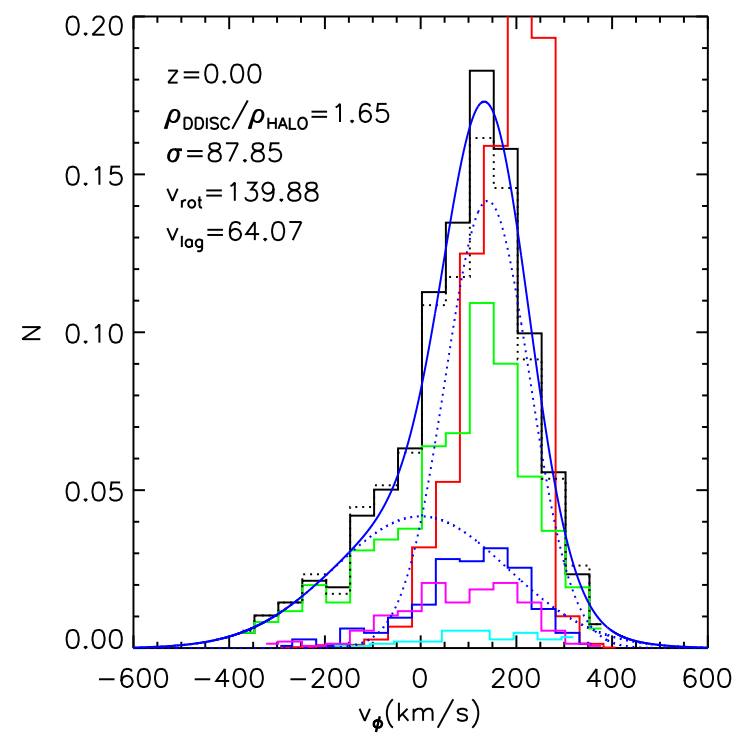

(a)

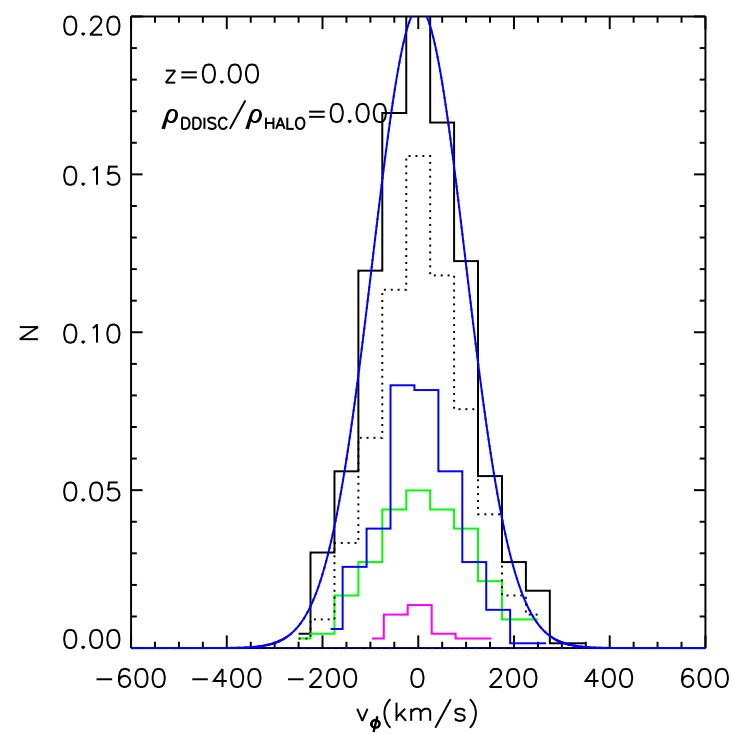

(b)

Figure 3.4: The distributions of rotational velocities $\mathrm{v}_{\phi}$ at the solar neighborhood for a simulated Milky Way mass galaxy. The lines show the dark matter (black), stars (red), a double Gaussian fit to the dark matter (blue; blue dotted), the matter accreted from the four most massive disrupted satellites (green, blue, magenta and cyan), and the sum of all matter accreted from these satellites (black dotted). The best fit double Gaussian parameters are marked in the top left. Figures taken from [89]. In (a) the baryons have been in included in the simulation leading to a strong double Gaussian distribution of the dark matter in the halo and the dark disc. (b) shows the simulation with dark matter only, showing that the inclusion of the baryons leads to the formation of the dark disc.

The kinematic properties of the dark disc can be estimated from the accreted stellar thick disc that forms concurrently. Stellar thick discs are found in the Milky Way and in all well-observed spiral galaxies [95, 96, 97, while at least one counter-rotating thick disc is strong evidence for an accretion origin [98]. However, thick discs can also form through heating of an underlying thin disc [99], or even directly from extended gas [100]. Indeed it is difficult in $\Lambda \mathrm{CDM}$ to obtain a thick disc massive enough from accretion alone [87. Thus it is assumed - based on the numerical models of [87] - that the dark disc's kinematics match the Milky Way's stellar thick disc. At the solar neighborhood, this gives a rotation lag $v_{\text {lag }}$ of $40-50 \mathrm{~km} / \mathrm{s}$ with respect to the local circular velocity, and dispersions of $\left(\sigma_{R}, \sigma_{\phi}, \sigma_{z}\right)=(63,39,39) \mathrm{km} / \mathrm{s}$ [87]. Since the dispersions are nearly isotropic and somewhat uncertain, the model used here for the velocity distribution of the dark disc is a simple 1D Maxwellian distribution, with a dispersion and lag, $\sigma=v_{l a g}=50 \mathrm{~km} / \mathrm{s}$, to show its general effect on direct detection. Improving on this assumption will involve untangling heated versus accreted components in the Milky Way stellar thick disc. This 
should become possible with future astronomical surveys like RAVE [101] and GAIA [102] that will provide full 6D phase space and chemical information for hundreds of thousands of individual stars.

It has to be emphasized that the dark disc must form in any hierarchical model of structure formation; it is not special to the $\Lambda \mathrm{CDM}$ simulations of [87]. However, $\Lambda \mathrm{CDM}$ is specific enough to predict the dark disc density with uncertainties owing only to the stochastic nature of the merging and accretion history. The dark disc is very different from dark matter streams [103, 104] that have a low filling factor (we are not likely to live in a stream), and are stochastic micro-structure. By contrast, the dark disc is the expected equilibrium end-state of dissolving satellites and the Earth must be embedded in one (if hierarchical formation is correct). Like the near-spherical dark matter halo, the dark disc is macro-structure.

\section{Direct Detection and the Dark Disc}

Direct detection experiments measure nuclear recoil rates above the detector's energy threshold [105]; here only Ge and Xe are considered. The detected elastic WIMP-nucleon recoils will range from a few to tens of $\mathrm{keV}$. The expected recoil rate per unit mass, unit nuclear recoil energy and unit time is [76]:

$$
\frac{d R}{d E}=\frac{\rho \sigma_{(\mathrm{WIMP}, \mathrm{N})}|F(E)|^{2}}{2 M_{\mathrm{WIMP}} \mu^{2}} \int_{v>\sqrt{m E / 2 \mu^{2}}}^{v_{\max }} \frac{f(\mathbf{v}, t)}{v} d^{3} v
$$

where $\rho$ is the local dark matter density $\left(\rho_{h}=0.3 \mathrm{GeV} / \mathrm{cm}^{3}\right.$ in the SHM), $\sigma_{(\text {WIMP,N) }}$ is the WIMP-nucleus scattering cross section, $F(E)$ is the nuclear form factor, $M_{W I M P}$ and $m$ are the masses of the dark matter particle and of the target nucleus, respectively, $\mu$ is the reduced mass of the WIMP-nucleus system, $v=|\mathbf{v}|$ and $v_{\max }$ is the maximal velocity in the earth frame for particles moving at the galactic escape velocity $v_{e s c}=544 \mathrm{~km} / \mathrm{s}[106$. Only the spin-independent scalar WIMP-nucleus coupling is considered, since it dominates the interaction (depending however on the dark matter particle) for target media with nucleon number $\mathrm{A} \gtrsim 30$ [48]. A simple 1D Maxwellian is used for the velocity distributions of particles in the dark disc and the SHM:

$$
f(\mathbf{v}, t) \propto \exp \left(\frac{-\left(\mathbf{v}+\mathbf{v}_{\oplus}(t)\right)^{2}}{2 \sigma^{2}}\right)
$$

where $\mathbf{v}$ is the laboratory velocity of the dark matter particle and the instantaneous streaming velocity $\mathbf{v}_{\oplus}=\mathbf{v}_{\text {circ }}+\mathbf{v}_{\odot}+\mathbf{v}_{\text {orb }}(t)$. This streaming velocity is the sum of local circular velocity $\mathbf{v}_{\text {circ }}=(0,220,0) \mathrm{km} / \mathrm{s}$, the peculiar motion of the Sun $\mathbf{v}_{\odot}=(10.0,5.25,7.17)$ $\mathrm{km} / \mathrm{s}$ [107] with respect to $\mathbf{v}_{\text {circ }}$ and the orbital velocity of the Earth around the Sun $\mathbf{v}_{\text {orb }}(t)$. In the SHM, the halo has no rotation and the dispersion $\sigma=\left|\mathbf{v}_{\text {circ }}\right| / \sqrt{2}$. For the dark disc, the velocity lag $\mathbf{v}_{\text {lag }}=(0,50,0) \mathrm{km} / \mathrm{s}$ replaces $\mathbf{v}_{\text {circ }}$ and a dispersion of $50 \mathrm{~km} / \mathrm{s}$ is adopted.

The lower relative velocities of the dark disc significantly increases the differential rate at low energies compared to the SHM rate (see figure 3.5). Detection of the dark disc 


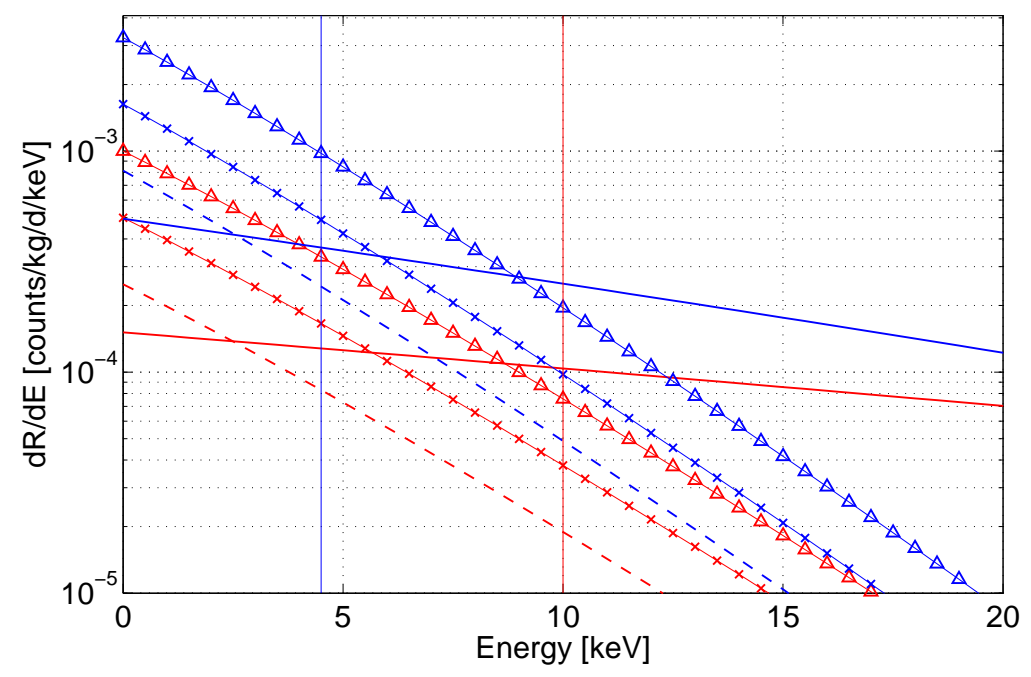

Figure 3.5: Differential recoil rates for Ge (red) and Xe (blue) targets, for $M_{W I M P}=100 \mathrm{GeV} / \mathrm{c}^{2}$ and $\sigma_{(\mathrm{WIMP}, \mathrm{N})}=10^{-8} \mathrm{pb}$ in the SHM (solid line) and the dark disc. Three different values of $\rho_{\mathrm{d}} / \rho_{\mathrm{h}}(0.5$ dashed, $1 \times$ and $2 \triangle)$ are shown. Vertical lines mark current experiment thresholds: XENON10 [108] (blue) using a Xe and CDMS-II [4] (red) using a Ge target.

depends crucially on the detector's low energy threshold. The differential rate for a specific WIMP target depends on $M_{W I M P}$. In figure 3.6, the energy below which the dark disc dominates the rate as a function of $M_{W I M P}$ is shown for three values of $\rho_{\mathrm{d}} / \rho_{\mathrm{h}}$. The total rate in a detector is the sum of the two contributions from the SHM and the dark disc, which dominate at high and low energies, respectively. For $M_{W I M P} \gtrsim 50 \mathrm{GeV} / \mathrm{c}^{2}$, the dark disc contribution lies above current detector thresholds, giving a much greater change in detection rate with recoil energy compared to the SHM alone.

The total rate in a detector using a Ge target is shown in figure 3.7 varying $\rho_{\mathrm{d}} / \rho_{\mathrm{h}}$ and $M_{W I M P}$. If the detectors threshold is sufficiently low even an extremely conservative dark disc with $\rho_{\mathrm{d}} / \rho_{\mathrm{h}}=0.1$ can be detected. Current germanium detectors achieve thresholds below $1 \mathrm{keV}$ [109, 110]. The details of the differential rate with energy, shown in figure 3.7. betrays both the contribution of the dark disc relative to the SHM and $M_{W I M P}$. This introduces a mass dependent characteristic shape of the differential rate which will improve the constraints on $M_{W I M P}$ upon detection.

The motion of the Earth around the Sun gives rise to an annual modulation of the event rate and recoil energy spectrum [111]. The annual modulation is more pronounced for the dark disc, since the relative change to the mean streaming velocity owing to the Earth's motion is larger $(\sim 19 \%)$ compared to the SHM $(\sim 6 \%)$. In Fig. 3.8 the residual integrated rates for a liquid xenon detector throughout a year is shown for three different $M_{W I M P}$ and two values of $\rho_{\mathrm{d}} / \rho_{\mathrm{h}}$. The residuals are calculated with respect to the mean counting rates in a given energy region.

The phase (defined at maximum rate) of the dark disc and the SHM differ because the Sun's motion is slightly misaligned to the dark disc. While the phase of each component 


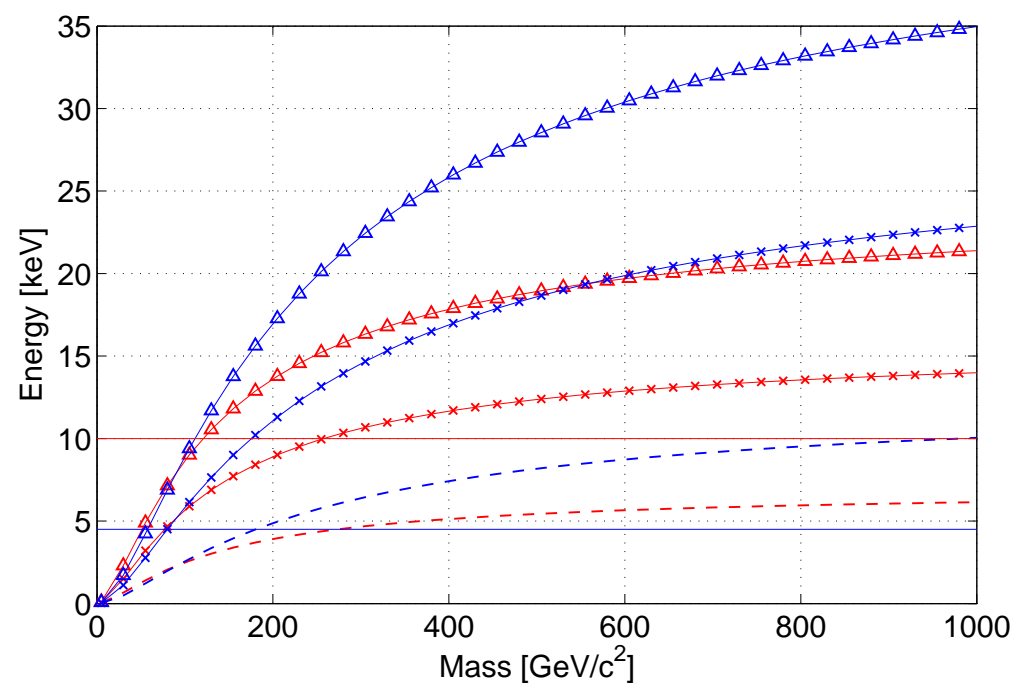

Figure 3.6: The recoil energy below which the signal is dominated by the dark disc (compared to the SHM) as a function of $M_{W I M P}$ for Ge (red) and Xe (blue) targets. Three different values of $\rho_{\mathrm{d}} / \rho_{\mathrm{h}}(0.5$ dashed, $1 \times$ and $2 \triangle)$ are shown. Horizontal lines mark current experiment thresholds: XENON10 [108] (blue) using a Xe and CDMS-II [4] (red) using a Ge target.

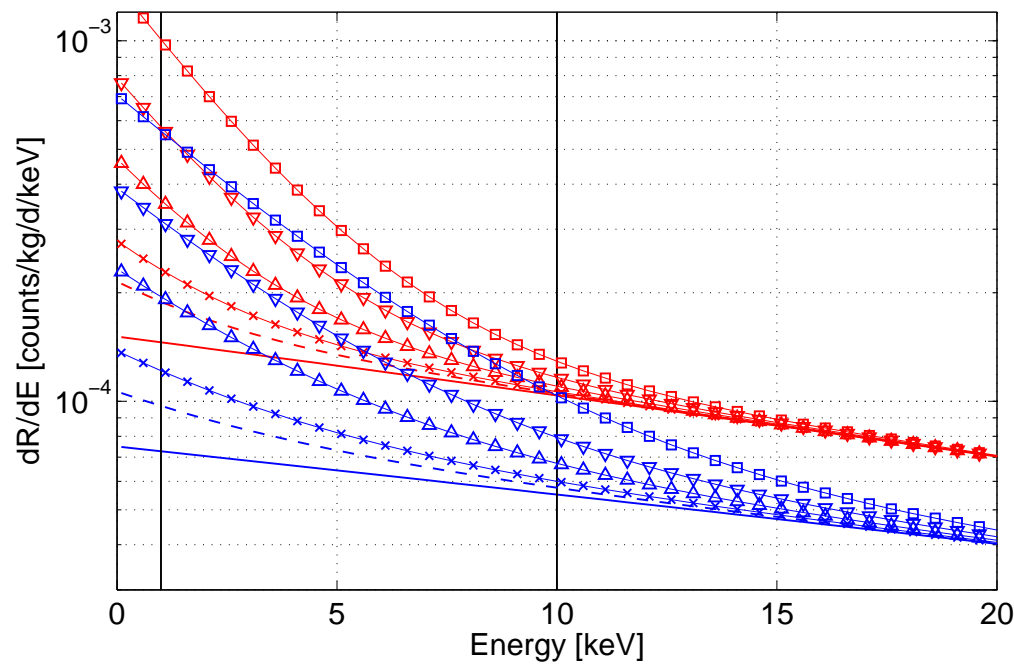

Figure 3.7: Total differential rate for a Ge target in the SHM (solid) and four different different values of $\rho_{\mathrm{d}} / \rho_{\mathrm{h}}(0.1$ dashed, $0.2 \times, 0.5 \triangle, 1 \nabla$ and $2 \square)$ are shown for two $M_{W I M P}$ $\left(100 \mathrm{GeV} / \mathrm{c}^{2}\right.$ (red) and $200 \mathrm{GeV} / \mathrm{c}^{2}$ (blue)). Vertical lines mark the current CDMS-II [4] threshold and a threshold of $1 \mathrm{keV}$. 

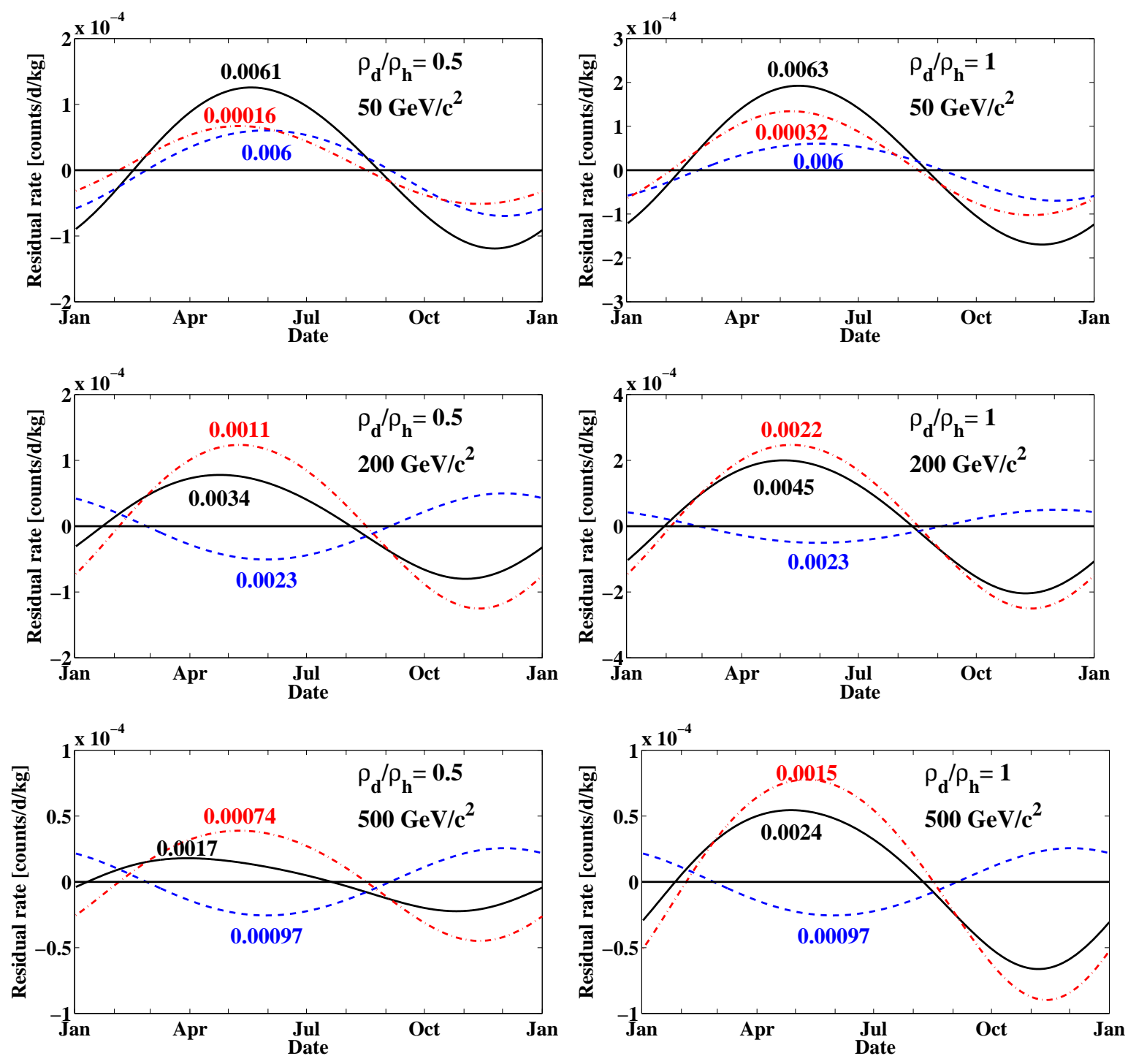

Figure 3.8: The annual modulation shown as the residual counting rate versus date for the XENON10 108 experiment (4.5 to $27 \mathrm{keV}$ ). The residuals are calculated with respect to the mean counting rates (given as numbers over each line) using a WIMP-nucleon cross section of $10^{-8} \mathrm{pb}$. The left/right column is calculated for $\rho_{\mathrm{d}} / \rho_{\mathrm{h}}=0.5 / 1$ and $M_{W I M P}$ (top to bottom) of $50 \mathrm{GeV} / \mathrm{c}^{2}$, $200 \mathrm{GeV} / \mathrm{c}^{2}$ and $500 \mathrm{GeV} / \mathrm{c}^{2}$. The (blue/dashed) line is the modulation signal obtained from the SHM, the (red/dot-dashed) line is the modulation signal from the dark disc and the (black/solid) line is the total modulation signal. The maximum of the dark disc contribution is shifted to May 9th compared to the SHM's maximum/minimum on May 30th. Note the different vertical scales in each of the figures of the plot array. 


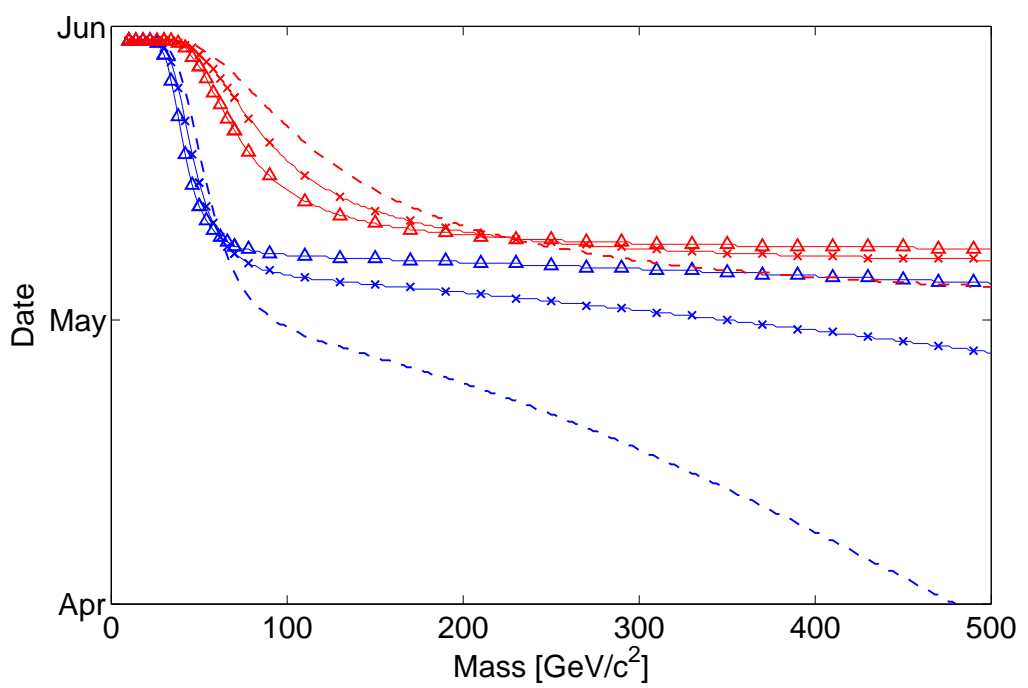

Figure 3.9: Phase shifts as a function of $M_{W I M P}$ in the energy range reported by the CDMS-II [4] experiment $(10-100 \mathrm{keV})$ (red/grey) and the XENON10 [108] experiment (4.5-27 $\mathrm{keV})$ (blue/black), for three different values of $\rho_{\mathrm{d}} / \rho_{\mathrm{h}}(0.5$ dashed, $1 \times$ and $2 \triangle)$.

does not depend on $M_{W I M P}$, their sum does because their amplitudes depend on $M_{W I M P}$. This dependency is shown in figure 3.9, for three values of $\rho_{\mathrm{d}} / \rho_{\mathrm{h}}$. The phase shift is determined by the relative contributions of each component. Figure 3.9 shows that the phase shift is largest for low $\rho_{\mathrm{d}} / \rho_{\mathrm{h}}$, since in this case the sum preferentially follows the halo modulation phase, while for higher $\rho_{\mathrm{d}} / \rho_{\mathrm{h}}$ the disc component dominates the modulation phase. This is a new effect introduced by the presence of the dark disc that allows $M_{W I M P}$ to be uniquely determined from the phase of the modulation signal, for given $\rho_{\mathrm{d}} / \rho_{\mathrm{h}}$. Notice that there is an amplitude flip for the SHM that occurs as $M_{W I M P}$ is increased, which is not seen for the dark disc. As $M_{W I M P}$ is lowered, the "crossing energy" at which the differential rates for minimal and maximal WIMP velocity are equal shifts to lower energies. For the dark disc, it remains close to, or below, current thresholds and so the amplitude flip is not seen. The main results of this study are :

1. The dark matter disc boosts the detection rates at low recoil energy. For $M_{W I M P} \gtrsim$ $50 \mathrm{GeV} / \mathrm{c}^{2}$, recoil energies of $5-20 \mathrm{keV}$ and $\rho_{\mathrm{d}} / \rho_{\mathrm{h}} \leq 1$, the rate is boosted by factors up to 2.4 for Ge and 3 for Xe targets. Comparing this with the rates at higher energy will constrain $M_{W I M P}$, particularly for $M_{W I M P}>100 \mathrm{GeV} / \mathrm{c}^{2}$.

2. The dark disc has a different annual modulation phase than the dark halo, while the relative amplitude of the two components varies with recoil energy and $M_{W I M P}$. As a result, the annual modulation signal varies uniquely with $M_{W I M P}$, for given dark disc properties (measurable by next generation surveys [101, 102]). 


\subsection{Experimental tasks}

Direct detection of WIMP interactions is a search for rare events which requires an efficient discrimination between background interactions and candidate events. Control of the background is thus mandatory for any direct detection experiment, regardless on which detection technology it is based. Below, the background restrictions for a direct detection experiment are discussed and an effective discrimination technique is introduced. Afterwards the leading technologies used in current state of the art direct detection experiments will be discussed and experiments using these technologies will be introduced.

\subsubsection{Backgrounds}

Based on the expected WIMP interaction rate, a direct detection experiment must be able to identify a spectrum of $\mathcal{O}(10) \mathrm{keV}$ nuclear recoils occurring at a very low rate. Unfortunately, this low-energy regime is dominated by background events from natural radioactivity and cosmic rays. Direct WIMP detection experiments are thus low-background experiments, demanding a superior protection from background events. The ultimate sensitivity of a direct detection experiment is determined by its rate of background events which are indistinguishable from WIMP candidates.

In a simplified picture, direct detection experiments are very simplistic event-counting experiments that operate for an exposure of $M D$ kilogram days and expect to observe $B$ background events which are indistinguishable from WIMP interactions. There are three general sensitivity classes in which the experiment can be operated, depending on the magnitude of $B$.

- Background-free: If $B \approx 0$ events, then any observed candidate events are evidence for WIMPs. If no candidate events are observed the experiment can set a $90 \%$ confidence level Poisson upper limit on the Wimp interaction rate of $r=\frac{2.3}{M D}$ events per kilogram-day, and the experiment's sensitivity improves in proportion to $M D$.

- Background subtraction: If $B$ is non-negligible, but well characterized from independent data (i.e. all background sources along with their spectrum are characterized and have negligible systematic errors), the background rate may be subtracted from the observed count rate. The experiment is thus searching for an excess of events above the known background. The accuracy of such a subtraction is limited by Poisson statistics giving an error on the subtraction of $\sigma_{B}=\sqrt{B}$. As the exposure increases $B \propto M D$ and thus $\sigma_{B} \propto \sqrt{M D}$. The number of excess candidate events needed to claim a detection scales as $\sigma_{B}$, so the experiments sensitivity grows only as $\sqrt{M D}$.

- Background limited: If the experiment has no knowledge of its backgrounds or great systematic uncertainties on the expected backgrounds the sensitivity will ultimately be limited. The systematical errors on the background count generally grow in proportion to $B$, while the statistical errors grow only as $\sqrt{B}$. Systematic errors therefor 
exceed statistical errors at large exposures. If the systematic uncertainty on $B$ exceeds the statistical uncertainty the sensitivity of the experiment no longer increases at all with increasing exposure, since the systematic error on the background rate constitutes an irreducible lower limit upon the rate of candidate events.

Due to these different scaling laws it is desired for a direct detection experiment to be operated as close to the background-free regime as possible.

Radioactive and cosmogenic background rates may be reduced by a variety of shielding and material-handling techniques [112]. However, some rate of background events invariably remains and modern direct detection experiments generally pursue an event-by-event discrimination to separate the WIMP signal from background events.

\subsubsection{Event-by-event discrimination}

Most modern direct detection experiments use event-by-event discrimination techniques to identify nuclear recoils from WIMP interactions (or neutrons) admits a far larger rate of electron recoils from radioactive decays and cosmogenic processes. Discrimination is achieved by measuring each event in two (or more) distinct detection channels and using their ratio to identify the recoil type. The most common of such channels are heat (phonons), ionization and scintillation light. The choice of detection channels carries tradeoffs in readout technology and experimental resolution. A sufficiently accurate discrimination can reduce an experiment's background rate enough, to maintain operation in the zero-background regime, even at very large WIMP-search exposures.

\subsubsection{Direct detection technologies}

The experimental realization of a direct detection experiment is mainly determined by the target material which determines the detection channels of an interaction. To date there are three main technologies used in direct detection experiments, which will be briefly reviewed below and experiments using this technology will be discussed.

\section{Solid-state detectors}

Solid-state detectors provide an excellent energy resolution and powerful discrimination against electron recoils. The Cryogenic Dark Matter Search (CDMS) experiment is currently the leading experiment in this category, since the CDMS experiment is discussed in detail in chapters 5 and 6 here other experiments using solid-state detectors are mentioned.

\section{EDELWEISS}

The EDELWEISS collaboration employs semi-conductor crystals (germanium crystals) operated at cryogenic temperatures in the search for dark matter interactions. The detection channels are ionization and phonon detection. The phonon detection is based on the measurement of the near-equilibrium temperature change of the entire crystal substrate. 
Recently the EDELWEISS collaboration has presented results based on a new detector design which uses an interleaved charge electrode design [113]. This design has been originally developed by the CDMS collaboration [114] but has been extended by the EDELWEISS collaboration [115]. Since this detector design allows the discrimination of the main background events in semi-conductor crystals, this technology is certainly to become the leading technology in the ionization readout of solid-state detectors in future experiments.

\section{CRESST}

The CRESST collaboration employs $\mathrm{CaWO}_{4}$ crystals instrumented with two thermometers to detect the thermal phonon signal and the scintillation light from a particle impact in the cryogenic substrate. The phonon signal is measured directly as a temperature rise of the crystal, while the scintillation light is detected by the temperature rise of a light-absorbing silicon wafer. CREEST has set limits on the spin-independent WIMP-nucleon cross section at the $5 \times 10^{-43} \mathrm{~cm}^{2}$ level [116] and is proceeding with a larger experimental installation.

\section{DAMA}

The DAMA collaboration operates an array of low-background $\mathrm{NaI}(\mathrm{Tl})$ scintillator crystals at the Gran Sasso Laboratory in Italy. Interactions in the crystals are identified by their scintillation light alone. The scintillation yield of a nuclear recoil is reduced compared to that of an electron recoil of similar energy by a "quenching factor" $\mathrm{Q}_{\mathrm{Na}}=0.3$ for $\mathrm{Na}$ recoils and $\mathrm{Q}_{I}=0.09$ for I recoils [117]. Without an independent measurement of the deposited energy, however, this difference cannot be used for background rejection. The DAMA detectors thus have no event-by-event discrimination between nuclear and electron recoils. The collaboration seeks to identify WIMP interactions on a statistical basis by searching for the expected annual modulation of the WIMP recoil spectrum.

The DAMA collaboration claims the observation of this annual modulation in two detector arrays, the DAMA/NaI and the DAMA/LIBRA setup. Taking both together, the modulation of the counting rate at low energies has been observed over 10 annual cycles with a combined exposure of 0.87 ton-years [118]. The interpretation of this result in terms of dark matter interactions will be addressed in Section 10.5 and Chapter 13.

\section{KIMS}

The KIMS collaboration operates an array of low-background CsI(Tl) scintillator crystals. Like in the DAMA experiment interactions in the crystal are identified by their scintillation light alone and no event-by-event discrimination is possible. Due to the use of two odd proton nuclides $\left({ }_{55}^{133} \mathrm{Cs}\right.$ and $\left.{ }_{53}^{127} \mathrm{I}\right)$ the detectors have a large sensitivity to spin-dependent WIMP interactions, providing one of the strongest limits on the WIMP-proton scattering cross section [119]. Since the detector technology is very similar to the one used by the DAMA collaboration, the KIMS collaboration proposes to search for the statistical annual modulation signature to test the DAMA claim. 


\section{Noble liquids}

Liquid noble detectors are large tanks of liquefied noble gases observed by photomultiplier tubes. Nobel liquids are excellent scintillators, with light yields approaching that of $\mathrm{NaI}(\mathrm{Tl})$ and long attenuation lengths for that light. The relative high boiling point of liquid noble gases make the cryogenic requirements far simpler than those needed for the phonon readout in cryogenic solid-state detectors. Nuclear and electron recoils can be distinguished in two distinct ways:

- Nuclear and electron recoils produce a different amount of ionization and scintillation in the liquid. The primary scintillation can be measured directly with phototubes, while the charge carriers can be drifted and extracted into a gas volume where they cause secondary proportional scintillation. Such a "dual-phase" detector thus observes two scintillation pulses for each interaction. The amplitudes of the two light signals identify the energy and recoil type of each interaction, while the time delay between them can be used to measure the event's position along the drift axis.

- The pulse shape of the primary scintillation signal can be a useful discriminant between electron and nuclear recoils. Each noble liquid has two scintillation time constants $\left(\tau_{1}\right.$ and $\tau_{3}$ ), corresponding to the de-excitation of singlet and tripled excimer states. Nuclear recoils populate the singlet state preferentially, since $\tau_{1}<\tau_{3}$ the tail of the primary scintillation pulse can be used as a discriminator if the time difference between $\tau_{1}$ and $\tau_{3}$ is large enough to be recognized by the readout channel. In addition the pulse shape has to be well reconstructed even at low energies, demanding a reasonable number of primary scintillation photons at the experiments recoil energy threshold.

\section{Xenon}

Liquid xenon is the most promising of the noble liquid targets. It has the highest boiling point, the largest light yield, no long lived radioisotopes and the scintillation light can be detected without wavelength-shifting. Its large atomic mass gives it a large cross section for spin-independent interactions. The high density of xenon allows for compact detectors and allows a very effective self-shielding: most background events cannot penetrate more than a few $\mathrm{cm}$ into the detector volume. On the other hand xenon is not well suited for pulse shape discrimination, due to its extremely short scintillation times $\left(\tau_{1}=2.2 \mathrm{~ns}\right.$ and $\left.\tau_{3}=21 \mathrm{~ns}\right)$.

Currently there are two experiments using a xenon target in a dual-phase detector. The XENON10 collaboration used a 15-kg detector and observed 10 candidate events (consistent with backgrounds) in a $136 \mathrm{~kg}$-day exposure [108. The ZEPLIN collaboration operated a $12 \mathrm{~kg}$ detector and observed 7 candidate events (consistent with backgrounds) in a $127.8 \mathrm{~kg}$-day exposure [120].

Several experiments are pushing xenon to yet higher masses and reduced backgrounds. The $100 \mathrm{~kg}$ XENON100 detector is currently taking data at Gran Sasso scaling up the basic design of the XENON10 experiment [121]. The $300 \mathrm{~kg}$ LUX experiment [122] adds an 
active water shield to aid against neutron rejection but uses the same detector technology as the XENON100 experiment. The XMASS collaboration is constructing a $800 \mathrm{~kg}$ singlephase detector, focusing on the self-shielding and good position reconstruction to eliminate backgrounds [123].

\section{Argon}

Liquid argon combines the good light yield of xenon with an extremely powerful pulse shape discrimination, made possible by argon's large difference in singlet and tripled decay times $\left(\tau_{1}=6 \mathrm{~ns}\right.$ and $\left.\tau_{3}=1590 \mathrm{~ns}\right)$ [124]. The main drawback of using argon as the target material is a large intrinsic background from radioactive ${ }^{39} \mathrm{Ar}$, demanding an enormous discrimination power $\left(\sim 10^{8}: 1\right)$ in a large-scale detector. Argon scintillates in the vacuum ultraviolet band. The photomultiplier readout of an argon target thus requires the use of wavelength-shifting fluorescent materials which unfortunately reduces the light yield. Current experiments based on an argon target are dual phase detectors providing an additional discriminator based on the detection of the primary scintillation light and the ionization.

The WARP collaboration has published results from a $2.3 \mathrm{~L}$ two-phase prototype detector at Gran Sasso, using both discrimination techniques [125]. The WARP and ArDM [126] collaborations are pursuing similar two-phase technology on a larger scale. The DEAP collaboration is operating a prototype $7 \mathrm{~kg}$ single-phase detector based solely on pulse-shape discrimination [127]. The recent discovery of underground argon deposits low in ${ }^{39} \mathrm{Ar}$ may open the door to multi-ton liquid argon detectors.

\section{Phase-transition detectors}

Recently the long known concept of "bubble chamber" detectors has been adopted in direct detection experiments. These phase transition detectors are based on the principle that an abrupt transition makes an effective detector. If a liquid is superheated to a metastable state above its boiling point, the energy deposited by a particle interaction can cause a very localized evaporation of the liquid. This boiling will only occur if enough energy is deposited in a small enough volume, i.e. the particle track must have an energy loss $(d E / d x)$ greater than a certain threshold. By tuning this threshold, phase transition detectors can be rendered insensitive to electromagnetic interactions while still remaining sensitive to nuclear recoils. Figure 3.10 shows representative photographs of particle events from the COUPP experiment, using a $\mathrm{CF}_{3} \mathrm{I}$ target.

Phase transition detectors only measure the count rate above threshold, since the creation of a bubble is a binary process: either enough energy was deposited to create a bubble, or not. To acquire information about the energy spectrum of the interaction data at a range of different energy thresholds must be acquired, since it is in general not possible to determine the energy deposited by the creation of a bubble. The COUPP collaboration reported competitive upper limits on the spin-dependent WIMP- proton interactions from a $1.5 \mathrm{~kg}$ pilot experiment [128]. 

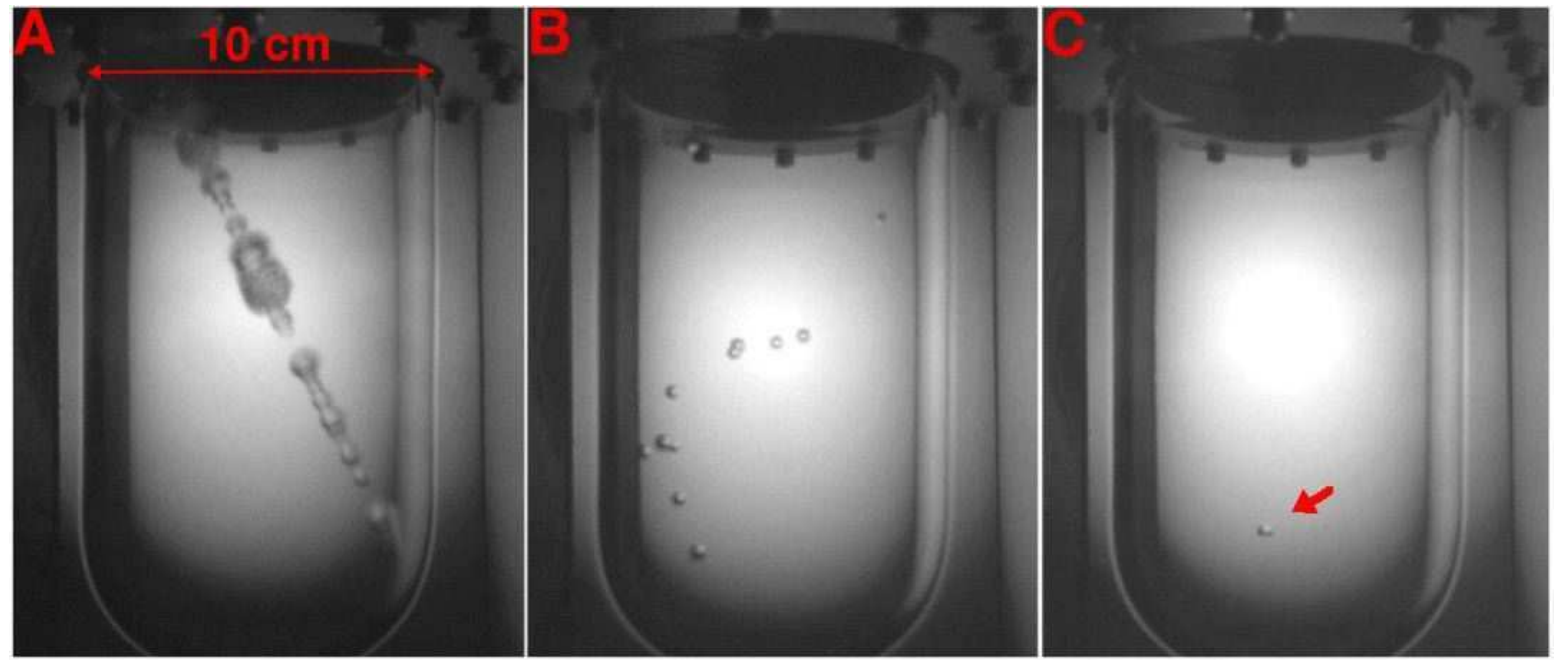

Figure 3.10: Photograph of three interactions in the COUPP bubble chamber. Shown are: a cosmic ray interaction detected in a run with very low $\mathrm{dE} / \mathrm{dx}$ threshold $(\mathrm{A})$, a neutron multiple-scatter event (B) and a neutron single-scatter event (C). Photographs taken from 128 . 


\section{Chapter 4}

\section{Indirect Detection Signals in Presence of the Dark Disc}

As disc galaxies form in a hierarchical cosmology, massive merging satellites are preferentially dragged towards the disc plane. The material accreted from these satellites forms a dark matter disc that contributes 0.25 - 1.5 times the non-rotating halo density at the solar position (see Section 3.2.1). Here, the importance of the dark disc for indirect dark matter detection in neutrino telescopes is discussed. Previous predictions of the neutrino flux from WIMP annihilation in the Earth and the Sun have assumed that Galactic dark matter is spherically distributed with a Gaussian velocity distribution, the standard halo model. Although the dark disc has a local density comparable to the dark halo, its higher phase space density at low velocities greatly enhances capture rates in the Sun and Earth.

\subsection{Indirect detection}

WIMPs may be detected indirectly by their annihilation products. The annihilation rate scales as the square of the WIMP density, so the most luminous sources are expected to be near the Galactic centre or the centre of dark matter sub-halos, where the dark matter density peaks [129, 130, 131, 132, 133, 134. In addition, the Sun and Earth capture WIMPs and may be seen as sources of WIMP annihilation [135, 136, 137, 138, 139]. In all cases, the annihilation rate is sensitive to the dark matter's phase space structure.

WIMPs can be gravitationally trapped inside the Sun and Earth by elastic scattering, if the final WIMP states have velocities below the escape velocity. To date, annihilation rates in the Sun and Earth have been estimated using the Standard Halo Model (SHM), which is modeled as a smooth, spherically symmetric density component with a non-rotating Gaussian velocity distribution (sometimes with an anisotropic velocity dispersion tensor). However, recent dark matter-only simulations show deviations from this picture in the form of many small amplitude density fluctuations on $\sim 100$ pc scales, a few large amplitude density fluctuations due to the presence of substructure (which makes up $\sim 0.5 \%$ of the mass at the solar circle), and relatively small deviations of the velocity distribution from 
Gaussian [140, 141]. Even if such structures were to survive in the presence of a baryonic disc and bulge, the indirect detection signal from the Earth and Sun is unlikely to deviate significantly from the SHM prediction. This is because the annihilation rate is sensitive to the phase space density averaged over long ( $\gtrsim 100 \mathrm{Myr})$ timescales. As a result, indirect detection by annihilation in the Earth and Sun is only sensitive to the local dark matter macrostructure.

There is at least one local macro-structural component beyond the SHM, which has been discovered in recent simulations of galaxy formation that include baryons. The baryonic disc of the Milky Way draws satellites closer to the disc plane by dynamical friction, where they are disrupted by tides 88 . This results in a thick disc of dark matter with a mid-plane density of 0.25 -1.5 times the local dark halo density and kinematics similar to the thick disc of stars [87, 86]. The dark disc boosts the flux in direct detection experiments at low energies and increases the annual modulation signal with an energy-dependent phase shift that will betray the mass of the dark matter particle [1].

Here the importance of the dark disc for the detection of neutrinos from WIMP annihilation in the cores of the Sun and Earth is studied. WIMPs can annihilate into a wide range of final products, of which muon neutrinos can escape and reach terrestrial detectors. On Earth, these muon neutrinos produce muons in charged current interactions with nuclei: $\nu_{\mu}+N \rightarrow \mu^{-}+X$. The ultra-relativistic muons can be detected by their Cerenkov radiation in large water or ice-based neutrino telescopes. So far, neutrino telescopes have found no evidence for high-energy neutrinos of astrophysical origin above the detected atmospheric neutrino background. The most stringent bounds on high-energy neutrinos from the Sun and Earth come from Super-Kamiokande [142], AMANDA [143] and IceCube [144]. Super-Kamiokande, a $50 \mathrm{kt}$ water Cerenkov detector, and AMANDA, located in the ice sheet at the Amundsen-Scott South pole station, have been taking data throughout the past decade. Their muon flux limits from the Earth and the Sun are of order $\Phi_{\mu} \sim 10^{3}-10^{4} \mathrm{~km}^{-2} \mathrm{yr}^{-1}$ for energies $E_{\mu}>1 \mathrm{GeV}$, where the tighter constraints apply to higher WIMP masses. These flux limits and those shown in the figures below are based on the assumption of a hard WIMP annihilation spectrum. IceCube, currently under construction at the site of the AMANDA experiment, has achieved a similar sensitivity with even a small fraction $(\sim 27 \%)$ of the construction completed, and is expected to have a 5-year sensitivity to flux from the Sun of $\Phi_{\mu} \sim 70 \mathrm{~km}^{-2} \mathrm{yr}^{-1}$ above a WIMP mass of $\sim 200 \mathrm{GeV}$. The expected 5-year sensitivity to flux from the Earth is $\Phi_{\mu} \sim 20 \mathrm{~km}^{-2} \mathrm{yr}^{-1}$.

\subsection{Capture from the dark disc}

For the study presented here, the distribution functions of both the SHM and dark disc are modeled as Gaussian,

$$
f(\mathbf{u})=\frac{1}{\left(2 \pi \sigma^{2}\right)^{3 / 2}} \frac{\rho}{M_{\chi}} e^{-\left(\mathbf{u}+\mathbf{v}_{\odot}\right)^{2} / 2 \sigma^{2}},
$$

where $\mathbf{u}$ is the heliocentric WIMP speed, $\sigma$ is the $1 \mathrm{D}$ velocity dispersion and $\mathbf{v}_{\odot}$ is the lag between the dark matter particles and the Sun. $\rho$ is the WIMP density at the solar 
circle and $M_{\chi}$ is the WIMP mass. For the SHM, $\left|\mathbf{v}_{\odot}\right|=220 \mathrm{~km} / \mathrm{s}$ and $\sigma=\left|\mathbf{v}_{\odot}\right| / \sqrt{2}$. For the dark disc, fiducial values of: $\rho_{\mathrm{d}}=\rho_{\mathrm{h}}$ and $\left|\mathbf{v}_{\odot}\right|=\sigma=50 \mathrm{~km} / \mathrm{s}$ are assumed, consistent both with the median of the ranges found in [87] and [86], and with the kinematics of the Milky Way thick disc stars. The sensitivity of the results to these parameters is discussed in Section 4.5.

The capture rate from a nuclear species $i$ per unit volume shell of a celestial body is given by 136

$$
\frac{d C_{i}}{d V}=\int_{0}^{u_{\max }} d u \int d \Omega_{w} f(\mathbf{u}) u w^{2} \sigma_{i} n_{i}
$$

where $f(\mathbf{u})$ is the velocity distribution normalized such that $\int f(\mathbf{u}) d^{3} \mathbf{u}=\rho / M_{\chi}$. The velocity $w$ at a given shell is related to the velocity at infinity $u$ and the escape velocity $v$ at the shell by $w=\sqrt{u^{2}+v^{2}}$. The WIMP-nucleus cross section is $\sigma_{i}$, and $n_{i}$ is the number density of nuclear species $i$. The upper limit of the integration is

$$
u_{\max }=2 \frac{\sqrt{M_{\chi} m_{i}}}{M_{\chi}-m_{i}} v,
$$

where $m_{i}$ is the mass of nuclear species $i$. This ensures that only WIMPs that can scatter to a velocity lower than the escape velocity $v$ are included. For fixed mass $m_{i}$ and escape velocity $v$ this upper cut off decreases with increasing $M_{\chi}$.

The annihilation rate per unit volume of WIMPs in the body is given by

$$
\frac{d \Gamma_{A}}{d V}=\left\langle\sigma_{A} v\right\rangle_{0} n_{\chi}^{2}(t, \mathbf{x})
$$

where $\left\langle\sigma_{A} v\right\rangle_{0}$ is the velocity-averaged annihilation cross section in the limit of non-relativistic speeds, and $n_{\chi}(t, \mathbf{x})$ is the number density of WIMPs in the body. If WIMPs quickly thermalize with nuclei in the body once captured, the number density of WIMPs in that body can be described by

$$
n_{\chi}(t, \mathbf{x})=N_{\chi}(t) \tilde{n}_{\chi}(\mathbf{x})
$$

where $\int d V \tilde{n}_{\chi}(\mathbf{x})=1$. In that case, the number $N_{\chi}(t)$ of WIMPs in the body is given by the solution to

$$
\dot{N}_{\chi}(t)=C-2 \Gamma_{A}
$$

if $M_{\chi} \gg m_{i}$, where the total capture rate is $C=\sum_{i} C_{i}$ [145, 137]. The factor of 2 in Eq. 4.6 reflects the fact that for self-annihilating particles, two WIMPs are destroyed in each annihilation. If the total capture rate $C$ is constant with time, the annihilation rate is given by

$$
\Gamma_{A}=\frac{C}{2} \tanh ^{2}(t / \tau)
$$


with the equilibrium time $\tau$ given by

$$
\tau=\left(C C_{A}\right)^{-1 / 2}, C_{A}=2\langle\sigma v\rangle_{0} \int d V \tilde{n}_{\chi}^{2}(\mathbf{x})
$$

For a WIMP of $M_{\chi} \sim 100 \mathrm{GeV}$ with purely spin-independent interactions with baryons, $C$ is about nine orders of magnitude greater for the Sun than for the Earth, while $C_{A}$ is about three orders of magnitude smaller [137]. Thus, equilibrium timescales tend to be orders of magnitude shorter in the Sun than in the Earth. If the spin-independent WIMP-proton cross section is $\sigma_{p}^{S I}=10^{-43} \mathrm{~cm}^{2}, \tau \sim 10^{8} \mathrm{yr}$ for the Sun and $\tau \sim 10^{11} \mathrm{yr}$ for the Earth [48. Therefore, if the age of the solar system is $t_{\odot} \approx 4.5 \mathrm{Gyr}, t_{\odot} / \tau \gg 1$ in the Sun and $\Gamma_{A}=C / 2$ and is constant. For this set of WIMP parameters, WIMP annihilation will have reached equilibrium in the Sun. However, in the Earth, $t_{\odot} / \tau \ll 1$, and $\Gamma_{A} \propto C^{2}$ and is growing with time. For some of the models that are considered, the interaction rate becomes low enough that equilibrium is broken in the Sun also. However, these models appear near the bottoms of the plots, far away from the interesting detection thresholds.

\subsection{The dark matter candidate}

For detailed calculations of capture and annihilation rates, a specific WIMP model for the particle's mass, scattering cross sections and annihilation channels has to be assumed. In supersymmetric extensions of the Standard Model with conserved R-parity, the lightest supersymmetric particle is a natural WIMP candidate. The lightest neutralino in the CMSSM is chosen as the dark matter particle. The CMSSM reduces the free parameters in supersymmetry to three parameters at a gauge unification scale: the gaugino mass $m_{1 / 2}$, the scalar mass $m_{0}$ and the tri-linear coupling $A_{0}$. At the electroweak scale, the ratio of the Higgs vacuum expectation values $\tan (\beta)$ and the sign of the Higgs/higgsino mass parameter $\mu$ are selected. Only the square of $\mu$ is calculated from the potential minimization conditions of electroweak symmetry breaking. The CMSSM parameter space was scanned in the range of $m_{1 / 2} \in\left[\begin{array}{ll}0 & 4\end{array}\right] \mathrm{TeV}, m_{0} \in\left[\begin{array}{ll}0 & 4\end{array}\right] \mathrm{TeV}, A_{0} \in\left[\begin{array}{ll}-7 & 7\end{array}\right] \mathrm{TeV}$ and $\tan (\beta) \in\left[\begin{array}{ll}20 & 65\end{array}\right]$ for $\mu>0$. The allowed parameter space, consistent with current experimental constraints is found with the publicly available SuperBayes package [146]. This Markov Chain Monte Carlo (MCMC) algorithm calculates the Bayesian posterior probability at each parameter point from the compatibility of the theoretical predictions with experimental constraints. The most relevant experimental constraints used in the MCMC are the cosmologically allowed relic density measured by the Wilkinson Microwave Anisotropy Probe (WMAP), electroweak precision observables and limits on the Higgs and lightest neutralino mass from colliders. All constraints listed in [146] have been used, except that the value for the dark matter density has been updated to the WMAP 5 year data release value, $\Omega_{D M} h^{2}=$ $0.1099 \pm 0.0062$, where $h$ is the Hubble constant in units of $100 h \mathrm{~km} / \mathrm{s} / \mathrm{Mpc}$ and $h=0.719$ is their best fit value [147]. Flat priors are used in the calculation of the Bayesian probability. Although the resulting allowed parameter space depends on the choice of priors, this study focuses on the relative change in flux caused by the addition of the dark matter disc 
and thus the choice of priors is not important. The chains used in this study contain a total of $0.9 \times 10^{6}$ samples. The posterior probability density functions presented below are normalized to their maximum values, and not to a total probability of 1 . Accordingly these are labeled as "relative probability densities". An interface of the SuperBayes MCMC algorithm to DarkSusy (v5.03) [148] routines is used to calculate the capture rate and the resulting muon flux at the Earth.

\subsection{Neutrino Flux from the Sun and Earth}

The measurable quantity in neutrino telescopes is the muon flux at the Earth's surface originating from neutrinos produced in WIMP annihilations. Thus the muon flux is a tracer of the neutrino flux emerging from the Sun's or Earth's interior which is induced by WIMP annihilations.

\subsubsection{The Sun}

In figure 4.1 the muon flux with an energy above $1 \mathrm{GeV}$ at the Earth resulting from WIMP capture and annihilation in the Sun is shown. The flux is shown as a function of $M_{\chi}$ for the SHM (left) and the dark disc (right) for $\rho_{\mathrm{d}} / \rho_{\mathrm{h}}=1$ and $\rho_{h}=0.3 \mathrm{GeV} / \mathrm{cm}^{3}$. The higher phase space density at low velocities for the dark disc strongly enhances the capture rate and hence the resulting muon flux at the detector site. The flux expected from the dark disc is larger by approximately an order of magnitude (depending on the specific model) compared to the flux expected from the SHM, since the capture rate increases by approximately an order of magnitude, and $t_{\odot} / \tau \gg 1$ for most of the models in the figure (see Section 4.2).

\subsubsection{The Earth}

Finding the muon flux from WIMP annihilation in the Earth is more complicated. Because the escape velocity of the Earth is small $(v \approx 15 \mathrm{~km} / \mathrm{s}$ at the centre), capture is only possible for low speed WIMPs unless the WIMP mass is nearly identical to that of one of the nuclear species in the Earth (Eq.(4.3); see also [136, 150]). Moreover, the capture rate is disproportionately sensitive to the lowest speed WIMPs since those WIMPs may be captured anywhere in the body, whereas higher speed WIMPs may only be captured at the centre where the escape velocity is largest. However, the low speed tail of the WIMP speed distribution is not precisely known; for speeds relative to the Earth of $u<72 \mathrm{~km} / \mathrm{s}$ (the speed of a WIMP at the escape velocity from the solar system, moving in the direction opposite to the Earth), the phase space may be occupied by WIMPs bound to the solar system as well as Galactic WIMPs streaming through the solar system on unbound orbits. Thus, the annihilation rate of WIMPs in the Earth depends on the density of WIMPs bound to the solar system, which has not yet been definitively determined. 

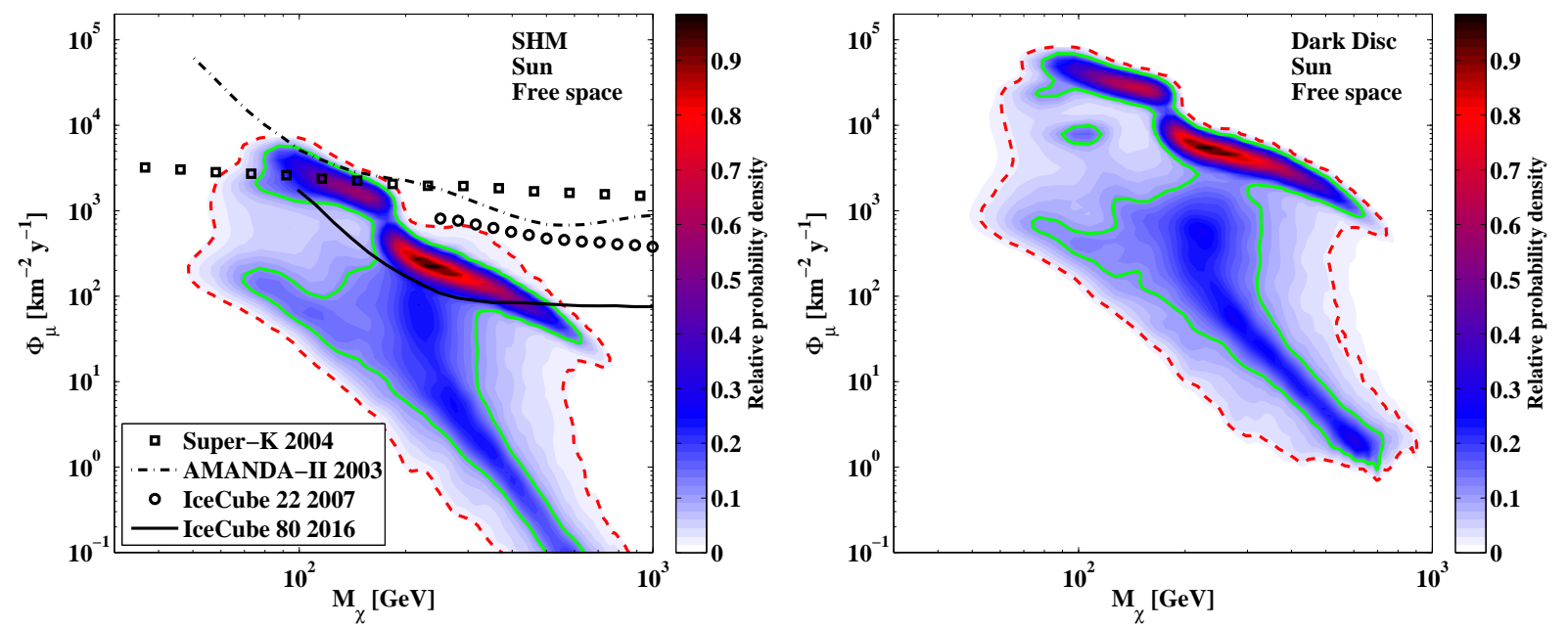

Figure 4.1: Muon flux $\Phi_{\mu}$ for $E_{\mu}>1 \mathrm{GeV}$ at the Earth's surface as a function of $M_{\chi}$ from neutrinos originating in the Sun, for the SHM (left panel) and the dark disc (right panel). The dark disc boosts the muon flux by an order of magnitude for $\rho_{\mathrm{d}} / \rho_{\mathrm{h}}=1$. Current experimental constraints on the muon flux from the Sun from Super-Kamiokande [142, AMANDA-II [143, 149] and IceCube22 [144] along with the expected sensitivity of IceCube80 are over-plotted on the left panel. The closed contours show $-95 \%$ (red/dashed) and 68\% (green/solid) - of the probability density of CMSSM models consistent with both astrophysical and collider constraints, and assuming flat priors. The color-bar gives the relative probability density (see Section 4.3 for details).

\subsubsection{Sensitivity to the population bound to the Solar System}

There is a spread in predicted bound WIMP distributions. Although the following studies predicted the bound WIMP distribution for the SHM, the results generalize to arbitrary dark matter distributions. On the high end, Gould [151] argued that the low speed WIMP distribution resulting from gravitational capture of WIMPs by the planets should be approximately the free space Gaussian distribution function of Eq. (4.1). This argument was based on treating WIMP-planet encounters as local, with the cumulative changes to WIMP speed treated in the random walk approximation. Also using the local approximation, Lundberg and Edsjö [152] found a smaller low speed WIMP distribution if they treated the Sun as being infinitely optically thick to WIMPs. Damour and Krauss [153] considered a population of long-lived WIMPs captured in the solar system by elastic scattering in the Sun, but neglected subsequent scatters of those WIMPs with solar nuclei. Bergström et al. [154] found that this population could boost the annihilation rate of WIMPs in the Earth by a factor of $\sim 100$ for $60 \mathrm{GeV}<M_{\chi}<130 \mathrm{GeV}$.

More recently, Peter [155, 156] has simulated $\sim 10^{10}$ WIMPs bound to the solar system by either gravitational capture or elastic scattering in the Sun. Orbits were integrated in a toy solar system consisting of the Sun and Jupiter. WIMP trajectories were followed using a modified symplectic integration algorithm, allowing for the possibility of further elastic scattering in the Sun. The orbits were integrated until the WIMPs were ejected 

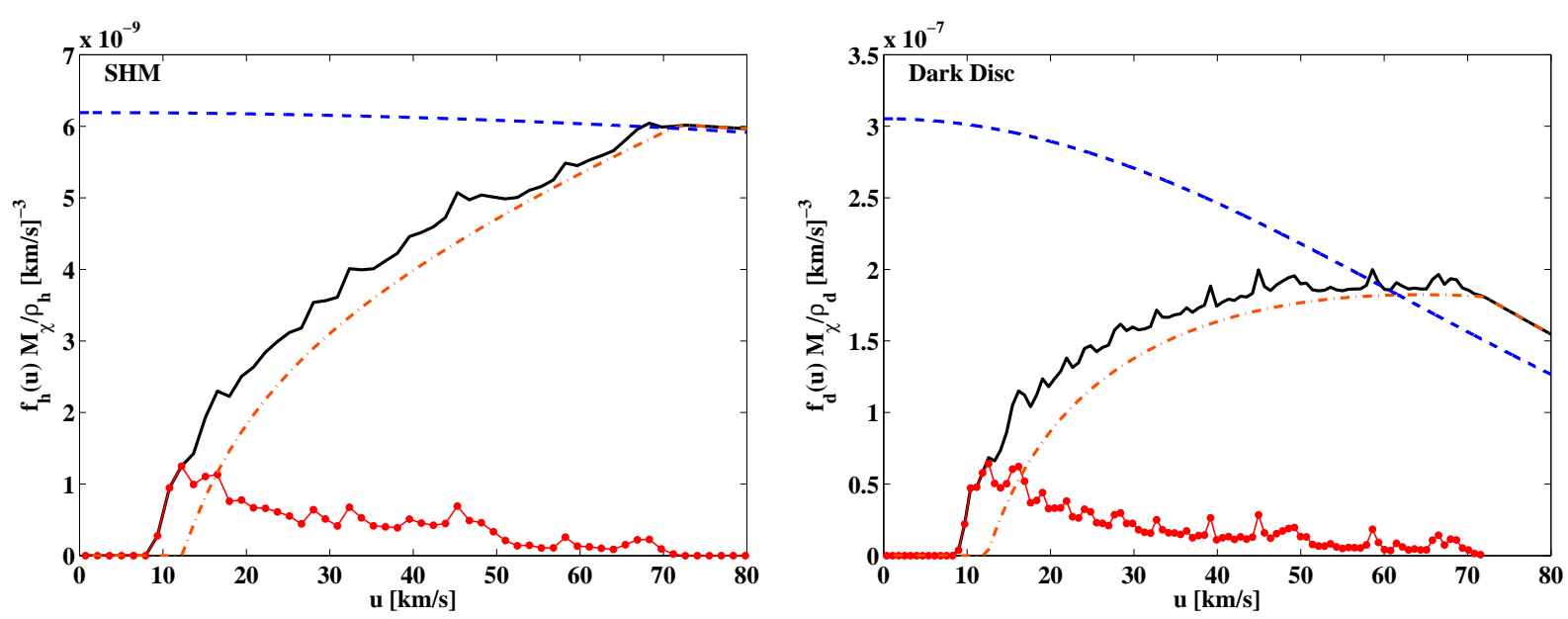

Figure 4.2: The phase space density at low velocities for the SHM (left panel) and the dark disc (right panel). The black/solid curve is the summed distribution of bound (red/dotted) and unbound (orange/dash-dotted) particles from the solar system simulations, used in the calculation of the capture rates. The blue/dashed line shows the distribution of the free space Gaussian approximation. Note the vertical scales of the two plots differ by two orders of magnitude.

or scattered onto orbits that no longer intersected the Earth. The phase space density distribution of bound WIMPs as a function of speed relative to the Earth is shown in figure 4.2 for both the SHM and the dark disc. Also shown in figure 4.2 are the phase space density distributions of only the Galactic WIMPs (unbound to the solar system) and the free space Gaussian approximation (denoted as "Free space" on relevant figures).

The bound velocity distribution is significantly smaller than predicted by Gould [151] and Damour and Krauss [153, and similar to that found by Lundberg and Edsjö [152]. While part of the difference is due to elastic scattering in the Sun (especially for the Damour and Krauss population), part of the difference is due to simulating orbits in a toy solar system. The cut-off in the velocity distribution at $u \sim 9 \mathrm{~km} / \mathrm{s}$ owes to the phase space below being inaccessible to WIMPs in the toy solar system due to the conservation of the Jacobi integral of motion; interaction with the inner planets is required to populate lower speeds. This cut-off in speed translates to a cut-off in muon flux above a particular WIMP mass. Solving Eq. (4.3) for $M_{\chi}$, and setting $m_{i}$ to the mass of ${ }^{56} \mathrm{Fe}$ (the dominant atomic species in the core of the Earth), we find that the muon flux is exactly zero for $M_{\chi}>700$ $\mathrm{GeV}$. The impact of the WIMPs bound to the solar system on the WIMP annihilation rate in the Earth depends crucially on how effective the inner planets are at populating the phase space below $u=9 \mathrm{~km} / \mathrm{s}$.

Given the uncertainty in the low speed WIMP distribution, the annihilation rates are calculated for both the Peter [155, 156] distribution functions and the free space Gaussian distribution function. These span a likely range of the true distribution function of low speed WIMPs. The Damour and Krauss [153] solar-captured distribution function is larger than the Gaussian distribution function for $30 \mathrm{~km} / \mathrm{s}<u<50 \mathrm{~km} / \mathrm{s}$. However Peter [155] 
finds that subsequent scattering in the Sun reduces the lifetime and phase space density of these WIMPs below the Gaussian phase space density.

In figure 4.3, the muon fluxes from WIMP annihilation in the Earth for both the SHM and the dark disc assuming a muon energy threshold of $1 \mathrm{GeV}$ is shown. For both distribution functions, the flux from the dark disc is two to three orders of magnitude above the SHM if $\rho_{\mathrm{d}} / \rho_{\mathrm{h}}=1$. This large increase is due to the fact that $t_{\odot} / \tau<1$, such that $\Gamma \propto C^{2}$. Thus, an increase in the capture rate of WIMPs in the Earth has a more dramatic effect on the muon flux than a similar enhancement in the capture rate of WIMPs in the Sun.

The predicted flux from WIMPs with $M_{\chi} \gtrsim 100 \mathrm{GeV}$ is quite sensitive to the low speed phase space density distribution. For the distribution function from the solar system simulations, a steep drop in flux is found due to the kinematic cut-off in the capture rate for $M_{\chi} \gtrsim 500 \mathrm{GeV}$. As a consequence, while the enhancement of the muon flux from the dark disc puts the search for WIMP annihilation in the Earth on the same level as the Sun for $M_{\chi} \lesssim 100 \mathrm{GeV}$, the prospects for detecting WIMPs of higher masses is unclear. Precision estimates of the low speed tail of the WIMP velocity distribution are necessary to determine the prospects for high mass WIMPs.

\subsection{Discussion}

Figure 4.4 shows the total flux from the Sun and the Earth (including capture from both SHM and dark disc components assuming $\rho_{\mathrm{d}} / \rho_{\mathrm{h}}=1$ ) along with current experimental constraints. The flux in both cases is dominated by the dark disc component. To be conservative, the lower bound of the expected muon flux from the Earth obtained using the phase space density distribution from the solar system simulations is shown. The inclusion of the dark disc component significantly improves the constraints on the allowed parameter space from current experiments. Large area neutrino telescopes such as IceCube will be sensitive to a large fraction of the allowed parameter space, providing a complementary search for dark matter to direct detection experiments.

Systematic uncertainties owing to the unknown density and velocity distribution of the dark disc are especially large for the Earth owing to high powers of these parameters in the calculation of the annihilation flux. For the results presented here, $\rho_{\mathrm{d}} / \rho_{\mathrm{h}}=1$ and $\sigma_{d}=50 \mathrm{~km} / \mathrm{s}$ with the mean lag $\left|\mathbf{v}_{\odot}\right|=\sigma_{d}$ was used. For the Earth the dependency is given by $\exp \left(-\left|\mathbf{v}_{\odot}\right|^{2} / \sigma_{d}^{2}\right)\left(\rho_{\mathrm{d}} / \rho_{\mathrm{h}}\right)^{2} / \sigma_{d}^{6}$ for masses $M_{\chi}>100 \mathrm{GeV}$, since the part of the WIMP phase space relevant for capture scales as $\exp \left(-\left|\mathbf{v}_{\odot}\right|^{2} /\left(2 \sigma_{d}^{2}\right)\right)\left(\rho_{\mathrm{d}} / \rho_{\mathrm{h}}\right) / \sigma_{d}^{3}$ and the flux depends on the capture rate squared. For the Sun the actual dependency is more complex, the capture rate scales as

$$
\frac{\left(\rho_{\mathrm{d}} / \rho_{\mathrm{h}}\right)}{\left|\mathbf{v}_{\odot}\right|}\left(2 \cdot \operatorname{erf}\left(\frac{\left|\mathbf{v}_{\odot}\right|}{\sqrt{2} \sigma_{d}}\right)-\operatorname{erf}\left(\frac{\left|\mathbf{v}_{\odot}\right|-v_{c u t}}{\sqrt{2} \sigma_{d}}\right)-\operatorname{erf}\left(\frac{\left|\mathbf{v}_{\odot}\right|+v_{c u t}}{\sqrt{2} \sigma_{d}}\right)\right)
$$

where $v_{\text {cut }} \sim 2000 \sqrt{\mathrm{GeV} / M_{\chi}} \mathrm{km} / \mathrm{s}$ is approximately the maximum speed of WIMPs which can be captured in the Sun. An empirical estimate for $\left|\mathbf{v}_{\odot}\right|=\sigma_{d}$ of the dependency gives a 

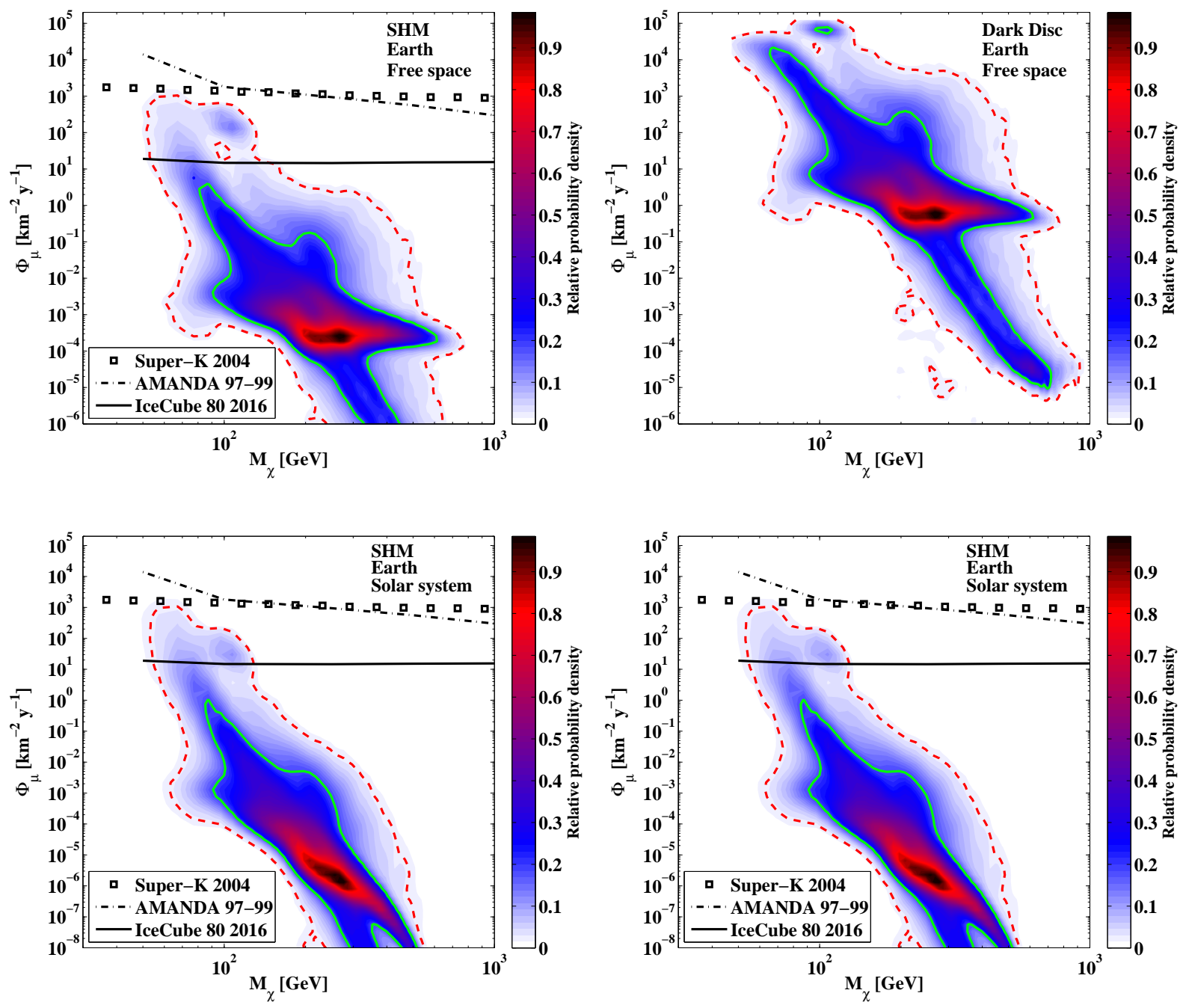

Figure 4.3: Muon flux $\Phi_{\mu}$ for $E_{\mu}>1 \mathrm{GeV}$ at the Earth's surface as a function of $M_{\chi}$ from neutrinos originating in the Earth. The top row is calculated with the free space Gaussian approximation of the velocity distribution, while for the bottom row the velocity distribution from the solar system simulations is used. The sharp decrease in the muon flux at high WIMP masses in the bottom row is caused by the kinematic cut-off of the capture rate (see text). Compared to flux from the SHM (left panels) the flux from the dark disc (right panels) is boosted by two to three orders of magnitude for $\rho_{\mathrm{d}} / \rho_{\mathrm{h}}=1$, depending on the specific model. Current experimental constraints on the muon flux from the Earth from Super-Kamiokande [142] and AMANDA-II [143, 149] along with the expected sensitivity of IceCube80 are compared to the flux expected from the SHM. The closed contours show - 95\% (red/dashed) and 68\% (green/solid) - of the probability density of CMSSM models consistent with both astrophysical and collider constraints, and assuming flat priors. The color-bar gives the relative probability density (see Section 4.3 for details). Note the vertical scales of the two bottom plots differ by two orders of magnitude as compared with the top plots. 

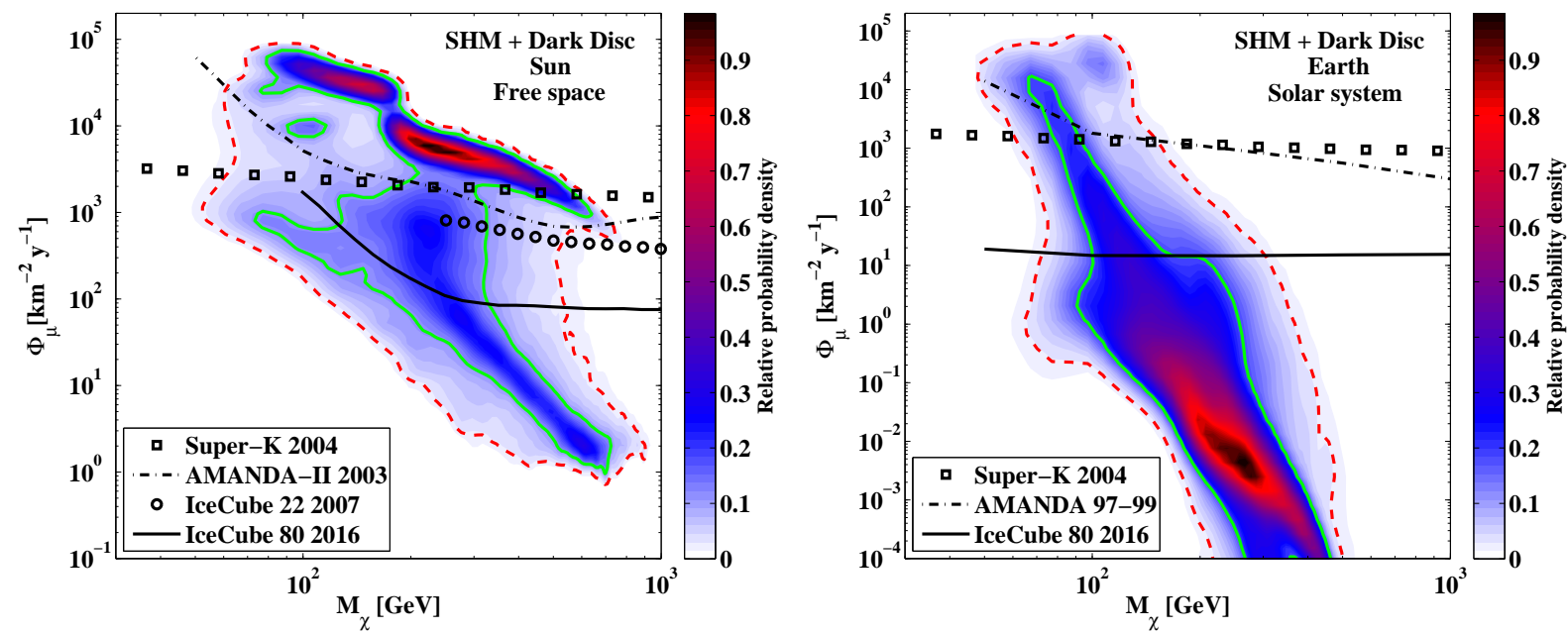

Figure 4.4: Total muon flux $\Phi_{\mu}$ for $E_{\mu}>1 \mathrm{GeV}$ at the Earth's surface as a function of $M_{\chi}$ from neutrinos originating in the Sun (left panel) and Earth (right panel). In both cases the muon flux is dominated by the dark disc component. Current experimental constraints on the muon flux from the Earth and Sun from Super-Kamiokande [142, AMANDA-II [143, 149] and IceCube22 [144] are shown. The enhanced flux allows these experiments to constrain a much larger portion of the CMSSM parameter space. The projected sensitivity of the IceCube80 experiment will probe a significant fraction of the allowed parameter space. The closed contours show $-95 \%$ (red/dashed) and 68\% (green/solid) - of the probability density of CMSSM models consistent with both astrophysical and collider constraints, assuming flat priors. The color-bar gives the relative probability density (see Section 4.3 for details)

scaling of $\left(\rho_{\mathrm{d}} / \rho_{\mathrm{h}}\right) / \sigma_{d}^{a}$ with $a \in\left[\begin{array}{ll}1 & 2\end{array}\right]$ depending on the particle's mass. This scaling differs from the scaling in the Earth owing to the flux being proportional to one power of the capture rate. Simulations have shown that all disc galaxies will have a dark disc, but the cosmic variance in its properties will be large. At the minimum extreme is a dark disc with $\rho_{\mathrm{d}} / \rho_{\mathrm{h}}=0.25$ and $\sigma_{d} \simeq 100 \mathrm{~km} / \mathrm{s}$ (corresponding to the lowest/highest value respectively, found in the simulations [86]). Even in this case, the annihilation signal from the Earth and Sun are both dominated by the dark disc rather than the dark halo. However, with such a large velocity dispersion, the scaling just described means that the dark disc does not lead to the large boosts that come from the median dark disc properties used in this study.

The median values for the dark disc properties exclude the most probable regions of the CMSSM parameter space. However, given the uncertainties in the dark disc properties it is not yet possible to convincingly exclude relevant CMSSM parameter space shown in figure 4.4.

Future surveys of our Galaxy like RAVE [101] and GAIA 102] will detect the local density of dark matter and may disentangle accreted stars (which will have nearly the same velocity dispersion as the dark disc) from those formed in-situ. In this case, it will be 
possible to infer the actual properties of the dark disc from these stars, and hence, make more robust predictions for the event rate in neutrino telescopes.

The main findings of this study are:

1. The dark disc significantly boosts the capture rate of dark matter particles in the Sun and Earth as compared to the SHM. This increase owes to the higher phase space density at low velocities in the dark disc. For the Sun, the expected muon flux from the dark disc with $\rho_{\mathrm{d}} / \rho_{\mathrm{h}}=1$ is increased by one order of magnitude relative to a pure SHM-generated flux. If the WIMP is the neutralino in the CMSSM, neutrino telescopes will explore a large fraction of the CMSSM parameter space.

2. For the Earth - where WIMP capture and annihilation are not in equilibrium - the increase in the muon flux is two to three orders of magnitude, although this depends sensitively on the distribution function of the dark disc. For the SHM alone, the flux from the Sun is far greater than that from the Earth. The enhancement from the dark disc puts the search for WIMP annihilation in the Earth on the same level as the Sun if $M_{\chi} \lesssim 100 \mathrm{GeV}$. For larger WIMP masses, the prospects for detecting muons from annihilation in the Earth requires better models of the density of WIMPs bound to the solar system.

\footnotetext{
${ }^{1}$ The considered WIMP model already has little parameter space below $100 \mathrm{GeV}$, but this extra sensitivity of detection below $100 \mathrm{GeV}$ owes to kinematics of solar system transport and capture, not the WIMP model.
} 


\section{Chapter 5}

\section{Cryogenic Dark Matter Search}

The small recoil energy deposition expected from dark matter interactions, coupled with an expected low event rate, requires an efficient background suppression. Active and passive shielding are used to reduce backgrounds produced outside the experimental apparatus. The experimental setup at the Soudan underground laboratory and the layout of the shielding to reduce external backgrounds is discussed in this chapter.

The CDMS experiment employs low temperature semiconductor crystals in the search for WIMPs, which are operated at millikelvin temperatures. Special techniques and custom made electronics are used to operate and read out the detectors at theses temperatures. A first stage of the CDMS experiment had been operated at the Stanford Underground Facility [157, 158], a tunnel $10.6 \mathrm{~m}$ beneath the Stanford University campus. The current stage of the experiment (CDMS-II) is operated at an underground laboratory to reduce muon-induced backgrounds.

\subsection{CDMS-II at the Soudan Underground Laboratory}

The CDMS-II experiment is located at the Soudan Underground Laboratory (SUL) in northern Minnesota, USA. The Soudan Underground Laboratory is a former iron mine which ceased operation in 1963, and is now operated by the state's Department of Natural Resources (DNR) as a joint experimental physics and tourist site. The access to the mine is provided by a single, two-cage shaft, which allows direct travel from the surface to the $27^{\text {th }}$ level. On the $27^{\text {th }}$ level, at a depth of $714 \mathrm{~m}$ below the surface two excavations host the CDMS-II and the MINOS neutrino oscillation experiment [159]. A schematically view of the underground experimental site is shown in figure 5.1 .

The underground site has been chosen to reduce the atmospheric muon flux, since muons that interact with nuclei in the surrounding material and the experimental apparatus can produce neutrons which produce signatures in the detectors, that are indistinguishable from WIMP candidate events. The rock overburden provides 2090 m.w.e 1 of shielding against cosmic radiation, reducing the muon flux by a factor of $\sim 50.000$ from its value at

${ }^{1}$ meters water equivalent 


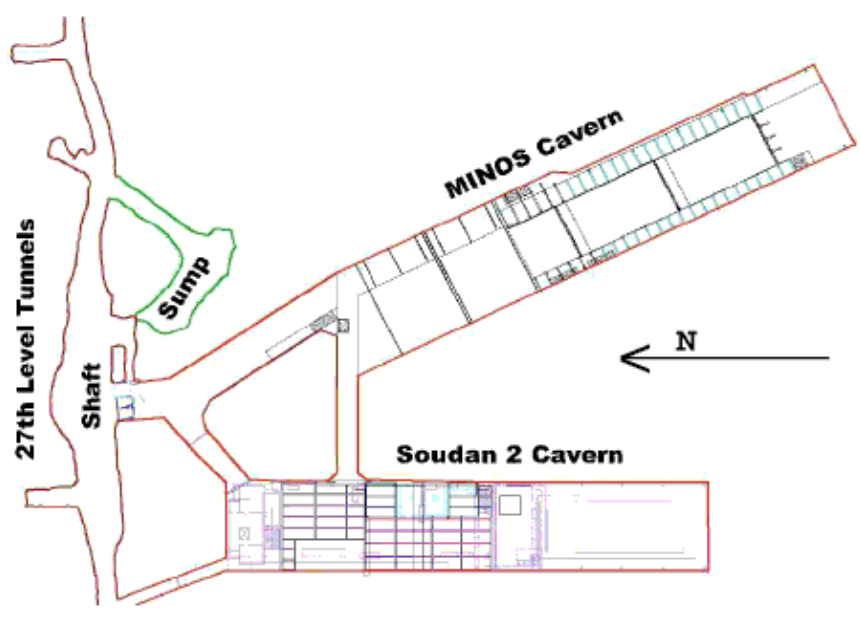

Figure 5.1: Schematic layout of the $27^{\text {th }}$ level of the SUL. The CDMS-II experiment is installed in the Soudan 2 Cavern.

the surface. This reduction in muon flux is accompanied with a corresponding reduction in muon-induced particle showers. Especially the reduction of neutrons generated in these showers is of importance for direct detection experiments, since neutron-induced nuclear recoils cannot be distinguished from their WIMP-induced counterparts. Experiments in underground laboratories have a much lower background rate of such events and hence a greater sensitivity than similar experiments at the surface.

\subsubsection{Cryogenics}

To reach the required $\mathrm{mK}$ temperatures for the operation of the detectors, they are cooled by an OXFORD Instruments $400 \mathrm{~S}^{3} \mathrm{He}^{-}{ }^{4} \mathrm{He}$ dilution refrigerator. The refrigerator has a rated cooling power of $400 \mu \mathrm{W}$ at $100 \mathrm{mk}$ and a base temperature below $10 \mathrm{mK}$ with no external load. The dilution refrigerator is built primarily of steel, generally with an unacceptable level of uranium, thorium and ${ }^{60} \mathrm{Co}$ contamination. Thus the refrigerator is mounted to the side of the experimental shielding (see figure 5.2). Its various temperature stages are connected through a custom made set of "tails" to the horizontally displaced cold volume known as the "icebox". The icebox and tails are connected through the icebox's surrounding shielding by a concentric set of copper cylinders called the "C-stem". A matching "E-stem " extends through the shield opposite the C-stem. This stem carries the detector stripline wiring from the icebox to the "E-box", a box shaped structure which connects the striplines to external cabling through vacuum sealed D-connectors.

Most of the dilution refrigerator's support plumbing is located outside of the experimental radio-frequency shielded room (RF-room hereafter), the so called "cryopad ". The circulation of the ${ }^{3} \mathrm{He}-{ }^{4} \mathrm{He}$ mixture is driven by a Pfeiffer rotary pump and a Roots blower connected in series. Before entering the fridge the mixture is circulated through a series of three cold traps to clean it of impurities. This cleaning circle consists of a liquid nitrogen cold trap on the cryopad, an external liquid helium cold trap on the cryopad, and an inner 
liquid helium cold trap within the helium bath of the refrigerator itself. All three of these traps are cleaned regularly during normal operation, generally on a monthly interval. The ${ }^{3} \mathrm{He}-{ }^{4} \mathrm{He}$ circulation loop is controlled and monitored by an Oxford Instruments Intelligent Gas Handling (IGH) unit, regularly reporting pressures, valve settings and cryogenic levels to a dedicated computer. The provided information is published on a web site for remote monitoring.

A Moore APACS automated industrial control system, located on the cryopad, monitors and directs numerous aspects of cryogenic operations, notably the daily refilling of the refrigerator's liquid helium and nitrogen baths. Refills occur automatically at a user predefined time each day, but can be manually initiated if necessary. APACS is interlocked to the data acquisition system, pausing the DAQ. APACS also regularly records temperatures, pressures, flow rates and other quantities; this provides a complete historical record of the cryogenic operations that can be accessed anywhere over the Internet through custom JAVA GUIs.

The installation of three additional detector arrays (called Tower) substantially increased the heat load on the icebox. This extra heat is mainly dumped at $4 \mathrm{~K}$ by radiation from 36 additional FETs and conduction through 19 new striplines (18 for detectors and one for icebox thermometry). Thermal modeling suggested that the heat leakage through the towers would have been sufficient to rise the detectors above the super conducting transition temperatures, rendering many readout channels inoperable. A Gifford-McMahon cryocooler from Sumitomo Heavy Industries was added as a second refrigeration system during this system upgrade. The cryocooler is a closed-circuit helium refrigerator that provides $1.5 \mathrm{~W}$ of cooling power at $4 \mathrm{~K}$ and $40 \mathrm{~W}$ at $77 \mathrm{~K}$ without the need for external cryogenics. The cryocooler is mounted on the E-Stem, driven by an external compressor outside the RF-room. Helium gas is driven to the head at high pressure $(\sim 20$ atmospheres) and compressed/expanded by a piston, providing cooling power. The unit has two cooling stages, cooling both the $77 \mathrm{~K}$ and the $4 \mathrm{~K}$ stages of the E-stem, intercepting the heat load of the striplines and preventing large temperature gradients across the $4 \mathrm{~K}$ layer. The cryocooler cold fingers are connected to the E-Stem through flexible copper couplings designed to limit the transmission of mechanical vibration while maintaining excellent thermal conductivity.

\subsubsection{Shielding of the experiment}

Any low background experiment requires an efficient shielding of the detectors from environmental background produced outside of the experimental apparatus. The CDMS setup consists of an active shielding component to shield against residual cosmic ray interactions and a passive component to shield against natural radioactivity.

\section{Active Scintillator Muon Veto}

The active veto system is intended to reject the remaining muon flux not stopped by the rock overburden. Muons could interact with nuclei in the passive shielding and produce 


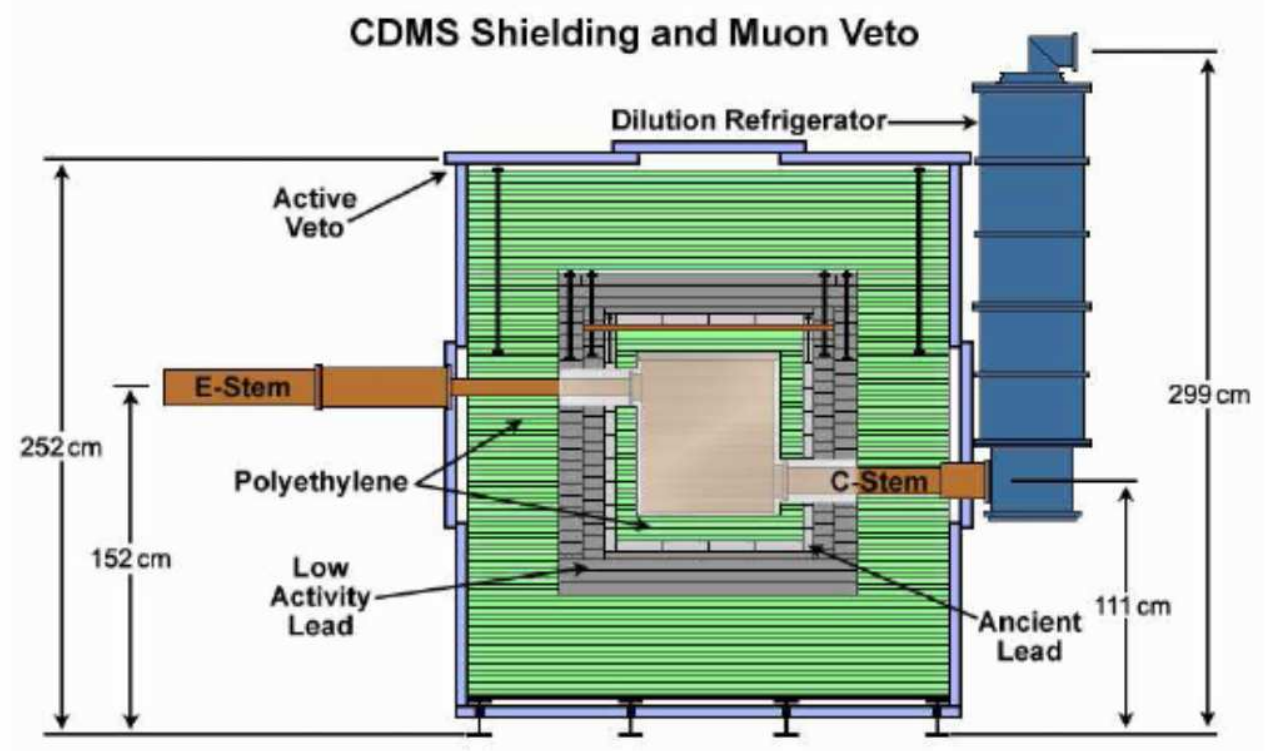

(a)

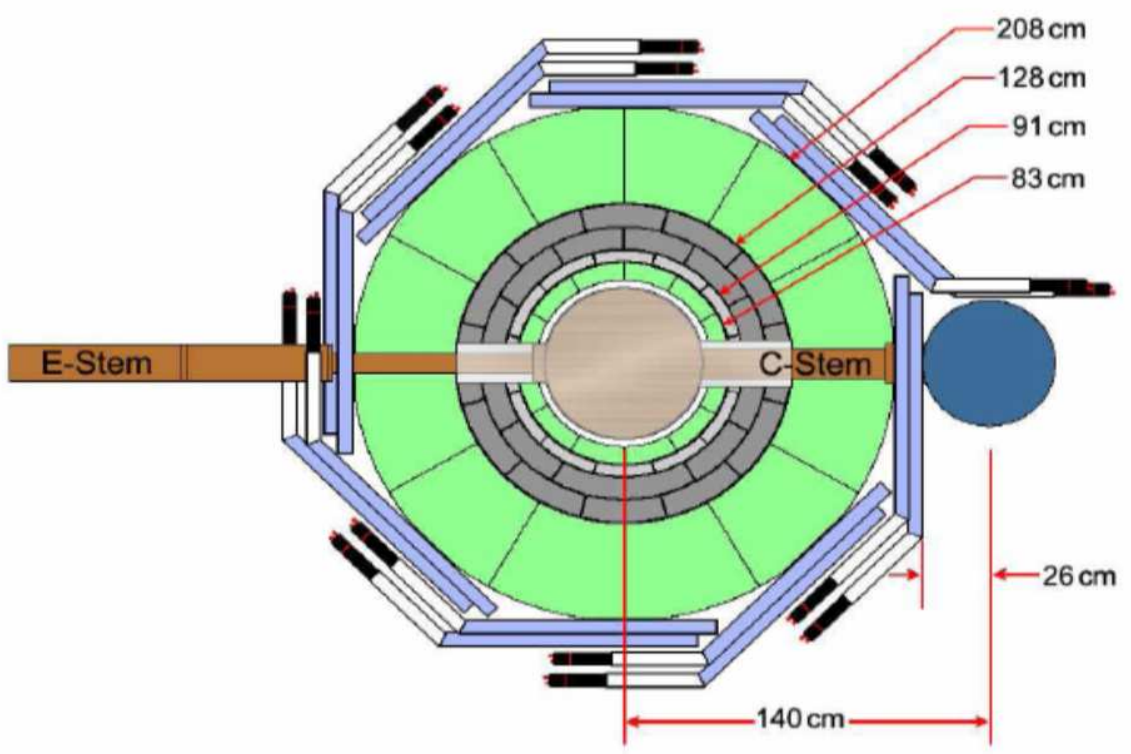

(b)

Figure 5.2: Schematic view of the (a) side and (b) top of the active veto and passive shielding. From the outside to the inside the figures show the veto panels (light blue) which enclose the outer polyethylene shield (green), the outer low radioactive lead shielding (gray), the inner ancient lead shield (light gray), the inner polyethylene shield (green) and finally the mu-metal shield (light metal colored). The E-stem and Cstem (brown) pass between the veto panels and the passive shielding and connect to the mu-metal shield. The C-stem connects to the dilution refrigerator (dark blue) providing the thermal contact between the cold layers and the fridge. 
neutrons in these interactions. The signature of a neutron scattering in one of the detectors is indistinguishable from the signature of a WIMP candidate. Hence a possible background from this source has to be suppressed.

The active muon veto consists of 40 BICRON BC-408 plastic scintillator panels surrounding the inner passive shielding (see figure 5.2). The panels are arranged such, that adjacent panels have a slight overlap to cover the whole experimental setup. Acrylic light guides direct the scintillation photons in the panels to the attached Hamamatsu R329-02 photo multiplier tubes (PMTs). The scintillator panel and light guide are wrapped in mylar foil for light isolation. The veto system also includes a source of blue light, transported to each panel with an optical fiber. Periodic pulsing of the blue light source (between data acquisition runs) allows calibration checks of the PMTs.

A minimum ionizing muon typically deposits $2 \mathrm{MeV} \mathrm{g}^{-1} \mathrm{~cm}^{2}$, hence the energy deposit is about $10 \mathrm{MeV}$ in the $5 \mathrm{~cm}$ thick veto panels with a density of $1.03 \mathrm{~g} \mathrm{~cm}^{-3}$. A muon passing through a scintillator generates after amplification approximately $100 \mathrm{pC}$ of collected charge. The veto efficiency for tagging through-going muons is $99.98 \pm 0.02 \%$.

\section{Passive Shielding}

The passive shielding consists of three parts: an outer layer of polyethylene, two layers of lead and an inner layer of polyethylene as can be seen in figure 5.2 .

The outer polyethylene layer is composed of one inch thick slabs that are designed to stick around the outer lead brick layer. The outer poly layer is $40 \mathrm{~cm}$ thick on the sides and $40.6 \mathrm{~cm}$ thick at the top and bottom. The purpose of the outer poly layer is to moderate the primary flux of neutrons from outside of the experimental apparatus down to energies that induce nuclear recoils below the detection thresholds of the detectors.

The lead reduces ambient gamma flux from neutral radioactivity outside of the experimental apparatus. The lead shield is composed of inner and outer layers of lead surrounding the detectors cylindrical. The outer layer consisting of low-activity lead has a thickness of $17.8 \mathrm{~cm}$ on the top, bottom and the sides. The inner lead layer with a thickness of $4.4 \mathrm{~cm}$ on the top, bottom and the sides is composed of ancient lead from a shipwreck off the coast of France (this is why is is often referred to as "french lead"). ${ }^{210} \mathrm{~Pb}$, the naturally occurring radioactive lead isotope with the longest half-life of 22.3 years, is no longer present in the ancient lead. The ancient lead is used to attenuate gamma backgrounds due to radioactive isotopes in the outer lead.

The inner layer of polyethylene shielding is inside the inner ancient lead and provides further neutron moderation. The inner poly is $10 \mathrm{~cm}$ thick on the side, $7.6 \mathrm{~cm}$ thick on the top, and $10.2 \mathrm{~cm}$ thick at the bottom. The inner polyethylene shielding also suppresses the cosmic-ray induced neutrons in the lead, but it is not very massive, so it does not increase the gamma flux significantly. The ordering of the passive shielding was determined by Monte Carlo simulations to be optimal in terms of suppression of neutron background.

Within the inner polyethylene layer, a can of mu-metal surrounding the detectors was placed to reduce the external magnetic field that might affect the performance of parts of the cold electronics, especially the SQUIDs (see Section 6.4.2 for a detailed description of 
the detector electronics). Any magnetic flux in the vicinity of the detectors would produce noise on the readout channels. Testing of the mu-metal shield with a Gauss probe showed, that it reduces the background magnetic field by about two orders of magnitude.

The detectors themselves are kept under vacuum, but the radon level in the mine air is relative high $\left(\sim 700 \mathrm{~Bq} / \mathrm{m}^{3}\right)$. The lead shield provides adequate protection of air outside the shied, but any substantial volume of air inside the shield increases the gamma background rate significantly. A continuous purge with dry nitrogen is installed to exclude mine air from the volume between the outer copper can and the mu-metal shield.

Within the shield, the icebox itself consists of a set of concentric copper cans heatsunk to the various temperature stages of the dilution refrigerator. These stages are: room temperature $(300 \mathrm{~K})$, nitrogen shield $(77 \mathrm{~K})$, helium bath $(4 \mathrm{~K})$, still $(\sim 1 \mathrm{~K})$, cold plate $(\sim 130 \mathrm{mK})$ and the mixing chamber $(\sim 40 \mathrm{mK})$. The detector housings and bulk of the cold hardware within the icebox are made from high-purity copper due to its excellent radio-purity, limiting the radioactive contamination near the detectors. The total thickness of copper surrounding the detectors is a few $\mathrm{cm}$, sufficient to shield the detectors from alpha and beta radiation from outside the cans.

\subsection{Cold hardware}

Each detector is connected to a system of cryogenic amplifiers, wiring and support structures known collectively as "cold hardware". Figure 5.3 illustrates a cross section of the Stanford icebox and cold hardware. Note that the icebox setup is different at Soudan, but the layout of the cold hardware is identical.

\subsubsection{Detector housing}

Each detector is housed within a hexagonal ring-shaped housing made of high-purity copper. The detector is mounted within its housing by a set of six flat Cirlex clamps. Detectors are mounted in a stack of six, each attached to a tower support structure. The housings of the detectors within the Tower do not have top or bottom lids, giving each detectors face a full view of the neighboring detector's face $3.5 \mathrm{~mm}$ away. This arrangement greatly increases the proportion of events, especially events due to radioactive surface contamination, which scatter in two neighboring detectors. Such events are easily rejected by an event multiplicity cut.

Each detector is connected to the readout electronics through a small detector interface board (DIB) located on one edge of its housing. The DIB plugs into the end of a side coax and couples the detector through ten Al wire-bond connections. The DIB also houses two infrared LEDs for clearing trapped charges in the detectors. The LED shines on the phonon face of its own detector and the charge face of the neighboring detector. 
Stack Assembly Cross Section

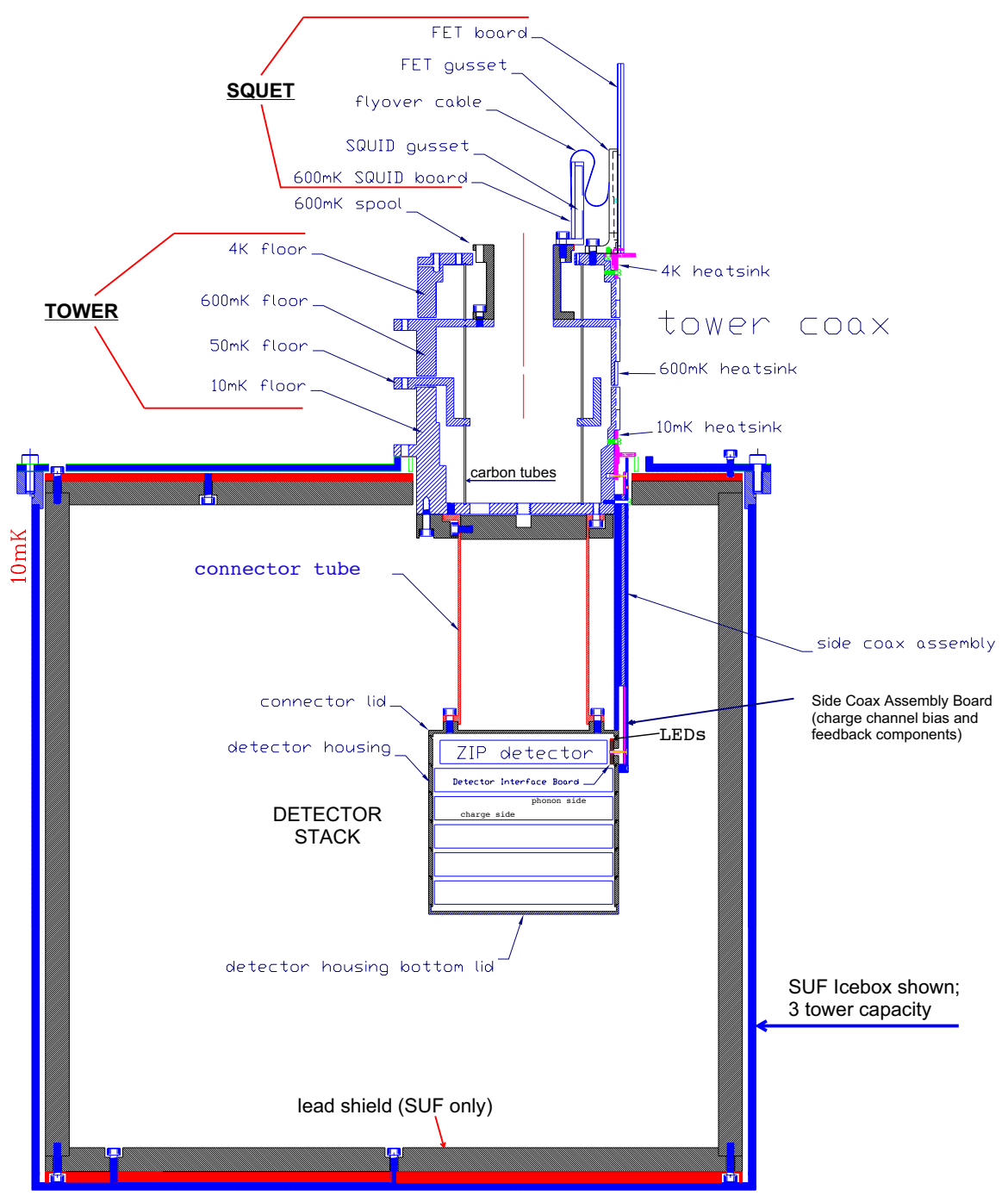

Figure 5.3: Cross section of the cold hardware within the icebox. Note that the icebox shown is that of the Stanford facility previously used by CDMS; the Soudan icebox can house seven towers and has no inner lead shield. Although the layout of the icebox is different the setup of the cold hardware is identical at Soudan. 


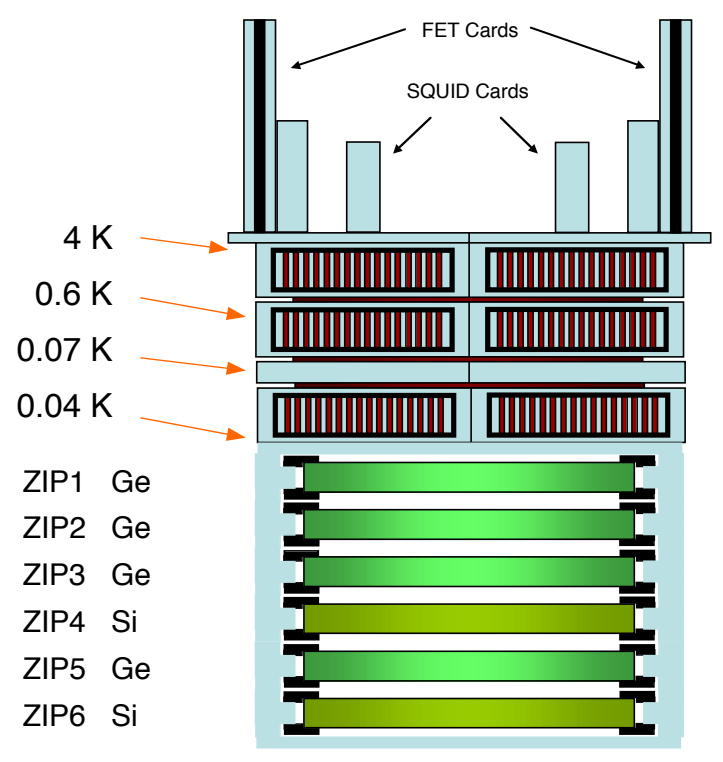

Figure 5.4: Layout of a specific Tower in the CDMS-II setup. The stacking of the detectors along with the various temperature stages of the Tower are shown. On top of the detector the cold readout electronics are mounted.

\subsubsection{Tower detector arrays}

A Tower, shown in cross section for a specific Tower in the Soudan setup in figure 5.4, is a hexagonal copper structure that supports the detector stack and connects it electrically to the SQUET cards (see Section 5.2.4). A Tower consists of four copper stages, each heat sunk to one of the icebox cans. A central graphite cylinder supports the four stages, holding them separate without excessive heat conduction between them. Each face of the hexagonal structure carries the bias and signal wires of one detector, connecting the side coax at the base temperature to the SQUET card mounted at $4 \mathrm{~K}$. The wires are heat-sunk to three of the temperature stages (base, still and $4 \mathrm{~K}$ ) and held under tension in vacuum channels along the Tower faces to limit their sensitivity to mechanical vibrations.

Note that individual detectors are identified based upon their Tower and stack position, e.g. "T3Z5" is the fifth detector (counting from the top of the stack) mounted in Tower 3.

\subsubsection{Side coax}

Each detector is connected to the base temperature stage of its respective Tower face by a connector called a side coax. The side coax also houses the coupling capacitors and bias feedback resistors of the detector's two charge amplifiers. The low temperature of the base stage lessens the Johnsons noise contributions to the ionization channel noise. Side coaxes are made in six distinct lengths so as to reach each of the six detector positions in the stack. 


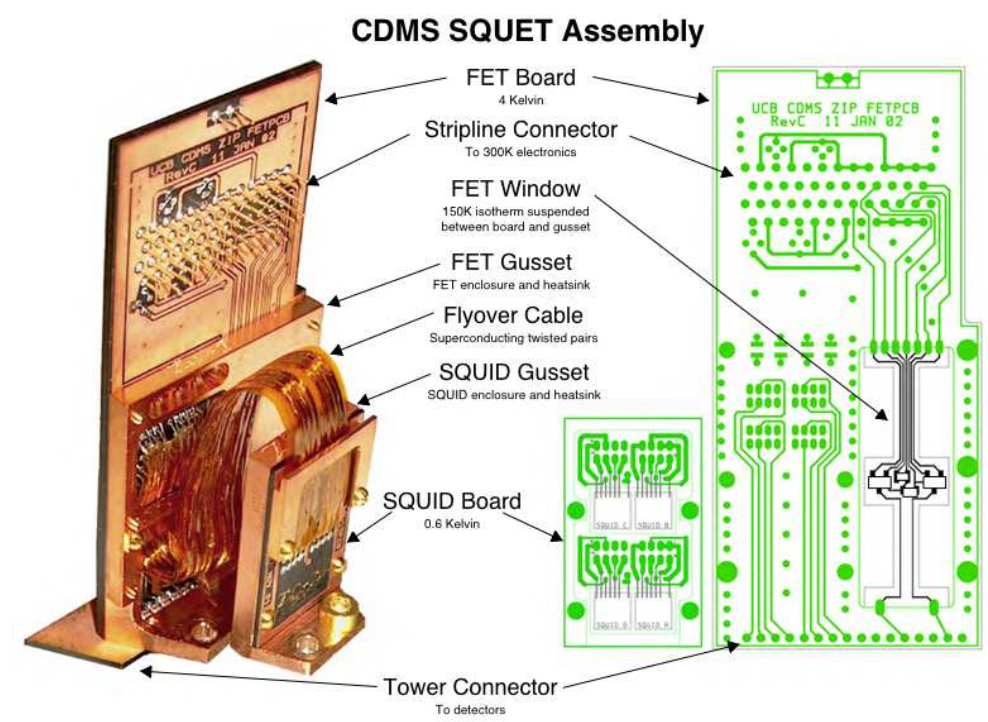

Figure 5.5: Photograph (left) and layout of the CDMS SQUET card (right). Pictures and annotations by Dennis Seitz.

\subsubsection{SQUET card}

On top of each tower face a SQUET ("SQUID and FET") card, shown in figure 5.5, is mounted. The SQUET is a combination of two circuits boards: a primary card housing the charge amplifiers' FETs and a second card containing the SQUIDs, shunt resistors and input and feedback coils of the phonon amplifiers (see Section 6.2.2 and 6.4.2 for details). The FETs are supported on a Kapton membrane within a copper gusset on the primary card. This arrangement enables the FETs to self heat to $\sim 140 \mathrm{~K}$ (the carriers within them would be frozen out at $4 \mathrm{~K}$ ) while protecting the rest of the $4 \mathrm{~K}$ stage from excessive infrared radiation from the hot FETs. The SQUID card is heat sunk to an extension of the still layer of the Tower to improve SQUID performance and reduce the Johnson noise of the shunt resistors. The two cards are joined by a flexible cable composed of twisted-pair superconducting niobium wires sandwiched between layers of Kapton tape.

\subsubsection{Stripline}

The warm and cold electronics are linked by a set of flat electrical cables called striplines. Each is a $3 \mathrm{~m}$ flexible circuit made of Kapton and copper, consisting of 50 copper traces sandwiched between two copper ground plates and surrounded by Kapton insulation. Each stripline connects the SQUET card of one detector through the E-stem to an E-box connector. The stripline bundle is heat-sunk at two thermal intercepts within the E-stem to limit heat flow between the room temperature and $4 \mathrm{~K}$ stage. Once inside the icebox the striplines fan out to connect to each individual detector SQUED as it is shown in figure 5.6. 


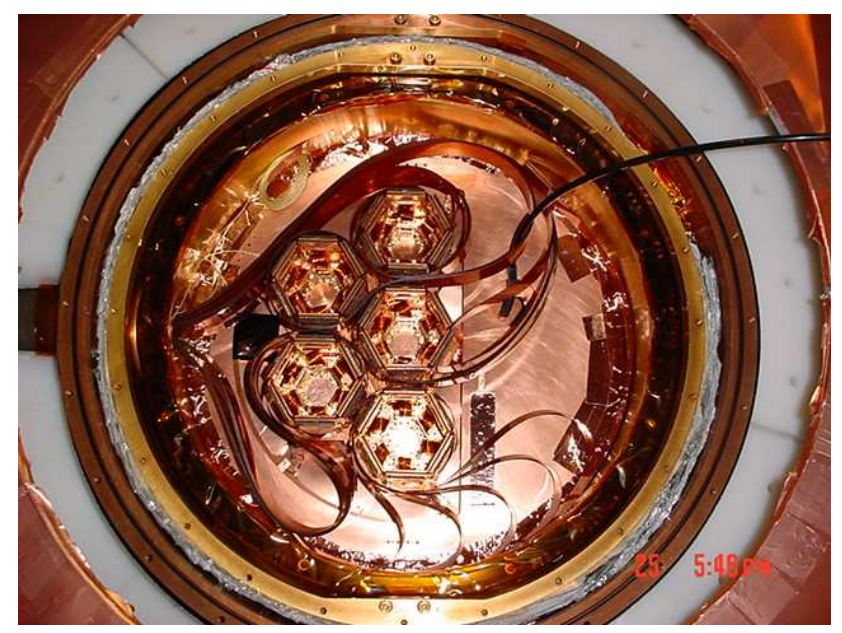

Figure 5.6: Final 5 Tower configuration of the Soudan icebox, Showing the SQUETs and striplines. The E-stem and warm electrons lie to the left of the photo, the C-stem and dilution refrigerator lie to the right.

\subsection{Warm electronics}

The actual readout of the signals from the detectors and recording of the data is installed at room temperature inside the RF room and the electronics room outside of the experimental clean room, to allow easy access to components and control of the experiment. These electronics are referred to as "warm electronics".

\subsubsection{Front-end boards}

Signals from the E-box pass through 50-wire cables to a rack of front-end boards (FEBs) in the RF room, one for each detector. Each FEB is a custom-made circuit board carrying the second- and later-stage amplifiers and other components of the ionization and phonon readout circuits, as well as control circuits for the detector LEDs (see Section 5.2.1), ionization electrode biasing, SQUID biasing, etc. The FEB also contains amplifiers for each readout channel to buffer and further amplify these signals before digitization. The FEBs are controlled through a GPIB interface, which is linked from the electronics room via fiber optics.

\subsubsection{Receiver trigger-filter boards}

Amplified signals from the FEB are send through the RF-room wall to a corresponding receiver trigger-filter (RTF) board in the electronics room. The RTF board conditions the FEB signals for digitization by baseline adjustment and applying a $336 \mathrm{kHz} 2$-pole Butterworth anti-aliasing filter. A Butterworth filter has a frequency response which is maximally flat in the passband (below the cut-off frequency), and rolls off towards zero 
in the stop-band (above the cut-off frequency) [160]. Each RTF also generates five logic trigger-signals that are used to actuate the digitizers. The four main trigger signals are denoted Qlo, Qhi, Plo and Phi. Plo is the primary event acquisition trigger, issued by a comparator which tests whether the sum of the four phonon signals exceeds its baseline by a software-defined amount, generally $3-4 \mathrm{mV}$. Phi is a similar test with a much higher threshold $(\sim 500 \mathrm{mV})$. Qlo and Qhi are analogues for Plo and Phi for the summed ionization channels. The fifth trigger signal, named Pwhisper, is a threshold similar to Plo but often with a lower threshold, intended for tagging multiply-scattered events.

\subsubsection{Data acquisition hardware}

The detector trigger signals and the equivalent ones from the veto panels pass to a trigger logic board (TLB) which determines when to issue a "global" trigger, i.e a signal to initiate the acquisition of the event, including the readout channels from all detectors and veto panels. The criteria for issuing such a trigger are user-defined, but include:

- A "random " trigger issued by the data acquisition system to measure the experimental noise.

- A "veto multiplicity" trigger initiated by a simultaneous hit on multiple veto panels (used for muon background characterization).

- A "detector trigger", initiated by a Plo trigger issued by any detector.

As a global trigger is received, the six readout channels of each detector are recorded by an array of 14-bit Struck SIS 3301 analog-digital converters and are written to disk. The digitizers operate at a sampling rate of $100 \mathrm{MHz}$, but 80-sample sequences are internally averaged to yield an output rate of $1.25 \mathrm{MHz}$ with a corresponding reduction in digitizer noise. When triggered, each digitizer records a 2048-sample (1.6 ms) trace. The trigger itself occurs in the 512 th bin, so each trace includes $409 \mu$ s preceding the trigger.

The photomultiplier signals from the 40 scintillator veto panels are processed by an analogous set of boards. A pulse-stretching filter network reshapes the very short (tens of ns) raw veto signals before digitization. The reshaped veto pulses are recorded by 12-bit Joerger VTR812 ADCs, each acquiring 1024 samples at $5 \mathrm{MHz}$. A set of comparators also issues trigger signals from the veto shield. These are fed to the trigger logic board for evaluation of global triggers.

All of the above trigger signals are also recorded by a set of Struck SIS 2400 TDCs. These boards record the time stamps of each trigger in a circular buffer with $1 \mu$ s resolution. This trigger history buffer is recorded along with each event written to disk, giving record of the several triggers immediately preceding and following the recorded trigger.

In addition to the event-specific data record, various experimental quantities are monitored by a slower data-acquisition system. This "slow DAQ" records the average values of the various signal channels and the mean trigger rates once per minute. The former are monitored for evidence of a SQUID losing flux lock, the latter for sudden changes that indicate changes in detector noise. 


\subsection{Data acquisition software and data handling}

The data acquisition system (DAQ) is controlled by an interlocking system of Java and $\mathrm{C}++$ programs run on a small cluster of computers in the Soudan electronics room. The DAQ software is highly modular, with each major function (GPIB communication, slow DAQ monitoring, high voltage control, etc.) handled by a dedicated program. Each major function is accessible through a graphical user interface (GUI), which is accessed from a "master GUI" called RunControl. When run from the mine or the surface building, the RunControl GUI allows full control of the data acquisition system. Experimental control from other remote locations is generally not allowed, but the RunControl GUI may be used remotely to monitor the experiment's status messages and settings.

The full readout of the experiment causes a non negligible DAQ deadtime - the time required to reset the system to accept a new global trigger - which limits the maximum rate at which the DAQ can acquire events. The data acquisition system can acquire event traces from all detectors at $\sim 20 \mathrm{~Hz}$. This limitation is of no concern for low-background operation of the experiment with an event rate of $\sim 0.3 \mathrm{~Hz}$, but is a significant restriction in calibration data runs. A typical calibration run with a ${ }^{133} \mathrm{Ba}$ source can have a trigger rate of $\gtrsim 200 \mathrm{~Hz}, 10$ times the rate at which the DAQ can actually acquire data from 30 detectors. In "selective readout" mode, the DAQ only records the digitizer output for those detectors which issued phonon triggers for a given event, rather than for all detectors and the veto shield as usual. Since only a few detectors trigger for each event, this greatly reduces the amount of data required to be recorded for an event. This not only saves disk space but also reduces the DAQ deadtime associated with data transfer, allowing for much faster data acquisition rates at the same total data throughput. ${ }^{133} \mathrm{Ba}$ calibration data sets are taken in selective readout mode, enabling acquisition rates of $\sim 70 \mathrm{~Hz}$ and reducing the amount of livetime spend on calibration. Low background runs and ${ }^{252} \mathrm{Cf}$ data (neutron source calibrations) are acquired in standard readout mode.

The raw event data acquired by the DAQ software is stored to local disks in the mine. As each raw event file is completed it is compressed and transferred to the surface. A copy of the compressed file is written to a digital backup tape in the mine. At the surface, the data are stored on disks at the Soudan Analysis Cluster (SAC). The SAC processes the incoming data through a data reduction package to produce a preliminary set of reduced quantities. These preliminary quantities are used by automated data quality checks and for any manual check of the experimental performance. The primary data processing occurs at Fermilab on the FermiGrid computing cluster. The raw data is transferred over the internet to the cluster and the final quantities used in the analysis are generated. The final data is then distributed from Fermilab to the home institutions of the collaboration. 


\section{Chapter 6}

\section{CDMS detectors}

The challenge for every dark matter direct detection experiment is the ability to identify candidate events amongst a far larger population of background interactions. The technical challenge is to realize this identification technique effectively for low energy depositions (keV-scale) in large mass detectors, while maintaining a stable performance over months of measuring time.

The Cryogenic Dark Matter Search experiment (see Chapter 5) employs low temperature germanium and silicon detectors to detect WIMPs via their elastic scattering off target nuclei. An interaction in the semiconductor crystals creates (regardless of the type of interaction) two signals: the ionization signal from electron-hole pairs created and the excitation of phonons in the crystal. Both signals are recorded by the ZIP 1 detectors used in the CDMS-II experiment.

The physics of an interaction in the crystals and the readout of the ionization and phonon signal are discussed in this Chapter. For the readout of the phonon signal it is necessary to operate the detectors at milli Kelvin temperatures, since the CDMS detectors use super conducting TES $\$ 2$ for the phonon detection. These sensors allow the collection of athermal phonons which carry information about where and when the initial particle interaction took place. The design of the phonon sensors provides information about the physical location of an event within the detector.

The type of interaction can be determined with theses two signals, since the ionization signal is different for nuclear recoils than for electron recoils from background gamma radiation. The basic principle of the background discrimination in CDMS detectors is introduced. Finally the main background in CDMS will be discussed, which is caused by a detector specific effect. The rejection of this background will be discussed in detail in Chapter 9

\footnotetext{
${ }^{1} \mathbf{Z}($ depth$)-$ sensitive Ionization- and Phonon- mediated

${ }^{2}$ Transition Edge Sensor
} 


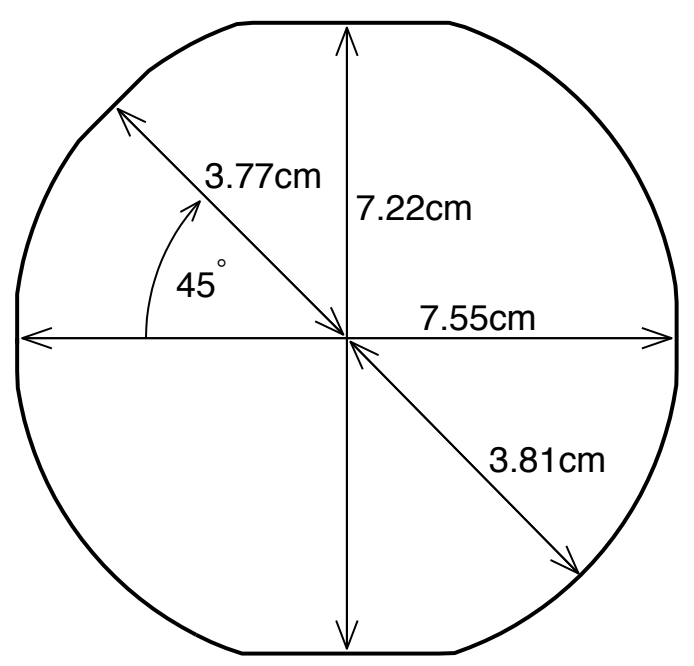

Figure 6.1: Physical layout of a ZIP detector substrate as seen from the top. The major flats are at the north and south, and the minor flats are at the east and west. The small fifth flat is $45^{\circ}$ west from north, indicating the (100) crystal axis.

\subsection{Crystal substrate characteristics}

Each ZIP detector is an almost cylindrical crystal made of germanium or silicon. The substrates are $1 \mathrm{~cm}$ thick and have a radius of $3.81 \mathrm{~cm}$. Out of alignment and handling reasons the crystals are not perfectly cylindrical in shape and show five flats at the outer edge (as shown in figure 6.1). Two major flats are at the north and south, separated by $7.22 \mathrm{~cm}$; the two minor flats are at the west and east position separated by $7.55 \mathrm{~cm}$; a small minor flat is located at the northwest position, with a distance of $3.77 \mathrm{~cm}$ to the center. The position of the fifth flat indicates the orientation of the crystal axis in the face-centered-cubic (fcc) crystalline structure. The vertical axis of each ZIP detector is aligned with the (001) crystal axis, and the fifth flat centered at $45^{\circ}$ west of the north position indicates the (100) crystal axis.

Most ZIP detectors used in the CDMS-II setup were made from $n$-type boules, with the exception of 8 -type Ge ZIPs in Towers 3, 4 and 5. The substrate material is prepared with low impurity and dislocation concentrations $\left(\sim 10^{11}\right.$ impurities $/ \mathrm{cm}^{3}$ and $<5000$ dislocations $/ \mathrm{cm}^{2}$ for $\mathrm{Ge}$ ) to ensure a good charge carrier transport.

The use of Ge and Si detectors is motivated by the different physical properties leading to different performance in terms of a dark matter signal and background rejection. The density of silicon is $2.33 \mathrm{~g} / \mathrm{cm}^{3}$, while germanium is much heavier at $5.34 \mathrm{~g} / \mathrm{cm}^{3}$. Each Ge ZIP has a mass of $\sim 230 \mathrm{~g}$, while each Si detector only has a mass of $\sim 100 \mathrm{~g}$. While having the same volume, the germanium exposure (defined as the mass times the measuring time) is always greater than the silicon exposure for a single ZIP detector. Due to its larger atomic mass, a Ge atom has a larger cross section for coherent WIMP-nucleus scattering than a Si atom. For all but the very light WIMP masses, Ge is expected to be more sensitive to 


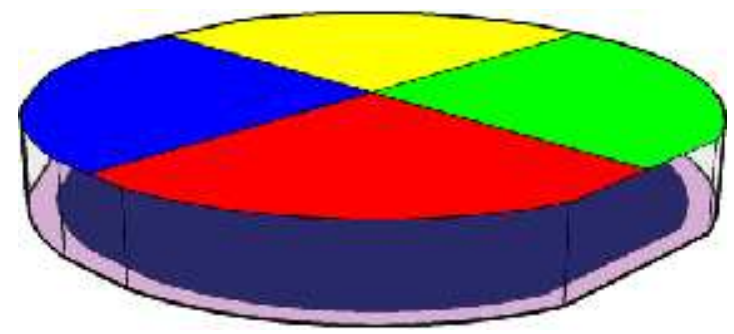

Figure 6.2: Schematic sensor configuration of a ZIP detector, showing the four phonon sensor quadrants on the top face and the two concentric ionization electrodes on the bottom face.

spin-independent WIMP interactions (for details see Section 3.1.2) than an equal mass of $\mathrm{Si}$. The substrates are natural silicon and germanium, with isotopic compositions shown in Table 6.1. All isotopes have even numbers of protons and neutrons except ${ }^{29} \mathrm{Si}(4.67 \%)$ and ${ }^{73} \mathrm{Ge}(7.73 \%)$, each of which has an unpaired neutron. These two isotopes give CDMS-II its sensitivity to spin-dependent WIMP interactions (for detail see Section 3.1.3). Since the cross section for elastic neutron scattering on $\mathrm{Si}$ is greater than on Ge the use of a mixture of $\mathrm{Ge}$ and $\mathrm{Si}$ is a hedge against a possible neutron background. A comparison of nuclear recoil rates in both detector substrates can statistically differentiate a WIMP signal from a neutron background.

\begin{tabular}{|c|c|c|c|c|}
\hline Isotope & Abundance & $\mathrm{p}$ & $\mathrm{n}$ & Notes \\
\hline \hline${ }^{28} \mathrm{Si}$ & $92.23 \%$ & 14 & 14 & \\
\hline${ }^{29} \mathrm{Si}$ & $4.67 \%$ & 14 & 15 & unpaired neutron \\
\hline${ }^{30} \mathrm{Si}$ & $3.10 \%$ & 14 & 16 & \\
\hline \hline${ }^{70} \mathrm{Ge}$ & $21.23 \%$ & 32 & 38 & \\
\hline${ }^{72} \mathrm{Ge}$ & $27.66 \%$ & 32 & 40 & \\
\hline${ }^{73} \mathrm{Ge}$ & $7.73 \%$ & 32 & 41 & unpaired neutron \\
\hline${ }^{74} \mathrm{Ge}$ & $35.94 \%$ & 32 & 42 & \\
\hline${ }^{76} \mathrm{Ge}$ & $7.44 \%$ & 32 & 44 & \\
\hline
\end{tabular}

Table 6.1: Isotopic composition of natural Si and Ge.

Each of the two detector faces is photolithographically patterned with sensor wiring, as shown schematically in figure 6.2. The bottom face (as the detectors are installed in the Soudan setup) is divided in two concentric ionization electrodes: an inner electrode (denoted as "Qinner") covering approximately $80 \%$ of the detector surface and an outer guard electrode $3 \mathrm{~mm}$ in width (denoted as "Qouter"). The top face of each detector is divided in four phonon sensor quadrants, each composed of 1036 tungsten transition edge sensors (TES) wired in parallel and fed by an array of aluminum quasi particle traps (see Section 6.4.1). The ionization and phonon sensors and their readout are discussed in detail in Section 6.2 and 6.4, respectively. 


\subsection{The ionization signal}

Particle interactions in a semiconductor crystal dissipate a portion of the deposited energy to the electron system of the crystal. These primary electrons may have sufficiently high momentum to liberate other electrons from their bound state to the conduction band, producing a cascade of charge carriers as the energy of the primaries is dissipated into the crystal. The process culminates in a population of lower-momentum electrons and holes in the vicinity of the particle track. This ionization proceeds in competition with phonon emission. The partition of energy is characterized by a quantity $\epsilon$, equal to the average incident energy required to create one electron-hole pair. Though the band gaps $E_{g}$ of Ge and $\mathrm{Si}$ are $0.734 \mathrm{eV}$ and $1.12 \mathrm{eV}$ at $0 \mathrm{~K}$, respectively, the average deposited energy required to generate an electron-hole pair is substantially greater because some of the energy is shed as optical and acoustic phonons.

The amount of energy needed to excite an electron-hole pair in a semiconductor is the temperature dependent band gap $E_{g}$. The temperature dependence of the band gap is commonly approximated by the Varshni relation [161]

$$
E_{g}(T)=E_{g}(0)-\frac{\alpha T^{2}}{T+\beta}
$$

The parameters for silicon and germanium are given in table 6.2 below.

\begin{tabular}{|c|c|c|c|}
\hline Material & $E_{g}(0)$ & $\alpha / 10^{-4} \mathrm{eV} \mathrm{K}^{-1}$ & $\beta / \mathrm{K}$ \\
\hline \hline $\mathrm{Si}$ & $1.170 \pm 0.001$ & $4.730 \pm 0.25$ & $636 \pm 50$ \\
\hline $\mathrm{Ge}$ & $0.7437 \pm 0.001$ & $4.774 \pm 0.30$ & $235 \pm 40$ \\
\hline
\end{tabular}

Table 6.2: Parameters of the Varshni equation for the temperature dependence of the band gap, values taken from Thurmond [162.

Most of the energy of any interaction, however, does not go into producing electron hole pairs . The number of charge pairs produced by an electron recoil is $N_{Q}=\frac{E_{R}}{\epsilon}$, where $E_{R}$ is the deposited recoil energy and $\epsilon$ is the average energy required to create one electron-hole pair, taking into account the competition with other processes.

A model developed by Shockley [163] gives:

$$
\epsilon=2.2 E_{i}+r E_{R}
$$

The threshold energy for ionization, $E_{i}$, and the number of optical phonon scatters per ionizing event, $r$, are empirically derived from the data; $E_{R}$ is the Raman phonon energy (the energy of the highest energy optical phonons). The model may be understood as partitioning the total energy into three portions: the band-gap energy required to create the charge pairs; the kinetic energy of the charges; and energy used to create optical phonons. An improvement of the Shockley model by Klein successfully explains the Fano factors in various materials as well as $\epsilon$ [164, 165]. The energy deposition proceeds in two 


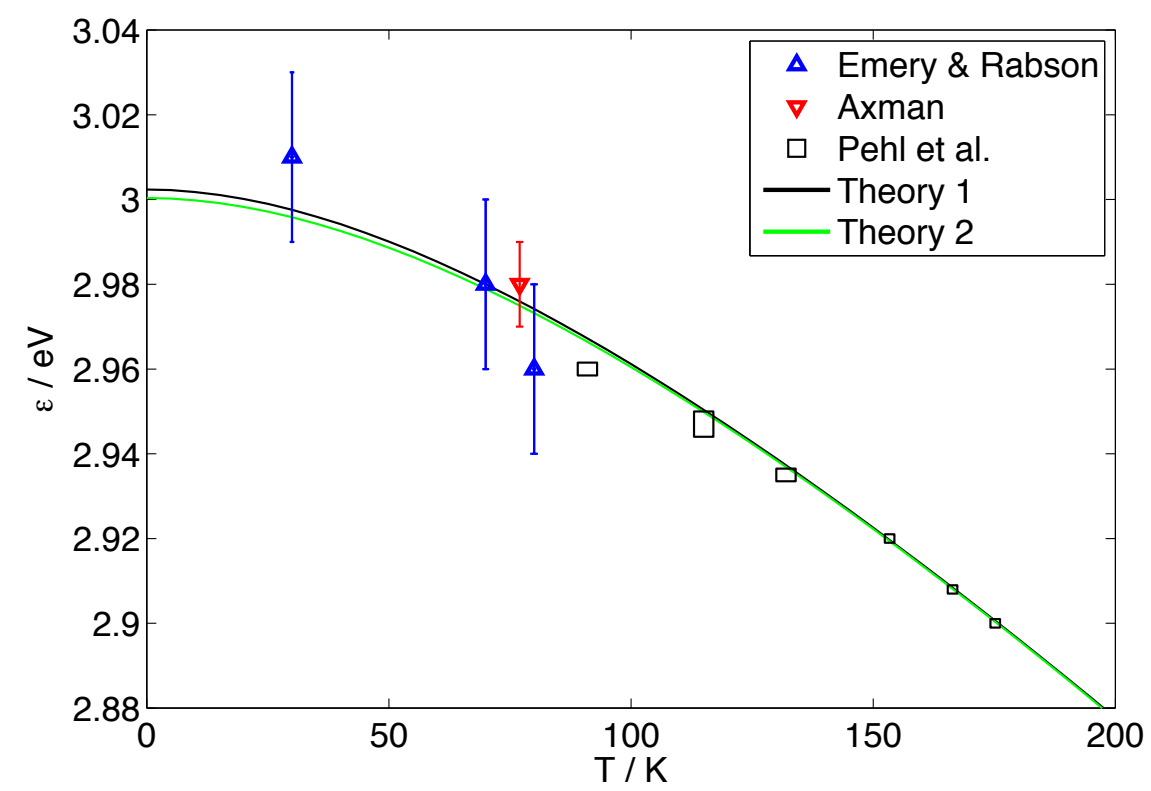

Figure 6.3: Ionization energy as a function of temperature for Ge. The black curve is as in Klein [165] and uses parameters from Varshni [161] and taking $r \hbar \omega_{R}=0.927 \mathrm{eV}$. The lighter, green curve adopts more recent band-gap parameters from Thurmond [162] and adjusts the optical phonon losses to $r \hbar \omega_{R}=0.918 \mathrm{eV}$ to maintain a good fit to the data. Figure taken from [166].

phases. The first, nearly instantaneous, phase is a cascade in which the high-energy incident particle knocks electrons into the conduction band; these create more charges, until the energy of the individual charges is below the ionization threshold. During this process, the energetic electrons and holes interact with the crystal lattice by emitting optical phonons. The second phase is the dissipation of the residual kinetic energy to acoustic phonon modes. The residual kinetic energy is related to the band gap by assuming that the carriers uniformly populate momentum space. This assumptionss gives the following expression:

$$
\epsilon=\frac{14}{5} E_{g}+r \hbar \omega_{R}
$$

It is observed that for many semiconductors $r \hbar \omega_{R}$ is of order $0.5-1.0 \mathrm{eV}$, independent of the band gap. According to this model the temperature dependence of $\epsilon$ comes only from the change in $E_{g}$, with constant optical phonon losses. The dependence of $\epsilon$ on temperature compares well with several data sets for Ge (see figure 6.3), as expected given the known temperature dependence of the band gap. Taking into account the parameters for silicon and germanium given in table 6.2 the values of the ionization energy appropriate for CDMSII ZIP detectors is calculated. A recent measurement for the band gap in germanium gives $r \hbar \omega_{R}=\bar{E}_{R}=0.918 \mathrm{eV}$. As the optical phonon losses are temperature-independent, the same value applies near $0 \mathrm{~K}$. We obtain $\epsilon_{G e}=3.00 \mathrm{eV}$ and $\epsilon_{S i}=3.84 \mathrm{eV}$ 


\subsubsection{Charge transport and collection}

If the initial electron hole pairs are left by themselves they will eventually recombine with one another across the gap or fall into localized states within the gap (so-called "traps"). CDMS-II uses an applied electric field to draw these charge carriers away from the particle track before they can recombine or diffuse to a trap. A bias voltage of $-3 \mathrm{~V}$ for Ge ZIPs, and $-4 \mathrm{~V}$ for $\mathrm{Si}$ is applied to the ionization electrodes with respect to the phonon sensors on the opposite side. At the millikelvin operation temperature of the detectors, all charge carriers are frozen out, hence no "depletion zone" exists and the electric field is nearly uniform. As the charges drift across the crystal, image charges are induced on the conducting electrodes. According to the Shockley-Ramo theorem [167, 168] the induced charge is equal to the charge drifted, so that the integrated current is a measurement of the ionization produced by the initial interaction. Since drifting a hole is equivalent to an electron drifting along the same track in opposite direction, a fully- collected electron-hole pair produces the same image charge as expected from a single electron drifting all the way across the detector.

If a carrier drifts only partway across the crystal the ionization signal will be reduced. The efficiency of the ionization readout channel depends on how likely a carrier will cross the crystal without recombining or becoming trapped. Both processes are more likely at very low electric fields, which allow more opportunity for carrier diffusion before reaching the electrodes. Shutt et al. [169] have shown that with low trap concentrations complete charge collection is possible in Ge with applied fields as low as $\sim 200 \mathrm{mV} / \mathrm{cm}$, well below those applied to CDMS-II detectors.

Charge carriers may still be trapped during drift if the crystal has a too high density of trapping centers. The trapping rate depends not only on the density of trapping centers but upon their character. Neutral impurity atoms and lattice defects are expected to have trapping cross sections orders of magnitude lower than those of charged centers. Out of this reason CDMS-II detectors are manufactured from highly pure substrate materials. At the low operating temperatures of the CDMS-II detectors donors and acceptors are ionized in equilibrium, providing enough charged traps to affect charge collection even at the high purity achieved in CDMS-II detectors. Fortunately, these and other charged trapping centers can be neutralized by exposure to a strong radioactive source or to infrared LED light [169, 171] . Both processes generate a large population of free charge carriers, and in the absence of an electric field these will quickly recombine. A small fraction of these charge carriers will diffuse to a charged trapping center and bind to it, neutralizing the center and reducing its trapping cross section. This neutralized configuration is not the equilibrium ground state, but at millikelvin temperatures it is stable over the $\sim 11$ hour duty cycle of a low-background data taking period.

The ionization side of the detectors is divided into two separate electrodes: a diskshaped inner electrode ( $80 \%$ of the detector area) and a thin, annular outer electrode (see figure 6.4 for an illustration). These two electrodes are read out separately. The inner electrode defines the fiducial volume in which charge collection is good, and the outer electrode forms a "guard ring" to reject events that may suffer from an incomplete charge collection due to trapping effects at the crystal edges. The charge contacts themselves 


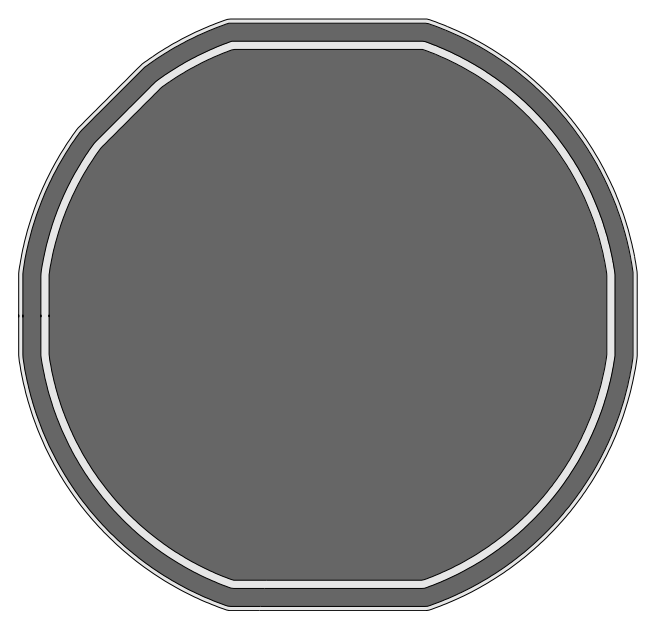

Figure 6.4: ZIP detector ionization side electrode layout.

take the form of $\mathrm{Al}$ grids covering the two detector faces, each separated from the detector bulk by a $\sim 40 \mathrm{~nm}$ layer of amorphous Si. The top electrode is the phonon sensor grid described in Section 6.4, held at electrical ground and covering 32\% of the detector face. The bottom electrode is an $\mathrm{Al}$ grid covering $16 \%$ of the face and connected to a low-noise charge amplifier. The grid coverage is deliberately kept low to avoid unnecessary phonon absorption far from TES sensors. The amorphous Si layer greatly improves the charge collection for near-surface events by blocking the back-diffusion of carriers into the nearby electrode [170].

\subsubsection{The ionization readout}

Each ionization channel is biased through a large resistance, and capacitively coupled to a FET amplifier (see Section 5.2.4). This ensures that the electrodes are well coupled to the readout for fast pulses, and coupled to the bias circuit over longer time periods. The bias and readout are thus decoupled. The ionization circuit is schematically shown in figure 6.5.

The charge bias is coupled to the detector by a large bias resistor $R_{b}=40 \mathrm{k} \Omega$, which does not dissipate much charge over the time scale of a pulse. Each electrode is connected to a coupling capacitor $\mathrm{C}_{\mathrm{c}}=300 \mathrm{pF}$, which passes the fast charge pulse, but keeps the bias and readout circuit relatively floating. Charge collected across the detector crystal quickly transfers to the feedback capacitor $\mathrm{C}_{\mathrm{fb}}$, producing a voltage spike at $\mathrm{V}_{\text {out }}$. This pulse decays as the capacitor drains through the feedback resistor $\mathrm{R}_{f b}$, producing an exponential falling edge of the voltage pulse. The fall-time $\tau_{\text {fall }}=40 \mu \mathrm{s}$ of the pulse is determined by the $R_{\mathrm{fb}}=40 \mathrm{M} \Omega$ and $\mathrm{C}_{\mathrm{fb}}=1 \mathrm{pF}$. Since the actual charge collection process takes less than a microsecond, the ionization pulse shape is solely determined by the electronics; all charge pulses have the same shape. Faster electronics would be able to measure the sub- $\mu$ s structure of the charge pulses enabling a better position reconstruction, but such electronics are not implemented in the current CDMS-II setup. 


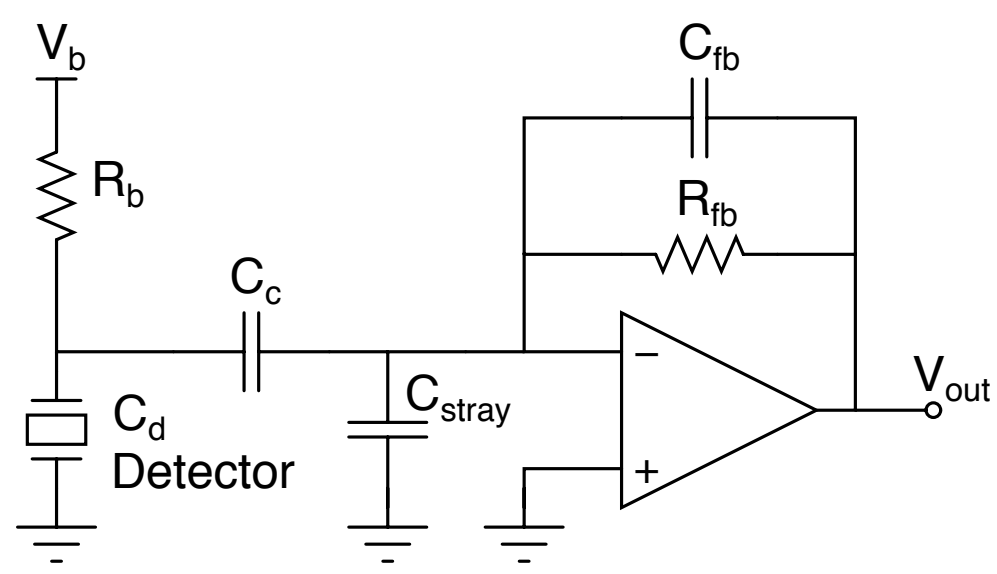

Figure 6.5: Simplified schematic of the CDMS-II ionization readout electronics. The ZIP detector has a capacitance $\mathrm{C}_{\mathrm{d}}=93 \mathrm{pF}$ for the inner electrode ( $36 \mathrm{pF}$ for the outer), and is coupled to the charge amplifier through a coupling capacitor $\mathrm{C}_{\mathrm{c}}=300 \mathrm{pF}$. The induced image charge on the detector surface also appears at the feedback capacitor $\mathrm{C}_{\mathrm{fb}}=1 \mathrm{pF}$. The amplifier adjusts the output voltage to keep zero potential difference between its two inputs. The charge on the feedback capacitor is bled off through the feedback resistor $R_{\mathrm{fb}}=40 \mathrm{k} \Omega$. There are also stray capacitances $\mathrm{C}_{\mathrm{s}}=100 \mathrm{pF}$. The detector is biased with a voltage $\mathrm{V}_{\mathrm{b}}$, through a bias resistor $\mathrm{R}_{\mathrm{b}}=40 \mathrm{k} \Omega$.

The theoretical noise performance of the charge amplifier is dominated by the noise of the amplifiers first stage JFET and by the Johnson noise in the feedback and bias resistor. In practice, there is also a very large contribution from pickup of ambient noise, which has been reduced by the use of an radio-frequency shielded experimental enclosure and a careful grounding scheme. An expression for the total output voltage noise as a function of frequency has been derived by Shutt [171]:

$$
\begin{aligned}
e_{0}^{2} & =|A(f)|^{2}\left\{e_{F E T}^{2}\left[\left(\mathrm{C}_{\mathrm{d}}+\mathrm{C}_{\mathrm{fb}}+\mathrm{C}_{\text {stray }}\right)^{2}(2 \pi f)^{2}+\left(\frac{1}{\mathrm{R}_{\mathrm{fb}}}+\frac{1}{\mathrm{R}_{\mathrm{b}}}\right)^{2}\right]\right. \\
& \left.+4 k_{B} T\left(\frac{1}{\mathrm{R}_{\mathrm{fb}}}+\frac{1}{\mathrm{R}_{\mathrm{b}}}\right)+i_{F E T}^{2}+i_{d}^{2}+i_{\mu}^{2}\right\}
\end{aligned}
$$

In this expression $T$ is the resistor temperature and $A(f)$ is the amplifier resistance

$$
A(f)=\frac{\mathrm{R}_{\mathrm{fb}}}{1+2 \pi i f \mathrm{R}_{\mathrm{fb}} \mathrm{C}_{\mathrm{fb}}} .
$$

Apart from the voltage noise $e_{F E T}$ at the FET input, there are also current noise contributions from the FET input $\left(i_{F E T}\right)$, detector leakage current $\left(i_{d}\right)$ and any microphonic effects on the detector or its wiring $\left(i_{\mu}\right)$.

The resistors are mounted on the detectors side coax board (see Section 5.2.3) at the base temperature stage, giving $T \sim 40 \mathrm{mK}$. The voltage noise at the JFET input has been 


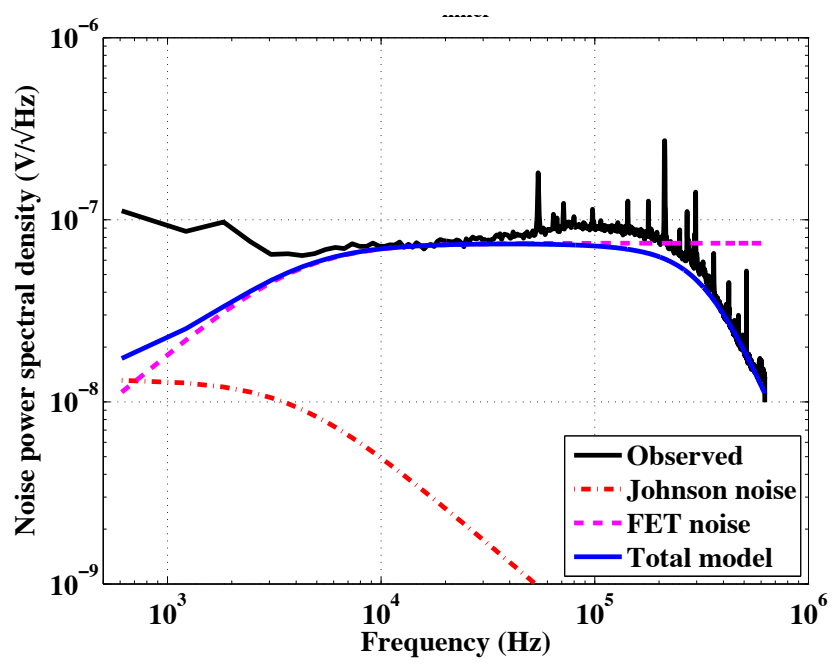

Figure 6.6: Observed ionization noise spectrum overlaid with model predictions following Eq. 6.4. The noise spectrum is rolled off at high frequencies by the warm electronics. A charge pulse has a power spectrum matching the shape of the Johnsons noise contribution. Figure taken from [173].

measured to be typically $0.5 \mathrm{nV} / \sqrt{\mathrm{Hz}}$ at $50 \mathrm{kHz}$ [172]. Figure 6.6] shows a representative noise spectrum with contributions from the model described above. At high frequencies peaks from various electronic noise pick up are clearly visible. The excess at low frequencies can be accounted for by a combination of electronic pickup, microphonic pickup of mechanical vibrations and $1 / f$ noise contributions from the JFET. The power spectrum of a charge pulse matches that of the Johnson noise; note that the JFET noise rolls off within the signal bandwidth, leaving low-frequency noise as the dominant contribution to the experimental resolution.

\subsubsection{Ionization pulse reconstruction}

The primary ionization pulse reconstruction algorithm used by the CDMS collaboration is optimal filtering. The optimal filter algorithm takes advantage of the special characteristics of ZIP ionization signals: each pulse from a ZIP detector is essentially of fixed shape, with time constants fixed by the electronics and predominantly Gaussian noise. The optimal filter is a frequency domain fit of a fixed template to the observed trace, accounting for variations in the noise power spectrum with frequency (for details on optimal filtering I refer the reader to [173, 174]). The templates to which the two ionization pulses are fit for each event are generated by averaging the traces from a selection of well-collected events from each detector. For well designed templates the $\chi^{2}$ returned by the optimal filter algorithm is an excellent goodness-of-fit parameter with which malformed pulses can be rejected. 


\subsection{Athermal Phonons}

When a particle interacts in a ZIP detector, phonons are generated through several distinct processes. These phonons are measured before the detector has had time to come to thermal equilibrium. These out-of-equilibrium phonons are often called "athermal-phonons". The phonon signal not only measures the deposited energy, since the prompt phonon signal retains a great deal of information on the interaction type and location. This additional information which is lost in an equilibrium detector is a vital contribution to the background rejection and event reconstruction of CDMS-II ZIP detectors.

The phonons released by a particle interaction are generated by three distinct mechanisms: primary phonons, recombination phonons and Luke phonons.

\section{Primary phonons}

The initially displaced nucleus or electrons deposit their kinetic energy as phonons at the interaction site as they return to rest. A recoil energy $E_{R}$ deposits $E_{\text {prim }}=E_{R}-$ $N_{Q} E_{g}$ as primary phonons, where $N_{Q}$ is the number of generated electron/hole pairs. This component consist of high energy, low-momentum optical phonons and lower-energy acoustic phonons that come from the residual kinetic energy of the charge carriers. The primary phonons carry information about the position, timing and other characteristics of the particle event.

\section{Recombination phonons}

An energy $E_{g}$ is stored in each electron-hole pair created upon the particle interaction. The charge carriers restore this energy to the phonon system by relaxing to the Fermi level at the detector surface, producing $E_{\text {relax }}=N_{Q} E_{g}$ of recombination phonons.

\section{Neganov-Trofimov-Luke phonons}

Neganov-Trofimov-Luke phonons can be emitted when charge carriers travel faster than the sound speed in the crystal; they are analogous to Cerenkov radiation. The imposed electric field does work on each electron-hole pair. This energy is dissipated to the crystal as phonon radiation. This mechanism proposed by Neganov and Trofimov [175] and by Luke [176], contributes additional energy to the crystal beyond that in the initial interaction. These phonons contribute an energy equal to the fields work on each charge:

$$
E_{\text {Luke }}=\sum_{i} q_{i} \int_{\text {path }_{i}} \vec{E} \cdot d \vec{x}
$$

where the sum is over each charge $i$ and the integral over the path taken by that charge. If the electric field is uniform and the charge carrier has elementary charge $e$ the integral can be written as a sum:

$$
E_{\text {Luke }}=e V_{b} \sum_{i} \frac{d_{i}}{d} .
$$


In the above expression $V_{b}$ is the detector bias, $d$ is the thickness of the crystal and $d_{i}$ is the distance traveled by charge carrier $i$. In case of a complete charge collection each electron-hole pair travels a distance $\mathrm{d}$ and the expression becomes:

$$
E_{\text {Luke }}=e V_{b} N_{Q}
$$

The measured ionization energy in the ZIP detectors is $E_{Q}=N_{Q} \epsilon$. The Luke contribution to the phonon signal can therefore be rewritten as:

$$
E_{\text {Luke }}=\frac{E_{Q} e V_{b}}{\epsilon}
$$

This remains true even when some charges are trapped before reaching the surface, since the measured charge signal is proportional to the number of charges and the Ramo potential that they drift through. When some charges get trapped in the crystal, the loss in the ionization signal is in the same proportion as the loss to Luke phonons. Assuming equal collection rates of all three of these populations the total phonon signal is given by:

$$
E_{P}=E_{\text {prim }}+E_{\text {recomb }}+E_{\text {Luke }}=E_{R}+\frac{E_{Q} e V_{b}}{\epsilon}
$$

The optical phonons have frequency $7-9 \mathrm{THz}$ in Ge, while the primary acoustic phonons have energies of a few $\mathrm{THz}$ [177].

\subsubsection{Phonon propagation}

Phonon propagation across a semiconductor crystal is determined by four processes [178, 179]: phonon-carrier scattering, phonon-phonon scattering, phonon-impurity scattering and an-harmonic phonon decay. Phonon-carrier and phonon-phonon scattering are essentially negligible at milikelvin temperatures, as no free carriers or phonons exist outside of the initial phonon "fireball". Thus the athermal phonons are subject to two types of scattering, both of which become stronger with increasing frequency. Isotope scattering is Rayleigh scattering on isotopic impurities, with a $\nu^{4}$ frequency dependence. For Ge and Si isotope scattering is independent of direction and phonon mode [180], with scattering time [181]

$$
\tau^{-1}=A_{0} \nu^{4}
$$

Reported values of $A_{0}$ are $3.67 \times 10^{-41} \mathrm{~s}^{3}$ for Ge [182] and $2.43 \times 10^{-42} \mathrm{~s}^{3}$ for Si [180]. This corresponds to a mean free path of $\sim 0.15 \mathrm{~mm}$ and $3.5 \mathrm{~mm}$ at $1 \mathrm{THz}$. An-harmonic decay occurs when a higher-energy phonon decays to two lower-energy phonons, each generally carrying away approximately half of the energy. The frequency dependency is $\tau^{-1} \propto \nu^{5}$.

Both processes are strongly frequency-dependent, hence the mean free path of lowfrequency phonons is much longer than that of high-frequency phonons. An-harmonic decay rapidly produces low-frequency phonons from the initial $\mathrm{THz}$ frequency up to a "bottleneck" frequency near $1.6 \mathrm{THz}$ at which isotope scattering begins to dominate. In this regime, the phonons undergo many isotope scatterings and occasional an-harmonic 
decays, so that the motion is a random walk with increasing step size. This mode of propagation is known as "quasi-diffusion". For phonons below about $350 \mathrm{GHz}(\mathrm{Ge})$ or $750 \mathrm{GHz}(\mathrm{Si})$, the path length is as long as the thickness of the crystal, and the phonons can reach the surface without scattering [183. Such low-energy modes propagate ballistically at the speed of sound. These "ballistic" phonons finally travel to the detector surface without significant further scattering.

The observed phonon signals in ZIP detectors are dominated by ballistic phonons which can reach the detector surface. Primary phonon detection is thus delayed by the processes described above: quasi-diffusion followed by ballistic propagation. Luke phonons are expected to be generated at ballistic frequencies. Recombination phonons are emitted at high energy, but at the surface of the detector they down convert rapidly through interaction with the metal films at the detector surface. Therefore recombination phonons are considered as ballistic as well.

\subsection{Phonon collection}

The top surface of each ZIP detector is divided into four phonon sensor quadrants. Each of the four arrays consists of 1036 tungsten (W) transition edge sensors (TES) for collecting and measuring phonons. The TESs are wired in parallel to act as a single quadrant sensor, identified as sensor A, B, C and D respectively. Figure 6.7 shows a schematic of the pattering of phonon sensors across the surface. Each TES is fed by a set of $350 \mu \mathrm{m}$-long aluminum (Al) collector fins. The TESs serve as a very sensitive thermometer and the aluminum fins concentrate the phonon energy from a wide area onto a tiny TES. The sensor element consisting of a TES and associated fins is referred to as a "QET", standing for Quasiparticle-trap-assisted Electrothermal-feedback Transition-edge sensor.

The TES's are configured to detect phonons collected in an array of aluminum fins arranged around each TES. If a phonon has at least twice the superconducting gap energy in Al: $2 \Delta=0.36 \mathrm{meV}$ it can break a Cooper pair in the $\mathrm{Al}$ fins to generate quasiparticles. This minimum energy is much greater than the typical energy $k_{B} T=3.4 \mu \mathrm{eV}$ of thermal phonons at $40 \mathrm{mK}$, thus only energetic phonons far from equilibrium can create Al quasiparticles. As the quasiparticles relax to the gap energy, they can break additional Cooper pairs as well as radiating sub-gap phonons back into the crystal. The quasiparticles diffuse through the Al fins, until they reach the end near the TES. The quasiparticles are collected from the Al fins into the W TES. There is a zone of overlap between the $\mathrm{Al}$ and W, with a superconducting gap smaller than that of $\mathrm{Al}$, but larger than that of $\mathrm{W}$. The minimum quasiparticle energy in the W TES $2 \Delta \simeq 25 \mu \mathrm{eV}$ is much lower than that in the fins. A quasiparticle that diffuses to the TES edge can thus enter the $\mathrm{W}$, but quickly loses enough energy to prevent its return to the $\mathrm{Al}$ fins. The quasiparticle energy in the $\mathrm{Al}$ fins is thus collected in the W TES. The phonon energy from a wide area is concentrated into a TES of low heat capacity, producing a much larger temperature change than would be achieved with the TES alone. The process of this quasiparticle trapping is illustrated in figure 6.8. 


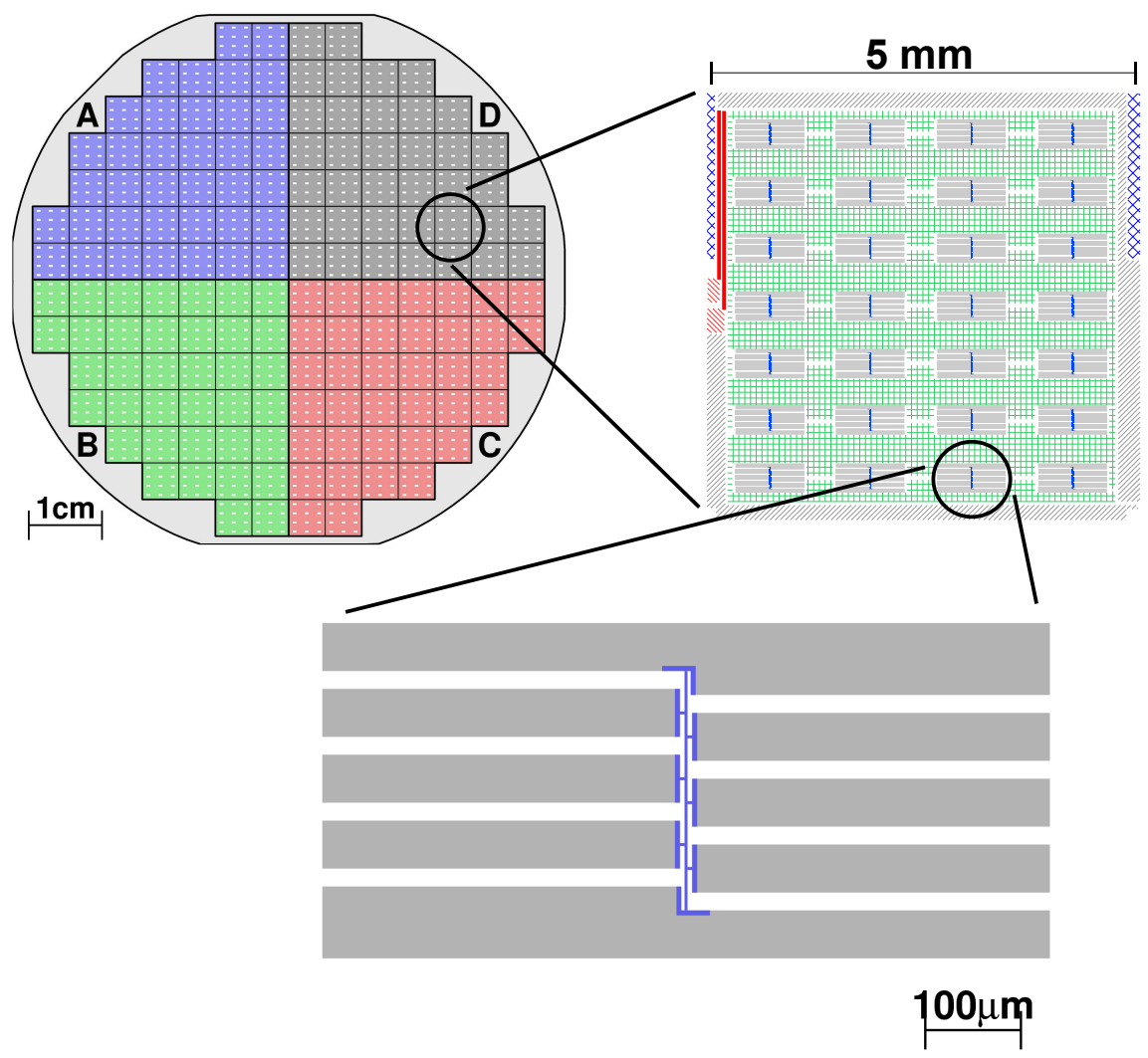

Figure 6.7: Schematic layout of the phonon sensors on a ZIP detector. Top left: Layout of the phonon side, illustrating the four sensor quadrants each consisting of $375 \mathrm{~mm} \mathrm{x}$ $5 \mathrm{~mm}$ dies. Top right: One of the 37 photolithography dies. Each die consists of 28 individual QETs. Bottom: A single QET, showing the $1 \mu \mathrm{m}$-wide W TES connected to $\mathrm{Al}$ collector fins.

\subsubsection{Transition-edge sensors}

A TES is a thin film of superconducting material maintained at its transition temperature $T_{c}$, part-way through its transition from normal to superconductivity conduction. Since the transition is very sharp, a TES is a very sensitive thermometer. A small temperature change of a few $\mathrm{mK}$ causes a macroscopic change in resistance which can be detected with an amplifier.

In CDMS ZIP detectors the crystal lattice of the tungsten film is heat-sunk to the crystal substrate which is cooled to $T_{0} \sim 40 \mathrm{mK}$. The electron system of the tungsten is heated by the Joule power $P_{J}$ of the electric current flowing through the TES, as well as by power $P$ from incoming quasiparticles. At these low energies electron phonon-interactions $\left(G_{e p}\right)$ are very weak, so the link between the two systems (the electron system and the substrate acting as a thermal bath) is very weak $\left(G_{e p} \propto T^{5}, G_{e p}<<G_{0}\right.$, where $G_{0}$ is the link of the phonon system to the thermal bath). In equilibrium the electron temperature $T_{e}$ finds a value such that the heat flow entering from Joule heating $\left(P_{J}=I V=V^{2} / R\right)$ 


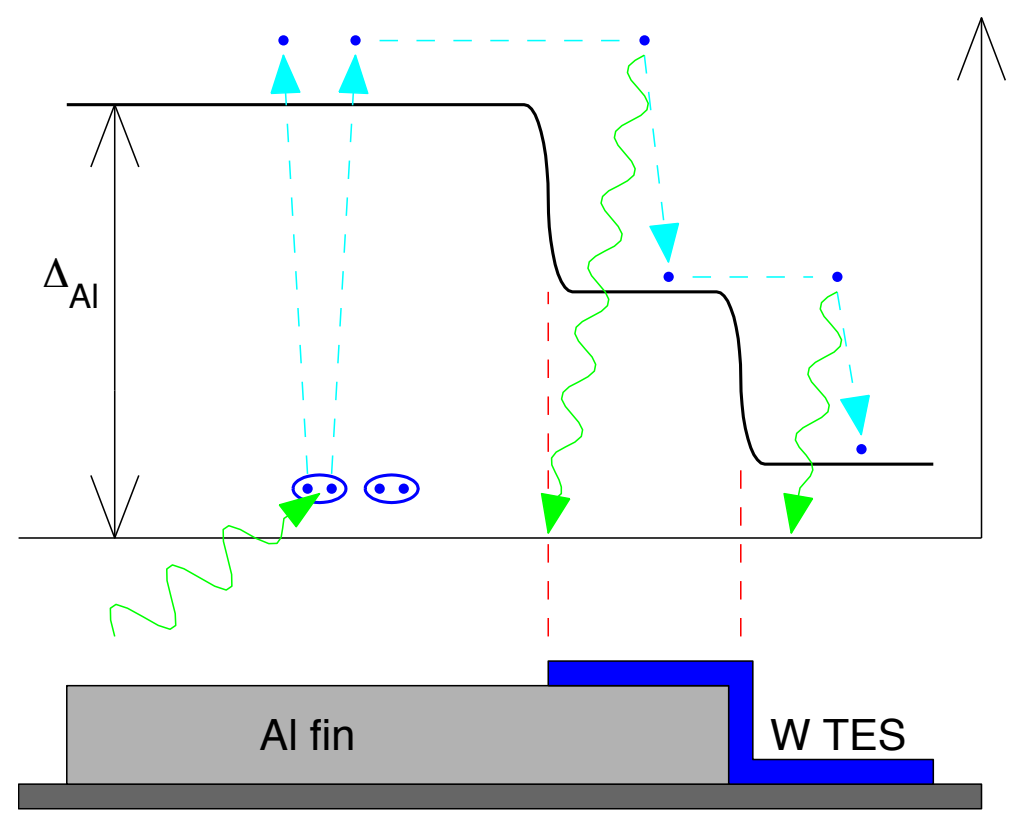

Figure 6.8: A phonon breaks a Cooper pair in the Al fin, creating quasiparticles above the $\mathrm{Al}$ gap. These quasiparticles diffuse to the Al-W overlap area where they lose energy by emitting phonons to relax to the smaller gap. They have no longer enough energy to diffuse back to the Al fin and are trapped. Finally they diffuse to the W TES and contribute to the observed phonon pulse.

and any constant external power matches that flowing away to the bath: $P_{J}+P=P_{e p} \simeq$ $G_{e p}\left(T_{e}-T_{0}\right)$. Since the TES temperature affects its resistance and thus the Joule power, the exact choice of bias point is set by the feedback between this thermal configuration and the bias circuit.

Stable operation can be achieved in a voltage biased configuration with negative electrothermal feedback (ETF) [184. If the bias voltage $V_{b}$ is fixed by the external circuit, any increase in the TES temperature (and thus resistance) leads to a decrease in Joule power. The reduction in Joule power acts as negative feedback, allowing the TES to cool back to its quiescent temperature after an energy deposition. Voltage-biased TESs are stable, and a large number of individual TESs can be biased in parallel with a single bias voltage. A TES with a higher $T_{c}$ will automatically draw a larger current, and one with a lower $T_{c}$ will draw a smaller current, so both are stably maintained within their transitions. This configuration allows TESs to be distributed across the entire detector surface without the complexity of biasing and reading each out separately.

Each TES is a tungsten film $1 \mu \mathrm{m}$ wide, $250 \mu \mathrm{m}$ long and $35 \mathrm{~nm}$ thick. The TES film are deposited by a Balzer sputterer as a mixture of $\alpha$-phase and $\beta$-phase tungsten, target for a transition temperature of $T_{c} \sim 120 \mathrm{mK}$. Following a technique developed by B. Young et al. [185, 186], implanted Fe ions are used to tune the transition temperatures of each sensor to produce a uniform $T_{c}$ near $80 \mathrm{mK}$ across the detector. 


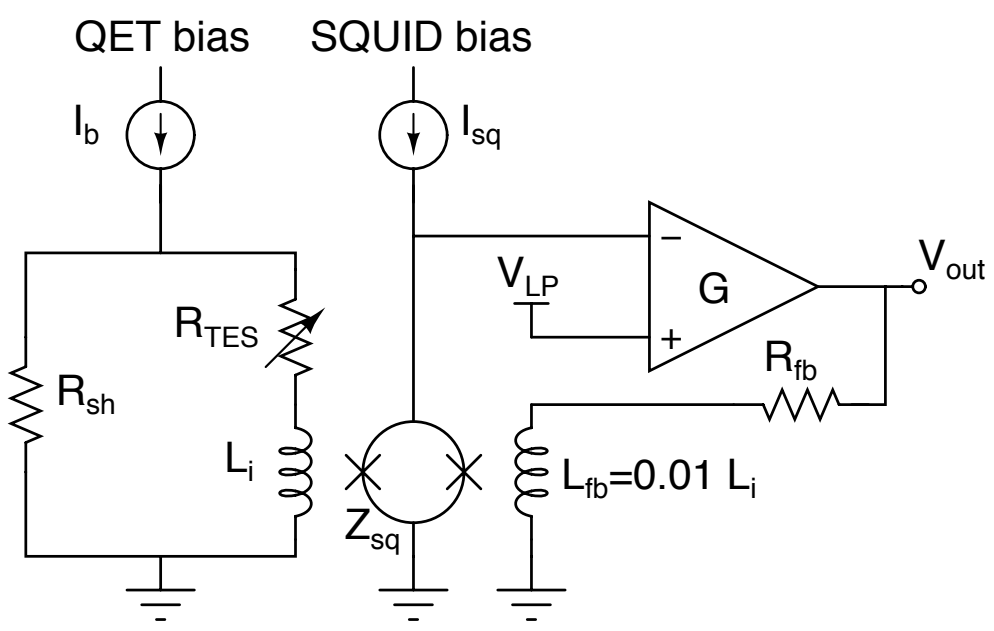

Figure 6.9: Simplified schematic of the phonon channel bias and readout electronics. The TES is shown as a variable resistance $\left(\mathrm{R}_{\mathrm{TES}}\right)$. It is voltage biased by being placed in parallel with a shunt resistor $R_{\text {sh }}=25 \mathrm{~m} \Omega$. This circuit creates stable, negative electrothermal feedback. The input coil $\mathrm{L}_{\mathrm{i}}=250 \mathrm{nH}$ couples the TES current to the readout SQUID. The integrating amplifier $\mathrm{G}$ adjusts its output voltage to cancel the flux through the SQUID. With $\mathrm{L}_{\mathrm{i}} / \mathrm{L}_{\mathrm{fb}}=100$ the resulting amplification is a factor of 10 . The feedback resistor $\mathrm{R}_{\mathrm{fb}}=1200 \mathrm{k} \Omega$ converts the current signal to a voltage.

\subsubsection{SQUID based TES readout}

The readout of the TES sensors must convert a small current through the TES into a voltage that can be digitized. Figure 6.9 shows a simplified schematic of the TES readout. The TES $\left(R_{\text {TES }}\right)$ is kept voltage biased by the bias current $I_{b}$ and the shunt resistor $R_{\text {sh }}$. Any change in the resistance of the TES produces a change in the input coil $\left(\mathrm{L}_{\mathrm{i}}\right)$ current $\Delta \mathrm{L}_{\mathrm{i}}$. The change in current results in a change of magnetic flux through a DC SQUID array $\left(\mathrm{Z}_{\mathrm{sq}}\right)$. The resulting change in SQUID voltage is amplified by an integrating amplifier $(\mathrm{G})$ and fed back to a second feedback coil $\left(\mathrm{L}_{\mathrm{fb}}\right)$. The feedback coil is arranged in negative feedback to keep the flux threading the SQUID constant. The feedback coil has a factor ten less turns as the input coil, so that $\mathrm{L}_{\mathrm{i}} / \mathrm{L}_{\mathrm{fb}}=100$. The amplifier must thus adjust its output voltage such that it drives a current $10 \Delta \mathrm{I}_{\mathrm{i}}$ to cancel the change in the SQUID flux. The feedback resistor $R_{\mathrm{fb}}$ in series with the feedback coil converts the current signal to a voltage, so that $\Delta V_{\text {out }}=\Delta I_{i} \times 10 \times R_{\text {fb }}$.

The largest sources of noise on the phonon signal are the Johnson noise of the TES and other resistive elements in the QET circuit. Thus, it is a good approximation, that the Johnson noise of the shunt resistor (operated at a temperature of $\sim 1 \mathrm{~K}$, rather than the TES at $40 \mathrm{mk}$ ) is the dominant contribution to the current noise in the input coil. The TES contributions to the noise are suppressed relative to this by the lower TES temperatures. 


\subsubsection{Phonon pulse reconstruction}

In CDMS there are two distinct aspects of phonon pulse analysis: energy reconstruction and pulse shape characterization.

\section{Energy reconstruction}

Phonon pulse energies are calculated using an optimal filter algorithm. A start time of the pulse and its amplitude are fit separately for each phonon sensor. Each detector uses a simple template based on a two exponential form. Since the phonon pulse shapes vary substantially with energy and position, while the template is the same, the resulting amplitude estimates have a significant position dependence and energy nonlinearity which must be corrected for.

An alternative method for determining the energy of each phonon pulse is the integral of each trace. This estimate has smaller position and energy dependence, but due to its poorer noise performance it is only used for high-energy events. These integral estimates are not used for any analysis reported in this work.

\section{Pulse shape characterization}

The phonon pulse shape parameters take the form of first-crossing times for particular thresholds. For each phonon pulse two parameters are determined

- The primary phonon rise-time is defined as the difference between the times at which the primary phonon pulse exceeds $10 \%$ and $40 \%$ of its maximum amplitude along its rising edge.

- The primary phonon delay is defined as the delay between the time at which the primary phonon pulse exceeds $20 \%$ of its maximum amplitude along the rising edged and the start time of the ionization pulse.

The crossing times of the phonon pulse are calculated with a time-domain walk algorithm, applied after filtering the pulse with a $50 \mathrm{kHz}$ (for Ge) low-pass Butterworth filter to reduce noise. This walked algorithm is thus vulnerable to noise of the phonon signal. This is generally negligible for the primary quadrant phonon pulses (defined as the quadrant with the greatest phonon pulse amplitude), but it can become a problem for much smaller phonon pulses on the opposite quadrant.

\section{Position reconstruction}

The approximate position of an interaction in the detectors may be reconstructed by comparing the phonon amplitudes and pulse shape parameters among the four phonon quadrants. With these quantities two position-reconstruction techniques can be applied: 
1. Phonon delay reconstruction: The phonons from an interaction should reach the nearest phonon sensors before the farther ones. Based on the relative start times of the largest phonon pulse and its two adjacent neighbors two position- reconstruction parameters are defined. For an event under phonon sensor B, these become:

$$
\begin{aligned}
x d e l & \equiv P B r 20-P C r 20 \\
y d e l & \equiv \text { PAr } 20-P B r 20
\end{aligned}
$$

Where $P * r 20$ (with $*=\mathrm{A}, \mathrm{B}, \mathrm{C}, \mathrm{D}$ ) is the $20 \%$ rising edge point of the phonon pulse. The position manifold produced by these two parameters is show in figure 6.10 (a). The plot shows calibration data taken with a source collimator with eight narrow holes, which can be clearly identified in the position reconstruction.

2. Phonon partition reconstruction: The energy of an event should be shed amongst the four quadrants depending on its position in the detectors. More energy should be deposited in the nearest phonon sensor than in the farther neighboring sensors. Based on the partitioning of the energy among the four quadrants two reconstruction parameters are defined:

$$
\begin{aligned}
\text { xppart } & \equiv \frac{(p c+p d)-(p a+p b)}{(p a+p b+p c+p d)} \\
\text { yppart } & \equiv \frac{(p a+p d)-(p b+p c)}{(p a+p b+p c+p d)}
\end{aligned}
$$

Where $p *$ (with $*=a, b, c, d)$ is the deposited phonon energy in sensor A,B,C and D respectively. These two parameters map the circular ZIP detector to an approximate square, but give the best position resolution due to the excellent amplitude resolution of the optimal filter algorithm. An example of this position manifold is shown in fig. 6.10 (b). The data is the same as in fig 6.10 (a).

These two position reconstruction techniques are a simple and intuitive way of determining the position of an interaction in the detectors. A more sophisticated position reconstruction algorithm has been developed by R.W. Ogburn [166]. This algorithm takes into account more parameters of the phonon pulses which carry a position dependency and reconstructs the position of the events based on an underlying model. For further details on this technique I referee the reader to [166]. The reconstructed position of events using this technique shows a significant improvement in the position resolution. Figure 6.11 (a) shows the reconstruction of events in a specific quadrant; the quadratic structure of the phonon sensors (shown schematically in fig 6.11 (b)) can clearly be identified by the color code. The color code shows the ionization yield which will be defined in the next section. The reduced ionization yield at this specific positions of the detector is a result of floating metal films on T1 and T2 detectors which affects the charge induced on the readout electrodes. These floating metal films have been removed from the detectors in 


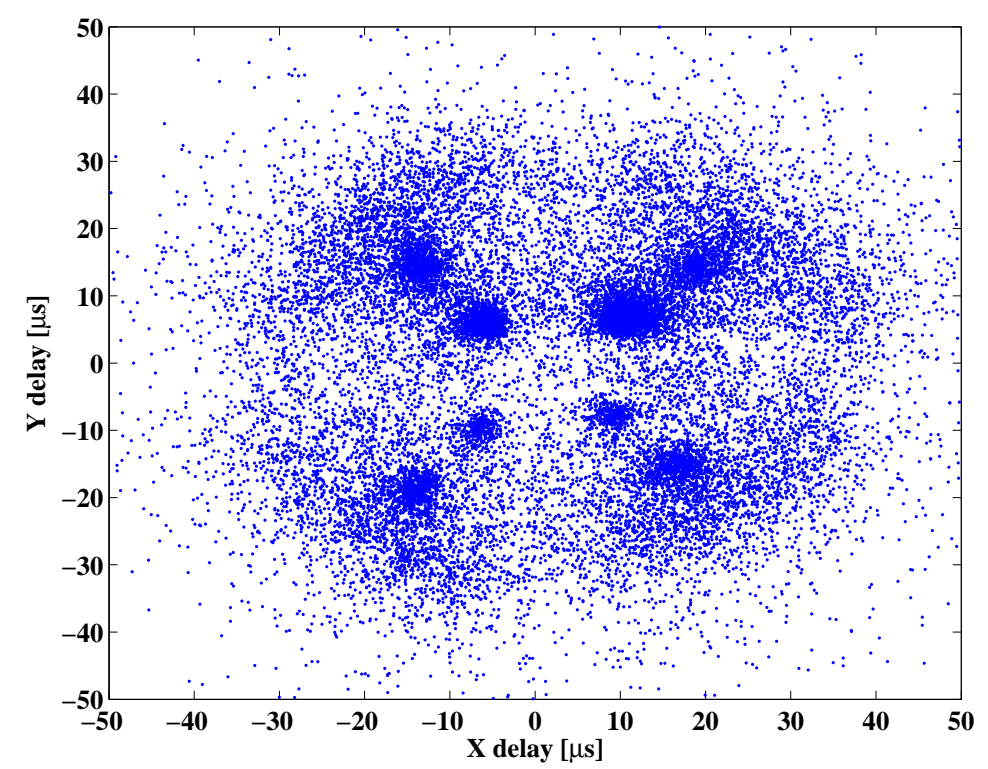

(a)

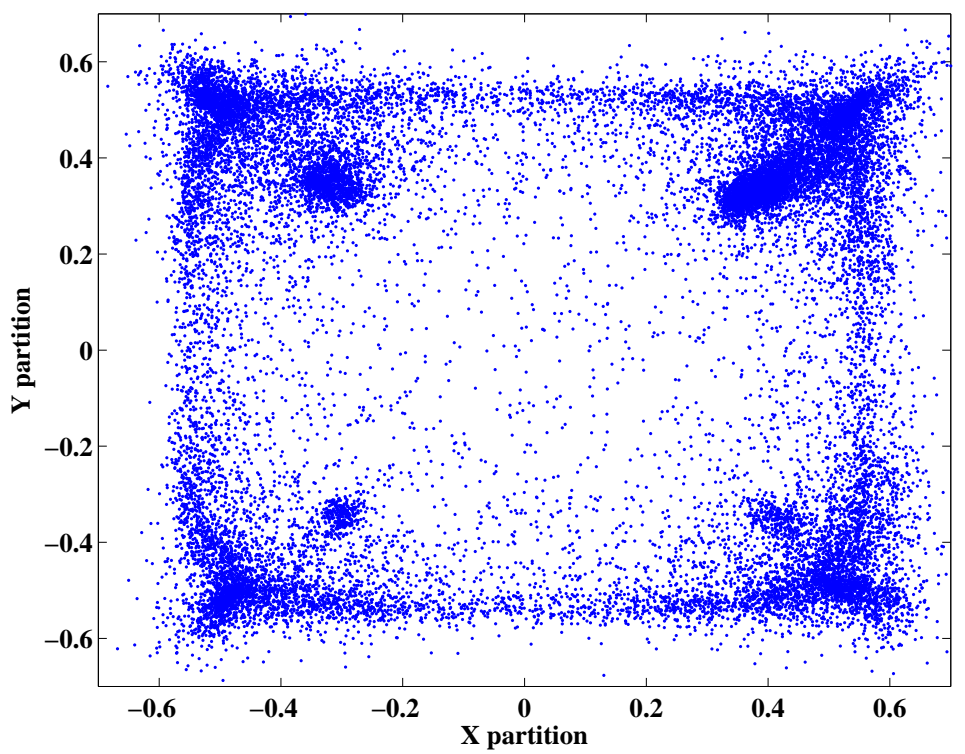

(b)

Figure 6.10: Position reconstruction plots for calibration data from a ${ }^{109} \mathrm{Cd}$ calibration of a ZIP detector, using (a) phonon delay and (b) phonon partition. The darker blobs indicate events from eight narrow holes in the source collimator. 


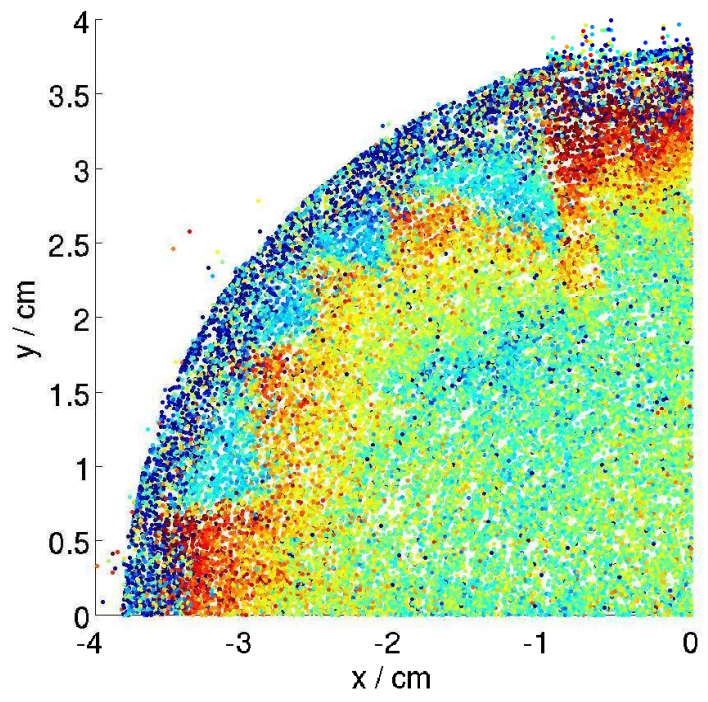

(a)
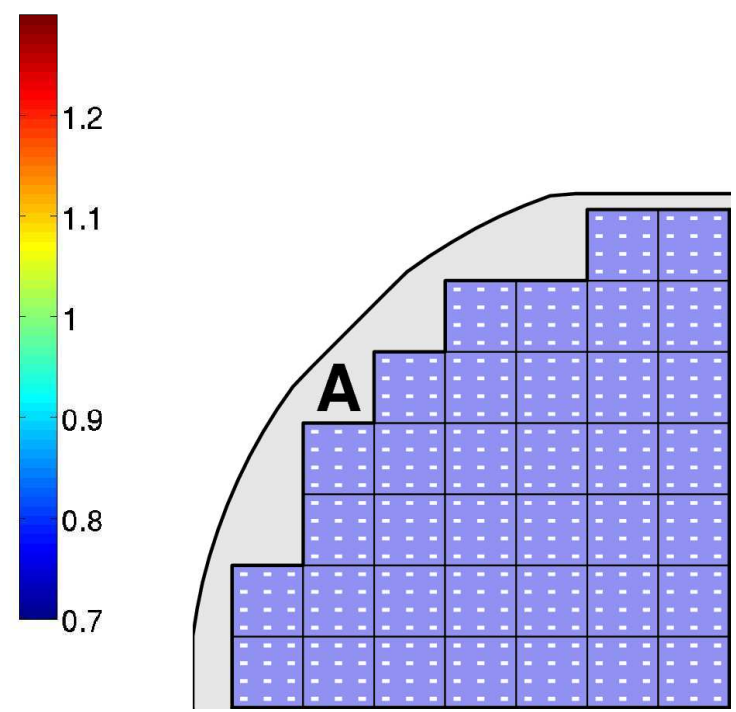

(b)

Figure 6.11: (a) Position reconstruction of events near the edges of the phonon sensor of a quadrant. The color code gives the ionization yield of the events. In the position reconstruction the quadratic layout of the phonon sensors, which is schematically shown in (b) can be clearly identified. Figure (a) taken from [166].

T3 to T5. Although this algorithm shows a better performance in position reconstruction its complexity makes it less favorable to be used in correcting for position dependencies of the phonon pulse parameters. Thus in the position correction of the phonon quantities the simpler position reconstruction techniques mentioned above are used. The position correction of the phonon quantities is discussed in more detail in Section 7.

\subsection{Background discrimination techniques}

The main reason for recording the ionization and phonon signal of an interaction is background discrimination. In the search for rare processes like the detection of dark matter it is mandatory to suppress backgrounds effectively. The main background in underground experiments comes from natural radioactivity resulting in electromagnetic interactions (e.g. scattering on atomic electrons) in the detectors. The signal expected from WIMP interactions on the other hand would be a recoiling nuclei.

A charged particle moving through a crystal lattice loses its energy either by liberating valence electrons or transferring kinetic energy to entire atomic cores. The relative rates for these two channels mainly depend on the particle's mass, charge and kinetic energy. Light charged particles like electrons deposit their energy almost completely in the electron system, while heavier particles like recoiling atomic nuclei can transfer a significant amount of energy to atomic cores. This division of energy has important implications for particle identification. Since recoiling nuclei transfer energy to the crystal lattice their ionization 


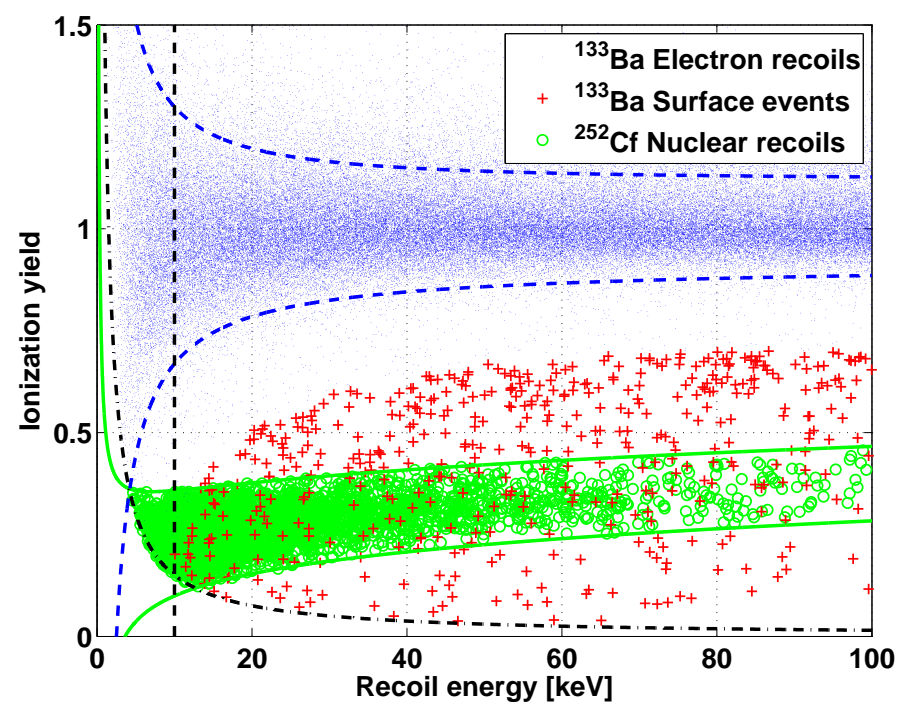

Figure 6.12: Ionization yield as a function of recoil energy for a representative detector. Three classes of events are shown: electron recoil events from a ${ }^{133} \mathrm{Ba}$ source calibration (blue points); nuclear recoils from a ${ }^{252} \mathrm{Cf}$ source calibration (green circles) and a population of electron recoil events with reduced ionization yield from the ${ }^{133} \mathrm{Ba}$ calibration (red crosses). The band show empirical fits to the distribution of electron and nuclear recoils respectively. A charge threshold cut is applied to the data (shown as the black/dash-dotted line). The vertical (black/dashed) line represents a threshold of $10 \mathrm{keV}$.

signal is suppressed with respect to an electron having the same kinetic energy. This effect is used to distinguish electron recoil events from nuclear recoil events.

By comparing the ratio of the ionization and phonon signal for each event a so called ionization yield can be defined:

$$
y \equiv \frac{E_{Q}}{E_{R}}=\frac{E_{Q}}{E_{P}-\frac{e V_{b}}{\epsilon} E_{Q}}
$$

The ionization yield is calibrated such that $y=1$ for electron recoils. Figure 6.12 shows the enormous discrimination power of this parameter. The bands shown in the figure are empirical fits to the distribution of electron and nuclear recoil events which will be discussed further in Chapter 7. The nuclear recoils are clearly separated from the electron recoil events for energies greater than $\sim 5 \mathrm{keV}$. At energies greater than $\sim 10 \mathrm{keV}$ less than one electron recoil in $10^{4}$ leaks into the nuclear recoil region which is defined by neutron calibration data.

The effect of a suppressed ionization signal from nuclear recoils is well understood, and a theoretical model of the energy-dependend ionization yield expected from recoiling nuclei in various crystal has been developed [187]. The model prediction can be parametrized as [76]:

$$
y\left(E_{R}\right)=\frac{k g\left(\epsilon\left(E_{R}\right)\right)}{1+k g\left(\epsilon\left(E_{R}\right)\right)}
$$


where, for a nucleus of atomic number $\mathrm{Z}$ (with a small correction ${ }^{3}$ to the formula given in [76]),

$$
\epsilon\left(E_{R}\right)=11.5 E_{R}(k e V) Z^{-7 / 3}, k=0.133 Z^{2 / 3} A^{-1 / 2}
$$

and the function $g\left(\epsilon\left(E_{R}\right)\right)$ is well described by

$$
g\left(\epsilon\left(E_{R}\right)\right)=3 \epsilon\left(E_{R}\right)^{0.15}+0.7 \epsilon\left(E_{R}\right)^{0.6}+\epsilon\left(E_{R}\right) .
$$

Particle interactions may suffer from a suppressed ionization signal if the interactions occur in the first few microns of the crystal surfaces, this ionization loss is sufficient to misclassify such events as nuclear recoils. These events, referred to as surface-events, can be identified as a third population with reduced ionization yield in figure 6.12. The reason for the suppressed ionization yield is an incomplete charge collection in a "dead-layer" of the crystals, which will be discussed in the next section.

\subsubsection{The dead-layer}

Surface-events can arise from incident low-energy electrons which have a very low penetration depth, or from Compton scattering within a near surface layer of the crystal. From figure 6.12 it is obvious that these surface events are the dominant background for the CDMS experiment. It is thus crucial to understand and reject these surface events at higher and higher exposures.

To understand the origin of the surface events, consider the case of an electron recoil near the detector surface. Half of the charge carriers from this event drift only a short distance, so the ionization signal is determined by the charge carriers which drift across the crystal. In principle the reduced ionization signal from an event interacting near the detector's surface could be caused by at least three mechanisms:

- Enhanced trap density: Due to damage from fabrication processes or chemical reactions with air, the crystal surface could contain a higher density of defects and other trapping sites. These traps could capture charge carriers from near surface events, preventing the drift across the crystal.

- Electrostatic induction: The polarization of a conducting surface by a nearby electric charge results in an attractive force on the charge. In CDMS detectors this force exceeds that of a $3 \mathrm{~V} / \mathrm{cm}$ drift field when an isolated electron approaches within $\sim 100 \mu \mathrm{m}$ of the conduction electrode. This scale is to large for the detectors used in CDMS, since surface events in CDMS are expected to interact in a layer with thickness $\sim 10 \mu \mathrm{m}$. However a realistic model would have to account for the polarization effect of both charge carrier types created very localized upon the interaction, which could drastically reduce the net attractive effect. Nonetheless this effect may affect the trapping and motion of charge carriers near the detector surface.

\footnotetext{
${ }^{3}$ The exponent of $\mathrm{A}$ in the formula for $\mathrm{k}$ has the wrong sign with respect to the original formula given in 187 .
} 
- Back-diffusion: Some charge carriers will reach the detector surface before the bias voltage draws them fully free of the initial potential created by the localized production of charge carriers. In particular a substantial fraction of charge carriers which would drift across the crystal will back-diffuse to the detector surface. A carrier reaching the metal electrode at the surface is expected to relax to the Fermi level almost immediately and not drift across the crystal.

From these three mechanism the back-diffusion is the most dominant source of the deadlayer effect. A work on ionization contacts by Shutt and collaborators [188] showed that an amorphous silicon layer between the bulk crystal and the electrode drastically reduced the occurrence of surface-events, consistent with a blocking of back-diffusion by the large pseudo-band-gap of the amorphous material. Although such layers are deposited on the CDMS detectors a dead layer effect still remains, either due to imperfect blocking or due to the other two mechanisms.

\subsubsection{Face dependency of surface events}

Extensive calibration studies of ZIP detectors showed that the occurrence of surface-events is different for the charge and phonon face of the detectors. In these studies the detectors phonon and charge face where illuminated with collimated ${ }^{109} \mathrm{Cd}$ sources (figure 6.10 shows the position of the sources on the detector). This data revealed a strong asymmetry between surface events on the two detector faces; the ionization yield of surface events on the phonon face is greatly reduced with respect to the charge face. For a more detailed description of this analysis I refer the reader to [172.

In the current setup at Soudan it is also possible to see this effect by selecting nearest neighbor double scatter events (these are events in which two adjacent detectors recorded a energy deposition). This event topology defines the face on which the event occurred. Figure 6.13 shows the yield distribution of surface events from ${ }^{133} \mathrm{Ba}$ calibration tagged by side. The origin of this phenomenon is not fully understood. Analysis of low energy $\mathrm{X}$-ray photons to infer the dependency of the ionization yield upon depth confirmed that the effect of the dead-layer is much bigger on the phonon face than on the charge face.

This effect has important implications for background from surface-events in the nuclear recoil band. The vast majority of surface events which could mimic nuclear recoils occur on the phonon face and only a small fraction on the charge face. Interestingly it turns out that the rejection of these surface-events using the phonon pulse shape information, is much more efficient for events on the phonon face than the charge face (this topic will be discussed further in Chapter 9). 


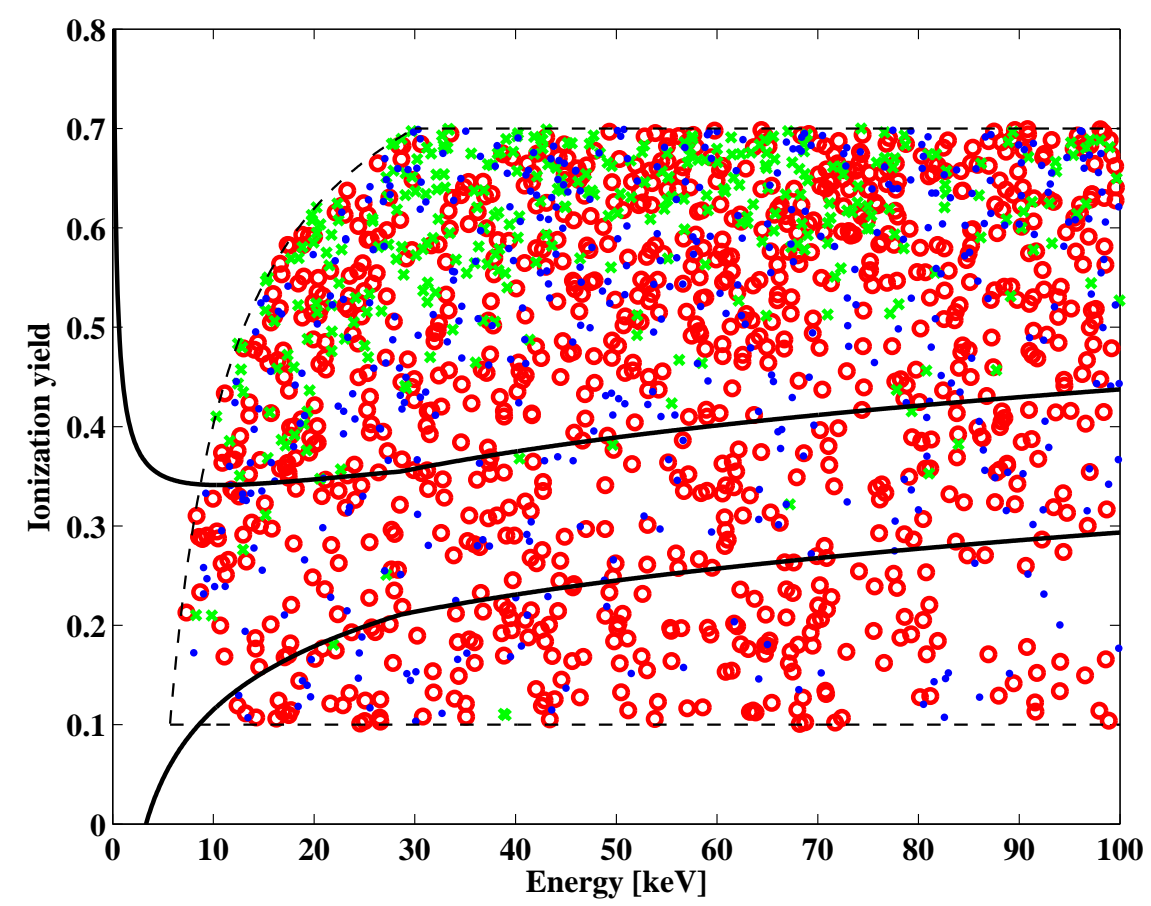

Figure 6.13: Distribution of surface-events from ${ }^{133} \mathrm{Ba}$ of a representative detector. Three event topologies are shown; charge face events (green crosses); phonon face events (red circles) and events to which no face can be assigned (blue dots). The black solid line indicates the $2 \sigma$ nuclear recoil band. Inside the nuclear recoil band many fewer charge face surface events appear than phonon face surface events. The dashed black line defines the selection of surface-events defined by several bounds. 


\section{Chapter 7}

\section{Calibration of the CDMS detectors}

In a search for very rare events at low energies it is mandatory to understand and characterize the detectors. For the CDMS ZIP detectors (described in chapter 6), the ionization and phonon energy scales are calibrated using the spectral lines of a ${ }^{133} \mathrm{Ba}$ source. The calibration sources can be inserted into in the experimental setup through two feedthroughs along the C-stem and the E-stem, such that the sources reside just outside of the icebox copper cans. In addition to the energy calibration, the detector response shows a position dependence with event location. This can be caused by possible manufacturing issues and geometrical properties of the crystals. For a uniform response of the detectors these position dependencies have to be corrected. In addition the phonon pulse parameters show an energy dependence related to the phonon readout which has to be corrected for as well.

For purposes of background rejection and expected signal region, the distributions of electron and nuclear recoils in the ionization yield parameter have to be defined. The ionization yield distribution of nuclear recoils is defined by the use of a ${ }^{252} \mathrm{Cf}$ neutron source. Since WIMPs are expected to give nuclear recoils, the distribution of the nuclear recoils from the calibration defines the expected signal region.

\subsection{Ionization signal}

The calibration of the ionization signal is performed on data taken during calibration runs with a ${ }^{133} \mathrm{Ba}$ source. This source has three high energy spectral lines at $302.853 \mathrm{keV}$, $356.017 \mathrm{keV}$ and $383.851 \mathrm{keV}$ which can be used for the calibration of the energy deposition. Higher energy lines are necessary such that the photons can penetrate the copper of the icebox cans. As described in section 6.2.3 the amplitude of the ionization pulses is determined by an optimal filter algorithm. The reconstructed quantity is called "OFvolts". Before these values can be calibrated to a physical meaningful energy deposition, a correction due to a position dependence of the ionization signal has to be applied. This position dependency is only of importance for the inner electrode, since the outer electrode is dominantly used as a veto electrode which does not directly affect the result of any analysis based on events in the fiducial volume, defined by the inner electrode. 


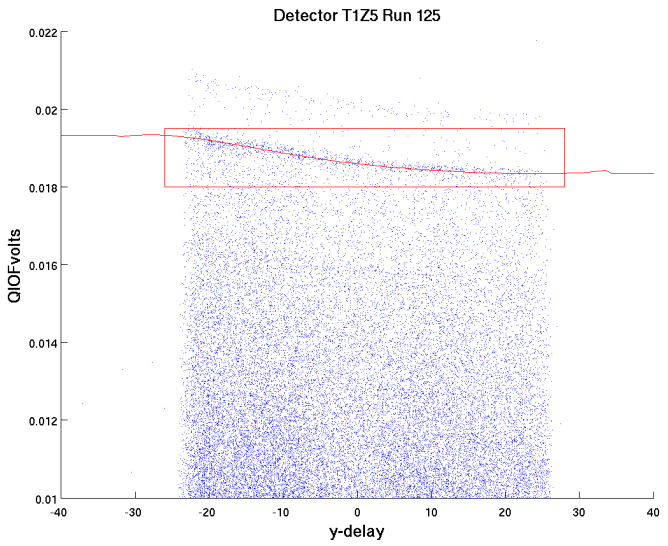

(a)

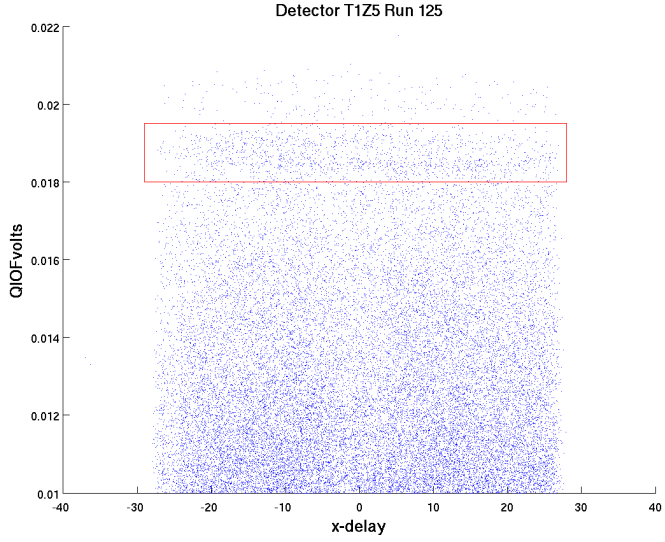

(b)

Figure 7.1: Position dependence of the optimal filter amplitudes (QiOFvolts, where Qi denotes the inner-electrode). Dependency on the y-direction and $\mathrm{x}$-direction are shown in (a) and (b) respectively. The red box indicates the population of events used for the correction of the position dependence which is modeled by a polynomial fit to the selected events, shown as the red line in figure (a). Figures provided by K. Sundqvist.

\subsubsection{Position dependence}

The amplitude of the ionization signal shows a dependency on the position of the interaction in the crystal, as shown in figure 7.1. This dependency is more pronounced in the ydirection of the crystals than in the x-direction. The position dependence is not understood quantitatively, but may result from a known y-dependence of the TES ion implantation or neutralization effects due to varying distance from the LEDs. The position reconstruction is performed by using the delay variable of the phonon signals discussed in Section 6.4.3,

To ensure a uniform calibration of the ionization signal throughout the detector volume this dependency has to be corrected. The correction is performed by selecting events which correspond to the $356 \mathrm{keV}$ line. To select these events, the second highest population of events in the reconstructed amplitude is taken. The selected region is shown as a red box in figure 7.1 (a). The distribution of events within the box are fitted to a polynomial as a function of the y-direction. The resulting fit is also shown as a red line in figure 7.1 (a). A correction factor is determined from the ratio of the polynomial value to a straight line. Each event is then multiplied by this correction factor to correct for the position dependence. After the correction of the y-direction dependence the same procedure is applied to the events as a function of the x-direction. Figure 7.1 (b) shows the selection of events (red box in the figure) as a function of the x-direction from which the correction factor is determined. The position dependency is not that strong anymore since most of the dependency has been corrected for in the y-direction.

Figure 7.2 shows the corrected amplitudes (which in the figure have already been calibrated for an energy deposition, see next Section) as a function of the y-direction (a) and 


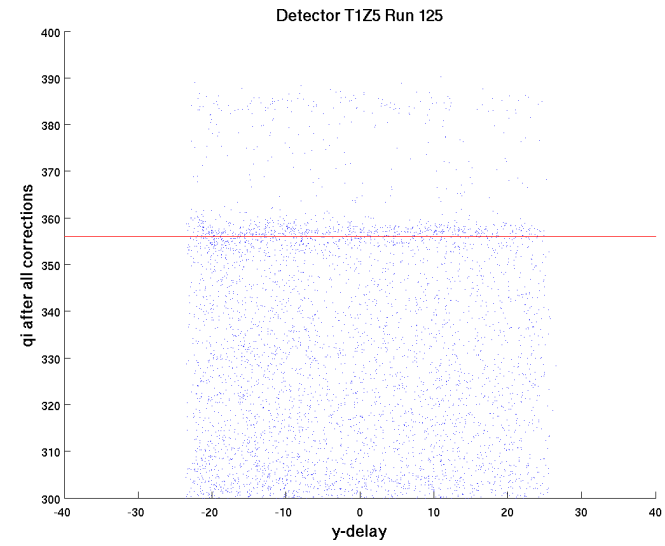

(a)

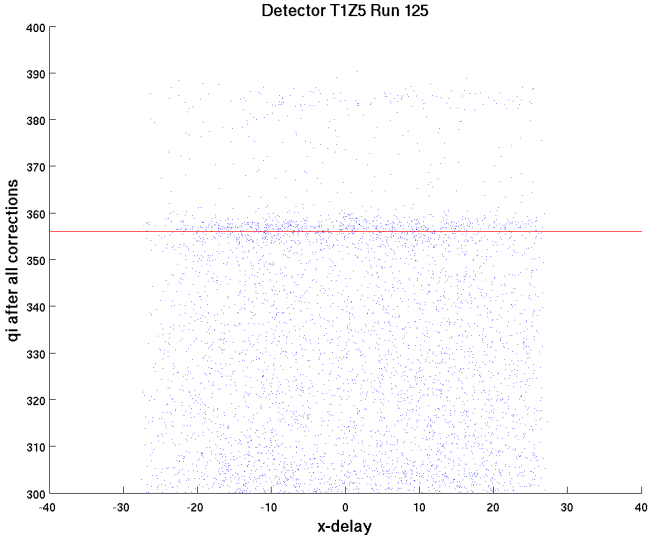

(b)

Figure 7.2: Position corrected ionization signal. The optimal filter amplitudes have been scaled to the energy in the inner electrode (denoted as qi in the figures). The correction results in a uniform distribution of the $356 \mathrm{keV}$ line along the $\mathrm{y}$-direction and $\mathrm{x}$ direction, shown in figure (a) and (b) respectively. Figures provided by K. Sundqvist.

$\mathrm{x}$-direction (b). As intended the amplitudes now have a uniform distribution as a function of direction. The density of events also shows a position dependence (there are more events at negative ydel (see Section 6.4.3) values than at positive), this is caused by a geometrical effect of the source position. The sources are positioned on two sides of the experimental setup to reduce the self-shielding effects of the detectors. However, there are remaining shielding effects so that the detectors are not uniformly illuminated, resulting in a position dependence of the event count. Since calibrations are taken regularly throughout the data taking, providing high calibration statistics on each detector, this dependence is of no concern for the calibration of the detectors.

\subsubsection{Energy calibration}

The overall calibration of the ionization signal is achieved by scaling the OFvolts values of the higher energy lines, dominantly the $356 \mathrm{keV}$ line, to the known energies. Figure 7.3)(a) below shows the comparison of the calibrated spectrum from ${ }^{133} \mathrm{Ba}$ calibration with a Monte Carlo simulation of the calibration run. The calibrated spectrum matches very well with the spectral lines and the Monte Carlo simulation.

The energy calibration of the outer electrode generally differs from the calibration of the inner electrode by a few percent. Not many of the $356 \mathrm{keV}$ events deposit their energy solely in the outer electrode thus the spectral lines are not so prominent in the outer electrode. To account for this discrepancy a small population of events shared between the two electrodes is used. Due to the low statistics, the calibration of the outer electrode is less precise than for the inner electrode, but since the outer electrode is mainly used as a charge veto the calibration is adequate. 


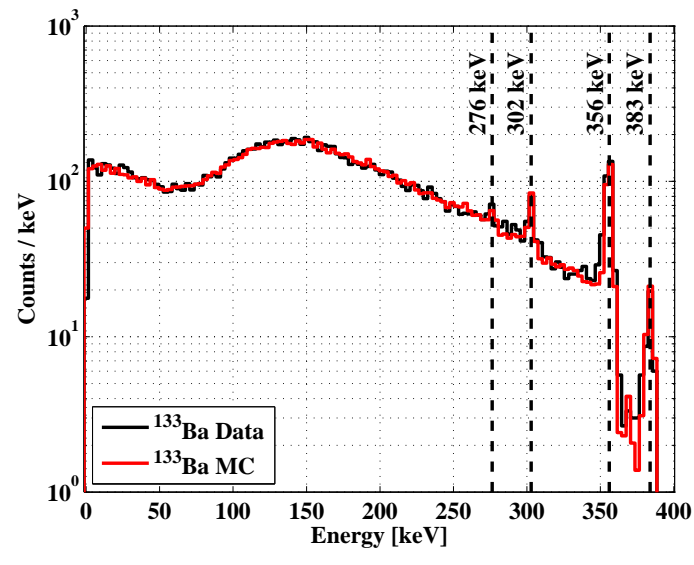

(a)

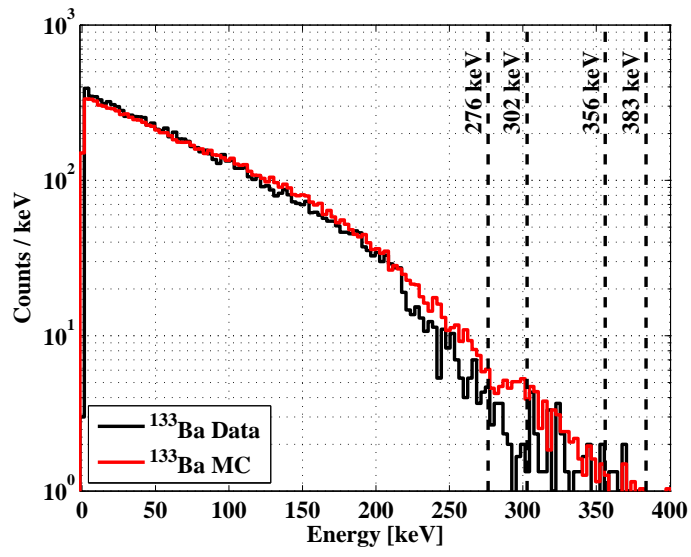

(b)

Figure 7.3: Comparison of a ${ }^{133} \mathrm{Ba}$ energy spectrum using calibrated ionization energies with a Monte Carlo simulation. The calibrated spectrum matches very well with the simulated spectrum for germanium detectors (a) as well as for silicon detectors (b). The high energetic lines are not visible in silicon due to the materials lower stopping power.

\subsubsection{Silicon detectors}

Due to the lower stopping power of silicon the energetic ${ }^{133} \mathrm{Ba}$ lines are not always visible in silicon detectors. Thus a more indirect calibration method is used. The calibration of the inner electrode for Si detectors uses $356 \mathrm{keV}$ events shared with neighboring detectors. This calibration scheme works out very well if the neighboring detector is a germanium detector, but works adequately for T2Z1 which is the only silicon detector without a neighboring germanium detector. In the absence of a charge line the calibrations of the inner and outer electrode are assumed to be equal and no charge position correction is performed. Figure 7.3 (b) shows a comparison of the calibrated ${ }^{133} \mathrm{Ba}$ spectrum in a silicon detector with a Monte Carlo simulation of the calibration run.

\subsection{Phonon signal}

The phonon amplitudes derived from the optimal filter algorithm are calibrated against the ionization signal. In a first step an overall calibration factor from ${ }^{133} \mathrm{Ba}$ calibration photons is determined such that the sum of the energy deposition in all four phonon sensors matches the ionization signal. The calibration factor is chosen based upon a $\chi^{2}$-minimization of a large event sample to avoid event by event bias. Although the phonon amplitudes contain a Luke phonon contribution this calibration defines an ionization yield parameter of 1 (equal energy in the phonon signal and ionization signal) for bulk photons. The calibrated phonon signal is shown in figure 7.4 for a representative detector.

Each of the four phonon sensors shows a slightly different phonon response for a given 


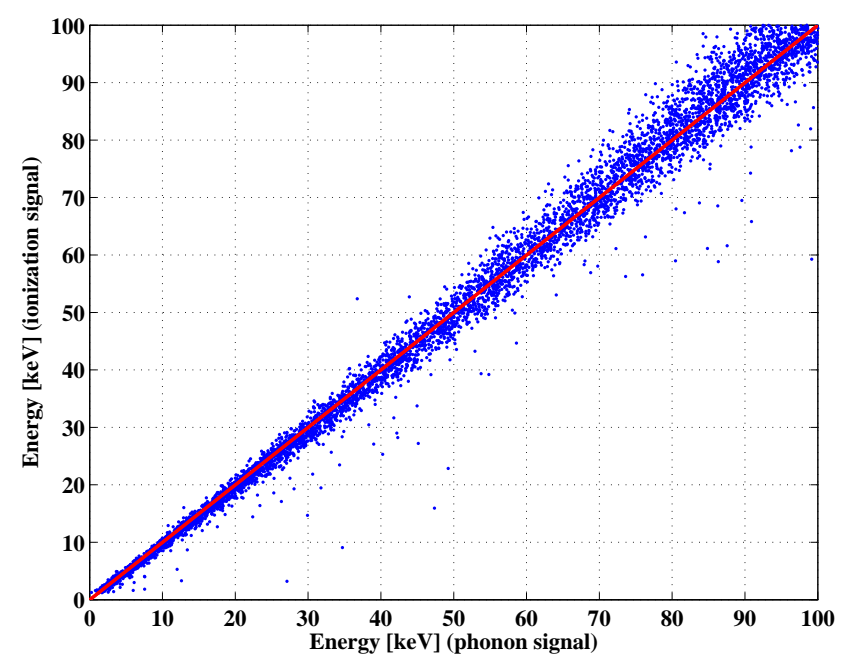

Figure 7.4: Calibration of the phonon signal to the ionization signal. The sum of the four phonon signals is calibrated such that the energy of the phonon signal matches the energy of the ionization signal. A large sample of photons from ${ }^{133} \mathrm{Ba}$ calibration data is used to determine the overall calibration factor.

event energy and location in the quadrant. To ensure a homogeneous response of the detector a relative calibration of the four sensors is performed. This calibration is chosen such that the phonon partition distributions (energy of a given phonon sensor divided by the total phonon energy) are aligned for all four quadrants. To ensure that the overall calibration factor is maintained these relative calibration factors are constrained to sum to a total value of 4 . The effect of this relative calibration can be seen in figure 7.5 (a) without the relative calibration the position reconstruction based on the partition of energy shows some slight offset and distortion at the edges. After the cross-calibration of the four phonon sensors the position reconstruction shows a uniform behavior for all four quadrants as can bee seen in figure 7.5 (b).

To correctly reconstruct the total phonon signal from these calibrations the calibrated phonon energy is multiplied by the Luke factor $\left(1+V_{b} e / \epsilon\right)$ derived from equation (6.10). The final recoil energy is constructed on an event by event basis from the total phonon signal and the ionization signal as given by equation (6.10). The comparison of the ${ }^{133} \mathrm{Ba}$ calibration spectra from a germanium detector with a Monte Carlo simulation shown in figure 7.6 validates the correct calibration of the phonon signals. The resolution of the phonon signal at high energies is worse than that of the ionization signal, since the TESs start saturating for high energy depositions. At lower energies the phonon signal resolution is comparable to the ionization signal, but always remains worse due to the applied Luke correction, transferring noise from the ionization signal to the phonon signal. 


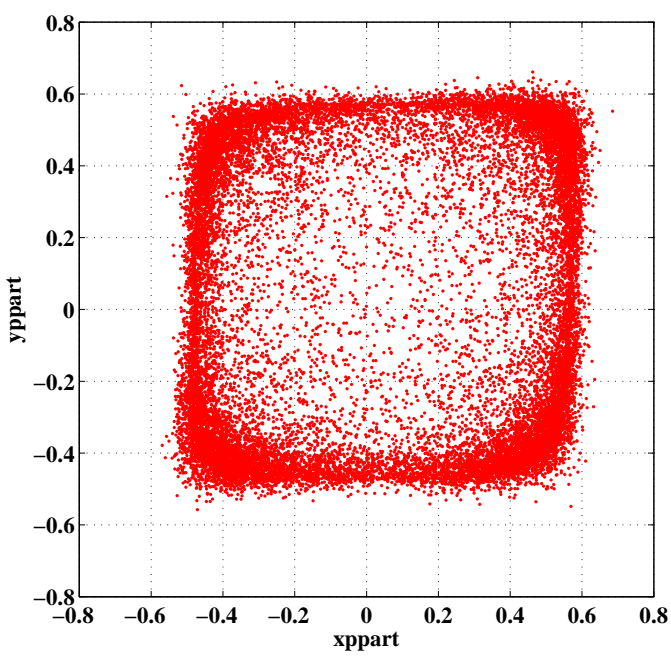

(a)

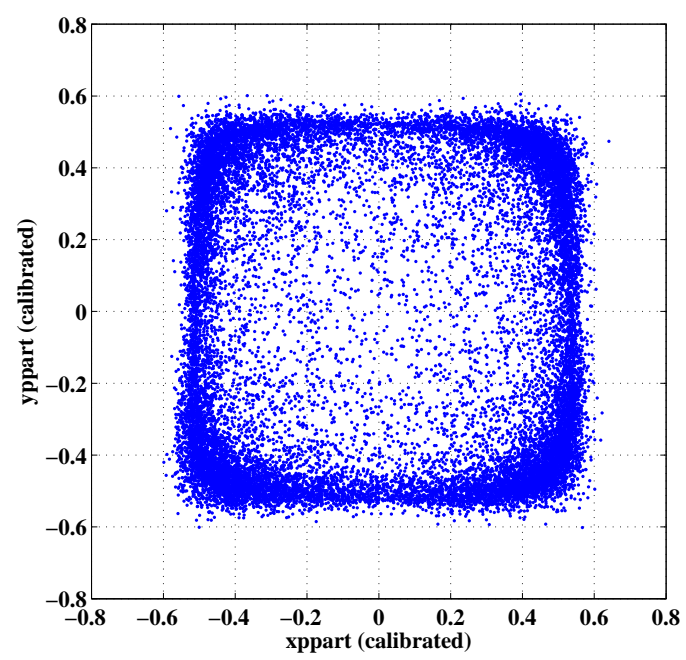

(b)

Figure 7.5: Position reconstruction based on phonon energy partition (see Section 6.4.3). The position reconstruction from non calibrated phonon sensors (a) shows some irregularities resulting from different responses of the four sensors depending on the event's location in the quadrant. Using a cross-calibration of the four sensors the position reconstruction shows a uniform behavior for all four quadrants (b).

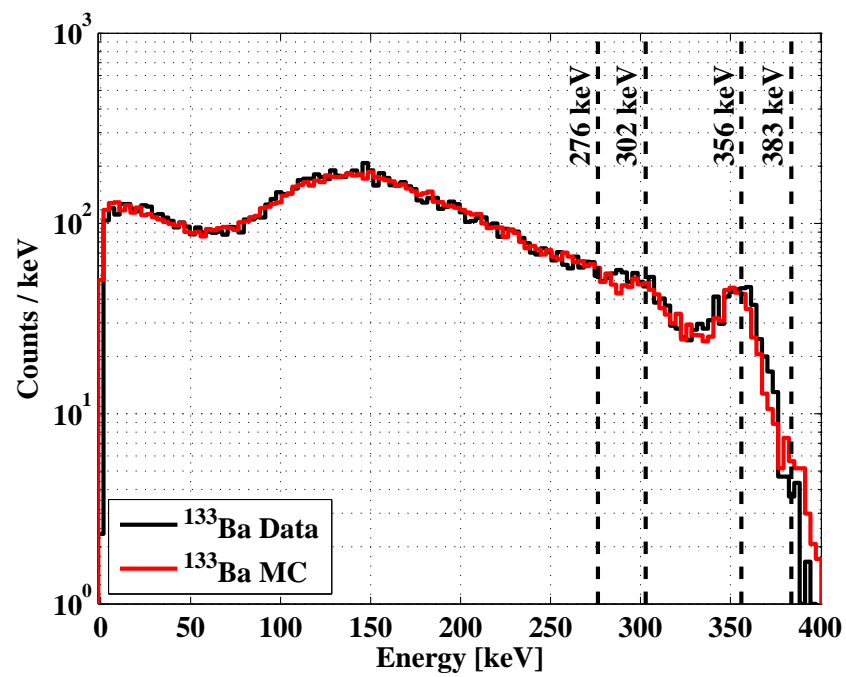

Figure 7.6: Comparison of a ${ }^{133} \mathrm{Ba}$ energy spectrum in a germanium detector using calibrated phonon energies with a Monte Carlo simulation. The resolution of the high energies lines is not as good as for the ionization signal, but the Monte Carlo spectrum matches the measured spectrum very well. 


\subsection{Energy and Position correction}

The phonon signal strongly varies with position and energy of an event. For a homogeneous response of the detectors, these variations have to be corrected for. All phonon pulse parameters and their dependencies refer to the primary quadrant phonon pulse which is defined as the one having the highest amplitude. After the discussion of the actual dependencies and how the position correction scheme minimizes these dependencies the actual position correction algorithm will be discussed.

\subsubsection{Energy dependence}

The delay and rise-time of the phonon pulses become slower with increasing energy like it can be seen in figure 7.7(a) for the primary quadrant delay and in figure 7.8(a) for the primary quadrant rise-time. The means of the distributions have been calculated in $5 \mathrm{keV}$ energy bins, shown as the red line in the figures to track the energy dependence. As the energy of an event increases, more and more TESs which are very near the event location begin to saturate. Due to their close proximity to the event location these TESs also have the fastest response. Thus, at high energies the composite TES signal for a quadrant is reweighed by saturation. The phonon pulse flattens and peaks later in time since the TESs further away from the event location have a significantly retarded response. The net effect is that timing parameters become slower as the event increases in energy. After the correction of the data, this energy dependence is minimized and the pulse shape quantities are nearly independent of the events energy, as can bee seen in figure 7.7 (b) for the delay and in figure 7.8 (b) for the rise-time. In the figures the means of the distributions have been calculated in energy bins of $5 \mathrm{keV}$ to track the energy dependence (shown as the green line in the figures). For comparison the line from the uncorrected data (shown in red) is also plotted in the figures. The correction of the data minimizes the energy dependence of the phonon pulse shape parameters and significantly improves the resolution.

\subsubsection{Position dependence}

Due to the propagation time of the phonons in the crystal the pulse shape parameters vary with the location of the event. As would be expected, events under a quadrants center are the quickest. As one moves towards the very center of the detector or near the edge of the detector the timing parameters are slower. To create a uniform response of the detector which does not depend on the events location this variation must be corrected for.

Neither the partition nor the delay parameters defined in section 6.4.3 are perfect for reconstructing the event location because both parameters are double valued. As the location of a particle interaction moves radially outward, a larger percentage of phonons are absorbed after bouncing off of the detector's cylindrical side wall. This physical process manifests itself by folding back the timing and energy radial estimates. However, it is possible to construct a monotonic event location using a combination of the delays and partition. In particular a 3-dimensional position coordinate using xppart, yppart and the 


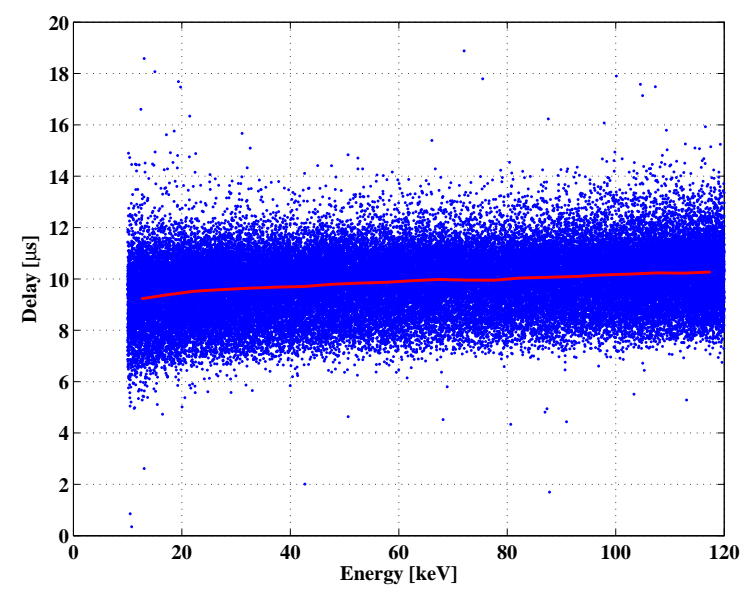

(a)

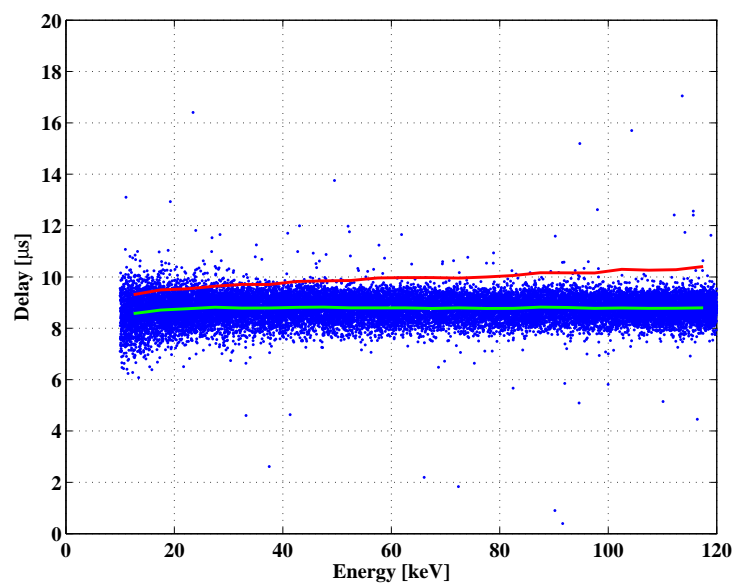

(b)

Figure 7.7: Energy dependence of the phonon pulse delay from ${ }^{133} \mathrm{Ba}$ calibration data. (a) The rise in the delay with energy is shown by the red line in the plot which tracks the mean of the delay distribution in $5 \mathrm{keV}$ bins. (b) Corrected data set with significantly reduced energy dependence and a much better resolution. The energy dependence is tracked by the green line in the plot, for comparison the uncorrected trend is also shown in the figure (red line). A software threshold of $10 \mathrm{keV}$ is imposed on the data.

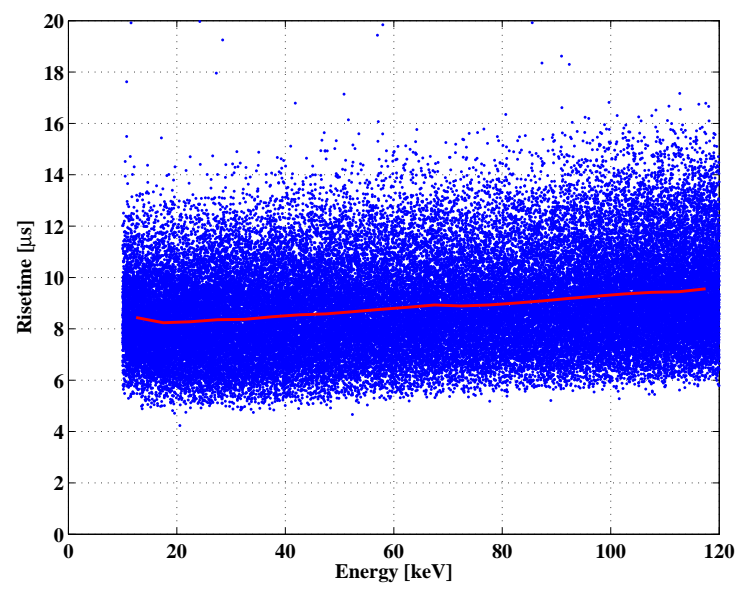

(a)

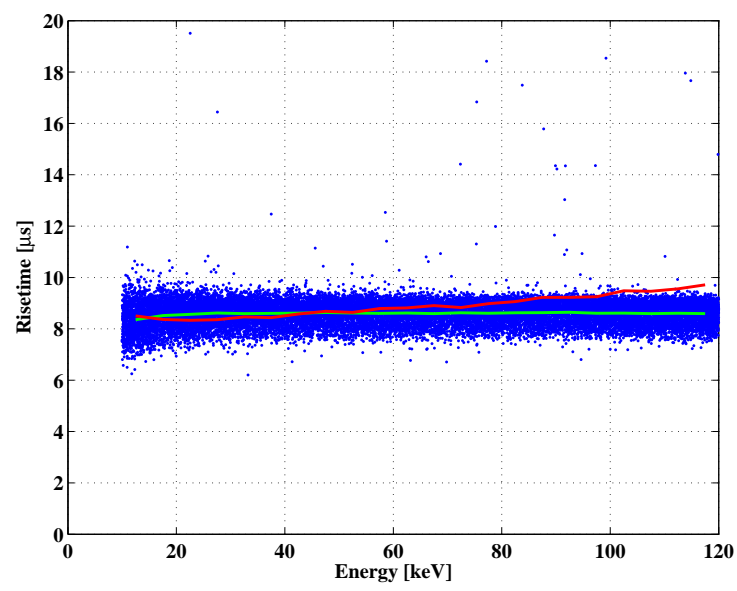

(b)

Figure 7.8: Energy dependence of the phonon pulse rise-time from ${ }^{133} \mathrm{Ba}$ calibration data. (a) The rise-time becomes slower with increasing energy, for illustration the red line tracks the mean of the distribution in $5 \mathrm{keV}$ bins. (b) Corrected data set as a function of energy. The mean of the distribution is tracked by the green line, for comparison the trend for the uncorrected data (red line) is shown. In addition the resolution is improved significantly. A software threshold of $10 \mathrm{keV}$ is imposed on the data. 


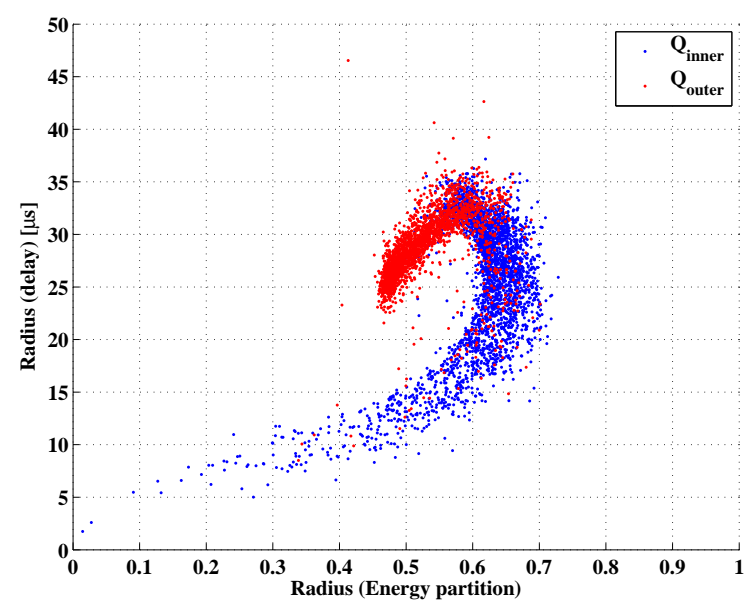

Figure 7.9: Slice of the 3-dimensional position manifold. Events under the inner-electrode (blue) and outer-electrode (red) at the edge of the detector are shown. This manifold breaks the degeneracy of the individual parameters for events at high radius.

delay radius $\left(r d e l=\sqrt{x d e l^{2}+y d e l^{2}}\right.$, see Section 6.4.3) can be constructed. This breaks most of the degeneracies in the reconstructed position. Some degeneracies can however still be observed at high radius. Figure 7.9 shows a slice from the 3-dimensional position coordinate in which polar coordinates have been used for the partition parameters. The figure shows the position of events under the central inner electrode and the outer electrode at the edge of the detectors. The fold-back for the individual parameters for events at high radius can clearly be seen and how the chosen position coordinates break this degeneracy.

The variation of the phonon pulse shape parameters with position in the manifold can be seen in figures 7.10 and 7.11 (a), colored by the respective pulse shape parameter. The data shown is the same data set from ${ }^{133} \mathrm{Ba}$ calibration data in an energy range from 10 $120 \mathrm{keV}$ used for figures 7.7 and 7.8. Events at large and small radius are considerably slower than those in the central parts of the detector. The spread in timing is far greater than the energy dependence of the pulse shape parameters. After the application of the position correction the response of the detector is homogenized throughout the volume, such that the pulse shape parameters do not show a strong position dependence. The corrected pulse shape parameters are shown in figure 7.10 and 7.11 (b) respectively.

\subsubsection{Phonon correction table}

These position- and energy-dependence is reduced by a phonon correction table. The basic principle of this method is to compare each events phonon parameter values against a look-up table describing how the mean of these parameters vary with event location and energy. The "Look-up Table Correction" homogenizes the detector's response throughout the entire crystal by comparing an event's parameter to those of its "nearest neighbors".

The look-up table of correction factors is derived empirically from the large sample of photon events from ${ }^{133} \mathrm{Ba}$ calibration runs. A separated table is defined for each ZIP 


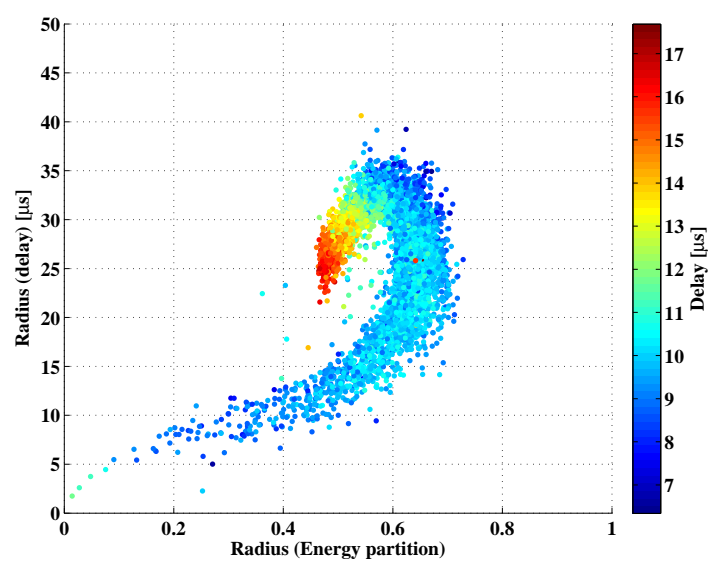

(a)

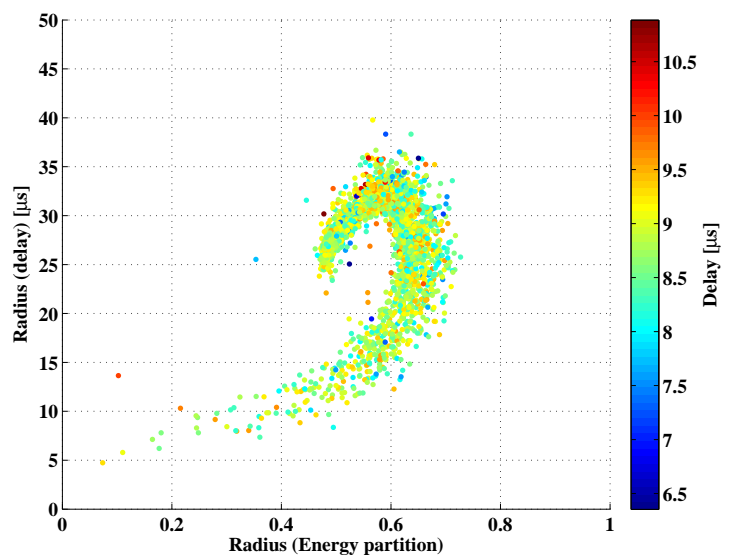

(b)

Figure 7.10: Position dependence of the phonon pulse delay. (a) Events at high and small radius are considerably slower than events in the central parts of the detector. (b) The correction scheme homogenizes the response of the detectors with respect to the events location. Note that the color scale changes from the left to the right panel.

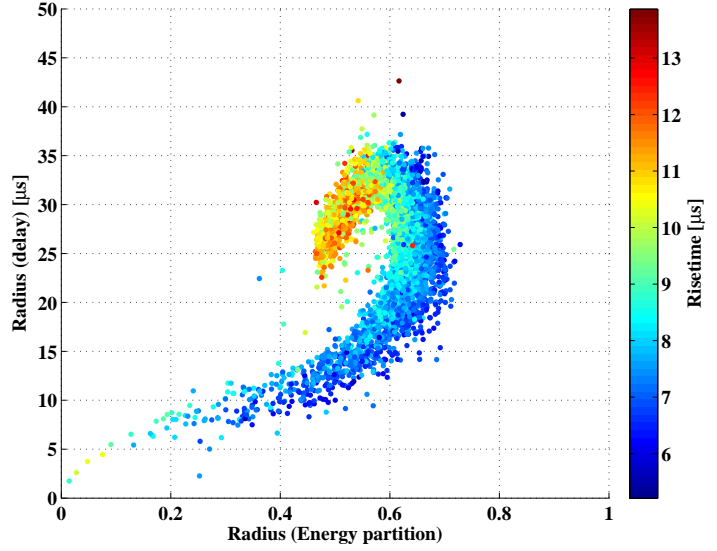

(a)

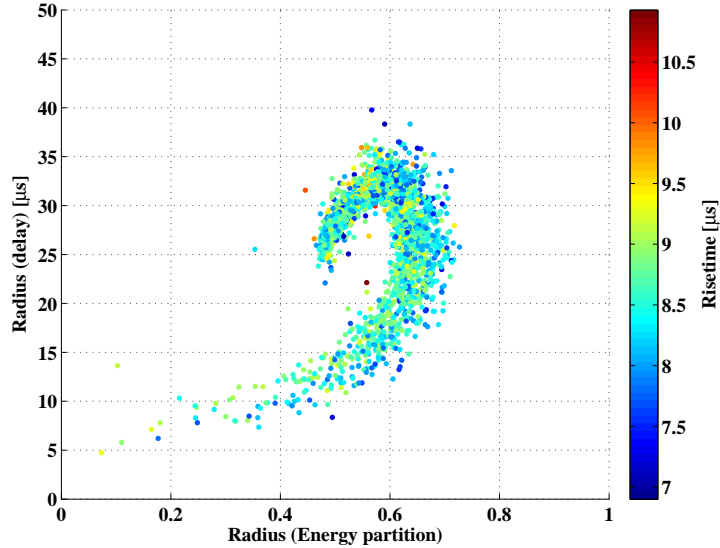

(b)

Figure 7.11: Position dependence of the phonon pulse rise-time. The rise-time strongly varies with event location (a) this variation is removed by the position correction scheme (b). Note that the color scale changes from the left to the right panel.

detector and for events beneath each of the four phonon sensors. Each event is located in a five-dimensional manifold defined by four position variables [xppart, yppart, xdel, ydel] (see Section 6.4.3) and the energy. Position within the manifold is defined as:

$$
\vec{q}=\operatorname{xppart} \vec{i}+\text { yppart } \vec{j}+\frac{x d e l}{L_{\text {del }}} \vec{k}+\frac{y d e l}{L_{\text {del }}} \vec{l}+\frac{\text { energy }}{L_{E}} \vec{m}
$$

where $[\vec{i}, \vec{j}, \vec{k}, \vec{l}, \vec{m}]$ are orthogonal unit vectors. $L_{d e l}$ and $L_{E}$ are weight factors that set 
the relative importance of partition, delay and energy quantities in determining the position of an event and its associated set of "nearest neighbors" within the manifold. The above metric on this five dimensional parameter space and the weight factors are chosen empirically to achieve good correction performance. The look-up table takes the form of a mapping between each of the calibration photon events and the mean values of the phonon parameters across a set of nearest neighbors events. The number of nearest neighbors affect the performance of the correction algorithm: a table with too few nearest neighbors is limited by statistical noise, while a table with too many nearest neighbors averages over a too large region in the manifold and misses local trends.

\subsubsection{Correction of the data}

For each single event either from low-background or calibration data, the nearest neighbors from the look-up table gammas according to the metric defined above are found. The lookup table provides a mean value of the quantity to be corrected over a set of neighboring events. For a particular event and parameter "RQ" (RQ stands for "Reduced Quantity" reflecting that these parameters are derived from the raw data) the corrected parameter "RQc" is defined as

$$
R Q c_{\text {event }}=R Q_{\text {event }} \cdot \frac{<R Q_{\text {cal }}>_{\text {all }}}{<R Q_{\text {cal }}>_{N N}}
$$

where $\left\langle R Q_{\text {cal }}>_{\text {all }}\right.$ is the mean over all calibration events, and $<R Q_{\text {cal }}>_{N N}$ stands for the mean of the parameter for those events in the nearest neighbor cluster drawn from the look-up table. Since every event is corrected with the same look-up table the overall mean only plays the role of a scaling factor included for convenience. The correction factor reflects how much the event differs from the mean of all events.

The correction table algorithm is vulnerable to degeneracies in the $5 \mathrm{D}$ manifold on which the look-up table is based. If two separated region of the detectors give similar values of the parameters used to measure the distance in the manifold, the set of nearest neighbors for a given event may include events from both detector regions. If these region have intrinsically different distribution of the parameter under consideration, erroneous corrections can occur. ZIP detectors unfortunately show substantial degeneracies between outer and inner detector radii in all of the parameters used to define the manifold. Though the combination of the parameters breaks most of the degeneracies troublesome regions of miscorrection remain. Events which are clearly misscorrected are rejected in the analysis. The classification of a potentially miscorrected event is based on a measure of the distance to the nearest neighbor events against which the event is corrected.

The correction table is defined on electron recoils and the same correction table is applied to nuclear recoils from the neutron calibration runs and the actual WIMP search data. Thus any systematic shifts in the parameters are the same for electron and nuclear recoils. 


\subsection{Ionization yield}

The ionization yield is the main discriminator between electron and nuclear recoils provided by the ZIPs (see section 6.5). To calibrate the ionization yield parameter and define the recoil type populations the ionization yield of electron recoils from ${ }^{133} \mathrm{Ba}$ calibration is calibrated to a value of 1 (see section (7.2). This calibration based on electron recoil events sets the scale for the ionization yield of nuclear recoils. To select interaction specific event populations the distribution of these events in the ionization yield vs. energy plane are defined.

\subsubsection{Electron-recoil band}

For the definition of the electron-recoil distribution data from ${ }^{133} \mathrm{Ba}$ calibrations is used. The electron recoil distribution is defined by fitting Gaussian distribution functions to the ionization yield profile in several energy bins.

Simple functional forms (given below) are fitted to the determined means and standard deviations as a function of the recoil energy.

$$
\mu_{E R}=a_{1} \cdot E^{a_{2}}[k e V] \quad \sigma_{E R}=\frac{b_{1}^{2} \cdot E^{b_{2}}[k e V]+b_{3}^{2}}{E[k e V]} .
$$

Due to different resolutions of the individual detectors the coefficients $a_{i}$ and $b_{i}$ are determined for each detector separately.

Using these functional forms a selection of events in the ionization yield vs. energy plane can be performed. Figure 7.12 shows the selection of electron recoils which lie in the $2 \sigma$ region of the electron-recoil distribution. The shape of the distribution implies to refer to this selection as the "electron-recoil band". The electron-recoil band widens at lower recoil energies, which is primarily caused by a fluctuation in noise which worsens the energy resolution of the ionization and phonon signal, determining the width of the ionization yield distribution.

\subsubsection{Nuclear-recoil band}

The nuclear-recoil band is defined from ${ }^{252} \mathrm{Cf}$ calibration data. Like for the electron-recoil band a Gaussian distribution function is fitted to the ionization yield profile in selected energy intervals. Due to the approximately exponential energy spectrum of nuclear recoils from the calibration runs, logarithmically spaced energy bins are chosen.

While the functional form fitted to the means of the Gaussian in the energy bins as a function of recoil energy is the same as used for the electron recoil band, the functional form used for the standard deviations is different.

$$
\mu_{N R}=a_{1} \cdot E^{a_{2}}[k e V] \quad \sigma_{N R}=b_{1} \cdot E^{b_{2}}[k e V] .
$$

At higher recoil energies $(\gtrsim 60 \mathrm{keV})$ the neutron statistics are very small and the primary selection of events in the energy bins may become polluted by low-yield electromagnetic 


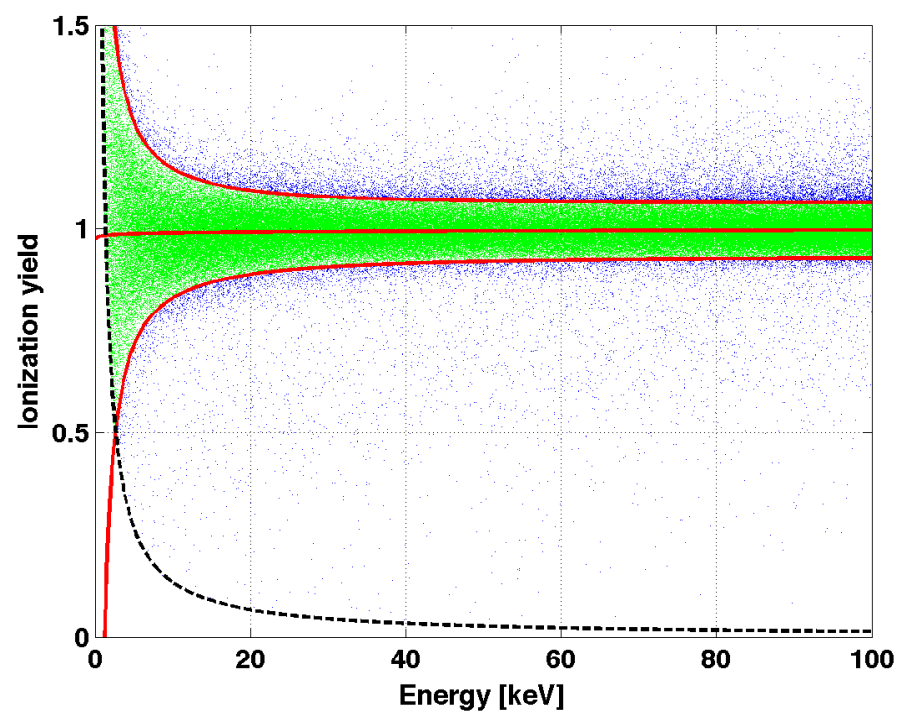

Figure 7.12: Electron-recoil selection by the ionization yield parameter. The centroid and the $2 \sigma$ region of the electron-recoil distribution are shown by the red lines. Selected events are shown in green. The dashed black line shows a threshold on the ionization signal applied to the ${ }^{133} \mathrm{Ba}$ calibration data shown in the figure.

events also present in the neutron calibration runs. This leads to a broadening of the nuclear-recoil band at higher energies. However the energy resolution should not significantly change neither for the ionization signal nor the phonon signal, thus the resolution of the ionization yield should not change either. In order to avoid a flaring of the band at higher energies the width of the nuclear recoil distribution is taken to be constant above a certain energy threshold value:

$$
\sigma_{N R}=\left\{\begin{array}{cl}
b_{1} \cdot E[k e V]^{b_{2}} & E \leq E_{c u t} \\
b_{1} \cdot E_{c u t}[k e V]^{b_{2}} & E>E_{\text {cut }}
\end{array}\right.
$$

The cutoff energies usually lie in the $20-40 \mathrm{keV}$ range (depending on the detector) with $40 \mathrm{keV}$ being a high threshold.

The $2 \sigma$ nuclear-recoil band is shown in figure 7.13. For illustration also the electronrecoil band is shown. At low recoil energies the electron-recoil band and nuclear-recoil band start to overlap, making a distinction between electron recoils and nuclear recoils based solely on the ionization yield parameter impossible. The dashed vertical line marks the position of the energy cut value above which the width of the distribution is kept constant. 


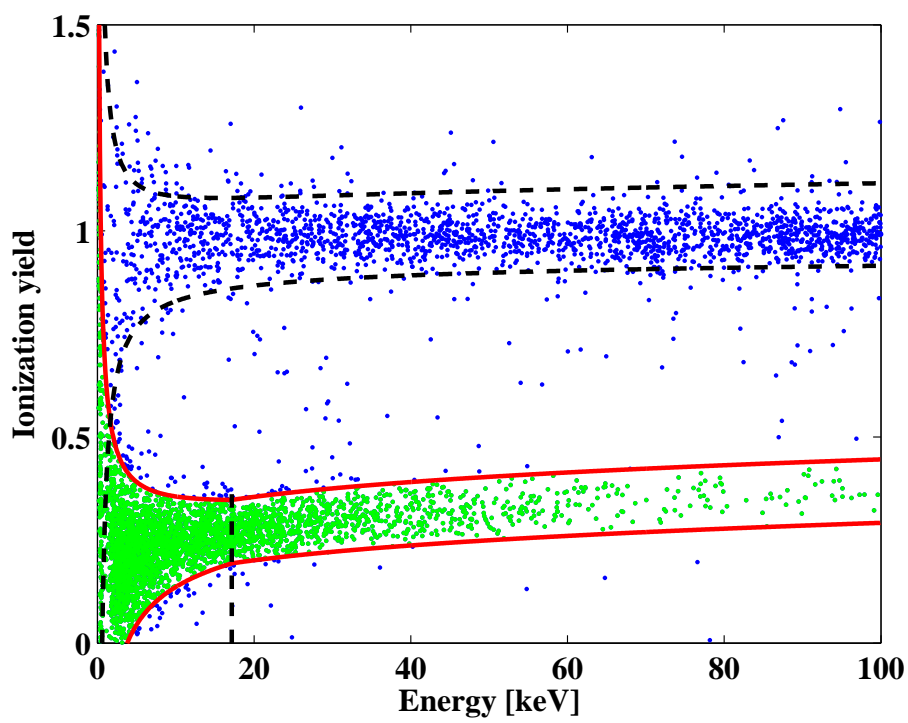

Figure 7.13: Nuclear-recoil selection by the ionization yield parameter. The red band shows the $2 \sigma$ nuclear-recoil distribution in which a signal from WIMP scattering is expected. Events in the selection band from ${ }^{252} \mathrm{Cf}$ neutron calibration are shown in green. For reference also the electron-recoil band is shown (black/dashed), illustrating the merging of the distributions at low energies. The vertical dashed line indicates the energy above which the width of the band is kept constant.

\subsubsection{Comparison with Lindhard prediction}

A validation of the correct ionization yield calibration of the detectors is the comparison of the centroids of the nuclear-recoil bands with the ionization yield predicted by the Lindhard model (see section 6.5). In general the agreement is very good as it is shown for the working germanium detectors in figure 7.14. For all detectors it is observed that the ionization yield centroid measured on calibration data is lower than the predicted value at lower recoil energies and higher at high recoil energies. This behavior is not understood quantitatively but there may be at least two reasons which may cause this effect. The simple formulas used to fit the centroid of the bands may not correctly account for the bending of the ionization yield as a function of recoil energy. In addition the mean of the ionization yield distribution at high recoil energy may be biased to higher ionization yield values, due to a pollution of the selected events by reduced ionization yield electron recoils. 

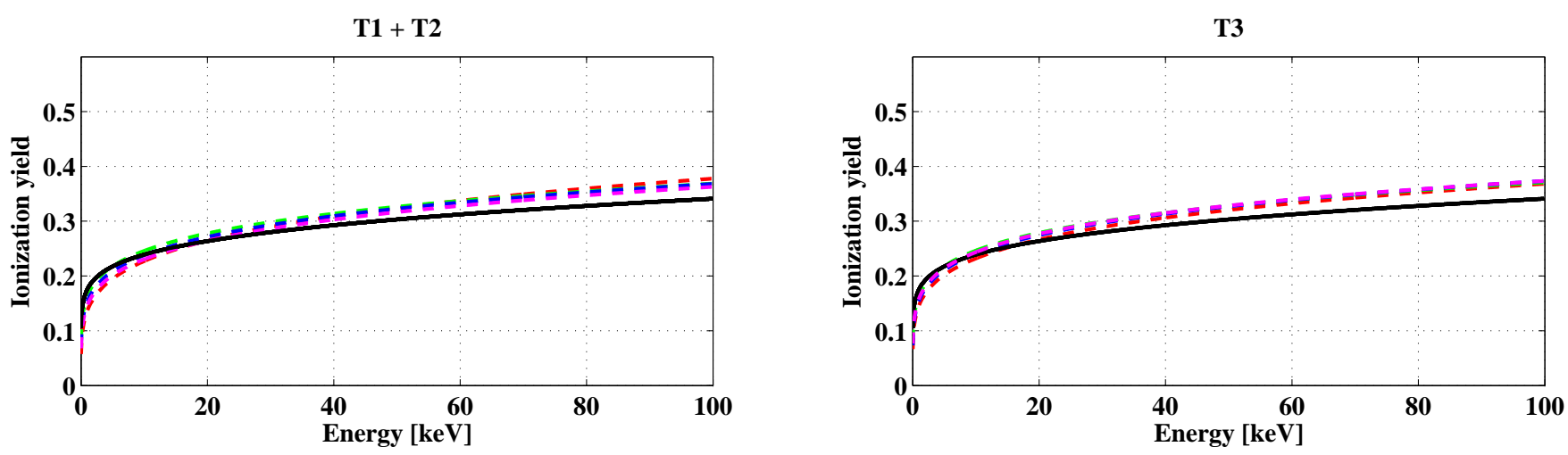

T4

T5
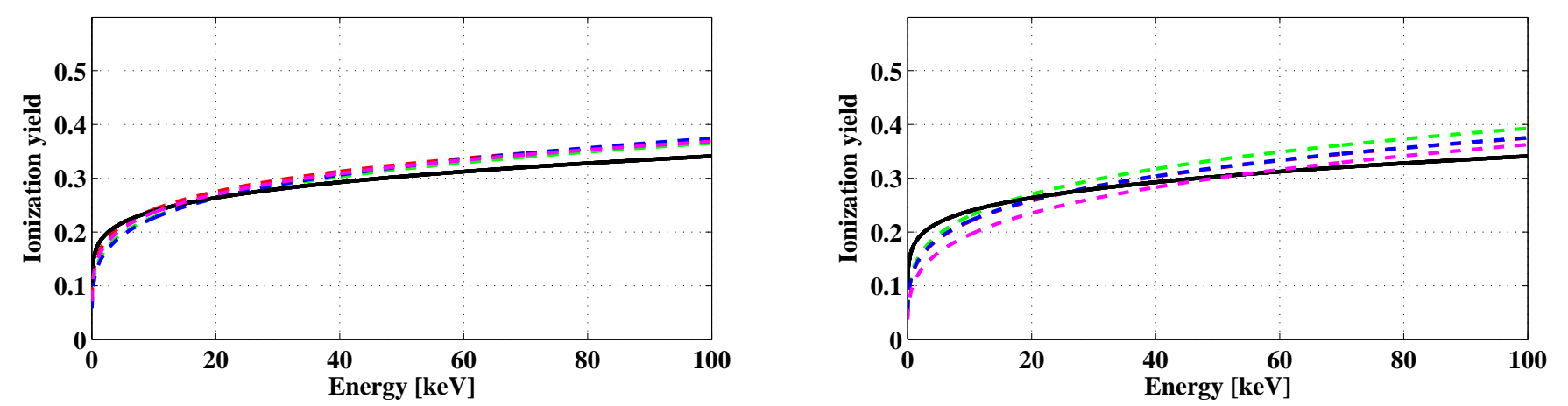

Figure 7.14: Comparison of the fitted nuclear-recoil distribution centroids with the Lindhard prediction. Each plot shows the fitted means of the nuclear-recoil band for working germanium detectors in one tower (tower 1 and 2 are grouped together) with the prediction of the reduced ionization yield from the Lindhard model (black line in each plot). 


\section{Chapter 8}

\section{Backgrounds}

The small recoil energy of dark matter interactions, together with an expected low event rate, makes an effective suppression of backgrounds necessary. Active and passive shielding (as described in Section 5.1.2) are used to reduce backgrounds produced outside the experimental apparatus, leaving decays of radioactive contamination inside the shielding as the dominant natural background. In addition cosmic ray induced neutrons can produce a nuclear recoil background in the detectors.

The radioactive decay of contamination of the materials in the experimental shielding can be identified by gamma-spectroscopy of the observed low-background data. In order to derive a concentration level of the various radioactive sources a simulation of the spectrum which matches the observed spectrum is performed. The gammas from the radioactive decays can produce events at the surface of the detectors which are the dominating background of the CDMS experiment. In combination with surface contamination of the crystals the total observed surface event rate can be explained. Finally the radioactive contamination can also be the source of neutrons which may be an irreducible background. To predict the rate of neutrons in the detectors, simulations of the neutron production processes and the detection of single-scatter nuclear recoils have to be performed.

As a last component cosmic rays interactions can produce an irreducible neutron background. Simulations are performed to determine the rate of such interaction in the detectors and allow to predict the expected single-scatter nuclear recoil background.

\subsection{Gamma background}

The gamma background in the CDMS-II experiment is caused by the contamination of the materials used inside the experimental apparatus. Much care in the selection of the materials which are close to the detectors has been taken to minimize the background from natural radioactivity. Most materials which were used in the construction of the experimental apparatus have been screened with a high purity germanium detector to quantify the level of contamination. This screening of the materials resulted mostly in upper limits on the concentration of a specific contamination. In addition to the selection of very 
clean materials, the inner volume (between the inner polyethylene layer and the mu-metal shield; see Section 5.1.2) of the experimental shield is purged with nitrogen to minimize the concentration of radon inside the experimental shield. The gamma background is mainly caused by the contamination of materials which are close to the detectors. These are the icebox vacuum cans and the cold hardware of the Towers which are both made of copper, since there is no shielding material in between these parts and the detectors. The copper itself adds some shielding from parts which lie further away from the detectors.

The main contribution to the gamma background arises from the naturally occurring ${ }^{238} \mathrm{U}$ and ${ }^{232} \mathrm{Th}$ isotopes and their subsequent decay products. In addition contamination with ${ }^{40} \mathrm{~K}$ and ${ }^{60} \mathrm{Co}$ could be identified. Since the germanium detectors in the towers have an excellent energy resolution in the ionization channel, they can be used for a detailed gamma spectroscopy of the spectrum observed in the low-background running mode. Figure 8.1 shows the co-added low-background data of all germanium detectors operated in Run 123128. The energies of several dominant lines in this spectrum are given, which are used to identify the contamination sources. All visible lines can be identified as being part of the decay chains mentioned above, and no unidentified lines are observed in the spectrum. Only the first bin shows a high excess in counting rate which is not associated with any spectral line from the isotopes mentioned above. This excess is caused by an intrinsic background of the germanium detectors, which results in a line at $10.36 \mathrm{keV}$ from the neutron activation of ${ }^{73} \mathrm{Ge}$ (the spectrum is shown in $15 \mathrm{keV}$ bins). Since this line is an intrinsic background of the detectors themselves, it will not be considered in this Section which is dealing with the gamma background from materials surrounding the detectors. This intrinsic background will be further discussed in Section 9.2.5.

In general the gammas emitted in the radioactive decay of the isotopes are of no concern in terms of a background source in the search for nuclear recoils, since they produce electron recoils in the detectors, and the CDMS detectors have an excellent discrimination power for electron recoils based on the ionization yield (see Section 7.4). However, these gammas can knock-off electrons in materials close to the detectors, which then could contribute to the surface-event background in the detectors, or they could directly Compton scatter in the surface layers of the detectors also increasing the surface-event background. In addition, the actual contamination levels of ${ }^{238} \mathrm{U}$ and ${ }^{232} \mathrm{Th}$ determine the experiments expected background from radiogenic neutrons. It is thus crucial to not only minimize the contamination levels of the materials which reduces the overall background of gammas, surface-events and neutrons, but also to determine the contamination levels such that predictions about the expected neutron background can be made. 


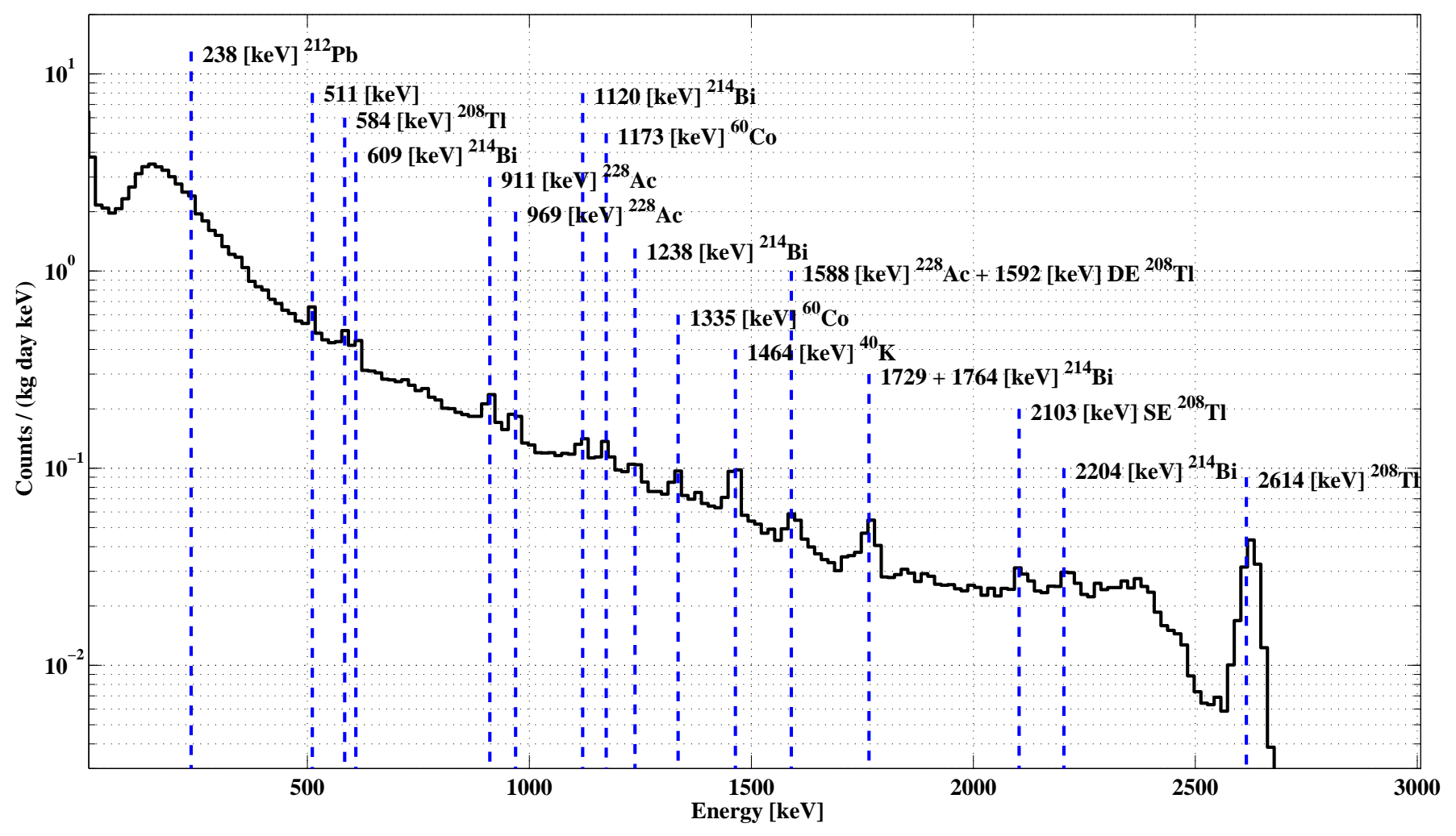

Figure 8.1: Low-background spectrum recorded with all germanium detectors. Prominent spectral lines are highlighted in the spectrum along with their energy and the decaying isotope. All lines can be identified to be part of either the ${ }^{238} \mathrm{U}$ or ${ }^{232} \mathrm{Th}$ decay chain or result from the decay of ${ }^{40} \mathrm{~K}$ or ${ }^{60} \mathrm{Co}$. In addition the single escape (SE) and double escape (DE) peaks from high energy lines can be identified. 


\subsubsection{Gamma background simulations}

Monte Carlo simulations using the GEANT4 simulation package [189, 190] have been performed to quantify the level of contamination of several parts of the experimental apparatus. In the simulations the decay of each isotope in the ${ }^{238} \mathrm{U}$ and ${ }^{232} \mathrm{Th}$ chains and the decay of ${ }^{40} \mathrm{~K}$ and ${ }^{60} \mathrm{Co}$ has been simulated using the G4RadioactiveDecay module. This module simulates the decay of a radioactive isotope taking into account the correct branching ratios of the decay channels. The isotropic decay of the isotopes has been spread over the whole volume of the considered material and part of the experimental apparatus. In the simulations the decay in each part of the Tower cold hardware, each icebox vacuum can and the inner polyethylene are simulated separately. This detailed simulation was performed to obtain the spectrum of all parts contributing to the total observed spectrum in the detectors. The relative contributions of each part could then be adjusted to match the observed spectrum observed in all five Towers, which enables the determination of the contamination levels of each part simulated.

The determination of the contamination levels was based on a global $\chi^{2}$-minimization between the summed simulated spectrum and the observed spectrum in each Tower. To keep the number of free parameters at a manageable level for the minimization routine some assumptions were made:

- The contamination levels of ${ }^{238} \mathrm{U}$ and ${ }^{232} \mathrm{Th}$ are assumed to be the same for all icebox cans, since they are made of essentially the same copper. Thus there are only two free parameters one for the ${ }^{238} \mathrm{U}$ and one for the ${ }^{232} \mathrm{Th}$ contamination level of the icebox cans.

- The contamination levels of ${ }^{40} \mathrm{~K}$ and ${ }^{60} \mathrm{Co}$ are assumed to be the same for any copper part in the simulations (icebox cans and Tower cold hardware). Thus there are only two global free parameters for the ${ }^{40} \mathrm{~K}$ and ${ }^{60} \mathrm{Co}$ contamination levels.

- In the minimization the contamination levels of ${ }^{238} \mathrm{U}$ and ${ }^{232} \mathrm{Th}$ have been fixed at the measured value for the inner polyethylene.

Thus, there are 14 free parameters in the minimization: two for the icebox cans, 10 for the Tower cold hardware (the contamination levels of ${ }^{238} \mathrm{U}$ and ${ }^{232} \mathrm{Th}$ are treated independently for each Tower) and two for the ${ }^{40} \mathrm{~K}$ and ${ }^{60} \mathrm{Co}$ contamination level of the copper.

Figure 8.2 shows the result of the global minimization by comparing the summed simulated spectrum, consisting of the spectrum of the individual parts also shown in the figure, with the observed spectrum for a representative Tower in the setup. First of all, the simulated spectrum matches the observed spectrum very well. From the simulated spectrum of the individual parts it can be seen, that the dominating contribution are the icebox cans with the second dominant contribution coming from the cold hardware of the Towers. The contribution from ${ }^{40} \mathrm{~K}$ and ${ }^{60} \mathrm{Co}$ as well as the contribution from the inner polyethylene are subdominant apart from the spectral lines of ${ }^{40} \mathrm{~K}$ and ${ }^{60} \mathrm{Co}$ visible in the spectrum. 


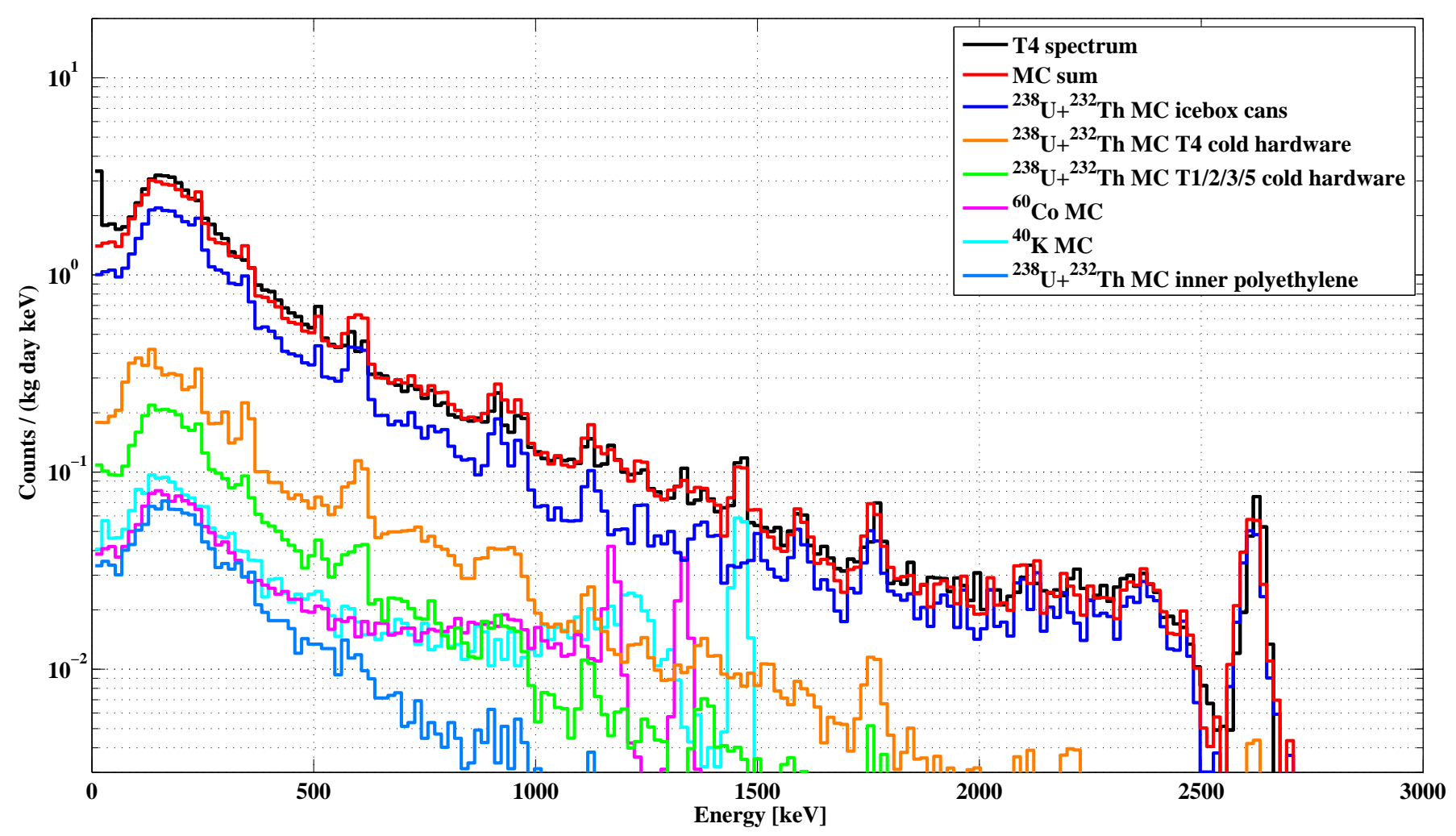

Figure 8.2: Comparison of the observed low background spectrum with the summed spectrum obtained from the simulations. The individual contributions from considered parts in the simulation are also shown in the figure. The simulated spectrum matches the observed one very well, and shows that the dominating contribution are the icebox cans, while the Tower cold hardware contributes subdominant, but non-negligible. Spectra are shown for a representative Tower. 
From the fit of the simulated spectrum to the observed spectrum only the activity of the corresponding isotope can be deduced. These activities can then be converted to a concentration level according to a prescription given in [112]. The resulting concentrations for the individual parts derived from the minimization are summarized in table 8.1. It has to be stressed, that some of the derived concentrations are not reliable, since in the minimization the scaling of the according spectra always reached a lower value prefixed in the minimization. Concentrations for which this pathology occurred are highlighted in red in table 8.1. Thus, it is conservative to assume that these concentrations are at the same level as the lowest concentration determined for the other Tower cold hardware parts. The errors quoted in the table are systematic errors based on starting the minimization routine with different starting values and lower and upper bounds on the single parameters. In addition there should be a $\sim 10 \%$ systematic error based on the selection efficiency of the inner-electrode cut applied for selecting the data shown in the spectra which affects the overall scaling of the spectra.

\begin{tabular}{|c||c|c|c|}
\hline Component & U conc. [ppb] & Th conc. [ppb] & Mass [kg] \\
\hline \hline T1 cold hardware & $0.03 \pm 0.02$ & $0.09 \pm 0.06$ & 4.98 \\
T2 cold hardware & $0.08 \pm 0.01$ & $0.09 \pm 0.06$ & 4.98 \\
T3 cold hardware & $0.18 \pm 0.03$ & $0.19 \pm 0.08$ & 4.98 \\
T4 cold hardware & $0.18 \pm 0.02$ & $0.22 \pm 0.08$ & 4.98 \\
T5 cold hardware & $0.27 \pm 0.01$ & $0.48 \pm 0.04$ & 4.98 \\
Copper icebox cans & $0.18 \pm 0.01$ & $0.56 \pm 0.06$ & 260 \\
Inner polyethylene & 0.12 & 0.12 & 120 \\
\hline \hline K conc. $[\mathrm{ppm}]$ & \multicolumn{3}{|c}{$0.106 \pm 0.002$} \\
Co conc. $[\mathrm{ppt}]$ & \multicolumn{3}{|c}{$0.027 \pm 0.004$} \\
\hline
\end{tabular}

Table 8.1: Contamination levels of materials within the inner lead shield as derived from the fit of the simulated spectrum to the observed low background spectrum. Concentration values highlighted in red are not reliable (see text). All concentrations are reported by mass: $1 \mathrm{ppm}=10^{-6} \mathrm{~g} / \mathrm{g}, 1 \mathrm{ppb}=10^{-9} \mathrm{~g} / \mathrm{g}, 1 \mathrm{ppt}=10^{-12} \mathrm{~g} / \mathrm{g}$. Note the different units for the ${ }^{40} \mathrm{~K}$ and ${ }^{60} \mathrm{Co}$ concentrations.

In general the concentration levels for the Tower cold hardware agree with each other, only the concentration levels of Tower 5 seem to be higher than for any other Tower's cold hardware. There are two explanations for this: either the cold hardware of Tower 5 has a higher concentration or there is a very localized source near Tower 5 which is accounted in the minimization for by increasing the concentration levels of the Tower's cold hardware. But it has to be stressed that the concentration levels returned by the minimization routine can explain the observed spectrum of each Tower very well. As a cross-check, figure 8.3 shows the combined low-background spectrum of all germanium detectors and compares this to the predicted spectrum from the minimization by properly taking into account the contribution from each component. 


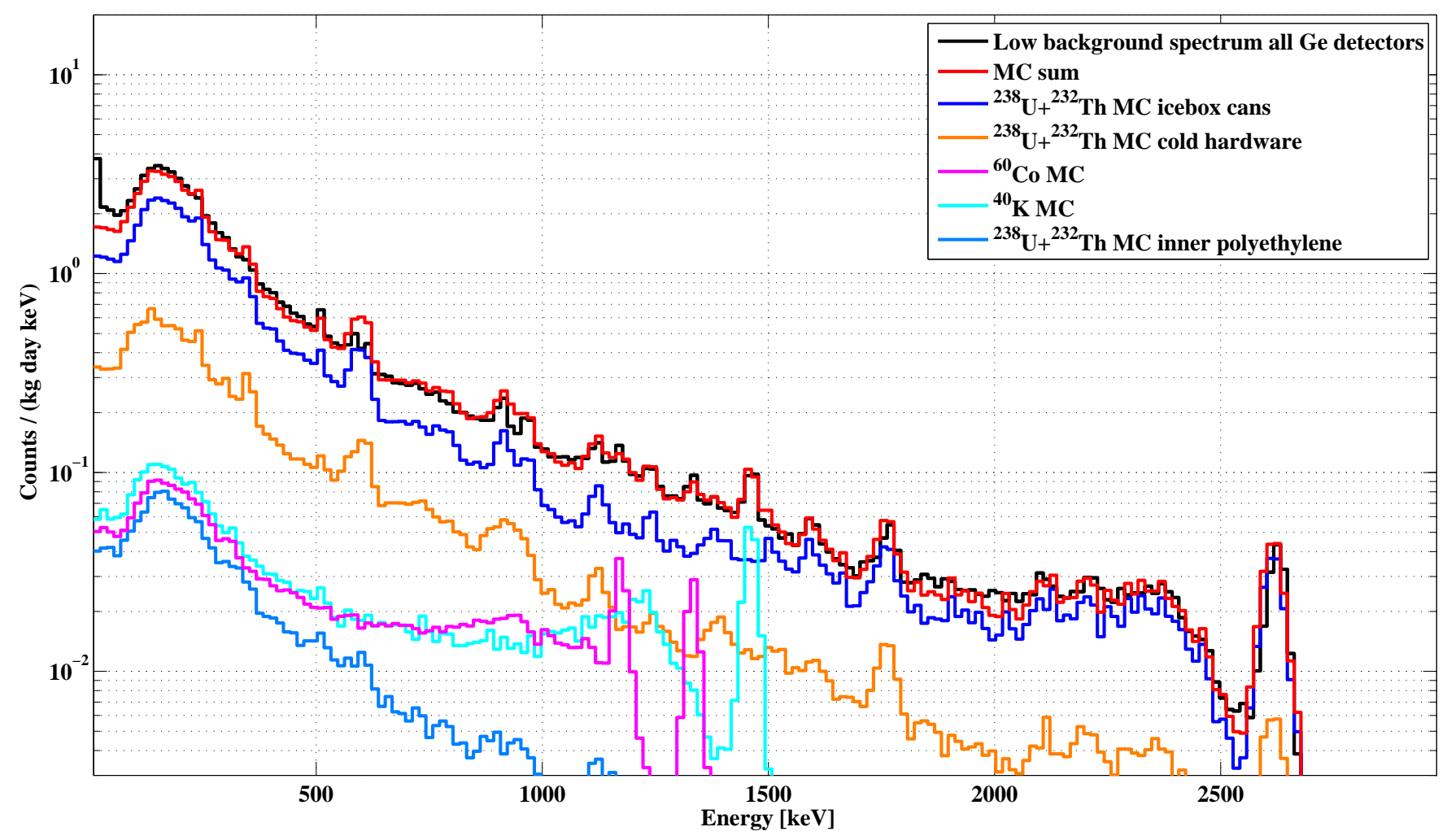

Figure 8.3: Comparison of the total observed low background spectrum with the total summed simulated spectrum in which the contribution of individual parts have been properly taken into account. The good match between the observation and simulation gives confidence in the reliability of the minimization scheme used for determining the contribution from the individual components. The mismatch between the observation and simulation at low energies is caused by neglecting contributions in the simulations (see text). 
Again the simulated spectrum completely matches the observed spectrum giving trust in the concentrations derived from the minimization. From the figure it can be seen, that the dominating contribution is caused by the icebox cans. If the icebox cans in a future experimental setup were made of the same ultra clean copper as the Tower cold hardware the overall background could be reduced by a factor of $\sim 3$. At the lowest energies the simulated spectrum does not match the observed spectrum. The first bin, including events from the $10.36 \mathrm{keV}$ line can not be explained since this contribution is simply not included in the simulations, but the simulations in general predicts a lower rate at energies below $\sim 70 \mathrm{keV}$. This may be in part due to neglecting surface contamination of the crystals which can give events at these energies. Since this study was primarily intended to explain the observed gamma spectrum based on the contamination of materials in the experimental apparatus, apart from the detectors themselves, and derive the concentrations of the associated isotopes, such localized contributions have not been considered in the simulations.

\subsection{Surface-event background}

For interactions occurring within tens of microns of the detector surface, diffusion of the charge carries into the incorrect electrode suppresses ionization collection [170, 191]. Thus electron recoils are classified into "bulk" and "surface-events". Surface-events are identified as those having an ionization yield more than $5 \sigma$ below the mean of the bulk electron-recoil band. A more detailed description of the identification of surface-events can be found in section 6.5.1.

Bulk events are dominantly due to Compton scattering of the ambient photon flux which extends up to $2.6 \mathrm{MeV}$ and is dominated by radioactive impurities in the experimental apparatus as described in the previous section. The key point is that bulk events arise from particles that scatter uniformly throughout the detectors, because they have long free mean paths. Surface-events can be caused by low-energy electrons incident on the detectors surface from outside, or Compton scattering within a near surface layer of the crystal. Such electrons may be liberated by Compton scattering of the ambient photon flux or may arise from decays of radioisotopes on the detector surfaces. Due to the prevalence in the environment and the emission of low energy electrons, a good candidate source is the $\beta$-emitting isotope ${ }^{210} \mathrm{~Pb}$. This isotope may be deposited on the surface of the detectors during production and testing and is referred to as surface contamination.

\subsubsection{Ambient gamma contribution}

A known contributor to the surface-event rate are electrons produced by the ambient flux of gammas. These gammas may liberate electrons from materials surrounding the detectors by Compton scattering which reach the detectors, or they could directly Compton scatter in the surface layers of the detectors. The regularly calibrations of the detectors with a ${ }^{133} \mathrm{Ba}$ source provide a large statistical sample of ambient gammas which is orders of 
magnitude higher than the ambient gamma flux during WIMP-search data taking. This highly elevated ambient gamma flux and the corresponding increase in the gamma-induced surface-event rate dwarfs any other contributions to the surface-event rate in calibration runs. This exposure thus allows to measure the relation between the gamma flux (as measured by the rate of bulk electron recoils) and the rate of gamma-induced surface events. For every bulk electron recoil in the $10-100 \mathrm{keV}$ range there are $8 \pm 4 \times 10^{-4}$ single-scatter surface-events. Using the ambient bulk electron recoil rate of 295 counts $/ \mathrm{kg} /$ day in WIMPsearch data a gamma-induced single-scatter surface-event rate of $(0.22 \pm 0.1)$ counts $/ \mathrm{kg} /$ day is obtained.

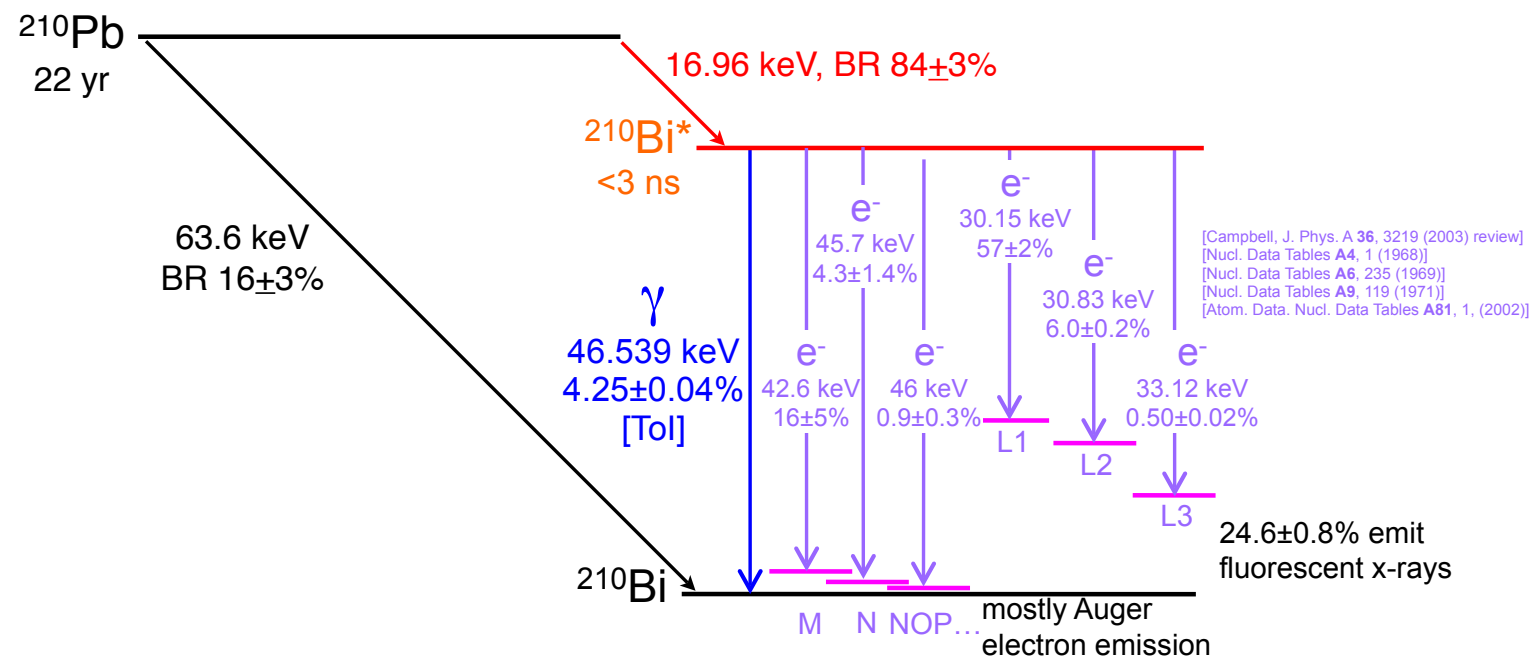

Figure 8.4: ${ }^{210} \mathrm{~Pb}$ decay scheme. A minority of the time, the decay is by $\beta$ emission to the ${ }^{210} \mathrm{Bi}$ ground state. A majority of the time, a $17 \mathrm{keV}$ endpoint $\beta$ decay occurs, leaving the ${ }^{210} \mathrm{Bi}$ daughter nucleus in an excited state. The remaining $46.5 \mathrm{keV}$ is emitted electromagnetically in various ways. Direct photon emission occurs a very small fraction of the time. Most of the time, internal conversion releases the nuclear excitation energy by ejecting a bound atomic electron from the atom. X-rays and Auger electrons are then emitted as the atomic electrons return to the ${ }^{210} \mathrm{Bi}$ atomic ground state.

\subsection{2 ${ }^{210} \mathrm{~Pb}$ surface contamination}

${ }^{210} \mathrm{~Pb}$ is a decay product of ${ }^{222} \mathrm{Rn}$, which is a gaseous daughter of the ubiquitous ${ }^{238} \mathrm{U}$ isotope. ${ }^{222} \mathrm{Rn}$ is airborne and decays with a half-life of 3.8 days. ${ }^{210} \mathrm{~Pb}$ may be produced in air and directly plate out onto the detector surfaces, or the parents of ${ }^{210} \mathrm{~Pb}$ may plate out on the detector surfaces and then decay to ${ }^{210} \mathrm{~Pb}$, implanting the ${ }^{210} \mathrm{~Pb}$ nucleus into the surface within tens of $\mathrm{nm} .{ }^{210} \mathrm{~Pb}$ decays with a half-life $\mathrm{T}_{1 / 2}=22.3$ years. A total of $63.6 \mathrm{keV}$ is available in the transition to the ${ }^{210} \mathrm{Bi}$ ground state. In $16 \%$ of the decays, the decay happens by $\beta^{-}$emission with the $63.6 \mathrm{keV}$ endpoint. For the other $84 \%$ of the time, 


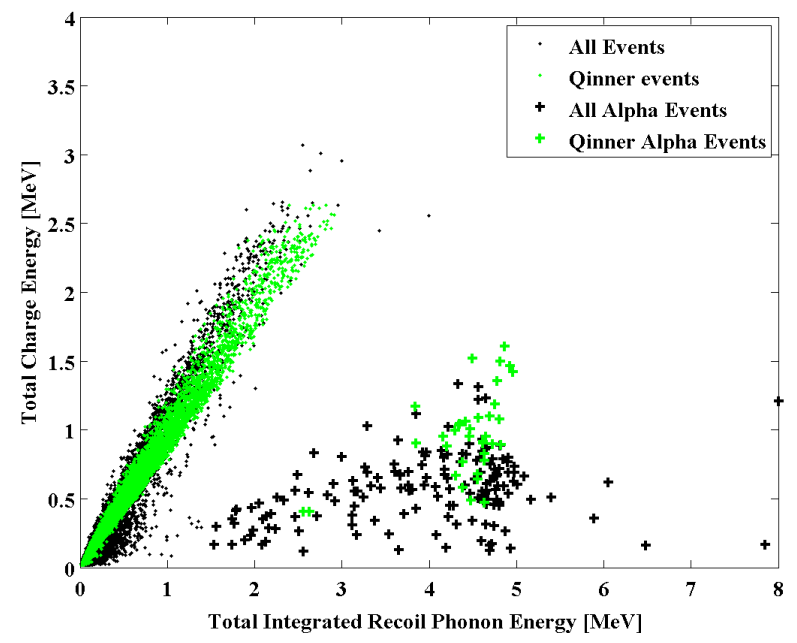

(a)

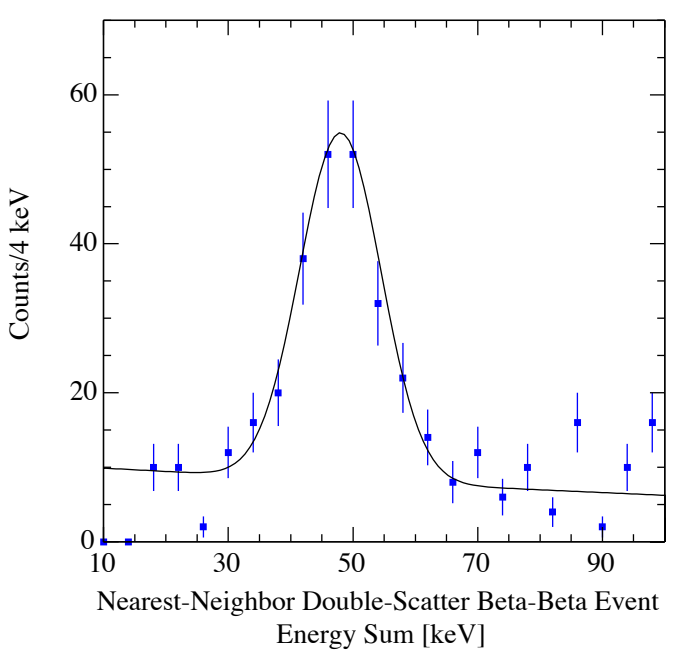

(b)

Figure 8.5: (a) Identification of $\alpha$ particles in the reconstructed ionization and phonon energy estimates in the $\mathrm{MeV}$ range. Inner-electrode $\alpha$ events cluster near $5 \mathrm{MeV}$ phonon energy and highly suppressed ionization yield, which is consistent with the ${ }^{210} \mathrm{Po} \alpha$ at $5.3 \mathrm{MeV}$. Figure provided by J. Cooley. (b) The broad (wider than detector resolution, due to combining different detectors) peak in the summed energy spectrum of nearest-neighbor double-scatter surface events. The position of the peak suggests the identification of the expected feature at $46.5 \mathrm{keV}$ from the ${ }^{210} \mathrm{~Pb}$ decay. Figure provided by R. Mahapatra.

the decay happens by $\beta^{-}$emission with a $17 \mathrm{keV}$ endpoint, followed by de-excitation of the daughter ${ }^{210} \mathrm{Bi}^{*}$ nucleus via emission of a $46.5 \mathrm{keV}$ photon or by internal conversion electron emission, followed by Auger-electron or X-ray emission. The details of the de-excitation are shown in figure 8.4. The ${ }^{210} \mathrm{Bi}$ daughter undergoes a $\beta^{-}$decay with a $1.16 \mathrm{MeV}$ endpoint and a half-life of $\mathrm{T}_{1 / 2}=5.01$ days to ${ }^{210} \mathrm{Po}$. ${ }^{210} \mathrm{Po}$ then decays with a half-life of $\mathrm{T}_{1 / 2}=138$ days to the stable ${ }^{206} \mathrm{~Pb}$ isotope, emitting an $\alpha$ particle with an energy of $5.3 \mathrm{MeV}$ while the nucleus recoils with $\sim 100 \mathrm{keV}$.

These various decays provide two event types that uniquely identify ${ }^{210} \mathrm{~Pb}$ as a surface event source. The $\alpha$-decay is the most unique signature, easily identified by the many $\mathrm{MeV}$ recoil energy of the $\alpha$ interaction with highly suppressed ionization yield since the $\alpha$ particle does not penetrate deep into the detectors. The identification of $\alpha$ particles is shown for a representative detector in figure 8.5(a). Roughly half of the time it is possible to identify the recoiling $100 \mathrm{keV}{ }^{206} \mathrm{~Pb}$ daughter in an adjacent detector as an event at roughly $100 \mathrm{keV}$ phonon recoil energy with negligible ionization signal. The single $\alpha$ events are observed at a rate of $\sim 0.4 /$ detector/day and the coincident $\alpha /$ recoiling-nucleus events at a rate of $\sim 0.2 /$ detector/day.

Another unique signature of the ${ }^{210} \mathrm{~Pb}$ decay is the emission of X-rays and Auger electrons by the de-excitation of the ${ }^{210} \mathrm{Bi}^{*}$ nucleus with a total energy of $46.5 \mathrm{keV}$. Because 
of the multiple particles emitted along with the non-negligible backscatter probability for low-energy electrons, all the energy is unlikely to be contained in one detector. Instead the summed energy spectrum of double-scatter surface events coincident between nearestneighbor detectors is considered. This selection does not only reduce a false combinatorial background but also shows the expected peak as can be seen in figure 8.5(b).

Most importantly there are highly correlated variations in the rates across detector pairs of these two unambiguously identifiable event classes, with no offset in either rate as shown in figure 8.6. These correlations strongly suggest that both classes of events arise from ${ }^{210} \mathrm{~Pb}$ decay and thus that ${ }^{210} \mathrm{~Pb}$ contamination of the detectors is a significant contribution to the surface event background.

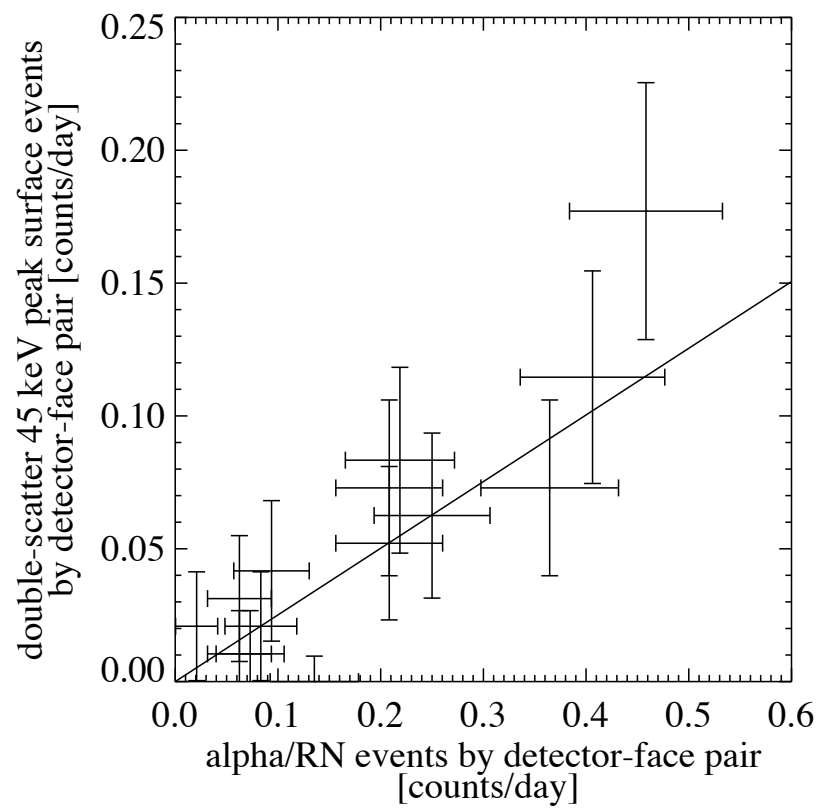

Figure 8.6: Correlation between nearest-neighbor double-scatter surface-event, $46.5 \mathrm{keV}$ sumpeak event rate and $\alpha$ /recoiling-nucleus event rate. Data points are shown for 14 out of 20 usable detector pairs. The correlation is strong, corroborating the identification of the $46.5 \mathrm{keV}$ peak due to ${ }^{210} \mathrm{~Pb}$. The overlaid line is based on the best-fit ${ }^{210} \mathrm{~Pb}$ contamination model; the data are in good agreement with the model. Figure provided by S. Golwala.

\section{Modelling the ${ }^{210} \mathrm{~Pb}$ activity}

The correlation shown in figure 8.6 allows to fit a ${ }^{210} \mathrm{~Pb}$-motivated contamination model to the data. The free parameters of the model are the ${ }^{210} \mathrm{~Pb}$ decay rates on the detectors and a number of global parameters dealing with the number of events of various types per ${ }^{210} \mathrm{~Pb}$ decay, geometrical factors, particle identification efficiencies, etc. Where possible these global parameters are defined by Monte Carlo simulations. The data points that constrain 
the model include the number of double-scatter surface-event/surface-event $46.5 \mathrm{keV}$ sumpeak events, $\alpha /$ recoiling-nucleus events and single-scatter surface events observed by each detector. Figure 8.6 shows the agreement between the data and the model.

The most important outputs of the fit are the ${ }^{210} \mathrm{~Pb}$ - sourced and non- ${ }^{210} \mathrm{~Pb}$ - correlated single-scatter surface-event rates listed in table 8.2. From these numbers two significant conclusions can be derived. First, the non- ${ }^{210} \mathrm{~Pb}$-correlated rate is consistent with the expected photon-induced single-scatter surface event rate discussed in the section above and also listed in the table. Thus, ${ }^{210} \mathrm{~Pb}$ and the ambient photon flux are sufficient to explain the entire observed surface event rate. Second, the ${ }^{210} \mathrm{~Pb}$-correlated rate drops by a factor of $2.5 \pm 2.5$ between Towers $1 / 2$ and Towers $3 / 4 / 5$, indicating better control of exposure to ${ }^{222} \mathrm{Rn}$.

\begin{tabular}{|c|c|}
\hline & $\begin{array}{c}10-100 \mathrm{keV} \\
10^{-3} \text { counts } / \mathrm{kg} / \text { day }\end{array}$ \\
\hline \hline All surface events, all towers & $371 \pm 183$ \\
\hline${ }^{210} \mathrm{~Pb}$, all towers & $240 \pm 183$ \\
\hline non- ${ }^{210} \mathrm{~Pb}$, all towers & $131 \pm 63$ \\
\hline gamma expected, all towers & $217 \pm 103$ \\
\hline \hline${ }^{210} \mathrm{~Pb}$, Tower $1 / 2$ & $377 \pm 166$ \\
\hline${ }^{210} \mathrm{~Pb}$, Tower $3 / 4 / 5$ & $149 \pm 131$ \\
\hline
\end{tabular}

Table 8.2: Various fitted rates from the ${ }^{210} \mathrm{~Pb}$ model. The rows list the total, as well as the ${ }^{210} \mathrm{~Pb}$-sourced and non ${ }^{210} \mathrm{~Pb}$ correlated single-scatter surface-event rate averaged over all towers in the full analysis range, as determined by the fit. The fourth line is the expected contribution to the non- ${ }^{210} \mathrm{~Pb}$-correlated component from the ambient photon flux. There is good consistency of the non- ${ }^{210} \mathrm{~Pb}$-correlated component as determined by the fit with the ambient photon flux expectation. The last two rows list the ${ }^{210} \mathrm{~Pb}$-sourced single-scatter surface event rate as determined by the fit subdivided by Towers, indicating that the ${ }^{210} \mathrm{~Pb}$ activity level is decreased by a factor of 2.5 between Tower $1 / 2$ and Tower $3 / 4 / 5$. 


\subsection{Neutron background}

Neutrons with $\mathrm{MeV}$ energies can produce nuclear recoils at $\mathrm{keV}$ energies in the detectors which would be identified as WIMP candidates. The detection of nuclear recoils from neutrons would ultimately limit the sensitivity of the experiment, since these would be indistinguishable from the expected signal of WIMP scatters. Thus, any direct detection experiment needs to evaluate the expected neutron background. There are two sources of neutron backgrounds: neutrons produced by natural radioactivity and neutrons produced by cosmic ray showers.

\subsubsection{Radiogenic neutrons}

Radioactive processes in and around the experimental apparatus can generate $\mathrm{MeV}$ neutrons, primarily through $(\alpha, \mathrm{n})$ reactions and spontaneous fission. The uranium and thorium decay chains are the main source of $\alpha$-particles in the materials used in the experimental setup. Uranium is the dominant source of neutrons from spontaneous fission, since the fission rate of thorium is essentially negligible. The expected flux of radiogenic neutrons thus depends upon the contamination levels of the various materials in and around the icebox, as well as geometric factors concerning the rate at which the neutrons reach the detectors. Radiogenic neutrons generated outside of the outer polyethylene shield are moderated enough to produce nuclear recoils below detection threshold in the detectors, while the detector substrates are pure enough ( $\ll 1$ ppt U/Th) to contribute negligibly.

The uranium and thorium contamination levels for the materials within the outer polyethylene shield are listed in table 8.3. These concentrations are provided by the screening of the materials based on gamma-counting measurements or the concentrations derived from the global fit to the observed gamma spectrum described in section 8.1.1. Note that the extrapolation from gamma rates to contamination levels often involves assumptions of secular equilibrium among the various elements and isotopes in a decay chain. For example the gamma rates from the uranium chain only directly give the contamination levels of isotopes after ${ }^{222} \mathrm{Rn}$ and not directly on the ${ }^{238} \mathrm{U}$ concentration which is the main source of radiogenic neutrons. So assuming secular equilibrium may introduce a substantial systematic error which is hard to quantify.

The contamination levels listed for the inner and outer lead are believed to be very conservative. The upper limits in the table are based upon null observation from gamma counts of the screening of lead samples. The EXO collaboration has set limits of $<10 \mathrm{ppt}$ $\mathrm{U} / \mathrm{Th}$ in lead [192] from the same sources as the lead used in the CDMS-II setup.

The listed contamination levels have been used to predict the nuclear recoil single scatter rates in the detectors. The neutron rates and energy spectra from $(\alpha, \mathrm{n})$ and spontaneous fission processes in the copper cold hardware, the copper icebox cans and he inner polyethylene shield were generated using the SOURCES4A simulation package [193]. Scaled by contamination levels and masses, neutrons from these spectra were propagated through a GEANT4 simulation to obtain the rate of nuclear recoil single-scatter events 194 . 


\begin{tabular}{|c||c|c|c|}
\hline Component & U concentr. [ppb] & Th concentr. [ppb] & Mass [kg] \\
\hline \hline Copper cold hardware & 0.16 & 0.25 & 14.95 \\
Copper icebox cans & 0.18 & 0.56 & 260 \\
Inner polyethylene & $<0.12$ & $<0.12$ & 120 \\
Inner lead & $<0.05$ & $<0.2$ & 1917 \\
Outer lead & $<0.05$ & $<0.4$ & 12190 \\
\hline
\end{tabular}

Table 8.3: Contamination levels of materials within the outer polyethylene shield. Upper limits on the concentration levels are from material screening while the levels for the copper parts have been derived from a global fit to the observed gamma spectrum (see table 8.1). The concentration of the cold hardware have been averaged for all five Towers. All concentrations are reported by mass: $1 \mathrm{ppb}=10^{-9} \mathrm{~g} / \mathrm{g}$.

For the lead $(\alpha, n)$ reactions are less significant due to the absence of light target nuclei for the $\alpha$-particle to be captured, thus only the fission neutrons from uranium are considered as a contribution to the nuclear recoil background. These simulations were carried out entirely within GEANT4, propagating neutrons generated from a ${ }^{252} \mathrm{Cf}$ spectrum. There may be a small systematic error by using a ${ }^{252} \mathrm{Cf}$ energy spectrum, however the binding energies of the two nuclei $\left({ }^{252} \mathrm{Cf} /{ }^{238} \mathrm{U}\right)$ are very similar, so the difference in the energy spectrum for spontaneous fission neutrons should not be a real concern in the prediction of the rates in the detectors. The results of these simulations were then normalized by the mass and upper limits on the contamination levels, the ${ }^{238} \mathrm{U}$ decay rate, and the branching ratio for spontaneous fission of ${ }^{238} \mathrm{U}$ [195].

The expected rates of single-scatter nuclear recoils within the $10-100 \mathrm{keV}$ range due to these various radiogenic sources are listed in table 8.4.

\begin{tabular}{|c||c|c|}
\hline Component & Ge rate $(\mathrm{n} / \mathrm{kg}$-years $)$ & Si rate $(\mathrm{n} / \mathrm{kg}$-years $)$ \\
\hline \hline Copper cold hardware & $3.6 \times 10^{-3}$ & $1.1 \times 10^{-2}$ \\
Copper icebox cans & $2.31 \times 10^{-2}$ & $6.0 \times 10^{-2}$ \\
Inner polyethylene & $<6 \times 10^{-3}$ & $<1.6 \times 10^{-2}$ \\
\hline Total copper/polyethylene & $2.67-3.27 \times 10^{-2}$ & $7.1-8.7 \times 10^{-2}$ \\
\hline Inner lead & $<3 \times 10^{-3}$ & $<2.4 \times 10^{-2}$ \\
Outer lead & $<7 \times 10^{-3}$ & $<5.3 \times 10^{-2}$ \\
\hline Total lead & $<1 \times 10^{-2}$ & $<7.7 \times 10^{-2}$ \\
\hline
\end{tabular}

Table 8.4: Expected rates in the energy range from 10-100 keV from radioactive contamination of the experimental setup components. These rates are based on the contamination levels given in table 8.3 . All statistical errors are negligible due to the large simulation size for determining the rates.

From the rates in the table it is obvious that the rate in the silicon detectors is much greater than in the germanium detectors, however the total germanium mass in the experimental setup $(\sim 4.5 \mathrm{~kg})$ exceeds the total mass of the silicon detectors $(\sim 1.1 \mathrm{~kg})$ by a 
similar factor. Thus, the increased cross section of silicon cannot be efficiently used as a veto of a possible neutron background in the germanium detectors, since the expected detection rates are on the same order of magnitude in both substrates. Only if both substrates detect nuclear recoils, suspicion on a possible neutron background would be raised, since the expected rate from WIMP interactions is much lower in silicon than in germanium.

\subsubsection{Cosmogenically produced neutrons}

The interaction of energetic cosmic-ray induced muons with the SUL cavern rock and materials of the experimental setup can generate neutrons at $\mathrm{MeV}$ energies which can produce $\mathrm{keV}$ nuclear recoils in the detectors. The neutrons can be generated through spallation (muon-induced nuclear disintegration) or various secondary processes within muon-induced hadronic and electromagnetic showers. At a depth of 2090 m.w.e, corresponding to the rock overburden at the experimental site the muon flux is reduced by a factor of $\sim 5 \times 10^{4}$ with respect to the surface flux. Nonetheless the remaining muon flux $(\sim 1$ muon per minute interacts in the experiments veto shield) produces a possible neutron background which must be evaluated.

Monte Carlo simulations have been performed to model the neutron production and propagation throughout the cavern rock and the experiment's shielding [196]. Two major software packages were used for the propagation of particles through matter: GEANT4 and FLUKA [197, 198, the latter was used in combination with a modified version of MCNPX [199] to model low-energy neutron scattering. An initial sample of muon energies and incident angles appropriate to the Soudan depth was generated using the MUSIC simulation package [200], based upon geology and geometry of the rock overburden and observation of the muon angular distribution. The main simulation propagated these muons through a $10 \mathrm{~m}$ thick shell of rock surrounding the experimental cavern to produce a series of particle showers, recording a sample of the particles which enter the cavern. These particles were then thrown in a simulation of the CDMS installation (detectors, shield and veto counters) to determine the number of muon induced nuclear recoil events.

The vast majority of cosmogenic nuclear recoil events in these simulations are accompanied by energy deposition in another active element of the CDMS apparatus (another detector or the veto counter), and would thus not be identified as WIMP candidates. Some of the events will not be identified as nuclear recoils in the real setup, since they are coincident with significant electromagnetic energy in the same detector. Of those nuclear recoils which are identifiable, most are part of events which deposit additional energy in other detectors and would thus not be identified as a single nuclear recoil event. Finally, the veto counter tags $\sim 98.8 \%$ of all events containing an identifiable nuclear recoil.

The simulation performance can be verified by testing the predictions of veto-coincident neutrons against experimental data. This benchmark study has been performed for the exposure of Run 123/4 of the CDMS-II experiment. The simulations predict 0.5 (1.4) veto-coincident neutron singles per kg-year of $\mathrm{Ge}(\mathrm{Si})$ exposure. For the exposure of Run $123 / 4$ the simulations predicted a total of 0.4 events. No such events were observed, in accordance with these expectations. The simulations also predicts 2.3 (7.9) nuclear recoils 
within multiple-scatter event per kg-year of exposure. This results in a total of 1.8 events in the considered exposure. In the data 5 apparent nuclear recoils within veto-coincident multiple scatter events have been observed, but it was estimated that $\sim 2$ of these events could be due to surface-events leaking into the nuclear recoil band. Again, the observation are consistent with the simulation within Poissonian fluctuations of the observed number of events.

Given the confidence in the predictions of the simulations, the results from the simulation can be used to estimate the cosmogenic neutron background. From the simulations the number of nuclear recoil single-scatters which are vetoed by the scintillator veto and those which are not vetoed are determined. Adding the statistics from all performed simulations, table 8.5 gives the number of vetoed and unvetoed nuclear recoil single-scatters in the germanium detectors in the energy range from $10-100 \mathrm{keV}$. The ratio of unvetoed to vetoed single-scatters can be multiplied by the number of vetoed nuclear recoil singlescatters observed in the data to estimate the number of expected unvetoed nuclear recoil single-scatters in the data. By using the ratio from the Monte Carlo, rather than the actual predicted rate from the simulations, the estimate is independent of any systematic effects in the scaling of the simulated livetime to the actual exposure of the analyzed data.

\begin{tabular}{|c||c|c|}
\hline & Unvetoed single-scatters & Vetoed single-scatters \\
\hline \hline $\mathrm{Ge}$ & 35 & 606 \\
\hline
\end{tabular}

Table 8.5: Number of unvetoed and vetoed nuclear recoil single-scatters in the 10-100 keV range recorded in the simulation of the cosmogenic neutron background. 


\section{Chapter 9}

\section{Analysis of WIMP - Search data}

In this Chapter the analysis of the data taken between July 2007 and September 2008, designated as Run 125 - Run 128 will be presented. Information from earlier runs of the CDMS-II setup (Run 123 and Run 124) are used, but the presented analysis focuses on the most recent data sample. The results from earlier runs will be included in Chapter 10, discussing the results of this WIMP search analysis.

This Chapter will give an overview of the WIMP search analysis, starting from the blinding of the data to ensure an unbiased analysis, the actual data selection and the definition of selection cuts applied to the data to extract a possible signal from WIMP interaction in the detectors. The cuts are grouped into data quality, reconstruction quality and event selection cuts. Each cut applied in the current analysis will be discussed in detail in the following sections. At the end of this Chapter the total exposure from this analysis and the signal efficiency of extracting WIMP induced nuclear-recoils from the data set are given. Note that the analysis presented here is a team effort from the whole CDMS collaboration.

\subsection{Blinding of the data}

In the search for a signal from WIMP interactions in the detectors, specific cuts on the event properties are defined to select only events which satisfy the expected signal. In order to define selection cuts in an unbiased way, a so-called "blind analysis" is performed on the data set. This means that the definition of any selection cut is made without the knowledge about the detailed characteristic of candidate or near-candidate events which may be present in the data. All selection cuts are defined on calibration data and WIMPsearch data outside of the expected signal region, so that there can be no fine-tuning of selection cuts to achieve a desired result. Blindness is maintained during the analysis by removing potential candidate events from the data by a blinding mask. The blinding mask represents a simplified and conservative version of the WIMP-search analysis. It is conservative in the sense that it removes more events from the data set than the final selection criteria would. Events satisfying the following criteria are removed from the data 
set until the final selection criteria have been defined:

- Veto anti-coincident: No signal in the scintillator veto shield within $50 \mu$ s preceding the global trigger of the event.

- Fiducial volume: Ionization signal in the outer electrode $\left(Q_{\text {outer }}\right)$ is consistent with the noise distribution of the electrode $\left(\left|Q_{\text {outer }}\right|<5 \mathrm{keV}\right)$.

- Energy range: The recoil energy lies in the interval from 5-130 keV.

- Ionization yield: Events lie within $3 \sigma$ of the nuclear recoil band.

- Single scatters: Phonon energy recorded only in the considered detector, the phonon energy in all other detectors is consistent with the noise distribution.

The blinding mask is applied to the data before the position correction of the data. Due to the less restrictive cut definitions and the poorer resolution of uncorrected phonon variables, these cuts are designed to substantially over-cover the true WIMP signal region. However, this procedure has some drawbacks. The phonon variables can change during the position correction of the data which may move events in or out of the blinding mask definition. In the current analysis it occurred that some events showed up in the expected signal region after the position correction of the data. The main reason for those events showing up in the expected signal region were the phonon energy and yield parameters which changed after the position correction of the data. Since these events could be valid candidates, a blinding mask based on the actual position corrected quantities was defined to remove those events from the analyzed data set. This small failure of the blinding procedure was not in conflict with the blinding philosophy, since the characteristics of these events were not studied in any detail and after removing them by an adequate blinding mask they did not affect the definition of any of the selection criteria. Since it seems that the position correction can change the properties of particular events it may be worth for future analyzes to apply the blinding mask after the data has been position corrected.

\subsection{Data quality}

At the top level of the analysis the data used for the analysis is selected, by requesting a good performance of the detectors and removing any time periods with suspicious run performances affecting the analysis. In the following the detailed data selection procedures and criteria for excluding specific periods of time from the analysis are given.

\subsubsection{Trigger criteria}

Any event considered in the WIMP search analysis should be acquired in the standard operation mode of the data acquisition system: a global trigger induced by a trigger of at least one detector (for trigger definitions see Sections 5.3.2 and 5.3.3). This selection is 
enforced by several cuts on the trigger information of a given event. Events which have an error in the trigger information are rejected, as are randomly triggered events taken at the beginning of each data series to monitor the noise performance of the detectors. A third rejected category are events triggered by the simultaneous hits in multiple scintillator paddles (acquired to monitor the veto counter performance).

\subsubsection{Detectors considered in the analysis}

Not all of the 30 detectors in the CDMS-II setup are used in the current WIMP-search analysis. Most importantly the Si detectors have not been analyzed in terms of a dedicated WIMP search analysis, since their sensitivity was not expected to justify the additional work needed. However the data from the silicon detectors remains blinded, such that it can be used in future analyzes. Hence, only the data from "good" germanium detectors is analyzed. The detector substrate positioning in the current CDMS-II setup is shown in table 9.1. Although all 30 detectors are used in screening for multiple-scatter events only a subset of the germanium detectors is considered in the current WIMP search analysis. Considered germanium detectors in this analysis are highlighted in green in table 9.1. A detailed description why specific detectors have been excluded from the analysis is given below.

\begin{tabular}{|c||c|c|c|c|c|}
\hline & T1 & T2 & T3 & T4 & T5 \\
\hline \hline Z1 & Ge & Si & Si & Si & Ge \\
Z2 & Ge & Si & Ge & Ge & Ge \\
Z3 & Ge & Ge & Si & Si & Si \\
Z4 & Si & Si & Ge & Ge & Ge \\
Z5 & Ge & Ge & Ge & Ge & Ge \\
Z6 & Si & Si & Ge & Ge & Ge \\
\hline
\end{tabular}

Table 9.1: Crystal substrates in the CDMS-II setup. "Good" germanium detectors are highlighted in green, while detectors having hardware problems and are thus not considered in a WIMP search analysis are highlighted in red.

Six detectors are excluded from all four data runs for a variety of reasons related to cold hardware and detector failures (these detectors are highlighted in red in table 9.1) :

- T1Z1: Disconnected phonon channels due to damaged tower wiring.

- T1Z3: Disconnected outer ionization channel amplifier.

- T1Z6, T2Z6: Poor neutralization due to broken LED wiring.

- T3Z1: Bistable performance of phonon sensor C. This phonon sensor frequently switches between a "good" and a "lazy" state, in which its phonon pulses are much smaller and slower. Without a proper calibration sample a detailed analysis to understand this behavior is impossible. 
- T5Z2: Disconnected inner ionization channel amplifier.

From the remaining 16 germanium detectors (highlighted in green in table 9.1) two detectors were excluded from the whole Run 125-128 analysis:

- T5Z1: A malfunctioning phonon sensor made the position correction of this detector very hard. In addition this detector has no good working neighboring detectors which could be used for vetoing nearest neighbor surface events, resulting in a high expected background.

- T5Z6: Extremely poor signal-to-noise for the phonon sensors, reducing the performance at low energies to unacceptable levels for a dedicated WIMP search analysis.

In the addition to the above several detectors have been excluded from specific runs. The reasons for excluding these detectors are given below.

\section{Run 127}

- T1Z5: The partial warm-up between Run 126 and Run 127 resulted in the loss of phonon channel $\mathrm{C}$ due to a superconducting short. This detector is excluded from the analysis after this run.

\section{Run 128}

- T2Z3: The partial warm-up between Run 127 and Run 128 resulted in the loss of phonon channel B due to a superconducting short. This detector is excluded from the analysis after this run.

- T2Z5, T4Z2: The statistics in calibration data were to low to perform a position correction on these detectors (see 9.2.3).

\subsubsection{Data selection}

In the search for very rare events which extends of months of data taking it is crucial to ensure the uniformity of the analyzed data. Detector performance can especially change after warm-up and cool-down, but also in-run variations of the detector performance are possible. In order to reject calibration data sets and WIMP search data with a reduced detector performance, Kolmogorov-Smirnov tests (KS-tests) are performed on the individual data sets. KS-tests are a commonly used statistical measure of similarity between distributions.

To benchmark the performance of the detectors KS-tests are performed on 7 variables

- Ionization yield

- Rise time of the primary phonon quadrant 
- Fall time of the phonon pulse

- Event radius based on phonon delay (see Section 6.4.3)

- Event radius based on phonon partition (see Section 6.4.3)

- Performance of the optimum filter ionization signal reconstruction (measured by the optimal filter $\chi^{2}$-value, see Section 6.2.3)

- Partition of the ionization signal between the inner and outer electrode

Each series was compared against each member of a random selection of 30 series which define the "standard" detector performance. For each parameter above, the $30 \mathrm{KS}$ comparison values for each data series were averaged together. Series which showed at least one average $\mathrm{KS}$ value lower than $2 \sigma$ below the mean of the average KS values are rejected from the analysis. In the calculation of the mean and standard deviation all series were taken into account, except those $10 \%$ of the series which showed the lowest KS values to avoid a widening of the standard deviation by extreme outliers.

\section{Similarity of the runs in this analysis}

At the beginning of the analysis, it had to be decided if the data should be position corrected as one large data set, or if there were variations between runs that necessitate the position correction of each run separately. The advantage of combining runs would be greater statistics, but if the position dependence varied between runs, then combining data from different runs would result in a loss of important information.

KS-tests were one tool used to get a reliable answer to this question. The statistics of R125, R126 and R127 were sufficient on each detector to position correct the data separately. However, R128 did not gather enough statistics to perform a position correction of the data on its own, thus it was intended to combine the data from R127 and R128 and perform a position correction for both runs together. A round of KS-tests on the combined data from R127 and R128 showed no clear difference in the detector performance except for two detectors, namely T2Z5 and T4Z2. For all but these two detectors the position correction was performed on the combined data set. Since no position correction was possible for the R128 data of detectors T2Z5 and T4Z2 this data has been excluded from the analysis.

\subsubsection{Helium films on the detectors}

During two time periods (December 2007 - January 2008; end of Run 125 and July 2008 August 2008; end of Run 127) multiple detectors showed a large increase in trigger rate. This increased trigger rate was caused by events showing only a phonon signal but no (or a very small) associated ionization signal. It is believed that this event pathology is caused by the settlement of helium films on the detectors. If a helium atom hits a detector, a phonon signal is released but no ionization signal, since the helium nucleus does not penetrate 
deep into the crystal. This results in a high rate of events with a low ionization yield value $(<0.1)$. A possible origin of these helium films is the increased temperature following the shutdown of the cryocooler. Helium frozen in the E-box (it was known that the vacuum of the E-box showed a leak) could have migrated into the icebox and to the detectors (for setup definitions see Chapter 5). The high trigger rates prevented a useful data taking for a dedicated WIMP search. In order to restore a stable WIMP search operation of the experiment the phonon triggers of affected detectors had been turned off, making these detectors useless for any WIMP search analysis out of the following reason:

- Detectors with deactivated phonon triggers can not be used for a WIMP-search data, because by definition of how the triggering occurs (see Section 9.2.1) these detectors would not record any single scatter event.

Thus the data from affected detectors has been removed from the analysis. Note however that affected detectors can still be used for vetoing multiple scattering events.

\subsubsection{Activation after neutron calibrations}

During the regularly performed neutron calibrations of the detectors, predominantly the copper from the detector supporting structure is activated. The decay time of the activation, matching a half-life of $12.7 \mathrm{~h}$ as can bee seen from figure 9.1, and the observation of an increased rate of the $511 \mathrm{keV}$ line suggest that ${ }^{64} \mathrm{Cu}$ with a half life of $12.7 \mathrm{~h}$ is the dominant contribution. Although ${ }^{64} \mathrm{Cu}$ decays via a $\beta^{+(-)}$-decay the solid angle to the detector faces is very small, thus up to now no conclusive result on an increased surfaceevent rate after neutron calibrations could be made. However, the gamma rates increase up to a factor of $\sim 6$ after the calibrations, potentially increasing ambient gamma induced surface-events during the activated time period. To ensure that no significant contribution from this activation is included in the final data set, all data taken within two days after a neutron calibration are not considered in the analysis.

Neutron capture on ${ }^{70} \mathrm{Ge}$ produces ${ }^{71} \mathrm{Ge}$ during the neutron calibrations. The X-rays and/or Auger-electrons following the electron capture decay of ${ }^{71} \mathrm{Ge}$ cause $10.36 \mathrm{keV}$ electron recoil events. Using this line, the energy calibration of the detectors (based on higher energy lines, see Section 7.1.2 and 7.2) at low energies can be verified and the energy resolution of the ionization and phonon channels can be determined. A fit to the line in the ionization channel and the phonon channel is shown for a representative detector in figure 9.2. The energy resolution of the ionization channel is $\leq 0.4 \mathrm{keV}$ on all considered detectors. For the phonon channel the energy resolution is $\leq 1 \mathrm{keV}$ with the exception of two detectors, namely T4Z6 and T5Z5, having a resolution of $\sim 1.5 \mathrm{keV}$. 


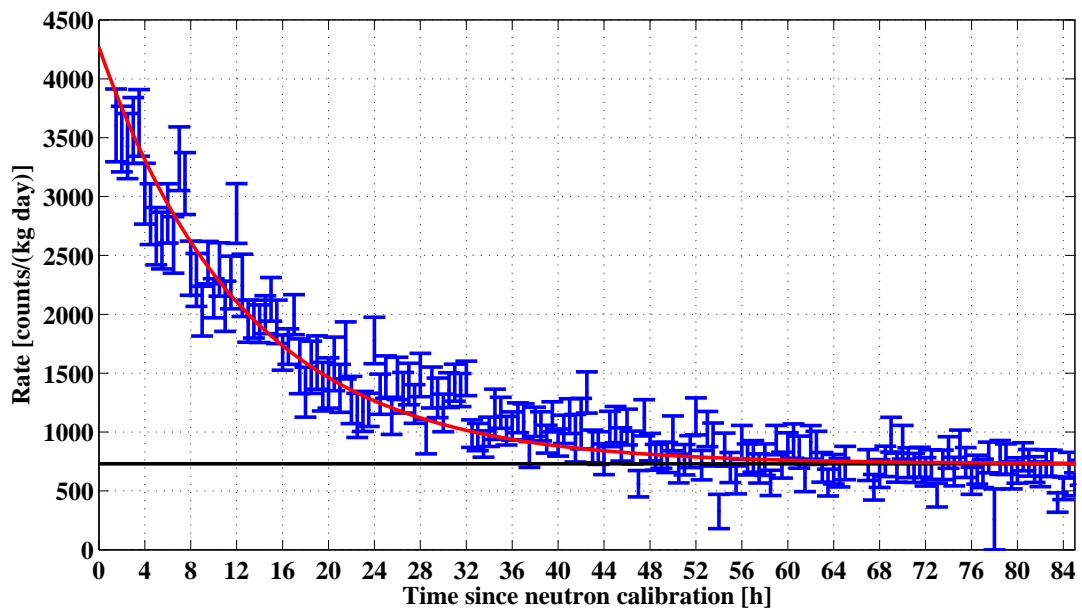

Figure 9.1: Total electron-recoil background rate in the energy range from $15-200 \mathrm{keV}$ as a function of time since the last neutron calibration. The time behavior of the detected rate is fitted by a constant background (shown in black) and an exponential decay component (the sum of the two components is shown in red). The decay constant of the exponential is given by $1 / 12.7 \mathrm{~h}^{-1}$ motivated by the half-live of ${ }^{64} \mathrm{Cu}$. The good match to the data shows that the dominant contribution to the increased rate after neutron calibrations is caused by ${ }^{64} \mathrm{Cu}$. Although the rate is increased by up to a factor of $\sim 6$ directly after the neutron calibration, the rate is close to the average value of normal operation mode after 48 hours.

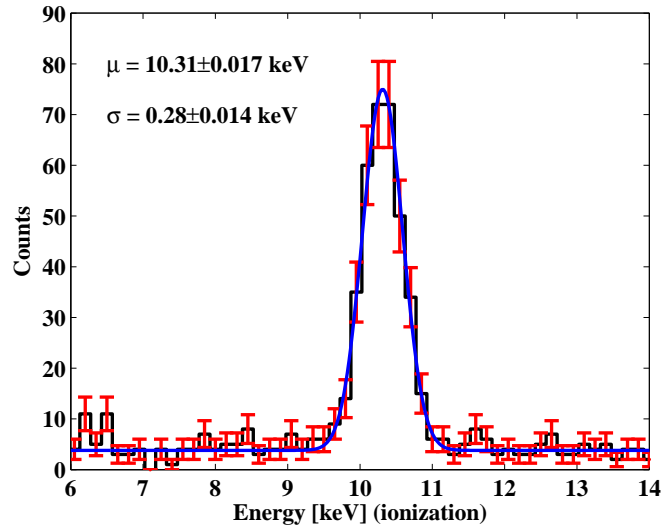

(a)

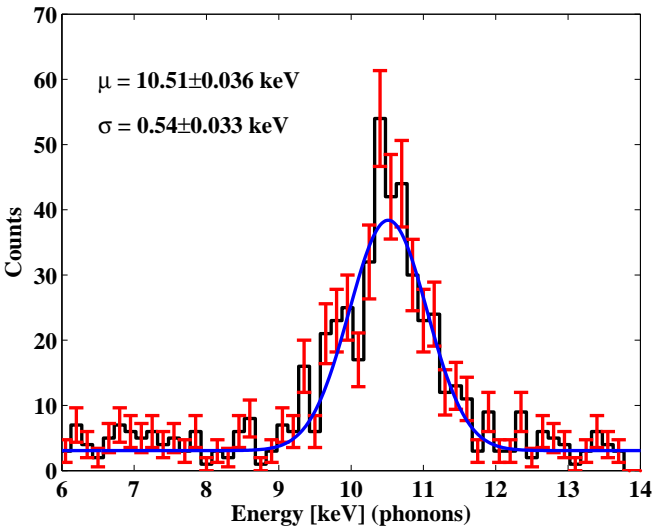

(b)

Figure 9.2: Position and resolution of the $10.36 \mathrm{keV}$ line from ${ }^{71} \mathrm{Ge}$ for the inner ionization electrode signal (a) and the phonon signal (b). The blue curve shows a fit consisting of the Gaussian with constant background to the data, from which the mean and resolution are determined. Fits are shown for a representative detector. 


\subsubsection{Neutralization state of the detectors}

If the detectors are left under voltage bias for an extended period of time, accumulated ionized trapping centers eventually lead to incomplete charge carrier collection. An incomplete charge collection would result in additional low yield events and thereby affect the ionization yield based discrimination of electron recoils. To reduce trapping effects in the bulk of the detectors, the detectors routinely undergo a neutralization procedure. During this procedure the detectors are grounded and illuminated with a LED mounted in the detector housing (see Section 5.2.1). The large number of electron-hole pairs created in the detectors diffuse until they neutralize ionized trapping centers or recombine. Neutralized trapping sites have a smaller trapping cross-section than if they were charged, therefore reducing bulk trapping effects in the detectors. The neutralization procedure is applied during the daily cryogenic transfers, after approximately 11 hours of WIMP search data taking, and after every barium calibration data set lasting for more than 30 minutes.

The neutralization state of the detectors was monitored offline for ${ }^{133} \mathrm{Ba}$ calibration and WIMP search data. Any period of time showing a loss in neutralization was excluded from the analysis. The procedure applied to the calibration and WIMP search data for identifying periods with a loss of neutralization are outlined below.

\section{${ }^{133}$ Ba calibration data}

All data series were divided into chunks with $10^{5}$ events. The fraction of low yield events (ionization yield $\in\left[\begin{array}{ll}0 & 0.8\end{array}\right]$ ) passing basic quality cuts and their standard deviation based on the confidence intervals from a binomial distribution were calculated for each chunk and for the whole series. The means of the fraction of low yield events over each run in units of chunks and series were calculated, assuming that the fractions of low yield events follow a normal distribution. For each chunk, the Poisson probability of observing as many low yield events or more in a particular chunk given the mean low yield event fraction was calculated:

$$
Q=1-\sum_{n=0}^{n_{o b s}-1} \frac{N^{n}}{n !} e^{-N}
$$

where $n_{\text {obs }}$ is the number of low yield events in the chunk and $N$ is the expected number of low yield events given the mean fraction of low yield events over each run.

Any series or chunk failing at least one of the following criteria was removed from the analysis

- A particular series failed the Poisson probability criteria $\left(Q<10^{-10}\right)$ as a whole or contained at least one chunk that failed this probability criteria.

- Chunks of data that showed an obvious loss of neutralization at the end of the series, especially if the series lies $2 \sigma$ above the run mean. 


\section{WIMP search data}

Due to the lower statistics in WIMP search data it was not possible to subdivide the data series into chunks, and thereby monitor the neutralization state within a series. Thus only the low yield event fraction passing quality cuts was calculated for each series. As for the ${ }^{133} \mathrm{Ba}$ calibration data the mean, standard deviations and Poisson probability criteria were calculated. Any series satisfying at least one of the following criteria were removed from the analysis:

- The fraction of low yield events in a series lies $2 \sigma$ above the run mean.

- The series failed the Poisson probability criteria $\left(Q<10^{-10}\right)$.

- The fraction of low yield events in a series is greater than $5 \sigma$ of a run averaged standard deviation taken over all series.

The livetime cut by this neutralization assessment is approximately $0.4 \%$.

\subsubsection{Periods of elevated charge noise}

Studies of the ionization channel noise stability identified periods of time with an increased noise. An increased ionization noise level would affect the resolution of the ionization start time and by this the delay of the phonon pulse, which is measured with respect to the ionization pulse. In addition the energy reconstruction at low energies can be significantly affected by a high noise level. To identify periods of high noise within a data series, ionization noise events are identified by requesting that the total phonon signal lies within $4 \sigma$ of the phonon noise distribution. The phonon noise distributions are determined for each data series individually by fitting a Gaussian distribution function to random triggered events recorded at the beginning of each series.

As a measure of the combined ionization noise, the summed signal of the inner and outer electrode is used. To reduce bias from an event by event spreading, the moving average over 20 events is calculated. For each series it is requested that the moving average of the summed ionization signal lies within $6 / \sqrt{20} \sigma$ of the ionization noise distribution which is calculated from the smoothed data. The factor $\sqrt{20}$ in this criteria takes into account the size of the averaged samples. Time periods which exceed this noise threshold are identified as high ionization noise periods. Identified time periods are rejected in the analysis with an additional 5 minute buffer before and after each interval which is also rejected. In addition to cutting periods with a high ionization channel noise, this cut also removes bursts of ionization noise events with reconstructed energies as high as $20 \mathrm{keV}$. This charge glitch event topology would not be cut by the standard electronic glitch cut, which searches for events with phonon triggers only but no associated ionization triggers (see Section 9.2.9 below). The reduction in live time of the cut is usually less than $0.5 \%$ on most detectors. The actual detector dependent loss in livetime is taken into account in the final calculation of the detector exposure (see Section 9.5). 


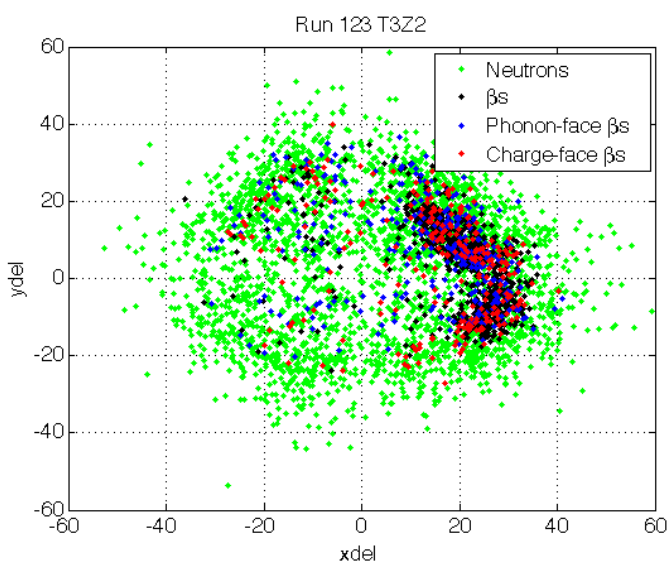

(a)

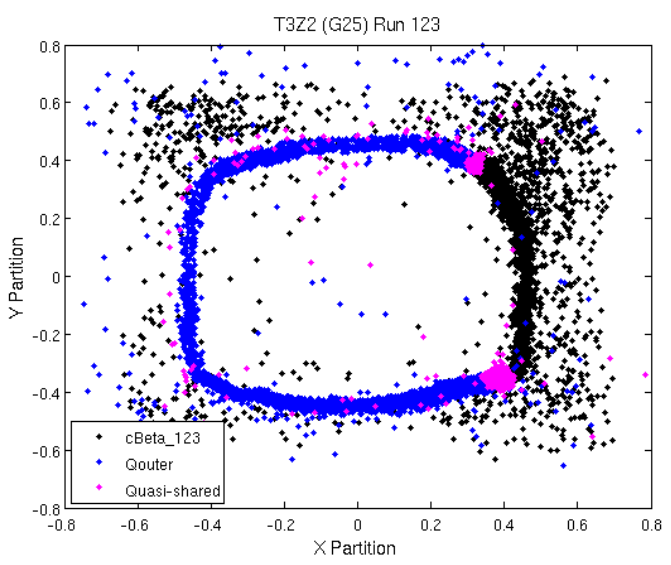

(b)

Figure 9.3: (a) Phonon delay plot for detector T3Z2, highlighting neutrons (green) and lowyield surface events (black). Face selected events are shown in blue (phonon-face) and red (charge-face). A huge excess in low yield events is seen in quadrants C and D (positive xdel). (b) The phonon partition plot illustrates a malfunctioning detector region causing the excess in low-events. Black indicates inner ionization electrode events (i.e negligible outer electrode signal), blue indicates outer ionization electrode events, and magenta indicates shared events. Figures taken from [173].

\subsubsection{Malfunctioning detector regions}

Detector T3Z2 showed a very localized anomalous behavior in the charge collection of the outer electrode. This region produced an excess of events at low ionization yield and slow phonon timing. This malfunctioning detector region was already identified in earlier runs of the experimental setup (Run 123/4).

As shown in the left plot of figure 9.3 , quadrants $\mathrm{C}$ and $\mathrm{D}$ show a huge population of low yield events. The right plot shows that this population is concentrated in an apparent gap of the outer ionization electrode. Events under the outer ionization electrode seem to deposit negligible charge in that electrode. It is likely that the outer electrode became disconnected or other effects prevent the charge collection on the outer electrode in this region. The malfunctioning charge collection in this particular detector region is not fully understood. The charge collection seems to be normal for quadrants A and B. To exclude any event from the analysis in this detector region the following selection criteria for this specific detector is defined:

- Only events with xdel $<0$ are considered in the analysis.

Since half of the detector is neglected in the analysis, generally the exposure of this detector is reduced by $50 \%$. The actual reduction in exposure is properly taken into account in the calculation of the total exposure (see Section 9.5) 


\subsubsection{Electronic glitches and trigger stability}

\section{Electronic glitches}

Electronic glitches may often trigger the phonon sensors of the detectors, but these glitches seldom show up on the ionization channels. To identify such glitch events the number of phonon triggers relative to the number of ionization triggers is investigated. For a single interaction in a single detector, there should only be one phonon trigger and in general an associated ionization trigger. The hardware readout is triggered by the phonon trigger so for very low ionization pulses the ionization trigger threshold may not be reached. Due to the multiple detector setup there is the possibility of a particle interaction in more than one detector resulting in multiple phonon/ionization triggers. Events which fulfilled one of the following conditions are not considered in the analysis:

- The subtraction of the number of ionization triggers from the number of phonon triggers is greater or equal to 4 and the number of phonon triggers is greater or equal to 4 .

- Three phonon triggers are recorded while no ionization trigger is recorded.

\section{Trigger stability}

The trigger rates in low background mode should be fairly low and stable in time. Any period of time with an elevated trigger rate of non random triggers may indicate electronics problems or an increase in phonon noise. Periods with an overall trigger rate $>0.7 \mathrm{~Hz}$ over at least 100 consecutive non-random events are considered as periods with high trigger rates and are removed from the analysis. The trigger rates are investigated for each detector separately to cut periods of high trigger rates on a specific detector but keep the data for non affected detectors. Such periods could only be identified in R125 and R127. Excluding these periods the reduction in livetime for R125 (R127) is $0.048 \%(0.018 \%)$

\subsubsection{MINOS neutrino beam}

An intense beam of neutrinos at $\mathrm{GeV}$ energies is currently directed from the Main Injector Facility at Fermilab to the far detector of the MINOS neutrino oscillation experiment at Soudan. Neutrinos from the beam may interact with the surrounding rock or shielding material and produce muons and associated neutrons, which may be detectable in the CDMS-II experiment. Such coincidences are not expected to produce a significant source of unvetoed background, but nonetheless events in coincidence with the beam are excluded from the analysis. For each event a GPS time stamp is recorded using a fiber optic link to the MINOS GPS receiver. After correction for the measured fiber optic delay time, these time stamps are compared against the time recorded of each beam dump. The cut excludes detector-triggered events within a $60 \mu \mathrm{s}$ window of a beam spill. A total of 72 events is observed in coincidence with the neutrino beam ( 81.2 are expected to occur due to a random coincidence). The reduction in livetime is $0.0016 \%$. 


\subsection{Reconstruction quality cuts}

In order to ensure that the events used in the analysis are reconstructed well a series of reconstruction quality cuts is defined which removes events with specific reconstruction pathologies. Any misfitted pulses or an abnormally high noise level can lead to a wrong reconstruction of energy and pulse shape characteristics.

\subsubsection{Ionization pulse reconstruction}

As a measurement of the reconstruction quality of the ionization pulses the charge optimal filter $\chi^{2}$ is used (see Section 6.2.3). Events with an abnormally high $\chi^{2}$-value are rejected. Large $\chi^{2}$-values mainly arise due to pile-up of multiple events within a single trace or electronic glitches in the ionization channel. If two particle events occur sufficiently close together in time (separated by less than the post-trigger digitization window of $\sim 1200 \mu \mathrm{s}$ ) in a single detector, the pulses from the two events can overlap in a digitizer trace. Such events are very rare at the low trigger rates of WIMP search data, but are very common in calibration data. Such distorted pulses are reconstructed incorrectly and are removed from consideration as WIMP candidates and in calibration data for detector characterization.

The distribution of the $\chi^{2}$-values (shown in figure 9.4(a)) is observed to flare out at higher energies, mainly due to the discreetness of the start time in the used optimal filter template pulse. The start time of the ionization pulse is only determined to the nearest digitizer bin, giving a resolution of $0.8 \mu \mathrm{s}(1 \mathrm{bin}=0.8 \mu \mathrm{s})$. This inaccuracy in the start time determination broadens the difference in $\chi^{2}$ between events with slightly different true start times. Furthermore the templates used in the optimal filtering retain some noise since they are produced by averaging over a finite number of real pulses. This noise enhances the $\chi^{2}$ slightly at high pulse amplitudes. A cut is set to exclude events with

$$
\chi^{2}>a+b \cdot Q_{\text {sum }}^{2}
$$

where $Q_{\text {sum }}$ is the summed ionization signal of the inner and outer electrode. The variables $a$ and $b$ are determined empirically by a fit to the distribution. For each run and each germanium detector the ${ }^{133} \mathrm{Ba}$ calibration data is divided into four energy bins $[2040] \mathrm{keV}$, [60 100] keV, [120 160] keV and [180 220] keV. The mean and standard deviation in each bin is determined by fitting a Gaussian distribution function to the $\chi^{2}$-values within an energy bin. The $3.5 \sigma$ deviations from each mean value are then fitted to the quadratic function shown above to determine the values of $a$ and $b$. An example fit to the distribution is shown in figure 9.4(a) the black line shows the setting of the cut, such that blue points pass the cut and red points fail the cut.

The efficiency of the cut is measured on WIMP search data simply by determining the fraction of events passing the cut in a given energy bin. An example of the efficiency as a function of energy is shown in figure 9.4(b). A functional form is fitted to the efficiencies to determine the energy dependency of the efficiency. The efficiencies are typically greater than $98 \%$ for energies greater than $\sim 10 \mathrm{keV}$, although there are some detectors with slightly lower efficiencies. The efficiencies are measured as a function of the energy deposited in 


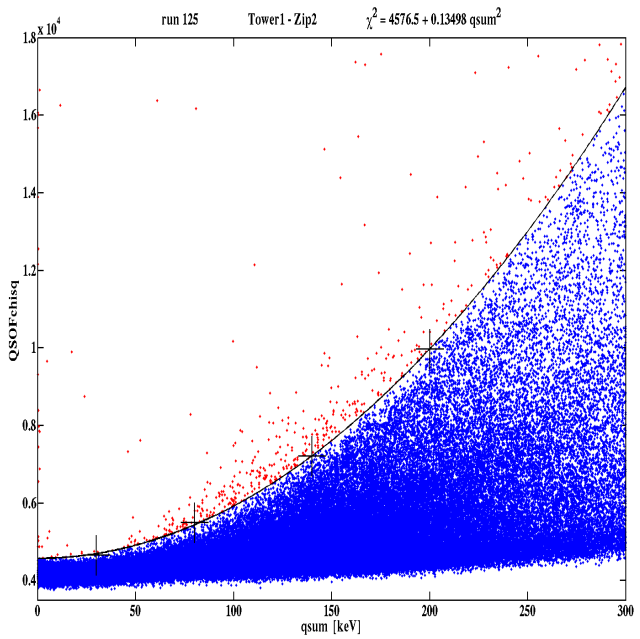

(a)

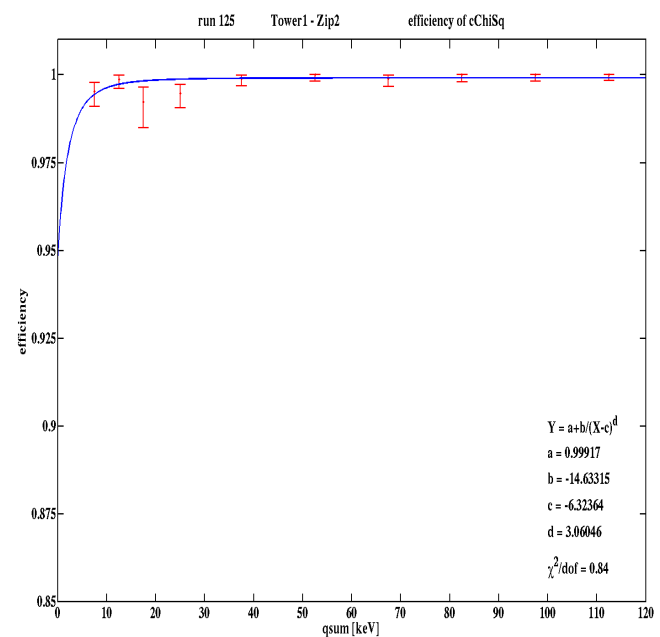

(b)

Figure 9.4: Definition and efficiency of the charge reconstruction quality cut. The cut is defined (a) by a quadratic form fitted to the $\chi^{2}$-values (QSOFchisq) as a function of summed ionization energy (qsum) (black line in the figure). Events passing the cut are shown in blue while events failing the cut are shown in red. (b)The efficiency of the cut is measured as a function of energy on WIMP search data. A functional from is fitted to the efficiency (red line). Figures provided by S. Arrenberg.

the summed ionization signal (for events within the fiducial volume (see Section 9.4.3) the summed ionization signal equals the inner electrode ionization signal). To obtain the efficiency as a function of phonon recoil energy for nuclear recoils the phonon energy is scaled by the centroid of the nuclear recoil band (see Section 7.4.2).

\subsubsection{Cryocooler cycle}

The cryocooler mounted to the E-stem (see Section 5.1.1) transmits substantial mechanical vibrations to the icebox during portions of its 1.2 seconds refrigeration cycle. The phonon channels and most ionization channels are essentially immune to these vibrations, but a few ionization channels show significant cryocooler induced microphonic noise pickup. The most sensitive channels are the inner ionization channels of T1Z4, T3Z2 and T3Z5 and the outer ionization channels of T1Z4, T2Z1, T2Z5, T3Z2 and T3Z5. Less sensitive channels are the inner ionization channels of T2Z3 and T2Z5 as well as the outer ionization channel of T4Z2. The difference in the average noise power spectrum of the inner ionization channel of T1Z4 with the cryocooler turned on and off is shown in figure 9.5. With the cryocooler in normal operation microphonic noise pickup is clearly visible at frequencies near and below $10 \mathrm{kHz}$. Since the resolution of the ionization signal is dominated by low frequency noise, this increased noise significantly affects the ionization pulse reconstruction (see Section 6.2.3). A higher noise level on the ionization channels does not only affect the resolution 


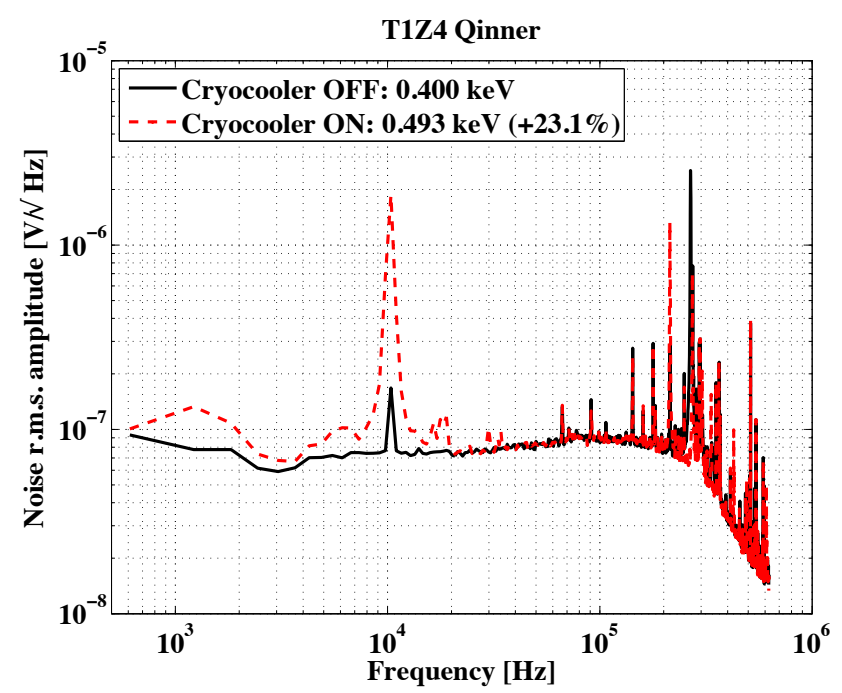

Figure 9.5: Noise power spectrum of T1Z4's inner ionization channel. The solid/black line shows the spectrum if the cryocooler is deactivated, while dashed/red shows the power spectrum with the cryocooler in normal operation. Cryocooler induced microphonic peaks are clearly visible near and below $10 \mathrm{kHz}$. The legend in the figure gives the r.m.s resolution of the ionization signal. Figure taken from [173].

of the reconstructed ionization energies (affecting the yield discriminator at low energies) but also the phonon delay which is measured with respect to the ionization pulse.

To remove periods with a high noise level the, standard deviation of the ionization pre-pulse baseline is investigated. This standard deviation is calculated for each event. For each series the distribution of the pre-pulse baselines is fitted by a Gaussian. Events are rejected in the analysis if the following cut criteria is fulfilled:

- The ionization pre-pulse baseline of an events lies $4 \sigma$ above the mean.

The series by series definition of the cut allows a tighter cut definition for low noise periods and prevents time periods with elevated baseline noise from being removed entirely. For some detectors, which show a large cryocooler sensitivity the cut is set more strictly:

- T2Z3: Inner ionization channel cut set at $3 \sigma$ above mean.

- T3Z5: Inner and outer ionization channel cut set at $3 \sigma$ above mean.

- T4Z5: Inner ionization channel cut set at $2.5 \sigma$ above mean.

For non cryocooler sensitive detectors the efficiencies are $\geq 99 \%$, while for sensitive detectors the efficiencies can be as low as 70\% (T3Z5). This efficiency is a reduction in livetime which is taken into account in the calculation of the final detector exposure (see section 9.5). 


\subsubsection{Phonon pre-pulse baseline}

Events showing an abnormally large variation in the phonon pre-pulse baseline (the first 400 digitizer samples in the trace) are rejected in the analysis. For each detector and phonon channel the standard deviation (PXstd, $\mathrm{X}=\mathrm{A}, \mathrm{B}, \mathrm{C}, \mathrm{D}$ ) of the pre-pulse baseline is calculated and the distribution is fit to a Gaussian. The cut is set to remove events for a given detector with PXstd more than $5 \sigma$ away from the mean of the channel. Since the samples on which the standard deviation is calculated occur well before the phonon trigger, they should just sample the noise on the given channel. In addition, phonon pulses also have substantially longer fall times than ionization pulses, so a trace can contain a phonon pulse tail from a previous trace, but not an ionization pulse tail. This cut serves as an additional protection against pile-up, as well as rejecting events with unusual phonon noise in general.

The phonon noise of the single channels varies substantially with time and there are periods where the noise variations lead to significant changes in the values of the pre-pulse standard deviations. For this reason, the thresholds for each channel were fit series-byseries to prevent cutting entire series with elevated noise and to cut more tightly on series with low noise. The efficiencies for this cut are measured on random triggers in the WIMP search data, by determining the fraction of random triggers which exceed the $5 \sigma$ threshold, and are $>99.9 \%$ for nearly all detectors.

\subsubsection{Phonon pulse start time}

In a multi detector setup it can happen that a particle hitting a detector issues a trigger, and a second unrelated particle hits another detector within the digitization time window. This reconstruction pathology is called "cross-detector pile-up". There is no overlap of the pulse traces like for usual pile-up events, so each pulse is properly formed, but the pulse in the second detector occurs unusually late within the digitization window.

Cross-detector pile-up is problematic since the data processing package uses fixed windows in the trace to search for the phonon and charge pulse. The package only searches for the start time of Ge ionization (phonon) pulses within a window of $[-100+10] \mu \mathrm{s}([-50$ $+200] \mu \mathrm{s})$ around the trigger time. The second particle event may occur outside of this search window. If a pulse lies outside of the optimal filter search window an incorrect start-time of the pulse will be selected, resulting in anomalously low amplitudes.

The rise-time of the phonon pulses is determined by a walking algorithm along the trace independently of the optimal filter, thus the primary phonon rise-time is generally correct for such events, while the primary phonon delay is anomalously long (the ionization start time is based upon the optimal filter and thus incorrect).

Such cross-detector pile-up events are rejected by enforcing that the walked start time of the primary phonon pulse lies within the optimal filter window for the ionization channel:

- PXr20 (where $\mathrm{X}=\mathrm{A}, \mathrm{B}, \mathrm{C}, \mathrm{D}) \in[-50,+10] \mu \mathrm{s}$ around the trigger time.

This corresponds to the overlap of the ionization and phonon search windows. 
This reconstruction pathology is very rare at the low acquisition rates of WIMP-search data but relative common during calibration runs. This selection cut has very good efficiency, essentially 100\% for nuclear recoils above $10 \mathrm{keV}$, but there is a small decrease in efficiency at very low energies which is taken into account in the final analysis efficiency (see Section 9.6).

\subsubsection{Position correction manifold quality}

Degeneracies in the phonon position correction manifold can lead to erroneous correction of the data (see section 7.3.2). Especially if an event lies far away from the nearest neighbors a miscorrection is expected, since it is corrected against a dissimilar population of events. These miscorrections are especially dangerous if the timing parameters of a surface-event are miscorrected, such that the surface-event mimics a nuclear-recoil. For each event a $\chi^{2}$-value based on the distance of the event in the manifold with respect to its nearest neighbors is calculated. Events with a $\chi^{2}$-value greater than a certain cut value are rejected in the analysis. The actual cut value has been determined by an optimization of accepting nuclear-recoils and rejecting as much surface-events with high $\chi^{2}$-values as possible. The efficiency of this cut is determined as a function of energy as part of the surface-event rejection cut (see Section 9.4.4).

\subsection{Event selection cuts}

A valid WIMP candidate event has to meet specific criteria defined in the analysis. These selection criteria are based on the recorded event properties and run conditions and are set to minimize the expected background in the signal region. Any of these criteria used in this analysis are defined in the following sections.

\subsubsection{Veto-anticoincident events}

The primary purpose of the plastic scintillator veto surrounding the experimental apparatus is to reject muons hitting the experimental setup. Such muons could produce neutrons in the experimental setup which would result in signals indistinguishable from WIMP interactions. Thus any recorded event in the detectors coincident with an activity in the scintillator veto is rejected in the analysis. On average one muon per minute is incident on the veto, while the mean veto rate is approximately $400 \mathrm{~Hz}$. This high veto rate is caused by ambient gammas in the experimental cavern. The detectors are protected against these gammas by additional passive shielding (see Section 5.1.2) and powerful discrimination (see Section 6.5), but these gammas would lead to an unacceptable loss in livetime if they were allowed to trigger the veto rejection cut.

The amplitude trace of every panel is recorded for every event in a time window of $[-120+20] \mu$ s relative to the global trigger. In addition the history of events exceeding a 


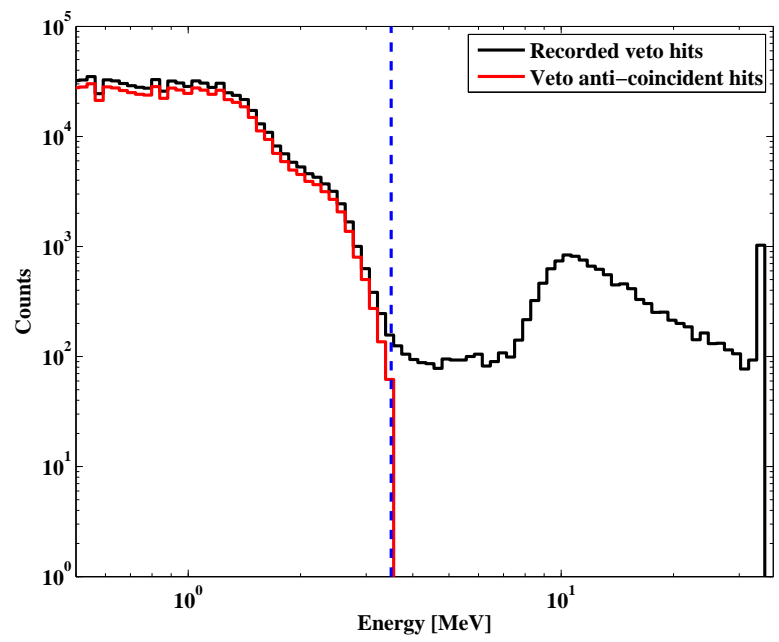

Figure 9.6: Histogram of WIMP search veto events for a representative veto panel. The black line shows all veto triggers with a muon peak around $10 \mathrm{MeV}$ deposited energy. The red line shows the veto anti-coincident events, predominantly gamma events rather than muon showers. The blue line indicates the energy corresponding to the fixed photoelectron signal threshold, as defined by the selection criteria in this analysis.

discriminator hardware threshold is recorded. Every event satisfying at least one of the following criteria is rejected from the analysis

- The photoelectron signal in at least one scintillator panel lies above a panel specific threshold within the recorded veto trace window.

- A discriminator threshold is exceeded at less than $50 \mu$ s before or during the event trigger

The veto trace threshold is set high enough to exclude essentially all gamma events, the cut position for one panel is plotted in figure 9.6, indicating a threshold near the upper edge of the ambient gamma distribution, but well below most muons and muon induced shower hits. The histogram of all recorded events (shown in black in the figure) shows a knee at the highest ambient gamma energy $(2.6 \mathrm{MeV})$ and a distinct muon peak around $10 \mathrm{MeV}$ the last bin in the histogram is caused by saturation.

Typical discriminator thresholds correspond to approximately $2 \mathrm{MeV}$. Thus, the majority of discriminator hits are not caused by muons but rather by high energetic ambient gammas. Since the trigger rate of the scintillator veto is approximately $400 \mathrm{~Hz}$ this corresponds to about a $2 \%$ probability of a veto activity in a randomly selected $50 \mu$ s window.

The analysis inefficiency of the veto-anticoincident cut is defined as the probability that an event caused by a WIMP is tagged as veto-coincident and thus rejected as a WIMP candidate. This inefficiency is calculated by finding the fraction of randomly-triggered events that are tagged as veto-coincident by the selection criteria mentioned above. From this a resulting efficiency of $97.88 \%$ is determined, this efficiency is introduced as a loss of live time into the analysis due to the scintillator shield event rate. 


\subsubsection{Single-scatters}

Due to their small interaction cross section WIMPs are expected to give a signal in a single ZIP only. Thus the other detectors are used as veto detectors to reject background events. A single-scatter is classified by a significant deposition of energy in one and only one detector. The energy deposition is measured on the total phonon signal including Luke phonon contributions (see Section 6.3). A valid WIMP candidate event should deposit energy above a certain threshold in the considered detector, and for all other detectors the recorded energy should be consistent with the expected noise fluctuations. Thus more strictly speaking the single-scatter cut is actually a multiple-scatter rejection cut. The single-scatter selection is defined by:

- The energy deposition in the considered detector lies more than $6 \sigma$ above the mean of the noise distribution.

- The recorded energy in all other detectors should lie within the $4 \sigma$ noise distribution.

The single detectors show a variation in noise performance from data series to data series. To account for the variations in the noise performance, the noise distribution, as measured on random triggers at the beginning of each series, has been fitted to a Gaussian distribution to determine the mean and resolution for each detector and data series. In figure 9.7 the variation of the noise distribution's mean values and resolutions for detectors in Tower 3 throughout the data taking period of Run 125-128 are shown. The individual runs are separated by time periods of warm-up and cool-down during which no data is taken. In particular there are end of run periods in Run 125 and Run 127 for a couple of detectors, showing a much worse noise resolution. This is partially caused by the settlement of helium-films on several detectors resulting in a high phonon trigger rates (see Section 9.2.4). Especially in Run 127 the bad end of run performance was caused by a bad performance of single phonon channels. Data series showing a bad performance have been identified by calculating the mean noise performance over the whole run and selecting series in which the performance is worse than $25 \%$ above the mean for each phonon channel. The following procedure has been adopted to deal with such particular series:

- If a phonon channel exhibits high noise, neglect this channel while screening for multiples. In this case put in the ionization signal as an additional veto.

- If more than two phonon channels exhibit high noise, neglect the phonon signal and use only the ionization signal as a veto.

For all runs the ionization signal on detectors T1Z1, T5Z5 and T5Z6 is checked for signals $4 \sigma$ above the mean noise threshold in addition to the phonon signal, since these detectors show a relative poor phonon performance.

The efficiency of the singles cut is measured on random triggers used as a proxy to determine how often a fluctuation in noise exceeds the $4 \sigma$ threshold. The mean efficiency of the single-scatter cut used in the analysis is calculated based on all randoms within a 


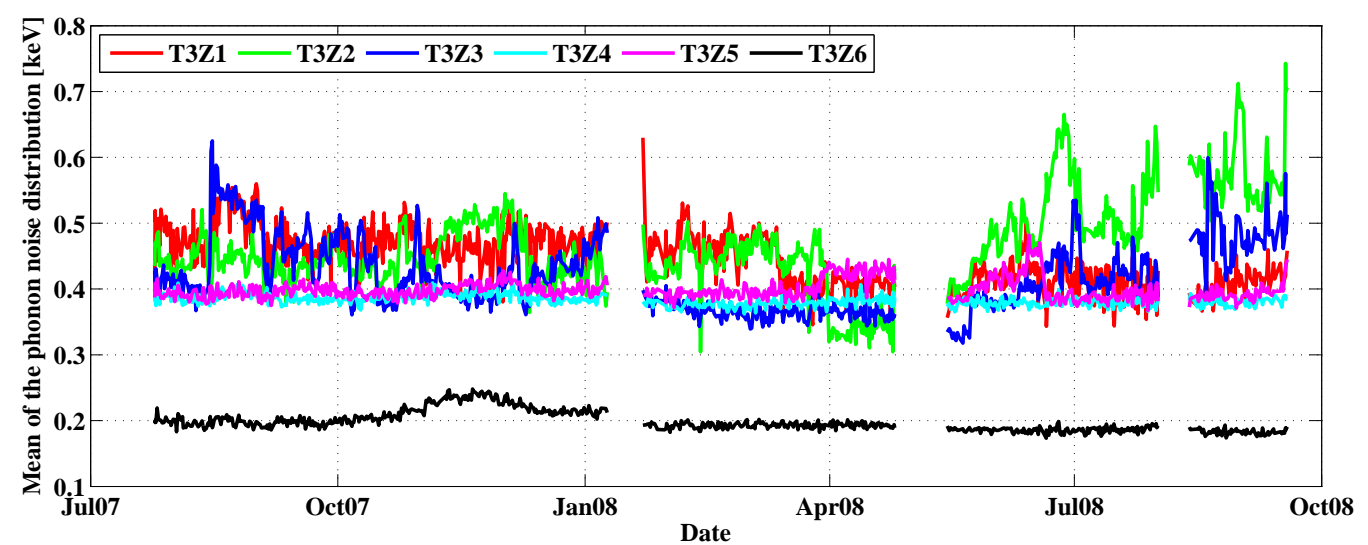

(a)

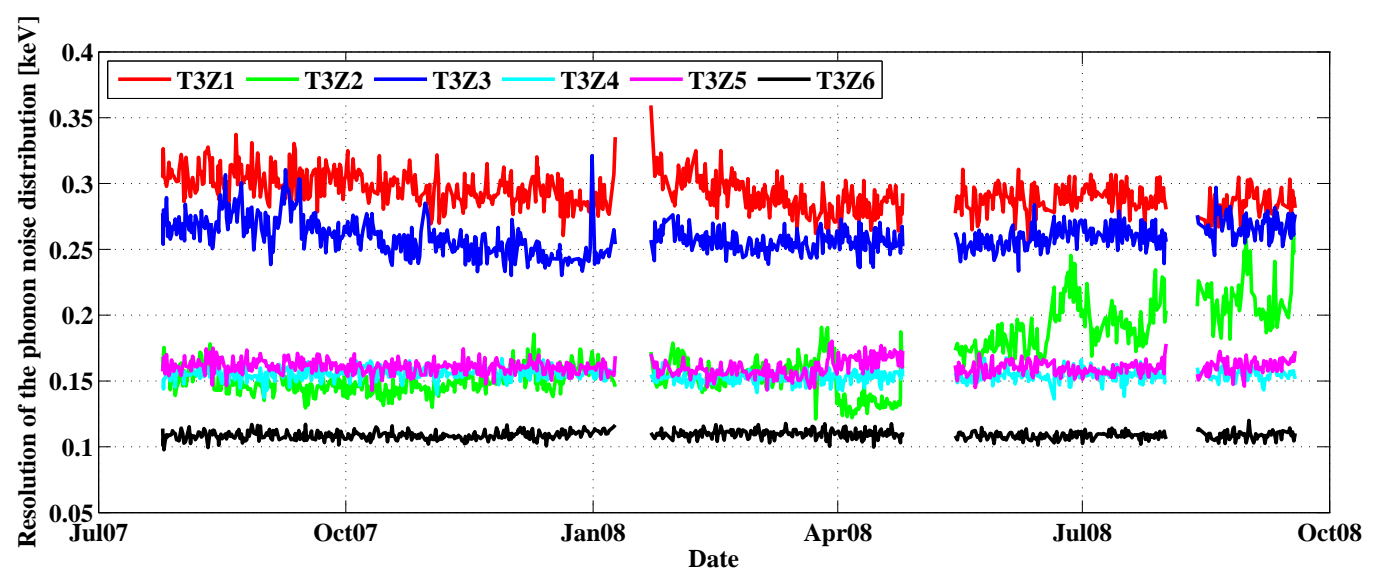

(b)

Figure 9.7: Variation of the mean and resolution of the noise distribution with time. The variation with time of the mean (a) and resolution (b) of the phonon noise distribution has to be taken into account in the rejection of multiple-scattering events. Data is shown for detectors in T3 throughout the data taking period of Run125-128. Time intervals in which no data is shown mark the warm-up and cool-down periods between the single runs.

run. Figure 9.8 below compares this mean efficiency with the efficiency calculated on a series by series basis. The mean efficiencies in Run 125 and Run 126 are generally around 99.4\%. In Run 127 many detectors suffer from poor performance of the single scatter cut at the end of the run due to helium-films, resulting in mean efficiencies slightly below $99 \%$. The mean efficiencies in Run 128 are close to $99 \%$. 


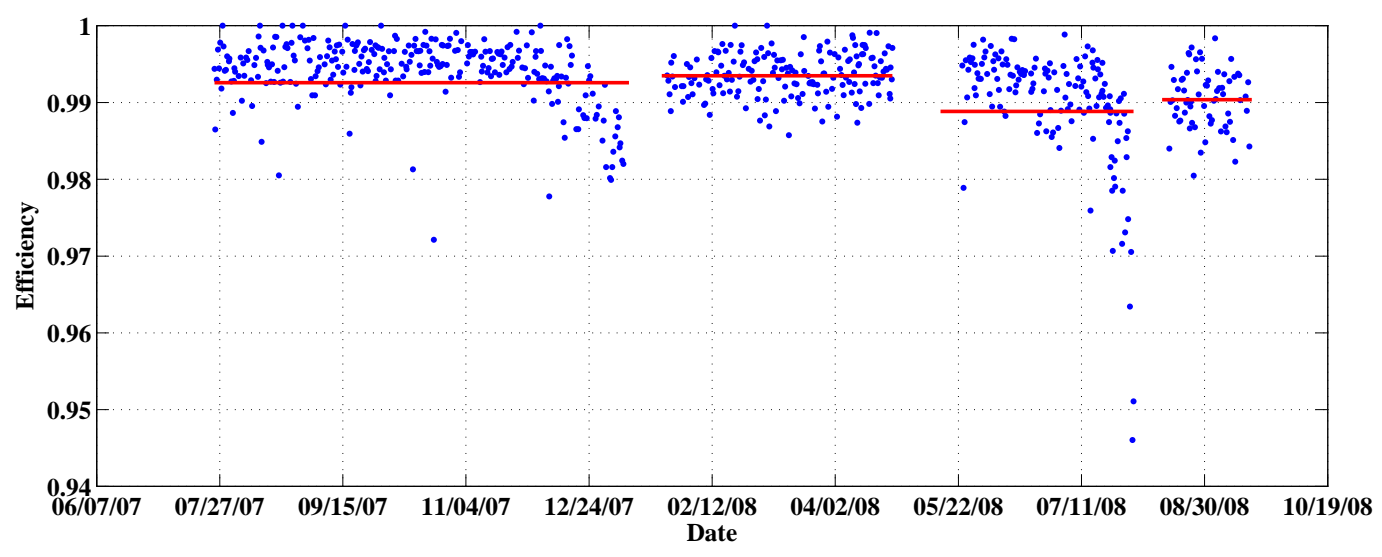

Figure 9.8: Efficiency of the singles cut throughout the data taking period. The efficiencies, calculated on a series by series basis (blue dots), are compared to the mean efficiencies calculated over the whole run (red line). Efficiencies are shown for a representative detector.

\subsubsection{Fiducial volume}

Events at very large radius, near the outer rim of the detectors may be corrupted by distortions of the electric field configuration, poor phonon sensor coverage and chargedeficit effects along the outer surface. In order to reject such events from the analysis, the relationship between the inner- and outer-electrode ionization signal is used. Any event with substantial energy in the outer-electrode is near the outer detector rim and is excluded from the WIMP search analysis. The fiducial volume based on the ionization signal is defined by requesting that:

- The ionization signal in the outer-electrode is consistent with the $2 \sigma$ noise distribution of the outer-electrode.

In the definition of the cut, Gaussian distribution functions are fitted iteratively to the outer-electrode ionization signal distributions from ${ }^{133} \mathrm{Ba}$ calibration data in bins of the inner-electrode ionization signal. The means of the distributions are fitted to a first order polynomial and the widths to a second order polynomial to define the cut as a function of the inner-electrode ionization signal. Any event which lies $2 \sigma$ away from the mean is rejected in the analysis.

The efficiency of the ionization based fiducial volume cut is measured on neutron induced nuclear recoils from ${ }^{252} \mathrm{Cf}$ calibration data in several energy bins. A first order polynomial is fitted to the measured efficiencies to obtain a functional form as a function of the recoil energy. The measured efficiencies are shown in figure 9.9 for a representative detector. On average the efficiency is $\sim 75 \%$ at $10 \mathrm{keV}$ recoil energy, dropping to $\sim 70 \%$ near $100 \mathrm{keV}$ recoil energy. These values are below the nominal value of $\sim 80 \%$ based upon the area of the detector face surface covered by the inner electrode (see section 6.2.1). 


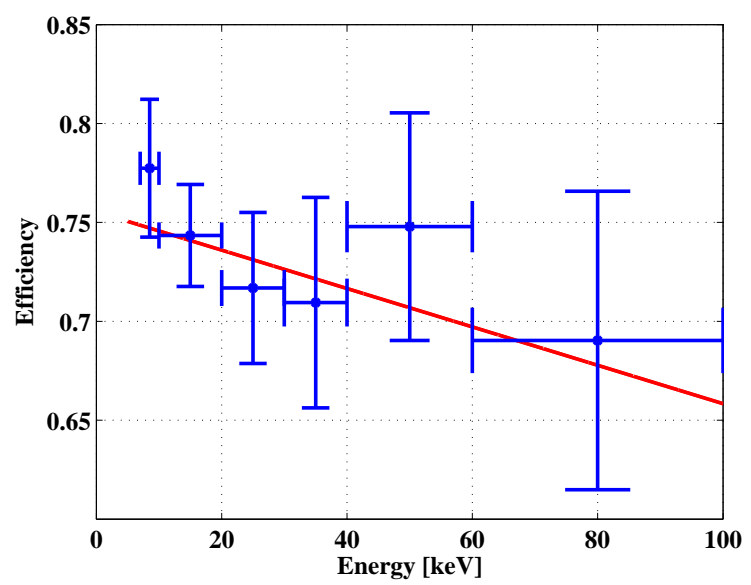

Figure 9.9: Efficiency of the ionization signal based fiducial volume cut. Measured efficiencies on nuclear recoils (blue) at several energies are fitted by a first order polynomial (shown in red). Results shown for a representative detector.

The reduced efficiency and energy dependency of the ionization signal based fiducial volume cut may be due to a combination of at least two effects:

- Any small charge sharing effect between the inner- and outer-electrode will lead to a decrease in efficiency at higher energies, since at high energies the ionization signal on the outer-electrode may exceed the cut set on the noise distribution of the outerelectrode.

- Neutrons used for the evaluation of the efficiency have a significant rate of internal multiple-scattering in a detector, leading to an increase in signals shared between the inner- and outer-electrode and thereby reducing the efficiency of the fiducial volume cut. In addition neutrons also have a finite attenuation length, and are more likely to interact in the outer detector regions than in the bulk volume.

Note that the latter effect does not apply for WIMPs, which are expected to interact uniformly in the detector volume and only scatter once, making the applied measurement of the fiducial volume efficiency a slight underestimate. To correct for this underestimation a correction factor has been determined from a dedicated Monte Carlo simulation. In a GEANT4 simulation the neutron calibration of the detectors are simulated. By varying a radial cut position, the fraction of nuclear-recoils passing the fiducial volume cut in the simulations is determined which matches the actual measured efficiency averaged over the energy range from $10-100 \mathrm{keV}$. This fraction is then compared to a volume fraction at the given radial cut position. The volume fraction is the efficiency expected for WIMPs which interact uniformly in the detector. The correction factor for measuring the fiducial volume with neutrons averaged over the 14 detectors used in this analysis is $95.48 \pm 0.82(\text { stat })_{-0.45}^{-0.65}($ sys $) \%$. This correction factor is taken into account in the final analysis efficiency (see Section 9.6) increasing the detection efficiency for WIMP interactions. 


\subsubsection{Surface-event rejection}

Contaminants like ${ }^{210} \mathrm{~Pb}$ on the detector surfaces and ambient gamma induced electrons generate electron recoils within the first few microns of the detector surface. Poor charge collection at the surface reduces the ionization yield of such events causing them to mimic nuclear-recoils. As a result, surface-events are a significant background source in CDMS-II (see Section 6.5.1 and Chapter 8). This background distribution can be rejected by phonon pulse time characteristics; surface-events are more prompt pulsed in time than events in the detector bulk. The timing characteristics are measured by several algorithms during the event reconstruction (see Section 6.4.3) and are corrected for any position/energy dependence (see Section 7.3 ).

To minimize the background from surface-events and maximize the signal efficiency (defined by maximizing the nuclear-recoil acceptance), a surface-event rejection cut can be tuned to the desired surface-event background in the blinded signal region (approximately given by the $2 \sigma$ nuclear-recoil band) and signal efficiency. For the definition of the cut, surface-event and bulk nuclear-recoil samples from ${ }^{133} \mathrm{Ba}$ and ${ }^{252} \mathrm{Cf}$ calibration data are used. The main difficulty in defining this rejection cut on calibration data are systematic differences in the surface-event population in the calibration sample and WIMP search data. The "wide surface-event sample" is selected by requesting that the ionization yield satisfies the following condition:

- Ionization yield less than the minimum of [ $5 \sigma$ below electron recoil band, 0.7] and is greater than 0.1 (for an illustration of the selection see figure 6.13).

\section{Difference in the ionization yield distribution}

The difference in the ionization yield distribution between the signal region in WIMP search data and the electron-recoil calibration sample is primarily due to the fraction of phonon and charge side events, since charge side events typically have a higher yield than phonon side events. Figure 9.10 illustrates the ionization yield distribution of charge and phonon side surface-events in low background data (a) and the ${ }^{133} \mathrm{Ba}$ calibration sample (b). For the figures data from all interior detectors has been taken into account. The distributions are given as a function of distance from the nuclear-recoil centroid measured in units given by the width of the nuclear-recoil distribution for each considered detector. The comparison of the two figures clearly shows that charge side surface-events are much less present in the calibration sample than in WIMP search data. This is mainly caused by an asymmetric illumination of the detectors during the calibration runs, related to the source location. In general, charge side surface events tend to have more slow timing outliers in the phonon pulse timing characteristics than phonon side events, leading either to a higher passing fraction of these events for a given cut or demanding a much more restrictive cut setting at the cost of signal efficiency. The distributions of charge side and phonon side events in a simple timing discriminator, the sum of the phonon pulse delay and rise-time (the choice of this simple discriminator will be discussed in detail below), are compared to the distribution of neutron induced nuclear recoils from ${ }^{252} \mathrm{Cf}$ calibration 


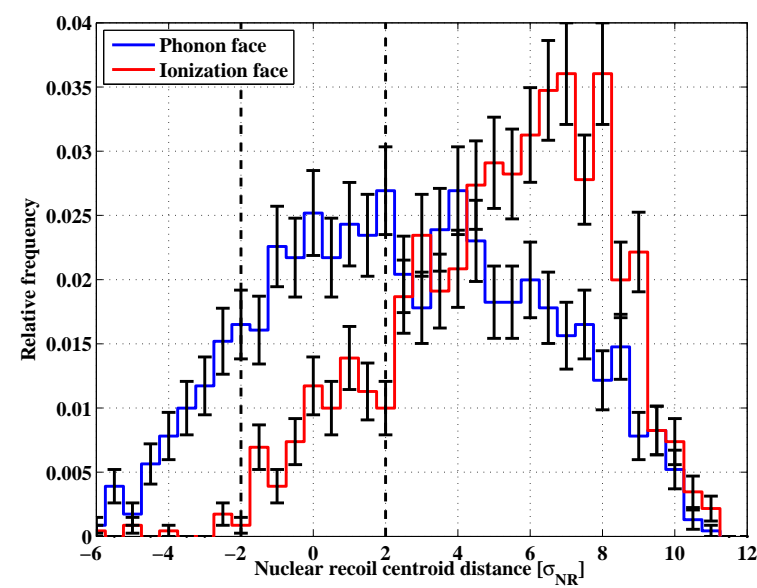

(a)

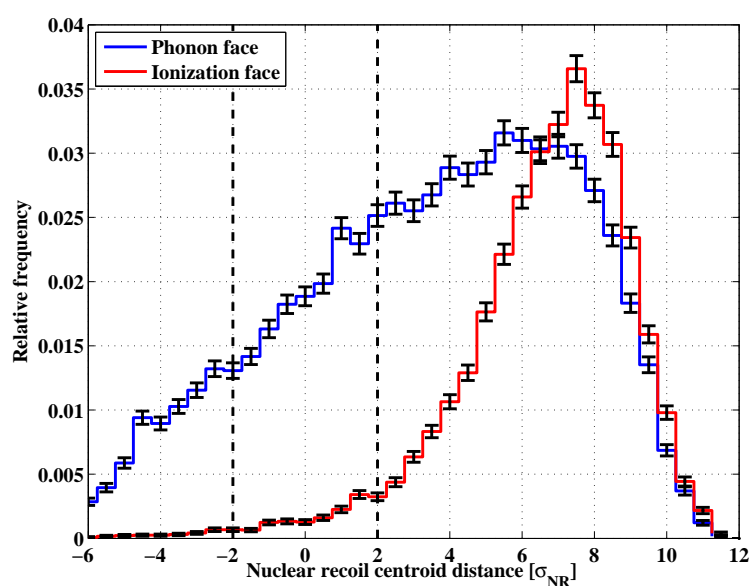

(b)

Figure 9.10: Ionization yield distributions of face selected surface-events in WIMP search (a) and ${ }^{133} \mathrm{Ba}$ calibration (b) data. The distributions are shown as a function of the distance from the nuclear-recoil centroid, measured in units of the width of the nuclear-recoil distribution. The black vertical lines enclose the approximate signal region. For the figures data from all interior detectors has been used.

data in figure 9.11. From this figure it is directly visible that the passage fraction of any rejection cut will be determined by long tails/slow outlier events in the calibration data.

\section{Difference in the energy distribution}

The background in the WIMP search signal region is given by single-scatter events, which show a significantly higher fraction of low energy events than the face selected ${ }^{133} \mathrm{Ba}$ calibration data sample. The energy distribution of single-scatters in the nuclear-recoil band from the Run 123/4 WIMP search data (the actual energy distribution of the background should not have significantly changed since the last analysis, since the background sources did not change) is compared to the face selected energy distributions from calibration data for a representative detector in this analysis in figure 9.12. In addition, the timing characteristics of low energy events flare because of an increased signal to noise ratio, leading to slower values in the timing characteristics, making them similar to bulk nuclear recoils. Cuts defined on ${ }^{133} \mathrm{Ba}$ data weight such events much less in the leakage estimate of single-scatter events passing the surface-event rejection cut in the signal region.

These two systematics cause the surface-event leakage estimate derived from the ${ }^{133} \mathrm{Ba}$ calibration data to be systematically lower than the expected leakage from WIMP-search side bands (the actual procedure for the leakage estimate will be discussed in Section 10.1.2). In order to account for these systematic differences, scaling factors between the distributions in the calibration sample and the WIMP search signal region derived from the previous analysis (Run 123/4) were used in this analysis, to allow a more precise setting of the rejection cut and an accurate leakage estimate. 


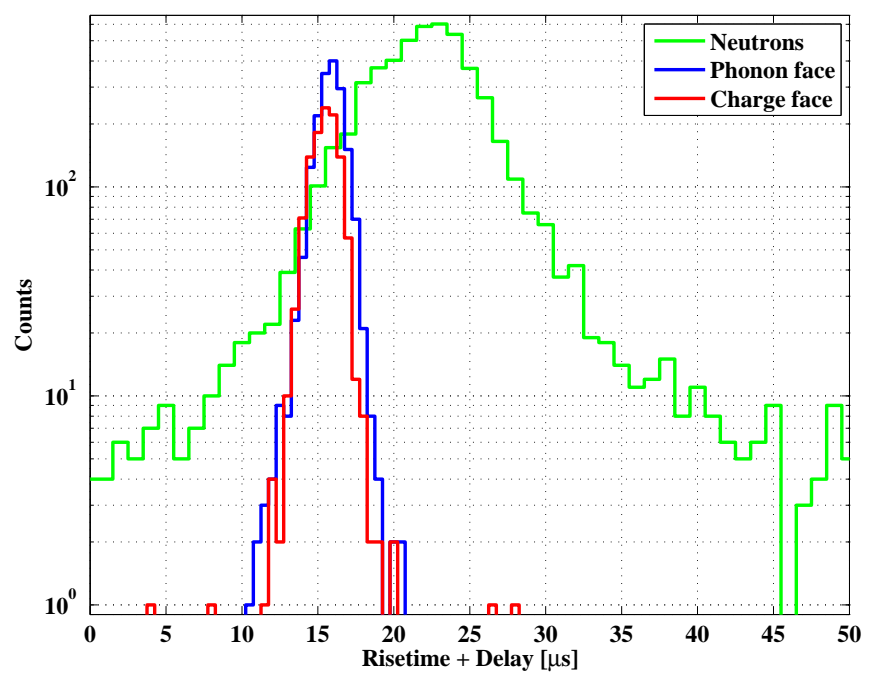

Figure 9.11: The distribution of face selected surface-events (blue/red histograms) are compared to the distribution of neutron induced nuclear recoils from ${ }^{252} \mathrm{Cf}$ data (green histogram). Rejecting all surface-events comes at the cost of a loss in nuclear-recoil acceptance. The passage fraction of any rejection cut is determined by long tails in the surface-event distributions, or by slow timing outliers like the two charge side events present in the figure. Data is shown for a representative detector.

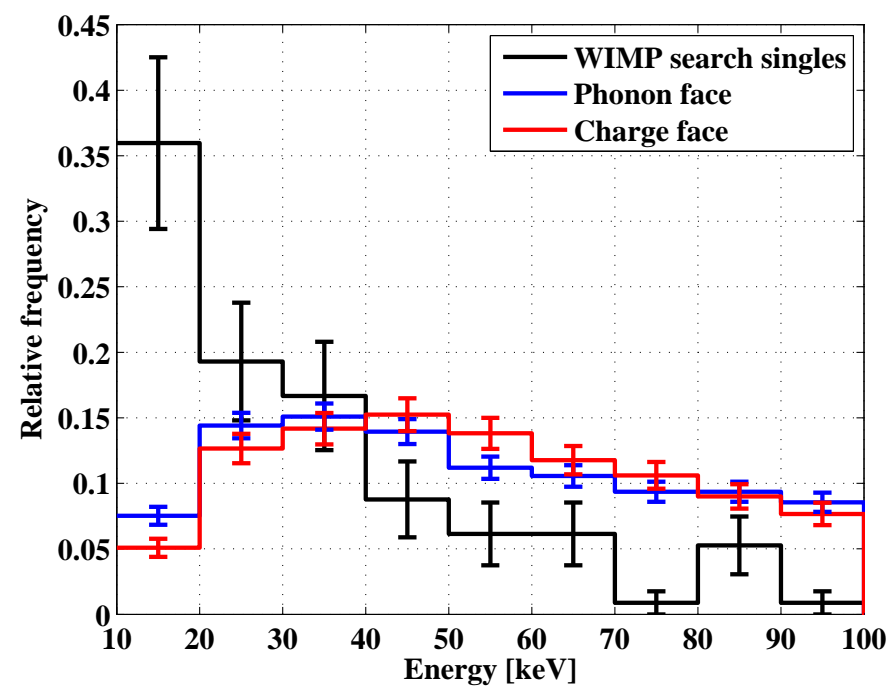

Figure 9.12: The energy distributions of face selected ${ }^{133} \mathrm{Ba}$ calibration surface-events for a representative detector are compared to the energy distribution of WIMP search single scatters in the nuclear-recoil band taken from the Run 123/4 data set. All detectors used in the Run 123/4 are combined to obtain the energy distribution of the WIMP search single-scatters. 
The purpose of the surface-event rejection cut is to keep the expected number of singlescatter events passing the cut $(\eta)$ in the signal region at a desired level (usually $\sim 0.5$ events) while maximizing the signal efficiency, as measured on neutron induced nuclear recoils. The total leakage $\eta$ is given by the sum of the leakages of all considered detectors $n_{i}$. The expected leakage of a single detector is defined as

$$
n_{i}=N_{i} \sum_{j, k} b_{j k} s_{j k}
$$

such that $n_{i}$ is the sum of the passage fractions for a given bin (in energy and face selection) $b_{j k}$, times a scaling factor $s_{j k}$, which gives the expected fraction of WIMP search nuclear recoil single-scatter events in that bin. The scaling factors $s_{j k}$, have been derived from ${ }^{133} \mathrm{Ba}$ calibration data and Run 123/4 WIMP search data. The indices $j$ and $k$ run over bins by face selection and energy (three energy bins are chosen: [10 20] keV, [20 30] keV and $\left.\left[\begin{array}{ll}30 & 100\end{array}\right] \mathrm{keV}\right) . N_{i}$ is the expected number of nuclear recoil single-scatters for the $i^{\text {th }}$ detector obtained by scaling the number of observed nuclear recoil single-scatters in the Run 123/4 data by the increased exposure of the current analysis.

To optimize the signal efficiency for a fixed target leakage over all considered detectors, the following functional form is used:

$$
f(t)=\left(1-\frac{\sum_{i} S A E_{i}\left(t_{i}\right)}{\left(\sum_{i} S A E_{i}\right)_{\max }}\right)^{2}+\left(1-\frac{\sum_{i} n_{i}\left(t_{i}\right)}{\eta}\right)^{2}
$$

where $S A E_{i}\left(t_{i}\right)$ is the spectrum averaged exposure (for a definition see Section 9.7) for the $i^{t h}$ detector given the threshold of the surface-event rejection cut $\left(t_{i}\right)$ on that particular detector. Hence the first term achieves maximum summed exposure and the second term accounts for the target leakage.

The surface-event rejection cut is based on the primary phonon risetime and the delay of the primary phonon pulse with respect to the ionization pulse. The cut has two components, each exploiting the strong positive correlation between the risetime and the delay. A "consistency cut" demands that the difference between these parameters is consistent with the ${ }^{252} \mathrm{Cf}$ calibration nuclear-recoil population. The consistency cut is set at $\pm 4 \sigma$ of the mean neutron value for each individual detector. This cut is primarily intended to reject "outlier" events for which one or both timing parameters are incorrectly reconstructed. The cut's main discrimination power comes from the "discrimination threshold" which demands that the sum of the two timing parameters is above a chosen value $t_{i}$. This servers to separate the slower nuclear recoils from the faster surface-event background. This threshold is varied for each detector separately in the optimization of the surface-event rejection cut (see equation 9.4). Figure 9.13(a) shows the distribution of neutron induced nuclear recoils from ${ }^{252} \mathrm{Cf}$ and face selected surface-events from ${ }^{133} \mathrm{Ba}$ calibration data in the two dimensional phonon pulse characteristics parameter space. The consistency cut is given by the two lines with positive slope in the figure while the lower line with negative slope marks the discrimination threshold. The upper line with negative slope defines an upper consistency cut. Events in parameter space enclosed by all selection criteria (all four 


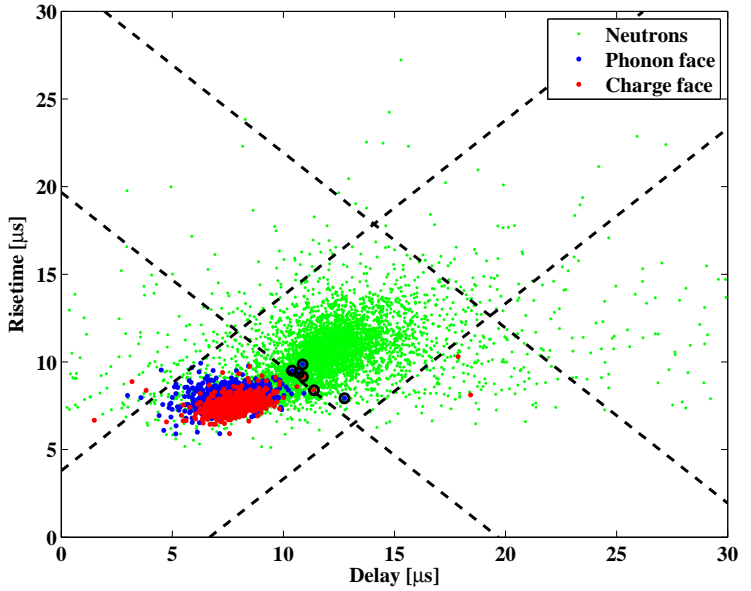

(a)

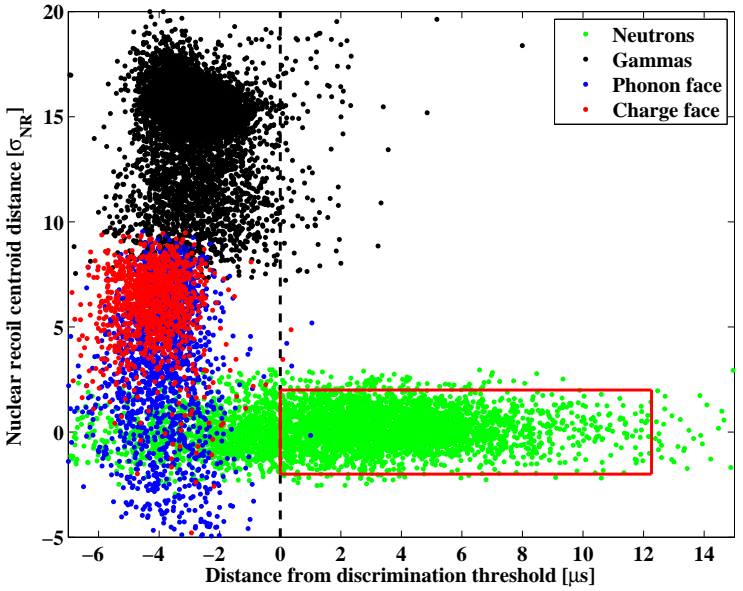

(b)

Figure 9.13: (a) Definition of the two dimensional surface-event rejection cut in the phonon pulse characteristics. Evens in parameter space enclosed by all selection criteria (represented by the black/dashed lines in the figure) are accepted. Lines with positive slope mark the consistency cut, while lines with negative slope define the discrimination threshold and an upper consistency cut. Surface-events passing the cut are highlighted by a black circle. (b) Normalized distributions of various event categories (see text). The red box displayed in the figure represents the signal region. Data shown for a representative detector.

lines in the figure) pass the surface-event rejection cut. Surface-events passing the cut are highlighted by a black circle in the figure. A normalized view of various event categories distributions is shown in figure 9.13(b). The distributions are normalized to the distance from the discrimination threshold and the distance from the nuclear-recoil band centroid measured in units of the width of the nuclear-recoil distribution. The signal region for this analysis is represented by the red box in the figure.

The final timing cut for this analysis has been set to achieve a target leakage of

$$
\eta=0.5
$$

The efficiency of the surface-event rejection cut as a function of energy is measured in several energy bins by the passage fraction of neutron induced nuclear recoils as it is shown in figure 9.14. The total efficiency of the surface-event rejection cut is the combined efficiency of the position correction manifold quality cut (see Section 9.3.5), the consistency cut and the discrimination threshold. The measured efficiencies are fitted by a functional form as shown in the figure. The mean efficiency for the interior detectors is $\sim 60 \%$, although there is a great detector by detector variation, only the two detectors at the bottom of the stack considered in this analysis (T3Z6 and T4Z6) show efficiencies as low as $\sim 10 \%$. These low efficiencies on these two detectors is intended, since they have a much higher background level (single-scatters in the nuclear-recoil band) than any of the 


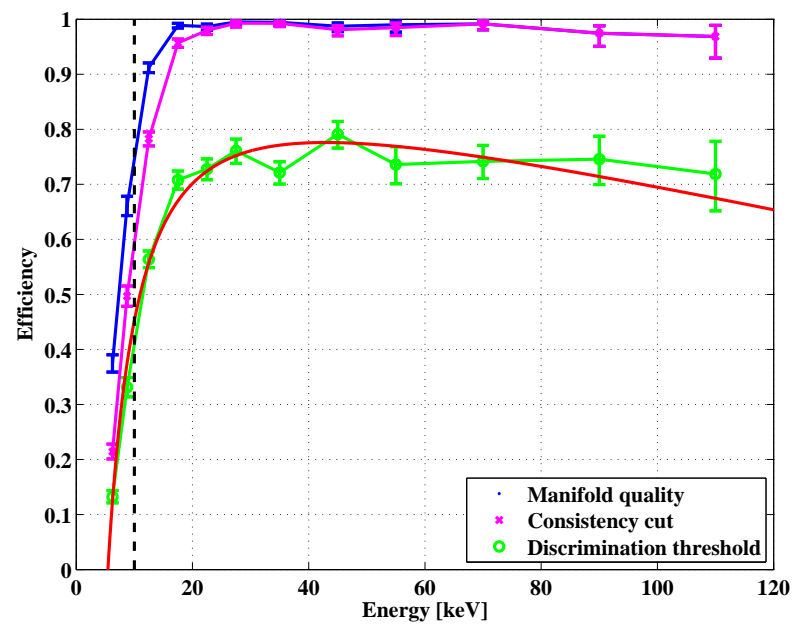

Figure 9.14: Efficiency of the surface-event rejection cut. The efficiencies measured on nuclear recoils in several energy bins are shown for the three cuts combined in the surfaceevent rejection cut. Each measured efficiency represents the total efficiency after adding the selection cut specified in the caption to the efficiency above. The measured efficiencies are fitted by a functional form (shown in red). Results shown for a representative detector.

interior detectors, due to the absence of a detector below vetoing additional multiples in the nuclear-recoil band. Thus, by cutting harder on these detectors the overall background level can be kept at the desired level while increasing the signal efficiency due to a looser cut setting for the interior detectors.

Other combinations of the timing characteristics of the phonon pulses have been studied, but non of these showed a significant improvement with respect to the chosen parameter combination. The main problem with simple $\chi^{2}$ based or more general higher dimensional Fishers's discriminant cut definitions, is the assumption that the used parameters follow a multi-dimensional Gaussian distribution. But clearly the data tells that this assumption is not true since the distributions show long tails and are sometimes not even Gaussian in the bulk of the distribution. If functional forms can be found which describe the data more accurately, better discrimination techniques like likelihood ratio tests could be used to increase the signal efficiency and improve the surface-event discrimination. Studies using generalized lambda distributions and kernel density estimators to obtain an accurate functional form of the distributions are ongoing and may be used in future analyzes.

\subsubsection{Ionization yield selection}

The scattering of a WIMP on an atomic nucleus results in a suppressed ionization signal with respect to particles scattering off electrons. Hence, any WIMP interaction is expected to be detected in the ionization yield distribution defined by nuclear recoils from the calibration of the detectors (see Section 7.4.2). It is thus demanded that, 


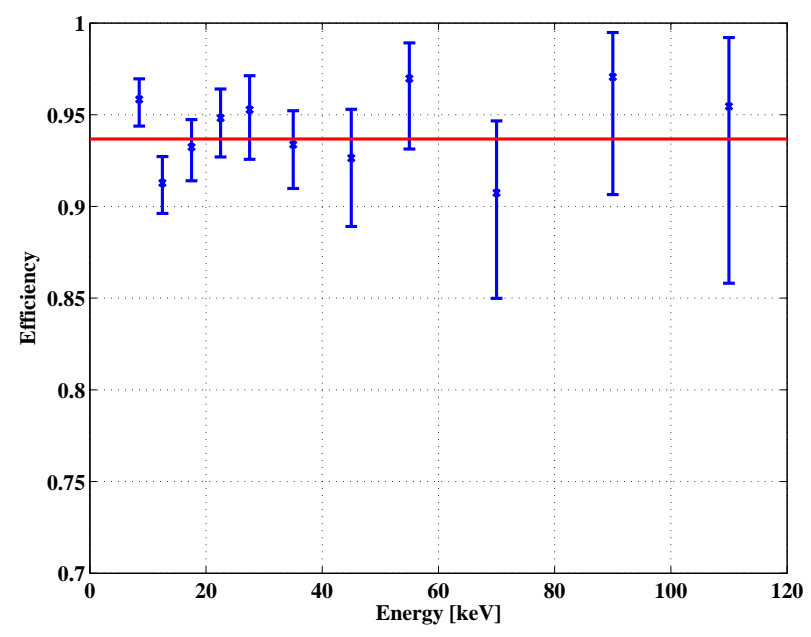

Figure 9.15: Efficiency of the ionization yield based nuclear-recoil selection. Efficiencies measured on nuclear recoils (blue) are fitted by a first order polynomial (shown in red). Efficiencies are shown for a representative detector.

- A valid WIMP candidate needs to have an ionization yield value which lies within $2 \sigma$ of the nuclear-recoil band centroid.

Variations in detector performance requested, that the nuclear recoil bands were determined for each run separately. The efficiency of selecting nuclear recoils is determined as a function of energy in several energy bins, by the ratio of events in the $2 \sigma$ and $4 \sigma$ nuclear recoil band. A linear function is fitted to the measured efficiencies to obtain a functional form of the efficiency as a function of energy. The determination of the nuclear recoil selection efficiency is illustrated in figure 9.15 for a representative detector. The efficiency for selecting nuclear recoils as measured from calibration data is usually $\simeq 94 \%$.

\subsubsection{Expected gamma leakage}

At low recoil energies, the nuclear-recoil band and the electron-recoil band are flaring to higher/lower ionization yields respectively, worsening the discrimination between electron recoils and nuclear recoils. Any non-surface electron recoil (primarily gammas interacting in the bulk of the crystals) which leaks from the electron-recoil band in the nuclear-recoil band may mimic a WIMP candidate event. Bulk electron recoils may have timing discriminator values passing the surface-event rejection cut (see Section 9.4.4), and thus the expected leakage of this event pathology should be kept at a negligible level. The expected leakage would be of purely statistical nature, by a random fluctuation in the reconstructed phonon and ionization signal. The expected gamma leakage can not easily be determined from the ${ }^{133} \mathrm{Ba}$ calibration data due to the presence of surface-events. Hence Monte Carlo simulations of gammas interacting in the bulk of the crystal are performed to determine the expected leakage. 

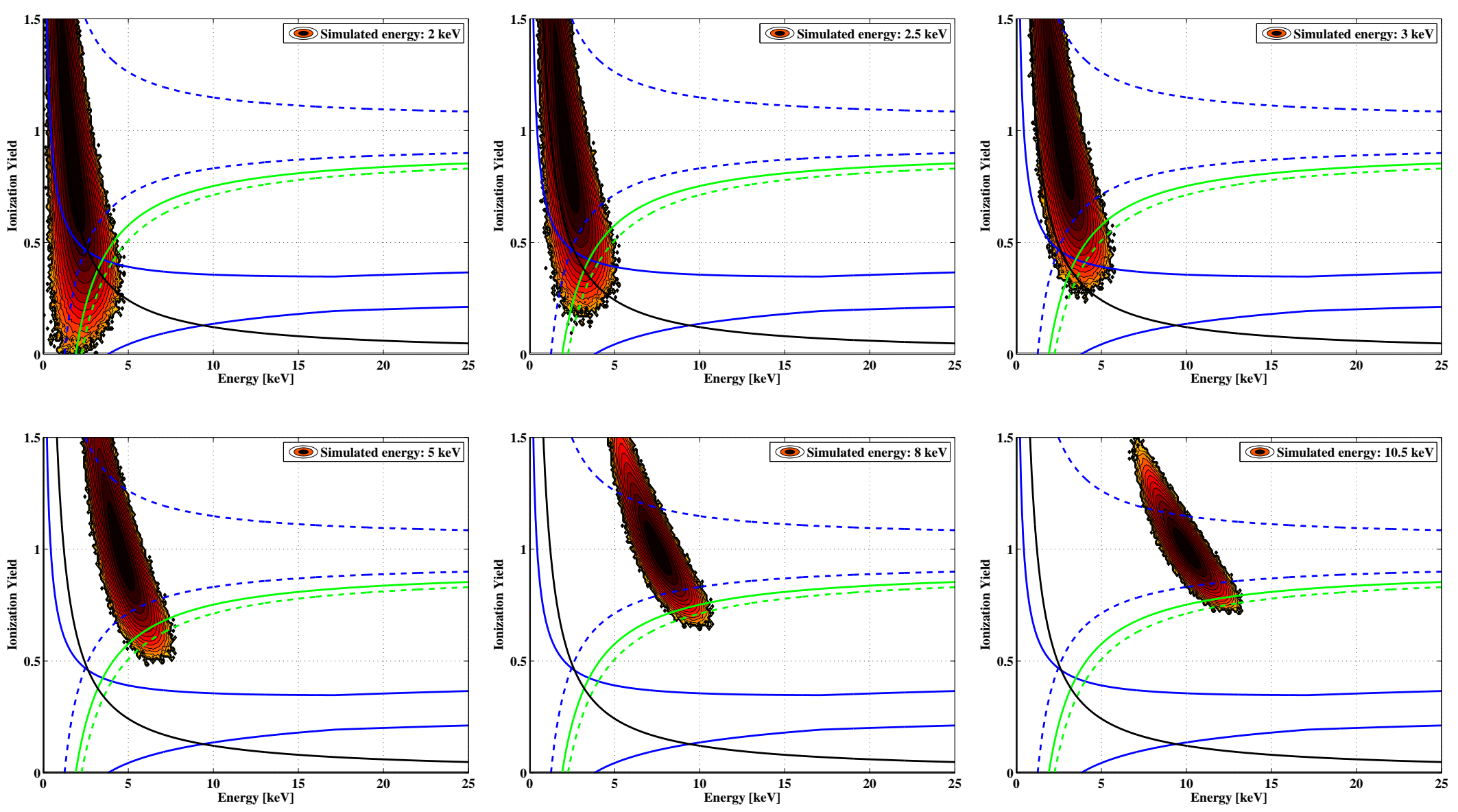

Figure 9.16: Yield distributions of the simulated energies for a representative detector for selected energies. The bending of the distribution to lower/higher yield is mainly caused by the slightly worse resolution of the phonon channel giving higher yield values at lower recoil energies and lower yield values at higher recoil energies. The true energy of the simulated events is given in each figure. The electron-recoil band (blue/dashed) and nuclear-recoil band (blue/solid) are shown along with a $3 \sigma$ (green/solid) and a $3.5 \sigma$ (green/dashed) below the electron-recoil band cut. In addition the ionization threshold cut is shown by the black/solid line. The main contribution to the leakage in the nuclearrecoil band are events with energies in the range from $1.5 \mathrm{keV}-5 \mathrm{keV}$, depending however on the specific detector performance. 
In the simulations $10^{8}$ events with an ionization yield of 1 have been generated. Energies in the range from $0-32 \mathrm{keV}$ in $0.5 \mathrm{keV}$ steps have been simulated. The phonon and ionization signal of these events have then been smeared by a Gaussian distribution function with width given by the width of the $10.36 \mathrm{keV}$ line (see Section 9.2.5). In this study the width at any energy is assumed to be given by the $10.36 \mathrm{keV}$ line. This assumption is conservative for energies below $10.36 \mathrm{keV}$ and the energy resolution is approximately constant below $10 \mathrm{keV}$. Also above $10 \mathrm{keV}$ the energy resolution should not dramatically change with energy. In any case leakage into the nuclear-recoil band is expected for energies well below $10 \mathrm{keV}$, thus, there is no contribution from higher energies to the leakage estimate. Figure 9.16 displays the yield distribution of some selected energies for a representative detector. From the figures it can be seen that the main contribution to the leakage in the nuclear-recoil band is caused by energies below $\sim 5 \mathrm{keV}$, depending however on the individual detector performance.

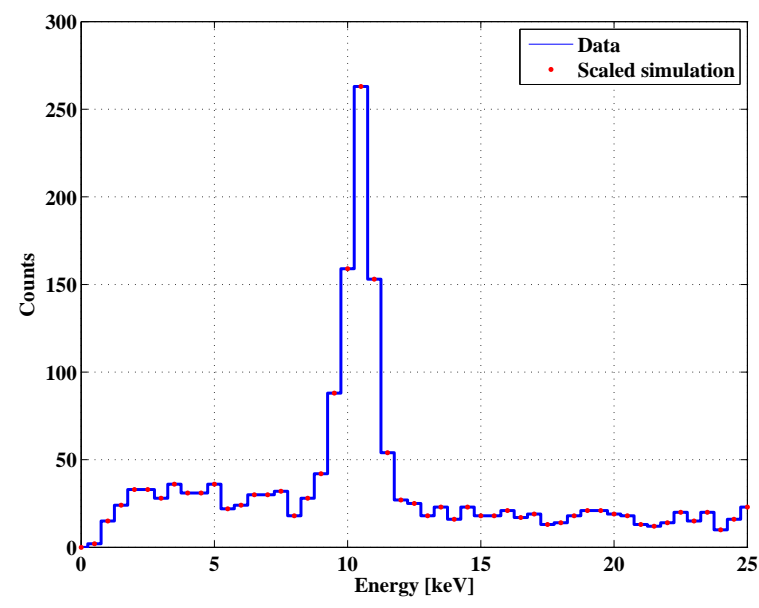

(a)

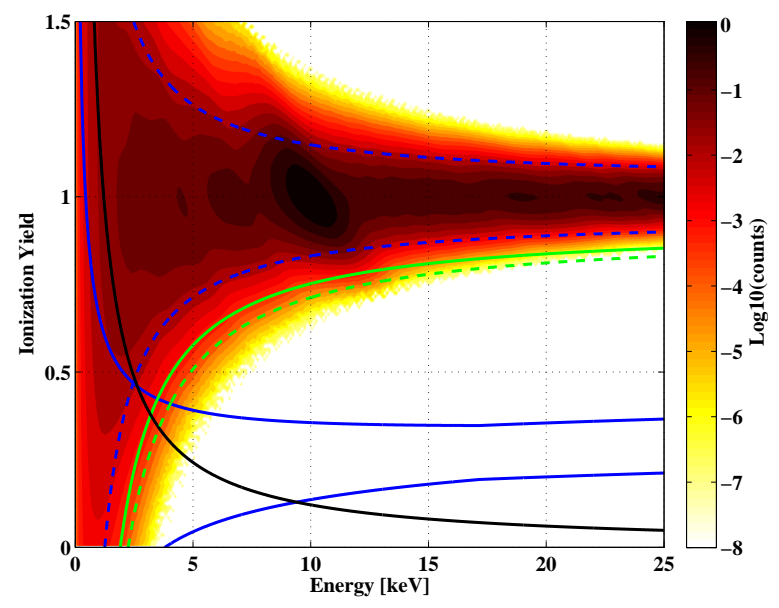

(b)

Figure 9.17: Scaling of the gamma simulations to the recorded low background spectrum. (a) The simulations are scaled to the observed low-energy spectrum in the electronrecoil band. (b) Scaled yield contours of the simulated gamma events. The electronrecoil band (blue/dashed) and nuclear-recoil band (blue/solid) are shown along with a $3 \sigma$ (green/solid) and a $3.5 \sigma$ (green/dashed) below the electron-recoil band cut. In addition the ionization threshold cut is shown by the black/solid line.

To predict the expected gamma leakage into the nuclear-recoil band the simulations have to be scaled to the actual measured low-background spectrum. The total leakage for the combined Run 125 - 128 data is estimated by adding the low-background spectra in the electron-recoil band and the simulations are scaled accordingly to match the spectrum in $0.5 \mathrm{keV}$ bins. The scaling factor for each of the simulated energies is found by minimizing the difference between the summed simulation spectrum and the measured spectrum. The comparison of the low-background spectrum with the scaled simulations is shown in figure 9.17(a), giving an excellent match between the simulation and the measured spectrum. For 


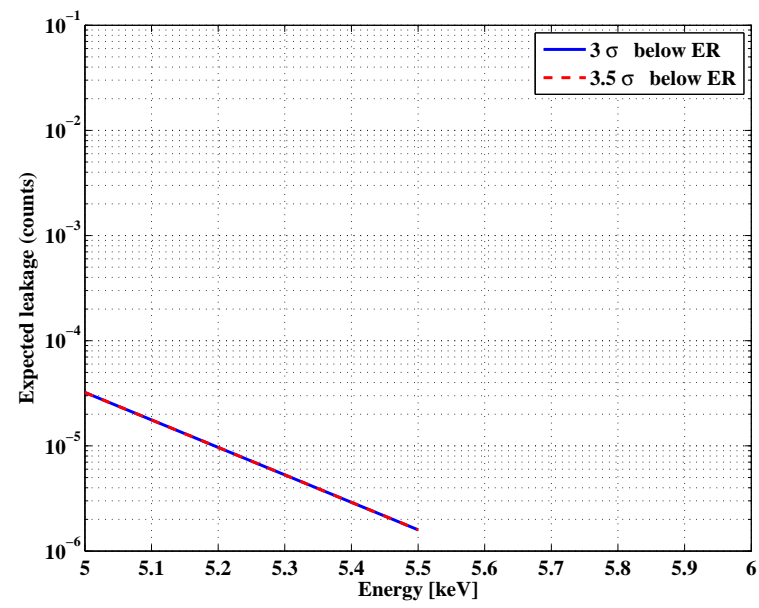

(a)

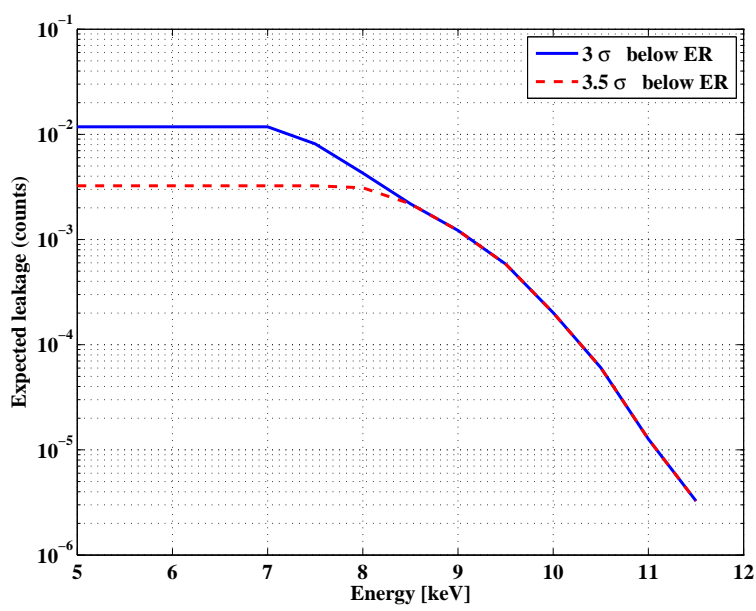

(b)

Figure 9.18: Cumulative expected gamma leakage in the nuclear-recoil band above threshold. For detectors with a good phonon and ionization channel resolution the expected cumulative leakage counts are of no concern with a reasonable threshold above $5 \mathrm{keV}$ (a). For detectors with a worse performance in resolution the expected leakage would be to high if too low thresholds are chosen (b). The difference in expected leakage between a $3 \sigma$ (blue/solid) and $3.5 \sigma$ (red/dashed) below the electron-recoil band cut is only visible at low thresholds. For reasonable thresholds of $10 \mathrm{keV}$ the expected gamma leakage is of no concern. The expected leakage vanishes in the $10^{-6}-10^{-5}$ region, since the simulations run out of statistics.

illustration figure 9.17(b) shows the contours of the expected counts from the simulation in the yield plane along with the electron-recoil and the nuclear-recoil band. In addition the ionization threshold cut and possible gamma rejection cuts are shown. The higher density of events from the $10.36 \mathrm{keV}$ line can be nicely seen in the figure.

The total gamma leakage into the nuclear-recoil band is determined by the cumulative counts above the ionization threshold cut and below the gamma rejection cut which lie in the $2 \sigma$ nuclear-recoil band. The cumulative leakage counts are found by summing the leakage above a specified energy threshold. This cumulative leakage is shown in figure 9.18 (a) for a representative detector showing a good performance in phonon and ionization energy resolution and for a representative detectors having a worse energy resolution in figure 9.18 (b). It can be seen from figure 9.18(b) that the difference between a $3 \sigma$ and a $3.5 \sigma$ below the electron-recoil band cut has only an effect on the expected leakage for thresholds well below $10 \mathrm{keV}$. A threshold of $10 \mathrm{keV}$ would in general result in a negligible gamma leakage contribution into the nuclear-recoil band, since for all working germanium detectors used in this analysis the expected number of leakage events is well below the $10^{-3}$ level. Given the results of this study a cut was defined requesting that:

- A valid WIMP candidate needs to have a yield value which is more than $3 \sigma$ below the electron-recoil distribution. 


\subsubsection{Analysis thresholds}

In order to reject noise events and define a reliable signal region at low energies certain thresholds have to be imposed in the analysis. In particular thresholds are defined on the inner-electrode ionization signal and the phonon recoil energy.

\section{Ionization threshold}

For the definition of an inner-electrode ionization threshold the noise distribution, as measured on random triggers at the beginning of each series is fitted by a Gaussian distribution function to determine the mean and resolution of the noise distribution. Like for the phonon channel (see Section 9.4.2) also the ionization channel shows a variation of the noise performance with time.

To account for this variation in noise performance, the ionization threshold cut is defined on a series-by-series basis. The ionization threshold cut is defined by the following criteria.

- The energy deposition in the considered detector lies more than $4.5 \sigma$ above the mean of the noise distribution.

The series dependent ionization thresholds for WIMP search data are shown for working germanium detectors used in the WIMP search analysis in figure 9.19, The ionization thresholds are generally below $\sim 2 \mathrm{keV}$, only cryocooler sensitive detectors (namely T2Z3, T3Z2 and T3Z5; see Section 9.3.2) show higher thresholds.

In addition to the series dependent thresholds a time independent threshold is defined by determining the mean and resolution of the noise distribution of all series in the data taking period. The ionization threshold is given by the criteria mentioned above. This threshold can be interpreted as a mean threshold over the course of the data taking period. The final ionization threshold combines both conditions by conservatively taking the maximum of both constraints, when applied in the analysis of the WIMP search data.

The efficiency of the ionization threshold cut for nuclear recoils is measured in combination with the electron recoil rejection cut described in Section 9.4.6. The efficiency of the combined selection criteria is given by the fraction of nuclear recoils in the $2 \sigma$ nuclearrecoil band which pass the two selection cuts. These efficiencies are measured in several energy bins. To obtain an analytical form of the efficiency as a function of energy the energy dependence is fitted to a functional form, as it is shown in figure 9.20 for a detector considered in this analysis.

\section{Recoil energy threshold}

A threshold on the phonon recoil energy is imposed to reject low energy events. At low energies the ionization yield bands start to overlap making a primary discrimination between electron recoils and nuclear recoils impossible (see Section 6.5). In addition events at low energies have noisy timing parameters (see section 9.4.4) leading to poor discrimination between surface-events and nuclear recoils. The standard WIMP search analysis can be 

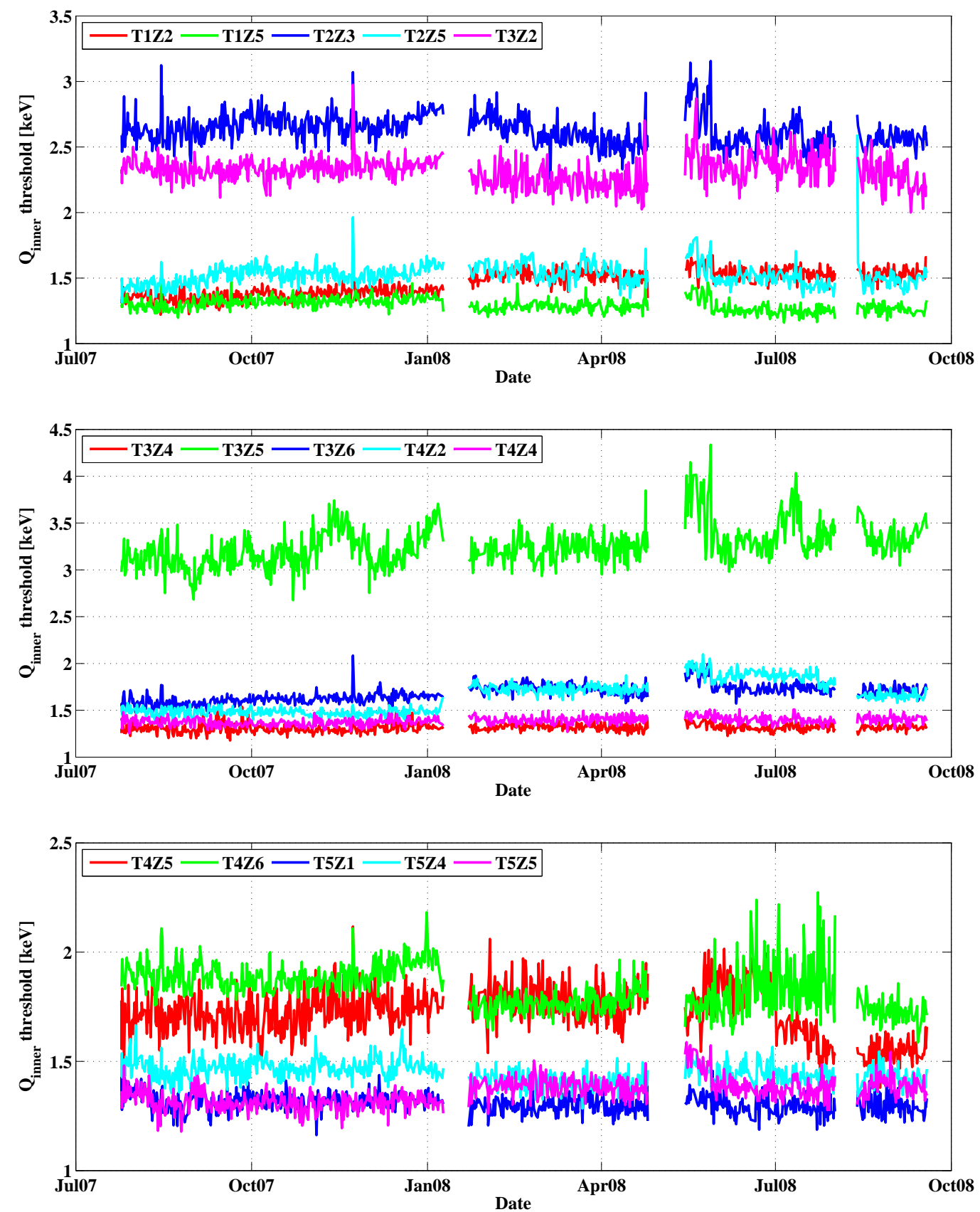

Figure 9.19: Variation of the ionization threshold with time. The three figures show the ionization threshold for the germanium detectors used in the WIMP search analysis. Time intervals in which no data is shown mark the warm-up and cool-down periods between the runs. 


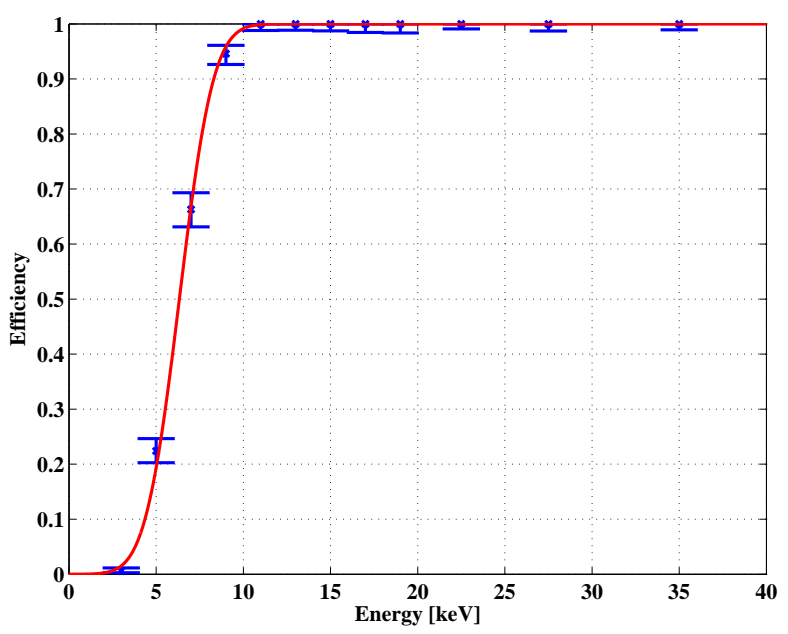

Figure 9.20: Combined efficiency of the ionization threshold and electron-recoil rejection cut. Efficiencies measured on nuclear recoils (blue) in several energy bins are fitted by a functional form (shown in red). Efficiencies are shown for a representative detector.

extended to at least $5 \mathrm{keV}$ [158, 166] and analyzes allowing a large background to $1 \mathrm{keV}$ or less. This analysis uses a conservative threshold of no less than $10 \mathrm{keV}$ in recoil energy for all germanium detectors. Different thresholds may be used in future analyzes. The thresholds in this analysis were chosen to ensure that the following conditions are satisfied:

- There should be at least a reasonable population of surface-events in the calibration data right above threshold in order to define a surface-event rejection cut which takes into account the timing parameters of events at energies close to the threshold.

- The expected leakage from the electron-recoil population in the nuclear-recoil band should be negligible (less than $10^{-2}$ ).

For the germanium detectors used in this analysis the face selected number of surfaceevents in the wide surface-event region in an energy range of $10-15 \mathrm{keV}$ is usually greater than 30 events, which was considered as a reasonable statistic to take the timing parameters of events at these low energies into account in the definition of the surface-event rejection cut. The electron-recoil leakage from the bulk events into the nuclear-recoil band for all detectors is less than $10^{-3}$ events, for thresholds greater than $10 \mathrm{keV}$ (see Section 9.4.6).

\subsection{Total exposure}

The exposure for each detector is calculated by the live time of each detector multiplied by the detector mass. The mass of the detectors varies from detector to detector due to individual production procedures. These variations are $\leq 10 \%$. The masses of the detectors as calculated from individual detector dimensions in the CDMS-II setup are given in table 9.2 (germanium detectors are highlighted in bold text). 


\begin{tabular}{|c||c|c|c|c|c|}
\hline & T1 & T2 & T3 & T4 & T5 \\
\hline \hline Z1 & $\mathbf{2 3 0 . 5}$ & 101.4 & 104.6 & 101.4 & $\mathbf{2 2 4 . 5}$ \\
Z2 & $\mathbf{2 2 7 . 6}$ & 104.6 & $\mathbf{2 3 1 . 2}$ & $\mathbf{2 3 8 . 9}$ & $\mathbf{2 2 9 . 5}$ \\
Z3 & $\mathbf{2 1 9 . 3}$ & $\mathbf{2 1 9 . 3}$ & 104.6 & 101.4 & 101.4 \\
Z4 & 104.6 & 104.6 & $\mathbf{2 3 8 . 9}$ & $\mathbf{2 3 4 . 6}$ & $\mathbf{2 2 4 . 5}$ \\
Z5 & $\mathbf{2 1 9 . 3}$ & $\mathbf{2 3 8 . 9}$ & $\mathbf{2 3 8 . 9}$ & $\mathbf{2 3 1 . 9}$ & $\mathbf{2 3 4 . 8}$ \\
Z6 & 104.6 & 104.6 & $\mathbf{2 3 1 . 7}$ & $\mathbf{2 3 8 . 9}$ & $\mathbf{2 2 3 . 6}$ \\
\hline
\end{tabular}

Table 9.2: Mass of the crystals in the CDMS-II setup.

The live time for each detector has been calculated by the following three criteria:

- All data quality selection cuts in Section 9.2 are imposed.

- Poorly-performing detector quadrants have been removed (see Section 9.2.8).

- Poor time periods on certain detectors are removed, primarily due to the cryocooler cycle sensitivity (see Section 9.3.2).

Multiplying the live time by the detector's mass gives the exposure for each detector which are given in table 9.3 and 9.4 separated by the four individual runs. Summed over all good detectors, the total germanium exposure of this analysis is $\mathbf{6 1 2 . 1 3} \mathrm{kg}$-days.

\begin{tabular}{|c||c|c|c|c|c||c||c|c|c|c|c|}
\hline R125 & T1 & T2 & T3 & T4 & T5 & R126 & T1 & T2 & T3 & T4 & T5 \\
\hline \hline Z1 & \multirow{2}{*}{21.89} & & & & & Z1 & & & & & \\
Z2 & & 8.01 & 22.15 & & Z2 & 13.14 & & 4.26 & 14.12 & \\
Z3 & & \multirow{2}{*}{17.90} & & & & Z3 & & 9.65 & & & \\
Z4 & & & 24.63 & 23.21 & 22.18 & Z4 & & & 14.13 & 13.85 & 13.09 \\
Z5 & \multirow{2}{*}{23.04} & 21.67 & 17.05 & 20.70 & 21.89 & Z5 & 13.45 & 12.36 & 8.24 & 11.88 & 13.23 \\
Z6 & & & 22.82 & 22.29 & & Z6 & & & 13.44 & 13.94 & \\
\hline
\end{tabular}

Table 9.3: Detector exposures of Run 125 and Run 126 in kg-days.

\begin{tabular}{|c||c|c|c|c|c||c||c|c|c|c|c|}
\hline R127 & T1 & T2 & T3 & T4 & T5 & R128 & T1 & T2 & T3 & T4 & T5 \\
\hline \hline Z1 & \multirow{2}{*}{8.48} & & & & & Z1 & & & & & \\
Z2 & & 3.61 & 8.18 & & Z2 & 4.60 & & 1.84 & & \\
Z3 & & \multirow{2}{*}{7.12} & & & & Z3 & & & & & \\
Z4 & & & 11.27 & 9.35 & 7.79 & Z4 & & & 5.23 & 4.60 & 4.84 \\
Z5 & & 9.43 & 6.71 & 9.44 & 9.60 & Z5 & & & 2.89 & 4.84 & 4.61 \\
Z6 & & & 9.31 & 10.22 & & Z6 & & & 5.03 & 4.91 & \\
\hline
\end{tabular}

Table 9.4: Detector exposures of Run 127 and Run 128 in kg-days. 


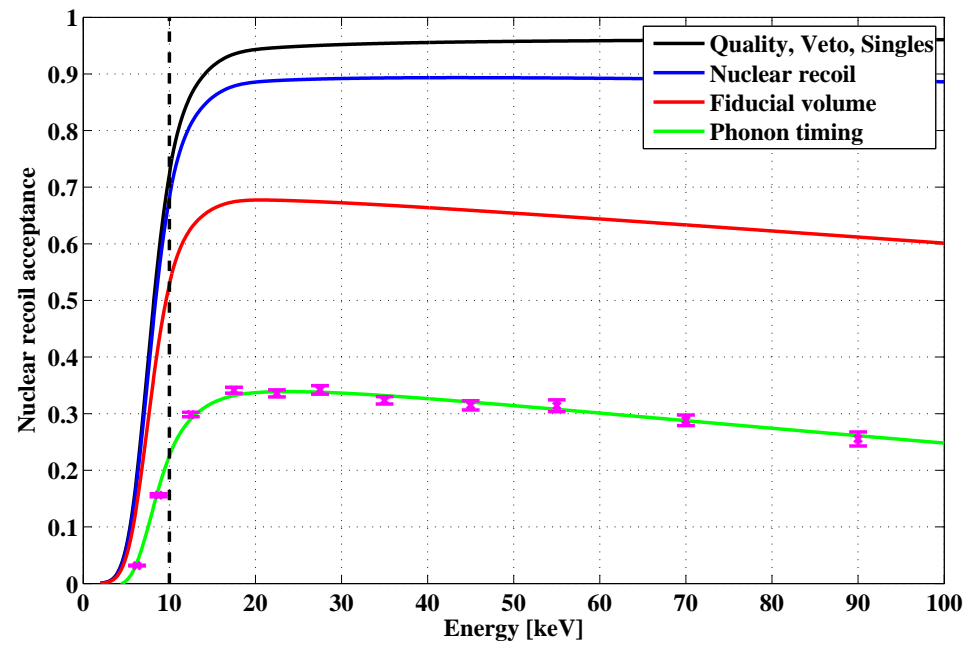

Figure 9.21: Nuclear recoil acceptance in the analysis as a function of recoil energy. Each curve represents the total efficiency after adding the selection cut specified in the caption to the efficiency curve above. The vertical dashed line marks the analysis threshold for this analysis. The magenta data points give the final efficiency measured directly on the data in several energy intervals.

\subsection{Analysis efficiency}

The efficiencies of the event reconstruction and event selection cuts for nuclear recoils are combined in the analysis to determine the signal efficiency. Efficiencies for each detector and run are given by the functional form fits to the measured values discussed above. The final analysis efficiency is a weighted average of the single cut efficiencies, in which the weight is given by the exposure contribution of a given detector in a specific run (see tables 9.3 and 9.4) to the total exposure of $612.13 \mathrm{~kg}$-days considered in this analysis. Figure 9.21 shows the cumulative analysis efficiency as a function of recoil energy after imposing the selection cuts given in the figure's caption. The efficiency for signal events has a maximum of $\sim 34 \%$ around $20 \mathrm{keV}$ recoil energy. It falls to $\sim 23 \%$ at $10 \mathrm{keV}$ recoil energy, due to the ionization signal threshold and the flaring of the electron-recoil band (see Section 9.4.7); and at $100 \mathrm{keV}$ recoil energy to $\sim 25 \%$ due to a drop in the fiducial volume (see Section 9.4.3). The largest cost in WIMP signal efficiency are the fiducial volume selection and the surface-event rejection cuts (see Section 9.4.3 and 9.4.4).

As a validation that the use of the functional forms does not overestimate/underestimate the actual signal efficiency, it has also been measured directly on the data in several energy bins. These efficiencies are shown as the magenta data points in figure 9.21 giving an excellent match to the analytical forms, and proving that statistical uncertainties are small. There may be greater systematic uncertainties in the analysis efficiency related to assumptions made in the surface-event rejection efficiency. Internal multiple scatters of neutrons should appear with unusually slow or fast timing parameters, some should fail the 
surface-event rejection consistency cut, and some should be miscorrected by the position correction. In addition there may be a slight bias to timing discriminator values at higher radius since neutrons have a finite attenuation length which should not be the case for WIMP interactions. However the magnitude of the net effect is unclear. Such systematic uncertainties can only be addressed with detailed Monte Carlo simulations of the phonon response of the detectors which are in progress.

\subsection{Spectrum averaged exposure}

It is not straight forward to compare the exposures of different experiments with different analysis efficiencies, since the shape of this function affect the sensitivity to WIMPs at a given mass. A useful measure of the experiment's exposure and thereby it's sensitivity is the WIMP-spectrum averaged exposure $(S A E)$ for a given WIMP mass. This spectrumaveraged exposure is defined by the exposure taking into account the analysis efficiency with respect to an ideal experiment with $100 \%$ efficiency. The value at a given WIMP mass is given by

$$
S A E=M T \frac{\int_{E_{\min }}^{E_{\max }} \epsilon(E) d R / d E(E) d E}{\int_{E_{\min }}^{E_{\max }} d R / d E(E) d E}
$$

where $M T$ is the total exposure, $E$ is the recoil energy, $\epsilon(E)$ is the efficiency as a function of recoil energy, $\left[E_{\min } E_{\max }\right]$ is the energy interval under analysis and $d R / d E$ is the expected recoil energy spectrum for a given WIMP mass.

For the current analysis the spectrum averaged exposure for a WIMP with a mass of $60 \mathrm{GeV} / \mathrm{c}^{2}$ is $185.3 \mathrm{~kg}$-days of germanium exposure. For comparison, the equivalent numbers for Run 123/4 were $119.9 \mathrm{~kg}$-days and $34 \mathrm{~kg}$-days for Run 119. Thus the germanium exposure in this analysis is roughly 1.5 (5.5) times greater than the WIMP exposure of Run 123/4 (Run 119). 


\section{Chapter 10}

\section{WIMP - Search results}

This Chapter presents the results of the blind analysis described in the previous Chapter. It begins describing the expected background from neutrons and surface events for the current exposure and unblinding of the signal region. Given the expected backgrounds the observation of two candidate events in the signal region cannot be interpreted as a statistical significant evidence for the detection of WIMP interactions. For each of the two candidate events a series of post unblinding checks is discussed validating that these events are valid nuclear recoil candidates without showing any degraded performance of the detectors which could potentially reject one of the candidate events.

The observation of two candidate events in the current analysis is taken into account in the calculation of upper limits on the WIMP-nucleon scattering cross section. In order to remain conservatively independent of any uncertainties in the background model, the analysis is performed without any background subtraction, which makes the result of the analysis independent of the expected and potentially observed backgrounds.

This analysis tests the DAMA signal hypothesis in a non standard WIMP scenario which will be briefly introduced. Finally, the future prospects of the CDMS experiment will be discussed.

\section{$10.1 \quad$ Expected backgrounds}

Given the low expected event rates from dark matter interactions in the detectors, the discovery potential of each direct detection experiment is determined by the expected backgrounds. Minimizing the expected backgrounds and uncertainties on these estimates is thus a crucial ingredient to any dark matter search analysis. The main background sources for the CDMS-II experiment have been summarized in Chapter 8 , here the determination of the expected background, in number of events in the signal region, for the current analysis is summarized. 


\subsubsection{Expected neutron background}

As outlined in Section 8.3 there are two sources of neutron background. From each of these two the expected number of neutron induced single-scatter events for the exposure under analysis has to be determined. For the radiogenic component, a simple scaling of the predicted rate by the simulations to the exposure under analysis provides the expected neutron background. For the cosmogenic neutron background, the actual data has to be taken into account. Both procedures providing the actual number of background events in the signal region are described below.

\section{Radiogenic neutrons}

The rate of nuclear-recoil single-scatter events in the germanium detectors from various radiogenic sources has been determined by Monte Carlo simulations as outlined in Section 8.3.1. The second column of table 10.1 summarizes the sum of these rates for the individual components of the experimental setup. The rates are given for events occurring in the fiducial volume and an energy range of $10-100 \mathrm{keV}$. To obtain the expected number of background events for the current analysis this rate has to be scaled to the analyzed exposure and taking into account the efficiency of the surface-event rejection cut for selecting nuclear-recoils. Since the efficiency of the surface-event rejection cut varies from detector to detector a conservative efficiency of $70 \%$ for selecting nuclear-recoils across all detectors is assumed in the calculation of the expected neutron background. Taking this efficiency into account the exposure of this analysis in kg-years becomes

$$
612.13 \text { [kg-days }] \times 0.7 / 365[\text { days }]=1.17[\mathrm{~kg} \text {-years }] .
$$

The third column in table10.1 gives the expected number of neutron background events obtained from scaling the rate in the second column by the exposure of this analysis given above.

\begin{tabular}{|c||c|c|}
\hline Component & Ge rate $(\mathrm{n} / \mathrm{kg}$-years $)$ & Expected background events \\
\hline \hline Copper cold hardware & $3.6 \times 10^{-3}$ & $4.2 \times 10^{-3}$ \\
Copper icebox cans & $2.31 \times 10^{-2}$ & $2.7 \times 10^{-2}$ \\
Inner polyethylene & $<6 \times 10^{-3}$ & $<7 \times 10^{-3}$ \\
\hline Total copper/polyethylene & $2.67-3.27 \times 10^{-2}$ & $3.1-3.8 \times 10^{-2}$ \\
\hline Inner lead & $<3 \times 10^{-3}$ & $<3.5 \times 10^{-3}$ \\
Outer lead & $<7 \times 10^{-3}$ & $<8.2 \times 10^{-3}$ \\
\hline Total lead & $<1 \times 10^{-2}$ & $<1.2 \times 10^{-2}$ \\
\hline
\end{tabular}

Table 10.1: Expected rates and number of single-scatter nuclear-recoils in the energy range from $10-100 \mathrm{keV}$ from radioactive contamination of the experimental setup components.

Since part of the expected neutron background is determined by measured contamination values while others are only derived from an upper limit on the contamination level, 
the total expected neutron background from radiogenic neutrons is

$$
n_{\text {radiogenic }}=0.031-0.05 \text { events. }
$$

Note that the assumption of a $70 \%$ efficiency of selecting nuclear-recoils is extremely conservative. Only the best performing detectors show efficiencies as high as 70\%. The correct exposure weighted detector averaged efficiency for selecting nuclear-recoils, averaged over the energy range of $10-100 \mathrm{keV}$ for this analysis is only $\sim 50 \%$. Hence assuming $70 \%$, the exposure may be overestimated by $\sim 30 \%$ and by this also the expected radiogenic neutron background. For future analyses it would be worth to use the correct exposure weighted efficiency instead of a conservative assumption to obtain an accurate number of the expected neutron background.

\section{Cosmogenic neutrons}

The estimation of the cosmogenic neutron background is based on a ratio of unvetoed to vetoed nuclear-recoil single-scatters which is determined from Monte Carlo simulations of cosmic rays interacting in the experimental cavern and apparatus (see Section 8.3.2). This ratio is then multiplied by the number of vetoed nuclear-recoil single-scatters observed in the data to estimate the expected number of unvetoed nuclear-recoil single-scatters in the data. To estimate the background for the current analysis also the observed number of vetoed nuclear-recoil single-scatters from the Run 123/4 exposure is taken into account, since the number of vetoed nuclear-recoil single-scatters is usually very small, and can thus be affected by large statistical fluctuations in the number of observed events. This can be easily seen by comparing the exposures and number of vetoed nuclear-recoil single-scatters observed in Run 123/4 and Run 125-128:

- Run 123/4: In an exposure of 510 kg-days used for looking for vetoed nuclear recoil single-scatters 0 events were observed.

- Run 125-128: In an exposure of 612 kg-days, 3 vetoed nuclear recoil single-scatters were observed.

Thus to obtain a reliable median value, the exposure weighted average of the number of observed vetoed nuclear-recoil single-scatters has been used to determine the expected unvetoed nuclear-recoil background from cosmogenic neutrons. Taking into account the results from the Monte Carlo simulations (see table 8.5) and the averaged efficiency for selecting nuclear-recoils of $50 \%$ the expected background becomes:

$$
n_{\text {cosmogenic }}=\left(\frac{612}{1122}\right) \times 3 \times\left(\frac{35}{606}\right) \times 0.5=0.04_{-0.03}^{+0.04} \text { events. }
$$




\subsubsection{Surface-event leakage}

Although the surface event rejection cut described in Section 9.4.4 has been tuned to an expected leakage of 0.5 events, it relies solely on the ${ }^{133} \mathrm{Ba}$ calibration data and an estimation of the nuclear-recoil single-scatters expected to be observed in the signal region. In order to obtain an accurate leakage estimation prior to unblinding of the signal region, the actual distribution of events in the signal region and sidebands surrounding the signal region has to be taken into account. The total expected leakage in this analysis is defined by:

$$
\eta=\sum_{i} r_{i} N_{i}
$$

where $r_{i}$ are the expected passing fractions of single-scatters in the nuclear-recoil band for each considered detector. $N_{i}$ denotes the number of single-scatters failing the timing cut on the $i^{t h}$ detector. By using the number of events which fail the timing cut rather than the total number of events before the timing cut, i.e. all single-scatters in the signal region, the expected leakage is statistically independent of the number of WIMP candidates.

The estimation of the expected surface-event leakage for this exposure is performed with three different, independent methods, which will be discussed below. In any of the three methods the single detectors are treated separately, to avoid systematics. In order to obtain an estimation of the error of the total expected leakage a Bayesian approach is used. This Bayesian estimate is based upon a Monte Carlo simulation, thrown in accordance with the observed event counts and a choice of prior distributions (for details on this Bayesian estimation framework I refer the reader to [173]).

\section{Multiple-scatters in the nuclear-recoil band}

Multiple-scatters in the nuclear-recoil band are expected to have the lowest systematic difference when compared to nuclear-recoil single-scatters, since the yield and face distributions should be the same as for single-scatters. However, the low statistics of the multiple-scatters in the nuclear-recoil band introduce a large statistical uncertainty on the expected leakage. The expected leakage is obtained by determining the passing ratios $r_{i}$ of equation (10.4):

$$
r_{i}=\frac{n_{i}^{m s}}{N_{i}^{m s}}
$$

where $n_{i}^{m s}$ are the nuclear-recoil multiple scatters passing the timing cut on the $i^{\text {th }}$ detector and $N_{i}^{m s}$ is the number of multiple-scatters in the nuclear-recoil band, failing the surfaceevent rejection cut. Although this method uses the population of events most similar to the one in the signal region, there are two possible systematics. The passage fraction between multiples and singles in the nuclear-recoil band may be different. Secondly the choice of the prior in the Bayesian framework may result in a bias or undercoverage of the expected leakage. Both effects lead to a systematic error on the total expected leakage. Taking these uncertainties into account, the final expected leakage for the internal detectors from this 
method becomes

$$
\eta_{1}^{\text {internal }}=0.65_{-0.29}^{+0.46} \text { (stat.) } \pm 0.13 \text { (syst.) events. }
$$

For the external detectors at the bottom of the stack (namely T3Z6 and T4Z6) this method is unreliable since there are no multiple-scatters on the exterior detector faces and the method additionally suffers from poor statistics.

\section{Single and multiple-scatters outside the nuclear-recoil band}

A second statistically independent estimate can be made by using single and multiple scatters in the wide surface-event selection (see Section 8.2), which are outside the nuclearrecoil band. This method suffers from systematic differences in the energy and face distributions with respect to the single-scatters in the nuclear-recoil band which have to be taken into account in an accurate leakage prediction. To correct for these differences the data is binned in two face and three energy bins: $10-20 \mathrm{keV}, 20-30 \mathrm{keV}$ and $30-100 \mathrm{keV}$. The passage fraction is measured in each energy bin separately and a weighted average of the passage fractions, based on the fraction of events which fall into each bin in the nuclear-recoil band is formed. The passing ratios of equation (10.4) are given by:

$$
r_{i}=\sum_{j, k} s_{i j k} \frac{w_{i j k}}{W_{i j k}} .
$$

In the above passing ratio the indices $j$ and $k$ run over bins by face selection and energy. The passing fraction in each bin is given by the ratio of events passing the surface-event rejection cut $w_{i j k}$ to the number of events failing the surface-event rejection cut in the bin $W_{i j k}$. The scaling fractions $s_{i j k}$ are determined from the number of multiple-scatters in the given bin which fall into the nuclear-recoil band to the total number of multiple-scatters in the nuclear-recoil band. Although this method uses a sample with higher statistics than the first method the systematics are much greater. The re-weighting described above tries to account for these systematic differences, however, there are residual systematics. Similar to the first method the passage fraction between multiples and singles in the wide surface-event selection may be different than for single-scatters in the nuclear-recoil band, and the choice of the prior in the Bayesian framework may result in a systematic bias.

In addition there maybe an incomplete re-weighting for events at low energy, possibly due to a lack of events with low ionization energies resulting from the selection criteria of the wide surface-event sample. Events at low ionization energies may suffer from a ionization pulse reconstruction pathology (this effect is discussed in detail in Section 10.3.1) which worsens the rejection capability of the surface-event rejection cut. This naturally results in a higher expected leakage. The additional leakage has been estimated by running artificial ionization pulses through the optimal filter algorithm to determine the worsening in the ionization delay resolution with decreasing pulse height (i.e. decreasing ionization energy). The distribution of the ionization delays of events at low ionization energy is then convolved with the resolution distributions obtained from the simulated pulses. Due to 
the resulting broadening of the distributions an additional leakage is determined, which is not accounted for by the standard re-weighting scheme described above. This procedure results in an addition leakage of 0.19 events summed over all internal detectors.

Taking into account all the effects described above, the resulting expected surface event leakage for the internal detectors becomes:

$$
\eta_{2}^{\text {internal }}=0.72_{-0.25}^{+0.56} \text { (stat.) } \pm 0.18 \text { (syst.) events. }
$$

For the detectors at the bottom of the stack a slight modification of the procedure described above has to be made since there are no tagged multiples on the detector's exterior charge face. Thus it is assumed, that all untagged events are charge side events. This assumption is conservative since it slightly overestimates the number of charge side events, which on average have a higher passage fraction than phonon side events. When calculating the fraction of events, $s_{i, j, k}$, which lie in each face/energy bin, a value for the fraction of untagged events which are actually untagged charge side multiples has to be assumed. To do this, the singles fraction in the nuclear-recoil band measured on the interior detectors is used to estimate the number of charge side multiples from the singles in the nuclear-recoil band for detectors at the bottom at the stack. This introduces additional systematic and statistical errors due to averaging over detectors and extrapolating this value to the bottom detectors, which are taken into account in the final leakage estimate.

Apart from this change in the procedure the systematic effects are similar to those for the interior detectors. Following the same procedure as described above to estimate the additional leakage due to low ionization energy events, an additional leakage of 0.04 events is taken into account.

The final surface-event leakage for the two detectors at the bottom of the stack from this method becomes:

$$
\eta_{2}^{\text {external }}=0.29_{-0.14}^{+0.33}(\text { stat. }) \pm 0.05 \text { (syst.) events. }
$$

\section{Surface-events from ${ }^{133} \mathrm{Ba}$ calibration data}

The procedure described above can also be applied to the high statistic ${ }^{133} \mathrm{Ba}$ calibration sample. Again the systematic differences between the distributions of events on which the passing ratio is measured and the single-scatter events within the nuclear-recoil band in WIMP search data have to be taken into account. However in this procedure the whole statistics of the wide surface-event selection can be used, since they are statistically independent of the population for which the leakage is to be determined. The passing ratios of equation (10.4) are given by:

$$
r_{i}=\sum_{j, k} s_{i, j, k} \frac{b_{i, j, k}}{B_{i, j, k}} .
$$

As in the paragraph above, the indices $j$ and $k$ run over bins by face selection and energy, and the scaling fractions $s_{i, j, k}$ are the same as in the method described above. The passing 
fraction in each bin is given by the ratio of events passing the timing cut $b_{i, j, k}$ to the number of events failing the surface-event rejection cut in the bin $B_{i, j, k}$. This procedure for estimating the leakage is similar to the procedure used to define the surface-event rejection cut (see Section 9.4.4), however the procedure of determining the weighting factors, $s_{i, j, k}$, is different and the total leakage is now determined by the actual number of single-scatters failing the surface-event rejection cut $\left(N_{i}\right.$ in equation (10.4) $)$ rather than based on an expected number of single-scatters in the nuclear-recoil band.

Remaining systematic differences are the same as in the second method and accounting for a widening in the ionization pulse delay resolution results in an additional leakage of 0.23 events, giving a total leakage estimate for the interior detectors of

$$
\eta_{3}^{\text {internal }}=0.69_{-0.10}^{+0.12} \text { (stat.) } \pm 0.19 \text { (syst.) events. }
$$

For the two detectors at the bottom of the stack the additional leakage due to low ionization energy events is 0.06 events, resulting in a total leakage estimate of

$$
\eta_{3}^{\text {external }}=0.14_{-0.04}^{+0.06} \text { (stat.) } \pm 0.06 \text { (syst.) events. }
$$

\section{Combined expected surface-event leakage}

The agreement of the expected leakage for all three methods described above is very good, especially the agreement of the method using calibration data with the methods using the actual WIMP search data shows, that by correctly taking into account systematic differences, the expected leakage can be derived from the calibration data. The final leakage estimate for the internal and external detectors is obtained by combining the expected leakages from the three methods. The combined leakage is:

$$
\begin{aligned}
& \eta^{\text {internal }}=0.66_{-0.09}^{+0.11}(\text { stat. }){ }_{-0.15}^{+0.16} \text { (syst.) events } \\
& \left.\eta^{\text {external }}=0.16_{-0.04}^{+0.06} \text { (stat. }\right)_{-0.05}^{+0.05} \text { (syst.) events }
\end{aligned}
$$

The total expected leakage for this exposure is calculated by combining the posterior distributions of the single leakage estimates. Adopting this procedure the resulting total expected leakage becomes:

$$
\eta=0.82_{-0.10}^{+0.12} \text { (stat.) }{ }_{-0.19}^{+0.20} \text { (syst.) events. }
$$

\subsection{Unblinding the signal region}

Prior to the unblinding of all considered detectors in this analysis (see Section 9.2.2), the blinding mask was lifted and the number of single-scatters in the nuclear-recoil band failing the surface-event rejection cut were counted. Table 10.2 summarizes the observed number of counts for each detector. These counts are the factors $N_{i}$ in equation (10.4) for the determination of the expected surface-event leakage. 


\begin{tabular}{|c||c|c|c|c|c|}
\hline & T1 & T2 & T3 & T4 & T5 \\
\hline \hline Z1 & & & & & \\
Z2 & 5 & & 6 & 10 & \\
Z3 & & 9 & & & \\
Z4 & & & 8 & 5 & 6 \\
Z5 & 9 & 7 & 4 & 10 & 9 \\
Z6 & & & 52 & 10 & \\
\hline
\end{tabular}

Table 10.2: Number of single-scatter events in the $2 \sigma$ nuclear-recoil band failing the surface-event rejection cut.

The observed total number of 150 of such events is consistent with the expected number of $119.43 \pm 15.29$ obtained by scaling the number of observed single-scatters in the nuclearrecoil band in the Run $123 / 4$ by the ratio of the live times of the two analyzes for each detector. The only exception from this is the high number of events (52) observed in T3Z6 which was much higher than the expected number of events of $19.27 \pm 7.87$. Since this detector is at the bottom of the stack it is expected that more single scatter-events are observed than for an interior detector due to the reduced power of rejecting multiplescatter events on the charge face of the detector. The background on detectors at the top or bottom of the stack is known to be higher than for an interior detector, and thus the surface-event rejection cut is set much stricter on these detectors. Since from the definition of the surface-event rejection cut the expected leakage on this detector was at an acceptable level and the increased number of observed single-scatter events just naturally increases the total expected surface-event leakage it was decided to keep the detector in the analysis and proceed with the unblinding. The complete unblinding was done by applying the surface-event rejection cut to the data resulting in the following observation:

- Two of the 150 observed nuclear-recoil single-scatter events passed the surface-event refection cut.

In addition, two near miss events which passed the surface-event rejection cut, but are outside of the $2 \sigma$ nuclear-recoil band signal region have been observed.

Since each detector shows some difference in the actual definition of the nuclear-recoil band and the setting of the surface-event rejection cuts' discrimination threshold, normalized yield and timing variables are defined to illustrate the distribution of events from all detectors in a single plot. The normalized yield is defined as the distance from the nuclearrecoil band centroid measured in units of the width of the nuclear-recoil band and the normalized timing variable is defined by the distance from the surface-event rejection cuts' discrimination threshold. Figure 10.1 shows the result of the unblinding in normalized yield and normalized timing variables. The red box in the figure represents the signal region, showing the two candidate events within the box and the two near miss events just outside of the box. Apart form the four events passing the surface-event rejection cut which are within or close to the signal region there are no events passing the surface-event rejection cut at higher or lower ionization yield. However, there are events distributed over the 


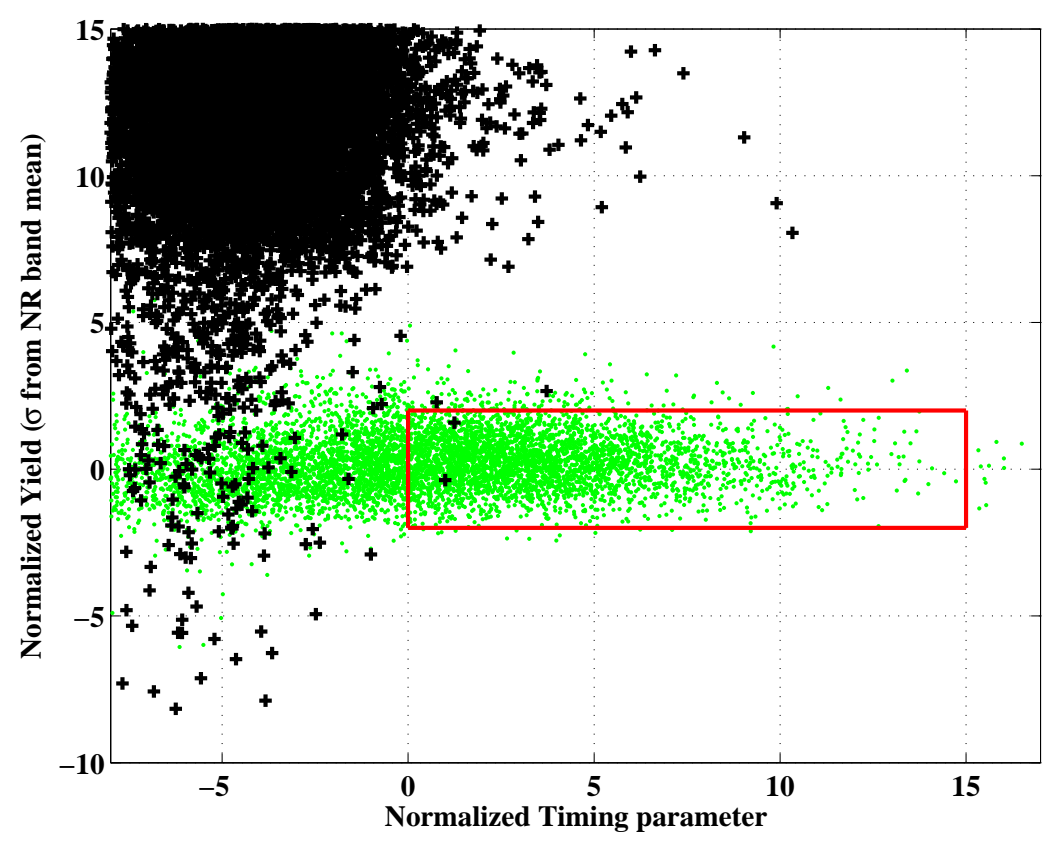

Figure 10.1: Distribution of WIMP search data (black crosses) passing all analysis cuts with exclusion of the surface-event rejection cut for all germanium detectors considered in this analysis in normalized timing and yield variables (see text). The red box in the figure represents the signal region, showing the two candidate events within the box and the two near miss events just outside of the box. For illustration of the distribution of nuclear-recoils in the normalized variables neutron induced nuclear-recoils from calibration data are shown as green dots.

whole range in ionization yield which are rejected by the surface-event rejection cut, which rises the question: Are these four events and especially the two candidate events tails of a background distribution, and if so why is this tail concentrated around the signal region? A drawback of this illustration is that it combines the data from all detectors which is nice for an overall visualization of the result, but may shadow the actual distributions of the events on a detector by detector basis which are very different due to different performances. The same normalized plots are shown in a plot array 10.2 separately for each detector. In most detectors the surface-event distribution is very well separated from the actual discrimination threshold. For the detectors on which no event passes the surface-event rejection cut, only T3Z2 shows a population of events close to the discrimination threshold. In the case of the two near miss events the situation is splitted. For the event occurring in T4Z2 there are events just below the discrimination threshold such that there may be the possibility for this event being part of a tail of this distribution. On the other hand the situation is completely different for the event observed in T4Z6, since it lies far away from the actual distribution of surface-events and there does not seem to be any surface-event which is close to the actual discrimination threshold. However, also the nuclear-recoil population expected at such high values of the timing discriminator is very low. 


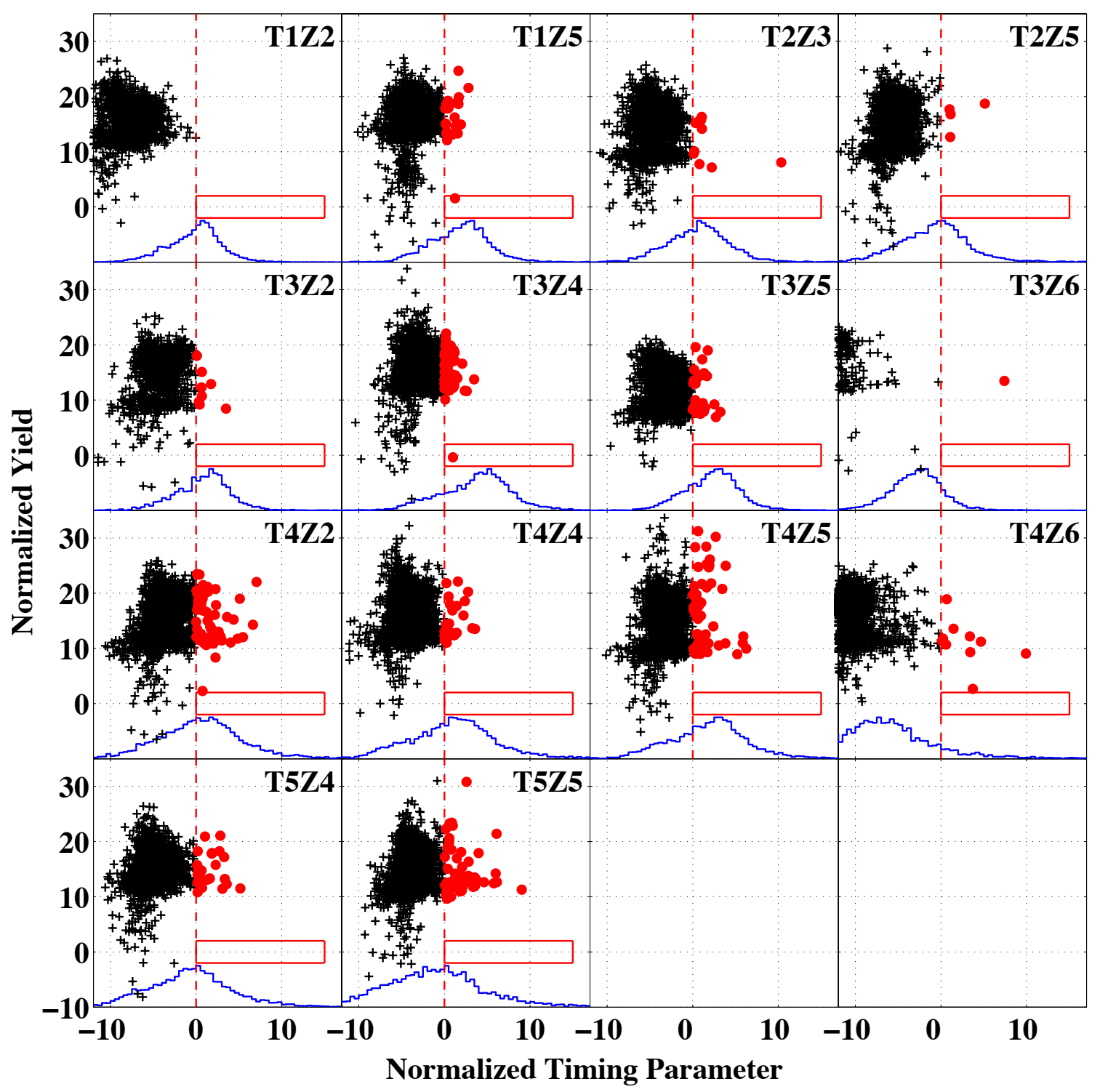

Figure 10.2: Distribution of WIMP search data (black crosses) in normalized yield and timing variables for each detector. Events passing the surface-event rejection cut are marked in red. The red box in the figures represents the signal region and the blue histogram in each plot shows the distribution in the timing variable of neutron induced nuclear recoils from calibration data. The candidate events have been observed in detectors T1Z5 and T3Z4. The near miss events are detected in T4Z2 and T4Z6. 
The position of the candidate events in ionization yield and timing are separately shown in figure 10.3. The left panel shows the position in ionization yield as a function of recoil energy of events in the low background data passing and failing the surface-event rejection cut. The candidate events are observed close to the upper edge of the nuclearrecoil band at an energy of $12.3 \mathrm{keV}$ for the candidate detected in T1Z5 (top) and at an energy of $15.5 \mathrm{keV}$ for the candidate observed in T3Z6, which lies close to the centroid of the nuclear-recoil band (bottom). The right panel displays the normalized variables, showing that both candidate events are well compatible with the nuclear-recoil distributions. The distribution of surface-events on these detectors may indicate that the candidate event occurring in T1Z5 may be part of the surface-event distribution tail, since there are a few events close to the discrimination threshold. The candidate event observed in T3Z4 however is a complete outlier, since it is well separated from the surface-event distribution and there is a huge gap in the timing variable between the candidate event and the distribution of surface-events.

Given the timing and yield parameters these two candidate events cannot be distinguished from nuclear recoils, like it would be expected from WIMP interactions in the detectors. On the other hand, there remains the possibility that these two candidate events are background events. Given the expect number of background events one can calculate the Poisson probability of observing as many candidate events as have been observed or more. Combining the expected background from surface-events and neutrons and taking into account the uncertainties on these estimates by marginalizing over the Bayesian posterior distributions results in the following statistical statement:

- The probability of observing two or more candidate events given the expected background is $23 \%$

With such a high probability of observing two candidate events it cannot be claimed that the result of this analysis can be interpreted as a significant detection of dark matter particles interacting in the detectors. Thus, the conclusion of this analysis is summarized in the statement below:

- The result of this analysis cannot be interpreted as a statistical significant evidence for WIMP interactions. However either event cannot be rejected as signal.

\subsection{Post unblinding checks of the candidate events}

Having unblinded the signal region, the running conditions in which the candidate events have been observed can be checked for any degraded performance of the experiment which potentially could reject one of the candidate events. The results of these performed checks are summarized below.

- No special running conditions nor any operator issues have been recorded in the run $\log$. 

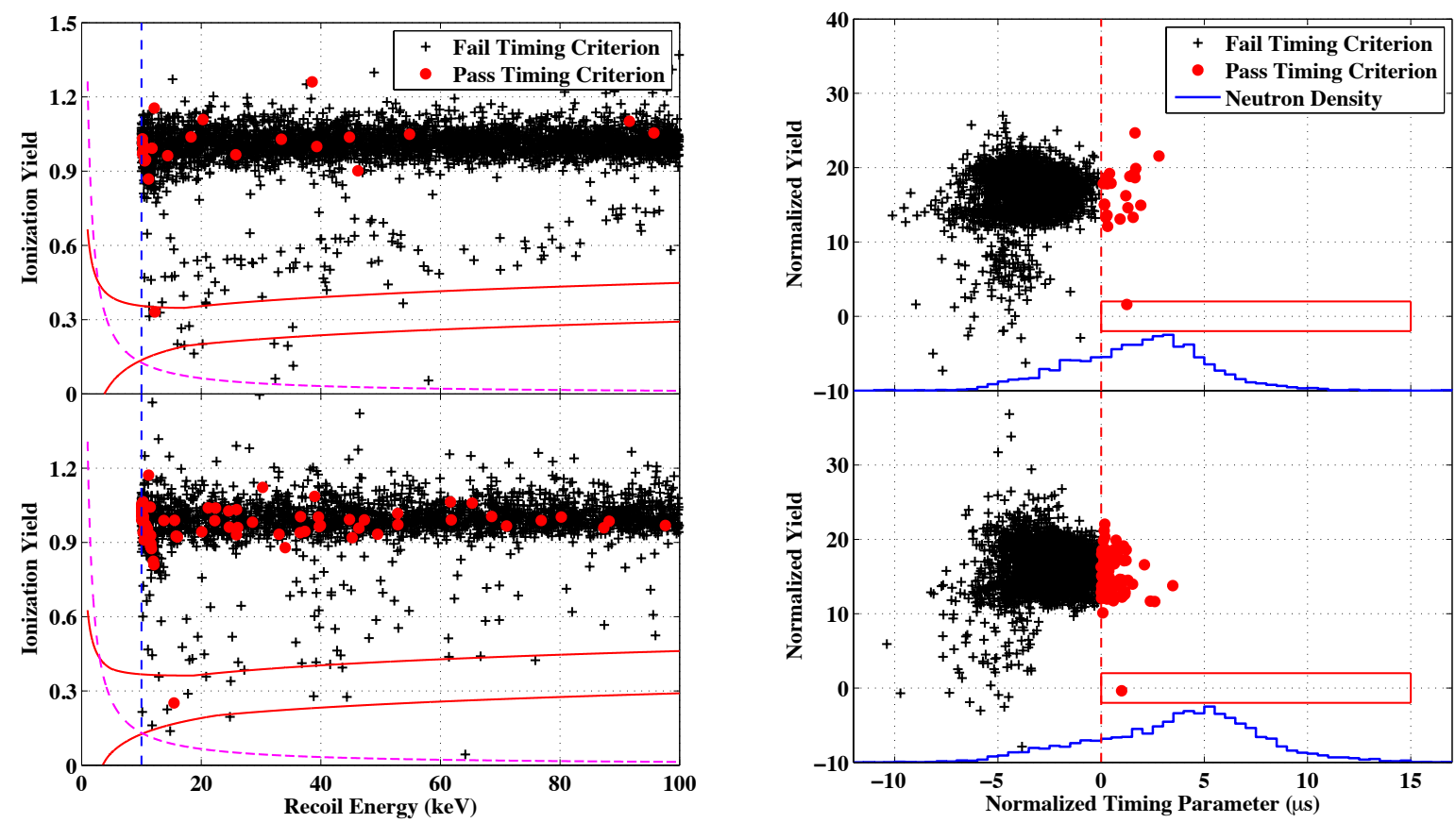

Figure 10.3: Left panel: Distribution of WIMP search data (black crosses) in the ionization yield vs. recoil energy plane. Events passing the surface-event rejection cut are shown in red. The top figure shows the position of the candidate event with an energy of $12.3 \mathrm{keV}$ close to the upper edge of the nuclear-recoil band in detector T1Z5. The candidate event observed in T3Z4 at an energy of $15.5 \mathrm{keV}$ lies close to the centroid of the nuclear-recoil band (bottom figure). Right panel: Distribution of WIMP search data (black crosses) in normalized ionization yield and timing variables. Events passing the surface-event rejection cut are shown in red. The red box in the figures shows the signal region containing the two candidate events. The blue histogram at the bottom of each figure shows the distribution of neutron induced nuclear recoils from calibration data. The top figure shows the distribution for detector T1Z5 and the bottom figure for detector T3Z4.

- The two data series in which the candidate events have been recorded did not show any unusual KS-values in any of the seven tested variables (see Section 9.2.3).

- Both candidate events are veto-anticoincident, selected by the software cut described in Section 9.4.1. An investigation of the recorded veto panel traces for the two candidate events did not show a significant activity in any of the panels. Any activity was well below the discriminator threshold and the veto activity is consistent with the ambient gamma background distribution.

- The neutralization criteria defined in Section 9.2.6 were checked for the two candidate data series. The low yield event fractions (T1Z5 candidate series: $0.012_{-0.01}^{+0.027}$. T3Z4 candidate series: $0_{-0.0}^{+0.031}$ ) are well compatible with the runs' best fit values (T1Z5: 
0.020 and T3Z4: 0.021 ). The Poisson probability criteria was calculated to $Q=0.503$ $(Q=0.703)$ for the T1Z5 (T3Z4) candidate series, well above the rejection threshold of $Q<10^{-10}$.

- At the time of detection of the two events, the noise levels of the phonon and ionization channels of the two detectors were typical. Also, the optimal filter $\chi^{2}$-values were well below threshold (see Section 9.3.2) and the phonon pre-pulse baseline was well within the defined acceptance region (see Section 9.3.3). In summary the detectors did not show an unusual noise performance upon the detection of the two candidate events.

- The reconstructed position of the two candidate events lies well within the central part of the detectors, such that they should not be affected by any particularities related to the phonon signal at high radius.

- The ambient gamma background induced electron recoil rate as well as the surfaceevent rate were consistent with the average rate of the run, such that there is no indication of an increased background rate in these two series.

- Both candidate events are valid single-scatter events, since none of the other 29 detectors exceeded the $4 \sigma$ rejection threshold (see Section 9.4.2).

In summary the two candidate events were recorded under usual running conditions of the experiments without any indication of a reduced performance which would reject these events as WIMP candidates.

However, the investigation of the raw data pulses revealed that there may be an issue with the used ionization pulse reconstruction algorithm which is discussed below.

\subsubsection{Ionization pulse reconstruction pathology}

A careful investigation of the raw data pulses of the two candidate events revealed that there may the a problem with the inner electrode ionization pulse reconstruction of the candidate event detected in T3Z4. Figure 10.4 shows the raw unfiltered data of the innerelectrode ionization signal. Overlaid on the data is the actual reconstructed pulse found by the optimal filtering algorithm. From the figure the suspicion may arise, that the start time of the pulse is actually determined to early by the algorithm. In the ionization pulse reconstruction algorithm the start time of the pulse is determined by selecting the ADC bin for which the summed amplitude of the inner and outer ionization electrode is maximal. The left panel in figure 10.5 shows the summed amplitude of the two signals as a function of the chosen ADC bin determining the start-time of the pulse. The red line in the figure indicates the ADC bin selected by the algorithm for the start-time, giving the maximal summed amplitude. The procedure of determining the pulse start time's bin by maximizing the summed amplitude is quite efficient, and for ionization pulses with an ionization energy $\gtrsim 6 \mathrm{keV}$ always gives the same result as a more computationally intensive algorithm which 


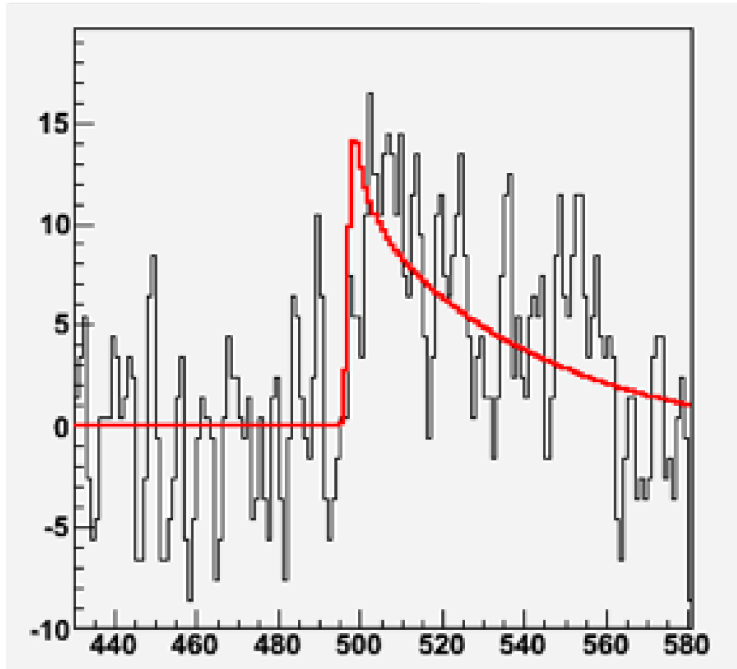

Figure 10.4: Raw unfiltered data of the inner-electrode ionization signal of the candidate event observed in T3Z4. The y-axis has arbitrary units, while the $\mathrm{x}$-axis is given in ADC bins. The red curve in the figure shows the fit to the data from the optimal filtering algorithm. From a visual comparison it may be concluded that the start time of the pulse is determined in a too early bin by the fitting routine. Figure provided by L. Hsu.
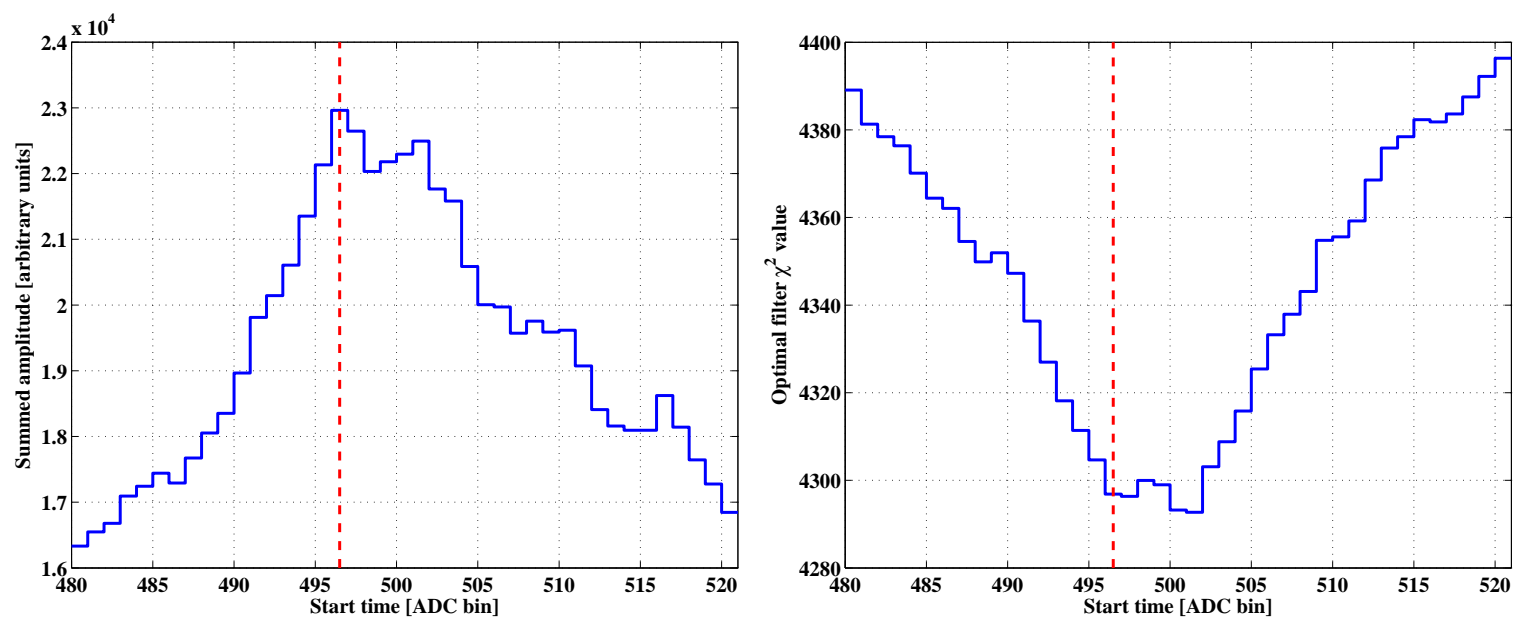

Figure 10.5: Left panel: Amplitude of the summed inner and outer-electrode ionization signal as a function of the start time bin of the ionization pulse. The start time is determined by the bin which maximizes the summed amplitude (marked by the red/dashed line). Right panel: Value of the optimal filter fit $\chi^{2}$ as a function of the start time bin of the ionization pulse. The chosen bin from the maximal amplitude algorithm is indicated by the red/dashed line, which is at a local minimum of the $\chi^{2}$ distribution, but the global minimum occurs 5 ADC bins later. 
searches for the minimum in the $\chi^{2}$-value of the fit. Because of computational processing speed the former algorithm is used as the standard algorithm in the data processing of this analysis. It turned out that a $\chi^{2}$-minimization method would have resulted in a later ADC bin for the start time of the pulse. This is shown in the right panel of figure 10.5. Although the chosen bin is a local minimum in the $\chi^{2}$ distribution, the global minimum occurs 5 ADC bins later. Since one ADC bin corresponds to $0.8 \mu$ s the start time of the ionization pulse based on the $\chi^{2}$ algorithm would have been shifted by $4 \mu \mathrm{s}$. This would have directly affected the phonon delay parameter which is measured with respect to the start time of the ionization pulse. Hence the timing discrimination parameter (sum of the delay and rise-time of the phonon pulse) would have been reduced by $4 \mu \mathrm{s}$. A reduction of $4 \mu \mathrm{s}$ in the timing parameter would have moved the event below the discrimination threshold of the surface-event rejection cut and close to the surface-event population observed in this detector (see bottom figure in the right panel of figure 10.3).

Although this ionization pulse reconstruction pathology would have removed one of the candidate events if a different reconstruction algorithm would have been used, the result of the analysis would be biased if the parameters of single events were fine-tuned. The effect of this reconstruction pathology could well go in the opposite direction, in the sense that events are reconstructed with a too short delay based on the amplitude maximizing procedure, while the use of the $\chi^{2}$ algorithm would lead to larger delays and thereby eventually pushing an event above the discrimination threshold of the current analysis. Only a unified treatment of all events with the same reconstruction algorithm would lead to an unbiased and reliable result. A reanalysis of the reprocessed data, which is being considered as a relevant analysis task, would be a valuable cross-check of the result of this analysis. However, this reconstruction pathology only worsens the rejection capability of the surface-event rejection cut at low ionization energies and thus increases the expected leakage for this analysis (see Section 10.1.2).

\subsection{Limits on the WIMP-nucleon elastic scattering cross-section}

Since the observation of two candidate events can not be interpreted as a statistical significant evidence for WIMP interactions, an upper limit on the rate of WIMP-nucleus elastic scattering, and thus on the WIMP-nucleus scattering cross section is set by the current analysis. The computation of the upper limit accounts for the experimental exposure (see Section 9.5) and the energy-dependent efficiency (see Section 9.6), as well as the energies of any observed candidate events. The finite energy resolution of the detectors is not taken into account, since studies showed that including the energy resolution does not significantly changes the expected recoil spectra and thus the resulting upper limit for WIMP masses above a few $\mathrm{GeV} / \mathrm{c}^{2}$. In a conservative approach no background subtraction is attempted, all candidate events are interpreted as WIMP signal events for the purpose of setting an upper limit. The statistical procedure used to calculate the upper limit is 
the "optimum interval method" [201, 202]. This technique sets an upper limit upon the normalization of a signal of known spectral shape in presence of an unknown background. In presence of candidate events the optimum interval method generally sets a stronger upper limit than the one obtained from Poisson statistics, since the expected energy distribution of a signal is taken into account. In the limit of a zero-background case the upper limit determined from the optimum interval method reduces to the usual Poisson-statistics upper limit. The optimum interval method sets one-sided limits and cannot be used to detect a signal. If the probability distributions of the expected backgrounds are known, a Feldman-Cousins technique [203] to set two sided limits could be used. Attempts for obtaining the probability distributions of the expected background have been made for studying the probabilities of the two candidate events observed in this analysis being part of the surface-event or nuclear-recoil distributions. Further development of this approach may allow for a full likelihood analysis of the expected backgrounds and signal resulting in a Feldman-Cousins like method for setting upper limits or even confidence intervals if a discovery can be claimed.

In the calculation of the expected WIMP interaction rate, needed as an input to the limit curve calculation, a standard halo model WIMP distribution is assumed. The WIMPs in the halo have velocities according to a Maxwellian velocity distribution with a characteristic velocity of $v_{0}=220 \mathrm{~km} / \mathrm{s}$ and a local density of $\rho_{0}=0.3 \mathrm{GeV} / \mathrm{c}^{2}$. These values are used as a conventional model in order to compare different experimental results within the same astrophysical model. When computing the expected recoil spectra from spinindependent interactions the Helm nuclear form factor is used (see equation (3.24) and [76]). For spin-dependent scattering the spin-structure functions given in [204] are used in the calculation of the expected spectra. The expected spectra are calculated for each WIMP mass separately to set a 90\% CL on the cross section for each mass, resulting in an upper limit curve on the cross section as a function of the WIMP mass.

\subsubsection{Spin-independent coupling}

The 90\% CL upper limit on the spin-independent WIMP-nucleon scattering cross section from the current CDMS-II data is shown as the red solid line in figure 10.6. The kink in the curve at $\sim 60 \mathrm{GeV} / \mathrm{c}^{2}$ is a result of the optimum interval method and the observation of the two candidate events. In the optimum interval method, the intervals in which the limit is set can switch based on the distribution of candidate events. This can result in a step like feature of the upper limit curve. Nonetheless for each mass the statistical correct 90\% CL upper limit is obtained. Although having a similar exposure than the combined data from previous analyzes of Soudan data (reanalysis of Run 118/119 [166] and the Run $123 / 124$ analysis [4 shown as the blue dashed line in the figure) the upper limit is weaker due to the observation of two candidate events at low recoil energies. Since for the elastic scattering scenario a possible signal is expected at low recoil energies the observation has a strong influence on the upper limit. The combined limit from all Soudan data is shown as the black line in the figure. The combination of the data is a naive combination of the exposures and observed events for each analysis. The final combined limit is weaker than 


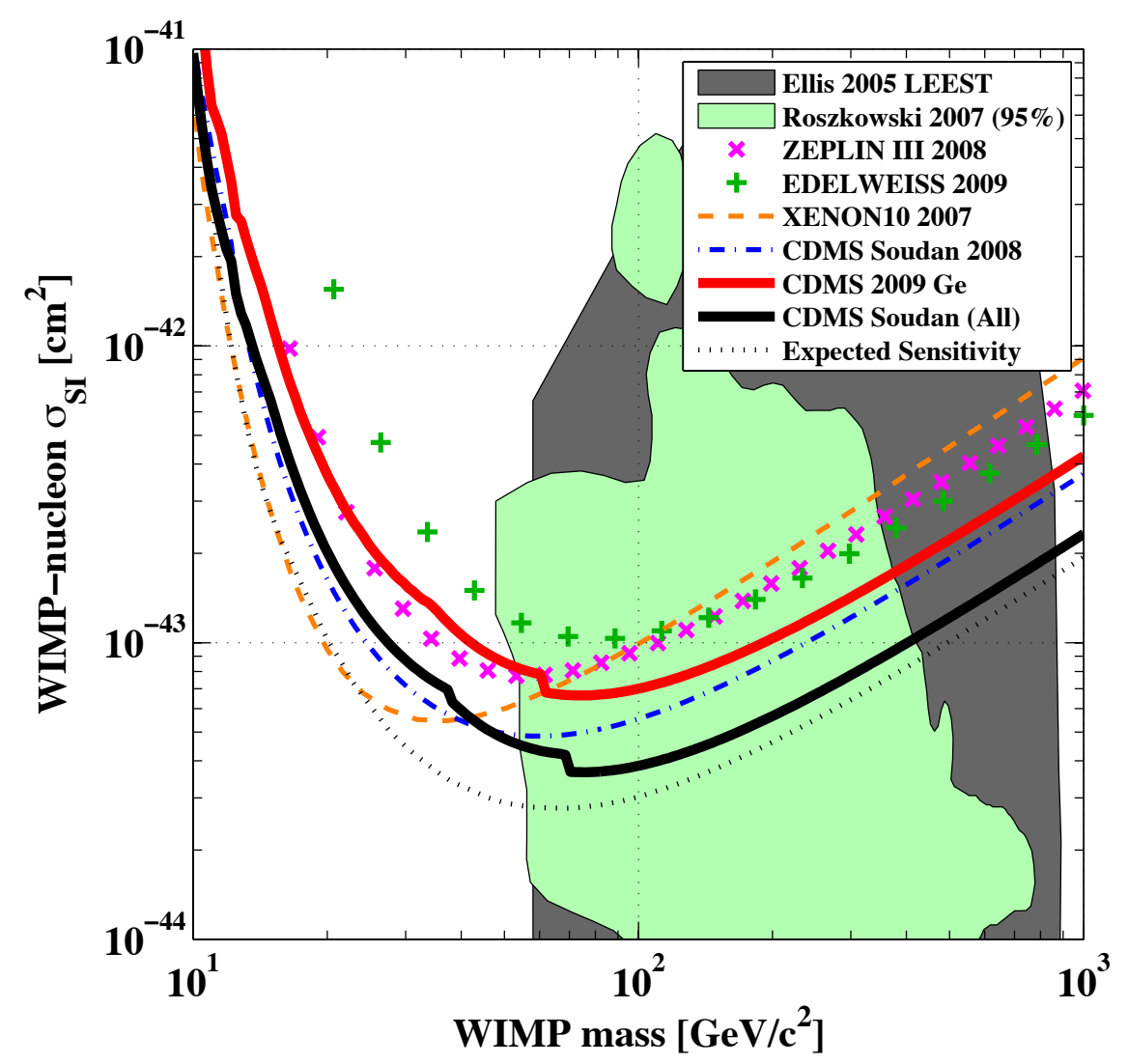

Figure 10.6: Upper limits (90\% C.L.) on the spin-independent WIMP-nucleon cross section as a function of the WIMP mass. The lines in the figure show the upper limit from the current CDMS-II analysis (red/solid), the combined sensitivity of previous Soudan data (blue/dash-dotted) and the combined upper limit from the CDMS-II experiment at Soudan (black/solid). The black/dotted curve gives the expected sensitivity of the combined data which would have been reached, if no candidate event had been observed in the current analysis. In addition upper limits from other leading experiments are shown (for references see text). The filled regions represent allowed parameter space from a broad range of MSSM and CMSSM models. 
the combined limit of the previous data at low WIMP masses, due to the inclusion of all four candidate events which have been observed. In the reanalysis of the Run 118/119 two events near threshold ( $5.3 \mathrm{keV}$ and $7.3 \mathrm{keV}$ ) have been observed [166] and the current analysis also observed two candidate events at $12.3 \mathrm{keV}$ and $15.5 \mathrm{keV}$. The Run $123 / 124$ analysis did not observe any candidate events. The figure also compares the combined upper limit against the results of other leading experiments. Results from EDELWEISS [113] (Ge target), ZEPLIN [120] (Xe target) and XENON10 [108] (Xe target) are shown. The combined CDMS upper limit is the strongest yet reported for WIMP masses $\gtrsim 44 \mathrm{GeV} / \mathrm{c}^{2}$. The dotted line in the figure indicates the expected sensitivity for the current exposure based on the estimated background combined with the observed sensitivity of past Soudan data. If the two candidate events observed in the current data would be interpreted as signal events the most likely values of the particles mass would reside in the region in which the difference between the actual and the expected sensitivity is largest.

In order to put this result in context with theoretical predictions, figure 10.6 also indicates two regions of theoretical and experimental interest. The dark gray filled region indicates the range of parameter space predicted in the LEEST (low-energy effective supersymmetry theory) framework, a broad selection of supersymmetry models [205]. This region represents the allowed parameter space of relatively unconstrained models, the actual allowed parameter space extends down to cross sections of $10^{-48} \mathrm{~cm}^{2}$, well below the figures' lower limit. The second green filled region indicates the allowed parameter space by more constrained CMSSM models, identified to be consistent with a variety of experimental results [206]. Current experiments are beginning to probe the most optimistic models of supersymmetry and future experiments will cut deep into the allowed parameter space.

\subsubsection{Spin-dependent coupling}

Figure 10.7 shows the 90\% CL upper limits on spin-dependent interactions. Following a convention 207 for comparing different experimental results, the upper limit is only shown for the WIMP-neutron coupling (i.e $a_{n}=1$ and $a_{p}=0$, see Section 3.1 .3 for reference). The figure shows the upper limit from the current analysis (shown in solid red) and the combined limit (solid black) with previous Soudan sensitivity (blue dashed) as in the spinindependent case. A comparison to results from other leading experiments namely ZEPLIN [208] and XENON10 [209] is shown in the figure. Although the spin-independent limit is stronger than any limit set based on a experiment using a Xe target (see figure 10.6) the limits for spin-dependent coupling are weaker due to a greater abundance of odd isotopes in $\mathrm{Xe}\left({ }^{129} \mathrm{Xe}(26.44 \%)\right.$ and $\left.{ }^{131} \mathrm{Xe}(21.18 \%)\right)$; the numbers in brackets give the natural abundance of each isotope), whereas Ge only has one odd isotope ${ }^{73} \mathrm{Ge}(7.73 \%)$ with a small natural abundance (see table 6.1). None of the current experimental sensitivities reaches the bounds of predictions of CMSSM parameters space, predicting cross sections dominantly below the $10^{-39} \mathrm{~cm}^{2}$ scale [206].

Limits on WIMP-proton scattering are dominated by dedicated experiments using oddproton nuclides, notably COUPP ( $\mathrm{CF}_{3} \mathrm{I}$ target) [128] and KIMS (CsI target) [119]. The constraints set from this analysis are not competitive with these result. Especially limits 


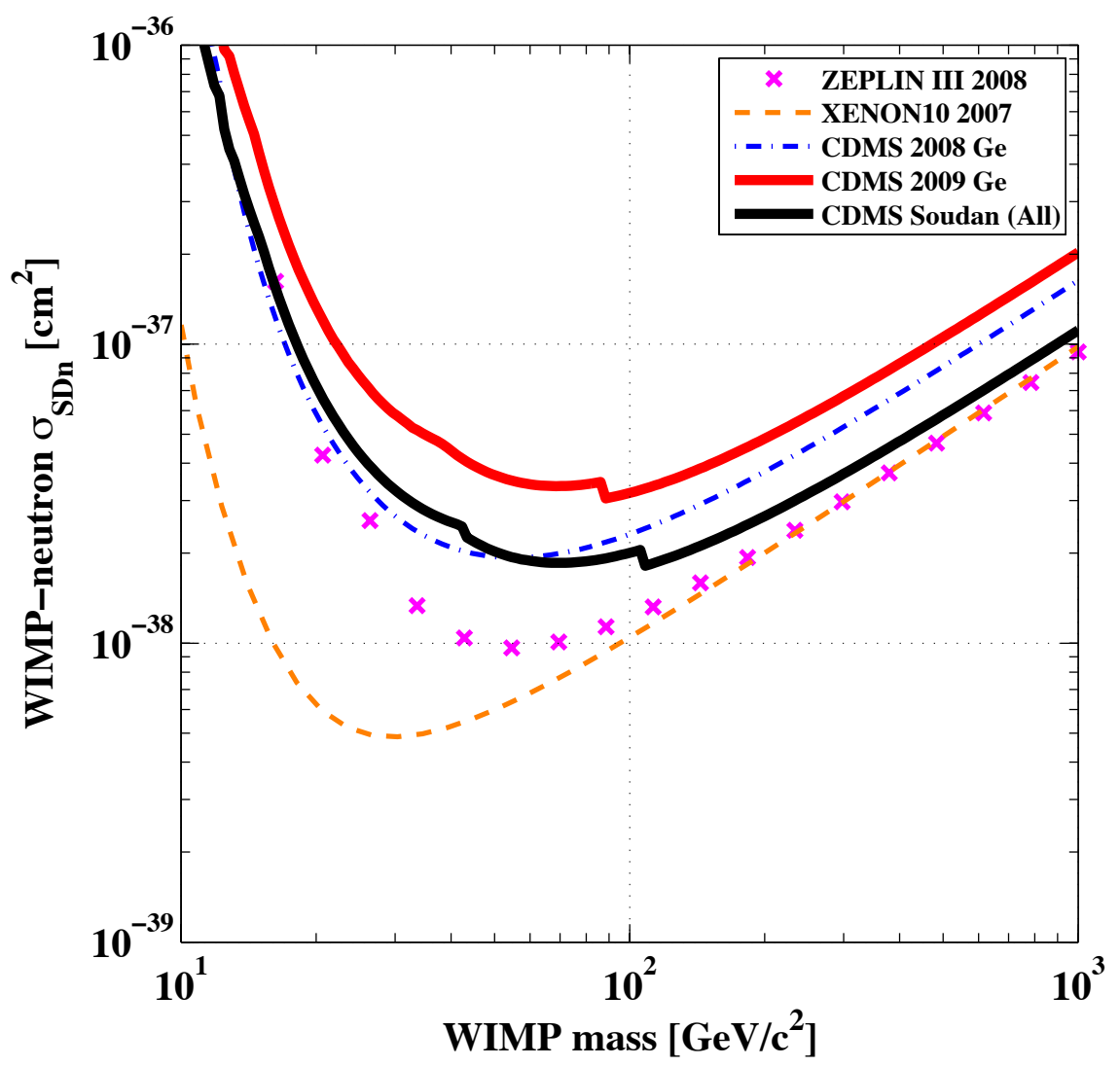

Figure 10.7: Upper limits (90\% C.L.) on the spin-dependent WIMP-nucleon cross section as a function of the WIMP mass, assuming a coupling solely to neutrons. The lines in the figure show the upper limit from the current CDMS-II analysis (red/solid), the combined sensitivity of previous Soudan data (blue/dash-dotted) and the combined upper limit from the CDMS-II experiment at Soudan (black/solid). In addition upper limits from other leading experiments are shown (for references see text). 
set from an indirect search for neutrino annihilation in the Sun by the Super-Kamiokande experiment [142] are roughly two orders of magnitude below the current CDMS sensitivity.

It is important to note that the assumption of a pure coupling to neutrons or protons is only a convention for comparing different experimental results but is not especially realistic. Most WIMP models predict spin-dependent cross sections upon protons and neutrons to be of similar magnitude, i.e $\left|a_{p}\right| \sim\left|a_{n}\right|$. The relative strengths of the various experimental constraints vary substantially with $a_{p} / a_{n}$, however assuming a pure coupling either to protons or neutrons sets a conservative upper limit on the expected cross section.

\subsection{DAMA result in light of the inelastic dark mat- ter model}

The movement of the Earth around the Sun would provide an annual modulation of the counting rate from dark matter particles, caused by the change in the relative velocity of the dark matter particle and the earthbound target (see Section 3.1.1). The DAMA collaboration claims the observation of such a modulation in two different $\mathrm{NaI}(\mathrm{Tl})$ scintillation detector arrays, the original DAMA/NaI setup [210] and the upgraded DAMA/LIBRA experiment [118]. Figure 10.8 shows the observed signal in the $2-6 \mathrm{keV}$ electron-equivalent energy range. The observed periodicity of $0.998 \pm 0.03$ years and phase of $144 \pm 8$ days is consistent with the expectation of a signal from dark matter particles distributed in the local halo according to the standard halo model. The DAMA collaboration claims that no known systematic detector effect could explain the modulation signal. However, the original interpretation of the DAMA result as a signal from WIMPs that would interact via nuclear recoils is inconsistent with other experimental results [4, 120, 108, 208, 209, 128, 119].

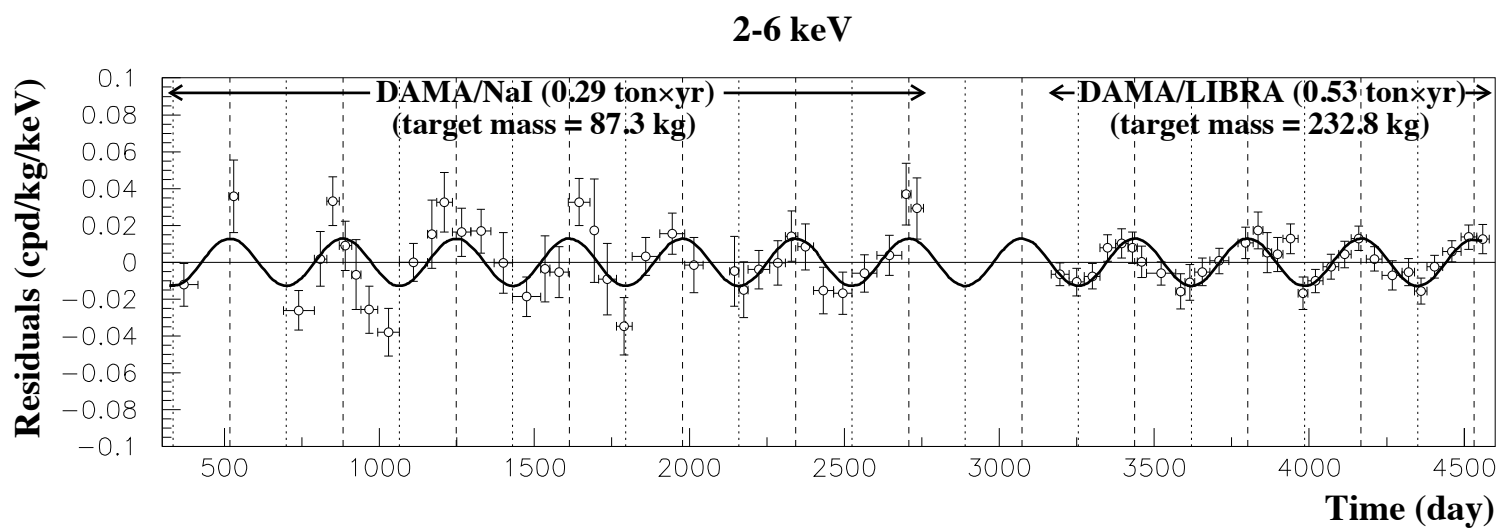

Figure 10.8: Residual rate of single hit scintillation events in the DAMA/NaI(LIBRA) setup. The rate clearly shows a modulation with time. Figure taken from [118].

However, in non-standard WIMP models the modulation signature can be made compatible with other direct detection results. In particular the so-called "inelastic dark matter" model [211, 212, 213] can explain the observed modulation while avoiding constraints 
from other experiments. The main difference between the inelastic dark matter model and the standard WIMP scenario is that the WIMP-nucleon scattering occurs via inelastic scattering with the dark matter particle transiting into an excited state, while elastic scattering id forbidden or highly suppressed. Thus, only WIMPs with enough energy to up-scatter into the excited state can scatter off nuclei in the detector. This minimal required energy translates into an increased minimal velocity the dark matter particles needs to have. Accordingly the lower bound of the phase space integral in equation 3.16 is updated to

$$
v_{\min }=\sqrt{\frac{1}{2 M_{T} E_{R}}}\left(\frac{M_{T} E_{R}}{\mu}+\delta\right)
$$

where $M_{T}$ is the mass of the target nuclei, $E_{R}$ is the recoil energy and $\mu$ is the reduced WIMP-nucleus mass. The new parameter introduced in the inelastic dark matter model is the mass-splitting $\delta$ between the ground and the exited state which increases the minimal velocity. Choosing $\delta=0 \mathrm{keV}$ leads to the usual elastic scattering scenario. Important consequences of this model for direct detection experiments are:

- The experiments probe the higher velocity part of the WIMP velocity distribution. So this model is much more sensitive to the cut-off imposed by the escape velocity than the conventional WIMP model.

- Heavier targets like I, Xe and W are favored over lighter targets.

- The differential event rates are significantly suppressed for lower recoil energies while the spectrum of conventional WIMPs rises roughly exponentially with lower recoil energies (see figure 10.9).

- The annual modulation is significantly enhanced.

Especially the last three points allow an interpretation of the observed modulation signature as a dark matter signal consistent with null observations from other direct detection experiments. However, the null observations in direct detection experiments allow to set constraints on the inelastic dark matter model parameter space. Since the parameter space is now three dimensional (mass, cross-section, mass-splitting) a direct comparison between two experiments is not easily visualized like in the standard WIMP scenario. In order to probe the parameter space allowed by the DAMA modulation signature a different approach is used.

First of all the DAMA allowed region in the mass-splitting and mass parameter space is determined by accepting each parameter point for which a cross section exists such that the expected rate is compatible at the $90 \% \mathrm{CL}$ with the DAMA data. The obtained allowed region is shown in green in figure 10.10. To set constraints on this allowed parameter space from the current analysis the 90\% CL exclusion limits for each parameter combination are calculated by the optimum interval method. If for a given choice of the mass-splitting and mass all DAMA allowed cross sections are excluded by the constraints set from this analysis, the parameter point is regarded as excluded. The resulting excluded parameter space is 


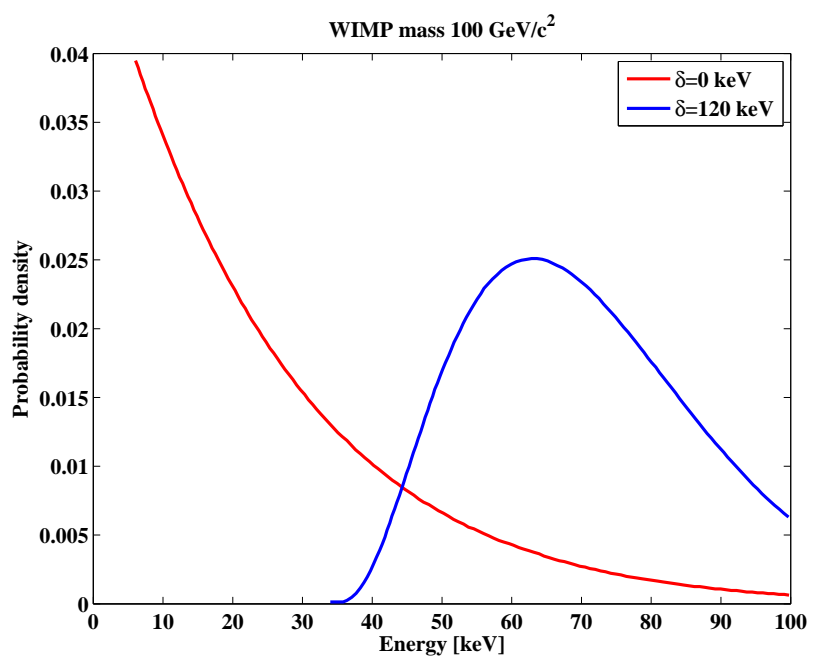

Figure 10.9: Comparison of the energy deposition probability densities for the elastic scattering scenario $\delta=0$ (red) and the inelastic scattering scenario for a mass splitting of $\delta=120 \mathrm{kev}$ (blue). For illustration the probability densities have been normalized in the range from $5-100 \mathrm{keV}$, and are shown for a WIMP mass of $100 \mathrm{GeV} / \mathrm{c}^{2}$. The main feature of the inelastic scattering scenario is, that the rate peaks at higher recoil energies and is completely suppressed at low recoil energies. Figure provided by S. Arrenberg.

plotted in black/hatched on top of the DAMA allowed parameter space in figure 10.10. The current result from the CDMS-II experiment excludes all but a narrow region of parameter space allowed by DAMA/LIBRA that resides at WIMP masses around $100 \mathrm{GeV} / \mathrm{c}^{2}$ and mass-splittings of $80-140 \mathrm{keV}$. In addition the plot shows the parameter space excluded by the XENON10 (red/hatched) result 214] calculated by the same procedure as described above.

It has to be stressed that the allowed regions and hence the excluded regions can vary substantially with the assumed galactic escape velocity. For the results shown in figure 10.10 an escape velocity of $544 \mathrm{~km} / \mathrm{s}$ has been assumed [215]. 


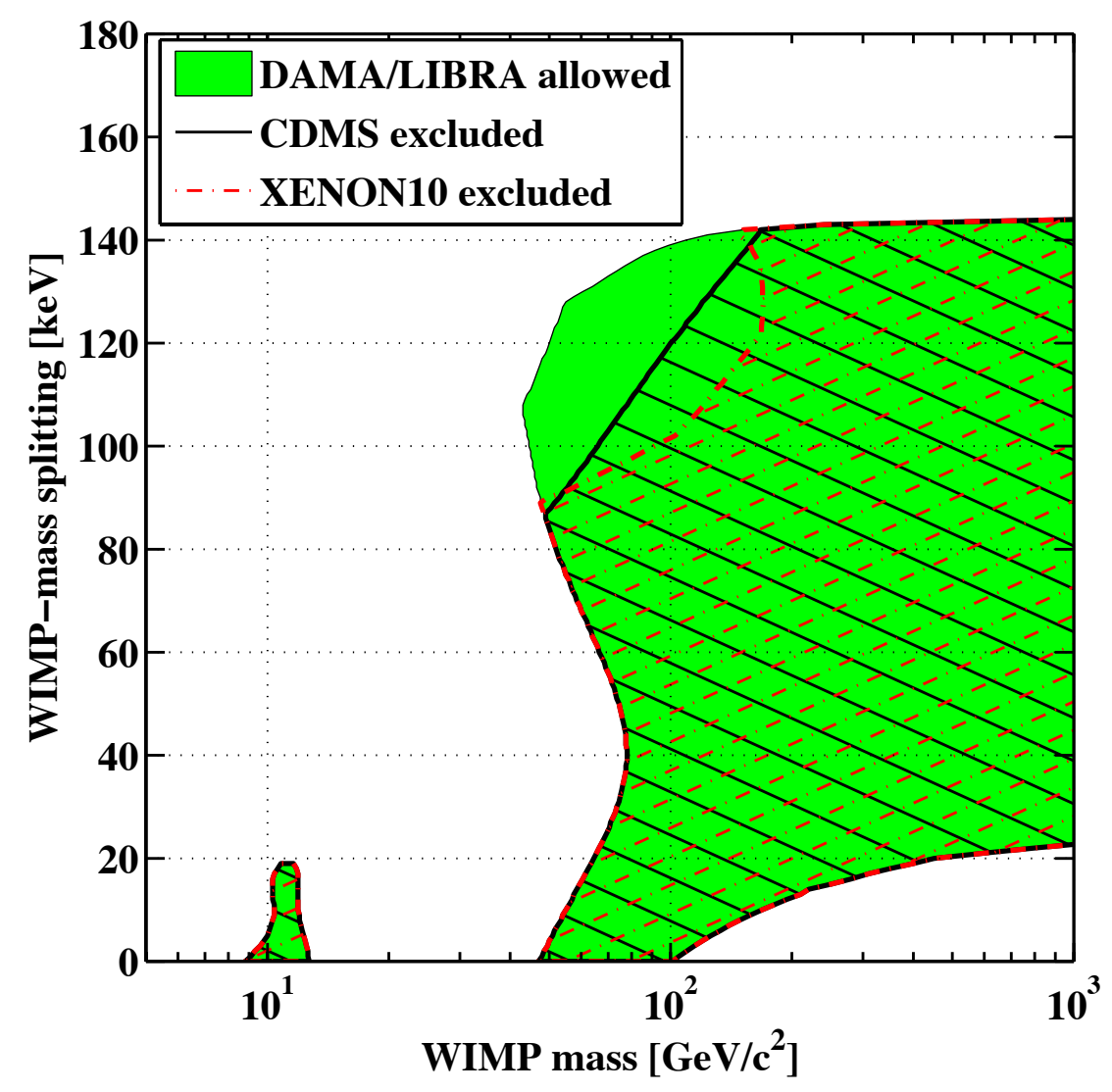

Figure 10.10: Allowed parameter space for which the DAMA modulation signature can be explained (green). Constraints on the allowed parameter space from this analysis [5] and the XENON10 result 214] are plotted as the hatched regions on top the allowed region. The current result from the CDMS-II experiment excludes all but a narrow region of parameter space allowed by DAMA/LIBRA that resides at WIMP masses around $100 \mathrm{GeV} / \mathrm{c}^{2}$ and mass-splittings of $80-140 \mathrm{keV}$. For details on how the parameter space is excluded see text. 


\subsection{Discussion and future prospects for CDMS}

The CDMS-II experiment currently sets world-leading upper limits on the WIMP-nucleon interaction, based on the combination of the data analysis described in this chapter with previous data from Soudan. The observation of two candidate events in the current exposure could not be interpreted as a significant evidence for WIMP interactions given a $23 \%$ probability that this result could be due to a fluctuation of the expected backgrounds. In the conservative approach of taking these two candidate events as signal events in the calculation of the upper limit on the WIMP-nucleon cross section the anticipated sensitivity of the combined data set was not reached.

The CDMS-II experiment has demonstrated a great capability of controlling backgrounds which is essential for the experiment's performance in future, longer data runs. The current analysis marks the end of the 5-Tower setup with $1 \mathrm{~cm}$ thick detectors at the Soudan site. To increase the sensitivity on the spin-independent cross-section beyond the $10^{-44} \mathrm{~cm}^{2}$ benchmark new detectors have been developed by the collaboration. These new ZIPs are fabricated on three-inch diameter, one-inch-thick (620g) Ge substrates, $2.5 \times$ thicker than the current detectors. The increased substrate thickness is a significant advantage, since the new detectors have $2.5 \times$ more detector mass for the same innerelectrode surface, thus reducing the surface-event rate at a given WIMP sensitivity by the same factor. The new detectors are also expected to have a lower intrinsic beta rate due to an improved handling to reduce radon exposure. In addition the arrangement of the aluminum collector fins around each TES has been optimized such that there is more aluminum within the quasiparticle-diffusion range of the TES, thereby improving the phonon collection. The layout of the phonon sensor is also changed to obtain a better event reconstruction. Rather than the four-quadrant design of current detectors the new ZIPs have three large inner sensors surrounded by an outer phonon guard ring. This sensor design shows a superior reconstruction of the radial position of events, thus reducing the possibility of miscorrections.

These detectors will be used in the next stage of the CDMS experiment, the SuperCDMS project [216]. As part of the SuperCDMS project, four Towers of the new detectors will be installed at the Soudan site alongside the best of the existing CDMS-II detectors. Each SuperCDMS Tower will only contain five one-inch ZIPs, surrounded by two $1 \mathrm{~cm}$ thick veto detectors at the top and bottom of the stack. This will improve self-shielding and allowing to avoid the difficulties faced with rejecting multiple scatters for detectors at the top or bottom of the stack. This stage will have a total of $\sim 15 \mathrm{~kg}$ germanium target mass, with an expected sensitivity reach on the spin-independent WIMP-nucleon crosssection of $\sim 5 \times 10^{-45} \mathrm{~cm}^{2}$ within a runtime of $\sim 2$ years. The first SuperCDMS Tower has been successfully deployed at the Soudan site to study the rejection capability of the new detectors and characterize their performance. Based on the experiment at Soudan and on continued research and development, a larger experiment of $100 \mathrm{~kg}$ target mass or more is expected to be proposed. This experiment would be located at the $6000 \mathrm{~m}$.w.e depth of SNOLAB [217] to reduce the neutron background to negligible levels. 


\section{Chapter 11}

\section{Constraining the WIMP scattering cross section and mass}

In this Chapter statistical tools are introduced, which can be used to determine the mass and cross section of WIMPs from the recorded scattered events of direct detection experiments. Due to the expected low statistics of the first detection, it is necessary to review the applicability of standard statistical tools like the determination of confidence levels with the likelihood function. Especially the treatment of background events in low counting statistics needs to be considered. In general the tools introduced in this chapter will follow the procedure of determining confidence levels described by Feldmann and Cousins in 203 .

Section 11.1 will review the determination of confidence levels with the likelihood function and discuss why this method is not accurate for low counting statistics. The approach for determining confidence levels with the method of [203] will be introduced. In Section 11.2 the proposed procedure to calculate confidence levels for direct dark matter experiments will be discussed. Especially the treatment of astronomical uncertainties on the input parameters needed for the calculations will be addressed by the use of a Markov Chain Monte Carlo algorithm.

\subsection{Determination of confidence regions}

A commonly used method for determining confidence regions on parameters $\theta$ is to use the shape of the likelihood function:

$$
\mathcal{L}(\theta)=\prod_{i=1}^{n} f\left(x_{i} ; \theta\right)
$$

where $f\left(x_{i} ; \theta\right)$ is the probability density function (p.d.f) of the data values $x_{i}$. The estimator on the parameter $\hat{\theta}$ is found by maximizing the likelihood function. In the large sample limit the likelihood function itself becomes Gaussian in form, centered around the maximum 
likelihood estimate $\hat{\theta}$ :

$$
\mathcal{L}(\theta)=\mathcal{L}_{\text {max }} \exp \left(\frac{(\theta-\hat{\theta})^{2}}{2 \sigma_{\hat{\theta}}^{2}}\right) .
$$

Thus changing the parameters $\theta$ by $\mathrm{N}$ standard deviations, the logarithm of the likelihood function (log-likelihood function) decreases by $\mathrm{N}^{2} / 2$ from its maximum value

$$
\log \left(\mathcal{L}\left(\theta \pm N \sigma_{\hat{\theta}}\right)\right)=\log \left(\mathcal{L}_{\max }\right)-\frac{N^{2}}{2}
$$

In fact it can be shown that even if the likelihood function is not a Gaussian function of the parameter, the central confidence interval $[\mathrm{a}, \mathrm{b}]=[\theta-\mathrm{c}, \theta+\mathrm{d}]$ can still be approximated by using equation (11.3).

In the case of $n$ parameters $\theta=\left(\theta_{1}, \ldots \theta_{n}\right)$ the likelihood function takes the form

$$
\mathcal{L}(\theta)=\mathcal{L}_{\text {max }} \exp \left[-\frac{1}{2}(\theta-\hat{\theta})^{T} V^{-1}(\theta-\hat{\theta})\right]=\mathcal{L}_{\text {max }} \exp \left[-\frac{1}{2} Q(\theta, \hat{\theta})\right] .
$$

Here $V^{-1}$ is the inverse of the covariance matrix and superscript $\mathrm{T}$ indicates the transposition. It can be shown, that if $\hat{\theta}$ is described by an $n$-dimensional Gaussian p.d.f, then the quantity $Q(\theta, \hat{\theta})$ is distributed according to a $\chi^{2}$ distribution for $n$ degrees of freedom. The statement that $Q(\theta, \hat{\theta})$ is less than some value $Q_{\gamma}$, i.e. that the estimate is within a certain distance of the true value $\theta$, implies $Q(\theta, \hat{\theta})<Q_{\gamma}$, i.e. that the true value $\theta$ is within the same distance of the estimate. Both cases therefor have the same probability

$$
P\left(Q(\theta, \hat{\theta}) \leq Q_{\gamma}\right)=\int_{0}^{Q_{\gamma}} f(z ; n) d z
$$

where $f(z ; n)$ is the $\chi^{2}$ distribution for $n$ degrees of freedom. The value $Q_{\gamma}$ is chosen to correspond to a given probability content

$$
\int_{0}^{Q_{\gamma}}(f)(z ; n) d z=1-\gamma
$$

That is,

$$
Q_{\gamma}=F^{-1}(1-\gamma ; n)
$$

is the quantile of order $1-\gamma$ of the $\chi^{2}$ distribution. The region of $\theta$-space defined by $Q(\theta, \hat{\theta}) \leq$ $Q_{\gamma}$ is called a confidence region with the confidence level 1- $\gamma$. As in the single parameter case, it can be constructed by finding the values of $\theta$ at which the log-likelihood function decreases by $Q_{\gamma} / 2$ from its maximum value,

$$
\log (\mathcal{L}(\theta))=\log \left(\mathcal{L}_{\max }\right)-\frac{Q_{\gamma}}{2}
$$

Values of the quantile $Q_{\gamma}$ for different values of the confidence level 1- $\gamma$ and number of parameters are given in table 11.1. From equation (11.4) it is obvious that this procedure 


\begin{tabular}{|c|ccccc|}
\hline & \multicolumn{5}{|c|}{$Q_{\gamma}$} \\
\cline { 2 - 6 } $1-\gamma$ & $\mathrm{n}=1$ & $\mathrm{n}=2$ & $\mathrm{n}=3$ & $\mathrm{n}=4$ & $\mathrm{n}=5$ \\
\hline 0.683 & 1.00 & 2.30 & 3.53 & 4.72 & 5.89 \\
0.90 & 2.71 & 4.61 & 6.25 & 7.78 & 9.24 \\
0.95 & 3.84 & 5.99 & 7.82 & 9.49 & 11.1 \\
0.99 & 6.63 & 9.21 & 11.3 & 13.3 & 15.1 \\
\hline
\end{tabular}

Table 11.1: Values of the quantile $Q_{\gamma}$ for different values of the confidence level $1-\gamma$ for $\mathrm{n}=1,2,3,4,5$ parameters.

is only accurate if the logarithm of the likelihood ratio is $\chi^{2}$ distributed,

$$
-2 \log \lambda(\theta)=-2 \log \left(\frac{\mathcal{L}(\theta)}{\mathcal{L}_{\text {max }}}\right)=Q(\theta, \hat{\theta}) .
$$

Since the signal sample in direct detection experiments is expected to be small $(\leq 10)$ in the first detections, it is questionable if $-2 \log \lambda(\theta)$ is $\chi^{2}$ distributed. In figure 11.1 the likelihood ratios for $10000 \mathrm{MC}$ experiments are shown for 5(a), 10(b), 20(c) and 30(d) signal events. The number of signal events in each experiment is treated as a poissonian number with the corresponding mean. Since two parameters are determined from the likelihood function (the mass and cross section of the dark matter particle) the likelihood ratios are expected to follow a $\chi^{2}$ distribution with 2 degrees of freedom, which is shown as the red line in the plots. It can be clearly seen that for low statistics $\lesssim 20$ the likelihood ratios are not $\chi^{2}$ distributed. For larger samples $\gtrsim 30$ the likelihood ratios are $\chi^{2}$ distributed. In figure 11.1 (a) the Poissonian distribution of the signal counts can be seen due to the strong peaks at specific values of the likelihood ratios. This is smoothened out in figure 11.1 (d), since the signal counts approach a Gaussian distribution. Thus the procedure described above is not applicable to low statistics (signal sample $\lesssim 30$ events). A statistical procedure is needed from which the accurate confidence regions can be constructed even in the low statistic case.

\subsubsection{Classical construction of confidence regions}

The classical construction of confidence intervals was first developed by Neyman [218]. Suppose one has $m$ observations of a variable $x$ which can be used to evaluate an estimator $\hat{\theta}$ for a parameter $\theta$, and that the value obtained is $\hat{\theta}_{o b s}$. Furthermore, suppose that one knows the p.d.f of $\hat{\theta}, \mathrm{g}(\hat{\theta} ; \theta)$, which contains the parameter $\theta$ as a parameter. From $\mathrm{g}(\hat{\theta} ; \theta)$ one can determine the value $u_{\alpha}$ such that there is a fixed probability $\alpha$ to observe $\hat{\theta} \geq u_{\alpha}$, and similarly the value $v_{\beta}$ such that there is a probability $\beta$ to observe $\hat{\theta} \leq v_{\beta}$. The values $u_{\alpha}$ and $v_{\beta}$ depend on the true parameter of $\theta$, and are thus determined by

$$
\alpha=P\left(\hat{\theta} \geq u_{\alpha}(\theta)\right)=\int_{u_{\alpha}(\theta)}^{\infty} g(\hat{\theta} ; \theta) d \hat{\theta}=1-G\left(u_{\alpha}(\theta) ; \theta\right)
$$




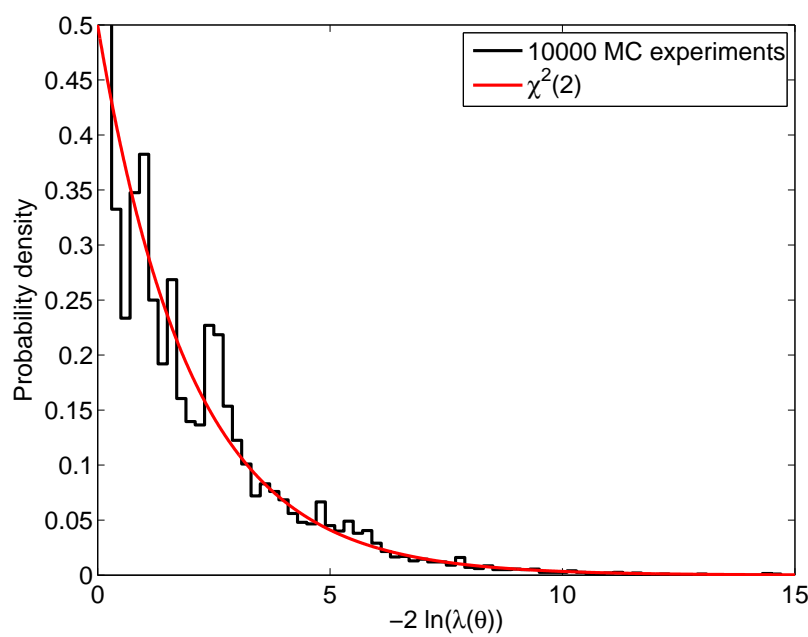

(a)

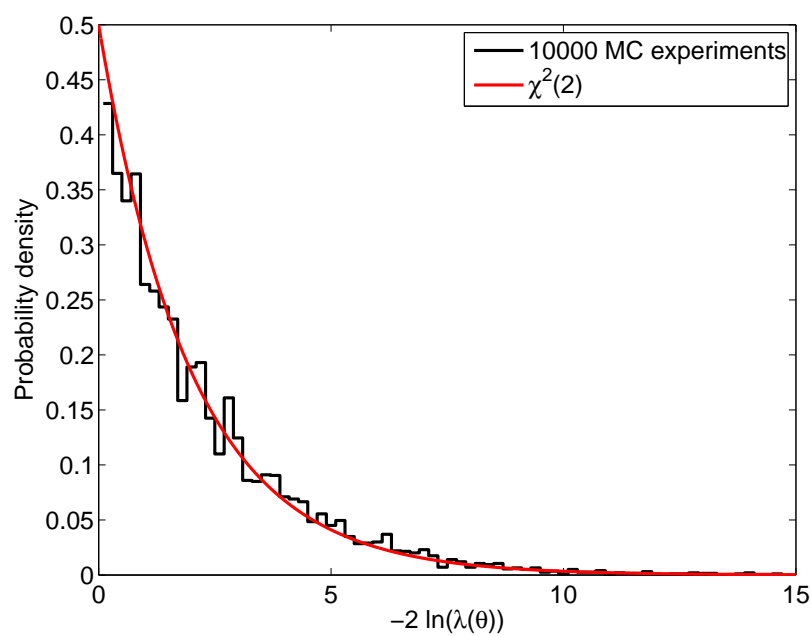

(c)

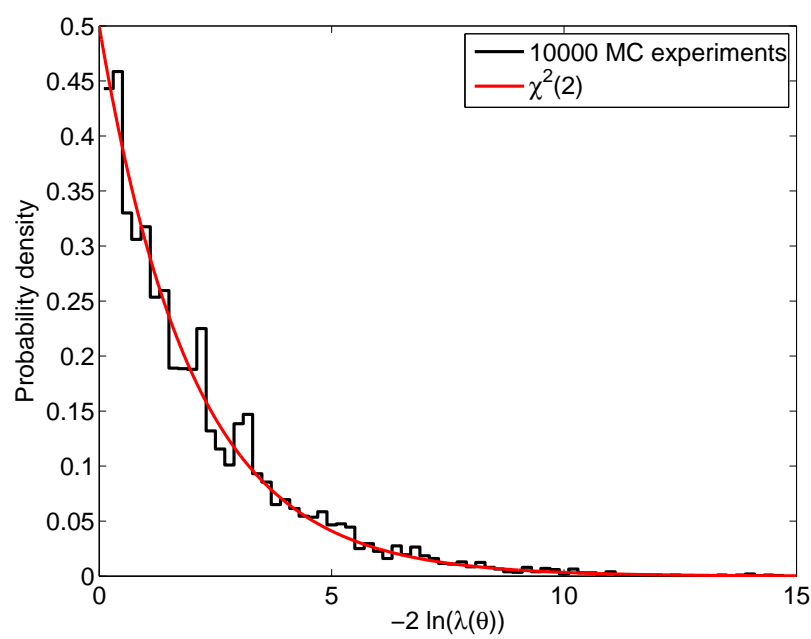

(b)

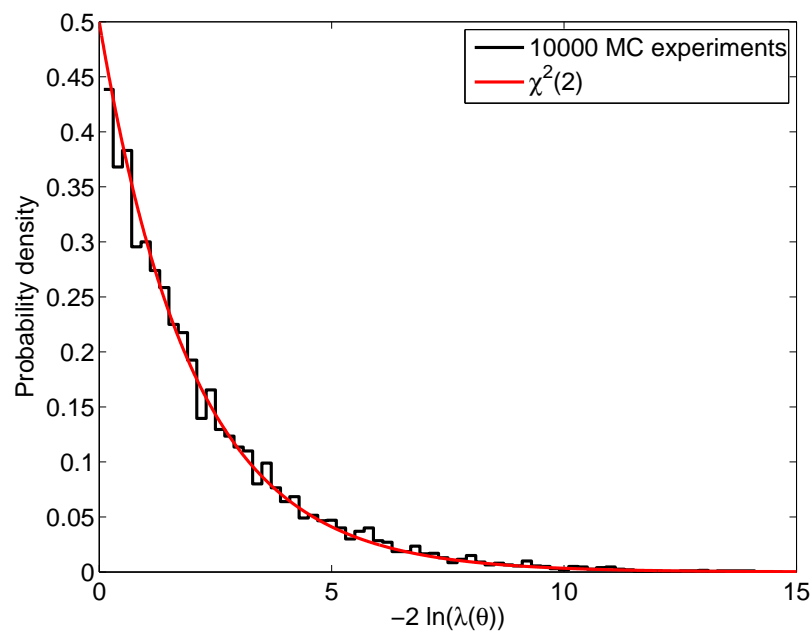

(d)

Figure 11.1: Likelihood ratios from 10000 Monte Carlo experiments for an expected mean number of signal events of 5(a), 10(b), 20(c) and 30(d). The red/solid curve is the $\chi^{2}-$ distribution for two degrees of freedom.

and

$$
\beta=P\left(\hat{\theta} \leq v_{\beta}(\theta)\right)=\int_{-\infty}^{v_{\beta}(\theta)} g(\hat{\theta} ; \theta) d \hat{\theta}=G\left(v_{\beta}(\theta) ; \theta\right)
$$

where $G$ is the cumulative distribution corresponding to the p.d.f $\mathrm{g}(\hat{\theta} ; \theta)$. The region between the two functions $u_{\alpha}(\theta)$ and $v_{\beta}(\theta)$ is called the confidence belt. The probability for the estimator to be inside the belt, regardless of the value $\theta$, is given by

$$
P\left(v_{\beta}(\theta) \leq \hat{\theta} \geq u_{\alpha}(\theta)\right)=1-\alpha-\beta \text {. }
$$


As long as $u_{\alpha}(\theta)$ and $v_{\beta}(\theta)$ are monotonically increasing functions of $\theta$ one can determine the inverse functions

$$
a(\hat{\theta}) \equiv u_{\alpha}^{-1}(\hat{\theta}), b(\hat{\theta}) \equiv v_{\beta}^{-1}(\hat{\theta})
$$

The inequalities

$$
\hat{\theta} \geq u_{\alpha}(\theta), \hat{\theta} \leq v_{\beta}(\theta)
$$

then imply respectively

$$
a(\hat{\theta}) \geq \theta, b(\hat{\theta}) \leq \theta
$$

Equations (11.10) and (11.11) thus become,

$$
P(a(\hat{\theta}) \geq \theta)=\alpha, P(b(\hat{\theta}) \leq \theta)=\beta
$$

or taken together,

$$
P(a(\hat{\theta}) \leq \theta \leq b(\hat{\theta}))=1-\alpha-\beta .
$$

The interval $[a, b]$ is called a confidence interval at a confidence level of $1-\alpha-\beta$.

Two-sided intervals (i.e both $a$ and $b$ specified) are not uniquely determined by the confidence level $1-\alpha-\beta$. One often chooses, $\alpha=\beta=\gamma / 2$ giving a so called central confidence interval with probability $1-\gamma$. Note that a central confidence interval does not necessarily mean that $a$ and $b$ are equidistant from the estimated value $\hat{\theta}$, but only that the probabilities $\alpha$ and $\beta$ are equal. In some situations one may only be interested in a one-sided confidence interval or limit. That is, the value $a$ represents a lower limit on the parameter $\theta$ such that $a \leq \theta$ with the probability $1-\alpha$. Similarly, $b$ represents an upper limit on $\theta$ such that $P(\theta \leq b)=1-\beta$.

\section{Poisson process with background}

Figures 11.2 (a) and (b) show classical confidence belts for a Poisson process with background when the observable $x$ is the total number of observed events $n$, consisting of signal events with mean $\mu$ and background events with known mean $b$. The p.d.f. is given by

$$
P(n \mid \mu)=\frac{(\mu+b)^{n}}{n !} e^{-(\mu+b)}
$$

For illustration the case where $b=1.0$ is used in these figures.

Since $n$ is an integer, the equations (11.16) can only be approximately satisfied. By convention one strictly avoids under-coverage and replaces the equalities in equations (11.16) with " $\geq "$. Thus the intervals over-cover. 


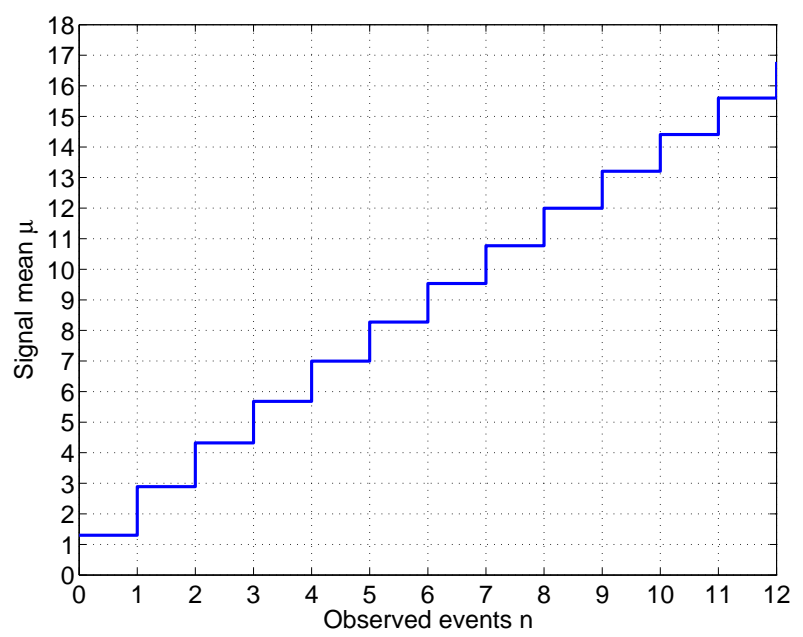

(a)

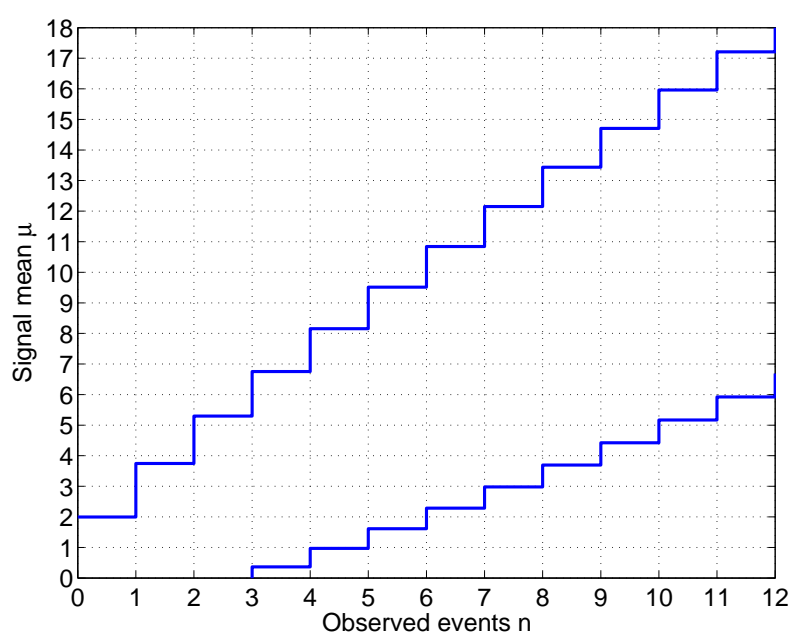

(b)

Figure 11.2: Classical confidence belt for 90\% C.L. upper limits (a) and central confidence intervals (b) for unknown Poisson signal mean $\mu$ in the presence of a Poisson background with known mean $b=1.0$.

\subsubsection{Confidence intervals from the Feldman-Cousins ordering principle}

In the example of a Poisson process with Poisson background for the assumed known mean $b=1.0$, the probability $P(n \mid \mu)$ to observe $n=0$ events is 0.135 if $\mu=1.0$, which is quite low. However, it is not so low when compared to the probability (0.368) of observing $n=0$ events with $b=1.0$ and $\mu=0.0$, which is the alternate hypothesis with the greatest likelihood. A ratio $R$ of likelihoods, in this case $0.135 / 0.368$, is used as an ordering principle when selecting those values of $n$ to place in the acceptance interval.

That is, for each $n$ let $\mu_{\text {best }}$ be the value of the signal mean $\mu$ which maximizes $P(n \mid \mu)$, and require $\mu_{\text {best }}$ to be physically allowed, i.e non negative in this case. Then $\mu_{\text {best }}=$ $\max (0, n-b)$. The probability is then given by $P\left(n \mid \mu_{\text {best }}\right)$. With this two probabilities the ratio

$$
R=\frac{P(n \mid \mu)}{P\left(n \mid \mu_{\text {best }}\right)}
$$

can be calculated on which the ordering principle is based. Table 11.2 summarizes these quantities for the construction of the acceptance interval for $\mu=1.0 . R$ is the ratio of two likelihoods: the likelihood of observing $n$ events given the actual signal mean $\mu$ and the likelihood of observing $n$ events given the best-fit physically allowed mean. Values of $n$ are added to the acceptance interval for a given $\mu$ in decreasing order of $R$, until the sum of the $P(n \mid \mu)$ meets or exceeds the desired C.L. This ordering, for values of $n$ necessary to obtain a total probability of $90 \%$, is shown in the column labeled "rank". Thus, the acceptance region for $\mu$ is the interval $n=[04]$. Like in the case of the classical construction the acceptance region contains a summed probability greater than $90 \%$ due 


\begin{tabular}{|cccccc|}
\hline $\mathrm{n}$ & $P(n \mid \mu)$ & $\mu_{\text {best }}$ & $P\left(n \mid \mu_{\text {best }}\right)$ & $\mathrm{R}$ & rank \\
\hline 0 & 0.1353 & 0 & 0.3679 & 0.3678 & 5 \\
1 & 0.2707 & 0 & 0.3679 & 0.7358 & 3 \\
2 & 0.2707 & 1 & 0.2707 & 1.0 & 1 \\
3 & 0.1804 & 2 & 0.2240 & 0.8054 & 2 \\
4 & 0.0902 & 3 & 0.1954 & 0.4616 & 4 \\
5 & 0.0361 & 4 & 0.1755 & 0.2057 & \\
6 & 0.0120 & 5 & 0.1606 & 0.0747 & \\
7 & 0.0034 & 6 & 0.1490 & 0.0288 & \\
\hline
\end{tabular}

Table 11.2: Quantities used in the confidence interval construction for signal mean $\mu=1$ in the presence of known mean background $b=1.0$.

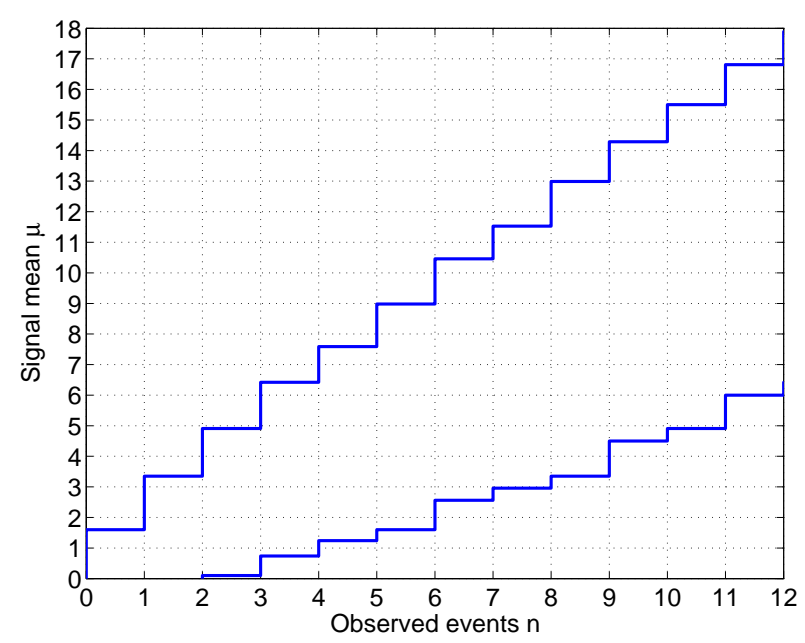

Figure 11.3: Confidence belt based on the Feldman - Cousins ordering principle, for 90\% C.L. confidence intervals for unknown Poisson signal mean $\mu$ in the presence of a Poisson background with known mean $b=1.0$.

to the discreetness of $n$. This is unavoidable and leads to confidence intervals which overcover. The construction of the confidence belt proceeds by finding the acceptance region for all values of $\mu$ for the given $b$. For the example case studied here, the resulting confidence belt is shown in figure 11.3. This confidence belt has to be compared to the confidence belts shown in figure 11.2.

In general the likelihoods used in the ratio $R$ can be replaced by the likelihood function constructed from the experimental data.

$$
R=\frac{P(\theta)}{P(\hat{\theta})}=\frac{\mathcal{L}(\theta)}{\mathcal{L}(\hat{\theta})}
$$

where $\theta$ is the parameter to be determined from the data and $\hat{\theta}$ is the best experimental estimator of this parameter which maximizes the likelihood function. In this general case, the ordering principle provides exact coverage. 


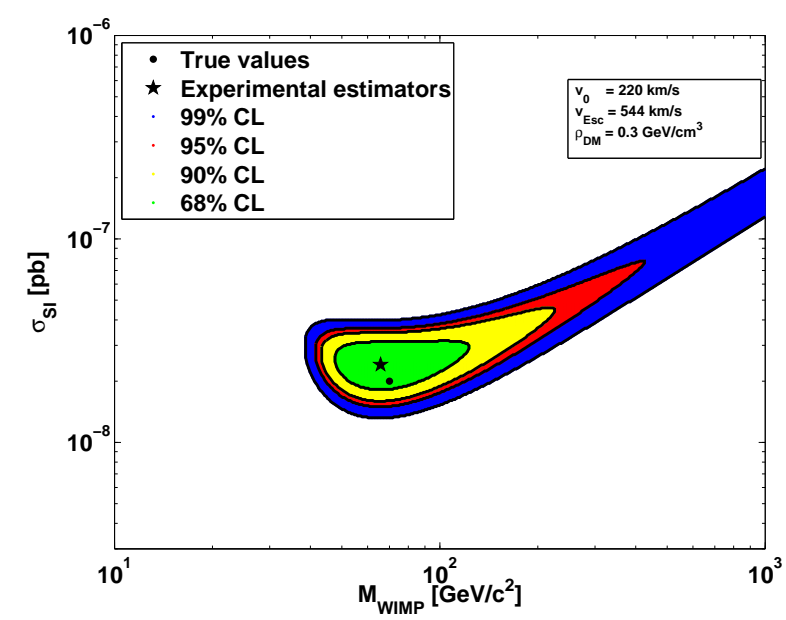

(a)

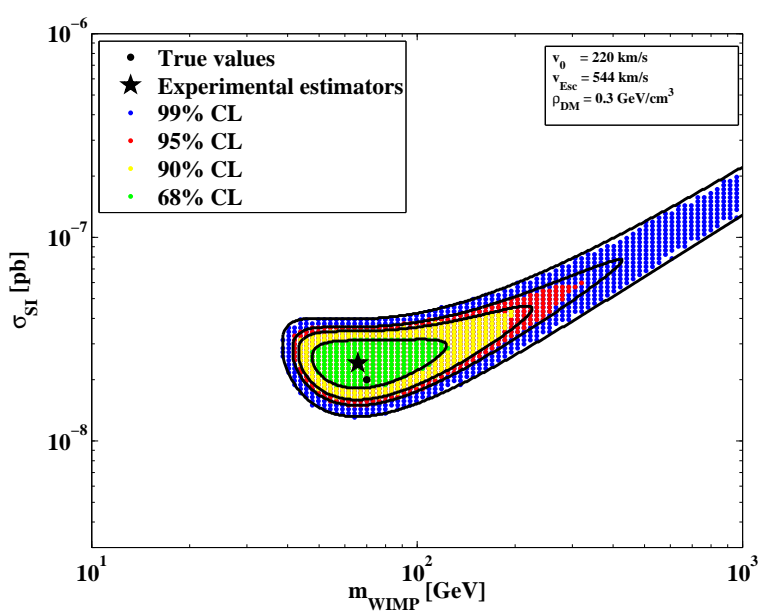

(b)

Figure 11.4: Confidence regions on the cross section and mass of the WIMP derived directly from the likelihood function (a) and from the Feldman-Cousins ordering principle (b), in the case of the detection of 30 WIMP scatters. The black solid lines in (b) are the same as in (a).

The application of this ordering principle to direct detection experiments will be discussed in the following Section. There will be two parameters to be determined from the experimental data. These are the mass of the dark matter particle and the scattering cross section. It is useful to study for which statistic the use of the Feldman-Cousins ordering principle is the only adequate procedure which provides the exact confidence regions on these parameters. As seen from figure 11.1, the likelihood ratios are approaching a $\chi^{2}$ distribution if the detected number of WIMP events is $\gtrsim 30$. Thus the confidence regions determined from the ordering principle and those derived directly from the likelihood function by equation 11.8 should match each other.

In figure 11.4 the case of the detection of 30 WIMP scatters is studied. Figure 11.4 (a) shows the confidence region derived directly from the likelihood function, and the confidence regions constructed from the ordering principle are shown in figure 11.4 (b). The black solid lines give the borders of the confidence regions derived from the likelihood function. The direct comparison of these borders with the confidence regions determined from the ordering principle in figure 11.4 (b) shows, that the confidence regions from both methods match each other. Although the confidence regions derived directly from the likelihood function show a slight over-coverage. This over-coverage will be reduced if higher statistics are used. Hence in the case of high statistics (detection of $\gtrsim 30$ WIMP scatters), the confidence regions derived directly from the likelihood function provide the correct confidence regions. 


\subsection{Application of the Feldman Cousins method}

Given $n$ events with energies $E_{n}$ which are considered as WIMP candidates, the likelihood function is constructed from the expected differential recoil spectrum 3.17. The p.d.f used for the likelihood function is the differential recoil spectrum normalized to the total energy range considered in the experiment:

$$
f\left(E_{n} ; \theta\right)=\frac{d R / d E_{n}(\theta)}{\int_{E_{t h}}^{E_{\text {max }}} d R / d E(\theta) d E},
$$

where $E_{t h}$ is the threshold of the experiment and $E_{\max }$ is the maximal recoil energy considered in the experiment. Since detector media are composed of $m$ isotopes the likelihood function has to take into account the relative contributions from each isotope weighted by its natural abundance $\eta_{m}$. In addition, the in general energy dependent detection efficiency $\epsilon(E)$ of the experiment has to be taken into account:

$$
f_{\text {sum }}\left(E_{n} ; \theta\right)=\frac{\sum_{j=1}^{m} \eta_{m} \epsilon\left(E_{n}\right) d R / d E_{n}(\theta)}{\sum_{j=1}^{m} \eta_{m} \int_{E_{\text {th }}}^{E_{\max }} \epsilon(E) d R / d E(\theta) d E} .
$$

Thus, the extended likelihood function can be written as:

$$
\mathcal{L}=\frac{\nu(\theta)^{n}}{n !} e^{-\nu(\theta)} \prod_{i=1}^{n} f_{\text {sum }}\left(E_{n} ; \theta\right),
$$

where for a given exposure $M D$ and $\epsilon(E)$

$$
\nu(\theta)=M D \cdot \int_{E_{t h}}^{E_{\max }} \epsilon(E) \frac{d R}{d E}(\theta) d E,
$$

is the total expected number of WIMP interactions. For fixed local dark matter density and velocities the parameters $\theta$ which are derived from the likelihood function are the WIMP mass $M_{W I M P}$ and the WIMP-nucleon cross section $\sigma$. We will focus here on the case of pure spin-independent WIMP - nucleus coupling with a cross section $\sigma_{S I}$.

For each parameter combination $\theta=\left(M_{W I M P}, \sigma_{S I}\right)$ a large number of MC experiments is performed and for each experiment the best estimators $\hat{\theta}$ are determined. In each MC experiment the number of detected events is poissonian fluctuated around the expected number of events. The energies of the events are determined from the expected differential recoil spectra in the detectors by an acceptance-rejection method. The likelihood ratio for each experiment is then calculated by:

$$
\lambda(\theta)=\frac{\mathcal{L}(\theta)}{\mathcal{L}(\theta)}
$$

For the experimental data, the best experimental estimators $\hat{\theta}_{\text {exp }}$ are determined, and the likelihood ratio at each parameter combination is calculated,

$$
\lambda\left(\theta_{\text {exp }}\right)=\frac{\mathcal{L}(\theta)}{\mathcal{L}\left(\hat{\theta}_{\text {exp }}\right)} .
$$




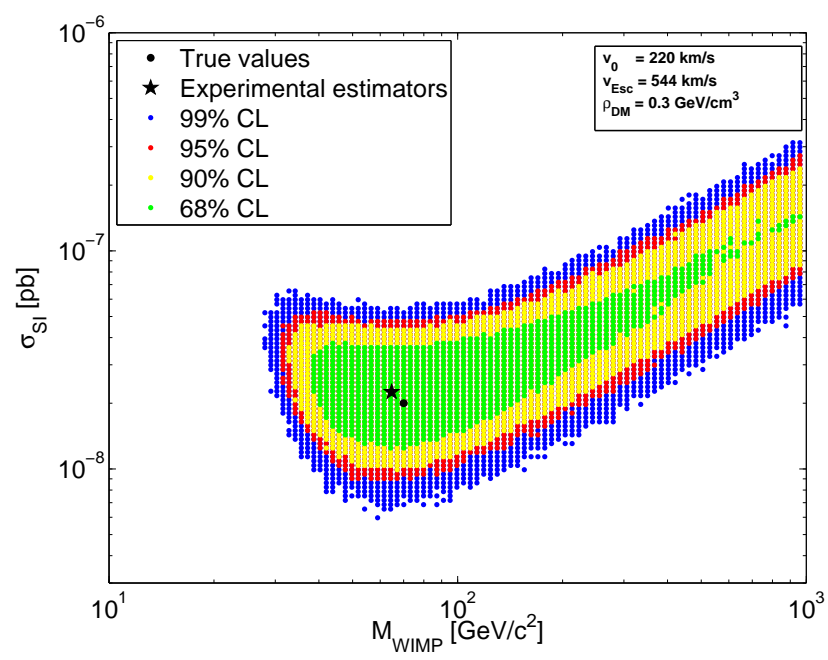

Figure 11.5: Confidence regions determined from eight signal events in germanium with a zero background assumption.

From the simulated $\lambda(\theta)$ a test criteria is constructed by determining the value $\chi_{C L}$ of the $\lambda(\theta)$ s for which $\alpha$ percent (where $\alpha$ is the confidence level) of the likelihood ratios are greater than this value $\chi_{C L}$. A certain parameter point is accepted in the confidence region for a confidence level $\alpha=1-\gamma$ if the value of $\lambda\left(\theta_{\exp }\right)$ is greater than $\chi_{C L}$.

Figure 11.5 shows the resulting confidence regions if eight events were detected in germanium detectors assuming typical parameters of the analysis performed with the CDMS experiment. A recoil threshold of $10 \mathrm{keV}$ has been assumed and a detection efficiency of $30 \%$ which is flat in energy. These confidence regions are determined under the assumption of zero background events.

\subsubsection{Treatment of backgrounds}

Direct detection experiments put a lot of effort in reducing their backgrounds down to a level of less than one expected background event in the signal region. For the last analysis of the CDMS-II data (Run 123/4) the expected background from surface-event leakage was:

$$
\eta_{\text {leak }}=0.59_{-0.27}^{+0.49}(\text { stat })_{-0.15}^{+0.32}(\text { sys. })
$$

The treatment of this background in the determination of the confidence regions is done under the assumption of a poissonian probability distribution of the number of leaking surface-events in the nuclear-recoil signal region. In each of the MC experiments the actual number of leaking surface-events is determined from a Poisson distribution with mean given by the expected number of the surface-event leakage. To each surface-event a random energy drawn from the energy distribution of singles in the nuclear-recoil region before applying the surface-event rejection cut (see figure 11.6) is assigned. The probability distribution of singles in the nuclear-recoil region $d S / d E_{n}$ is described by the sum of an 


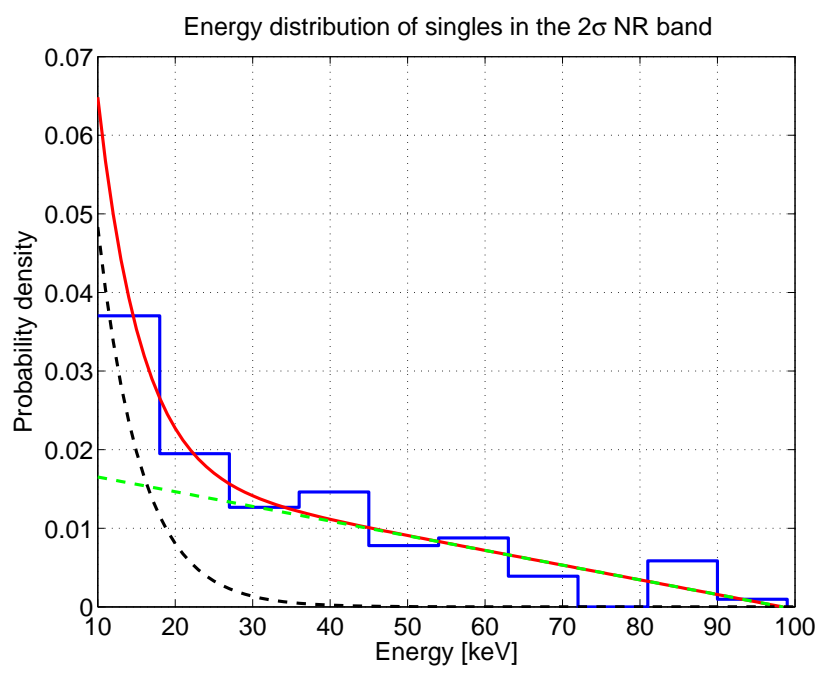

Figure 11.6: Energy distribution of singles in the nuclear-recoil signal region before applying the surface-event rejection cut.

exponential and a linear term. The likelihood function (11.23), including a term describing the contribution from the surface-event leakage, can be written as:

$$
\begin{aligned}
\mathcal{L}= & \frac{\left(\nu(\theta)+\eta_{\text {leak }}\right)^{n}}{n !} e^{-\left(\nu(\theta)+\eta_{\text {leak }}\right)} \times \\
& \prod_{i=1}^{n}\left(\frac{\nu(\theta)}{\left(\nu(\theta)+\eta_{\text {leak }}\right)} f_{\text {sum }}\left(E_{n} ; \theta\right)+\frac{\eta_{\text {leak }}}{\left(\nu(\theta)+\eta_{\text {leak }}\right)} f_{\eta_{\text {leak }}}\left(E_{n}\right)\right)
\end{aligned}
$$

where $\eta_{l e a k}$ is the expected number of leaking surface-events and

$$
f_{\eta_{l e a k}}\left(E_{n}\right)=\frac{d S / d E_{n}}{\int_{E_{t h}}^{E_{\max }} d S / d E d E} .
$$

The effect of considering several values of the mean expected surface-event leakage on the confidence regions is shown in figure 11.7. As expected, the inclusion of the expected number of leaking surface-events in the determination of the confidence regions leads to larger confidence regions. Although the effect is not big, the $68 \%$ confidence region extends to higher masses as the number of expected background events is increased. In general this effect should get smaller as the statistics of the detected events increases, while the expected background is kept at the same level.

A second source of backgrounds are nuclear recoils from neutron scatters. In general there are two sources which could cause neutron scatters in direct detection experiments. As described in Chapter 8, these are neutrons induced by muon interaction and neutrons from $(\alpha, \mathrm{n})$ reactions and spontaneous fissions of radioactive contamination of the materials used to construct the experimental apparatus. Since direct detection experiments are usually installed in laboratories with a great overburden of rock to reduce the muon induced 


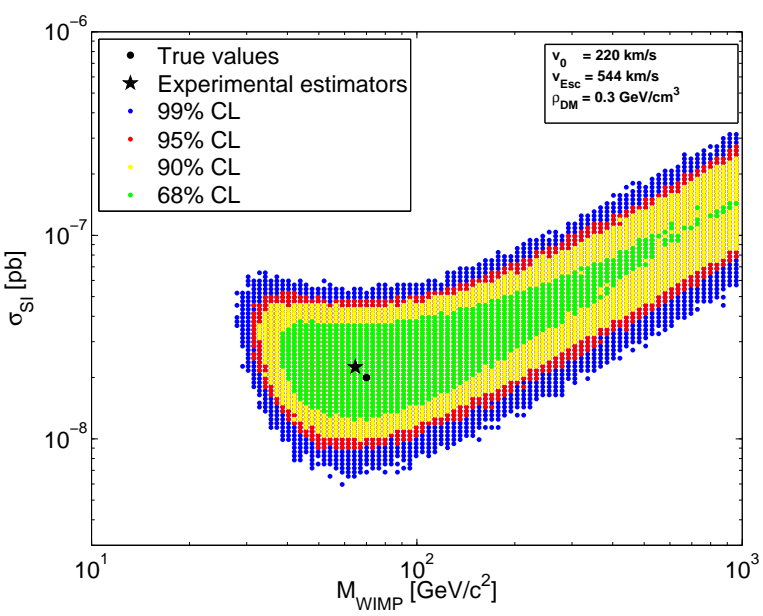

(a)

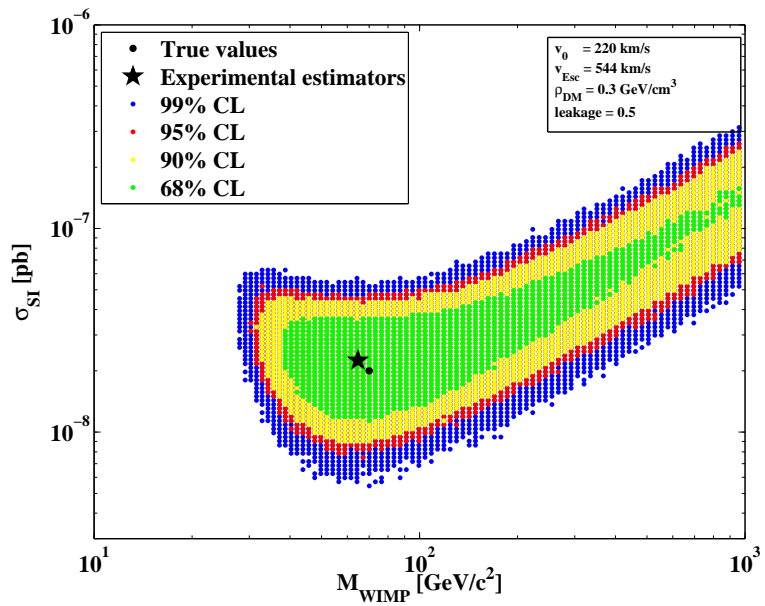

(c)

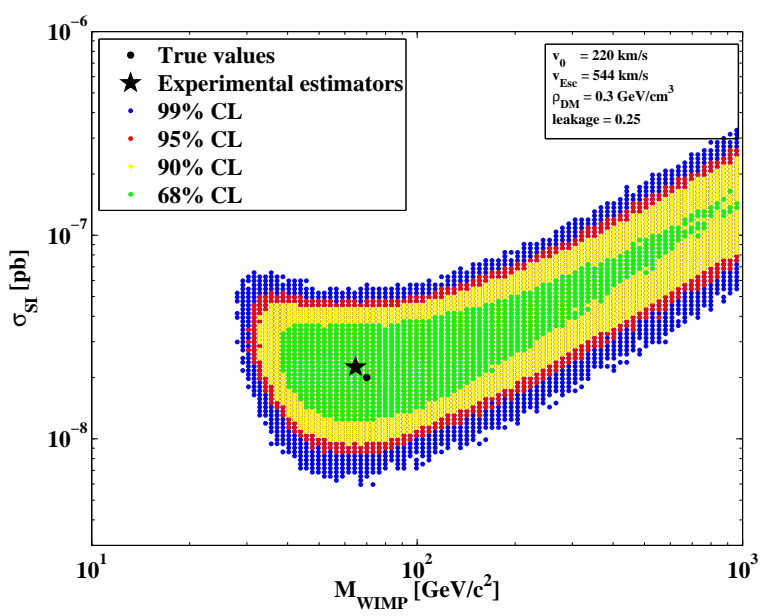

(b)

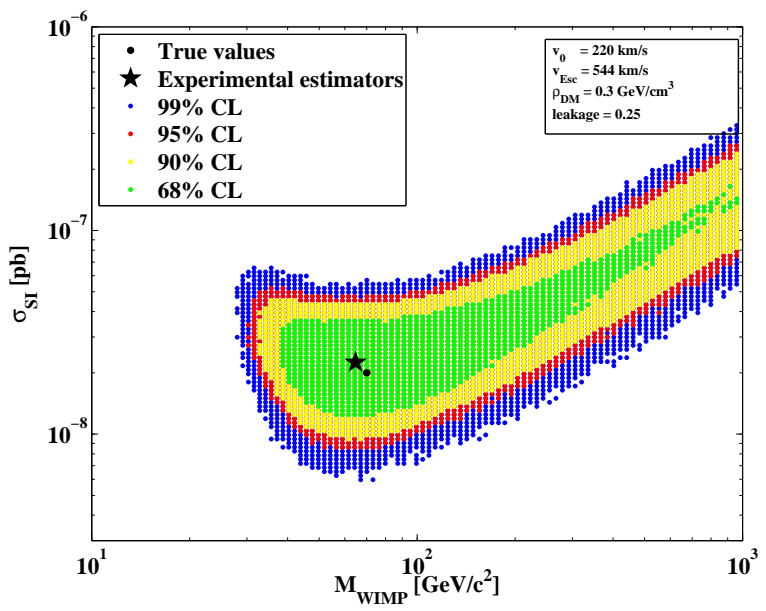

(d)

Figure 11.7: Confidence regions for the example of the detection of eight events and different values of the expected number of surface-events leaking into the signal region. (a) No leakage, (b) 0.25 events leakage, (c) 0.5 events leakage and (d) 1 event leakage.

component and use very clean materials, the actual number of expected neutron induced nuclear recoils as well as their energy distribution has to be determined by Monte Carlo simulations. Usually only an upper limit on the expected rate of neutron induced nuclear recoils is set. The expected neutron background can be incorporated into the likelihood function in the same way as the surface-event background. In this study only a background from surface-events is considered for simplicity, but in the application to real data all terms describing the background of the experiment have to be taken into account. 


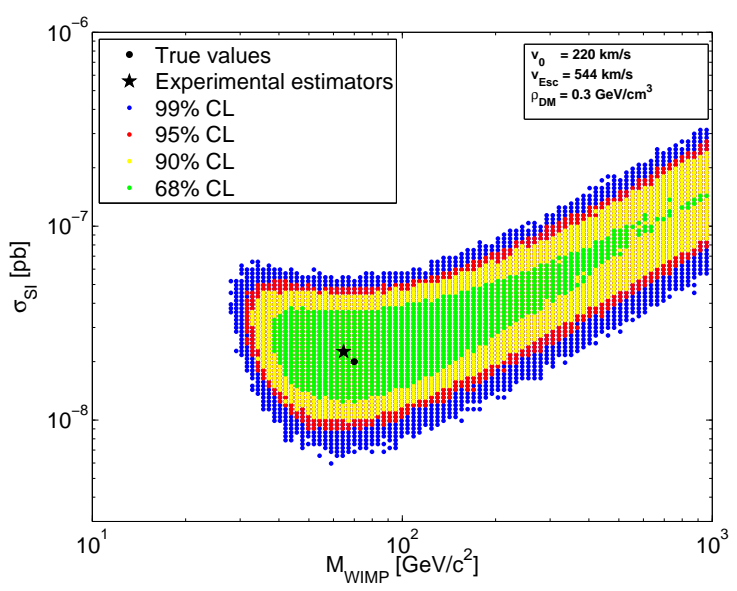

Figure 11.8: Confidence levels for the detection of eight events under a zero background assumption. In the generation of the Monte Carlo experiments used for the determination of the acceptance regions as well as in the maximization of the likelihood function the same values of the circular velocity, given in the figures caption, are used.

\subsubsection{Uncertainties on astronomical parameters}

The main uncertainty in the likelihood function is the dependency of the differential rate on astronomical input parameters. These are the velocity dispersion of the dark matter particles usually set to the galactic rotation velocity for an isotropic Maxwellian velocity distribution, the galactic escape velocity and the local dark matter density. The recoil energy imparted to a nucleus struck by a WIMP is determined by the kinematics of the scattering process, hence, changing the input parameters $\left(v_{0}\right.$ and $\left.v_{\text {esc }}\right)$ the differential recoil spectrum will change. In figure 11.8 the confidence levels have been constructed under the assumption, that the velocity parameters used to find the best fit parameters to the experimental data are the true values. But this assumption has no general validity, and the resulting confidence levels will depend on the choice of these nuisance parameters.

As an example, the confidence levels in figure 11.9 (a) and (b) have been obtained by generating the energies for the Monte Carlo experiments in the Feldman-Cousins method with a different circular velocity than is used in the likelihood analysis of these experiments. In figure 11.9 (a) a circular velocity of $200 \mathrm{~km} / \mathrm{s}$ has been used to generate the differential recoil spectra whereas in figure 11.9 (b) a circular velocity of $240 \mathrm{~km} / \mathrm{s}$ has been used. In both cases the likelihood analysis is done under the assumption of a circular velocity of $220 \mathrm{~km} / \mathrm{s}$. From the comparison of the two figures with the confidence regions shown in figure 11.8 it can be seen, that by overestimating the circular velocity in the analysis, the confidence regions extend to higher masses, since to match the observed rate under the assumption of a higher velocity the particle's mass has to be increased. This is caused by the fact that the total observed rate is proportional to the circular velocity and to the inverse of the particle's mass. Thus the effect goes in the opposite direction if the circular velocity assumed in the analysis lies below the true value.

Changing the escape velocity has little impact on the actual confidence levels, but it 


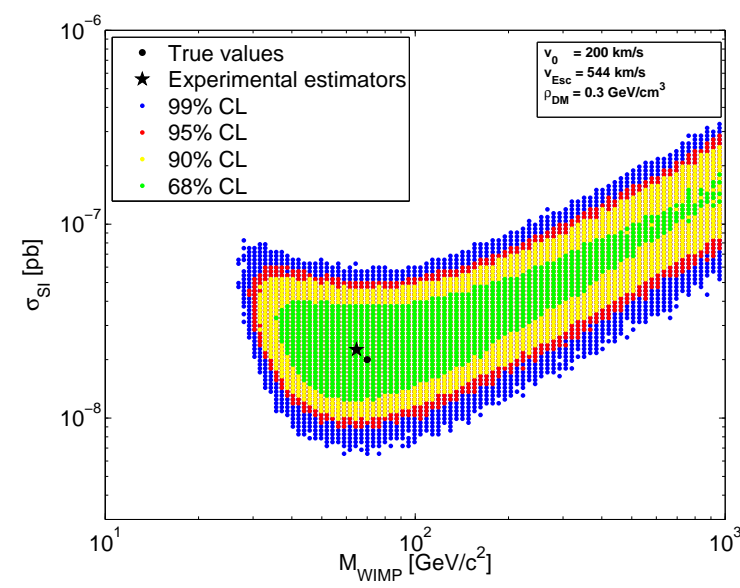

(a)

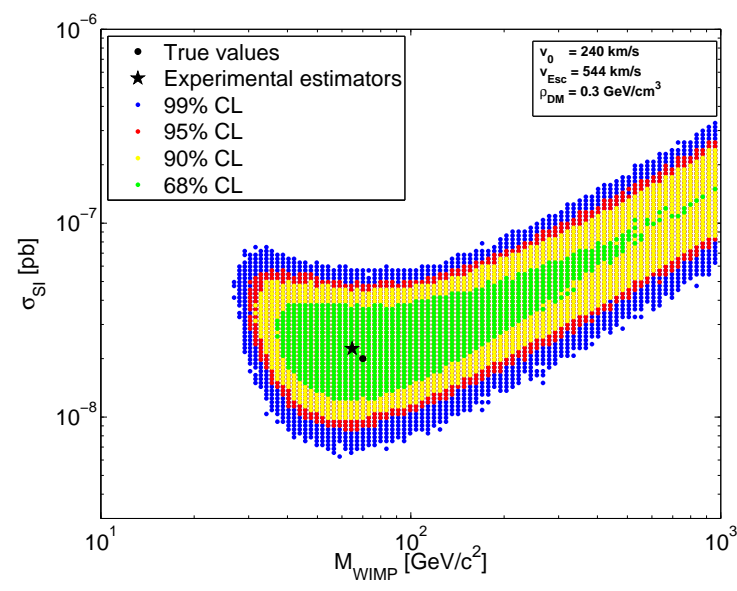

(b)

Figure 11.9: Confidence levels for the detection of eight events under a zero background assumption. In the generation of the Monte Carlo experiments used for the determination of the acceptance regions, the values of the circular velocity, given in the figures caption, are used. In the maximization of the likelihood function a circular velocity of $v_{0}=220 \mathrm{~km} / \mathrm{s}$ is assumed resulting in a bias to higher (a) and lower (b) masses in the determined confidence regions.

changes the sharp mass cut at low masses, since this is only determined by the restriction that a certain particle mass has a maximal recoil energy. If the experiment detects one or more events with energy above this maximal energy, the particle's mass must allow this momentum transfer, and hence a lower limit can be set on the WIMP mass by the detected energies.

\subsubsection{Applying Markov Chains to sample over nuisance param- eters}

The Markov Chain Monte Carlo (MCMC) technique [219] is a useful tool to effectively sample high dimensional probability distributions. One simple MCMC algorithm is the so called Metropolis Hastings Sampler [220, 221]. The actual confidence regions of the probability distribution are determined by the frequency of points for a given parameter combination. This frequency approach is valid in the case that the MCMC is able to sample the whole probability distribution to obtain the correct normalization which is given by the sum of all samples. As a toy-model figure 11.10 shows the determination of the confidence regions of a correlated two dimensional Gaussian distribution. In this case the MCMC samples the whole parameter space, and thus the confidence regions obtained from the MCMC coincide with the analytical confidence regions.

Since the actual confidence regions on the WIMP mass and the WIMP-nucleon cross section determined from an experiment depend on the choice of the astrophysical and 


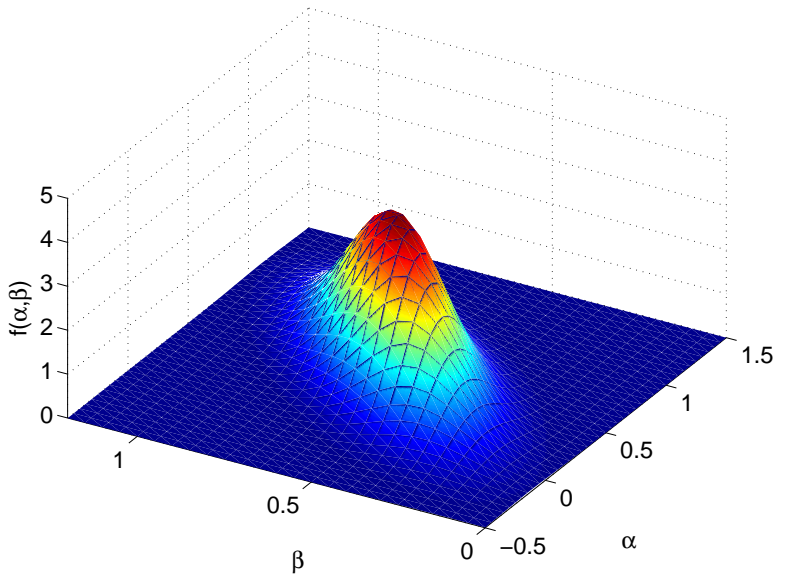

(a)

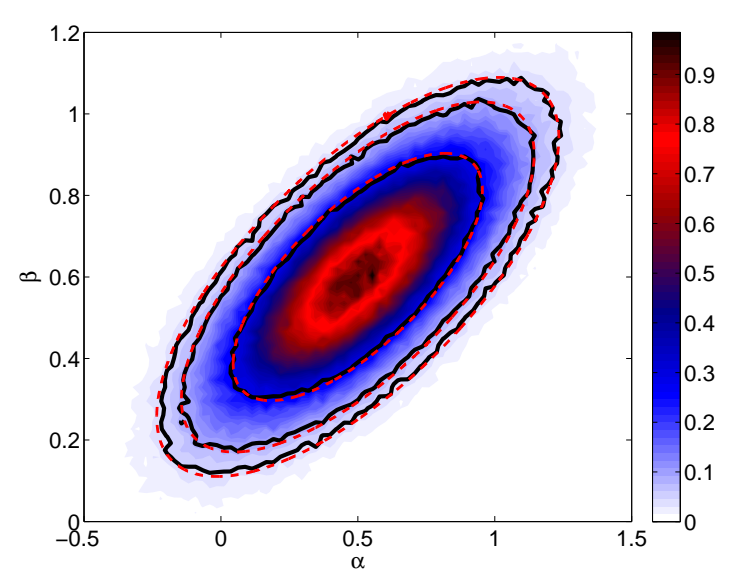

(b)

Figure 11.10: (a) Correlated two dimensional Gaussian distribution for which the confidence regions are determined by a MCMC. The confidence regions from the MCMC shown in (b) as black lines coincide with the analytical determined confidence regions (red/dashed lines). The color code shows the relative probability density. The jagged structure of the confidence regions from the MCMC is a result of the finite sample size in the chain and smoothens as the sample size is increased.

experimental input parameters, a MCMC is used to sample over the nuisance parameters

$$
\Psi=\left\{v_{0} ; v_{\text {esc }} ; \eta_{l e a k} ; \rho\right\}
$$

The resulting confidence regions would thus incorporate any experimental or astrophysical systematics.

Before introducing the corresponding MCMC algorithm we have to take care of a restriction of the MCMC. To illustrate this restriction we return to the example of a detection of 30 events without background and fixed astrophysical parameters (figure 11.4(a)). Since the accurate determination of the confidence regions by a MCMC depends on the correct determination of the normalization, it is not surprising, that the confidence regions will not be correctly determined, if only a part of the distribution is sampled. This could either be caused by a long tail of the distribution, like in the case of low statistics for which the confidence regions are not closed for high masses, or by an arbitrary cut-off in one of the parameters at which the sampling is stopped. The frequency confidence regions from a MCMC for the example considered here are shown in figure 11.11. In the case of non-closed confidence regions (a) the confidence regions are far greater than the analytical ones and thereby show a great over-coverage. This over-coverage can be reduced by introducing a cut-off in one of the parameters like the mass of the particle. Although the confidence regions in (b) are now closer to the analytical ones, they directly depend on the arbitrary cut-off mass.

Given this dependency the frequency approach is not useful for the purpose of determining the correct confidence regions. From the analytical method (either the likelihood 


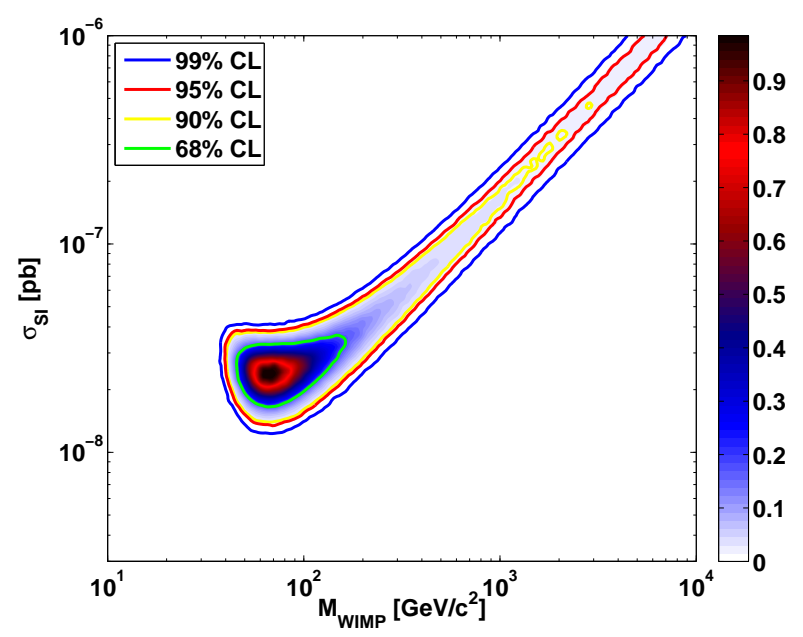

(a)

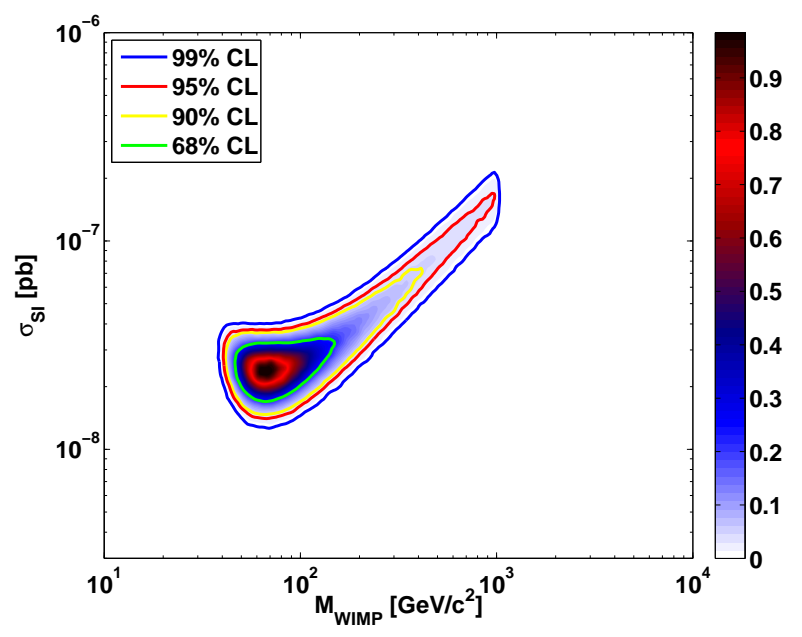

(b)

Figure 11.11: Frequency confidence intervals for the example of a detection of 30 events. In (a) the confidence regions greatly over-cover the analytical confidence regions, due to a wrong normalization. In (b) this over-coverage is reduced by an arbitrary cut-off in the particle's mass, causing a strong dependency of the confidence regions on the choice of the cut-off.

approximation or the Feldman Cousins approach) we know for each parameter point the correct confidence level for a given choice of nuisance parameters. The idea is now to use the MCMC in a further step to sample over the nuisance parameters, but determine the confidence levels for each point from the analytical method. In the MCMC algorithm the nuisance parameters used in the determination of the confidence level are drawn from analytical probability density distributions. The acceptance of a parameter point in the MCMC is determined from the combined $\chi^{2}$-values of the parameter point and the nuisance parameter distributions. For this the confidence level of a parameter point is converted to a $\chi^{2}$-value using a gaussian approximation. The resulting confidence level of a parameter point of interest (here in the mass - cross section parameter space) at the end of the MCMC sampling is then determined by the average value of the analytical confidence levels over all samples at the considered parameter point. In the limiting case of infinite statistics this results in a confidence level of each parameter point which has been marginalized over the nuisance parameters. The results of this approach, applied to the same MCMC run discussed above, are shown in figure 11.12. The use of the analytical values for each point breaks the dependence on the normalization as well as the dependence on an arbitrary cut-off mass. The verification of this approach of providing the correct confidence regions is given in figure 11.13. The confidence regions obtained from the MCMC run (a) are compared in (b) to the confidence regions obtained from the analytical method (see figure 11.4 (a)). The confidence regions from the MCMC exactly match those obtained from the likelihood function. 


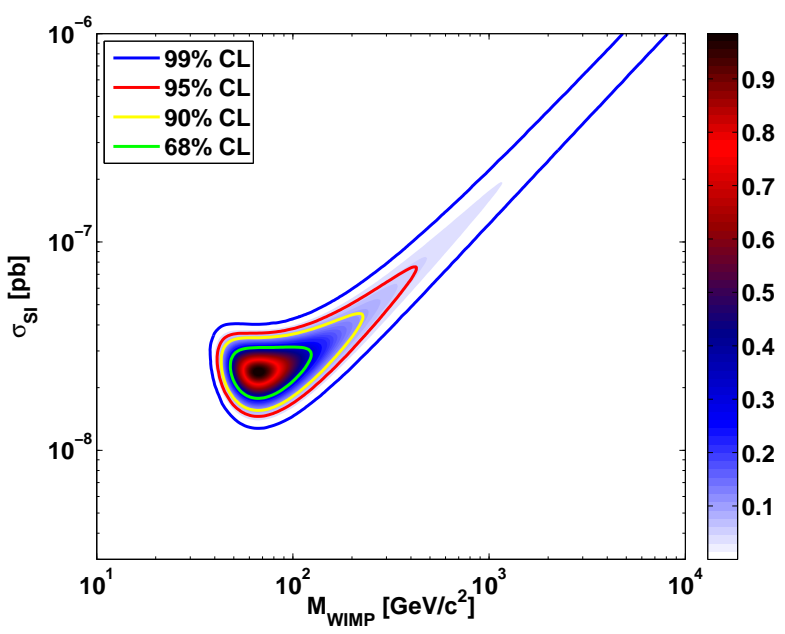

(a)

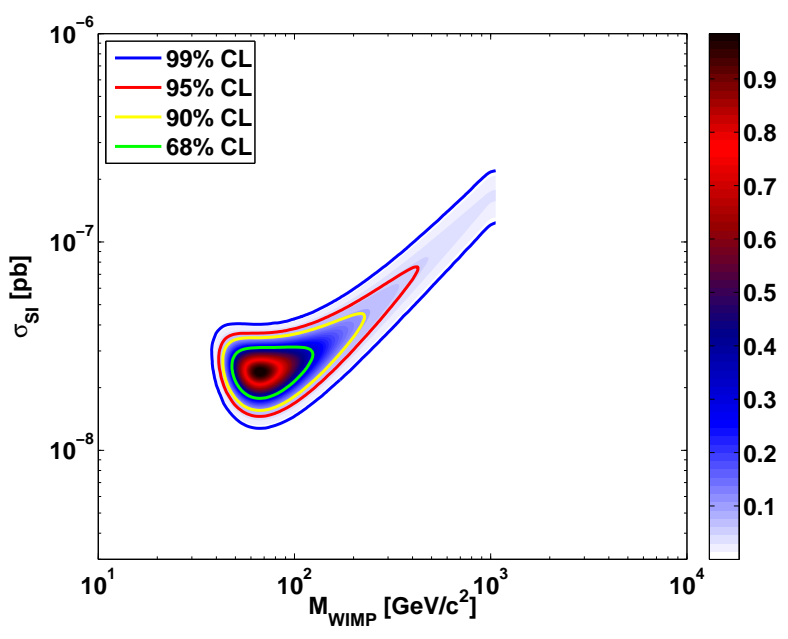

(b)

Figure 11.12: Confidence regions from the analytical approach in the MCMC. This approach breaks the dependency on the normalization (a) and an arbitrary cut off (b).

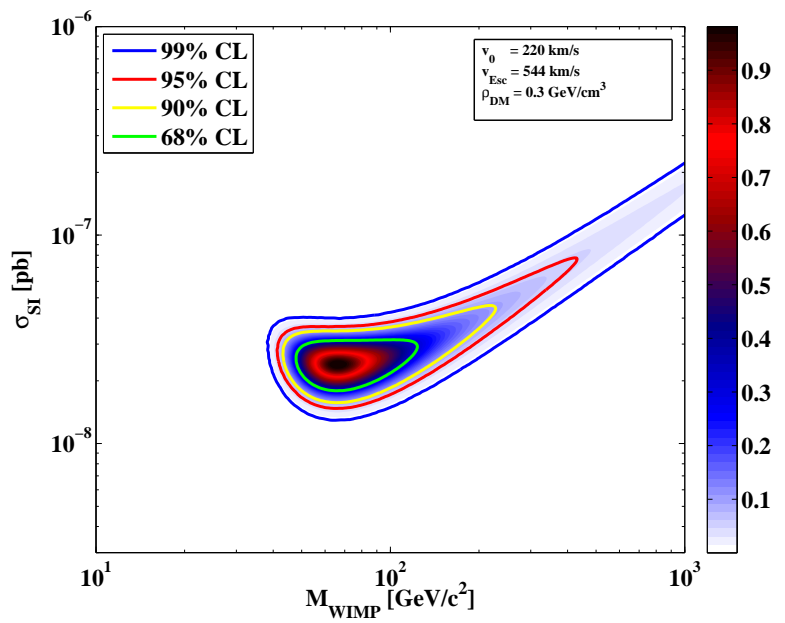

(a)

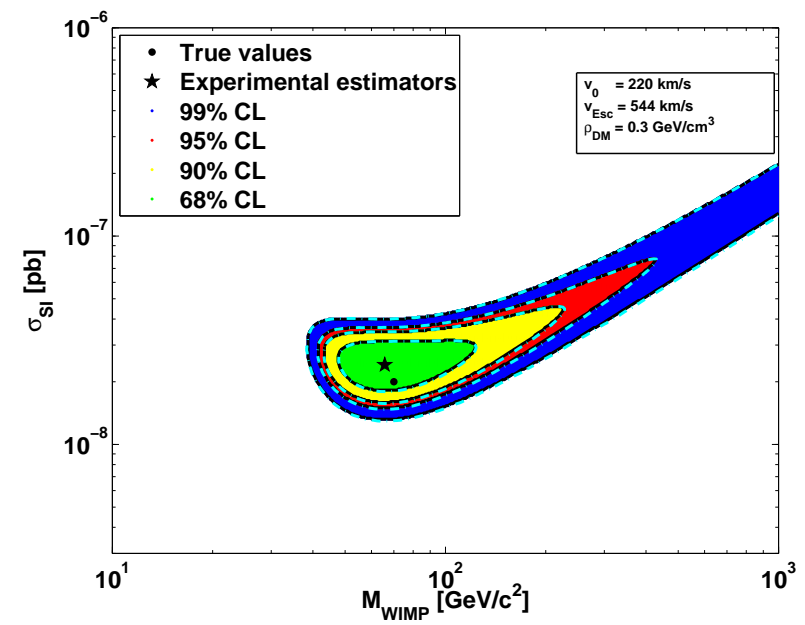

(b)

Figure 11.13: Comparison of the confidence regions obtained from a MCMC run and the analytical method (a) with the confidence regions obtained directly from the likelihood function (b). The contours of the MCMC confidence regions are shown as (cyan/dashed) lines in (b). 


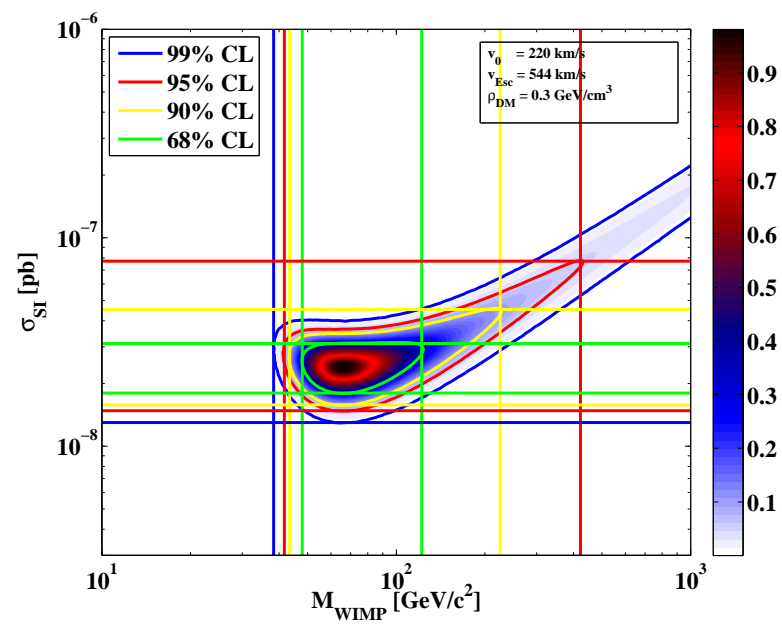

Figure 11.14: Upper and lower limits on the mass and cross section can be derived from the acceptance region by finding the highest/lowest values of the considered parameters accepted at a given confidence level. The straight lines in the figure mark those values for the given confidence levels (indicated by color). The astrophysical parameters used in the determination of the confidence level are indicated in the figure's caption.

From the obtained acceptance regions upper and lower limits on the particle's mass and cross section can be determined by finding the highest/lowest values of the considered parameter accepted at a given confidence level. This procedure is graphically illustrated in figure 11.14 in the case for which the astrophysical parameters have been fixed in the determination of the acceptance regions. The straight lines mark the lowest/highest value of the respective parameters on the figures axes. Table 11.3 summarizes these upper and lower limits on the particle's mass and cross section.

\begin{tabular}{|c|c|c|c|c|c|c|c|c|}
\hline & \multicolumn{7}{|c|}{ Confidence interval } \\
\cline { 2 - 8 } & \multicolumn{2}{|c|}{$68 \%$} & \multicolumn{2}{|c|}{$90 \%$} & \multicolumn{2}{c|}{$95 \%$} & \multicolumn{2}{c|}{$99 \%$} \\
\hline Mass $\left[\mathrm{GeV} / \mathrm{c}^{2}\right]$ & 47.9 & 122.1 & 43.5 & 225.5 & 41.7 & 422.6 & 38.3 & - \\
$\sigma_{S I}\left[10^{-8} \mathrm{pb}\right]$ & 1.8 & 3.11 & 1.58 & 4.53 & 1.48 & 7.73 & 1.3 & - \\
\hline
\end{tabular}

Table 11.3: Lower (left column) and upper (right column) limits of a given confidence interval for the detection of 30 events with fixed astronomical input parameters $v_{0}=220 \mathrm{~km} / \mathrm{s}$, $v_{\text {esc }}=544 \mathrm{~km} / \mathrm{s}, \rho_{D M}=0.3 \mathrm{GeV} / \mathrm{cm}^{3}$ and no expected leakage $\left(\eta_{\text {leak }}=0\right)$.

Having verified that the MCMC sampling returns the correct acceptance regions one can start to sample over the single nuisance parameters to study how the uncertainties on the astrophysical input parameters and the expected background affect the upper and lower limits on the particle's properties. With this it is possible to quantify the systematical error introduced in the lower and upper limits on the particle's mass and cross section due to the astrophysical input parameters and the experiment's background. In order to study these 


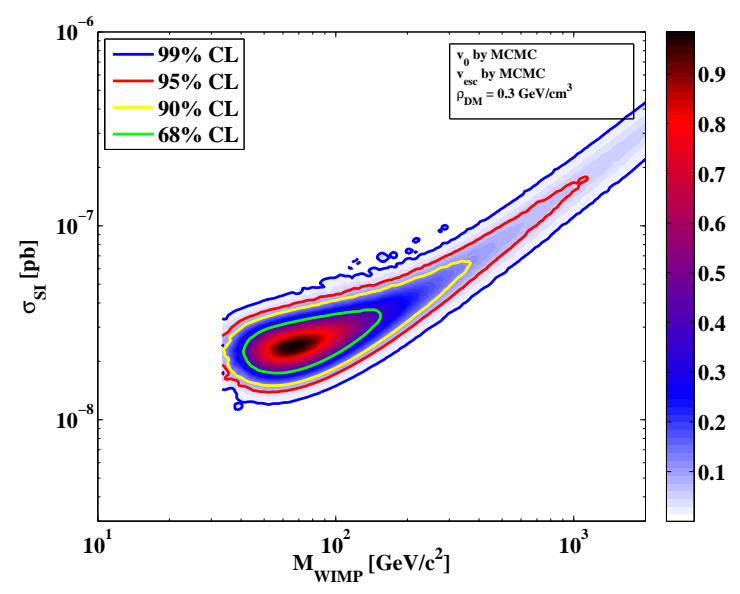

Figure 11.15: Acceptance regions in which the astrophysical input parameters have been sampled by the MCMC algorithm.

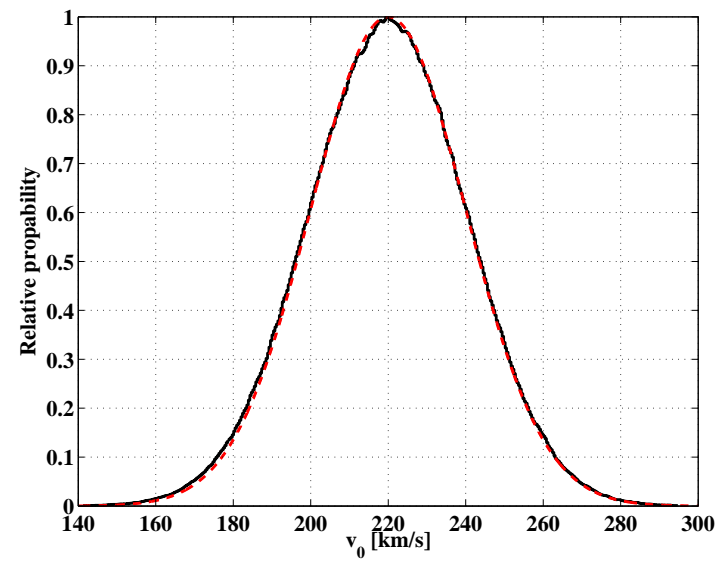

(a)

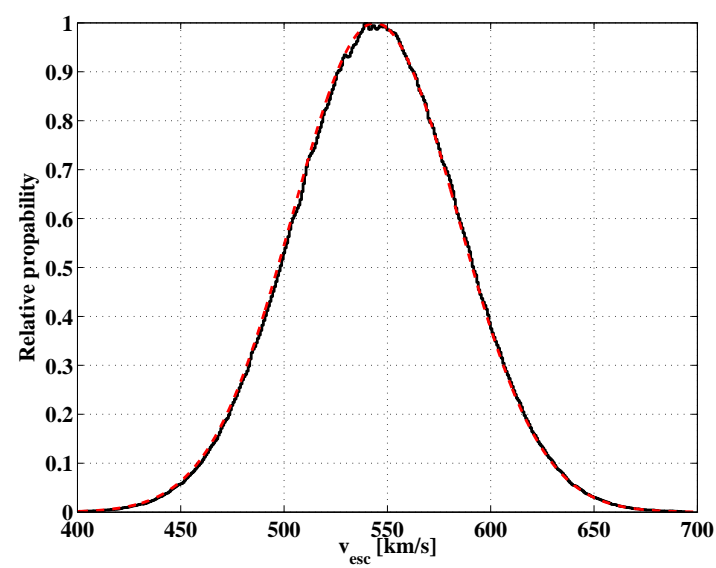

(b)

Figure 11.16: Comparison of the relative probability distributions (shown in black) sampled by the MCMC with the analytical input distributions (red/dashed). The relative probability distributions are normalized to the highest sample frequency. (a) Circular velocity and galactic escape velocity (b).

systematic uncertainties, a detection of 30 events in a germanium target is considered with a detection threshold of $10 \mathrm{keV}$ and a detection efficiency of $30 \%$ which is flat in energy. The exposure is not of great importance in this study, since it would only change the overall scaling of the cross section, but does not affect the systematic uncertainties on the confidence regions of the cross section.

Figure 11.15 shows the acceptance regions, in which the astrophysical parameters have been marginalized over by the MCMC algorithm. From a visual comparison with figure 11.14 it can be seen, that by including the uncertainties on the circular velocity and 


\begin{tabular}{|c|c|c|c|c|c|c|c|c|}
\hline & \multicolumn{7}{|c|}{ Confidence intervall } \\
\cline { 2 - 9 } & \multicolumn{2}{|c|}{$68 \%$} & \multicolumn{2}{|c|}{$90 \%$} & \multicolumn{2}{c|}{$95 \%$} & \multicolumn{2}{c|}{$99 \%$} \\
\hline Mass $\left[\mathrm{GeV} / \mathrm{c}^{2}\right]$ & 41.5 & 151.7 & 34.0 & 364.6 & 33.4 & 1135.3 & 33.4 & - \\
$\sigma_{S I}\left[10^{-8} \mathrm{pb}\right]$ & 1.75 & 3.65 & 1.51 & 6.55 & 1.4 & 18 & 1.14 & - \\
\hline
\end{tabular}

Table 11.4: Lower (left column) and upper (right column) boundary of a given confidence interval for the detection of 30 events with fixed expected leakage $\left(\eta_{\text {leak }}=0\right)$ and varying the astronomical input parameters $v_{0}$ and $v_{e s c}$ by the MCMC. The local density is fixed to $\rho_{D M}=0.3 \mathrm{GeV} / \mathrm{cm}^{3}$.

\begin{tabular}{|c|c|c|c|c|c|c|c|}
\hline & \multicolumn{7}{|c|}{ Confidence intervall } \\
\hline & & & & & & $\%$ & $99 \%$ \\
\hline Relative uncertainty & $13 \%$ & $24 \%$ & $22 \%$ & $62 \%$ & $20 \%$ & $169 \%$ & $13 \%$ \\
\hline
\end{tabular}

Table 11.5: Relative uncertainties on the lower (left column) and upper (right column) boundary of a given confidence interval from varying the astronomical input parameters $v_{0}$ and $v_{\text {esc }}$ by the MCMC. The relative uncertainties have been calculated with respect to the values obtained from fixing the astrophysical input parameters (see table 11.3).

the galactic escape velocity, the acceptance regions broaden, preferentially accepting higher particle masses. The correct sampling of the astrophysical input parameters can be verified by comparing the relative probability distributions returned from the MCMC algorithm with the distributions functions of the parameters used in the MCMC algorithm. This comparison is shown for the circular velocity in figure11.16 (a) and for the galactic escape velocity in figure 11.16 (b). The relative probability distributions (normalized to the highest frequency) match the analytical functions. For the distribution of the astrophysical parameters a Gaussian distribution with a mean and standard deviation corresponding to the measured values and their associated uncertainties have been used: $v_{0}=220 \pm 20 \mathrm{~km} / \mathrm{s}$ and $v_{\text {esc }}=544 \pm 40 \mathrm{~km} / \mathrm{s}$. The upper and lower limits on the particle's mass and cross section obtained by sampling the distributions of the astrophysical input parameters are given in table 11.4. By comparing these numbers to the numbers in table 11.3, it can be seen that the constraints on the cross section are not strongly influenced by the uncertainties on the velocity input parameters, whereas the constraints on the particles mass are strongly affected by the uncertainty on the velocities since both affect the differential recoil spectrum, determined by the scattering kinematics. The systematical uncertainties for a given confidence level are summarized in table 11.5.

The systematic uncertainty introduced by the expected background is far less, if the expected background is much smaller than the number of signal events. In this study an expected background given by equation (11.27) has been assumed. In order to obtain a probability density function of the expected background, the statistical and systematical uncertainties on the expected background have been added in quadrature, to provide the standard deviations of a Gaussian distribution above and below the central value. The resulting acceptance regions from a MCMC sampling from this background distribution 


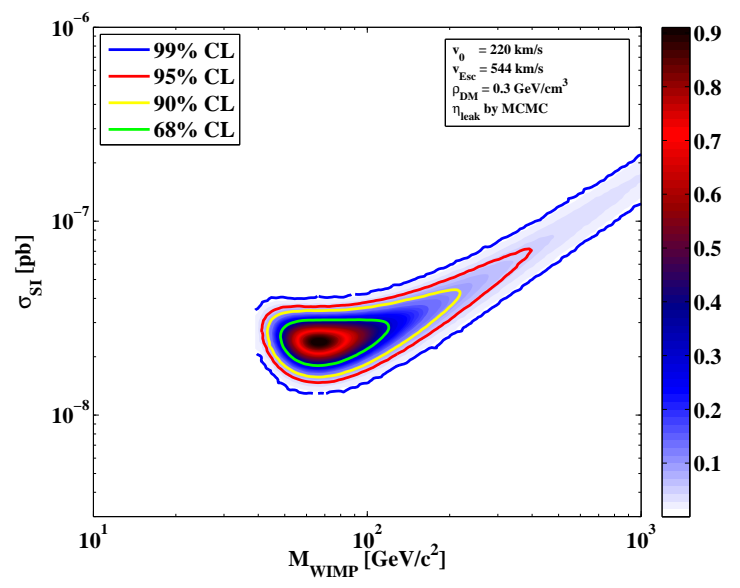

(a)

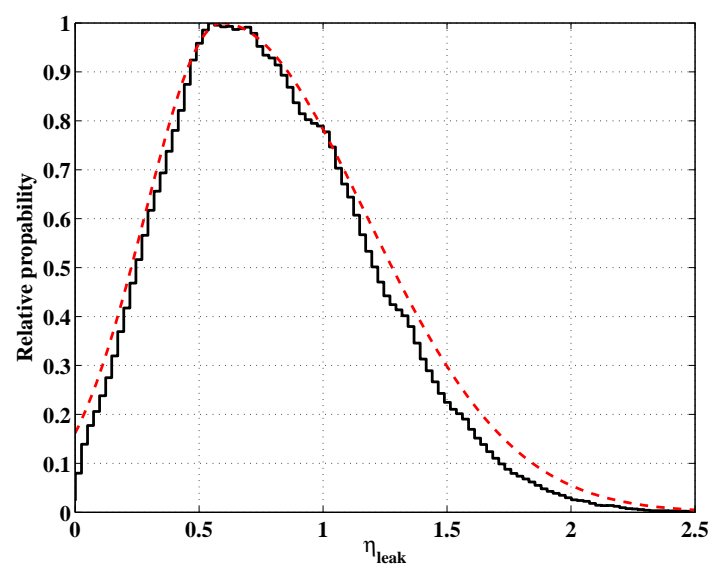

(b)

Figure 11.17: (a) Acceptance regions in which the background parameter has been sampled by the MCMC algorithm. The astrophysical parameters are kept fixed. (b) Comparison of the relative probability distribution (shown in black) of the background events sampled by the MCMC with the analytical input distribution (red/dashed). The relative probability distribution is normalized to the highest sample frequency.

\begin{tabular}{|c|c|c|c|c|c|c|c|c|}
\hline & \multicolumn{7}{|c|}{ Confidence intervall } \\
\cline { 2 - 9 } & \multicolumn{2}{|c|}{$68 \%$} & \multicolumn{2}{|c|}{$90 \%$} & \multicolumn{2}{c|}{$95 \%$} & \multicolumn{2}{c|}{$99 \%$} \\
\hline Mass $\left[\mathrm{GeV} / \mathrm{c}^{2}\right]$ & 48.3 & 118.7 & 43.8 & 217.6 & 41.3 & 390.9 & 38.9 & - \\
$\sigma_{S I}\left[10^{-8} \mathrm{pb}\right]$ & 1.82 & 3.08 & 1.58 & 4.42 & 1.49 & 7.07 & 1.29 & - \\
\hline
\end{tabular}

Table 11.6: Lower (left column) and upper (right column) boundary of a given confidence interval for the detection of 30 events with fixed astronomical input parameters $v_{0}=220 \mathrm{~km} / \mathrm{s}, v_{\text {esc }}=544 \mathrm{~km} / \mathrm{s}, \rho_{D M}=0.3 \mathrm{GeV} / \mathrm{cm}^{3}$ and varying the expected leakage $\eta_{\text {leak }}$ by the MCMC.

while keeping the astrophysical input parameters fixed is shown in figure 11.17(a). The comparison between the sampled background distribution and the input distribution is shown in figure 11.17(b). The upper and lower limits on the particle's mass and cross section obtained by sampling the expected background distribution are given in table 11.6. By comparing these numbers to the numbers in table 11.3, it can be seen that the constraints on the cross section and mass are not strongly influenced by the inclusion of the expected background and associated uncertainties.

This study shows that even in the case of a relative high statistic detection of WIMP interactions (30 events), the astrophysical input parameters introduce a large systematic uncertainty on the upper and lower limits of the particle's mass. It has to be noted that these uncertainties will likely depend on the actual true mass of the particle and will be reduced as the detection statistics increase to an even higher number of events. Further 
studies are needed to quantify the dependence of the uncertainties on the particle's mass and the detection statistics.

The MCMC approach offers the possibility to combine the results from different experiments using different detection media. Since the differential recoil spectrum is determined by the kinematics of the scattering process, as well as material dependent form factors, the combination of two (or more) different detection media is expected to improve the constraints on the particle's mass. This should also be the case if the total number of detected events in the two target materials is equal to a number of events in a single detection media from which the confidence regions are determined.

This study did not account for the uncertainties in the local dark matter density $\rho$, since this value is not constrained very well. So far only lower and upper bounds on the local density are reported ranging from $\rho \sim 0.1-0.8 \mathrm{GeV} / \mathrm{cm}^{3}$. For the studies presented here a value of $0.3 \mathrm{GeV} / \mathrm{cm}^{3}$ has been assumed. Assuming a uniform distribution of the local density within the bounds given above (this is a conservative assumption, since no probability density distribution of the local density is known), the uncertainties on the cross section will directly scale with the range of the allowed local densities. That is, the lower bound on the cross section will extend to cross sections 0.8/0.3 times lower and respectively to values $0.3 / 0.1$ greater than the cross sections obtained from assuming a local density of $0.3 \mathrm{GeV} / \mathrm{cm}^{2}$. Thus it may be better to not directly constrain the cross section, but the product of the cross section and the local density $\zeta=\sigma \cdot \rho$. The actual bound on the cross section can then be obtained from this parameter by the multiplication of the actual scaling factor appropriate for the assumed local dark matter density. Once the local dark matter density is at least constrained to a central value, and a probability density distribution can be assigned to the values, this parameter can also be considered in the MCMC sampling. 


\section{Chapter 12}

\section{Search for Solar Axions with the CDMS Experiment}

The axion offers a solution to the strong $\mathrm{CP}$ problem as has been discussed in Section 2.2. If this particle exists, the Sun would be a strong source of axions. At the beginning of this Chapter the production mechanism in the Sun's interior and the detection of axions in crystal detectors via the Primakov effect will be discussed.

The first axion search result from the CDMS-II experiment will be presented. Data taken between October 2006 and July 2007 designated as Run 123 and Run 124 of the CDMS-II experiment is considered in this analysis. The selection cuts, optimized for a performance at low energies, will be discussed along with the evaluation of the detection efficiencies for extracting a possible axion signal from the data. In order to search for a signal from solar axion conversions in the detectors a likelihood analysis is performed.

\subsection{Solar Axions}

In the search for solar axions the axion model is confined to hadronic axion models, where these particles do not interact with electrons at the tree level. Such axions can be efficiently produced in the interior of stars only by processes involving their coupling $g_{a \gamma \gamma}$ to photons. In general, pseudoscalar particles are considered which interact only by a two-photon vertex. The interior of stars is expected to be a powerful source of axions due to the high abundance of photons and strong electromagnetic fields. The fluctuating electromagnetic field of the charged particles in the plasma may convert black body photons to axions. Thus the astronomical closest source of axions is our Sun. With the standard solar model the differential solar axion flux at the Earth can be calculated. The flux of solar axions as a function of axion energy is shown in 12.1 The differential axion spectrum at the Earth can be well approximated by [222]:

$$
\frac{d \Phi_{a}}{d E_{a}}=\frac{6.02 \cdot 10^{14}}{\mathrm{~cm}^{2} \mathrm{~s} \mathrm{keV}}\left(\frac{g_{a \gamma \gamma} \cdot 10^{8}}{G e V^{-1}}\right) E_{a}^{2.481} e^{-E_{a} / 1.205}
$$

where $E_{a}$ is the energy of the axion in $\mathrm{keV}$. 


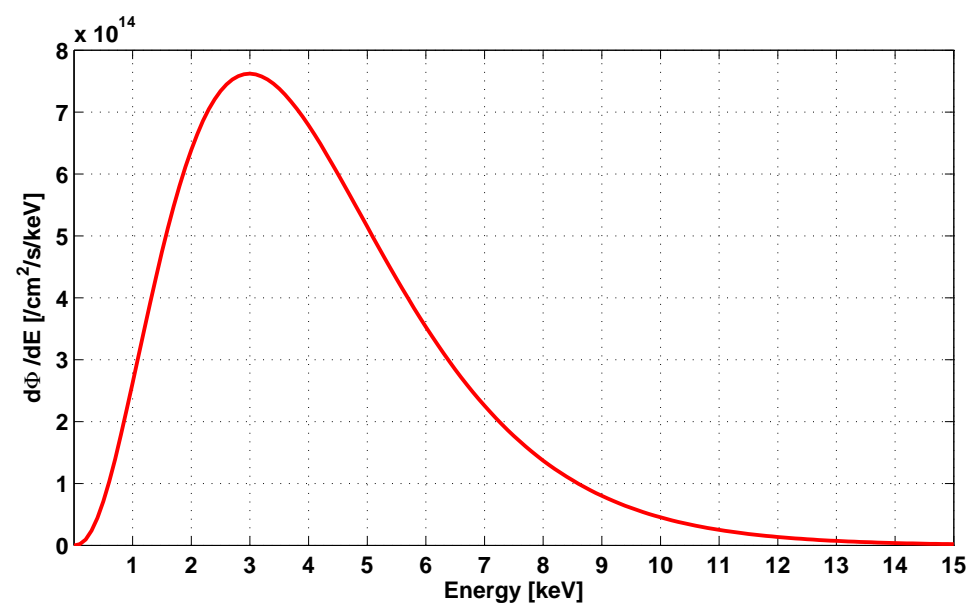

Figure 12.1: Spectral flux of solar axions at the Earth.

\subsection{Expected conversion rate}

The solar axions may be converted back to photons by the Primakov effect in the strong electric field of a nucleus. For very light axions with a mass below the keV scale the Primakov process in a periodic lattice is coherent, similar to Bragg reflection of X-rays. This leads to the Bragg condition, namely that the momentum transferred to the crystal must be a reciprocal lattice vector $\vec{G}$. The expected counting rate in a crystal detector in the energy range $E_{1}<E<E_{2}$ is given by [223, 224]:

$$
R\left(E_{1}, E_{2}\right)=(2 \pi)^{3} 2 c \hbar \frac{V}{v_{c}^{2}} \sum_{\vec{G}} \frac{d \Phi}{d E_{a}} \frac{1}{|\vec{G}|^{2}} \frac{g_{a \gamma \gamma}}{16 \pi^{2}}\left|F_{c}(\vec{G}) S(\vec{G})\right|^{2} \sin ^{2}(2 \theta) W
$$

where $V$ is the Volume of the crystal, $v_{c}$ is the volume of the elementary cell of the lattice and $2 \theta$ is the scattering angle. The atomic form factor is given by:

$$
F_{c}(\vec{q})=\frac{Z e k^{2}}{r^{-2}+|\vec{q}|^{2}}
$$

where $\vec{q}$ is the transferred momentum $|\vec{q}|=2 k \sin (\theta)$ while $k \equiv|\vec{k}| \simeq E_{a}$ is the axion momentum, $Z=32$ for germanium, $e$ is the elementary charge and $r$ is the screening length of the atomic electric field. The term $W$ is the detector energy resolution function:

$$
W=\frac{1}{2}\left[\operatorname{erf}\left(\frac{E_{a}-E_{1}}{\sqrt{2} \Delta}\right)-\operatorname{erf}\left(\frac{E_{a}-E_{2}}{\sqrt{2} \Delta}\right)\right]
$$

where $\Delta$ is the detector's energy resolution. The structure functions $S(\vec{G})$ of the crystal are given by:

$$
S(\vec{G})=\sum_{i, j} \exp ^{i \vec{a}_{i}^{j} \vec{G}}
$$


where the sum runs over the atomic species $j$ forming the crystal lattice with the basis vectors $i$. The face-centered-cubic (fcc) crystal structure of germanium can be described by a two atomic basis with the basis vectors

$$
\overrightarrow{a_{1}^{1}}=\left(\begin{array}{l}
0 \\
0 \\
0
\end{array}\right), \overrightarrow{a_{1}^{2}}=\frac{a}{4}\left(\begin{array}{l}
1 \\
1 \\
1
\end{array}\right)
$$

where $a=5.66 \AA$ is the primitive cell size of germanium. For each basis the crystal lattice is generated by the primitive vectors

$$
\overrightarrow{x_{1}}=\frac{a}{2}\left(\begin{array}{l}
0 \\
1 \\
1
\end{array}\right), \overrightarrow{x_{2}}=\frac{a}{2}\left(\begin{array}{l}
1 \\
0 \\
1
\end{array}\right), \overrightarrow{x_{3}}=\frac{a}{2}\left(\begin{array}{l}
1 \\
1 \\
0
\end{array}\right), \overrightarrow{x_{4}}=\frac{a}{2}\left(\begin{array}{l}
0 \\
0 \\
0
\end{array}\right)
$$

The structure functions (12.5) can then be written as:

$$
\begin{aligned}
S(\vec{G}) & =\sum_{i, j} \exp ^{i \vec{a}_{i}^{j} \vec{G}}=\sum_{i} \exp ^{i\left(\overrightarrow{x_{i}}+\overrightarrow{a_{1}^{1}}\right) \vec{G}}+\sum_{i} \exp ^{i\left(\overrightarrow{x_{i}}+\overrightarrow{a_{1}^{2}}\right) \vec{G}} \\
& =\sum_{i} \exp ^{i \overrightarrow{x_{i} \vec{G}}}+\exp ^{i a_{1}^{2} \vec{G}} \sum_{i} \exp ^{i \overrightarrow{x_{i} \vec{G}}}=\left(1+\exp ^{i a_{1}^{2} \vec{G}}\right) \sum_{i} \exp ^{i \overrightarrow{x_{i} \vec{G}}}
\end{aligned}
$$

with the reciprocal lattice vectors

$$
\vec{G}=\frac{2 \pi}{a}\left(\begin{array}{c}
h \\
k \\
l
\end{array}\right)
$$

the structure functions are given by:

$$
S(\vec{G})=\left(1+\exp ^{i \frac{\pi}{2}(h+k+l)}\right) \times\left(1+e x p^{i \pi(h+k)}+e x p^{i \pi(h+l)}+e x p^{i \pi(k+l)}\right) .
$$

The second term of the structure functions gives a selection criteria for the values of $(h, k, l)$ :

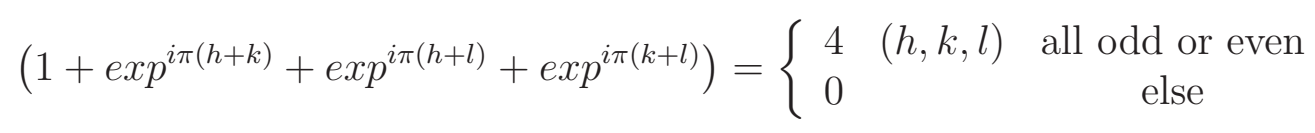

The most important reciprocal lattice vectors contributing to the inverse Primakov conversion of axions in a germanium crystal are given in table 12.1 .

When the Primakov conversion satisfies the Bragg condition, $\vec{q}=\vec{G}$, and the crystal interacts in a coherent way. The Bragg condition implies that in (12.2):

$$
E_{a}=\hbar c \frac{|\vec{G}|^{2}}{2 \hat{u} \cdot \vec{G}}
$$

where the unitary vector $\hat{u}$ points toward the Sun. Since the position of the Sun changes with daytime this term induces a time dependence of the expected signal. The expected 


\begin{tabular}{|c|c|c||c|c|c|}
\hline$(h, k, l)$ & $E_{0}(\mathrm{keV})$ & mult & $(h, k, l)$ & $E_{0}(\mathrm{keV})$ & mult \\
\hline \hline$(1,1,1)$ & 1.89 & 8 & $(5,5,1)$ & 7.78 & 24 \\
$(2,2,0)$ & 3.08 & 12 & $(7,1,1)$ & 7.78 & 24 \\
$(3,1,1)$ & 3.62 & 24 & $(6,4,2)$ & 8.16 & 48 \\
$(4,0,0)$ & 4.36 & 6 & $(5,5,3)$ & 8.37 & 24 \\
$(3,3,1)$ & 4.75 & 24 & $(7,3,1)$ & 8.37 & 48 \\
$(4,2,2)$ & 5.34 & 24 & $(8,0,0)$ & 8.72 & 6 \\
$(3,3,3)$ & 5.66 & 8 & $(7,3,3)$ & 8.92 & 24 \\
$(5,1,1)$ & 5.66 & 24 & $(8,2,0)$ & 8.99 & 24 \\
$(4,4,0)$ & 6.17 & 12 & $(6,6,0)$ & 9.25 & 12 \\
$(5,3,1)$ & 6.45 & 48 & $(5,5,5)$ & 9.44 & 8 \\
$(5,3,3)$ & 6.45 & 24 & $(7,5,1)$ & 9.44 & 48 \\
$(4,4,4)$ & 7.55 & 8 & $(7,5,3)$ & 9.93 & 48 \\
\hline
\end{tabular}

Table 12.1: The integers $(h, k, l)$ of the components of $\vec{G}$ in units of $2 \pi / a . E_{0}$ is the minimum energy for which a zero rest mass particle can Bragg scatter with momentum transfer $|\vec{q}|=|\vec{G}|$, and mult is the multiplicity or number of reciprocal lattice vectors in each family of planes represented by $(h, k, l)$. Table taken from [223]

conversion rate as a function of daytime and energy is shown in figure 12.2, for one specific germanium detector in the CDMS-II setup. The conversion rate is given by the color-code in the figure. The Bragg condition creates a unique pattern in time and energy which can be seen as a "fingerprint" of solar axion conversions. This fingerprint is determined by the position of the sun with respect to the reciprocal lattice vectors. It can also be seen from the figure that the higher the energy, the more fine structure in time is visible, since the number of reciprocal lattice vectors for which the Bragg condition can be fulfilled increases with increasing energy (see table 12.1).

The germanium crystals in the CDMS-II setup have different alignments with respect to true north. Within the cryostat the 30 CDMS detectors are mounted in five towers of six detectors each. The vertical axis of each tower is aligned with the (001) axis of the detectors. The (110) axis that defines the major flat on each substrate is rotated by $60^{\circ}$ with respect to its neighbors above and below, such that the detectors form a helix within each tower. Figure 12.3 shows a schematic sketch of the alignment of the detectors crystal planes with respect to true north. The orientation of the (110) crystal axis with respect to true north is precisely determined by the detector interface board (DIB) located at the detectors major flat. The position of the DIBs for each detector is known from the installation of the detectors in the cryostat.

Table 12.2 summarizes the azimuthal offset of the (100) axis for each germanium detector. In the table only 16 of the 19 detectors in the CDMS-II setup are listed, since three detectors suffer from readout failures or broken channels. Thus, these three detectors are not considered in the analysis. Three groups of detector alignments could be identified which have the same, or a by $180^{\circ}$ rotated alignment of the (100) axis to true north. The 


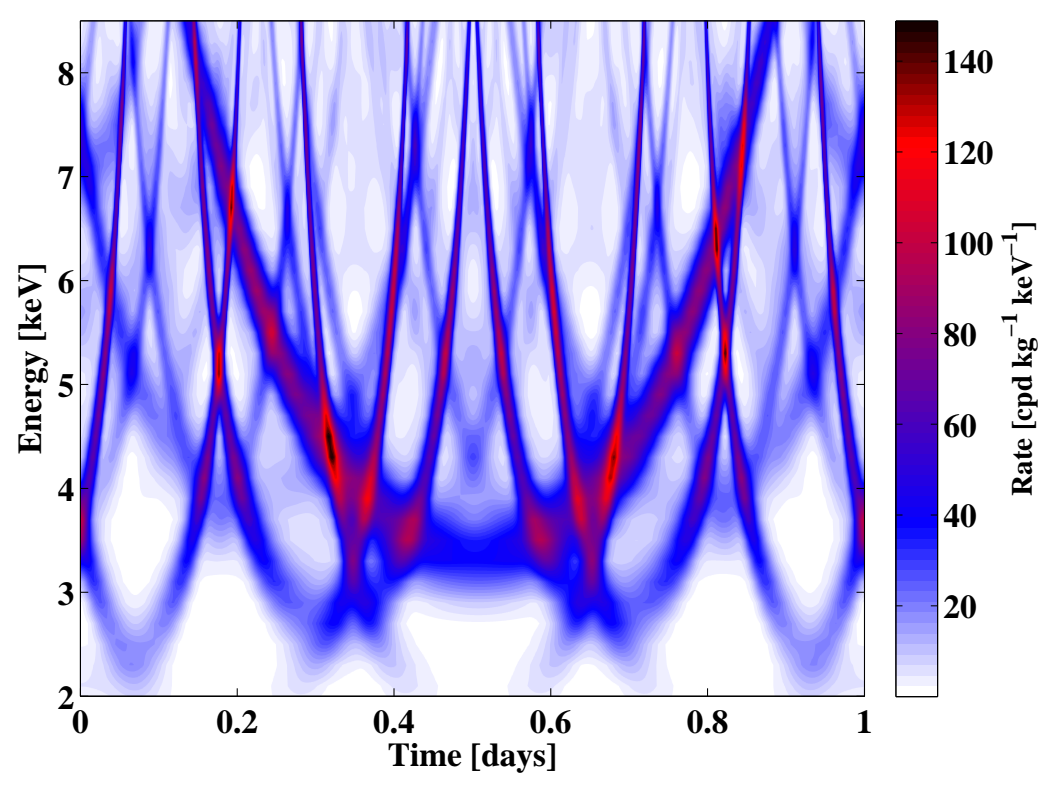

Figure 12.2: Expected conversion rate of solar axions as a function of daytime and energy. The color-code gives the conversion rate at a given daytime and axion energy. The Bragg condition creates a unique conversion pattern in time and energy which can be seen as an "fingerprint" of solar axion conversions.

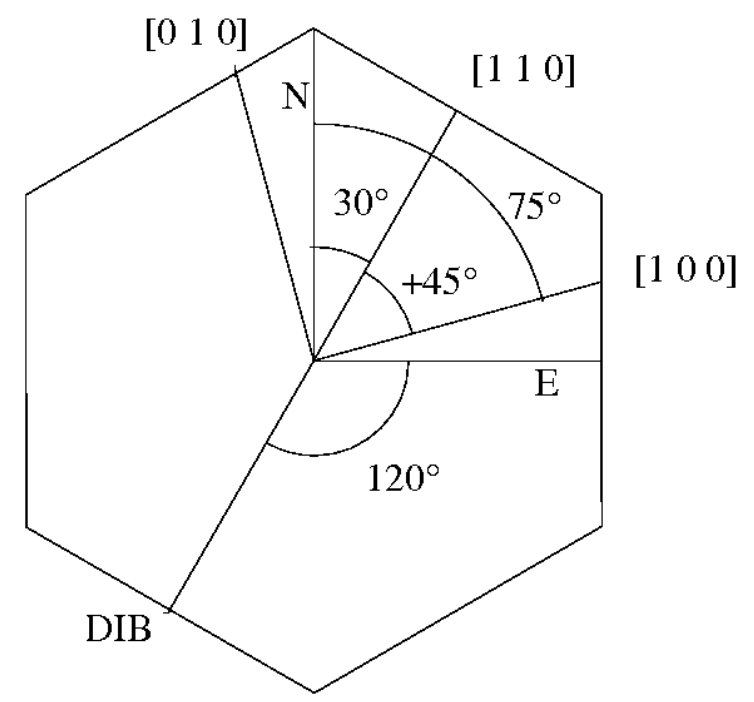

Figure 12.3: Sketch of the alignments of different crystal planes with respect to true north. The [110] plane, normal to the (110) axis is aligned with the detector interface board (DIB) located at the major flat of the crystals. The detectors DIB is rotated by $60^{\circ}$ with respect to the DIB of the neighboring detectors above and below. With this information the alignment of all crystal axes can be precisely determined for each detector. 
rotation of $180^{\circ}$ does not change the expected conversion rate since for a given daytime and energy interval the same reciprocal lattice vectors contribute to the conversion rate.

\begin{tabular}{|c|c|c||c|c|c|}
\hline Detector & Offset & Group & Detector & Offset & Group \\
\hline \hline T1Z2 & $135^{\circ}$ & $\mathrm{A}$ & T2Z5 & $195^{\circ}$ & $\mathrm{B}$ \\
T1Z5 & $315^{\circ}$ & $\mathrm{A}$ & $\mathrm{T} 3 Z 6$ & $15^{\circ}$ & $\mathrm{B}$ \\
$\mathrm{T} 2 Z 3$ & $315^{\circ}$ & $\mathrm{A}$ & $\mathrm{T} 4 Z 2$ & $15^{\circ}$ & $\mathrm{B}$ \\
$\mathrm{T} 3 Z 2$ & $135^{\circ}$ & $\mathrm{A}$ & $\mathrm{T} 4 Z 5$ & $195^{\circ}$ & $\mathrm{B}$ \\
$\mathrm{T} 3 Z 5$ & $315^{\circ}$ & $\mathrm{A}$ & $\mathrm{T} 5 Z 5$ & $15^{\circ}$ & $\mathrm{B}$ \\
$\mathrm{T} 4 Z 4$ & $135^{\circ}$ & $\mathrm{A}$ & $\mathrm{T} 3 Z 4$ & $255^{\circ}$ & $\mathrm{C}$ \\
$\mathrm{T} 5 Z 1$ & $135^{\circ}$ & $\mathrm{A}$ & $\mathrm{T} 4 Z 6$ & $255^{\circ}$ & $\mathrm{C}$ \\
T5Z4 & $315^{\circ}$ & $\mathrm{A}$ & $\mathrm{T} 5 Z 6$ & $75^{\circ}$ & $\mathrm{C}$ \\
\hline
\end{tabular}

Table 12.2: Azimuthal offset of the (100) axis from true north for 16 of the 19 geranium detectors in the CDMS-II setup. Three detectors are not considered due to readout problems or broken channels. Three groups of detector alignments could be identified which have the same or an alignment rotated by $180^{\circ}$.

For the different detector alignments the Bragg condition will be fulfilled by different reciprocal lattice vectors, providing for the same energy and daytime a different rate of solar axion conversions. These different orientations of the crystals provide an in-situ check of the conversion rate making a false positive result of detection extremely unlikely. The uncertainty in the absolute azimuth orientation of the crystal planes is dominated by an estimated $3^{\circ}$ uncertainty in the exact angular position of the tower axes with respect to the central axis of the cryostat. The uncertainty of the zenith angle measurement is estimated to be less than $1^{\circ}$. Figure 12.4 shows the expected conversion rate for two detectors (left and right column). In addition, the changing zenith angle of the Sun throughout the year creates a monthly changing pattern of the conversion rate in the crystals, which is shown in the figure for three months from the top to the bottom.

The position of the Sun at the Soudan Underground Laboratory has been calculated with a routine based on an algorithm by Reda and Andreas [226]. The precision of the algorithm is at \pm 0.0003 degrees which is sufficient for the calculations of the expected conversion rate. With the algorithm the position of the Sun can be calculated at any given geodesic location. The geodesic location of the Soudan Underground laboratory is latitude $47.815^{\circ} \mathrm{N}$, longitude $92.237^{\circ} \mathrm{W}$ and altitude $210 \mathrm{~m}$ below sea level. The geodesic north of the CDMS experimental cavern was measured in 1999 by the Fermilab Alignment Group 227. A line connecting two survey points along the central axis of the cavern was found to be $0.165^{\circ} \mathrm{E}$ from true north. By extension, the main horizontal axis of the CDMS cryostat was found to be $0.860 \pm 0.018^{\circ} \mathrm{E}$ from true north. 

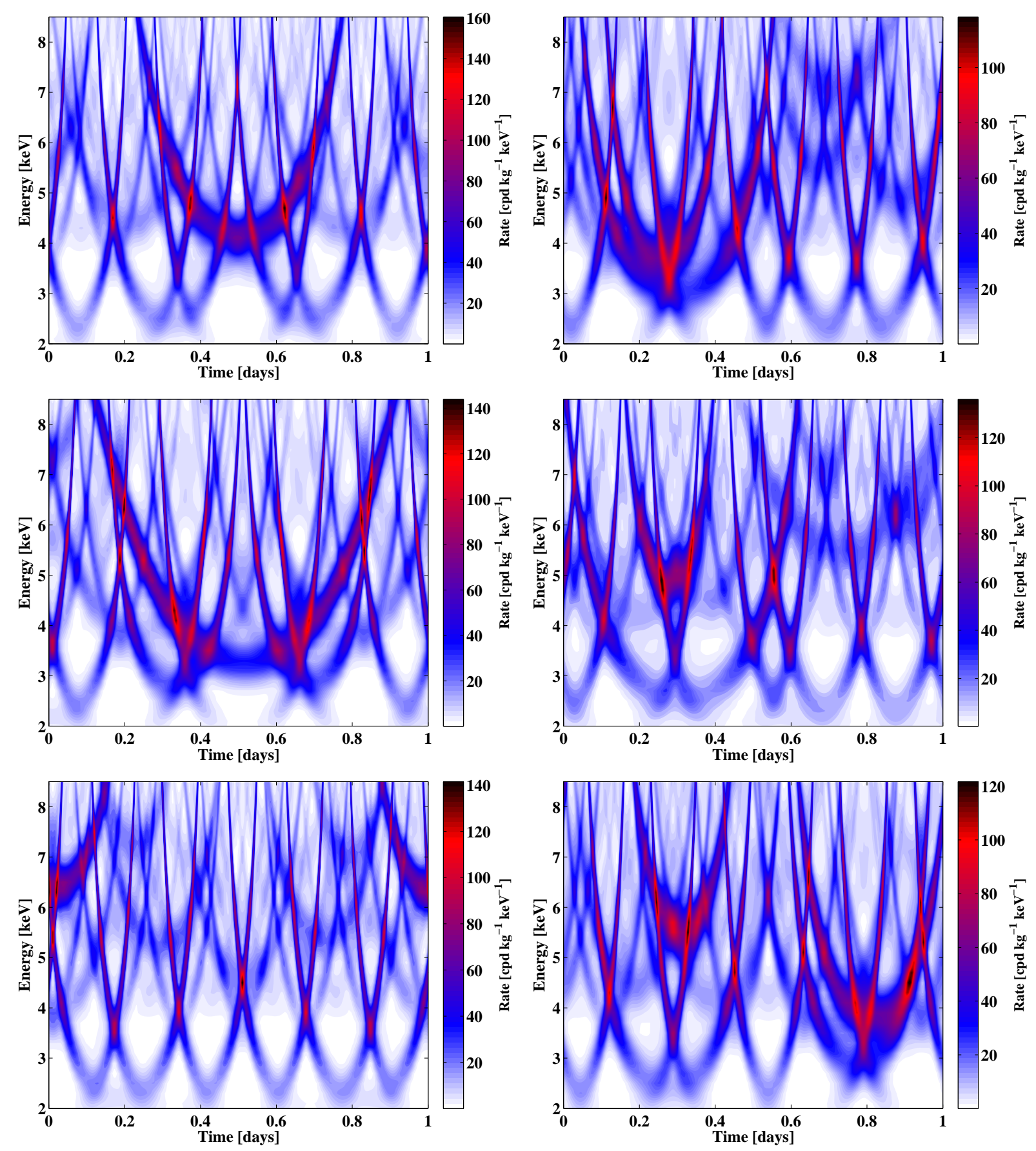

Figure 12.4: Expected conversion rate of solar axions as a function of daytime and energy for two different detector alignments (left and right column). The Sun's changing zenith angle introduces a monthly variation of the unique patterns, shown from top to bottom for the months November 2006, January 2007 and April 2007. 


\subsection{Solar axion search analysis}

To search for a signal of axion conversions in the data an expected signal region has to be determined. As outlined in Chaper 6 the CDMS ZIP detectors are capable of distinguishing between electron and nuclear recoils. This discrimination is achieved by the ionization yield parameter. The low background data from a net germanium exposure of $443.2 \mathrm{~kg}$-days used in this analysis is shown in figure 12.5(a) in the ionization yield versus energy plane. The interaction type selection is shown by the two bands in the figure representing the $2 \sigma$ regions of electron recoils and nuclear recoils. In the standard WIMP search analysis the signal is expected to be detected as a nuclear recoil, thus the nuclear recoil band defines the expected signal region (green region in figure 12.5(b)) and the events in the electron recoil distribution are considered as background events. Since the X-rays from the inverse Primakov conversions of axions in the crystal will be detected as electron recoils, the expected signal region for the search of solar axions is the electron-recoil distribution (green region in figure 12.5 (c)). This analysis therefore uses the background of the CDMSII experiment to perform a different physics analysis as the CDMS-II experiment was designed for, demonstrating a multipurpose feature of this experiment. The standard WIMP search analysis of the Run 123/4 data [4] evaluated the detection efficiencies down to an analysis threshold of $10 \mathrm{keV}$ recoil energy. Since the flux of solar axions peaks at lower energies (see figure 12.1) it was necessary to adjust and evaluate the efficiency of selection cuts for an optimal performance at energies below $10 \mathrm{keV}$.

\subsubsection{Data selection}

The same data selection criteria in terms of good data series as in the Run 123/4 WIMP search analysis have been used, expect that some detectors from Run 124 not considered in the WIMP search analysis have been used in this analysis. These detectors have been excluded from the WIMP search analysis since they showed deviations of their performance critical for a WIMP search analysis. The main criteria for a solar axion search is a good energy resolution and calibration at low energies and a clear selection of electronrecoils. Thus it has been checked for suspicious detectors, that the yield distributions of the detectors in Run 123 and Run 124 are the same and the position and resolution of the $10.36 \mathrm{keV}$ line are as expected and lie within statistical fluctuations. The exposure used in this analysis is $443.2 \mathrm{~kg}$-days with respect to the $397.8 \mathrm{~kg}$-days used in the WIMP search analysis. Two detectors (namely T2Z5 and T3Z6) from R124 have been excluded from the analysis due to a bad neutralization state which would result in a loss of events in the electron-recoil distribution, since the reduced ionization signal lowers their yield parameter. Detector T5Z6 has been excluded for both data sets due to an awful trigger efficiency at low energies. This trigger behavior is mainly caused by the small phonon amplitudes on this detector leading to the effect that a considerable amount of energy has to be deposited to efficiently trigger this detector. Other good event selection criteria have been the same as for the WIMP search analysis (for a summary of the selection criteria see Chapter 9). 


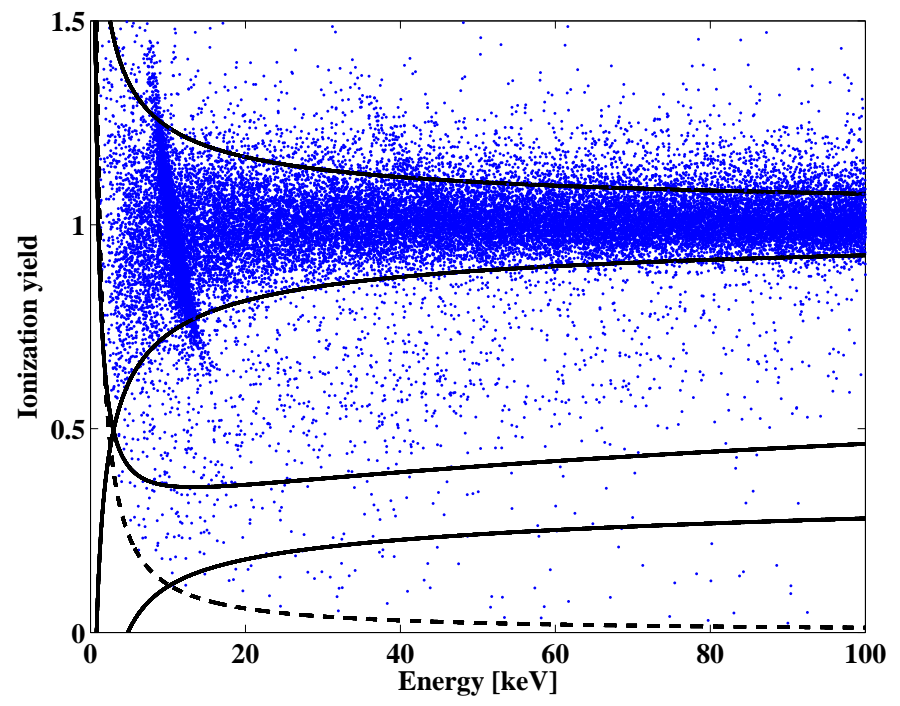

(a)

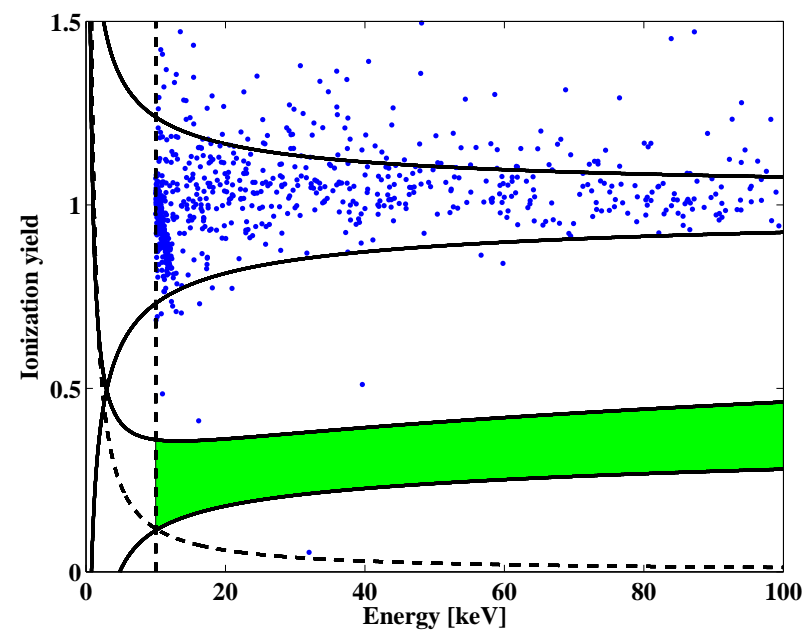

(b)

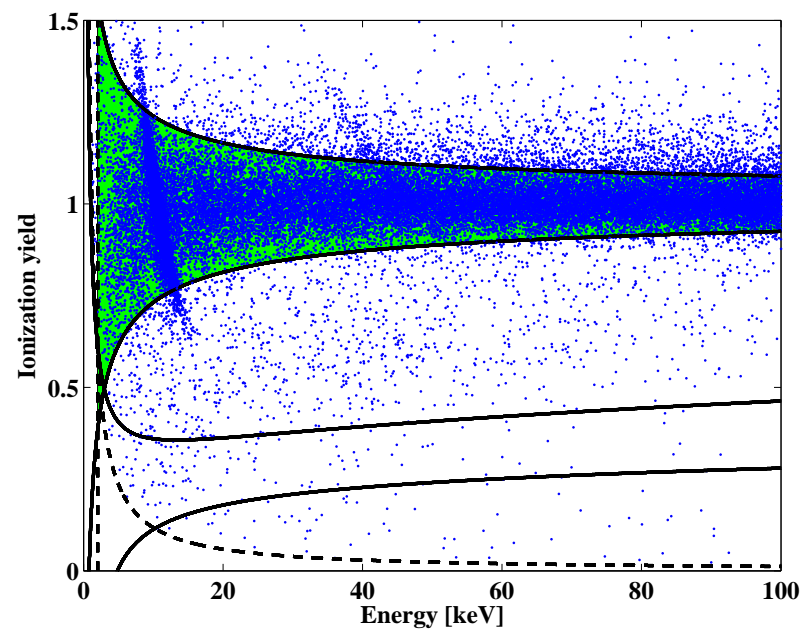

(c)

Figure 12.5: (a) Low background data of all germanium detectors considered in this analysis in the ionization yield vs. energy plane, the electron and nuclear-recoil band are given in solid black. The dashed line is the charge threshold of this analysis. The standard WIMP search analysis searches for nuclear recoils in the signal window defined by the nuclear-recoil band shown in green in figure (b). Here the surfaceevent rejection cut has been applied and the analysis threshold of the Run 123/124 analysis, shown as black/dashed line is applied. (c) Shows the selection of electron recoils considered in this analysis without any surface-event rejection cut applied and a much lower analysis threshold of $2 \mathrm{keV}$. These events are normally considered as background in the WIMP search analysis, but in the search for axions this selection (shown in green) defines the expected signal region. 


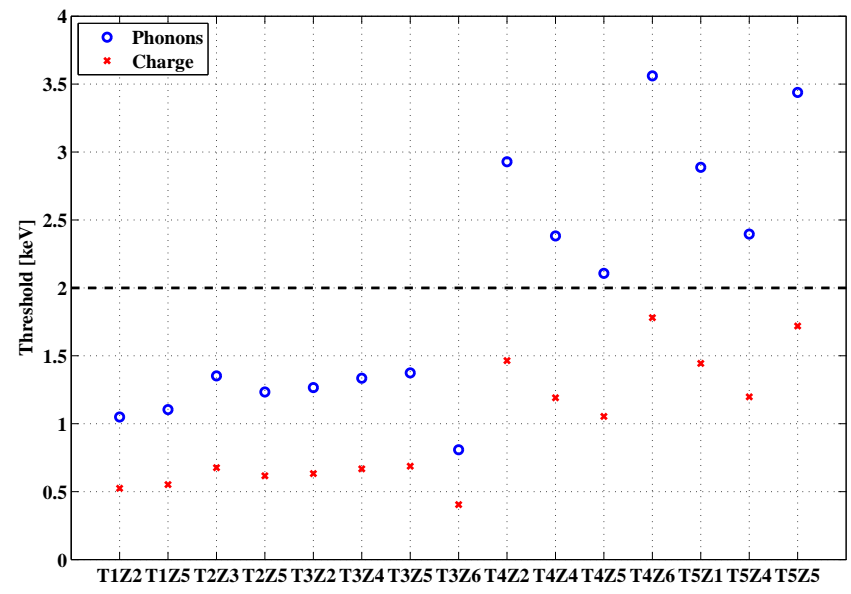

Figure 12.6: Singles cut threshold for the detectors used in this analysis. The total phonon thresholds (primary phonon + luke phonon) are given as blue circles. For electron recoils with an ionization yield of 1 these phonon thresholds translate to a threshold in ionization which is half the phonon threshold (red crosses).

\section{Electron-recoil band}

The electron-recoil band definition has not been changed from the calibration of the detectors see 7.4 . Only the selection efficiency of electron recoils has been determined as a function of energy for energies below $10 \mathrm{keV}$. This selection efficiency is determined by the ratio of events in the $4 \sigma$ and $2 \sigma$ electron-recoil band definition and is typically at $95 \%$ which is expected from a Gaussian distribution in ionization yield.

\section{Singles cut}

Since the X-rays from the inverse Primakov conversion of axions will only be detected in a single crystal the singles cut has been applied to the data selection. This reduces the Compton background from environmental radioactivity at energies below $10 \mathrm{keV}$. Some detectors, especially the later ones show small phonon pulses and thus a significantly higher energy deposition is necessary to exceed the $6 \sigma$ threshold of a single scatter selection (see section 9.4.2). The mean total phonon thresholds of the detectors used in this analysis are shown in figure 12.6. In a first order approximation for electron recoils with an ionization yield of 1 this total phonon signal consists of a primary phonon signal and a luke phonon signal of the same size. Thus the effective threshold in the ionization signal set by the singles cut is half the threshold of the total phonon threshold. The resulting thresholds on the ionization signal are shown as red crosses in figure 12.6. All ionization thresholds lie below an energy of $2 \mathrm{keV}$. Hence it was not necessary to change the singles cut for an optimization of selection efficiency at low energies, since other parameters determined an analysis threshold of $2 \mathrm{keV}$ in ionization energy as will be discussed below. 


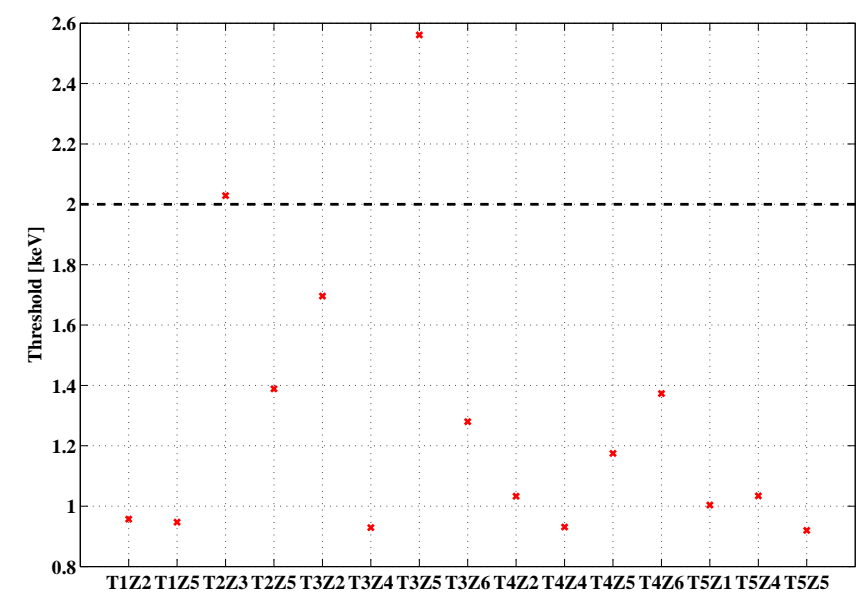

Figure 12.7: Ionization threshold for the detectors used in this analysis. Only two detectors exceed a threshold of $2 \mathrm{keV}$ due to their increased noise level. These two detectors are known to have an increased noise level due to their cryocooler cycle sensitivity.

\section{Ionization threshold and above nuclear-recoil band}

In order to reject noise events the charge threshold has been set at the $3 \sigma$ value above the mean of the noise distribution. This value is lower by one $\sigma$ than in the standard WIMP search analysis (see section 9.4.7) to obtain a higher detection efficiency at low energies. The thresholds in ionization energy are shown in figure 12.7 only two detectors namely T2Z3 and T3Z5 exceed a threshold of $2 \mathrm{keV}$ due to a higher noise level caused by the cryocooler sensitivity of these detectors (see section 9.3.2). In addition, due to the yield selection the charge threshold cut affects the selection efficiency of electron recoils as a function of energy. The rejection of nuclear recoils is achieved by rejecting events which lie below the $3 \sigma$ nuclear-recoil band upper band edge. To determine the selection efficiency in yield an analytical approximation is used. It is assumed that both, the electron recoil and the nuclear-recoil band are correctly described by a Gaussian distribution function for a given fixed energy. This approximation is well motivated and differences from a Gaussian distribution are small. Figure 12.8(a) shows this procedure in the ionization yield vs. energy plane for a specific detector, the yellow area is cut by the two selection criteria, reducing the selection area of the electron recoils (the $2 \sigma$ electron-recoil band is shown in green) at low energies. The selection efficiency of electron recoils is now defined as the fraction of the electron-recoil distribution which is neither cut by the charge threshold nor the above nuclear recoil definition. The combined selection efficiency for a specific detector is shown in figure 12.8 (b). 


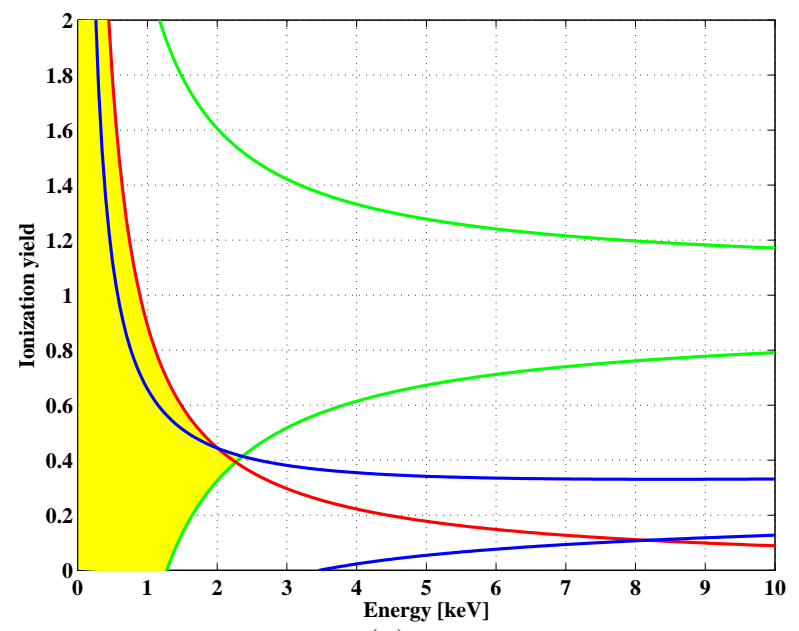

(a)

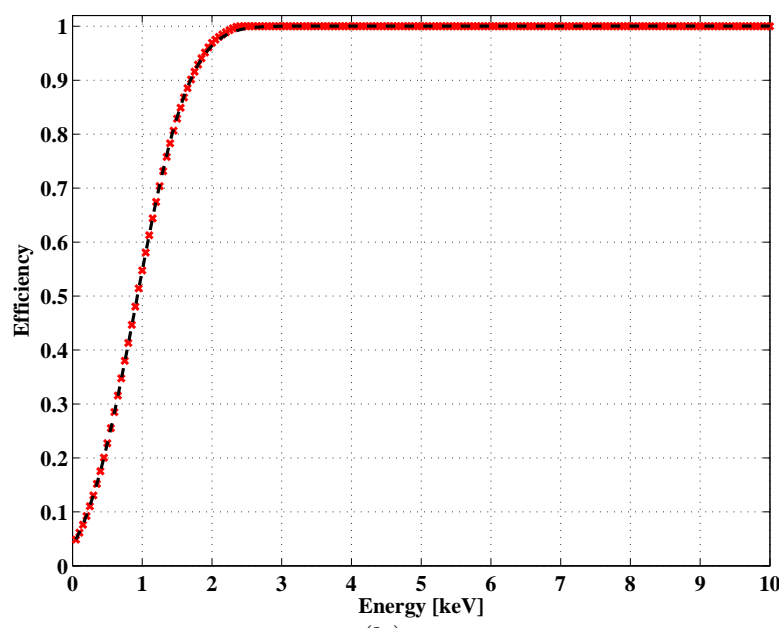

(b)

Figure 12.8: (a) Cut region (yellow/filled region) in the yield plane which lies below the charge threshold cut (red/solid line) and the upper edge of the $2 \sigma$ nuclear-recoil band (blue/solid line). The green band shows the $2 \sigma$ electron-recoil band. (b) Selection efficiency for electron recoils as a function of energy for the noise and nuclear-recoil rejection cut. The red crosses give the efficiency calculated at given energies, while the black dashed line shows an empirical fit to the this data. Plots shown for a representative detector.

\section{Charge reconstruction}

As a measurement of the reconstruction quality and thus the reliability of the charge signal from the optimal filter algorithm the $\chi^{2}$ - value of the fit is used. For further details on the definition of the cut I referr the reader to section 9.3.1. The same procedure is used here, but this analysis focuses on the low-energy signals for which the efficiency of the cut has been calculated as it is show in figure 12.9 (a). An empirical function is fitted to the measured efficiencies to obtain the efficiency as a function of energy.

\section{Fiducial Volume}

The fiducial volume for the selection of solar axion conversion photons is determined in the same manner as for nuclear recoils from WIMP interactions (see section 9.4.3). No changes to this fiducial volume cut are made with respect to the one used in the WIMP search analysis, only the efficiency as a function of energy has been determined for energies below $10 \mathrm{keV}$. Again it is assumed that neutrons provide a uniform illumination of the detectors, and hence the fraction of neutron induced nuclear recoils should be a valid measure of the fraction of volume accepted by the fiducial volume cut. The efficiency as a function of energy is fitted to a straight line as it is shown in figure 12.9(b). 


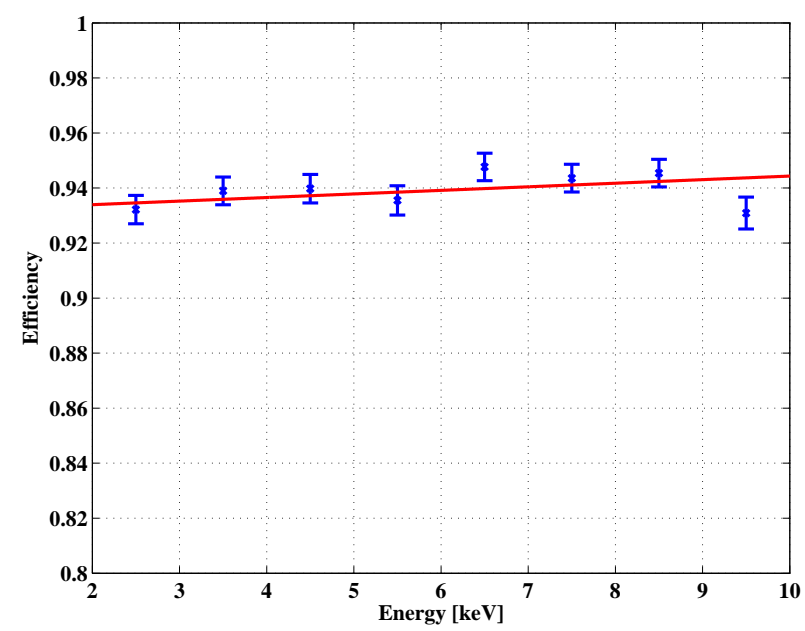

(a)

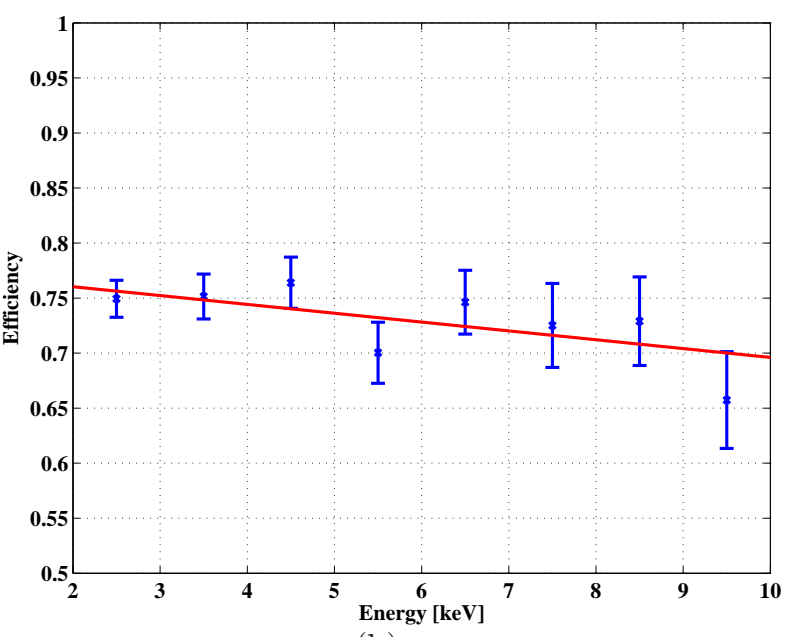

(b)

Figure 12.9: (a) Efficiency of the charge $\chi^{2}$ rejection cut as a function of energy measured on data (blue crosses). The red line shows an empirical fit to this data to describe the efficiency as a function of energy. (b) Selection efficiency of events in the fiducial volume defined by the inner-electrode cut. An empirical function (red/line) is fit to the measured efficiency (blue crosse). Plots shown for a representative detector.

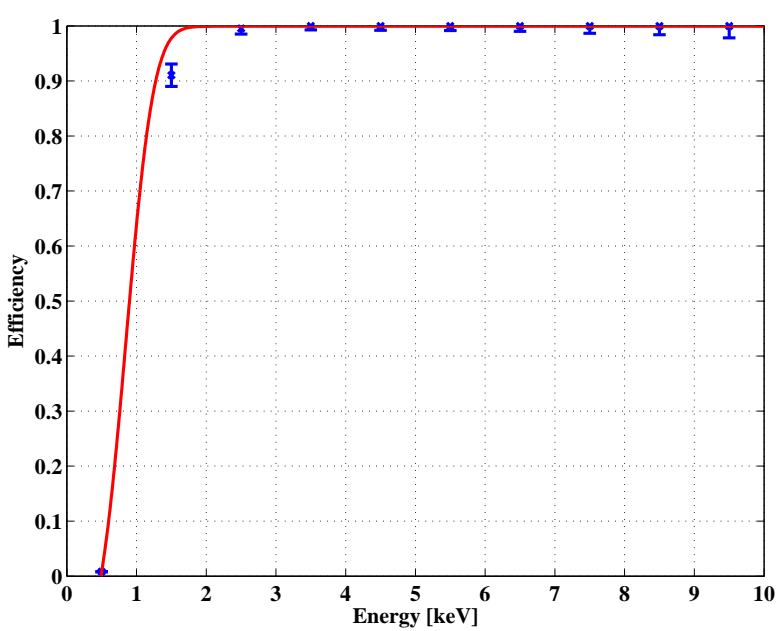

(a)

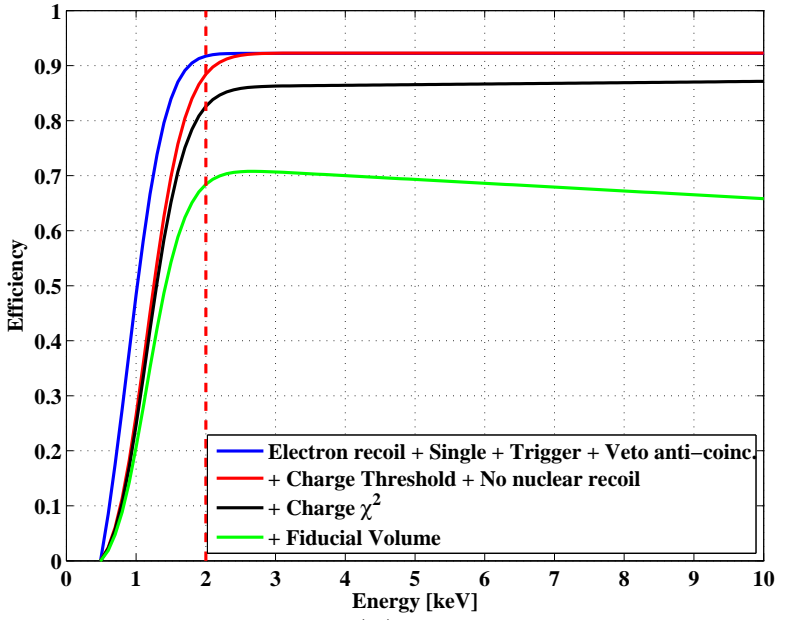

(b)

Figure 12.10: (a) Phonon trigger efficiency as a function of energy. The red line shows an empirical fit to the measured efficiency (blue crosses) to describe the efficiency as a function of energy. (b) Combined efficiency as a function of energy after applying various selection cuts. Below $2 \mathrm{keV}$ (horizontal red-dashed line) the detection efficiency rapidly degrades. The final efficiency is given by the nethermost green line. Plots shown for a representative detector. 


\section{Trigger efficiency}

The trigger efficiency has been measured in a finer energy binning than for the WIMP search analysis, to obtain a reasonable measurement of the efficiency as a function of energy to the lowest energies. Figure 12.10 shows the measured efficiencies and a functional form fitted to these efficiencies as a function of energy. For most detectors the trigger efficiencies are close to $100 \%$ for energies greater than $2 \mathrm{keV}$, only detectors in Tower 4 and 5 show some reduced performance below $4 \mathrm{keV}$ which is likely caused by the degraded phonon performance of these detectors with respect to the others.

The final efficiency for each detector is given by the combination of all these event selection efficiencies. The combined efficiency as a function of energy after applying various selection cuts is shown in figure 12.10 (b). The green line in the figure gives the final detection efficiency used in this analysis.

\section{Veto anti-coincident}

To ensure that the selected events are not due to residual cosmic ray interactions, they are required not to be coincident in time with activity in the veto shield surrounding the apparatus. The efficiency of this event selection has been determined as described in section 9.4 .1 to be $97.6 \%$ for this analysis.

\section{Neutron activation}

The regularly performed neutron calibrations of the detectors result in an activation of the materials surrounding the detectors (see section 9.2.5). The effect on the background of electron recoils originating from this activation has been measured by investigating the count rate as a funtion of time after the neutron calibration. Figure 12.11 shows the rate at low energies (from 2 to $8.5 \mathrm{keV}$ ) as a function of time after the last neutron calibration. The mean low background counting rate (shown as the black line in the figure) is determined from data sets taken within five days before the actual neutron calibration. A fit to the rate as a funtion of time is performed, including an exponential decaying component from ${ }^{64} \mathrm{Cu}$ which is the main isotope produced in the activation of the materials. In the fit the half-live is fixed a 12.7 hours to determine the initial rate originating from the neutron activation. The fit is shown as the red line in figure 12.11, The green line in the figure shows only the decaying component determined from the fit. Extrapolating this decaying rate to a time of three days after the neutron calibrations results in a residual rate from the neutron activation of the materials of $0.21 \pm 0.02$ [counts/(kg day)] which is only about $2 \%$ of the mean counting rate. Thus data sets taken within three days after a neutron calibration are not considered in this analysis making a contribution to the background rate from neutron activation negligible. 


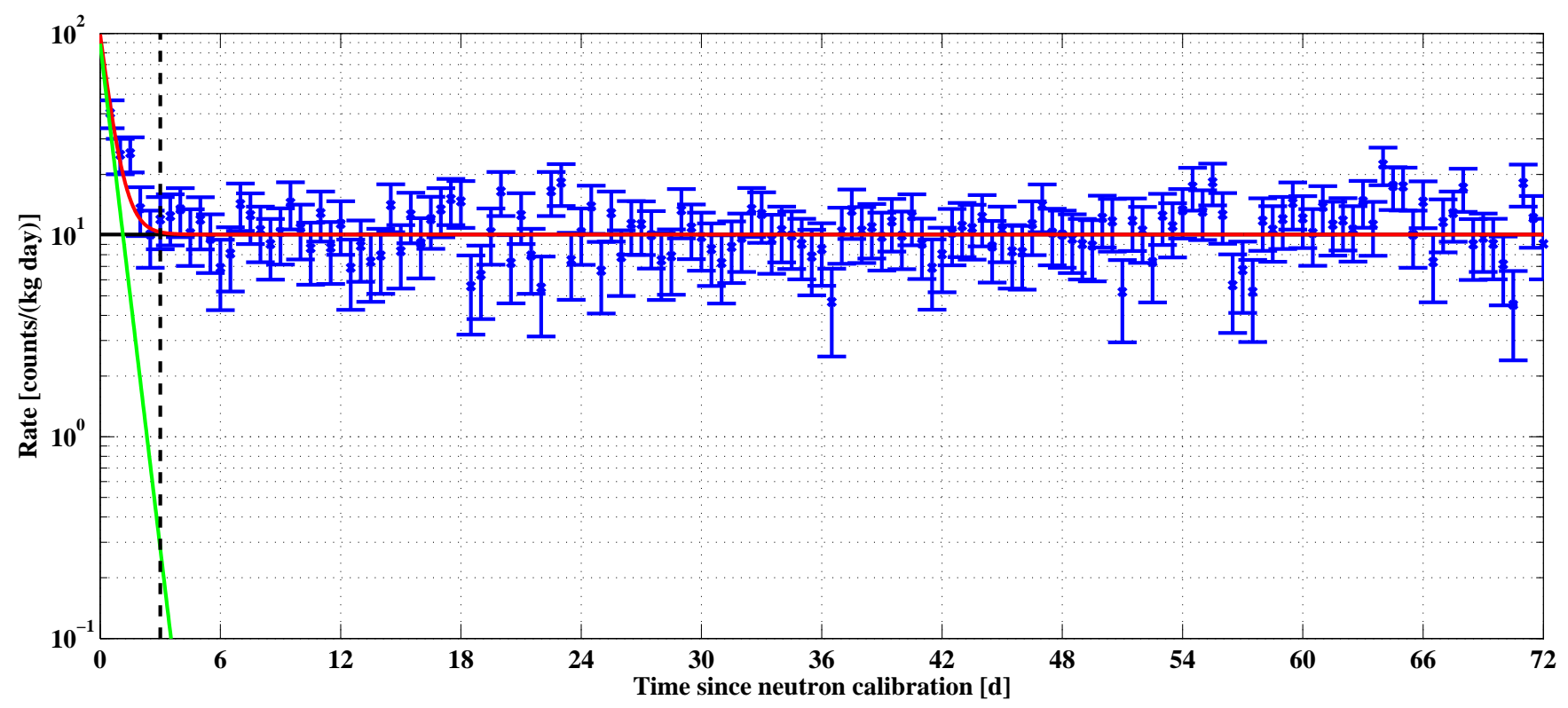

Figure 12.11: Rate as a funtion of time since the last neutron calibration. The black line gives the mean rate determined from data sets taken within five days before the calibration. The red line is a fit to the rate including an exponential function describing the decay rate of ${ }^{64} \mathrm{Cu}$ with an half live of $12.7 \mathrm{~h}$. The green line shows only this decaying component. The time threshold of 3 days after the neutron calibration is shown as vertical (dashed/black) line. 


\subsubsection{Low-energy electron-recoil spectrum}

It is interesting to investigate the combined low-energy spectrum to identify possible backgrounds and to set a reasonable analysis window to search for conversion of solar axions. For this purpose the exposure weighed combined efficiency for all detectors used in this analysis has been determined. This is simply done by summing the final efficiencies with a weight given by the detector's contribution to the total exposure. The resulting efficiency is shown in figure 12.12. The detection efficiency is better than $\sim 60 \%$ for energies greater than $\sim 4 \mathrm{keV}$. This is far greater than the typical efficiency for the WIMP search analysis due to the fact that no surface-event rejection cut is used in this analysis. The detection efficiency is dominated by rejection of events with an ionization signal in the detector annular guard electrode (fiducial volume selection).

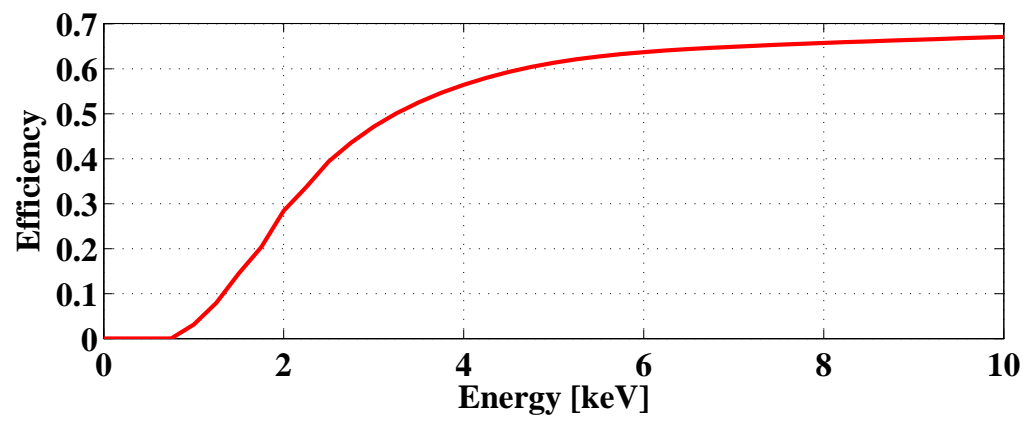

Figure 12.12: Exposure weighted detection efficiency as a function of energy.

The summed background spectrum of all considered detectors, taking into account the detection efficiency, is shown in figure 12.13. The prominent $10.36 \mathrm{keV}$ line is caused by $\mathrm{X}$-rays and Auger-electrons from the electron capture decay of ${ }^{71} \mathrm{Ge}$, produced by neutron capture on ${ }^{70} \mathrm{Ge}$ during ${ }^{252} \mathrm{Cf}$ calibrations of the detectors. There may also be a contribution from ${ }^{68} \mathrm{Ge}$ from cosmogenic activation of the detectors, but this contribution should be subdominant considering the time the detectors spend underground and the regularly performed neutron calibrations. Taking into account the spectral flux of solar axions (see figure 12.1) and the fact that the backgrounds are increasing rapidly below $2 \mathrm{keV}$, and approaching the $10.36 \mathrm{keV}$ line, an analysis window ranging from 2 to $8.5 \mathrm{keV}$ has been defined. The choice of the upper edged of the analysis window is motivated by the fact that the flux of solar axions is small above this energy and the background contribution from the $10.36 \mathrm{keV}$ line is negligible since it is about $5-6 \sigma$ away, given the typical energy resolution of the detectors of $0.3-0.4 \mathrm{keV}$. The inset in figure 12.13 shows an enlargement of this analysis window. The excess in event rate around $6.5 \mathrm{kev}$, visible in the inset, is likely caused by remnant ${ }^{55} \mathrm{Fe}$ decays from cosmogenic activation of the detectors. The de-excitation of ${ }^{55} \mathrm{Mn}$ following the electron-capture decay of ${ }^{55} \mathrm{Fe}$ yields a total of $6.54 \mathrm{keV}$ electron-recoil events.

Although this contribution is taken into account in the likelihood analysis described in the next section it should be of no concern for a search for solar axions, since the decay 


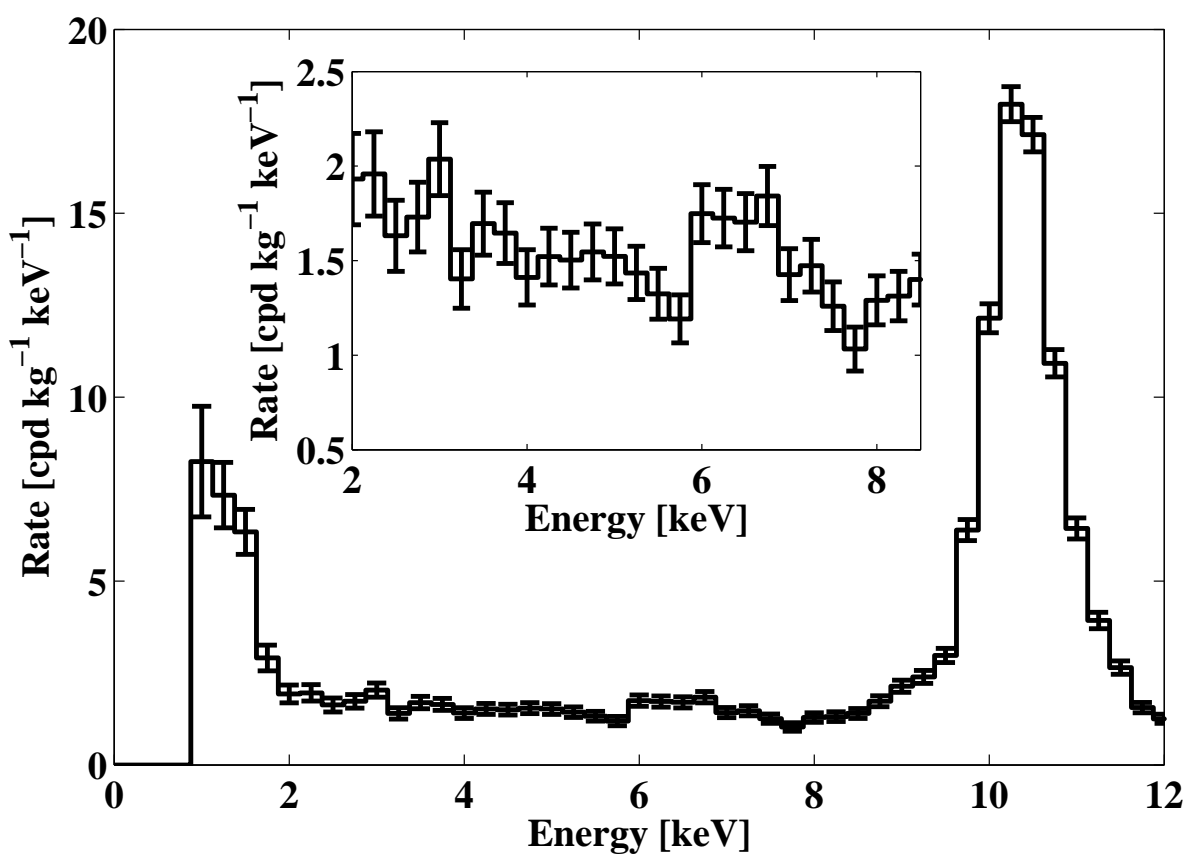

Figure 12.13: Efficiency corrected, co-added low-energy spectra. The spectral line at $10.36 \mathrm{keV}$ is used for the energy calibration of the detectors. The inset shows an enlargement of the analysis window from 2 to $8.5 \mathrm{keV}$. The excess in rate at $\sim 6.5 \mathrm{keV}$ is likely caused by the detection of remnant ${ }^{55} \mathrm{Fe}$ decays from cosmogenic activation.

does not follow the pronounced structure in time which is expected from the conversion of solar axions (see Section 12.2). The origin of this background contribution is discussed in more detail in the next Chapter.

The energy resolution of the detectors as a function of energy has been obtained by a fit to the ionization noise distribution and the resolution of the $10.36 \mathrm{keV}$ line. The energy resolution has been determined for each detector separately. The detector-averaged r.m.s energy resolution $\sigma(E)$ below $10 \mathrm{keV}$ is given by:

$$
\sigma(E)=\sqrt{(0.293)^{2}+(0.056)^{2} E}[\mathrm{keV}]
$$

\subsubsection{Likelihood analysis}

A maximum likelihood analysis has been carried out to search for a detection of photons from solar axion conversions in the crystals. The strongly pronounced pattern in time of these conversions (discussed in Section 12.2) makes an unbinned likelihood analysis in time the most promising procedure to extract a possible signal.

The event rate per unit measured energy $(E)$, per unit time $(t)$ and per detector $(d)$ of solar axion conversions with background is expressed by

$$
R(E, t, d)=\varepsilon(E, d)[\lambda \mathcal{R}(E, t, d)+B(E, d)],
$$


where $\varepsilon(E, d)$ is the detection efficiency which has been measured as a function of energy for each individual detector, $\mathcal{R}(E, t, d)$ is the expected event rate for a coupling constant $g_{a \gamma \gamma}=10^{-8} \mathrm{GeV}^{-1}$, and $\lambda=\left(g_{a \gamma \gamma} \cdot 10^{8} \mathrm{GeV}\right)^{4}$ is the scale factor for the actual value of $g_{a \gamma \gamma} . B(E, d)$ is the background model described by

$$
\begin{aligned}
B(E, d) & \equiv C(d)+D(d)+H(d) / E \\
& +\frac{\eta_{6.54}}{\sqrt{2 \pi} \sigma_{6.54}} e^{\left(-\frac{(E-6.54 \mathrm{keV})^{2}}{2 \sigma_{6.54}^{2}}\right)},
\end{aligned}
$$

where $C(d), D(d)$ and $H(d)$ are free parameters. The Gaussian term describes a contribution from ${ }^{55} \mathrm{Fe}$ decays at an energy of $6.54 \mathrm{keV}$ and unknown total rate $\eta_{6.54}$. The width of the Gaussian $\sigma_{6.54}$ has been fixed at the measured energy resolution. The background model is assumed to be independent of time since the expected counting rate from solar axion conversions varies with daytime and the background from ambient gamma induced electron recoils is uniform in time throughout a day.

The fitting is done by maximizing the unbinned log likelihood function with respect to $\lambda$ and the background parameters for each individual event $i$ and detector $j$. To derive the used unbinned likelihood function for a specific detector we start with a standard likelihood function from which the number of signal events $\mu_{s}$ and number of background events $\mu_{b}$ should be extracted from the data:

$$
\log (\mathcal{L})=-\left(\mu_{s}+\mu_{b}\right)+\sum_{i} \log \left(\mu_{s} f_{s}(i)+\mu_{b} f_{b}(i)\right)
$$

where $f_{s, b}(i)$ is the probability of event $i$ being a signal/background event. The probability density distributions $f_{s, b}(i)$ can be expressed by:

$$
f_{s}(i)=\frac{\mathcal{R}\left(E_{i}, t_{i}, d\right)}{\left(\mathcal{R}_{T}(d) / M D\right)} \quad f_{b}(i)=\frac{B\left(E_{i}, d\right)}{B_{T}(d)},
$$

where $\mathcal{R}_{T}(d)$ is the total sum of the signal rate $(\mathcal{R}(E, t, d))$ over energy and time, $B_{T}(d)$ is the total sum of the background rate $B(E, d)$ over energy and $M D$ is the exposure under analysis. Since the number of observed events $\mu_{s, b}$ are given by:

$$
\mu_{s}=\lambda \cdot \mathcal{R}_{T}(d) \quad \mu_{b}=B_{T}(d) \cdot M D,
$$

the likelihood function (12.18) can be written as:

$$
\begin{aligned}
\log (\mathcal{L}) & =-\left(\lambda \cdot \mathcal{R}_{T}(d)+B_{T}(d) \cdot M D\right) \\
& +\sum_{i} \log \left(\lambda \cdot \mathcal{R}_{T}(d) \cdot \frac{\mathcal{R}\left(E_{i}, t_{i}, d\right)}{\left(\mathcal{R}_{T}(d) / M D\right)}+B_{T}(d) \cdot M D \cdot \frac{B\left(E_{i}, d\right)}{B_{T}(d)}\right) .
\end{aligned}
$$

The exposure $M D$ is a constant factor in the likelihood function and does not depend on the parameters of interest, thus a reduced likelihood function which neglects the constant term $\log (M D)$ in the second part of the likelihood function can be defined: 


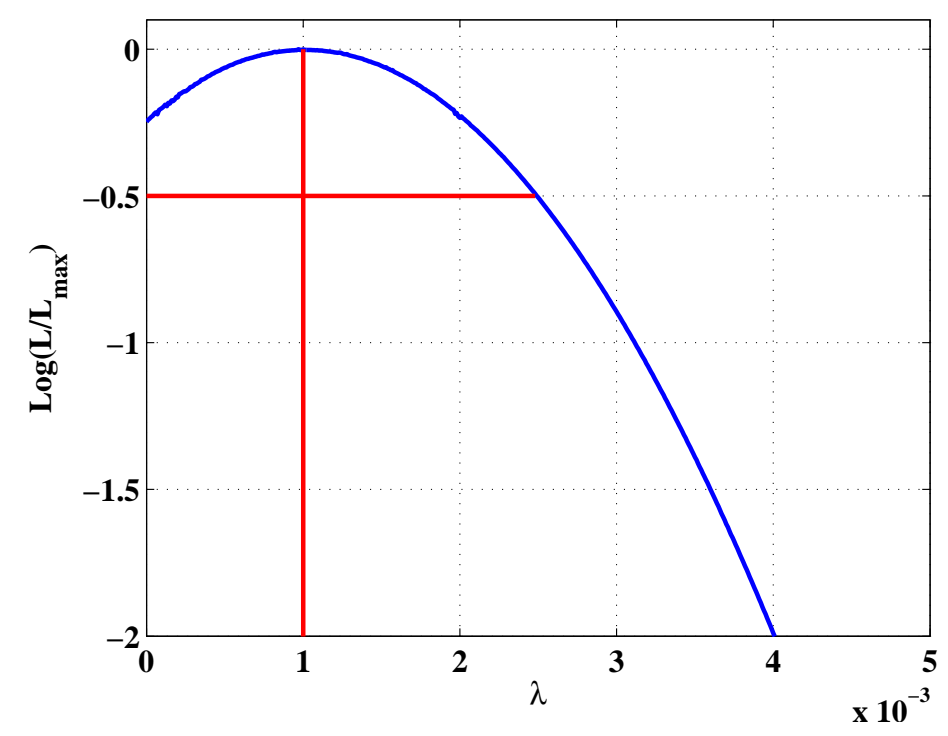

Figure 12.14: Profile likelihood function for the scaling parameter $\lambda$. The maximum likelihood estimator is compatible with zero within the error, found at the value of $\lambda$ at which the profile likelihood function is reduced by $1 / 2$ from its maximum value.

$$
\log (\mathcal{L})=-\left(\lambda \cdot \mathcal{R}_{T}(d)+B_{T}(d) \cdot M D\right)+\sum_{i} \log \left(\lambda \cdot \mathcal{R}\left(E_{i}, t_{i}, d\right)+B\left(E_{i}, d\right)\right) .
$$

Taking into account the detection efficiency $\varepsilon(E, d)$ and using notation (12.15) for the event rate the likelihood function can be written as:

$$
\log (\mathcal{L})=-R_{T}(d)+\sum_{i} \log \left(R\left(E_{i}, t_{i}, d\right)\right)
$$

Finally summing over the detectors considered in the analysis the likelihood function can be expressed by:

$$
\log (\mathcal{L})=-R_{T}+\sum_{i, j} \log \left(R\left(E_{i}, t_{i}, d_{j}\right)\right)
$$

where $R_{T}$ is the total sum of the event rate $(R)$ over energy, time and detectors. The maximum likelihood estimator for the scaling factor $\lambda=(1.0 \pm 1.5) \times 10^{-3}$ is compatible with zero. The error on the scaling parameter has been found by determining the value of $\lambda$ at which the profile likelihood [228, 229, 230] has been reduced by $1 / 2$ from its maximum value. This is a standard procedure for determining the $68 \%$ confidence level from a likelihood function:

$$
\log (\mathcal{L}(\lambda))=\log \left(\mathcal{L}_{\max }(\lambda)\right)-\frac{1}{2}
$$

No indication of solar axion conversions to photons is observed. The profile likelihood function from which the scaling parameter has been determined is shown in figure 12.14. 
The profile likelihood function is found by fixing the scaling parameter at a given value and performing the maximization of the likelihood function with respect to the background parameters only. The profile likelihood marginalizes over the nuisances background parameters and can be treated as a real likelihood function to determine errors and confidence levels of a maximum likelihood estimator.

Given a null observation an upper limit on the coupling constant $g_{a \gamma \gamma}$ can be set. The upper limit on the scaling factor $\lambda$ is obtained in a bayesian approach (using a flat prior) by integrating the profile likelihood in the physical allowed region $(\lambda>0)$ until the integral reaches $95 \%$ of the total area:

$$
0.95=\frac{\int_{0}^{\lambda_{95}} \mathcal{L}(\lambda)}{\int_{0}^{\infty} \mathcal{L}(\lambda)}
$$

This procedure ensures a physical interpretable limit even if the maximum likelihood estimator turns out to be negative. In such a statistically occurring case the upper limit could be set to strict if a classical construction would be used.

The upper limit at a $95 \%$ CL on the coupling constant

$$
g_{a \gamma \gamma}<2.47 \times 10^{-9} \mathrm{GeV}^{-1}
$$

derived from the upper limit on the scaling factor is the only laboratory bound based on the accurate measurement of all crystal orientations of the detectors. None of the previous crystal based solar axion search experiments (SOLAX/COSME/DAMA) measured their crystal orientations [231, 232, 233], and thus their limits are penalized by picking the least sensitive orientation for their limit on the axion-photon coupling. The result of this analysis is compared to other experimental limits in figure 12.15. In the figure the upper limit from this analysis is given by the red solid line. As well as the results from other crystal based experiments the upper limit is independent of the axion mass for masses below the keV scale. At higher masses the coherence condition is not fulfilled anymore as it is the case for the searches with helioscopes shown in blue (CAST) and cyan (Tokyo helioscope). Although the sensitivity of crystal based experiments is not competitive to the sensitivity of helioscopes at low masses, the high mass region of allowed axion models (shown as the yellow band in the figure) around $1 \mathrm{eV}$ can be probed quite effectively.

As mentioned in section 12.2, the main systematic uncertainty on this result is the estimated $3^{\circ}$ uncertainty in the exact angular position of the tower axes with respect to central axis of the cryostat. To determine the effect of this uncertainty the likelihood analysis has been carried out for different alignments of the tower axes with respect to the central axis of the cryostat. In this systematic study the position of the tower axes with respect to true north has been varied by $\pm 4^{\circ}$ in $1^{\circ}$ steps from the measured value of $0.860 \pm 0.018^{\circ} \mathrm{E}$ from true north. The systematic uncertainty on the upper limit as a function of the offset from true north is shown in figure 12.16. All systematical changes to the alignment of the detectors with respect to true north resulted in a higher upper limit on the axion-photon coupling constant, than the actual measured value. Thus only an upper systematic uncertainty is given by the highest value of the upper limit. This 


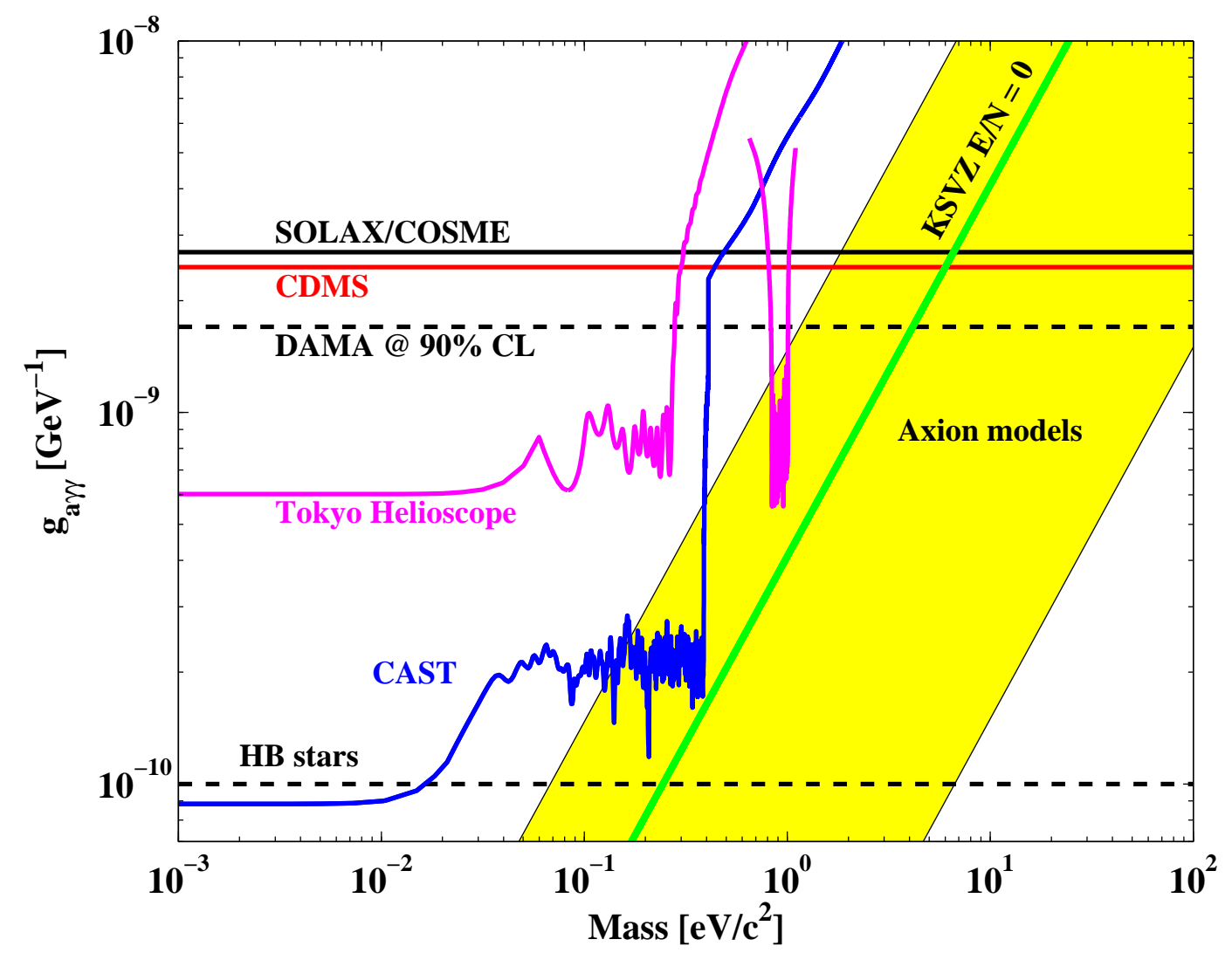

Figure 12.15: Comparison of the 95\% C.L. upper limit on $g_{a \gamma \gamma}$ achieved in this analysis (red/solid) with other crystal search experiments (SOLAX/COSME [231, 232. (black/solid) and DAMA (upper black/dashed) 233]) and helioscopes (Tokyo heslioscope (magenta/solid) 234] and CAST (blue/solid) [225]). The constraint from Horizontal Branch stars (lower black/dashed) is also shown [235]. The yellow band shows the range of axion models [73], including the DFSZ model [71, 72] and the KSVZ model [69, 70] shown as the green line. 


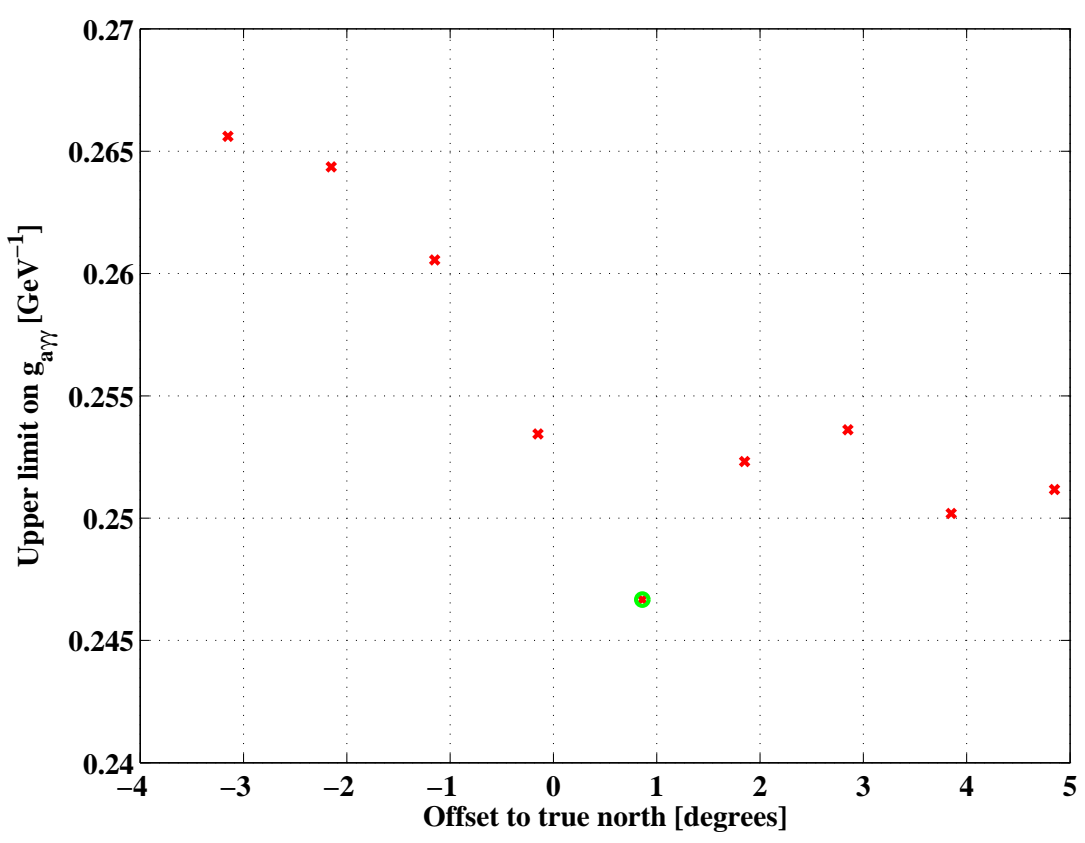

Figure 12.16: Systematic uncertainty on the upper limit on the coupling constant $g_{a \gamma \gamma}$ due to the uncertainty in the azimuthal alignment of the detectors. The limit has been calculated for several azimuthal offsets (red points) from the measured position of $0.860 \pm 0.018^{\circ} \mathrm{E}$ from true north (highlighted by the green circle).

systematic study showed that the upper limit on the solar axion coupling constant could be as high as:

$$
g_{a \gamma \gamma}<2.65 \times 10^{-9} \mathrm{GeV}^{-1} .
$$

After opening the icebox for the installation of the first SuperTower the alignment of the detector axes has been measured more precisely to be $1.24 \pm 0.6$ West from true north which lies in the expected uncertainty and would set a limit close to

$$
g_{a \gamma \gamma}<2.6 \times 10^{-9} \mathrm{GeV}^{-1}
$$

as can be seen from figure 12.16. These measurements were not available at the time the analysis was performed but should definitely be used in future searches for solar axions with the CDMS-II experiment. 


\subsection{Discussion}

The search for solar axions may resolve the strong CP problem arising in QCD. In this analysis it has been shown that the coherent Primakov conversion in crystals provides an efficient procedure to search for solar axion conversion fingerprints, due to a unique time and energy dependent signature of solar axion conversions. This analysis did not show a hint of solar axion conversions in the detectors and sets an upper limit on the axion photon coupling constant of $g_{a \gamma \gamma}<2.4 \times 10^{-9} \mathrm{GeV}^{-1}$ at the $95 \% \mathrm{CL}$. The result lies below the current best limit set by germanium detectors from the SOLAX experiment [231, 232]. The advantages of the CDMS-II experiment are the low background rate and the precise knowledge of the crystal orientations. The dominating factor in the sensitivity of crystal based experiments to solar axion conversions is the electromagnetic background these experiments are facing. Thus in order to increase the sensitivity beyond the benchmark of $g_{a \gamma \gamma}<1 \times 10^{-9} \mathrm{GeV}^{-1}$ this background has to be decreased significantly. Nevertheless, this analysis inspires the prospect that future large crystal detector arrays may provide competitive sensitivity on the photon axion coupling constant. A 100-kg SuperCDMS style experiment, with substantially reduced gamma background level $(\sim 0.1$ counts $/ \mathrm{kg} / \mathrm{day} / \mathrm{keV})$ would improve the sensitivity beyond this benchmark, making searches for solar axions with crystal based experiments competitive to the sensitivity of helioscopes in the high mass region $\left(m_{\text {axion }} \leq 1 \mathrm{keV} / \mathrm{c}^{2}\right)$, where most of the relevant parameter space lies. Other crystal based experiments like the GERDA 236 or MAJORANA 237] experiment could also reach these sensitivities, if the alignment of the crystals are known and sufficiently low thresholds can be reached. 


\section{Chapter 13}

\section{Low-energy electron-recoil spectrum analysis}

The WIMP search analysis is in some way a model dependent analysis since it is only looking for nuclear recoils. If a dark matter interaction would not produce a nuclear recoil it may evade detection in the WIMP search analysis. The other possible interaction type is that dark matter particles produce an electron recoil in the detectors. Although the background is orders of magnitude higher when searching for electron recoils rather than nuclear recoils it is worth to take a look at the low-energy electron-recoil spectrum. In order to search for an excess rate above background the low-energy electron-recoil spectrum background has to be characterized and an assumption on the spectral shape of a possible signal has to be made. Having done so, one may derive constraints either on a particular candidate model or a general constraint on the rate above background.

There are some motivations for looking at the low-energy electron-recoil spectrum. First ,there are theoretically motivated pseudoscalar dark matter candidates which would be detected as electron recoils. Signals from these particles would show up in the lowenergy range depending however on their mass. Second, if the annual modulation signature observed by DAMA/LIBRA is not induced by nuclear recoils but electron recoils the investigation of the low-energy electron-recoil spectrum helps to identify or constrain possible models which could explain the modulation signature. Finally, in a very model independent way on can look for a modulation of the counting rate at the lowest energies, just like the DAMA experiment does. This can set constraints on a modulated counting rate and test the DAMA claim in a model independent analysis. 


\subsection{CDMS low-energy electron-recoil spectrum}

The data and selection cuts applied in the general analysis of the low-energy electron-recoil spectrum are the same as described in section 12.3, in which also the analysis efficiency as a function of energy has been determined. Here the spectrum is analyzed in terms of possible background contributions and possible excess rates above background. Figure 13.1 shows the low-energy electron-recoil spectrum as in figure 12.13. In addition a simple fit to the spectrum is shown in the figure. The fit incorporates known spectral lines at $10.36 \mathrm{keV}$ and $8.98 \mathrm{keV}$. The latter originates in the decay of remnant ${ }^{65} \mathrm{Zn}$ from cosmogenic activation of the detectors. The fit also incorporates a spectral line corresponding to an excess of events observed near $6.5 \mathrm{keV}$. Each peak is fit by a Gaussian distribution function with width fixed at the detectors measured energy resolution (see section 12.3.2). For reference table 13.1 gives the observed rates in an energy interval from $2-8.5 \mathrm{keV}$.

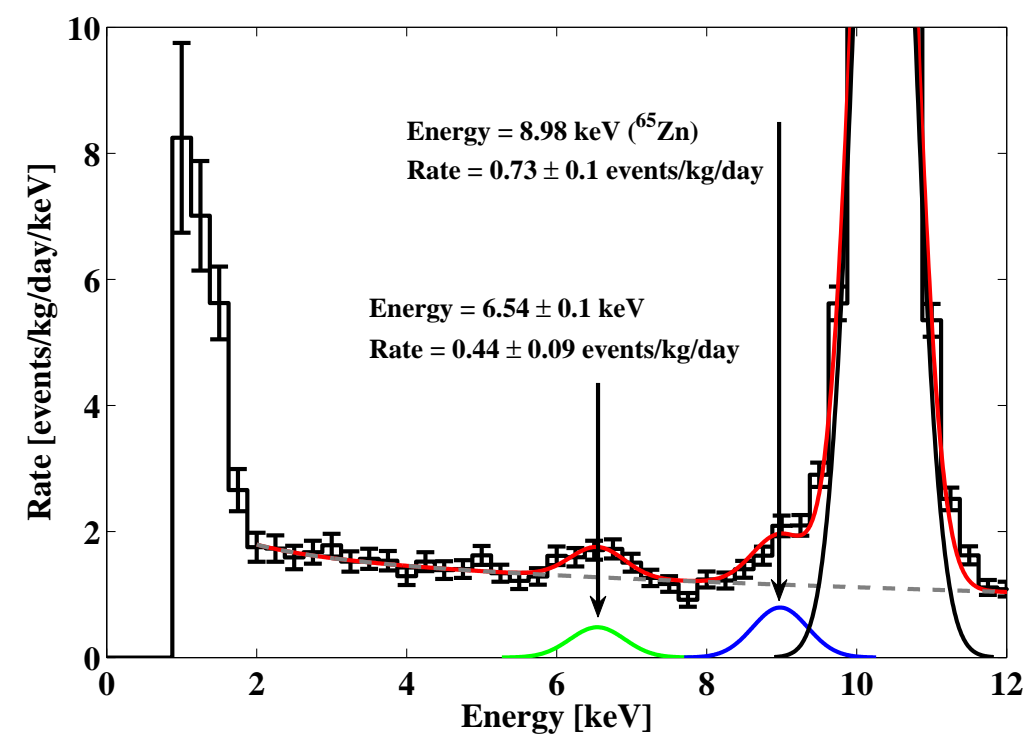

Figure 13.1: Fit (red line) to the efficiency corrected low-energy spectrum consisting of a background model (gray/dashed) and three Gaussian distribution functions describing the $10.36 \mathrm{keV}$ line from ${ }^{71} \mathrm{Ge}$ (black), the $8.98 \mathrm{keV}$ line from ${ }^{65} \mathrm{Zn}$ (blue) and a line at the energy of ${ }^{55} \mathrm{Mn}$ (green, see text). The total counting rate of the latter two lines is given in the figure..

The excess in event rate near $6.5 \mathrm{keV}$ is likely caused by the de-excitation of ${ }^{55} \mathrm{Mn} .{ }^{55} \mathrm{Mn}$ can be produced by electron capture of remnant ${ }^{55} \mathrm{Fe}$ from cosmogenic activation. The deexcitation of ${ }^{55} \mathrm{Mn}$ results in a spectral line at $6.54 \mathrm{keV}$, matching exactly the energy of the corresponding peak in the spectrum. While at the surface the detectors were exposed to fast neutrons from cosmic-ray showers. Gamma-rays from isotopes produced in Ge by these fast cosmic-ray neutrons have been observed in the CoGeNT experiment, which uses a p-type contact germanium detector providing an excellent energy resolution [110]. The most dominant lines in their spectrum are from ${ }^{65} \mathrm{Zn}$ with an energy of $8.98 \mathrm{keV}$ and ${ }^{68,71} \mathrm{Ge}$ 


\begin{tabular}{|c|c||c|c||c|c|}
\hline Energy & Rate & Energy & Rate & Energy & Rate \\
\hline 2.0 & $1.93 \pm 0.24$ & 4.25 & $1.52 \pm 0.15$ & 6.5 & $1.70 \pm 0.15$ \\
2.25 & $1.96 \pm 0.22$ & 4.5 & $1.50 \pm 0.15$ & 6.75 & $1.84 \pm 0.16$ \\
2.5 & $1.63 \pm 0.19$ & 4.75 & $1.55 \pm 0.15$ & 7.0 & $1.43 \pm 0.14$ \\
2.75 & $1.73 \pm 0.18$ & 5.0 & $1.52 \pm 0.15$ & 7.25 & $1.47 \pm 0.14$ \\
3.0 & $2.04 \pm 0.19$ & 5.25 & $1.43 \pm 0.14$ & 7.5 & $1.26 \pm 0.13$ \\
3.25 & $1.40 \pm 0.15$ & 5.5 & $1.32 \pm 0.13$ & 7.75 & $1.03 \pm 0.12$ \\
3.5 & $1.70 \pm 0.17$ & 5.75 & $1.19 \pm 0.13$ & 8.0 & $1.29 \pm 0.13$ \\
3.75 & $1.65 \pm 0.16$ & 6.0 & $1.75 \pm 0.15$ & 8.25 & $1.31 \pm 0.13$ \\
4.0 & $1.41 \pm 0.15$ & 6.25 & $1.73 \pm 0.15$ & 8.5 & $1.40 \pm 0.13$ \\
\hline
\end{tabular}

Table 13.1: Rate [events/kg/day/keV] in the $2-8.5 \mathrm{keV}$ energy range.

with an energy of $10.36 \mathrm{keV}$, which are both also visible in our spectrum. Calculations of the production rate of cosmogenic isotopes show that ${ }^{55} \mathrm{Fe}$ is produced in Ge [242]. The long half-life of ${ }^{55} \mathrm{Fe}$ of $\mathrm{T}_{1 / 2}=2.73$ years allows a remaining activity of this isotope in the detectors. Since the activation stopped when the detectors were moved underground, the time evolution of this counting rate would determine if it is caused by ${ }^{55} \mathrm{Fe}$ isotopes. However, the uncertainties in the production rate and on the time the detectors spent at the surface are likely too great to give a reliable constraint on the total rate expected from the de-excitation of ${ }^{55} \mathrm{Mn}$.

A profile likelihood analysis has been performed in order to search for an excess of event rate above background. The event rate per unit measured energy $(E)$ and per detector $(d)$ including background was written as:

$$
R(E, d)=B(E, d)+A(E, d)
$$

The background $B(E, d)$ is assumed to be of the form

$$
\begin{aligned}
B(E, d) & =\varepsilon(E, d) \cdot\left[C(d)+D(d) E+\frac{H(d)}{E}\right] \\
& +\eta \cdot \varepsilon(E, d) \cdot \frac{\lambda_{6.54}}{\sqrt{2 \pi} \sigma_{6.54}(d)} e^{-\left(\frac{E-6.54}{\sqrt{2} \sigma_{6.54}(d)}\right)^{2}}
\end{aligned}
$$

where $C(d), D(d)$ and $H(d)$ are free parameters determined by the fit routine and $\varepsilon(E, d)$ is the energy-dependent detection efficiency. The Gaussian represents a contribution from ${ }^{55} \mathrm{Fe}$ decays at an energy of $6.54 \mathrm{keV} . A(E, d)$ represents a spectral line at a given energy $E_{0}$. Thus, a Gaussian distribution function multiplied with the efficiency is used:

$$
A(E, d)=\varepsilon(E, d) \cdot \frac{\lambda_{0}}{\sqrt{2 \pi} \sigma_{0}(d)} e^{-\left(\frac{E-E_{0}}{\sqrt{2} \sigma_{0}(d)}\right)^{2}}
$$

Since there is no constraint on the ${ }^{55} \mathrm{Fe}$ contribution to the spectrum no subtraction of a possible background contribution was performed. The reason for introducing the additional 
factor $\eta$ in (13.2) is that, while scanning over the recoil energy and approaching the $6.54 \mathrm{keV}$ background peak, the fit function actually consists of a sum of two Gaussians at the same energy. Thus, it serves as a weight suppressing the importance of the ${ }^{55} \mathrm{Fe}$ rate in the background model $B(E, d)$. The parameter $\eta$ has been varied in steps of 0.1 between 0 and 1 and the most conservative of these limits for each energy has been chosen in this study.

The fit was performed by a maximization of the unbinned log-likelihood function

$$
\log (\mathcal{L})=-R_{T}+\sum_{i, j} \log R\left(E_{i}, d_{j}\right)
$$

where the sum goes over events $(i)$ and detectors $(j)$, with respect to $\lambda_{0}$ and the background parameters. $R_{T}$ denotes the total sum of the event rate $(R)$ over energy and all detectors. No statistically significant excess of the event rate above background is observed. A Bayesian 90\% confidence level (CL) upper limit on the total counting rate $\lambda_{0}$ was set, by integrating the profile likelihood function in the physically allowed region $\left(\lambda_{0}>0\right)$.

The search for an excess rate has been performed in steps of $0.05 \mathrm{keV}$ in an energy range from 2 to $8.5 \mathrm{keV}$, a finer binning of $0.025 \mathrm{keV}$ is used in the energy intervals from 1.4 to $2 \mathrm{keV}$ and 8.5 to $9 \mathrm{keV}$. Although the analysis window remained fixed at 2 to $8.5 \mathrm{keV}$ like in the search for solar axions, the extension to search for an excess rate at energies which lie outside the actual analysis window is possible due to the Gaussian distribution function assumed for the excess rate. However, the further away from the actual window, the contribution from the signal Gaussian in the analysis window decreases and thus the upper limits on a possible signal contribution increase. Figure 13.2 shows the upper limits on the excess rate obtained from the likelihood analysis at each mass/energy step. The color-code gives the deviation of the maximum likelihood estimator $\hat{\lambda}$ from $\lambda=0$ in Gaussian sigmas. These Gaussian sigmas are determined from the value of the profile likelihood function at 0 . From this value the corresponding quantile of a $\chi^{2}$ distribution with one degree of freedom is calculated:

$$
2 \log \left(\frac{\mathcal{L}(0)}{\mathcal{L}(\hat{\lambda})}\right)=-Q_{\gamma} .
$$

The quantile is then translated to the probability of a Gaussian distribution covered by a specified choice of standard deviations.

$$
\int_{0}^{Q_{\gamma}} \chi^{2}(z, 1) d z \equiv P \equiv \frac{1}{\sqrt{2 \pi}} \int_{-\sigma}^{\sigma} e^{-x^{2} / 2} d x
$$

These sigmas are counted negative if $\hat{\lambda}$ lies in the unphysical negative region $(\hat{\lambda}<0)$ and positive if $\hat{\lambda}>0$. Statistically it is possible that the maximization returns a negative value of the total rate as long as this estimator is compatible with zero within its uncertainites. From the color-code it is visible that $\hat{\lambda}$ is consistent with zero with less than a $3 \sigma$ deviation. Hence no excess rate above background could be identified. For negative estimators $\hat{\lambda}<0$ the procedure of integrating the profile likelihood in the physical allowed region $(\lambda>0)$ 


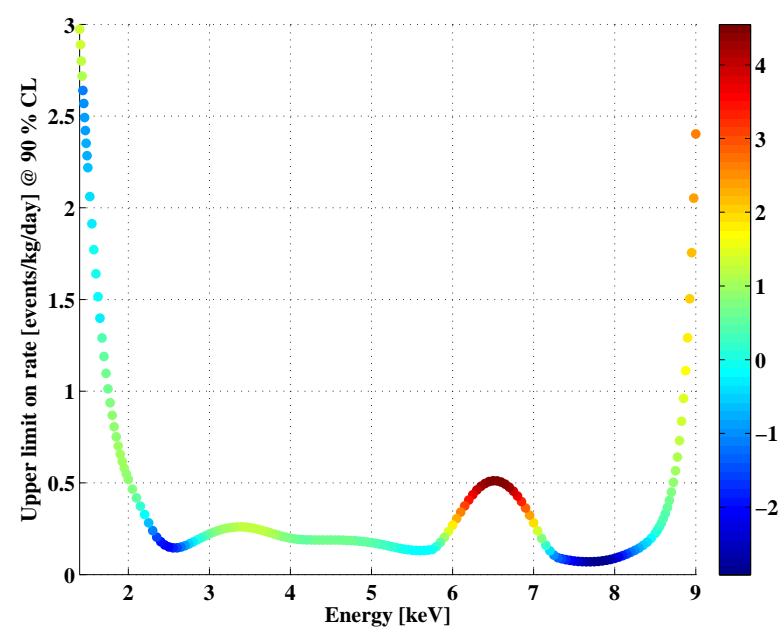

(a)

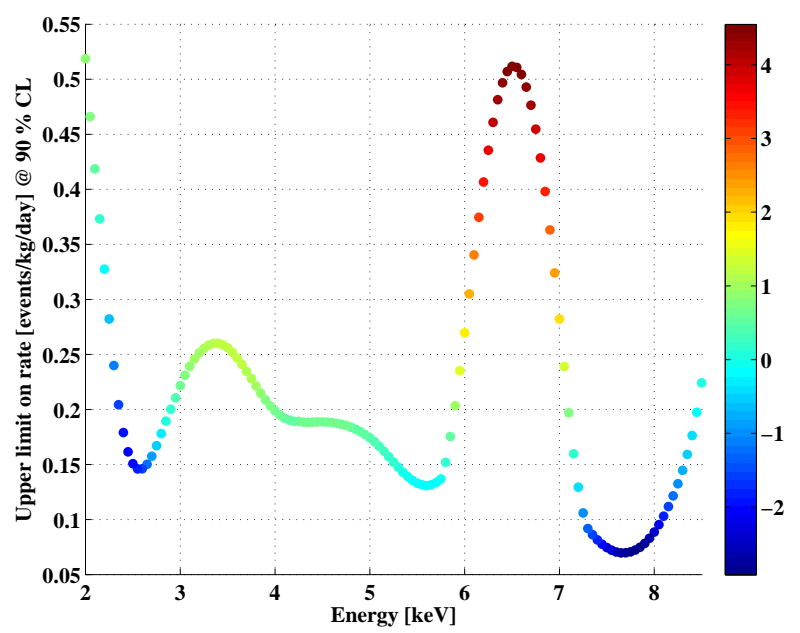

(b)

Figure 13.2: (a) Upper limit on an excess rate above background at a 90\%CL. The color code gives the compatibility of the maximum likelihood estimator for the excess rate with $\lambda=0$ measured in Gaussian sigmas (see text). (b) The same upper limits as in (a) showing an enlargement of the 2 to $8.5 \mathrm{keV}$ region. The presence of a peak around $6.5 \mathrm{keV}$ is clearly visible with a total rate which is incompatible with zero by more than $4 \sigma$.

results in a valid upper limit on the counting rate. An enlargement of the 2 to $8.5 \mathrm{keV}$ range is shown in figure 13.2 (b). Here it is obvious that $\hat{\lambda}$ is inconsistent with zero around $6.5 \mathrm{keV}(\sim 4 \sigma)$ which clearly indicates the presence of a peak in the data. As discussed above there is a candidate for this detected excess in counting rate, but the upper limits shown are set conservatively on the total rate observed without any attempt to subtract a possible background contribution. In figure 13.3 the upper limits on the counting rate are over-plotted to the observed low-energy spectrum for illustration.

\subsection{Pseudoscalar dark matter}

As discussed in Chapter 2 the leading dark matter candidate is the so called WIMP with a mass of $\mathcal{O}(10-1000) \mathrm{GeV}$. However persistent problems in understanding small-scale gravitational clustering properties in cold dark matter simulations and on galactic scales has motivated variants of the standard WIMP dark matter picture, where the dark matter may have masses down to the $\mathrm{keV}$ range. Candidates are for example bosonic super-WIMPs 239. In axion models which exhibit late phase transitions (below the QCD scale) one can find multiple pseudoscalars with axion-like couplings to matter, which are viable dark matter candidates [240]. These particles can have significantly higher masses than in the conventional invisible axion models (see Section 2.2).

There may be more axion-like particle models which can be referred to as pseudoscalar 


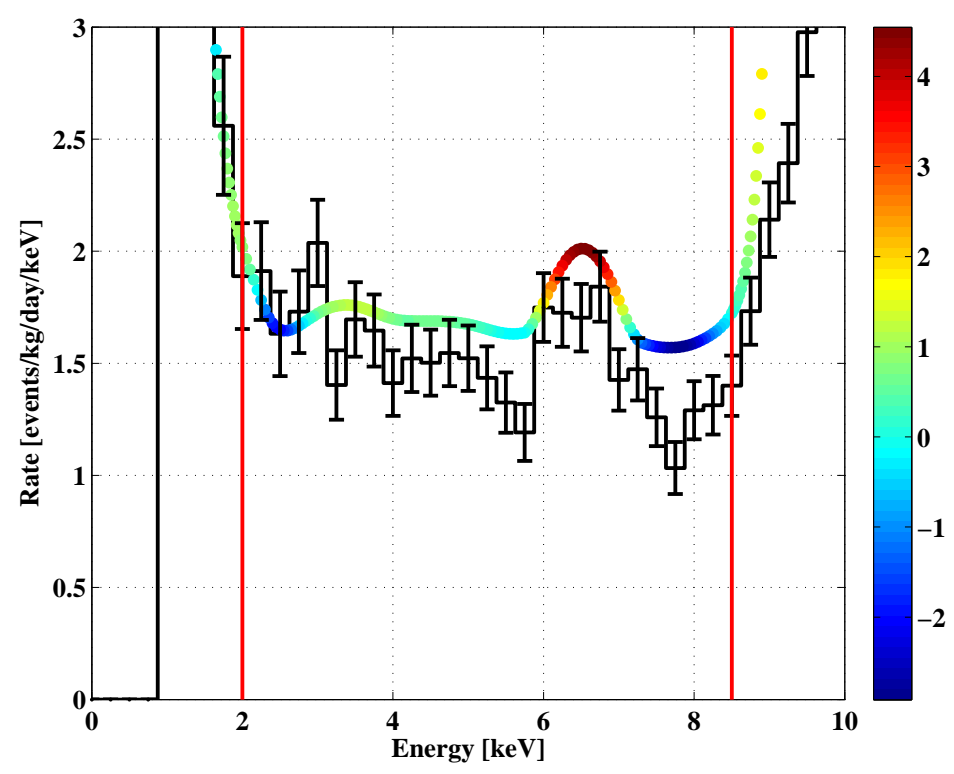

Figure 13.3: For illustration the upper limits from figure 13.2 are plotted together with the observed low-energy electron recoil spectrum from figure 13.1. Note that the upper limits are shifted by an arbitrary value for illustration.

dark matter. It should be noted that these axion-like particles do not include the invisible axion models discussed in section 2.2.3. Axion-like particles are particles having similar phenomenology with ordinary matter as the axion, but which allow values for the coupling constant and for the mass significantly different from those foreseen in the DFSZ and KSVZ models.

These non-relativistic particles would materialize in the CDMS detectors via the "axioelectric effect". If the pseudoscalar particle has a nonzero coupling to electrons $\left(g_{a \bar{e} e}\right)$ it can be absorbed by a bound electron of an atom exciting or ionizing it. The detected sum of the kinetic energy of the photoelectron and of the energy produced by X-rays and Auger electrons in the rearrangement of the atomic shell is given by the particles mass. This effect is phenomenologically similar to the ordinary photoelectric effect.

The absorption cross section can thus be related to the photoelectric cross section. Assuming a local Galactic dark matter density of $0.3 \mathrm{GeV} / \mathrm{c}^{2} / \mathrm{cm}^{3}$, the expected event rate is given by [239]:

$$
R[\text { counts } / \mathrm{kg} / \text { day }]=1.2 \times 10^{43} A^{-1} g_{a \bar{e} e}^{2} m_{a} \sigma_{p e}
$$

where $m_{a}$ is the particles mass in $\mathrm{keV} / \mathrm{c}^{2}, A=73$ for germanium and $\sigma_{p e}$ is the photoelectric cross section in $\mathrm{cm}^{2}$ per atom.

The upper limits on an excess rate above background set in the previous section can be converted into an upper limit on the coupling to electrons shown in figure 13.4. The resulting upper limit curve excludes significant new pseudoscalar parameters space in the mass range between 1.4 and $9 \mathrm{keV} / \mathrm{c}^{2}$, and sets the world leading upper limit on the coupling constant in this mass range. 
The results are inconsistent with the interpretation of the DAMA signature due to a pseudoscalar dark matter particle. It should be noted that the DAMA region should be understood with caution. The allowed region is based on an analysis of the modulation amplitude. However, the non-relativistic speed of particles distributed in the local halo causes the conversion rate to be independent of the particle's velocity. This is caused by the fact that the conversion rate is proportional to $\sigma \times v$ where $\sigma$ is the interaction cross section, which for such inelastic processes is proportional to $1 / v$. Thus the annual modulation of the counting rate is highly suppressed [239]. Therefore the DAMA modulation signal is too large to be interpreted by pseudoscalar dark matter particle interactions in the detectors.

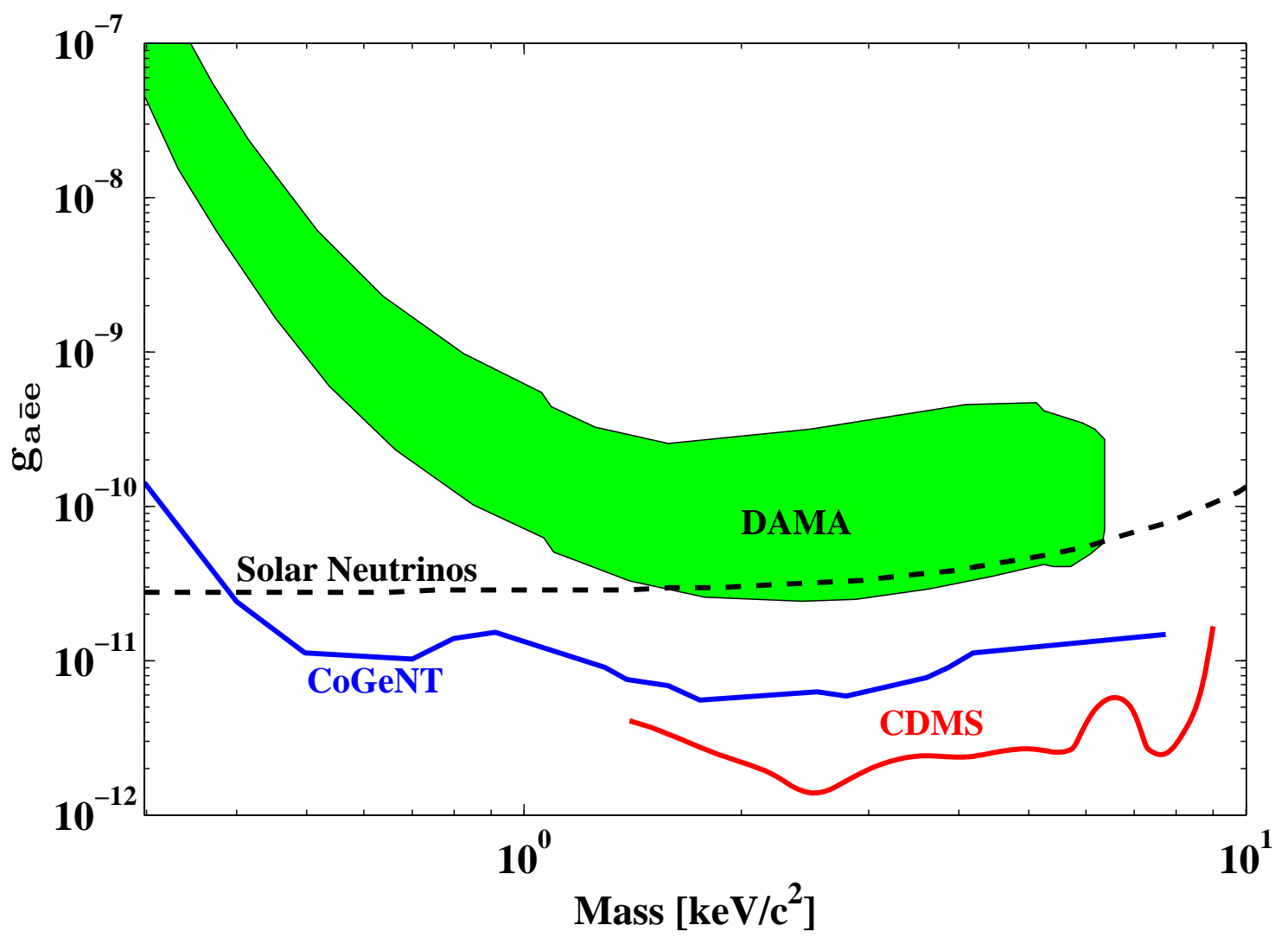

Figure 13.4: The allowed region (green/filled) from a Galactic axion interpretation of the annual modulation signature observed by the DAMA experiment [238] is shown. The $90 \%$ C.L. upper limits on the $g_{a \bar{e} e}$ coupling constant from this work (red/solid) and the CoGeNT experiment (blue/solid) [110] completely exclude the DAMA allowed region. The indirect constraints from astrophysical bounds (black/dashed) are also shown [241. 


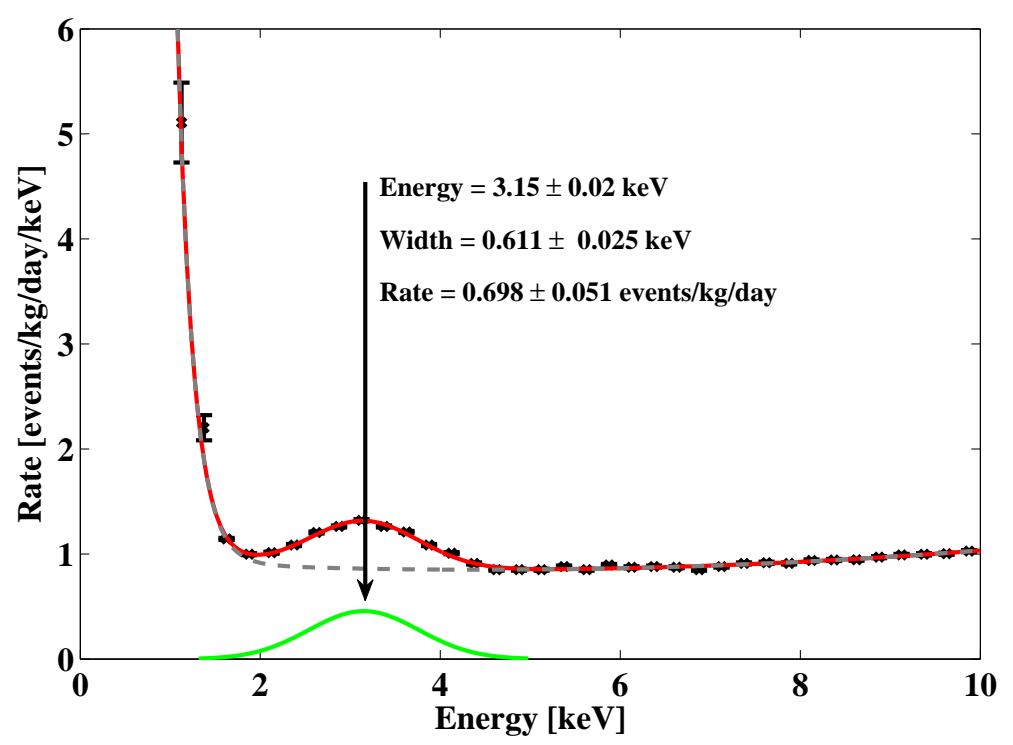

Figure 13.5: Fit (red) to the published DAMA/LIBRA low-energy spectrum [118, consisting of a background model (gray/dashed) and a Gaussian distribution function (green). The parameters of the Gaussian are given in the figure.

\subsection{Electromagnetic conversion dark matter}

The annual modulation signature observed by DAMA [118 may be interpreted as the conversion of a dark matter particle into electromagnetic energy in the detector. In this case it should be possible to observe the corresponding signal in the electron-recoil spectrum of CDMS. The upper limits on an excess rate presented in this chapter should thus help to identify or constrain possible models which can explain the annual modulation signature observed by DAMA. The total counting rate above background observed by DAMA/LIBRA in the claimed signal region has been obtained from a fit to their spectrum consisting of a Gaussian and a background model shown in figure 13.5 giving a total rate of $0.698 \pm 0.051$ events $/ \mathrm{kg} /$ day. A direct comparison between the $90 \%$ CL upper limits from this analysis (black/solid) and the total rate above background observed by DAMA (black data point with $2 \sigma$ error bars in the figure) is shown in figure13.6. At the energy of the DAMA peak $(3.15 \mathrm{keV})$ the observed rate is inconsistent with the upper limit on the rate in CDMS of 0.246 events $/ \mathrm{kg} /$ day. Though the peak of figure 13.5 may contain a contribution from the decay of ${ }^{40} \mathrm{~K}$ and the subsequent de-excitation of ${ }^{40} \mathrm{Ar}$ resulting in a spectral line at $3.2 \mathrm{keV}$, no information is supplied on the actual rate of such a background [243]. Thus, no subtraction is performed, which would reduce the difference between the upper limit from CDMS and the excess rate in DAMA.

Currently there are two studies on the ${ }^{40} \mathrm{~K}$ background contribution to the total rate in the DAMA spectrum. The DAMA collaboration performed an analysis, showing that the ${ }^{40} \mathrm{~K}$ rate is a dominant component 244]. This leads to high ratios of the modulated 
to unmodulated signal counting rate which are inconsistent with the expected ratio in a standard halo model. On the other hand, an independent Monte Carlo based analysis of the DAMA spectrum claims that the ${ }^{40} \mathrm{~K}$ contamination of the crystals [243] are too small, to account for the excess rate observed in the unmodulated spectrum [245]. In order to account for this background contribution these discrepancies need to be resolved.

The event rates in the CDMS and DAMA detection media may differ depending on the coupling of the dark matter particle. Thus, the upper limits in Ge have to be scaled to the expected rate in $\mathrm{NaI}$ in order to perform a comparison in a particular model. For an electromagnetic conversion of a dark matter particle, the particle velocity is essentially irrelevant (in contrast to the calculation for nuclear recoils, where the energy threshold provides a minimum velocity for the phase space integral). Thus, the annual modulation signature is only caused by a change in the particle flux over the course of the year. The total counting rates per unit mass of such a conversion in the case of a Ge and a NaI target are related by the following condition:

$$
\frac{R_{N a I}}{R_{G e}}=\frac{A_{G e}}{A_{I}+A_{N a}} \cdot \frac{\sigma_{I}+\sigma_{N a}}{\sigma_{G e}}
$$

where $A_{i}$ is the atomic mass of the nuclei, and $\sigma_{i}$ is the total cross section per atom of the interaction. The detection efficiencies in both materials should be very close to $100 \%$ at these low energies; thus, effects of a material and detector geometry dependent detection efficiency are neglected in the following.

The total cross section will depend on the coupling of the dark matter particle to the detection media. For an electromagnetic conversion a $\mathrm{Z}^{2}$ (where $\mathrm{Z}$ is the atomic number) scaling of the cross section is natural and is thus considered in the comparison of the rate limits in Ge from this analysis with the rate observed by DAMA. Another scaling can be trivially considered. This is a more general model than the one considered in the search for pseudoscalar dark matter discussed in the previous section. The scaled rate limits in $\mathrm{NaI}$ at a 90\% CL are given in Fig.13.6 (blue/dashed line). The total counting rate observed by DAMA/LIBRA remains greater than the upper limit at $3.15 \mathrm{keV}$.

Under standard halo assumptions a conservative upper limit on the modulation amplitude is $\pm 6 \%$ if the modulation is caused by a change in the particle flux only [76]. Note, that if the conversion cross section is inversely proportional to the dark matter particle velocity (as inelastic cross sections tend to be [239]) the annual modulation amplitude is highly suppressed. The insert in figure 13.6 compares the unscaled upper limit (black/solid) and the $\mathrm{Z}^{2}$ scaled upper limit in $\mathrm{NaI}$ (blue/dashed) on the modulation amplitude with the $2 \sigma$ regions of the annual modulation amplitude observed by DAMA (NaI+LIBRA) in the 2 - $4 \mathrm{keV}$ (red/filled) and $2-6 \mathrm{keV}$ (green/hatched) energy range [118]. The upper limits on the modulation amplitudes are a factor of $\sim 2$ less than observed by DAMA.

It has to be mentioned that the actual scaling between $\mathrm{Ge}$ and $\mathrm{NaI}$ has to be provided by a specific model, but the analysis of the low-energy electron-recoil spectrum helps to identify or constrain possible models which can explain the annual modulation signature observed by DAMA. 


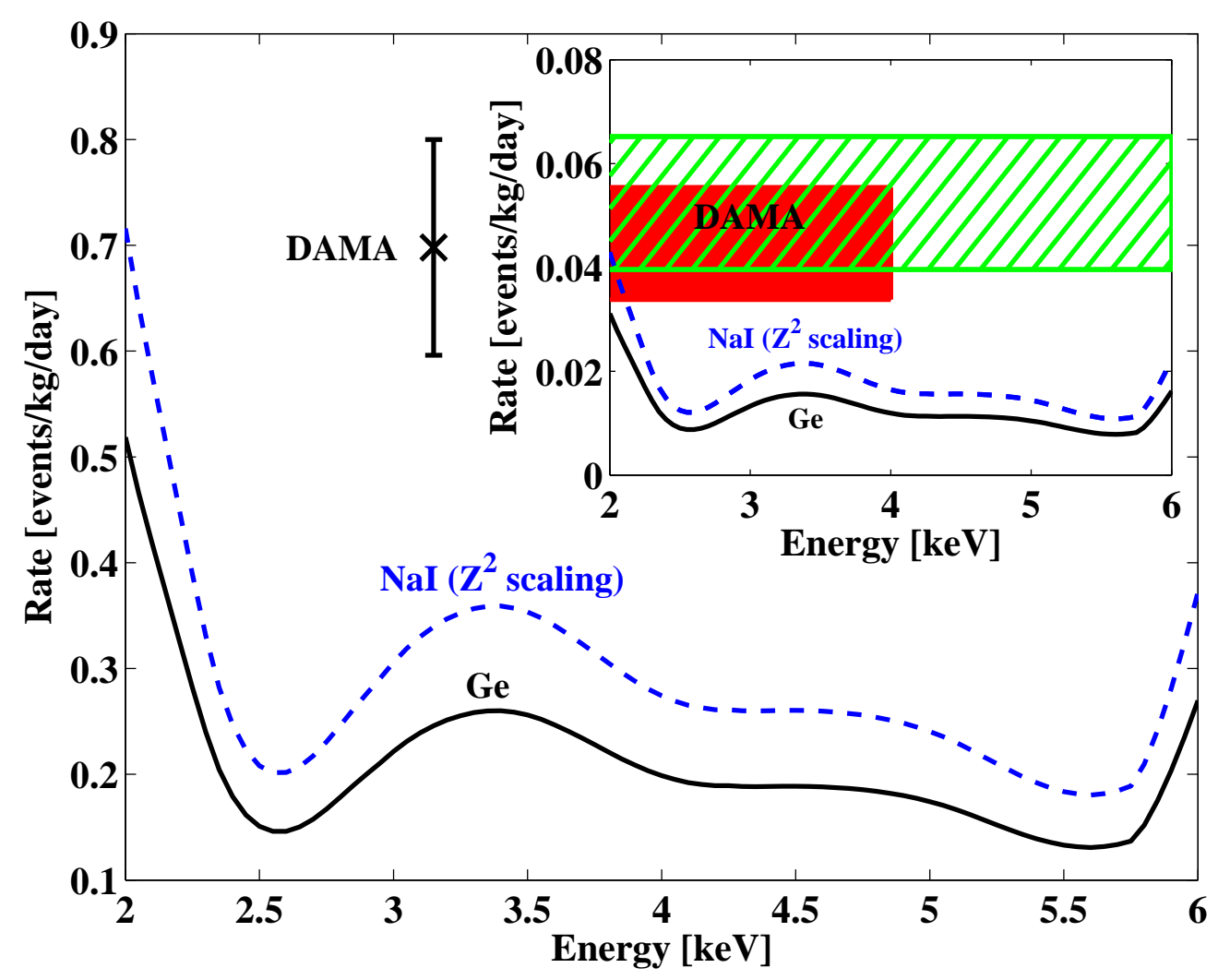

Figure 13.6: 90\% CL upper limit on the total counting rate in Ge from this analysis (black/solid). The corresponding upper limit on the total counting rate in NaI under the assumption of a $\mathrm{Z}^{2}$ scaling of the conversion cross section (see text) is also shown (blue/dashed). The black data point with $2 \sigma$ error bars gives the total counting rate of the $3.15 \mathrm{keV}$ peak of DAMA/LIBRA derived from a fit to their spectrum (see Fig.(13.5). The insert compares the upper limit on the modulation amplitude assumed to be $6 \%$ of the unscaled upper limit (black/solid) and the $\mathrm{Z}^{2}$ scaled upper limit in $\mathrm{NaI}$ (blue/dashed) with the $2 \sigma$ regions of the annual modulation amplitude observed by DAMA (NaI+LIBRA) in the $2-4 \mathrm{keV}$ (red/filled) and $2-6 \mathrm{keV}$ (green/hatched) energy range. 


\subsection{Annual modulation}

In a more general framework the CDMS low-energy electron recoil data can be directly investigated for the hint of a modulation of the detected rate. In a first order approximation the detected rate in CDMS with a possible contribution from a dark matter particle distributed in the local halo can be written as:

$$
R(t)=R_{0}+R_{\text {mod }} \cdot \cos \left(\frac{\left(t-t_{0}\right)}{365} \cdot 2 \pi\right)
$$

where the total rate is parametrized in a constant counting rate $R_{0}(E)$ which may also include a time independent contribution from backgrounds and a modulated counting rate given by an amplitude $R_{\text {mod }}$ and a specified phase $t_{0}$. The modulated counting rate should only be caused by a possible dark matter signal. The phase $t_{0}$ and time $t$ are given here by specified days in a year.

To search for such a periodicity in the data, the observed counts in the $2-4 \mathrm{keV}$ range are binned in time bins as it is shown in figure 13.7 for the R123/4 data (a bin size of two weeks was chosen). The number of counts observed in a given time interval $i$ and energy interval $\left(E_{1}, E_{2}\right)$ is given by:

$$
\nu_{i}\left(t_{i} ; R_{0}, R_{\text {mod }}, t_{0}\right)=M D_{i} \int_{E_{1}}^{E_{2}} \epsilon(E) \frac{d R_{0}}{d E}+\frac{d R_{\text {mod }}}{d E} \cdot \cos \left(\frac{\left(t_{i}-t_{0}\right)}{365} \cdot 2 \pi\right) d E
$$

with the energy dependent detection efficiency $\epsilon(E)$ and the exposure $M D_{i}$ of the time interval. For simplicity the value of the cosine function is assumed to be constant over the time bin. This approximation is good if the chosen time bins are small enough, meaning that the velocity of the earth does not largely change over the time bin. Otherwise a second integration over the time bin has to be introduced.

To find the best estimators for $R_{0}, R_{\bmod }$ and the phase $t_{0}$ a binned likelihood maximization can be performed:

$$
\log \left(\mathcal{L}\left(R_{0}, R_{\text {mod }}, t_{0}\right)\right)=-\nu_{\text {tot }}\left(R_{0}, R_{\text {mod }}, t_{0}\right)+\sum_{i}^{N} n_{i} \log \left(\nu_{i}\left(t_{i} ; R_{0}, R_{\text {mod }}, t_{0}\right)\right)
$$

where $n_{i}$ are the actual number of counts observed in the $N$ time bins and $\nu_{\text {tot }}\left(R_{0}, R_{\text {mod }}, t_{0}\right)$ is the total number of expected counts summed over all time bins. It is interesting to maximize the Likelihood function with $R_{\text {mod }}$ set to zero to see how well the data is described by a constant counting rate. The result of this maximization gives $\hat{R}_{0}=1.77$ counts $/ \mathrm{kg} / \mathrm{day} / \mathrm{keV}$ which is shown as the (red/dashed) horizontal line in figure 13.7. From simply visually comparing this result with the observed counts there seems to be agreement that the data could be described by a constant and no modulation is seen in the data. On the other hand it would be interesting to search for a hint of a modulation of the data for an arbitrary phase $t_{0}$ and determine the acceptance regions of $t_{0}$ and $R_{\text {mod }}$ which are compatible with the data. In order to obtain the acceptance regions at a given confidence level a Feldman-Cousins approach is used [203. 


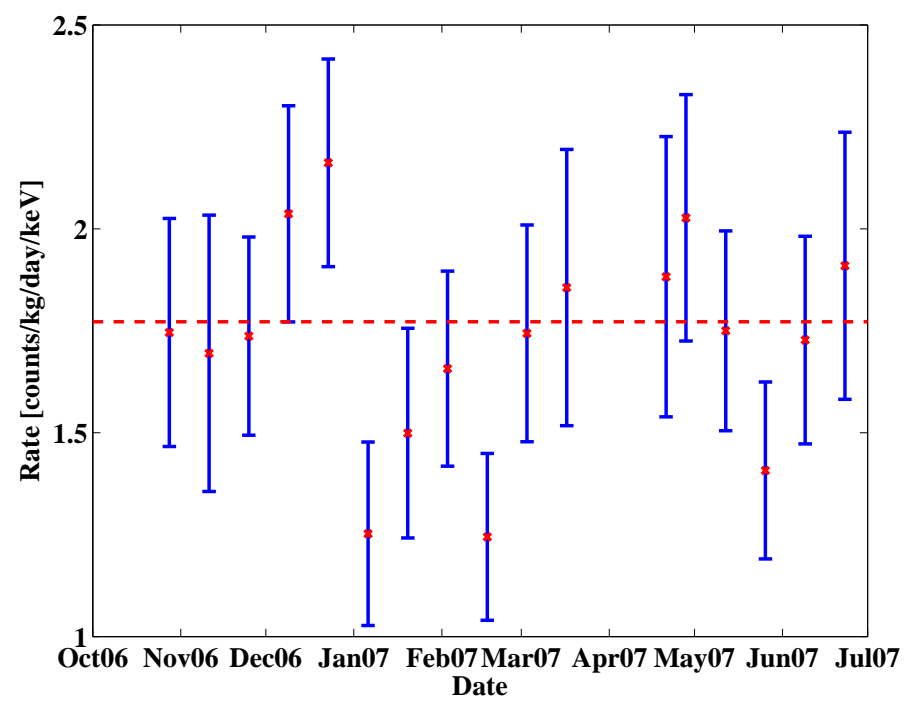

Figure 13.7: Count rate as a function of date in 2 week bins for R123/4 data in the $2-4 \mathrm{keV}$ range. The horizontal (red/dashed) line gives the value of the constant counting rate from a maximum likelihood fit to the data under the assumption that no modulation term is present in the data. This fit seems to describe the data well, so that there does not seem to be a hint of a modulation.

For each parameter point $\left(R_{0}, R_{\text {mod }}, t_{0}\right)$ in the three dimensional parameter space a large number of Monte Carlo experiments is generated. This is done by calculating the expected counts for each time bin $i$ and add a Poisson fluctuation to the number of counts. For each experiment the likelihood ratio:

$$
\lambda=\frac{\mathcal{L}\left(R_{0}, R_{\text {mod }}, t_{0}\right)}{\mathcal{L}\left(\hat{R}_{0}, \hat{R}_{\text {mod }}, \hat{t}_{0}\right)}
$$

is calculated, where $\left(\hat{R}_{0}, \hat{R}_{\text {mod }}, \hat{t}_{0}\right)$ are the maximum likelihood parameters for the considered MC experiment. For the actual data a second likelihood ratio is calculated

$$
\lambda_{\text {Data }}=\frac{\mathcal{L}\left(R_{0}, R_{\text {mod }}, t_{0}\right)}{\mathcal{L}\left(\hat{R}_{0}, \hat{R}_{\text {mod }}, \hat{t}_{0}\right)}
$$

where $\left(\hat{R}_{0}, \hat{R}_{\text {mod }}, \hat{t}_{0}\right)$ are the maximum likelihood parameters for the data. A certain parameter point is accepted by the data at a given confidence level $\alpha$, if (1- $\alpha)$ percent of the simulated likelihood ratios are smaller than the experimental likelihood ratio at this point. For the determination of the acceptance regions $10000 \mathrm{MC}$ experiments have been evaluated at each considered parameter point.

The slice through the three dimensional parameter space, showing the acceptance regions for a fixed constant counting rate $R_{0}$ (fixed at the best fit value of the data $R_{0}=\hat{R}_{0}$ ) is shown in figure13.8 (a). The color code in the figure shows the confidence level at which the parameter space is accepted. Contours for the 68\% CL and 90\% CL are also shown in 


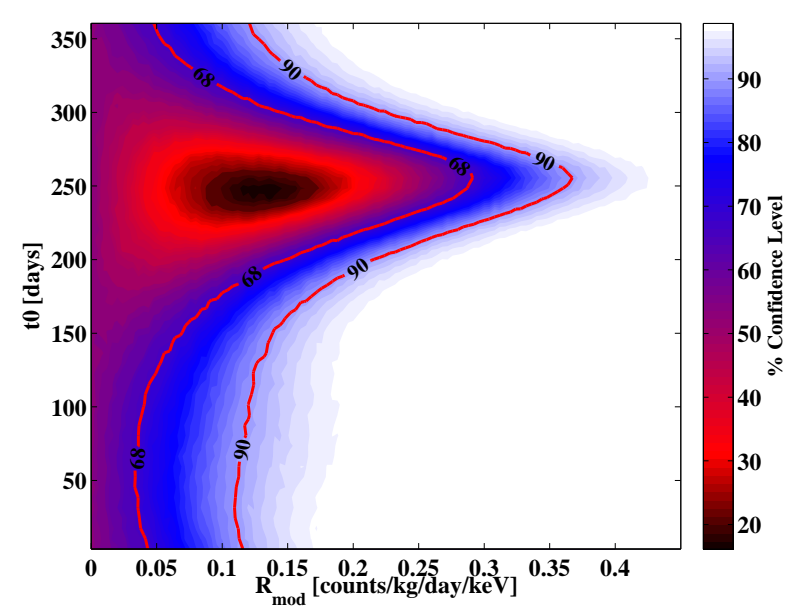

(a)

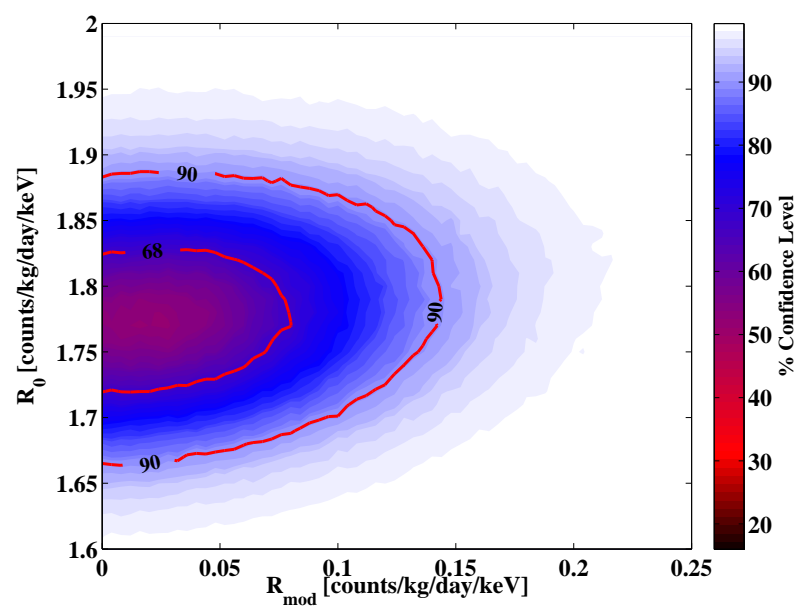

(b)

Figure 13.8: Slices through the three dimensional acceptance region space determined from a Feldman Cousins ordering principle. (a) Slice for a fixed value of $R_{0}=\hat{R}_{0}$ showing a best fit region at $t_{0} \approx 250$ days which is incompatible with the phase in a SHM. The modulation term is consistent with zero for any phase, thus no hint of a modulation is observed in the current data set. The sensitivity to a modulation term in a slice fixed at the phase expected in the SHM $t_{0}=152.5$ is shown in (b). In both figures the color code gives the confidence level of the acceptance region and contours for a $68 \%$ CL and $90 \%$ CL are shown.

the figure. The best fit point lies at a phase of $t_{0} \approx 250$ days corresponding to the 7 th of September. This phase significantly differs from the expected phase from a standard halo model which lies on May 30th. In any case the modulation amplitude $R_{\text {mod }}$ is consistent with zero for all phases, thus no modulation of the data is observed.

Nevertheless we can compare the sensitivity of CDMS on a possible modulation amplitude in the SHM framework. This is what is shown in figure 13.8 (b) which shows a second slice of the three dimensional parameter space now for a fixed phase of $t_{0}=152.5$ days which is expected in the SHM. From this figure a modulation amplitude of $\gtrsim 0.15$ counts $/ \mathrm{kg} /$ day $/ \mathrm{keV}$ can be excluded at a $90 \% \mathrm{CL}$. This figure shows that with the current data the CDMS experiment is not really sensitive to a modulation term in the expected rate, especially if one aims to probe the modulation amplitude observed by DAMA/LIBRA of $0.0223 \pm 0.0027$ counts $/ \mathrm{kg} /$ day $/ \mathrm{keV}$ in the $2-4 \mathrm{keV}$ bin. More exposure per time interval would be needed to gain a better sensitivity on a possible modulation which should be achievable with a larger detector mass. In addition the longer runtime of the experiment during R125-128 should in combination with R123/124 increase the sensitivity to a modulation term since this data covers approximately 2.5 years. 


\section{Chapter 14}

\section{Conclusions}

The identification of the nature of dark matter is yet an unanswered question of modern astrophysics. The detection of dark matter particles either by their direct interaction with terrestrial detectors or by their annihilation products in neutrino telescopes depends on their local phase scape density in the vicinity of the Earth. The formation of dark matter halos in galaxies evolution can to date only been modeled by large N-body simulations. If in these N-body simulations the baryonic matter is included, new macroscopic dark matter structures can be formed, apart from the usual isothermal halo. A prominent example of such a structure is the recently predicted formation of a dark matter disc. Particles in this disc are much slower with respect to the Earth and Sun than particles in the dark halo. As has been shown in this thesis, the lower velocities of such particles significantly alter the expected recoil spectrum in direct detection experiments. A very predictive signature of the dark disc is the shift of the phase of the annual modulation signature expected to be observed at high statistic records of dark matter interactions. This shift would not only verify the existence of the dark disc, but would also allow to uniquely determine the dark matter particle's mass from the phase of the annual modulation.

The higher phase-space density of the dark disc at low velocities significantly increases the gravitational capture rate of dark matter particles in the Sun and Earth. An increased capture rate results in a higher density of particles increasing the self-annihilation rate. Particles from these annihilation processes emerging from the interior of the Sun and Earth can be detected in terrestrial detectors, such as neutrino telescopes. Given the median values of the dark disc, it has been shown in this thesis, that the expected muon fluxes in neutrino telescopes are significantly increased for the Sun and the Earth. These increased fluxes set the search for dark matter with neutrino telescopes at a comparable sensitivity as current direct detection experiments, providing a complementary search for dark matter particles. It has to be noted, that the actual properties of the dark disc are subject to large uncertainties since the complete merger history of the Milky Way is unknown. However, the properties of the dark disc can be derived from future large surveys of the Milky Way. Even if the properties of the dark disc turn out to be different than the median values assumed in this thesis, the expected muon fluxes in neutrino telescopes originating in the Sun will be dominated by the dark disc rather than the dark halo. 
A major requirement for direct detection experiment is to reduce background interactions as much as possible, since the sensitivity and the scientific reach of the experiment is determined by the expected background in the signal region. It is thus not only crucial to shield the detectors against any environmental backgrounds but also to identify the sources of background within the shielding. A global fit of gamma spectra obtained from Monte Carlo simulations of the material's radioactive contamination to the observed gamma spectrum has been performed to derive the contamination levels of ${ }^{238} \mathrm{U}$ and ${ }^{232} \mathrm{Th}$. These two isotopes are of special interest since their decays are sources of neutrons which would produce an irreducible background in the detectors. The derived contamination levels have thus been used to predict the rate of radiogenic neutron interactions in the detectors. For the detectors used in the CDMS experiment the dominating background are surface-events. A primary source of surface-events is ${ }^{210} \mathrm{~Pb}$ surface contamination of the crystal substrates. However, the surface-event rate from the contamination can not fully account for the total observed surface-event rate. It has been shown in this thesis that the non- ${ }^{210} \mathrm{~Pb}$ related surface event rate can be explained by ambient gamma induced surface events. In order to decrease the surface-event background not only the ${ }^{210} \mathrm{~Pb}$ contamination but also the ambient gamma induced rate has to be decreased, since both contribute on a comparable level. The ${ }^{210} \mathrm{~Pb}$ contamination can be reduced by reducing the exposure of the detectors to air as much as possible, and there are indications that a reduction in this rate has been achieved for the later Towers. The ambient gamma induced rate can only be reduced by a reduction of the overall ambient gamma flux, achievable by a careful selection of the materials used in the experimental setup. The simulation of the ambient gamma spectrum shows that the dominating contribution is the copper of the icebox cans, and if the icebox cans were made out of the same copper as the Tower cold hardware, the ambient gamma flux and thus the ambient gamma induced surface-event rate could be reduced by a factor of $\sim 3$.

The primary analysis of the CDMS experiment is dedicated to the search for nuclear recoils from WIMP interactions in the detectors. In the blind analysis of the Run 125-128 data an expected background of $\eta=0.82_{-0.10}^{+0.12}$ (stat.) ${ }_{-0.19}^{+0.20}$ (syst.) surface-events leaking into the signal region, and less than 0.1 neutron induced nuclear-recoils $(0.031-0.05$ events from radiogenic neutrons and $0.04_{-0.03}^{+0.04}$ events from cosmogenic neutrons) has been achieved. The detection of two candidate events in the signal region cannot be interpreted as a statistically significant detection of WIMP interactions, since there is a $23 \%$ probability to observe two events in the signal region given the expected backgrounds. Hence an upper limit on the WIMP-nucleon interaction cross section could be set by this analysis. Taking these two candidate events into account in the calculation of the upper limit the sensitivity of the Run 125-128 data is comparable to the combined sensitivity of all previous data taken at Soudan. Combining this result with all previous CDMS-II data gives an 90\% confidence level upper limit on the WIMP-nucleon spin-independent cross section of $3.8 \times 10^{-44} \mathrm{~cm}^{2}$ for a WIMP mass of $70 \mathrm{GeV} / \mathrm{c}^{2}$. The combined upper limits place world-leading upper limits on the WIMP-nucleon spin-independent cross section for WIMP masses above $\sim 44 \mathrm{GeV} / \mathrm{c}^{2}$. In addition, this result constraints the allowed parameters of the inelastic dark matter model which can resolve the discrepancy between the annual modulation signature observed by 
DAMA and the null observation of other direct detection experiments to a narrow region of WIMP masses around $100 \mathrm{GeV} / \mathrm{c}^{2}$ and mass-splittings of $80-140 \mathrm{keV}$.

The CDMS-II experiment has demonstrated a great capability of controlling backgrounds which is essential for the experiment's performance in future, longer data runs. The current analysis marks the end of the 5-Tower setup with $1 \mathrm{~cm}$ thick detectors at the Soudan site. To increase the sensitivity on the spin-independent cross section beyond the $10^{-44} \mathrm{~cm}^{2}$ benchmark, new detectors have been developed by the collaboration. These detectors will be used in the next stage of the CDMS experiment, the SuperCDMS project. As part of the SuperCDMS project, four Towers of the new detectors will be installed at the Soudan site alongside the best of the existing CDMS-II detectors. This stage will have a total of $\sim 15 \mathrm{~kg}$ germanium target mass, with an expected sensitivity reach on the spinindependent WIMP-nucleon cross-section of $\sim 5 \times 10^{-45} \mathrm{~cm}^{2}$ within a runtime of $\sim 2$ years. The first SuperCDMS Tower has been successfully deployed at the Soudan site to study the rejection capability of the new detectors and characterize their performance. Based on the experiment at Soudan and on continued research and development, a larger experiment of $100 \mathrm{~kg}$ target mass or more is expected to be proposed, to increase the sensitivity down to cross sections of $\sim 3 \times 10^{-46} \mathrm{~cm}^{2}$.

The data recorded with the CDMS experiment can not only be analyzed in terms of a dark matter signal from WIMP interactions but also in search for other weakly interacting particles. One such particle is the axion, which could solve the strong CP problem arising in QCD. The strong correlation between the position of the Sun on the sky with respect to the crystal lattice vectors and the expected conversion rate provides a "fingerprint" of solar axion conversions in the crystal. In the search for solar axion conversions in crystal based experiments a precise knowledge of the crystal lattice orientation is thus mandatory. The knowledge of the detectors alignment has been achieved for the first time in the CDMS-II setup reducing any systematic uncertainty on the expected conversions rate. In contrast to the search for nuclear-recoils, in which in general a threshold of $10 \mathrm{keV}$ in phonon energy is set to reduce surface-event leakage in the nuclear-recoil band, a detection threshold of $2 \mathrm{keV}$ in ionization energy for electron-recoils has been achieved in this analysis. Such a low threshold is a major requirement in the search for solar axions, since the flux of solar axions at the Earth peaks around $4 \mathrm{keV}$. Given the precise knowledge of the crystal orientations the performed unbinned likelihood analysis resulted in a null observation of solar axion conversions. The upper limit on the photon axion coupling constant of

$$
g_{a \gamma \gamma} \leq 2.4 \times 10^{-9} \mathrm{GeV}^{-1} @ 95 \% \mathrm{CL}
$$

for axion masses less than $0.1 \mathrm{keV} / \mathrm{c}^{2}$ lies below the current best limit set by germanium detectors. However, the electron-recoil background is still too high to achieve sensitivities below the benchmark of $g_{a \gamma \gamma} \leq 1 \times 10^{-9} \mathrm{GeV}^{-1}$. To achieve this sensitivity and make the search for solar axions with crystal based experiments competitive to helioscopes, gamma background levels of $\sim 0.1$ counts $/ \mathrm{kg} /$ day $/ \mathrm{keV}$ have to be achieved. This could become possible with a 100-kg SuperCDMS style experiment or other large germanium crystal array experiments like the GERDA and MAJORANA experiment, if in these two experiments the alignment of the crystals is known and low enough thresholds can be reached. 
In general a signal from dark matter interactions could be detected as an electron-recoil. These signatures would be rejected in the standard WIMP search analysis, searching for nuclear-recoils and rejecting any electromagnetic interaction as background. The analysis of the low-energy electron-recoil spectrum of the CDMS-II experiment did not show any statistically significant excess in detected rate above the background model in an energy range from $2-8.5 \mathrm{keV}$. The upper limits on the counting rate could thus be used to set constraints on the coupling of pseudoscalar dark matter particles. This analysis sets the world-leading experimental upper limits on the axio-electric coupling constant in a mass range from $1.4-9 \mathrm{keV} / \mathrm{c}^{2}$ and excludes the interpretation of the DAMA annual modulation signature in terms of axion interactions for axion masses above $1.4 \mathrm{keV} / \mathrm{c}^{2}$.

Analyses of the low-energy electron-recoil spectrum are of special interest in light of the DAMA claim which is inconsistent with null observations of other experiments, if the modulation signature is interpreted as the detection of nuclear-recoils. In the framework of an electromagnetic conversion of the dark matter particle, the upper limits on the counting rate above background are thus compared to the total rate above background observed by DAMA, which has been determined from a fit to their spectrum. The $90 \%$ confidence level upper limit of 0.246 events $/ \mathrm{kg} /$ day at $3.15 \mathrm{keV}$ is lower than the total rate above background observed by DAMA. It has to be noted that the direct comparison between the upper limits obtained from the germanium crystals used in CDMS and the rate observed in the $\mathrm{NaI}(\mathrm{Tl})$ crystals operated by DAMA may have systematic uncertainties, based on the scaling of the cross section between the two materials. In absence of a specific particle model to provide the scaling in cross section a simple scaling with the atomic number $\left(Z^{2}\right.$ scaling) has been assumed. With this assumption the observed rate in DAMA remains higher than the upper limit from CDMS. Different scalings can easily be considered if a specific theory motivates the existence of such a particle and provides the actual scaling. In addition, a large systematic uncertainty is introduced in this comparison due to the presence of a ${ }^{40} \mathrm{~K}$ background in the DAMA crystals which results in X-rays detected at $3.2 \mathrm{keV}$. So far the magnitude of this background is unknown, but it certainly decreases the difference between the observed rate in DAMA and the upper limit set by CDMS. The modulated rate however should not be biased by a contribution of the ${ }^{40} \mathrm{~K}$ background. In the standard halo model a conservative assumption is, that the modulated rate is $6 \%$ of the unmodulated rate. The upper limits on the total rate above background can thus be scaled to an upper limit on the modulation amplitude. These upper limits are a factor $\sim 2$ less than observed by DAMA, constraining some possible interpretations of the observed modulation signature.

The direct search for an modulated counting rate contribution in the CDMS low-energy spectrum sets upper limits on the modulation amplitude which are not yet constraining enough to probe the DAMA claim. This analysis has been performed with the Run 123/4 data, adding the Run125-128 data in this analysis could significantly increases the sensitivity to a modulation term since the combined data covers approximately 2.5 years of data taking. 


\section{Acknowledgment}

I would like to thank Prof. Dr. Laura Baudis, my dissertation advisor, who has offered me the opportunity to work on a leading front in astroparticle physics. Her insights on cosmology and particle physics have been an invaluable guidance throughout the years of my PhD studies. She always treated me as a valued colleague, and I appreciate the opportunities she has given me to set my own research goals.

I would like to express my gratitude to the whole CDMS Collaboration. It has been a great pleasure to work with you. It would be impossible to give proper thanks to everyone whose support and instructions I have enjoyed. I would especially like to thank Prof. Dr. Bernard Sadoulet, Prof. Dr. Sunil Golwala, Prof. Dr. Steven Yellin, Prof. Dr. David O. Caldwell, Dr. Jeffrey Filipini and Dr. Jonghee Yoo, for useful discussions on statistics and analysis, as well as problems in physics, leading to new analysis ideas.

Many thanks to Prof. Dr. George Lake and Dr. Justin Read for useful discussions about the formation and properties of the Milky Way's dark disc and the provided input, resulting in collaborative publications. I also enjoyed the work with Dr. Annika. H. G. Peter in predicting the capture rates from the dark disc in the Sun and Earth, thanks for the great work you have done.

I want to thank Dr. Teresa Marrodan Undagoitia and Sebastian Arrenberg for reading the manuscript of this thesis and providing useful suggestions for improvement.

It has been a pleasure to be a member of the Zürich group. I would like to give particular thanks to Sebastian Arrenberg with whom I spend hours on discussions and working on the analysis. Thanks to all current and former members of the Zürich group: Dr. Alfredo Davide Ferella, Dr. Aaron Manalaysay, Dr. Teresa Marrodan Undagoitia, Dr. Roberto Santorelli, Dr. Marc Schumann, Dr. Eirini Tziaferi, Sebastian Arrenberg, Ali Askin, Annika Behrens, Francis Froborg, Marijke Haffke, Alexander Kish and Michal Tarka.

My lasting gratitude to my family and friends: without your support and encouragement I would not have made it. Thanks for all the good times hanging around just shooting the breeze. 


\section{Bibliography}

[1] T. Bruch, J. Read, L. Baudis, G. Lake, ApJ 696, 920 (2009).

[2] T. Bruch, A. H. G. Peter, J. Read, L. Baudis, G. Lake, Phys. Lett. B 674, 250 (2009).

[3] CDMS Collaboration, in preparation.

[4] Z. Ahmed et al., Phys. Rev. Lett. 102, 011301 (2009).

[5] Z. Ahmed et al., Science DOI: 10.1126/science.1186112 (2010).

[6] Z. Ahmed et al., Phys. Rev. Lett. 103, 141802 (2009).

[7] Z. Ahmed et al.Phys. Rev. D 81, 042002 (2010).

[8] M. Milgrom, Astrophys. J. 270, 365 (1983).

[9] J. D. Bekenstein, Phys. Rev. D 70, 083509 (2004).

[10] E.W. Kolb and M. Turner, The Early Universe, Westview Press (1990).

[11] T. Padmanabhan, Structure Formation in the Universe, Cambridge University Press (1993).

[12] S. Dodleson, Modern Cosmology, Academic Press (2003).

[13] E. Hubble, Proceedings of the National Academy of Science 15, 3 (1929).

[14] M. Kowalski et al., Astrophys. J. 686(2), 749 (2008).

[15] E. Komatsu et al., Astrophys. J. Suppl. 180, 330 (2009).

[16] P. Coles and F. Lucchin, Cosmology: The Origin and evolution of Cosmic Structure, John Wiley \& Sons Ltd. (2002).

[17] J. Bechtold, arXiv:astro-ph/0112521 (2001).

[18] C. Amsler et al., Phys. Lett. B667, 1 (2008).

[19] M. R. Nolta et al., Astrophys. J. Suppl. 180, 296 (2009). 
[20] A. R. Liddle and D. H. Lyth, Cosmological inflation and large-scale structure, Cambridge University Press (2000).

[21] F. Zwicky, Helv. Phys. Acta 6, 110 (1933).

[22] F. Zwicky, Astrophys. J. 86, 217 (1937).

[23] M.C. Weisskopf et al., Exper. Astron. 16, 1 (2003).

[24] F. Jansen et al., Astron. and Astrophys. 365, L1 (2001).

[25] P. Schneider, J. Ehlers and E. E. Falco, Gravitational Lenses, Springer-Verlag (1992).

[26] R. Narayan and M. Bartelmann, arXiv:astro-ph/9606001 (1996).

[27] J. P. Kneib et al., Astron. Astrophys. 303, 27 (1995).

[28] J. P. Kneib et al., Astrophys. J. 471, 643 (1996).

[29] D. Clowe et al., Astrophys. J. 648, 109 (2006).

[30] M. Bradac et al., Astrophys. J. 687, 959 (2008).

[31] Y. Sofue and V. Rubin. Annu. Rev. Astron. Astrophys. 39, 137 (2001).

[32] A. Klypin, H. Zhao and R. S. Somerville, Astrophys. J. 573, 597 (2002).

[33] W. Dehnen and J. Binney, Mon. Not. Roy. Astron. Soc. 294, 429 (1998).

[34] J. Wees and J. Bagger, Princeton University Press (1992).

[35] M. Drees, R. Godbole and P. Roy, World Scientific (2004).

[36] H. Baer and X. Tata, Cambridge University Press (2006).

[37] P. Binetruy, Oxford University Press (2006).

[38] N. Arkani-Hamed, S. Dimopoulos and G. R. Dvali, Phys. Lett. B 429, 170 (2005).

[39] L. Randall and R. Sundrum, Phys. Rev. Lett. 83, 3370 (1999).

[40] J. L. Feng, A. Rajaraman and F. Takayama, Phys. Rev. Lett. 91, 011302 (2003).

[41] G. D. Starkmann, A. Gould, R. Esmailzadeh and S. Dimopoulos, Phys. Rev. D 41, 3594 (1990).

[42] D. J. H. chung, E. W. Kolb and A. Riotto, Phys. Rev. Lett. 81, 4048 (1998).

[43] A. Kusenko and M. E. Shaposhnikov. Phys. Lett. B 418, 46 (1998).

[44] S. N. Ahmed et al., Phys. Rev. Lett. 92, 181301 (2004). 
[45] Y. Ashie et al., Phys. Rev. D 71, 112005 (2005).

[46] D. G. Michael et al., Phys. Rev. Lett. 97, 191801 (2006).

[47] T. Araki et al., Phys. Rev. Lett. 94, 081801 (2005).

[48] G. Jungman, M. Kamionkowski and K. Griest, Phys. Rep. 267, 195 (1996).

[49] M. E. Peskin and D. V. Schroeder, Addison Wesley (1995).

[50] J. F. Donoghue, E. Golowich and B. R. Holstein, Cambridge University Press (1992).

[51] H. Georgi and S. L. Glashow, Phys. Rev. Lett. 32, 438 (1974).

[52] D. I. Kazakov, arXiv:hep-ph/0012288 (2000).

[53] M. Shiozawa et al., Phys. Rev. Lett. 81, 3319 (1998).

[54] Y. Hayato et al., Phys. Rev. Lett. 83, 1529 (1999).

[55] K. Kobayashi et al., Phys. Rev. D 72, 052007 (2005).

[56] S. Dimopoulos and H. Georgi, Nucl. Phys. B 193, 150 (1981).

[57] S. Dawson, arXiv:hep-ph/9712464 (1997).

[58] G. L. Kane, C. F. Kolda, L. Roszkowski and J. D. Wells, Phys. Rev. D 49, 6173 (1994).

[59] S. R. Coleman and E. J. Weinberg, Phys. Rev. D 7, 1888 (1973).

[60] A. A. Belavin, A. Polyakov, A. Schwartz and Y. Tyupkin, Phys. Lett. B 59, 85 (1974).

[61] J. E. Kim, Physics Reports 150, 1-177 (1987).

[62] J. E. Kim and G. Carosi, arXiv:0807.3125v2[hep-ph].

[63] C. A. Baker et al., Phys. Rev. Lett. 97, 131801 (2006).

[64] R.D. Peccei and H.R. Quinn, Phys. Rev. Lett. 38, 1440 (1977).

[65] R.D. Peccei and H.R. Quinn, Phys. Rev. D 16, 1791 (1977).

[66] H. Georgi and L. Randell, HUTP-86/A025 (1986).

[67] S. Weinberg, Phys. Rev. Lett. 40, 223 (1978).

[68] F. Wilczek, Phys. Rev. Lett 40, 279 (1978).

[69] J. E. Kim, Phys. Rev. Lett. 43, 103 (1979). 
[70] M. A. Shifman, A. I. Vainshtein and V. I. Zakharov, Nucl. Phys. B 166, 493 (1980).

[71] M. Dine, W. Fischler and M. Srednicki, Phys. Lett. B 104, 199 (1981).

[72] A. R. Zhitnitskii, Sov. J. Nucl. Phys. 31, 260 (translation)(1980).

[73] S. L. Cheng, C. Q. Geng and W. T. Ni, Phys. Rev. D 52, 3132 (1995).

[74] M. W. Goodman and E. Witten, Phys. Rev. D 31, 3059 (1985).

[75] A. Kurylov and M. Kamionkowski, Phys. Rev. D 69, 063503 (2004).

[76] J. Lewin and P. Smith, Astropart. Phys. 6, 87 (1996).

[77] S. Arrenberg, private communication.

[78] M. T. Ressel et al., Phys. Rev. D 48, 5519 (1993).

[79] V. Dimitrov, J. Engel and S. Pittel, Phys. Rev. D 51, 291 (1995).

[80] J. Engel, Phys. Lett. B 264, 114 (1991).

[81] J. Navarro, C. Frenk and S. White, ApJ 490, 493 (1997) .

[82] B. Moore et al., ApJL 499, L5 (1998).

[83] M. Kamionkowski and A. Kinkhabwala, Phys. Rev. D 57, 3256 (1998).

[84] A. M. Green, Phys. Rev. D 66, 83003 (2002).

[85] M. Kamionkowski and S. Koushiappas, Phys. Rev. D 77, 103509 (2008).

[86] J. Read et al., Mon. Not. Roy. Astron. Soc. 397, 44 (2009).

[87] J. Read, G. Lake, O. Agertz and V. Debattista, Mon. Not. Roy. Astron. Soc. 389, 1041 (2008).

[88] G. Lake, AJ 98, 1554 (1989).

[89] J. Read et al., Proc. of Science (idm2008), 048 (2008).

[90] J. Oort, Bull. Astr. Soc. Neth. 6, 24 (1932).

[91] J. Oort, Bull. Astr. Soc. Neth. 15, 45 (1960).

[92] J. Bahcall, ApJ 287, 926 (1984).

[93] J. Holmberg and C. Flynn, Mon. Not. Roy. Astron. Soc. 313, 209 (2000).

[94] T. Statler, ApJ 344, 217 (1989). 
[95] D. Burstein, ApJ 234, 829 (1979).

[96] G. Gilmore and N. Reid, Mon. Not. Roy. Astron. Soc. 202, 1025 (1983).

[97] P. Yoachim and J. Dalcanton, AJ 131, 226 (2006).

[98] P. Yoachim and J. Dalcanton, ApJ 624, 701 (2005).

[99] S. Kazantzidis et al., arXiv:astro-ph/0708.1949 (2007).

[100] C. Brook, D. Kawata, B. Gibson and K. Freeman, ApJ 612, 894 (2004).

[101] M. Steinmetz et al., AJ 132, 1645 (2006).

[102] M. Perryman et al., A\&A 369, 339 (2001).

[103] K. Freese, P. Gondolo, H. J. Newberg and M. Lewis, Phys. Rev. Lett 92, 111301 (2004).

[104] C. Savage, K. Freese and P. Gondolo, Phys. Rev. D 74, 043531 (2006).

[105] L. Baudis, Int. J. Mod. Phys. A 21, 1925 (2006).

[106] M. C. Smith et al., Mon. Not. Roy. Astron. Soc. 379, 755 (2007).

[107] W. Dehnen and J. Binney, Mon. Not. Roy. Astron. Soc. 298, 387 (1999).

[108] J. Angle et al., Phys. Rev. Lett. 100, 021303 (2008).

[109] S. T. Lin et al., Phys. Rev. D 79, 061101 (2009).

[110] C. Aalseth et al., Phys. Rev. Lett. 101, 251301 (2008).

[111] A. Drukier, K. Freese and D. Spergel, Phys. Rev. D 33, 3495 (1986).

[112] G. Heusser, Ann. Rev. Nucl. Part. Sci. 45, 543 (1995).

[113] E. Armengaud et al., arXiv:0912.0805v1 [astro-ph.CO] (2009).

[114] P. L. Brink et al., Nucl. Instrum. Meth. A 559, 414 (2006).

[115] A. Broniatowski et al., Journal of Low Temperature Physics 151, 830 (2008).

[116] G. Angloher et al., Astropart. Phys. 31, 270 (2009).

[117] R. Bernabei et al., Phys. Lett. B 389, 757 (1996).

[118] R. Bernabei et al., Eur. Phys. J. C 56, 333 (2008).

[119] H. S. Lee et al., Phys. Rev. Lett. 99, 091301 (2007). 
[120] V. N. Lebedenko et al., Phys. Lett. D 80, 052010 (2009).

[121] E. Aprile and L. Baudis, Proc. of Science (idm2008), 018 (2008).

[122] S. Fiorucci et al., arXiv:0912.0482v1 [astro-ph.CO] (2009).

[123] A. Minamino et al., arXiv:0912.2405 [astro-ph.CO] (2009).

[124] M. G. Boulay and A. Hime, Astropart. Phys. 25, 179 (2006).

[125] P. Benetti et al., Astropart. Phys. 28, 495 (2008).

[126] A. Rubbia, J. Phys. Conf. Ser. 39, 129 (2006).

[127] M. G. Boulay et al., arXiv:0904.2930v1 [astro-ph.IM] (2009).

[128] E. Behnke et al., Science 319, 933 (2008).

[129] J. E. Gunn et al., ApJ 223, 1015 (1978).

[130] J. Silk and M. Srednicki, Phys. Rev. Lett. 53, 624 (1984).

[131] G. Lake, Nature 34, 39 (1990).

[132] M. Kuhlen, J. Diemand, P. Madau, ApJ 686, 262 (2008).

[133] V. Springel et al., Nature 456, 73 (2008).

[134] L. E. Strigari, ApJ 678, 614 (2008).

[135] K. Freese, Phys. Lett. B 167, 295 (1986).

[136] A. Gould, ApJ 321, 571 (1987).

[137] K. Griest, D. Seckel, Nucl. Phys. B 283, 681 (1987).

[138] L.M. Krauss, K. Freese, D.N. Spergel, W.H. Press , ApJ 299, 10001 (1985).

[139] J. Silk, K. Olive, M. Srednicki, Phys. Rev. Lett. 55, 257 (1985).

[140] M. Vogelsberger et al., arXiv:0812.0362v1 [astro-ph] (2008).

[141] M. Zemp et al., Mon. Not. Roy. Astron. Soc.394, 641 (2009).

[142] S. Desai et al., Phys. Rev. D 70, 083523 (2004).

[143] T. DeYoung et al., Journal of Physics: Conference Series 136, 022046 (2008).

[144] R. Abbasi et al., Phys. Rev. Lett. 102, 201302 (2009).

[145] D. N. Spergel, W. H. Press, ApJ 294, 663 (1985). 
[146] R. Ruiz de Austri, R. Trotta, L. Roszkowski, JHEP 0605, 002 (2006).

[147] E. Komatsu et al., Astrophys. J. Supp. 180, 330 (2008).

[148] P. Gondolo et al., JCAP 0407, 008 (2004).

[149] A. Achterberg et al., Astropart. Phys. 26, 129 (2006).

[150] A. Gould, Astrophys. J. 328, 919 (1988).

[151] A. Gould, Astrophys. J. 368, 610 (1991).

[152] J. Lundberg, J. Edsjö, Phys. Rev. D 69, 123505 (2004).

[153] T. Damour, L. M. Krauss, Phys. Rev. D 59, 063509 (1999).

[154] L. Bergström et al., JHEP 8, 10 (1999).

[155] A. H. G. Peter, Phys. Rev. D 79, 103531 (2009).

[156] A. H. G. Peter, Phys. Rev. D 79, 103533 (2009).

[157] D. Abrams et al., Phys. Rev. D 66, 122003 (2002).

[158] D. S. Akerib et al., Phys. Rev. D 68, 082002 (2003).

[159] I. Ambats et al., NUMI-L-337 (1998).

[160] S. Butterworth, Experimental Wireless 7, 536 (1930).

[161] Y. P. Varshni, Physica 34, 149 (1967).

[162] C. D. Thurmond, J. Electrochem. Soc. 122, 1133 (1975).

[163] W. Shockley, Solid State Electronics 2, 35 (1961).

[164] C. A. Klein, Journal of Applied Physics 39, 2029 (1968).

[165] C. A. Klein, IEEE Transactions Nucl. Sci. 15, 214 (1968).

[166] R. W. Ogburn, PhD thesis, Stanford University, 2008.

[167] W. Shockley, J. Appl. Phys. 9, 635 (1938).

[168] S. Ramo, Proc. IRE 27, 584 (1939).

[169] T. Shutt et al., Phys. Rev. Lett. 69, 3531 (1992).

[170] T. Shutt et al., Nucl. Instrum. Meth. A 444, 340 (2000).

[171] T. Shutt, PhD thesis, University of California, Berkeley, 1993. 
[172] V. Mandic, PhD thesis, University of California, Berkeley, 2004.

[173] J. P. Filippini, PhD thesis, University of California, Berkeley, 2008.

[174] S. Golwala, PhD thesis, University of California, Berkeley, 2000.

[175] B. S. Neganov and V. N. Trofimov, J. Exp. Theor. Phys. 28, 328 (1978).

[176] P. N. Luke, Journal of Applied Physics 64(12), 6858 (1988).

[177] A.R. Al-Derzi, R.L. Johnston, J.N. Murrell and J.A. Rodriguez-Ruiz, Molecular Physics 73, 265 (1991).

[178] H. Maris, Phys. Rev. B, (1990).

[179] H. Maris and S. Tamura, Phys. Rev. B, (1993).

[180] S. Tamura, J.A. Shields and J.P. Wolfe, Phys. Rev. B 44, 3001 (1991).

[181] J.A. Shields, S. Tamura and J.P. Wolfe, Phys. Rev. B 43, 4966 (1991).

[182] S. Tamura, Phys. Rev. B 27, 858 (1983).

[183] B.A. Young, B. Cabrera and A.T. Lee, Phys. Rev. Lett. 64, 2795 (1990).

[184] K. D. Irwin, Applied Physics Letters 66(15), 1998 (1995).

[185] B. A. Young et al., Journal of Applied Physics 91(10), 6516 (2002).

[186] B. A. Young et al., Journal of Applied Physics 86(12), 6975 (1999).

[187] J. Lindhard, M. S. V. Nielsen and P. Thomsen, Mat. Fys. Medd. Dan. Vid. Selsk. 33(10), 1-42 (1963).

[188] T. Shutt et al., Nucl. Instrum. Meth. A 444, 340 (2000).

[189] S. Agostinelli et al., Nucl. Instrum. Meth. A 506, 250 (2003).

[190] J. Allison et al., IEEE Trans. Nucl. Sci. 53, 270 (2006).

[191] T. Shutt et al., AIP Conf. Proc.: Low Temperature Detectors 605, 513 (2002).

[192] D. Leonard et al., Nucl. Instrum. Meth. A 591, 490 (2008).

[193] W. B. Wilson et al., Radiat. Prot. Dosimetry 115, 117 (2005).

[194] M. Tarka, Diploma thesis, RWTH-Aachen (2007).

[195] X. Qui, PhD thesis, University of Minnesota, in preperation.

[196] R. Hennings-Yeomans, PhD thesis, Case Western Reserve University (2008). 
[197] A. Fasso et al., arXiv:hep-ph/0306267 (2003).

[198] A. Fasso, A. Ferrari, J. Ranft and P. R. Sala, CERN-2005-10, INFN/TC_05/11, SLAC-R-77 (2005).

[199] http://mcnpx.lanl.gov/ .

[200] V. A. Kudryavtsev, arXiv:0810.4635 (2008).

[201] S. Yellin, Phys. Rev. D 66, 032005 (2002).

[202] S. Yellin, arXiv:0709.2701v1 [physics.data-an] (2007).

[203] G. J. Feldman and R. D. Cousins, Phys. Rev. D 57, 3873 (1998).

[204] V. Dimitrov, J. Engel and S. Pittel, Phys. Rev. D 51, 291 (1995).

[205] J. R. Ellis, K. A. Olive, Y. Santoso and V. C. Spanos, Phys. Rev. D 71, 095007 (2005).

[206] L. Roszkowski, R. Ruiz de Austri and R. Trotta, JHEP 07, 075 (2007).

[207] D. R. Tovey et al., Phys. Lett. B 488, 17 (2000).

[208] V. N. Lebedenko et al., Phys. Rev. Lett. 103, 151302 (2009).

[209] J. Angle et al., Phys. Rev. Lett. 101, 091301 (2008).

[210] R. Bernabei et al., Phys. Lett. B 480, 23 (2000).

[211] D. Smith and N. Weiner, Phys. Rev. D 64, 043502 (2001).

[212] D. Tucker-Smith and N. Weiner, Phys. Rev. D 72, 063509 (2005).

[213] S. Chang, G. D. Kribs, D. Tucker-Smith and N. Weiner, Phys. Rev. D 79, 043513 (2009).

[214] J. Angle et al., Phys. Rev. D 80, 115005 (2009).

[215] M. C. Smith et al., Mon. Not. Roy. Astron. Soc. 379, 755 (2007).

[216] R. W. Schnee et al., arXiv:astro-ph/0502435 (2005).

[217] A. J. Noble, New Astron. Rev. 49, 311 (2005).

[218] J. Neyman, Philos. Trans. R. Soc. London A 236, 333 (1937).

[219] R. M. Neal, Technical Report CRG-TR-93-1, Dept. of Computer Science, University of Toronto, available from www.cs.toronto.edu/ radford/res-mcmc.html 
[220] N. Metropolis et al., Journal of Chemical Physics 21, 1087 (1953).

[221] W. K. Hastings, Biometrika 57, 97 (1970).

[222] E. Arik et al., JCAP 0902:008 (2009).

[223] R. J. Creswick et al., Phys. Lett. B 427, 235 (1998).

[224] S. Cebrián et al., Astropart. Phys. 10, 397 (1999).

[225] E. Arik et al., arXiv:0810.4482.

[226] I. Reda and A. Andreas, National Renewable Energy Laboratory Technical report NREL/TP-560-34302 (2003). The document is available at www.osti.gov/bridge .

[227] Fermilab Alignment Group survey at Soudan Underground Laboratory, drawing H5.1, MN1002/3 (1999).

[228] M.S. Bartlett, Biometrica 40, 306 (1953).

[229] D. N. Lawley, Biometrica 43, 295 (1956).

[230] S. A. Murphy, A. W. Van Der Vaart, Journal of the American Statistical Association 95, 449 (2000).

[231] F. T. Avigone III et al., Phys. Rev. Lett. 81, 5068 (1998).

[232] A. Morales et al., Astropart. Phys. 16, 325 (2002).

[233] R. Bernabei et al., Phys. Lett. B 515, 6 (2001).

[234] M. Minowa et al., Phys. Lett. B 668, 93 (2008).

[235] G. G. Raffelt, Stars as Laboratories for Fundamental Physics, The University of Chicago Press (1996).

[236] S. Schönert et al., Nucl. Phys. B 145, 242 (2005).

[237] C. E. Aalseth et al., arXiv:0811.2446 [nucl-ex] (2008).

[238] R. Bernabei et al., Int. J. Mod. Phys. A 21, 1445 (2006).

[239] M. Pospelov, A. Ritz and M. Voloshin, Phys. Rev. D 78, 115012 (2008).

[240] D. B. Kaplan and K. M. Zurek, Phys. Rev. Lett, 96, 041301 (2006)

[241] P. Gondolo and G. G. Raffelt, arXiv:0807.2926v3 [astro-ph].

[242] D. -M. Mei, Z.-B. Yin and S. R. Elliot, arXiv:0903.2273v1 [nucl-ex]. 
[243] R. Bernabei et al., Nucl. Instrum. Meth. A 592, 297 (2008).

[244] R. Bernabei et al., arXiv:0912.4200 [physics.ins-det] (2009).

[245] V. Kudryavtsev, M. Robinson and N. J. C. Spooner, arXiv:0912.2983 [hep-ex] (2009). 